\title{
Hydrogen detection near surfaces and shallow interfaces with resonant nuclear reaction analysis
}

\author{
Markus Wilde and Katsuyuki Fukutani \\ Institute of Industrial Science, The University of Tokyo \\ 4-6-1 Komaba, Meguro-ku, 153-8505 Tokyo, Japan
}

This review introduces hydrogen depth profiling by nuclear reaction analysis (NRA) via the resonant ${ }^{1} \mathrm{H}\left({ }^{15} \mathrm{~N}, \alpha \gamma\right){ }^{12} \mathrm{C}$ reaction as a versatile method for the highly depth-resolved observation of hydrogen $(\mathrm{H})$ at solid surfaces and interfaces. The technique is quantitative, non-destructive, and readily applied to a large variety of materials. Its fundamentals, instrumental requirements, advantages and limitations are described in detail, and its main performance benchmarks in terms of depth resolution and sensitivity are compared to those of elastic recoil detection (ERD) as a competing method. The wide range of ${ }^{1} \mathrm{H}\left({ }^{15} \mathrm{~N}, \alpha \gamma\right){ }^{12} \mathrm{C}$ NRA applications in research of hydrogen-related phenomena at surfaces and interfaces is reviewed.

Special emphasis is placed on the powerful combination of ${ }^{1} \mathrm{H}\left({ }^{15} \mathrm{~N}, \alpha \gamma\right){ }^{12} \mathrm{C}$ NRA with surface science techniques of in-situ target preparation and characterization, as the NRA technique is ideally suited to investigate hydrogen interactions with atomically controlled surfaces and intact interfaces. In conjunction with thermal desorption spectroscopy, ${ }^{15} \mathrm{~N}$ NRA can assess the thermal stability of absorbed hydrogen species in different depth locations against diffusion and desorption. Hydrogen diffusion dynamics in the near-surface region, including transitions of hydrogen between the surface and the bulk, and between shallow interfaces of nanostructured thin layer stacks can directly be visualized. As a unique feature of ${ }^{15} \mathrm{~N}$ NRA, the analysis of Doppler-broadened resonance excitation curves allows for the direct measurement of the zero-point vibrational energy of hydrogen atoms adsorbed on single crystal surfaces.

Keywords: Nuclear reaction analysis, Hydrogen depth profiling, Surface and interface analysis, Hydrogen adsorption, Hydrogen absorption, Hydrogen diffusion

Corresponding authors:

M.Wilde (wilde@iis.u-tokyo.ac.jp), K. Fukutani (fukutani@iis.u-tokyo.ac.jp) 


\section{Contents}

\section{Introduction}

\section{Hydrogen depth profiling by resonant nuclear reaction analysis}

2.1 Principles of resonant NRA

2.1.1 Fundamentals

2.1.2 Merits of $\left.{ }^{1} \mathrm{H}\left({ }^{15} \mathrm{~N}, \alpha \gamma\right)\right)^{12} \mathrm{C}$

2.1.3 Energy loss and stopping power

2.1.4 Straggling

\subsection{Instrumental Aspects}

2.2.1 Ion beam generation (accelerator)

2.2.2 Measurement apparatus and sample

2.2.3 Depth resolution and range

2.2.4 $\gamma$-detection, background, and sensitivity

2.2.5 Radiation effects

2.2.6 Quantitation and calibration

2.2.7 Data analysis and simulation

\subsection{Comparison to ERDA}

2.3.1 Performance (depth resolution, sensitivity, probing range)

2.3.2 Versatility

\section{Applications}

\subsection{Surface Hydrogen}

3.1.1 A note on adsorption experiments with atomic hydrogen

3.1.2 Metals: (W(100), W(110), Rh(111), Ir(111), Ni(110), Ni(100), Ni(111), $\mathrm{Pt}(111), \mathrm{Cu}(111), \mathrm{Cu}(100), \mathrm{Cu}(110), \mathrm{Ag}(111)$; Ultrathin films and (surface) alloys: $\mathrm{Au} / \mathrm{Ir}(111), \mathrm{Au} / \mathrm{Pt}(111), \mathrm{Ag} / \mathrm{Pt}(111), \mathrm{Pt}(111)-\mathrm{Sn}(2 \times 2)$, $\left.\mathrm{Pt}(111)-\mathrm{Sn}(\mathrm{V} 3 \times \sqrt{3}), \mathrm{Mo}_{0.75} \mathrm{Re}_{0.25}, \mathrm{Pt}_{0.5} \mathrm{Ni}_{0.5}\right)$

3.1.3 Semiconductors (Diamond, $\mathrm{Si}(111), \mathrm{Si}(100), \mathrm{Si}_{(1-\mathrm{x})} \mathrm{Ge}_{\mathrm{x}}(100)$ )

3.1.4 Insulators $\left(\mathrm{ZnO}\left((10 \overline{1} 0), \mathrm{SrTiO}_{3}(001)\right)\right.$

\subsection{Absorbed Hydrogen}

3.2.1 General aspects of hydrogen absorption at metal surfaces

3.2.2 NRA observation of absorbed hydrogen

3.2.3 Metals

3.2.3.1 Titanium(0001) 
3.2.3.2 Zirconium(0001), (1010)

3.2.3.3 Palladium(100)

3.2.3.4 $\mathrm{Pd}_{70} \mathrm{Au}_{30}(110)$

3.2.3.5 Hydrogen trapping at internal defects

3.2.3.6 Modified surface layers

3.2.3.7 Thin films and superlattices

3.2.3.8 Nanoparticles

3.2.4 Semiconductors: $\mathrm{H}$ in thin films

3.2.5 Insulators

3.2.5.1 $\mathrm{H}$ in near-surface regions of oxides and minerals

3.2.5.2 H-redistribution in electrochromic multilayers

3.2.5.3 $\mathrm{H}$ in ultrathin dielectric films $\left(\mathrm{SiO}_{2}, \mathrm{HfSiO}_{x}, \mathrm{HfO}_{2}, \mathrm{SiON}\right)$

\subsection{Interfacial hydrogen}

3.3.1 $\mathrm{H}$ at MOS interfaces and its relation to device reliability

3.3.2 $\mathrm{H}$ at metal/silicon interfaces

3.3.3 $\mathrm{H}$ at metal-insulator interfaces

\section{Conclusions}

\section{Appendix A}

Stopping cross sections of ${ }^{15} \mathrm{~N}$ at 6.385 and $13.35 \mathrm{MeV}$ in elemental targets

\section{Appendix B}

Elastic recoil detection (ERD)
a. Principles
b. Sensitivity
c. Depth resolution
d. Advanced detection systems

\section{References}




\section{Acronyms}

\begin{tabular}{|c|c|}
\hline AES & Auger electron spectroscopy \\
\hline BGO & Bismuth germanium oxide $\left(\mathrm{Bi}_{4} \mathrm{Ge}_{3} \mathrm{O}_{12}\right)$ \\
\hline CRB & Cosmic radiation background \\
\hline CVD & Chemical vapor deposition \\
\hline $\operatorname{ERD}(A)$ & Elastic recoil detection (analysis) \\
\hline DFT & Density functional theory \\
\hline FET & Field effect transistor \\
\hline FGA & Forming gas annealing \\
\hline HAS & Helium atom scattering \\
\hline $\mathrm{H}_{\mathrm{at}}$ & Atomic hydrogen \\
\hline$H-E R D(A)$ & High-resolution elastic recoil detection (analysis) \\
\hline HIERD & Heavy ion elastic recoil detection \\
\hline HREELS & High resolution electron energy loss spectroscopy \\
\hline IAEA & International Atomic Energy Agency \\
\hline IRAS & Infrared absorption spectroscopy \\
\hline LAPW & Linear augmented plane wave \\
\hline LEED & Low energy electron diffraction \\
\hline LERS & Low energy recoil scattering \\
\hline MBE & Molecular beam epitaxy \\
\hline MOS & Metal-oxide-semiconductor \\
\hline$N R(A)$ & Nuclear reaction (analysis) \\
\hline PSD & Position sensitive detector \\
\hline RBS & Rutherford backscattering \\
\hline PIXE/PIGE & Particle induced X-ray/Gamma-ray emission \\
\hline $\mathrm{ROI}$ & Region of interest (energy integration window in $\gamma$-spectroscopy) \\
\hline SNICS & Source of negative ions by Cs sputtering \\
\hline SSBD & Silicon surface barrier detector \\
\hline SSD & Solid state detector \\
\hline STM & Scanning tunneling microscopy \\
\hline TC & Transmission channeling \\
\hline
\end{tabular}




$\begin{array}{ll}\text { TD(S) } & \text { Thermal desorption (spectroscopy) } \\ \text { TOF } & \text { Time-of-flight } \\ \text { UHV } & \text { Ultrahigh vacuum } \\ \text { UPS } & \text { Ultraviolet photoelectron spectroscopy } \\ \text { XPS } & \text { X-ray photoelectron spectroscopy }\end{array}$

\section{Symbols}

\begin{tabular}{|c|c|}
\hline$\alpha_{i}$ & Incidence angle (ion beam direction relative to the surface normal) \\
\hline$A$ & $\begin{array}{l}\text { Mass ratio }\left(M_{2} / M_{1}\right) \text { of incident }\left(M_{1}\right) \text { and scattered ion }\left(M_{2}=M_{s}\right) \text { or } \\
\text { recoiled target atom }\left(M_{2}=M_{r}\right)\end{array}$ \\
\hline$B$ & Magnetic flux [Gauss] \\
\hline$c$ & Speed of light in vacuum $\left[2.998 \times 10^{8} \mathrm{~m} \mathrm{~s}^{-1}\right]$ \\
\hline$c_{b}$ & Bulk $\mathrm{H}$ concentration $\left[\mathrm{cm}^{-3}\right]$ \\
\hline$c_{\text {ref }}$ & Reference $\mathrm{H}$ concentration in a standard for sensitivity calibration \\
\hline$d$ & Depth in the target (measured perpendicular from the surface) [nm] \\
\hline$D$ & $\mathrm{H}$ diffusion coefficient $\left[\mathrm{m}^{2} \mathrm{~s}^{-1}\right]$ \\
\hline$\delta_{D}$ & Gaussian width parameter of Doppler broadening distribution [keV] \\
\hline$\delta_{b}$ & Gaussian width parameter of incident beam energy distribution [keV] \\
\hline$\delta E_{\mathrm{d}}$ & Channel width of ERD detection energy \\
\hline$\delta_{G}$ & Total Gaussian energy broadening (Doppler, straggling, beam) [keV] \\
\hline$\delta_{s}(z)$ & Gaussian width parameter of energy straggling distribution [keV] \\
\hline$\Delta E$ & Energy loss (due to ion stopping in a target material) [keV] \\
\hline$\Delta E_{\mathrm{b}}$ & Beam energy spread (FWHM) [keV] \\
\hline$\Delta E_{\mathrm{tot}}$ & Total energy width (FWHM) of the NRA instrumental function [keV] \\
\hline$\Delta h$ & Thickness of a thin film or height of an adlayer [nm] \\
\hline$\Delta H_{\mathrm{s}}$ & Enthalpy of $\mathrm{H}$ solution $[\mathrm{eV}]$ \\
\hline$\Delta \phi$ & Work function change $[\mathrm{eV}]$ \\
\hline$\Delta \Omega$ & Detector solid angle $[\mathrm{sr}]$ \\
\hline$e$ & Elemental charge $\left(1.60218 \times 10^{-19} \mathrm{As}\right)$ \\
\hline$\varepsilon$ & Stopping cross section $\left[\mathrm{eV} \mathrm{cm}{ }^{2} / 10^{15}\right.$ atoms] or $\left[\mathrm{MeV} /\left(\mathrm{mg} / \mathrm{cm}^{2}\right)\right]$ \\
\hline E & Energy (general) [eV] \\
\hline
\end{tabular}




\begin{tabular}{|c|c|}
\hline$E_{\text {ads }}$ & Adsorption energy [eV] \\
\hline$E_{\mathrm{d}}$ & Detection energy channel (ERD) \\
\hline$E_{\text {des }}$ & Activation energy for desorption [eV] \\
\hline$E_{\text {diff }}$ & Activation energy of $\mathrm{H}$ diffusion [eV] \\
\hline$E_{\mathrm{H}}$ & Zero-point vibrational energy of surface-adsorbed $\mathrm{H}$ atoms \\
\hline$E_{\mathrm{i}}$ & Incident ion beam energy in NRA \\
\hline$E_{1}$ & Incident projectile ion energy in ERD and RBS \\
\hline$E_{\mathrm{R}}$ & Resonance energy (in a nuclear reaction cross section) \\
\hline$E_{\mathrm{r}}$ & Energy of a recoiled target atom \\
\hline$E_{\mathrm{T}}$ & Kinetic (translational) energy \\
\hline$E_{\text {th }}$ & Threshold energy (of an endothermic nuclear reaction) \\
\hline$F\left(E, E_{\mathrm{i}}, Z\right)$ & $\begin{array}{l}\text { NRA instrumental function; its convolution with the } \mathrm{H} \\
\text { depth distribution yields the NR excitation curve } Y\left(E_{\mathrm{i}}\right)\end{array}$ \\
\hline$\phi$ & Fluence (ion beam exposure) [ions $\left./ \mathrm{cm}^{2}\right]$ \\
\hline$\Gamma$ & Width of a nuclear reaction cross section resonance [keV] \\
\hline$\theta_{\mathrm{H}}$ & Hydrogen surface coverage [ML] \\
\hline$\theta_{\mathrm{R}}$ & Recoil angle (ERD, laboratory system) \\
\hline$K$ & $\gamma$-detection efficiency factor \\
\hline$k_{\mathrm{B}}$ & Boltzmann constant \\
\hline$L_{D}$ & (Thermal) diffusion length in a time interval $\Delta t, L_{D}=(D \cdot \Delta t)^{1 / 2}[\mathrm{~m}]$ \\
\hline$m_{\mathrm{e}}$ & Electron rest mass \\
\hline$m_{\mathrm{H}}$ & Proton mass \\
\hline$M_{1}$ & Mass of the projectile ion \\
\hline$M_{2}$ & Mass of a target atom (scattered or recoiled) \\
\hline$m_{C}$ & Mass of a carbon ion \\
\hline$m_{\mathrm{H}}$ & Mass of a proton \\
\hline$m_{\mathrm{N}}$ & Mass of a nitrogen ion \\
\hline$M_{\mathrm{R}}$ & Mass of a recoiled target atom \\
\hline$n_{\mathrm{H}}$ & Hydrogen number density $\left[\mathrm{cm}^{-3}\right]$ \\
\hline$N$ & Atomic density $\left[\mathrm{cm}^{-3}\right]$ \\
\hline$N_{\mathrm{i}}$ & Incident beam flux $\left[\mathrm{cm}^{-2} \mathrm{~s}^{-1}\right]$ \\
\hline
\end{tabular}




\begin{tabular}{|c|c|}
\hline$N_{\mathrm{S}}$ & Surface areal density [atoms $\mathrm{cm}^{-2}$ ] \\
\hline$q$ & Charge state (projectile ion) \\
\hline$Q_{\mathrm{i}}$ & Acquisition time-integrated incident beam charge $[\mathrm{C}]$ \\
\hline$Q_{s}$ & Straggling cross section $\left[\mathrm{eV}^{2} / \mathrm{nm}\right]$ \\
\hline$R$ & Probing depth range $[\mu \mathrm{m}]$ \\
\hline$s_{0}$ & Initial sticking coefficient \\
\hline$S$ & Stopping power $(\mathrm{dE} / \mathrm{dz})\left[\mathrm{keV} \mathrm{nm^{-1 } ]}\right.$ \\
\hline$S_{S}$ & Solution entropy $[\mathrm{eV} / \mathrm{K}]$ \\
\hline$\sigma(E)$ & Nuclear reaction cross section [barn] \\
\hline$\sigma_{\text {off }}$ & Off-resonant nuclear reaction cross section \\
\hline$\sigma_{\mathrm{R}}$ & Nuclear reaction cross section (resonance amplitude)[barn] \\
\hline$\sigma_{\mathrm{r}}$ & Rutherford recoil cross section [barn] \\
\hline$\sigma_{s}$ & Straggling cross section [keV] \\
\hline$T$ & Temperature $[\mathrm{K}]$ \\
\hline$T_{\mathrm{S}}$ & Surface temperature $[\mathrm{K}]$ \\
\hline$v$ & ion velocity $\left[\mathrm{m} \mathrm{s}^{-1}\right]$ \\
\hline$V_{\mathrm{i}}$ & Injection voltage (accelerator ion source) [keV] \\
\hline$V_{\mathrm{T}}$ & Terminal voltage (Tandem accelerator) [keV] \\
\hline$v_{0}$ & Bohr velocity $\left(2.188 \times 10^{6} \mathrm{~m} \mathrm{~s}^{-1}\right)$ \\
\hline$\Omega_{\mathrm{s}}$ & Bohr straggling cross section $\left[\mathrm{cm}^{2}\right]$ \\
\hline$Y\left(E_{\mathrm{i}}\right)$ & Excitation function of the nuclear reaction ( $\gamma$-yield vs. $E_{\mathrm{i}}$ ) \\
\hline$z$ & Ion trajectory length below the target surface, $z=d / \cos \alpha_{i}[\mathrm{~nm}]$ \\
\hline Z & Nuclear charge \\
\hline$Z_{1}$ & Nuclear charge of the projectile ion \\
\hline$Z_{2}$ & Nuclear charge of a target element \\
\hline
\end{tabular}

\section{Units}

C Coulomb (1 C = $1 \mathrm{As})$

$\mathrm{L} \quad$ Langmuir (unit of gas exposure: $1 \mathrm{~L}=1.33 \times 10^{-4} \mathrm{~Pa} \cdot \mathrm{s}$ )

$\mathrm{ML} \quad$ Monolayer (relative unit of $\mathrm{H}$ coverage)

$\mathrm{mb} \quad$ Millibarn, cross section unit. $1 \mathrm{mb}=10^{-3}$ barn $=10^{-27} \mathrm{~cm}^{2}$ 


\section{Introduction}

The great wealth of surface and interface interactions of hydrogen and water has caught the keen attention of the Surface Science community for a long time [1-8]. The weak Van-der-Waals forces (and surface-catalyzed ortho-para conversion processes [9-16]) in the physisorbed state of molecular $\mathrm{H}_{2}$ are of lasting fundamental interest, and recently heightened from the viewpoint of hydrogen in energy applications [17, 18]. Dissociative chemisorption [19-24], surface diffusion, and reaction of adsorbed $\mathrm{H}$ atoms with coadsorbates enable hydrogenation catalysis and fuel cell reactions. Recombination of $\mathrm{H}_{2}$ is important in astrophysics as well as in photocatalysis and electrochemistry. Hydrogen surfactant effects in epitaxy and passivation of semiconductor interfaces impact fabrication and reliability of electronic devices. Adsorption of hydrogen or water can tremendously change the physical and chemical properties of the substrate surface, giving rise to work function shifts, relaxation lifting, reconstruction, conductivity, hydrophilicity, corrosion, etc. Owing to its light mass, the dynamics of hydrogen are subject to quantum effects that may influence the rate of reactions, diffusion, and surface penetration at low temperatures.

Many materials also exhibit volume solubility for hydrogen [25-27]. Bulkdissolved hydrogen may dramatically affect the electrical and mechanical properties of its host [27-29]. Metal hydride formation, essential for hydrogen storage $[18,30]$ and optically switchable windows [31-33], the embrittlement of technical alloys [34], 
getter pumping and $\mathrm{H}_{2}$-outgassing in the ultrahigh vacuum (UHV) environment [35, 36], the permeation through diffusion membranes for $\mathrm{H}_{2}$ purification [37], $\mathrm{H}$-induced conductivity [38, 39], or, conversely, dopant passivation in semiconductors [40], are but a few prominent examples to highlight the diversity of bulk hydrogen-material interactions and their enormous technological impact. Interfacial hydrogen is recognized to influence the electrical performance of Schottky-contacts [41-43] and semiconductor devices [44-46].

Despite its ubiquitous abundance, hydrogen behaves rather elusive in standard surface analysis, as only a few techniques can detect it directly. Since hydrogen has only a single electron and interacts weakly with common analytical electron or X-ray probes, it is barely visible by low energy electron diffraction (LEED) and cannot be detected by photoemission (UPS, XPS) and Auger electron spectroscopy (AES). Widely utilized Thermal Desorption Spectroscopy (TDS) removes hydrogen from the sample and is in this sense destructive to the original adsorption (or absorption) state. Vibrational techniques such as reflection absorption infrared spectroscopy (RAIS), high resolution electron energy loss spectroscopy (HREELS), and helium atom scattering (HAS) are non-invasive, hydrogen-sensitive, and capable of identifying surface-adsorbed $\mathrm{H}$ species, but they are neither quantitative nor able to reveal the presence and depth location of hydrogen below surfaces [47] [48]. Hence, the distinction of hydrogen species on a surface from those situated closely below a surface or deeper in the bulk and their respective quantifications pose delicate analytical challenges. 
This review therefore introduces hydrogen depth profiling by nuclear reaction analysis (NRA) via the resonant ${ }^{1} \mathrm{H}\left({ }^{15} \mathrm{~N}, \alpha \gamma\right){ }^{12} \mathrm{C}$ reaction as a powerful tool for the investigation of hydrogen near solid surfaces and interfaces, which can ideally complement conventional methods of surface analysis and overcome the said limitations with respect to hydrogen detection. Resonant ${ }^{1} H\left({ }^{15} \mathrm{~N}, \alpha \gamma\right){ }^{12} \mathrm{C}$ NRA determines hydrogen surface coverages and absorbed concentrations quantitatively, non-destructively, and, in particular at $6.385 \mathrm{MeV}$, with high depth resolution. The specific nuclear reaction renders ${ }^{15} \mathrm{~N}$-NRA sensitive to the proton only, i.e., it is selective to the nucleus of the ${ }^{1} \mathrm{H}$ isotope, yet indifferent towards its chemical oxidation state.

Among NRA variants that use other nuclear reactions, the resonant ${ }^{1} \mathrm{H}\left({ }^{15} \mathrm{~N}, \alpha \gamma\right){ }^{12} \mathrm{C}$ technique at $6.385 \mathrm{MeV}$ with energy monochromatized ${ }^{15} \mathrm{~N}$ ion beams is appreciated for its excellent near-surface depth resolution in the order of a few nanometers, for its high sensitivity ( $\mathrm{H}$ concentrations of $\sim 10^{18} \mathrm{~cm}^{-3}$ ( 100 at. ppm) in bulk materials and $\sim 10^{13} \mathrm{~cm}^{-2}$ ( 1\% of a monolayer) on surfaces), and a reasonably versatile indepth probing range of at least $2 \mu \mathrm{m}$ [49-53]. As the measurement delivers absolute hydrogen concentrations, ${ }^{15} \mathrm{~N}-\mathrm{NRA}$ is frequently applied as a reference for the calibration of other $\mathrm{H}$-sensitive detection methods, especially infrared absorption spectroscopy $[54,55]$. An eminent advantage that distinguishes NRA from other forms of depth-resolved elemental analysis such as sputter profiling or Secondary Ion Mass Spectroscopy (SIMS) is its ability to obtain the in-depth $\mathrm{H}$-concentration information non-destructively. Thus the benefits of ${ }^{1} \mathrm{H}\left({ }^{15} \mathrm{~N}, \alpha \gamma\right){ }^{12} \mathrm{C}$ NRA for hydrogen analysis at surfaces and interfaces are near at hand: Subtle distinctions of surface- 
adsorbed and near-surface absorbed $\mathrm{H}$ are routinely achieved on a depth scale of a few nanometers $[51,52,56,57]$. Under grazing ${ }^{15} \mathrm{~N}$ ion incidence conditions, the depth resolution can be enhanced further to below $1 \mathrm{~nm}$ [58], allowing for monolayer depth distinctions in particular situations [59]. Even the discrimination of surface-adsorbed from volume-absorbed hydrogen in size-controlled nanocrystals has recently been achieved [60]. NRA is particularly powerful in investigating hydrogen diffusion processes in the near surface region, including transitions of hydrogen between the surface and the subsurface crucial to the hydrogen absorption phenomenon. Especially in combination with TDS unambiguous identifications of thermal desorption features and assignments to hydrogen in different depth locations become possible, thus allowing for a depth-resolved assessment of the thermal stability of respective $\mathrm{H}$ species against diffusion and desorption $[61,62]$. The essentially non-destructive nature of the NRA measurement enables repeated analysis of the same specimen conditioned by in-situ treatments. Evidently this is an enormous advantage in studies of adsorbed and absorbed hydrogen states at well-defined surfaces that are in principally sensitive to air contact, as well as for the investigation of hydrogen interactions with intact interfaces.

Besides delivering depth information, for which NRA has been devised primarily, the ${ }^{15} \mathrm{~N}$ technique is also suited for the analysis of surface hydrogen, and its powerful potential can be exploited fully on well-defined single crystal targets. As a unique feature in addition to the quantitative coverage information ${ }^{15} \mathrm{~N}$ NRA provides, the pronounced Doppler Effect in the nuclear reaction kinematics allows for direct 
measurements of the zero-point vibrational energy of hydrogen atoms in their chemical bonds to the surface [63-67]. This quantity is entirely inaccessible with other methods of vibrational spectroscopy that probe vibrational excitations. Through the zero-point energy the degree of vibrational anharmonicity of the hydrogen adsorption potential between the ground and first excited state can be assessed, which is related to the activation barrier for surface diffusion and to the debated possibility that the hydrogen wave function is delocalized on certain transition metal surfaces $[68,69]$. To a limited extent, the zero-point vibrational energy can even be used to discriminate different chemically bound species of surface hydrogen [70].

In view of these capabilities for the analysis of surface and interfacial hydrogen, it is striking that NRA applications to single crystals or thin film systems with welldefined interfaces, in contrast to mere bulk $\mathrm{H}$ concentration evaluations, have remained comparatively rare until rather recently, and that apparently the potential of ${ }^{15} \mathrm{~N}$-NRA for surface-near hydrogen analysis is not as widely recognized in the Surface Science community as it might deserve. As we will show, the instrumental techniques for ion beam analysis and UHV-based surface characterization are well compatible, and the combination of NRA with standard Surface Science methods is a versatile and powerful one.

Since its introduction by Lanford in 1976 [49], NRA via the $6.385 \mathrm{MeV}$ resonance of the ${ }^{1} \mathrm{H}\left({ }^{15} \mathrm{~N}, \alpha \gamma\right){ }^{12} \mathrm{C}$ reaction has matured into a well-established ion beam technique for the quantitative measurement of hydrogen depth distributions [51, 52, 71, 72]. Historically, NRA was first mainly applied to determine $\mathrm{H}$ concentrations in 
bulk materials and thin films. A large body of literature describes NRA measurements of volume-dissolved hydrogen, related to phenomena such as hydrogen-induced embrittlement [73], the retention of $\mathrm{H}$ isotopes in plasma-exposed fusion reactor wall materials [74-76], the $\mathrm{H}$ content in CVD fabricated films, or the depth-range and internal diffusion of ion implanted hydrogen. Such studies were seldom concerned about the surface quality of their targets, which might bear uncontrolled surface layers, modified by natural oxidation, contamination, or various treatements. The resulting hydrogen profiles often showed ill-defined 'near-surface regions', where the $\mathrm{H}$-content differed considerably from the value deep in the bulk. Focusing on this $\mathrm{H}$ bulk content, many such studies simply disregarded the surface region altogether. A notable exeption is the thickness evaluation of near-surface hydration layers found on weathered silicate materials $[50,77]$ with ${ }^{15} \mathrm{~N}$ NRA, which has later been proposed as a method for age dating of natural minerals or of glass or quartz artifacts [78-80] (Section 3.2.5.1). The depth-resolving powers of ${ }^{15} \mathrm{~N}$ NRA have also been applied to observe $\mathrm{H}$ redistribution associated with electrochromic transitions in multilayer systems $[81,82]$ (Section 3.2.5.2). NRA measurements of hydrogen at surfaces, however, did not emerge before the late 1980s after the discovery of the Doppler Effect that broadens the width of the nuclear reaction resonance [63-66].

The first truly surface-analytical applications of ${ }^{1} \mathrm{H}\left({ }^{15} \mathrm{~N}, \alpha \gamma\right){ }^{12} \mathrm{C}$ NRA allowing for measurements of absolute $\mathrm{H}$ coverages and of the zero-point vibrational energy of $\mathrm{H}$ at well-defined in-situ prepared single crystal targets were developed in Japan by Murata and Komaki $[65,83]$. This first 'marriage' of ${ }^{1} \mathrm{H}\left({ }^{15} \mathrm{~N}, \alpha \gamma\right){ }^{12} \mathrm{C}$ NRA with Surface Science instrumentation has largely promoted the recent progress in research of 
hydrogen in the near-surface region of atomically controlled targets. At first, $\mathrm{H}$ adsorbed on $\mathrm{W}(100)$ and $\mathrm{W}(111)$ was examined as a well-known benchmark system in Surface Science, which demonstrated the potential of the NRA technique to characterize hydrogen adsorbed on single crystal surfaces $[65,83]$. Next, it was confirmed that new scientific information on hydrogen at surfaces and interfaces can be obtained with NRA using the $\mathrm{Pb} / \mathrm{H} / \mathrm{Si}(111)[42,59]$ and $\mathrm{Ag} / \mathrm{H} / \mathrm{Si}(111)[43]$ model systems (Section 3.3.2). This was followed by the successful real space observation of the zero-point vibrational motion of $\mathrm{H}$ atoms chemisorbed on $\mathrm{Si}(111)$ and $\mathrm{Pt}(111)$ surfaces through a ${ }^{1} \mathrm{H}\left({ }^{15} \mathrm{~N}, \alpha \gamma\right){ }^{12} \mathrm{C}$ Doppler broadening analysis [67] (Section 3.1). Later, also the possibilities to study the 'dynamical' behavior of surface and interfacial hydrogen, such as transient and strongly temperature dependent nearsurface hydrogen distributions observable after hydrogen absorption at metal single crystals $[61,84]$ (the first half of Section 3.2 .3 ), the thermally driven migration of hydrogen atoms between the bulk and the surface of $\mathrm{H}$-absorbing metals [62] (Section 3.2.3.1), as well as hydrogen redistribution phenomena between interfaces of microelectronic device structures $[45,46]$ (Section 3.3.1) were added to the portfolio of successful ${ }^{15} \mathrm{~N}$ NRA applications to the material science of hydrogen at surfaces, at interfaces, and in nanostructured systems (e.g., Section 3.2.3.8).

The present Surface Science Report focuses on ${ }^{1} \mathrm{H}\left({ }^{15} \mathrm{~N}, \alpha \gamma\right){ }^{12} \mathrm{C}$ NRA applications that investigate hydrogen at and within a 'close' range of well-characterized solid surfaces and shallow interfaces. An important criterion to select literature for this review has hence been the sample quality. Surfaces should have been as clean and structurally controlled as possible (ideally single-crystalline), and interfaces been 
abrupt and comprised by layers of uniform thicknesses. In order to exclude an enormous amount of literature on ${ }^{15} \mathrm{~N}$ NRA applications that simply determined the $\mathrm{H}$ content in the volume of materials, we (arbitrarily) restricted our region of interest to a depth of $\sim 100 \mathrm{~nm}$ below the surface.

A second important objective of this review in addition to describing the ${ }^{15} \mathrm{~N}$ NRA technique has been to provide a comprehensive (although by far not exhaustive) introduction to the rich 'materials science' of hydrogen at solid surfaces and interfaces. To this end, as well as in order to highlight the relevance of the information that NRA of hydrogen near surfaces and interfaces has contributed in many research fields, due effort has been paid throughout to outline the scientific background for the reviewed NRA investigations. In order to cover as completely as possible the full range of NRA applications in the surface science of hydrogen, we included also several ${ }^{2} \mathrm{D}\left({ }^{3} \mathrm{He}, \mathrm{p}\right){ }^{4} \mathrm{He}$ NRA studies at well-defined targets (e.g., Table 3 in Sect. 3.1.2, Sections 3.2.3.2, 3.2.3.5, and 3.2.3.6). NRA via the D-specific, nonresonant ${ }^{2} \mathrm{D}\left({ }^{3} \mathrm{He}, \mathrm{p}\right)^{4} \mathrm{He}$ reaction principally provides no depth resolution but quantifies the $D$ content in a $\sim 0.5 \mu \mathrm{m}$ deep near-surface region (cf. Sect. 2.1.2), so that - in absence of absorbed deuterium - it can determine absolute surface coverages of adsorbed D. (These ${ }^{3} \mathrm{He}$ NRA studies did not require depth resolution but could as well have been performed with $\left.{ }^{15} \mathrm{~N} N R A\right)$. We hope that the substantial information on important aspects of hydrogen interactions with surfaces and interfaces of selected materials compiled in this review will be appreciated as helpful by many interested readers. 
The article is organized as follows: Chapter 2 will first provide a thorough description of the relevant fundamentals and instrumental aspects of the ${ }^{1} \mathrm{H}\left({ }^{15} \mathrm{~N}, \alpha \gamma\right){ }^{12} \mathrm{C}$ NRA technique for surface applications. A brief comparison to its main competitor will follow, namely high-resolution Elastic Recoil Detection (H-ERD), which (under optimum provisions) can achieve similar performance as ${ }^{15} \mathrm{~N}$ NRA in terms of depth resolution and sensitivity. Chapter 3 then reviews (mainly $\left.{ }^{1} \mathrm{H}\left({ }^{15} \mathrm{~N}, \alpha \gamma\right){ }^{12} \mathrm{C}\right)$ NRA applications to hydrogen adsorbed at surfaces, followed by absorbed hydrogen in shallow bulk regions $(<100 \mathrm{~nm}$, including thin films, multilayers, and nanoparticles), and, finally, at interfaces. Each subchapter contains sections for different classes of analyzed material such as metals, semiconductors, and insulators. Further subsections then single out particular materials or hydrogen interaction phenomena investigated by (mainly ${ }^{1} \mathrm{H}\left({ }^{15} \mathrm{~N}, \alpha \gamma\right){ }^{12} \mathrm{C}$ ) NRA. Chapter 4 draws brief conclusions. 


\section{Hydrogen depth profiling by resonant nuclear reaction analysis}

This chapter describes the principle and experimental requirements of hydrogen depth profiling by resonant nuclear reaction analysis. It is meant as a practical compendium providing the relations necessary to enable even the reader without profound experience in ion beam analysis or nuclear physics to measure, analyze, and interpret resonant NRA data without extensive need for external references. Several original papers and earlier reviews offer general introductions of $\mathrm{H}$ depth profiling by resonant NRA [49-53, 72, 85-90]. Surface-analytical NRA applications with a particular focus on high-resolution measurements at atomic-level controlled single crystal and interface targets have appeared only rather recently $[56-58,65,83$, 91].

\section{$2.1 \quad$ Principles of resonant NRA}

\subsubsection{Fundamentals}

In the laboratory a nuclear reaction can be induced by colliding an accelerated projectile nucleus (a) with a resting target nucleus $(A)$ at an energy that exceeds the Coulomb barrier between the two nuclei. In general, a stable residual nucleus (B) is formed in one direct or several intermediate steps under emission of one or several 
particles (B b') (protons, $\alpha$-particles, $\beta$-emission, $\gamma$-rays, etc.) that carry characteristic energies. The general notation for a nuclear reaction $(a+A \rightarrow B+b)$ is $A(a, b) B$. In nuclear reaction analysis (NRA) one determines the concentration of a nucleus $(A)$ in a specimen by bombarding the target with accelerated projectile ions (a) and detecting the particles (b) emitted in the $A(a, b) B$ nuclear reaction. The reaction yield, $Y_{B}$ determines the sensitivity to detect an areal density of $(A)$ nuclei $\left(n_{A}\left[\mathrm{~cm}^{-2}\right]\right)$ and is proportional to the flux $\left(N_{\mathrm{i}}\left[\mathrm{cm}^{-2} \mathrm{~s}^{-1}\right]\right)$ of incident projectiles and to the energydependent cross section of the nuclear reaction, $\sigma(E)\left[\mathrm{cm}^{2}\right]: Y_{\mathrm{b}}(E)=\sigma(E) N_{\mathrm{i}} n_{\mathrm{A}}$. Certain collision energies are resonant to excited levels of stable nuclei that may form as reaction intermediates and are referred to as compound nuclei. At such energy resonances $\left(E_{R}\right)$ the reaction cross section $\sigma_{R}=\sigma\left(E_{R}\right)$ can be enhanced by several orders of magnitude. Owing to a multitude of excited states in light nuclei $(Z<20)$ that are accessible in particle collisions at several MeV energy $[92,93][94]$, a vast variety of reaction channels exists, but only a very few have practical importance. In general, nuclear reaction resonances suited to analytical depth profiling have the following characteristics: (1) a large resonant cross section $\sigma_{R}$ and a small offresonance cross section ( $\rightarrow$ sensitivity), (2) a narrow resonance width $(\Gamma)$ ( $\rightarrow$ depth resolution), (3) a low resonance energy $E_{\mathrm{R}}(\rightarrow$ accelerator size required to generate the projectile beam), and (4) a large separation from the next resonance at higher energy $(\rightarrow$ probing depth range).

Table 1 lists several resonant nuclear reactions applicable to hydrogen $(H)$ detection that fulfill the above criteria more or less ideally. The data are compiled from studies that explored the potential of various nuclear reactions for $\mathrm{H}$ profiling 
$[51,53,71,85,86,89]$. The product $\sigma_{R} \Gamma$ is proportional to the integral resonant cross section and hence defines the achievable sensitivity for $\mathrm{H}$ detection (Eqs. (2.11) and (2.12)); these reaction yield values are also given relative to $\sigma_{R} \Gamma$ of ${ }^{1} \mathrm{H}\left({ }^{15} \mathrm{~N}, \alpha \gamma\right){ }^{12} \mathrm{C}$ at $6.385 \mathrm{MeV}$ for comparison. The $\Gamma / S$ entry is a rough indicator for the near-surface depth resolution ( $S$ is the stopping power of the ion projectile). The actually realized resolution is worse due to the Doppler broadening effect that depends on the $\mathrm{H}$ vibration frequency on a specific sample material $[64,66,95]$ (Section 2.2.3). When the Doppler broadening width is known, the achievable near-surface resolution can be evaluated from the listed $\Gamma$ and $S$ values through Eq. (2.15) at $z=0$.

\begin{tabular}{|c|c|c|c|c|c|}
\hline Reaction & ${ }^{7} \mathrm{Li}+\mathrm{H}$ & ${ }^{15} \mathrm{~N}+\mathrm{H}$ & ${ }^{15} \mathrm{~N}+\mathrm{H}$ & ${ }^{19} \mathrm{~F}+\mathrm{H}$ & ${ }^{19} \mathrm{~F}+\mathrm{H}$ \\
\hline Resonance energy [MeV] & 3.07 & 6.385 & 13.35 & 6.418 & 16.4 \\
\hline $\begin{array}{l}\text { Cross section }\left(\sigma_{R}\right) \\
\text { at resonance [mbarn] }\end{array}$ & 4.8 & 1650 & 1050 & 88 & 440 \\
\hline $\begin{array}{l}\text { Resonance width }(\Gamma) \\
{[\mathrm{keV}]}\end{array}$ & 81 & 1.8 & 25.4 & 44 & 86 \\
\hline$\sigma_{\mathrm{R}} \Gamma[\mathrm{mbarn} \cdot \mathrm{keV}]$ & 389 & 2970 & 26700 & 3870 & 37800 \\
\hline Relative yield & 0.13 & 1.0 & 9.0 & 1.3 & 12.7 \\
\hline$S=\mathrm{d} E / \mathrm{d} z$ in $\mathrm{Si}[\mathrm{keV} / \mathrm{nm}]$ & 0.442 & 1.45 & 1.35 & 1.94 & 1.94 \\
\hline$\Gamma / S[\mathrm{~nm}]$ & 183 & 1.2 & 18.8 & 22.6 & 44.3 \\
\hline $\begin{array}{l}\text { Energy of next } \\
\text { resonance[MeV] }\end{array}$ & 7.11 & 13.35 & 18.0 & 9.1 & 17.6 \\
\hline Gamma-ray energy [MeV] & $14.75,17.75$ & 4.43 & 4.43 & $6.13,6.98,7.12$ & $6.13,6.98,7.12$ \\
\hline
\end{tabular}

Table 1. Characteristics of nuclear reaction resonances useful for $\mathrm{H}$ detection with NRA. Data from Refs. [41, 43, 72].

The first resonance of the ${ }^{1} \mathrm{H}\left({ }^{15} \mathrm{~N}, \alpha \gamma\right){ }^{12} \mathrm{C}$ reaction at $6.385 \mathrm{MeV}$ has been by far most widely applied for surface-near $\mathrm{H}$ depth profiling owing to its particularly narrow $(\Gamma=1.8 \mathrm{keV})[96,97]$, intense $\left(\sigma_{\mathrm{R}}=1650 \mathrm{mbarn}\right)$, and isolated shape [89, 98-100]. As shown in Fig. 1, the ratio of the on-resonant to the nearby off-resonance crosssection (77 $\mu$ barn) is larger than $10^{4}$, earning it the attribute of 'Mona Lisa of resonances' from Amsel, one of the founding fathers of resonant NRA [100]. 


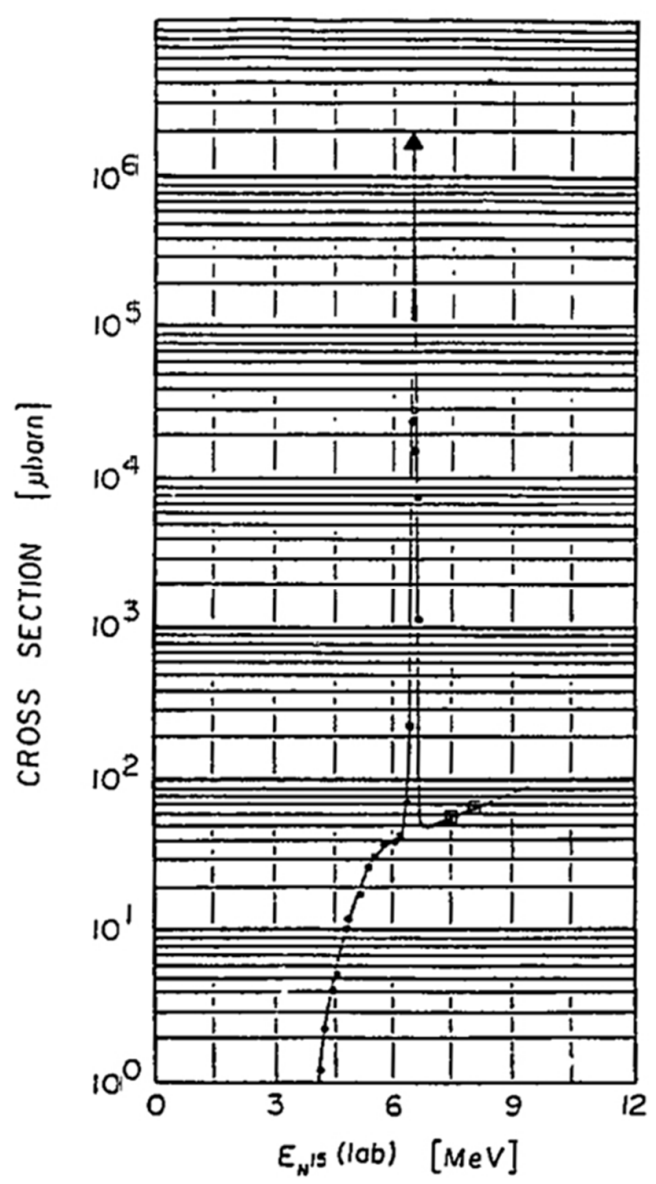

Fig. 1. The narrow resonance of the ${ }^{1} \mathrm{H}\left({ }^{15} \mathrm{~N}, \alpha \gamma\right){ }^{12} \mathrm{C}$ nuclear reaction cross section at 6.385 MeV. The resonance peak is more than four orders of magnitude larger than the nearby off-resonant cross section. Reproduced from Ref. [41] with permission from Elsevier.

Together with the large separation to the next resonance at $13.35 \mathrm{MeV}$, these properties provide for an outstanding near-surface depth resolution ( $1-5 \mathrm{~nm}$, Section 2.2.3), good sensitivity ( $100 \mathrm{ppm}$, Section 2.2 .4 ), and a practical probing depth range (2-4 $\mu \mathrm{m}$, Section 2.2 .3$)$ at an energy that can still be realized with moderately sized accelerators. All near-surface high resolution work reviewed herein has been performed with this reaction. We therefore choose ${ }^{1} \mathrm{H}\left({ }^{15} \mathrm{~N}, \alpha \gamma\right){ }^{12} \mathrm{C}$ as the most representative example to describe in the following the principles of hydrogen depth profiling with resonant NRA. A brief comparsion to other resonant nuclear reactions in Table 1 will be given in Section 2.1.2. 
The hydrogen depth profiling method by resonant NRA via ${ }^{1} \mathrm{H}\left({ }^{15} \mathrm{~N}, \alpha \gamma\right){ }^{12} \mathrm{C}$ is schematically illustrated in scheme (2.1) and in Fig. 2. The analyzed sample is subjected to a beam of accelerated and energy-monochromatized ${ }^{15} \mathrm{~N}$ ions that may induce the following nuclear reaction with protons in the target:

$$
{ }^{15} \mathrm{~N}+{ }^{1} \mathrm{H} \rightarrow{ }^{16} \mathrm{O}^{*} \rightarrow{ }^{12} \mathrm{C}+\alpha+\gamma
$$

At the resonance energy $E_{R}=6.385 \mathrm{MeV}$, an excited ${ }^{16} \mathrm{O}$ nucleus is formed as the intermediate compound, which undergoes an $\alpha$-decay into the first nuclear excited state of ${ }^{12} \mathrm{C}$ [101]. The latter finally relaxes to the ${ }^{12} \mathrm{C}$ ground state by releasing characteristic $\gamma$-radiation of $4.4389 \mathrm{MeV}$ [102]. The emitted $\gamma$-rays (and the $\alpha$ particles) provide signals proportional to the $\mathrm{H}$ concentration at a certain depth in the target. For reasons explained in Section 2.2.4, $\gamma$-detection is preferred in practice. The residual ${ }^{12} \mathrm{C}$ nucleus retains a large forward velocity component in the incidence direction of the ${ }^{15} \mathrm{~N}$ projectiles and is deposited into the target (at much larger depth than the analyzed region).

Information on the $\mathrm{H}$ depth location is obtained as follows. The resonance in the ${ }^{1} \mathrm{H}\left({ }^{15} \mathrm{~N}, \alpha \gamma\right){ }^{12} \mathrm{C}$ reaction cross section at $E_{\mathrm{R}}=6.385 \mathrm{MeV}$ with a width of only $1.8 \mathrm{keV}$ $[95,96,98]$ is extremely sharp (Fig. 1). From the peak value at $E_{R} \sigma(E)$ falls steeply to a 4 orders of magnitude smaller off-resonance cross section [51], setting a very narrow energy window for the nuclear reaction. Only ${ }^{15} \mathrm{~N}$ projectiles incident with the energy $E_{\mathrm{i}}=E_{R}$ can thus undergo the nuclear reaction with $\mathrm{H}$ atoms on the target surface (Fig. 2). The MeV ${ }^{15} \mathrm{~N}$ ions penetrate into the target and experience energy 
loss by electronic stopping while travelling through the material (they eventually come to rest at an implantation depth of several $\mu \mathrm{m}$, far beyond the analyzed depth region of interest).

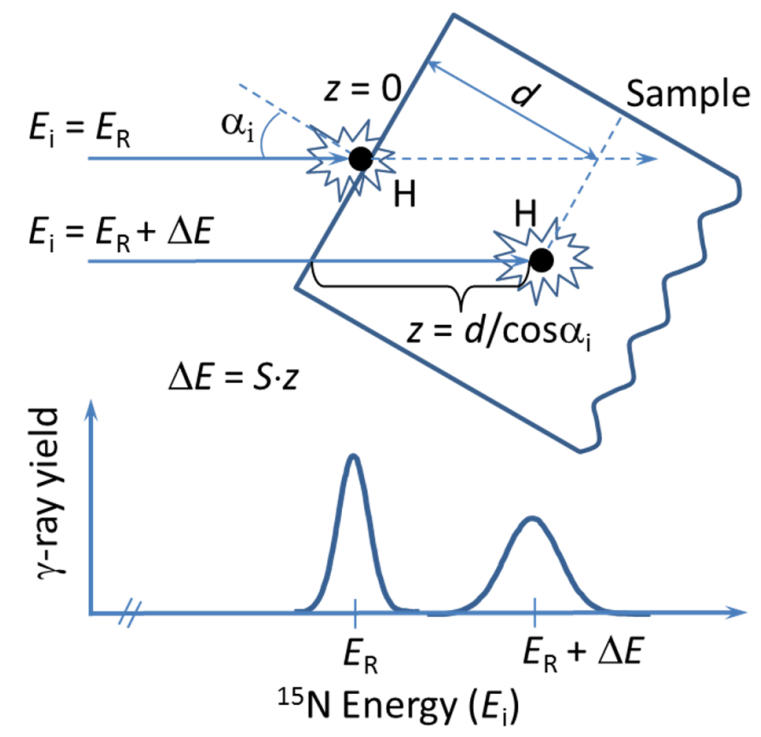

Fig. 2. Depth-resolved $\mathrm{H}$ detection with ${ }^{1} \mathrm{H}\left({ }^{15} \mathrm{~N}, \alpha \gamma\right){ }^{12} \mathrm{C}$ NRA. ${ }^{15} \mathrm{~N}$ ions accelerated to $E_{\mathrm{i}}$ strike the sample. For $E_{\mathrm{i}}=E_{\mathrm{R}},{ }^{15} \mathrm{~N}$ reacts with $\mathrm{H}$ on the surface, whereas for $E_{\mathrm{i}}=E_{\mathrm{R}}+\Delta E,{ }^{15} \mathrm{~N}$ reacts with $\mathrm{H}$ in a depth $d=\Delta E \cdot \cos \alpha_{\mathrm{i}} / S$. The excitation curve $Y\left(E_{\mathrm{i}}\right)(\gamma$-ray yield as a function of the incident ${ }^{15} \mathrm{~N}$ energy $\left(E_{\mathrm{i}}\right)$ ) reveals the $\mathrm{H}$ depth distribution in the target convolved with the instrumental function. The latter broadens with increasing depth due to energy straggling of the ${ }^{15} \mathrm{~N}$ ion.

This energy loss $\Delta E$ is proportional to the trajectory length $z$ that the ion has propagated inside the target ( $z=0$ at the surface) and to the material specific stopping power $S=(\mathrm{d} E / \mathrm{d} z)$ :

$$
\Delta E=S \cdot Z
$$

Stopping powers for ${ }^{15} \mathrm{~N}$ near $6.4 \mathrm{MeV}$ range in the order of $\sim 1-4 \mathrm{keV} / \mathrm{nm}[103$, 104] for elemental targets, so that the energy loss by penetrating only a few atomic 
layers suffices to shift the ${ }^{15} \mathrm{~N}$ ion energy out of the nuclear reaction energy window spanned by the narrow cross section resonance. When the ${ }^{15} \mathrm{~N}$ beam strikes the target at an angle $\alpha_{i}$ with the surface normal $\left(0 \leq \alpha_{i}<90^{\circ}\right)$, the trajectory length to penetrate into a depth $d$ below the surface is $z=d / \cos \alpha_{i}(d$ is measured in the surface normal direction). Thus a ${ }^{15} \mathrm{~N}$ incident energy $E_{\mathrm{i}}=E_{\mathrm{R}}+\Delta E$ corresponds to a well-defined probing depth $d=\Delta E \cos \alpha_{i} / S$ inside the target (Fig. 2). To obtain hydrogen depth profiles the incident energy of the ${ }^{15} \mathrm{~N}$ ions is scanned stepwise to position the resonant reaction energy window at varying depths within the target while collecting the $\gamma$-yield. The experimental excitation curve $Y\left(E_{i}\right)$ (reaction yield versus incident projectile energy) thus displays the depth distribution of the hydrogen density projected into the ion beam direction, $n_{H}(z)$, yet in a convolution (*) with an effective instrumental function $F\left(E, E_{\mathrm{i}}, z\right)$ :

$$
Y\left(E_{i}\right)=K N_{i} \int_{0}^{\infty} F\left(E, E_{i}, z\right) * n_{H}(z) d z
$$

$K$ denotes the $\gamma$-detection efficiency of the experimental system, which includes the quantum efficiency and solid angle of the detector, as well as its position relative to the beam axis (Section 2.2.4). $F\left(E, E_{i}, z\right)$ is defined in Eq. (2.4) and reflects the broadening of the reaction energy window beyond the nuclear reaction resonance width $\Gamma$ by three physical effects that independently cause variations of the ${ }^{15} \mathrm{~N}-\mathrm{H}$ collision energy: (i) the energy distribution of the incident ion beam, $B\left(E, E_{i}\right)$, Eq. (2.6), (ii) a Doppler effect due to zero-point vibration of the $\mathrm{H}$ atoms within their bonds to the target, $D(E)$, Eq. (2.7), and (iii) trajectory length dependent energy straggling of the ${ }^{15} \mathrm{~N}$ projectiles in the target material, $\mathrm{S}(E, z)$, Eq. (2.9). In the instrumental 
function $F\left(E, E_{i}, z\right)$ the nuclear reaction cross section $\sigma(E)$ is convolved with these three spectral components:

$$
F\left(E, E_{\mathrm{i}}, z\right)=\sigma(E)^{*} B\left(E, E_{\mathrm{i}}\right)^{*} D(E) * S(E, z)
$$

The cross section resonance of the nuclear reaction has the Lorentzian line shape of a Breit-Wigner dispersion function, where $E_{R}, \sigma_{R}$, and $\Gamma$ denote the resonance energy, the maximum, and the spectral width of the cross section, respectively:

$$
\sigma(E)=\sigma_{R} \frac{\Gamma^{2} / 4}{\Gamma^{2} / 4+\left(E-E_{R}\right)^{2}} .
$$

The incident ion beam energy distribution (as delivered by the accelerator) can usually be approximated by a Gaussian function with a width parameter $\delta_{\mathrm{B}}$ (standard deviation) [96]:

$$
B\left(E, E_{i}\right)=\frac{1}{\sqrt{2 \pi} \delta_{B}} \exp \left(-\frac{\left(E-E_{i}\right)^{2}}{2 \delta_{B}^{2}}\right)
$$

The experimentally accessible beam energy spread $\Delta E_{\mathrm{B}}$ is the full width at half maximum (FWHM) of this distribution:

$$
\Delta E_{\mathrm{B}}=[8 \ln (2)]^{1 / 2} \delta_{\mathrm{B}}=2.3548 \delta_{\mathrm{B}} .
$$

(In the special situation that the ion beam is monochromatized, $B(E)$ has a triangular shape (cf. Fig. 3), which reflects the transmission function of the entrance and exit slits of the energy analyzer and the approximately linear relationship between the beam energy and small distances from the center beam axis in the focal plane of the analyzing sector magnet (Section 2.2.1): 


$$
B\left(E, E_{i}\right)= \begin{cases}0 & E \leq E_{\mathrm{i}}-\Delta E_{\mathrm{B}} \\ 1+\left(E-E_{\mathrm{i}}\right) / \Delta E_{\mathrm{B}} & E_{\mathrm{i}}-\Delta E_{\mathrm{B}}<E \leq E_{\mathrm{i}} \\ 1-\left(E-E_{\mathrm{i}}\right) / \Delta E_{\mathrm{B}} & E_{\mathrm{i}}<E<E_{\mathrm{i}}+\Delta E_{\mathrm{B}} \\ 0 & \mathrm{E} \geq E_{\mathrm{i}}+\Delta E_{\mathrm{B}}\end{cases}
$$

The spatial confinement of the hydrogen atoms inside their chemical bond potentials causes an inevitable uncertainty of their momentum according to the Heisenberg relation, and the wave function of the resulting zero-point vibration is Gaussian in momentum space (assuming a harmonic oscillator model for the nuclear motion). Although the zero-point vibrational energy $E_{\mathrm{H}}$ of the $\mathrm{H}$ nuclei is small (some tens of meV) compared to the nuclear reaction energy $E_{R}$, the resulting Doppler shift of the ${ }^{15} \mathrm{~N}-\mathrm{H}$ collision energy translates into a sizeable Gaussian broadening $D(E)[63$, $64,66,95$ ] of several keV FWHM (cf. Section 3.1.3, $\mathrm{Si}_{1-\mathrm{x}} \mathrm{Ge}_{\mathrm{x}}$ ):

$$
D(E)=\frac{1}{\sqrt{2 \pi} \delta_{D}} \exp \left(-\frac{E^{2}}{2 \delta_{D}^{2}}\right)
$$

As derived in Ref. [64], the width parameter $\left(\delta_{D}\right)$ of this Doppler-broadening distribution relates as follows to $E_{\mathrm{H}}, E_{\mathrm{R}}$, and the masses of the $\mathrm{H}\left(m_{\mathrm{H}}\right)$ and ${ }^{15} \mathrm{~N}\left(m_{\mathrm{N}}\right)$ nuclei $[67,95]$ :

$$
\delta_{D}=\sqrt{\frac{2 m_{N} E_{H} E_{R}}{m_{H}}}
$$

i.e., the Doppler broadening width $\Delta E_{D}=2.3548 \delta_{D}(F W H M)$ increases in proportion to the square root of the $\mathrm{H}$ zero-point vibrational energy $E_{\mathrm{H}}$. 
Along the trajectory of the probing ions in the target, the center of their energy distribution decreases linearly with the propagation length $z$ from the surface due to electronic stopping (Eq. 2.2). Straggling, an energy fluctuation caused by multiple weak scattering interactions of the ion with the target electrons, simultaneously broadens this energy distribution. Due to the stochastic nature of the straggling process the energy broadening function is Gaussian and its width parameter increases in proportion to the square root of the ion trajectory length in the analyzed material $\left(\delta_{s}(z) \propto(z)^{1 / 2}\right.$, Section 2.1.4) $[105,106]$ :

$$
S(E, z)=\frac{1}{\sqrt{2 \pi} \delta_{S}(z)} \exp \left(-\frac{(E-\Delta E(z))^{2}}{2\left(\delta_{S}(z)\right)^{2}}\right)
$$

Since all broadening components (i)-(iii) are Gaussian [approximately also for monochromatized beams] they convolve into a Gaussian broadening function $G\left(E, E_{\mathrm{i}}\right.$, $z)=B\left(E, E_{\mathrm{i}}\right) * D(E) * S(E, z)$ with a spectral width parameter $\delta_{\mathrm{G}}{ }^{2}=\delta_{\mathrm{B}}{ }^{2}+\delta_{\mathrm{D}}{ }^{2}+\left(\delta_{\mathrm{S}}(z)\right)^{2}$ Convolution with the Lorentzian reaction resonance $\sigma(E)$ has to be done numerically [96], which finally yields the instrumental function $F\left(E, E_{\mathrm{i}}, z\right)=\sigma(E) * G\left(E, E_{\mathrm{i}}, z\right)$ in shape of a Voigt profile. There is no analytical expression for the spectral width of the Gaussian-broadened Lorentzian resulting from this convolution, but Briere et al. have suggested a numerical approximation for the resulting total energy width $\Delta E_{\text {tot }}$ (FWHM) of the instrumental function as [107]:

$$
\Delta E_{\text {tot }}=0.56 \Gamma+\left[8 \ln (2)\left(\delta_{\mathrm{B}}^{2}+\delta_{\mathrm{D}}^{2}+\left(\delta_{\mathrm{S}}(z)\right)^{2}\right)\right]^{1 / 2}
$$

With the known components $\sigma(E), B\left(E, E_{\mathrm{i}}\right), D(E)$, and $S(E, z)$ of the instrumental function $F\left(E, E_{i}, z\right)$, the $H$ concentration distribution $n_{H}(\mathrm{~d})$ can be determined from 
experimental excitation curves $Y\left(E_{\mathrm{i}}\right)$ by numerical deconvolution (Section 2.2.7). Resonance width parameters $(\Gamma)$ of several nuclear reactions relevant to $\mathrm{H}$-profiling are listed in Table 1 . The incident ion beam energy spread $\Delta E_{\mathrm{B}}$ must be determined experimentally [108] or can be calculated from the resolution of the accelerator's energy analyzer (Section 2.2.1). Refer to Section 2.1.4 for methods to estimate the straggling cross section $\delta_{\mathrm{s}}(z)$.

For surface hydrogen the Doppler broadening function $D(E)$ can be determined experimentally by providing a target on which hydrogen is present exclusively on the surface. Since the H solubility in crystalline silicon is immeasurably small [109], the NRA excitation function obtained from a H-terminated $\mathrm{Si}(111)(1 \times 1)$ surface shown in Fig. 3 is a good example for this situation. A perfectly symmetric resonance profile centered at $E_{\mathrm{R}}=6.385 \mathrm{MeV}$ is observed, its integral being proportional to the surface $\mathrm{H}$ coverage according to Eq. (2.11) (below). $D(E)$ is obtained through numerically deconvoluting $\sigma(E)$ and $B\left(E, E_{\mathrm{i}}\right)$ from the experimental yield curve. (In practice, the known $\sigma(E)$ and $B\left(E, E_{\mathrm{i}}\right)$ functions are convolved with a series of test Gaussian $D(E)$ functions of various width parameters ( $\delta_{D}$ in Eq. (2.7)) until the $\delta_{D}$ value is found for which the total convolution result $Y\left(E_{\mathrm{i}}\right)=\sigma\left(E_{i}\right) * B\left(E, E_{\mathrm{i}}\right)^{*} D(E)$ adequately reproduces the experimental data.) For the example in Fig. 3, this analysis yields $\delta_{D}=4.9 \mathrm{keV}[67]$. It is seen in Fig. 3 that the Doppler broadening $\left(\Delta E_{D}=2.3548 \delta_{D}=11.4 \mathrm{keV}, \mathrm{FWHM}\right)$ is by far the dominant contribution to the total width of the surface resonance curve, which therefore appears almost Gaussian in shape (the Lorentzian tails of the actual Voigt profile become visible by plotting the $\gamma$-yield on a logarithmic scale). 


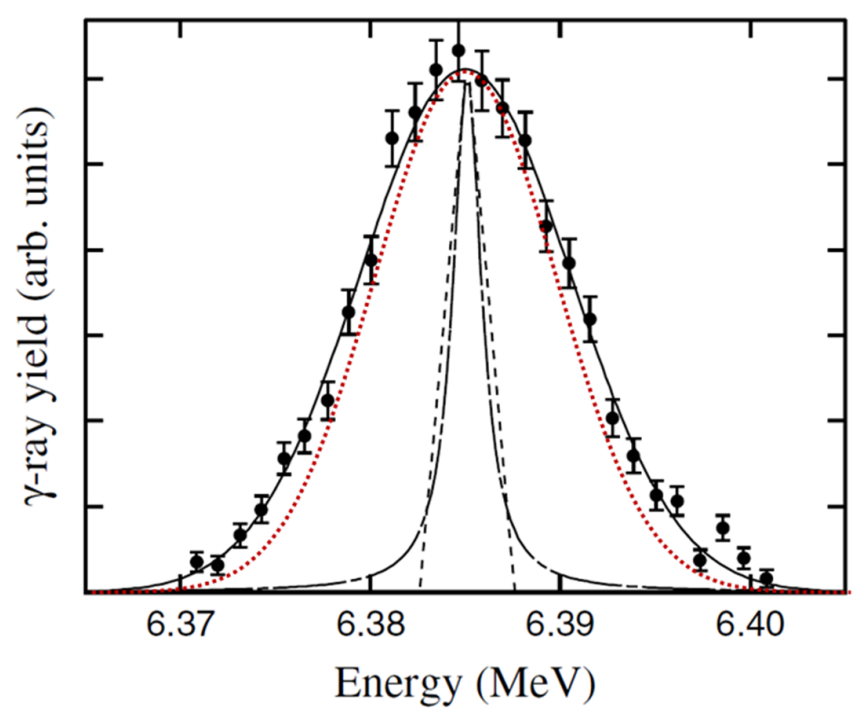

Fig. 3. Spectral decomposition of a surface resonance excitation curve (filled symbols) measured at $\mathrm{Si}(111)(1 \times 1)-\mathrm{H}$ with ${ }^{1} \mathrm{H}\left({ }^{15} \mathrm{~N}, \alpha \gamma\right){ }^{12} \mathrm{C}$ NRA near $E_{\mathrm{R}}=6.385 \mathrm{MeV}$ in surface-normal ${ }^{15} \mathrm{~N}$ incidence, showing the nuclear resonance (Lorentzian, $\Gamma=1.8 \mathrm{keV}$, long-short-dashed line), the incident ion beam energy distribution (triangular, $\Delta E_{\mathrm{B}}=3 \mathrm{keV}$, dashed line), the Doppler broadening (Gaussian, red dotted line), and their convolution (solid line through data points). Data from Ref. [67].

Since the Doppler broadening effect is so significantly manifested in the surface resonance excitation curves, the zero-point vibrational energy of surface-adsorbed $\mathrm{H}$ atoms $\left(E_{\mathrm{H}}\right)$ can be determined directly from the width parameter $\delta_{\mathrm{D}}$ via Eq. (2.8.) [63$65,67,95]$. This is a unique feature of resonant ${ }^{1} \mathrm{H}\left({ }^{15} \mathrm{~N}, \alpha \gamma\right){ }^{12} \mathrm{C}$ NRA at $6.385 \mathrm{MeV}$. More examples of this application for the systems $H / W(100), H / \operatorname{Ir}(111), H / P t(111)$, $\mathrm{H} / \mathrm{Rh}(111), \mathrm{H} / \mathrm{Si}(111)$, and $\mathrm{H}$ adsorbed on SiGe(100) alloy surfaces are discussed in Sections 3.1.2 and 3.1.3.

The vibrational frequency of low-coordinated $\mathrm{H}$ at surfaces and interfaces is expected to be harder than that of bulk-dissolved $\mathrm{H}$ in interstitial sites. Hence, the Doppler broadening width parameter of surface $\mathrm{H}$ determined above must be 
regarded an upper limit estimate for $\delta_{\mathrm{D}}$ of $\mathrm{H}$ absorbed below the surface of a given material. Measuring the Doppler width of the latter directly with NRA seems impossible due to the difficulty to provide a target in which absorbed $\mathrm{H}$ would assume a delta function like depth distribution (confined to a single atomic layer such as on the surface or at an interface). If available, substituting $\mathrm{H}$ zero-point energies $\left(E_{H}\right)$ deduced in a harmonic approximation from vibrational spectroscopic data of $\mathrm{H}$ in bulk materials into Eq. (2.8) may thus provide a more appropriate estimate of $\delta_{D}$ for interstitial hydrogen below the surface.

(a) Surface $\mathrm{H}$
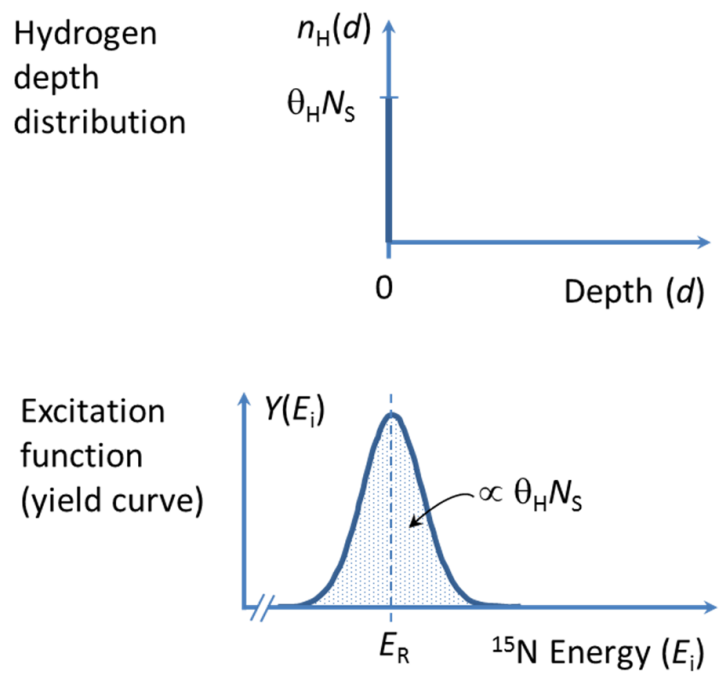

(b) Bulk H
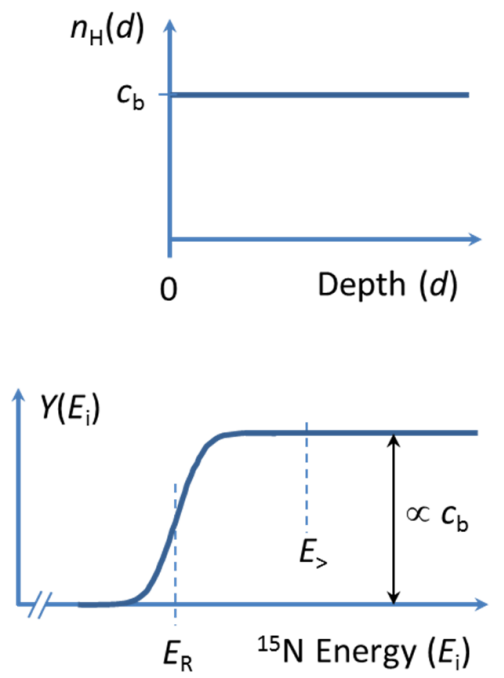

Fig. 4. Principle shape of the NRA excitation function $Y\left(E_{i}\right)$ resulting from (a) $\mathrm{H}$ situated exclusively on the surface and (b) from a uniform $\mathrm{H}$ concentration in the target volume (bulk).

For the quantitative determination of hydrogen surface coverages and volume concentrations it is instructive to consider two idealized limiting cases of hydrogen depth-concentration distributions $n_{H}(d)$ : 
1.) Surface hydrogen: The first situation is a surface layer of adsorbed $H$ atoms without any hydrogen below the surface (as in the above example). Let $N_{\mathrm{S}}$ be the (substrate) surface areal density [atoms $/ \mathrm{cm}^{2}$ ] and $\theta_{H}$ the hydrogen coverage in monolayers (ML). Then the $\mathrm{H}$ distribution is $n_{\mathrm{H}}(d)=\theta_{\mathrm{H}} \cdot N_{\mathrm{S}} \cdot$ at the surface $(d=0)$ and zero elsewhere $(d \neq 0)$, and Eq. (2.3) becomes:

$$
\begin{aligned}
& Y\left(E_{\mathrm{i}}\right)=K \cdot N_{\mathrm{i}} \cdot \theta_{\mathrm{H}} \cdot N_{\mathrm{S}} \cdot F\left(E, E_{\mathrm{i}}, 0\right), \text { and } \\
& \int Y\left(E_{i}\right) d E_{i}=K N_{i} N_{S} \sigma_{R}\left(\frac{\pi \Gamma}{2}\right) \theta_{H} .
\end{aligned}
$$

The characteristic excitation curve of surface hydrogen is a peaked profile centered at $E_{\mathrm{R}}$ that reflects the Gauss-Lorentz (Voigt) shape of the instrumental function (Fig. $4(a)$ ). The integrated $\gamma$-yield under the profile is proportional to the number of hydrogen atoms on the surface, $\theta_{H} \cdot N_{S}$. By normalizing the yield $Y\left(E_{i}\right)$ to the number of incident ${ }^{15} \mathrm{~N}$ ions $\left(N_{\mathrm{i}}\right)$ the hydrogen coverage can be evaluated quantitatively once the $\gamma$-detection efficiency of the setup $(K)$ has been determined through calculation or calibration with a standard of known $\mathrm{H}$ concentration (Section 2.2.6).

2.) Bulk hydrogen: The second situation to consider is a homogeneous $\mathrm{H}$ volume density $c_{b}\left[a t o m s / \mathrm{cm}^{3}\right]$ in the target, i.e. a rectangular concentration jump at the surface $(d=0): n_{H}(d)=c_{b}$ for $d>0$. Convolving this $H$ distribution with the instrumental function yields an excitation curve $Y\left(E_{\mathrm{i}}\right)$ that rises in a sigmoid shape and asymptotically approaches an intensity plateau as depicted in Fig. 4 (b), attaining 
its half-height at $E_{\mathrm{R}}$. At incident projectile energies sufficiently far above $E_{\mathrm{R}}\left(E_{>}\right)$, the $\gamma$-yield becomes constant and is then proportional to the bulk $\mathrm{H}$ concentration:

$$
Y\left(E_{>}\right)=K N_{i} \sigma_{R}\left(\frac{\pi \Gamma}{2}\right) c_{b} / S
$$

Equation (2.12) shows that the $\mathrm{H}$ volume concentration $c_{b}$ in a target can be evaluated by a single measurement of the $\gamma$-yield at any projectile energy $\left(E_{>}\right)$ sufficiently far above the rising surface edge of the excitation curve (Fig. 4). In such measurements (such as for sensitivity calibration, Section 2.2.6) it is always recommendable to experimentally verify the energy $E_{>}$at which $Y\left(E_{i}\right)$ attains the bulk plateau height, since the $\mathrm{H}$ distribution may be inhomogeneous in the near-surface region (Section 3.2.3.6). If the $H$ concentration in the target is ideally depthindependent, one may estimate $E_{>}$from the nuclear reaction resonance width $(\Gamma)$. The ${ }^{1} \mathrm{H}\left({ }^{15} \mathrm{~N}, \alpha \gamma\right){ }^{12} \mathrm{C}$ cross section width near the $6.385 \mathrm{MeV}$ resonance $(\Gamma=1.8 \mathrm{keV})$ is much smaller than the Gaussian width components in the NRA instrumental function (convolved Doppler and beam energy broadenings, typically $\delta_{\mathrm{G}} \sim 6.5 \mathrm{keV}$, Fig. 3), yet its broad Lorentzian wings still contribute significant $\gamma$-yield at energies quite far above $E_{R}$. The cumulative integral of the Lorentzian (Gauchy distribution) indicates that the full plateau height is approached except for a small percentile $(p)$ when $E_{>}$is chosen as: $E_{>}=E_{R}+1 / 2 \Gamma \cdot \tan [((1-p)-0.5) \pi]$. If one accepts $1 \%$ error $(p=0.01)$, this evaluates $E_{>}$to $E_{R}+29.4 \mathrm{keV}\left(E_{R}+404 \mathrm{keV}\right)$ for the $6.835 \mathrm{MeV}(13.35 \mathrm{MeV}, \Gamma=25.4$ $\mathrm{keV}$ ) resonance, which is considerably larger than even a $3 \delta_{\mathrm{G}}$ interval of the Gaussian component. 
Note that Eq. (2.12) resembles Eq. (2.11), but that the stopping power $S=(d E / d z)$ enters as an additional factor resulting from substituting the integration variable from $z$ to $E$ in Eq. (2.3) ( $d z=d E / S)$. This means that if two materials contain the same absolute $\mathrm{H}$ density, the one with the larger stopping power will give a smaller $\gamma$-yield. The physical implication is that a large stopping power shifts the energy of the projectile ion away from the resonance within a short traveling distance, thus reducing the probability of the ion to encounter $\mathrm{H}$ atoms while its energy is within the resonant reaction window.

\subsubsection{Merits of $\left.{ }^{1} \mathrm{H}\left({ }^{15} \mathrm{~N}, \alpha \gamma\right)\right)^{12} \mathrm{C}$}

Having laid out the principles of resonant NRA, the suitability of the various nuclear reaction resonances in Table 1 for $\mathrm{H}$ profiling can now be compared. As noted, the well isolated first resonance of the ${ }^{1} \mathrm{H}\left({ }^{15} \mathrm{~N}, \alpha \gamma\right){ }^{12} \mathrm{C}$ reaction at $E_{\mathrm{R}}{ }^{(1)}=6.385$ $\mathrm{MeV}$ offers the most versatile combination of favorable properties. Its narrow resonance width $\left(\Gamma^{(1)}=1.8 \mathrm{keV}\right)$, large cross section $\left(\sigma_{R}=1650\right.$ mbarn, Fig. 1$)$, and large separation to the next resonance provide excellent near-surface depth resolution ( $\sim 1-5 \mathrm{~nm})$, high sensitivity ( $100 \mathrm{ppm}$, Section 2.2 .4$)$, and a reasonable probing range $(2-4 \mu \mathrm{m}$, Section 2.2.3). At the expense of near-surface depth resolution, also the considerably wider $\left(\Gamma^{(2)}=25.4 \mathrm{keV}\right)$ second resonance of ${ }^{1} \mathrm{H}\left({ }^{15} \mathrm{~N}, \alpha \gamma\right){ }^{12} \mathrm{C}$ at $\mathrm{E}_{\mathrm{R}}^{(2)}=13.35 \mathrm{MeV}$ is available, which offers $\sim 9$ fold increased sensitivity owing to its larger cross section integral $\left(\propto \sigma_{R} \Gamma\right)$. 
This is demonstrated in Fig. 5, which compares the NRA profiles of a $\sim 40 \mathrm{~nm}$ thick hydrogenated SiN layer on a $\mathrm{H}$-free $\mathrm{Si}(100)$ backing recorded with the first and second ${ }^{1} \mathrm{H}\left({ }^{15} \mathrm{~N}, \alpha \gamma\right){ }^{12} \mathrm{C}$ resonances, respectively. Observe the strongly increased $\gamma$ yield in the $13.35 \mathrm{MeV}$ profile (Fig. 5 (b)). The intensity ratio of two curves [I(13.35 $\mathrm{MeV}) /((6.385 \mathrm{MeV})]$ amounts to $8.7 \pm 0.2$, which reflects the integral ratio of the cross-section resonances. The much inferior depth resolution of the $13.35 \mathrm{MeV}$ profile is also apparent. The slight inclination of the $\mathrm{H}$ plateau concentration against the surface of the thin SiN film - clearly seen in the high-resolution yield curve at 6.385 MeV - induces only a barely recognizable asymmetry in the $13.35 \mathrm{MeV}$ profile [110]. This resolution disadvantage of the broader $13.35 \mathrm{MeV}$ resonance will never vanish but be less significant for probing $\mathrm{H}$ in deeper target regions where ${ }^{15} \mathrm{~N}$ straggling becomes the major resolution limiting factor (Sections 2.1.4 and 2.2.3).
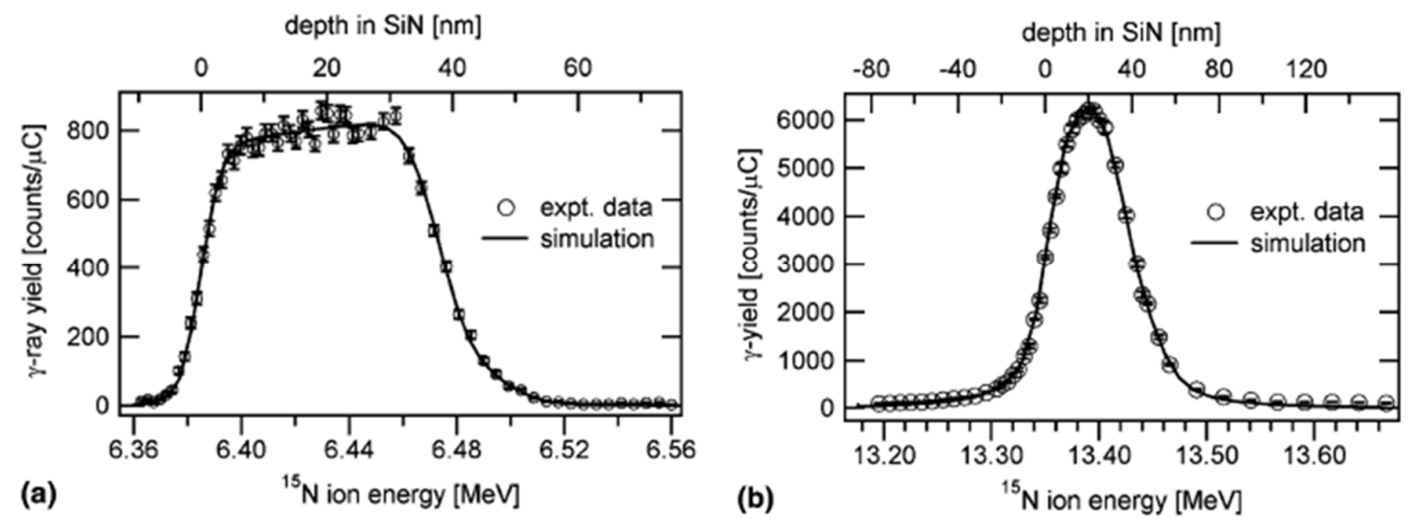

Fig.5. Hydrogen depth profiles of $\mathrm{H}: \mathrm{SiN} / \mathrm{Si}(100)$ measured with the ${ }^{1} \mathrm{H}\left({ }^{15} \mathrm{~N}, \alpha \gamma\right){ }^{12} \mathrm{C}$ resonances at (a) $6.385 \mathrm{MeV}$ and (b) at $13.35 \mathrm{MeV}$. Bold lines represent simulated yield curves based on the same $\mathrm{H}$ depth distribution. Reproduced from Ref. [110] with permission from Elsevier.

The larger $\gamma$-yield at 13.35 MeV can be useful to determine small H bulk contents, yet as explained in Section 2.2.4, disturbing $\gamma$-yield due to the interaction of surface 
$\mathrm{H}$ with the non-resonant ${ }^{1} \mathrm{H}\left({ }^{15} \mathrm{~N}, \alpha \gamma\right){ }^{12} \mathrm{C}$ reaction cross section above $\sim 10 \mathrm{MeV}$ may become a concern for such measurements, since the ratio of the resonant to the offresonant cross section near $13.35 \mathrm{MeV}$ is far less favorable than at $6.385 \mathrm{MeV}$ (Fig. 10) $[98,100,110,111]$. Also the probing depth range of $13.35 \mathrm{MeV}$ resonant NRA is shorter as compared to that of the $6.385 \mathrm{MeV}$ resonance, since a third resonance follows already at $18.0 \mathrm{MeV}$ and the ${ }^{15} \mathrm{~N}$ stopping power is slightly (up to $20 \%$ ) smaller at $13.35 \mathrm{MeV}$ than at $6.385 \mathrm{MeV}$ (Table 1 and Section 2.2.3).

The 6.42 MeV and 16.44 MeV resonances of the ${ }^{1} \mathrm{H}\left({ }^{19} \mathrm{~F}, \alpha \gamma\right){ }^{16} \mathrm{O}$ reaction have inferior near-surface depth resolution and shallower probing depth ranges (Section 2.2.3) relative to the respective resonances of ${ }^{1} \mathrm{H}\left({ }^{15} \mathrm{~N}, \alpha \gamma\right){ }^{12} \mathrm{C}$, but especially the 16.44 $\mathrm{MeV}$ resonance has a large cross section that enables highly sensitive $\mathrm{H}$ detection. Several applications of ${ }^{1} \mathrm{H}\left({ }^{19} \mathrm{~F}, \alpha \gamma\right){ }^{16} \mathrm{O}$ are reported [86, 112-116], among them also the notably very first demonstration of hydrogen profiling measurements with resonant NRA by Leich and Tombrello in 1973 [117] that even preceeded Lanford's application of the ${ }^{1} \mathrm{H}\left({ }^{15} \mathrm{~N}, \alpha \gamma\right){ }^{12} \mathrm{C}$ reaction by a few years [49]. For a direct comparison of ${ }^{19} \mathrm{~F}$ and ${ }^{15} \mathrm{~N}$ NRA see Ref. [112]. Compared to the ${ }^{15} \mathrm{~N}$ and ${ }^{19} \mathrm{~F}$ reactions, the 3.07 MeV resonance of the ${ }^{1} \mathrm{H}\left({ }^{7} \mathrm{Li}, \gamma\right)^{8} \mathrm{Be}$ reaction offers neither a particularly favorable energy width for high-resolution near-surface $\mathrm{H}$ profiling nor a suitably large resonance cross section for highly sensitive measurements, although the explorable depth range $(\sim 6 \mu \mathrm{m})$ is the largest of the listed resonances owing to the small stopping power of the ${ }^{7} \mathrm{Li}$ projectiles [53]. Worth mentioning is also the ${ }^{1} \mathrm{H}\left({ }^{18} \mathrm{O}, \alpha\right){ }^{15} \mathrm{~N}$ reaction (not listed in Table 1), which has a $43 \mathrm{keV}$ wide resonance of $75 \mathrm{mbarn}$ at 11.25 MeV and produces $\alpha$ particles in an energy region of low background $(<4.3$ 
$\mathrm{MeV}$ ), and can thus be used for rather sensitive bulk $\mathrm{H}$ concentration measurements $(<10$ ppm) $[53,118]$.

Although this review focuses primarily on highly depth-resolving resonant ${ }^{1} \mathrm{H}\left({ }^{15} \mathrm{~N}, \alpha \gamma\right){ }^{12} \mathrm{C}$ NRA, we should mention that also a widely used nuclear reaction technique for deuterium profiling exists, namely $D\left({ }^{3} \mathrm{He}, \mathrm{p}\right)^{4} \mathrm{He}$ NRA $[119,120]$. The $\mathrm{D}\left({ }^{3} \mathrm{He}, \mathrm{p}\right)^{4} \mathrm{He}$ reaction produces protons of $\sim 13 \mathrm{MeV}$ and $\alpha$ particles of 2-4 MeV energy that are easily identified $[51,119,120]$. The $D\left({ }^{3} \mathrm{He}, \mathrm{p}\right)^{4} \mathrm{He}$ cross section has a very broad ( 350 keV) peak ( 900 mbarn total) at $650 \mathrm{keV}$ [121]. Since furthermore the stopping powers for the product He particles and protons are small, the reaction offers almost no depth resolution ( $\sim 500 \mathrm{~nm}$ near the surface). Nonetheless, $\mathrm{D}\left({ }^{3} \mathrm{He}, \mathrm{p}\right)^{4} \mathrm{He}$ NRA can be conducted with small accelerators (even with $400 \mathrm{keV} \mathrm{He} \mathrm{H}^{3+}$ ) and has thus found wide application in studies of important hydrogen-material interactions. The large resonance width allows quantifying the $D$ content in the entire near-surface region [122], which has been instrumental in establishing fundamental surface properties such as absolute D saturation coverages (Chapter 3.1, Table 3) for materials that do not absorb hydrogen, as well as D uptake/release and relocation kinetics in hydrogen absorbing (or D-implanted) materials (cf. Sections 3.2.3.4-6). Notably, Davis and Norton evaluated the differential cross section at 750 $\mathrm{keV}$ to $(54.5 \pm 1.7) \mathrm{mbarn} \mathrm{sr}^{-1}$ and performed the first quantitative measurements of D coverages at Pt surfaces [123]. For detailed descriptions of the $D\left({ }^{3} \mathrm{He}, \mathrm{p}\right)^{4} \mathrm{He}$ NRA technique refer to Refs. [119] and [120], which also provide excellent general introductions to hydrogen depth profiling with NRA. 


\subsubsection{Energy loss and stopping power}

In order to convert the energy abscissa of experimental NRA excitation curves $(\gamma-$ yield vs. incident ${ }^{15} \mathrm{~N}$ energy, Figs. 2 and 4$)$ into a probing depth scale $\left(d=\Delta E \cos \alpha_{i} / S\right)$, it is necessary to know the specific energy loss $S=(\mathrm{d} E / \mathrm{d} z)$ of the projectiles. The stopping power $S$ is also required to derive absolute $\mathrm{H}$ concentrations from $\gamma$-yield measurements at bulk targets (Eq. 2.12); hence it is essential for the sensitivity calibration of the experimental system described in Section 2.2.6. A table with $S$ values for ${ }^{15} \mathrm{~N}$ at $6.385 \mathrm{MeV}$ in all elemental targets is provided in Appendix A (data from Ref. [124]). An extensive body of experimental $(\mathrm{d} E / \mathrm{d} z)$ values has been compiled in large databases, such as the frequently cited 'Stopping cross section values for energetic ions in all elements' by Ziegler $[103,104]$. This database is included in a simulation program for the calculation of electronic stopping and ranges, SRIM (The Stopping and Range of lons in Matter). The database is expanded and the software updated regularly [125-127] and can be obtained free of charge for non-commercial research purposes from the SRIM website [128]. Stopping cross section curves of light ions in many elements and compounds are also made available in graphic form on the website maintained by Paul at Linz University [129].

The stopping power is characteristic for any given ion projectile-material combination and in principle energy-, more precisely, velocity-dependent [130]. Several theories of the stopping process have been developed by Bohr [105, 131, 132], Bethe [133], Bloch [134, 135], Lindhard and Scharff [136], and Firsov [137]. 'Slow' ions traveling at velocities much smaller than the Bohr velocity $\left(v_{0}=e^{2} /\left(4 \pi \varepsilon_{0} \hbar\right)\right.$ $=2.188 \times 10^{6} \mathrm{~m} / \mathrm{s}$, or $25 \mathrm{keV} / \mathrm{amu}$ ) of the valence electrons (i.e., $v \ll<0.1 v_{\mathrm{o}}$ ) tend to 
get neutralized by electron capture, so that the interaction with the target atoms is one of neutral elastic collisions. This so-called 'nuclear stopping' decreases with $1 / E$. At 'intermediate' velocities, i.e., $v \sim 0.1 v_{0}$ to $Z_{1}^{2 / 3} v_{o}\left(Z_{1}\right.$ is the atomic number of the ion), the probing particles attain an equilibrium charge state by charge exchange processes with the target material [138]. They propagate at velocities corresponding to multiples of $v_{0}$, which results in energy loss by collision-induced excitations of target electrons. This energy loss process is proportional to the velocity or $E^{1 / 2}$ and referred to as 'electronic stopping'. At yet much higher velocities $\left(v>>v_{0}\right.$, 'swift' ions) the projectiles become fully stripped of their electrons, after which the stopping cross section decreases with increasing energy as the interaction time gets shorter [133]. The result is an overall energy dependence of the electronic stopping cross section that shows a characteristic broad maximum ('Bragg peak') near velocities of $\sim 1-2 \mathrm{MeV} / \mathrm{amu}$ for light nuclei in most elements $[103,129]$. The SRIM program package for electronic stopping and range calculations uses a semiempirical parameterization of the effective charge state $[125,126]$.

In the intermediate energy range of typical NRA projectiles $\left({ }^{15} \mathrm{~N}\right.$ of $E_{\mathrm{i}} \geq 6.4 \mathrm{MeV} ; v$ $\left.\geq \sim 4 v_{0}\right)$, the dominant energy loss mechanism is electronic stopping. The nuclear stopping cross section amounts to less than $1 \%$ of the electronic stopping cross section and is hence safe to neglect. Stopping powers for ${ }^{15} \mathrm{~N}$ at $6.385 \mathrm{MeV}$ are well known in all elements and range from $\sim 1$ to $\sim 4 \mathrm{keV} / \mathrm{nm}$ for light $(\mathrm{C})$ and heavy (Pt) elements, respectively $[103,124]$. Although $S$ is principally energy-dependent, the stopping-induced energy losses in shallow near-surface $H$ profiling $(z \leq 100 \mathrm{~nm})$ are so small $(\Delta E \leq \sim 0.4 \mathrm{MeV})$ that the $(\mathrm{d} E / \mathrm{d} z)$ value of ${ }^{15} \mathrm{~N}$ at $6.4 \mathrm{MeV}$ can safely be 
regarded as a material-specific constant in the applications reviewed herein (surface approximation). Note that at a given velocity, $v=\left(2 E / M_{1}\right)^{1 / 2}$, the energy loss by electronic stopping scales with the square of the ion's atomic number, $Z_{1}{ }^{2}$, so that the $(d E / d z)$ values for ${ }^{15} \mathrm{~N}$ are to be taken from data tables or stopping cross section charts of ${ }^{14} \mathrm{~N}[103]$.

Except for a limited number of compounds, the databases contain the stopping cross sections $(\varepsilon)$ of ions in elemental targets. The stopping cross section for compound materials can be estimated in a first approximation, the so-called Bragg Rule, as a linear combination of the respective cross sections of the constituting elements [139]:

$$
\varepsilon\left(A_{n} B_{m}\right)=n \varepsilon_{A}+m \varepsilon_{B}, \text { where } n+m=1 .
$$

Bragg's rule assumes that the stopping process is independent of the bonding state of the valence electrons between the atoms in a material. This assumption is generally well fullfilled for the velocity range of ${ }^{15} \mathrm{~N}$ projectiles used for NRA on heavier targets. Noticeable deviations from Bragg's additivity rule may occur, however, when strong hybridizations in compounds of light elements are present such as in organic hydrocarbons $[140,141]$ or amorphous hydrogenated silicon carbide [142], especially for light projectile ions at low energy, such as He used in ERD or RBS. To account for the chemical bonding state of elements in compounds, Ziegler and Mayonan introduced corrections to the Bragg rule that consider (atomic) cores and bonds (electrons) separately (CAB model) [140]. The accuracy of different semi-empirical parameterizations of the energy loss in simulation codes relative to 
experimental stopping power data is still subject of ongoing research [143-145]. The SRIM program is being refined continuously to achieve an ever better agreement of the simulations with the experimental data [126].

In converting experimental $\mathrm{NR}$ excitation curves into $\mathrm{H}$ concentration depth profiles, the stopping power quantitatively affects the depth scale (Eq. 2.2) as well as the bulk $\mathrm{H}$ content (Eq. 2.12). If the target contains a sizeable $\mathrm{H}$ concentration (on the order of several atomic \%), the material's stopping power will in turn depend on this $\mathrm{H}$ content. To determine stopping power and $\mathrm{H}$ concentration in such cases, the ( $d E / d z)$ value is first to be approximated with Bragg's rule for a roughly estimated $H$ content. This approximate stopping power is then used to derive a first $\mathrm{H}$ concentration value from the experimental NRA data. In a second iteration of the analysis this $\mathrm{H}$ concentration is used to calculate a refined $(\mathrm{d} E / \mathrm{d} z)$ value, with which the $\mathrm{H}$ content is evaluated again. After a few repetition cycles the process should converge to produce consistent values of $\mathrm{H}$ content and stopping power.

\subsubsection{Straggling}

Straggling becomes the dominant limitation of the depth resolution (Section 2.2.3) from probing depths beyond several $\mathrm{nm}$, the precise value depending on the stopping power and straggling cross section of the projectile in the target. Straggling is a complicated stochastic process related to multiple scattering of the ions with electrons in the target. It depends on both the analyzing ion beam and the target material, i.e., on the atomic mass $\left(M_{1}\right)$, atomic number $\left(Z_{1}\right)$, energy $\left(E_{1}\right)$, and charge state $(q)$ of the projectile in the target, as well as on the target element $\left(Z_{2}\right)$, its 
number density $N$ [atoms $\mathrm{cm}^{-3}$ ], and its electronic structure $[111,130]$. The collisions between the projectile and the target electrons are strongly correlated, i.e. not independent of previous interactions. Various theories have been developed to describe the process in different projectile velocity regimes and for light as well as for heavier ions [105, 146-149]. Bohr's theory [105] assumes that the target atoms are randomly distributed, that the energy loss during a single collision is much smaller than the width of the energy loss distribution, and that the projectile velocity is much greater than the orbital electron velocity of the target atoms, i.e., $v>>$ $v_{\mathrm{o}} / \mathrm{Z}_{2}{ }^{1 / 2}\left(v_{\mathrm{o}}=e^{2} /\left(4 \pi \varepsilon_{0} \hbar\right)=2.188 \times 10^{6} \mathrm{~m} \mathrm{~s}^{-1}\right)$. In this high velocity regime the energy distribution is Gaussian, with an almost velocity-independent width parameter (the Bohr straggling cross section): $\Omega_{s}{ }^{2}=Z_{1}^{2} Z_{2} e^{4} N \Delta h /\left(4 \pi \varepsilon_{0}{ }^{2}\right) . N \Delta h$ gives the density (in atoms $/ \mathrm{cm}^{2}$ ) of the target penetrated by a thickness $\Delta h$ [130]. At medium and low projectile energies the assumption of velocity-independent straggling is not valid. The Lindhard-Scharff theory [147] extends Bohr's theory by adding a correction factor for medium- and low-energy projectiles, where $v^{2} \leq 3 Z_{2} v_{o}^{2}$ [130]. The Hvelplund theory [149] applies for heavy ions in the low-velocity range, i.e., $v<$ $v_{0} Z_{1}^{2 / 3}$, where the electronic stopping is proportional to the projectile velocity, and when $Z_{1}$ and $Z_{2}$ do not differ by more than a factor of 4 . This theory takes the binding of target electrons and their spatial distribution into account [106, 111].

Unfortunately, rather few systematic investigations of straggling cross sections for ${ }^{15} \mathrm{~N}$ projectiles in solid targets exist in the NRA relevant energy range, and the agreement with the above theoretical models is sometimes far from satisfactory. Rud et al. compiled a larger body of experimental straggling FWHM values of ${ }^{15} \mathrm{~N}$ at 
6.5 MeV in various elemental targets ( $C, A l, C u, G e, A g, S n, B i)$ and found that the straggling width (FWHM) divided by $Z_{2}^{0.39 \pm 0.02}$ scales proportional to the square root of the penetrated thickness [106]. Hjörvarsson and Ryden measured the straggling of 6.5-10 MeV ${ }^{15} \mathrm{~N}$ in Au thin films and found that their data matched Rud's results very closely as seen in Fig. 6 . Here, the broken line is a fit to Rud's empirical formula [111]:

$$
\delta_{S}=2.03 Z_{2}^{0.39} \text { thickness }^{1 / 2}
$$

with $\delta_{s}$ in $\mathrm{keV}$ and the thickness in $10^{16}$ atoms $/ \mathrm{cm}^{2}$.

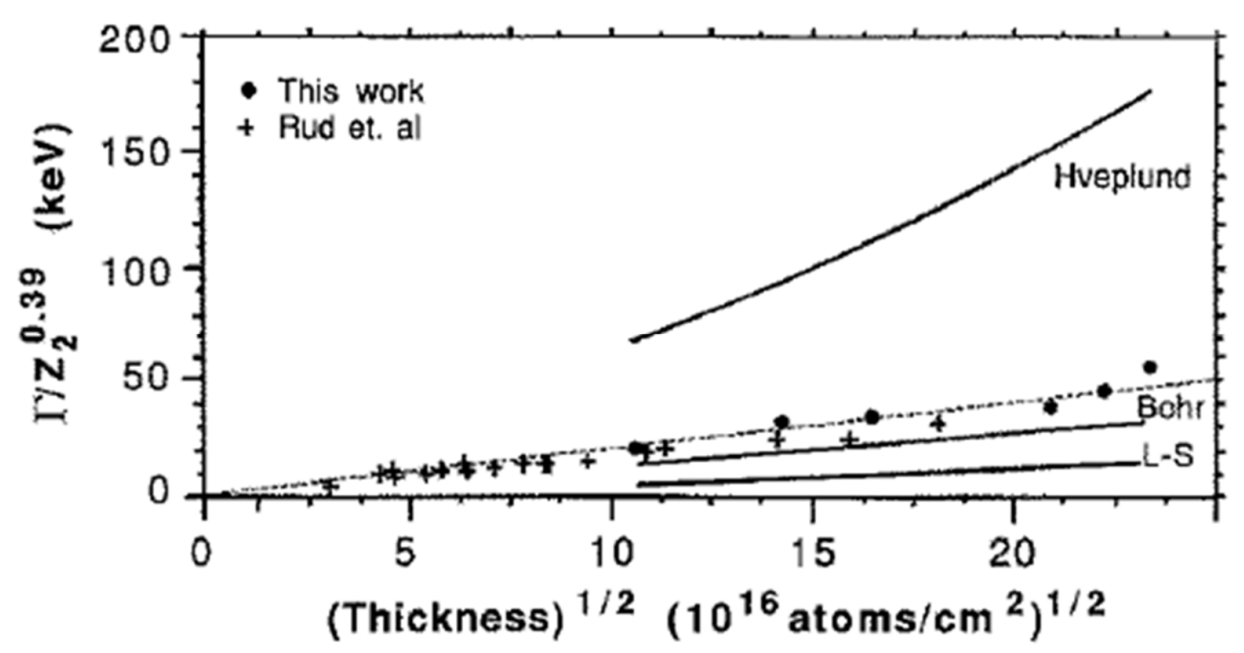

Fig. 6. Comparison of experimental straggling width parameters of $6.4 \mathrm{MeV}{ }^{15} \mathrm{~N}$ from Refs. [106] $(+)$ and [111] $(\bullet)$ with theoretical estimations of maximum electronic straggling. The dotted line represents the empirical formula by Rud (Eq. (2.14)). Reproduced from Ref. [111].

For ${ }^{15} \mathrm{~N}$ projectiles incident on Au above $6.4 \mathrm{MeV}, v>4 v_{0}$, so in terms of the above theories, the Bohr theory should apply at ${ }^{15} \mathrm{~N}$ energies much higher than 5 
$\mathrm{MeV}$ and the Lindhard-Scharff theory should be valid for ${ }^{15} \mathrm{~N}$ energies below $85 \mathrm{MeV}$. The validity range of the Hvelplund theory for ${ }^{15} \mathrm{~N}$ in Au ends at energies below 5 MeV and also $Z_{2} / Z_{1}>4$, so this model is not expected to describe the straggling well for NRA conditions, which is indeed the case as the comparison to Hjörvarsson's and Rud's data in Fig. 6 shows. The Bohr and Lindhard-Scharff theories, on the other hand, appeared to underestimate the straggling effect. A few years later Briere and Biersack [150] as well as Goppelt-Langer et al. [151] independently made similar measurements of $6.4 \mathrm{MeV}{ }^{15} \mathrm{~N}$ ion straggling in thin elemental overlayers on $\mathrm{H}$ terminated Si-backings for several elements $\left(6 \leq Z_{2} \leq 79\right)$, and, surprisingly, obtained rather good agreement of their data with the Bohr straggling theory (Fig. 7).

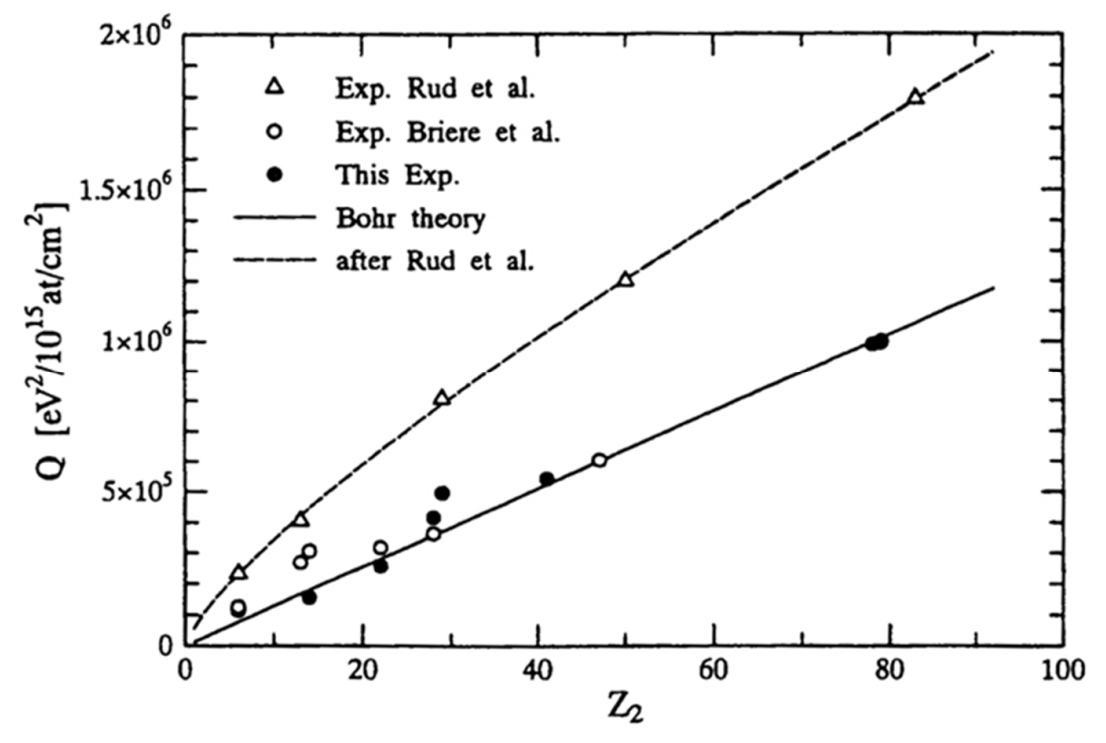

Fig. 7. Comparison of experimental straggling width parameters for $6.4 \mathrm{MeV}{ }^{15} \mathrm{~N}$ obtained by Rud et al. [106], Briere and Biersack [150], and Goppelt-Langer et al. [151] (•) with the Bohr straggling theory (solid line) and Rud's empirical $Z_{2}{ }^{0.39}$ dependence (Eq. (2.14), broken line). Reproduced from Ref. [151]. 
Ref. [151] notes that this agreement should be regarded as coincidental rather than as a justification for the Bohr theory in this energy range. The Bohr theory assumes the ions to be fully stripped, which is not the case for $6.4 \mathrm{MeV}{ }^{15} \mathrm{~N}$, where the equilibrium charge state is about +5 , so charge state fluctuations are expected to contribute to straggling. To our knowledge these discrepancies still stand unresolved as of today, suggesting that gaps exist in the systematic understanding of the straggling process and that apparently also the experimental determination of straggling parameters can have its pitfalls, especially for ultrathin films. Non-uniform film thicknesses, i. e., surface or interface roughness, reactive interfacial diffusion, or island formation come to mind as possible error sources. Fukutani et al. even found indications that the Rud formula may underestimate the straggling width in ultrathin $\mathrm{Pb}$ deposits on $\mathrm{H}$-terminated $\mathrm{Si}(111)$ surfaces [59].

For the important $\mathrm{H}$ profiling work with $\mathrm{SiO}_{2} / \mathrm{Si}$ systems reviewed in Chapter 3.3.1, Briere and Bräunig established that the energy straggling for $6.4 \mathrm{MeV}^{15} \mathrm{~N}$ ions in glow discharge deposited amorphous $\mathrm{SiO}_{2}$ films expectedly scales as a function of the square root of the film thickness, i.e., $\sigma_{s}=\left(Q_{s} \Delta h\right)^{1 / 2}$, where the straggling cross section $Q_{s}=1.06 \times 10^{6} \mathrm{eV}^{2} / \mathrm{nm}$ was again found to be in good agreement with the classical Bohr theory of fully stripped ions [107].

\section{$2.2 \quad$ Instrumental aspects}

\subsubsection{Ion beam generation (accelerator)}

The $\mathrm{MeV}$ ion beams required for nuclear reaction analysis are generated by single stage or tandem accelerators. Several groups have been performing ${ }^{1} \mathrm{H}\left({ }^{15} \mathrm{~N}\right.$, 
$\alpha \gamma)^{12} \mathrm{C}$ NRA internationally and described their accelerator and measurement facilities to various degrees of detail in the literature, to which we shall refer the interested reader (some of these installations appear to have been decommissioned or to no longer be in active use): Amsel (Paris) [152-154], Wagner and Rauch (Frankfurt) [77, 155, 156], Kalbitzer (Heidelberg) [98, 157, 158], Herrmann and Grambole (FZ Rossendorf) [159], Krauser, Bräunig, Briere, and Weidinger (HMI) [107, 160, 161], Thomas and Fallavier (Cedex) [162], Lanford (SUNY-Albany) [51], Davis (LLNL) [163, 164], Allred, White, Clark, and Appleton (Oak Ridge Natl. Lab.) [112], Saris et al. (FOM, Amsterdam) [165], Pick, Dienes, Davenport, Ruckman, and Strongin (Brookhaven) [166], Hjoervarsson (Uppsala) [167], Andersen, Rud, Bøttiger, Jensen, and Stensgaard (Aarhus) [168], Berheide, Becker, Schulte, Rolfs (Bochum) [118, 169171], Schwickert, Uhrmacher, Lieb (Göttingen) [172, 173], Yamamoto and Naramoto (JAERI, Takasaki) [151, 174], Sasa (Tsukuba) [116], and de Almeida and Baumvol (Rio Grande do Sul) $[175,176]$.

Here, we give an exemplary description of the vertically mounted $5 \mathrm{MV}$ van-deGraff tandem accelerator (5UD Pelletron, National Electrostatics Corporation) in the MALT microanalysis laboratory at the Department of Nuclear Engineering and Management of the University of Tokyo [177].

\section{Accelerator}

The MALT accelerator system is schematically illustrated in Fig. 8. It features a negative ion source in which $\mathrm{Cs}$-sputtering of a mixed $\mathrm{Ti}^{15} \mathrm{~N}+\mathrm{C}$ cathode material generates an intense $(\sim 1 \mu \mathrm{A})$ and highly stable beam of primary $\mathrm{C}^{15} \mathrm{~N}^{-}$ions which are injected from a negative ion source potential $\left(V_{i}\right)$ into the first acceleration stage. To 
produce ${ }^{15} \mathrm{~N}^{2+}$ beams of $6.385 \mathrm{MeV}$ the terminal is held at a potential $V_{\mathrm{T}}(\approx 2.47 \mathrm{MV})$. At the terminal the accelerated $\mathrm{C}^{15} \mathrm{~N}^{-}$ions pass through a stripper gas canal where collision-induced charge exchange with low pressure Ar gas leads to their break up into mainly ${ }^{15} \mathrm{~N}^{2+}$ and ${ }^{15} \mathrm{~N}^{3+}$ ions in addition to $\mathrm{C}^{2+}, \mathrm{C}^{3+}$, and a smaller number of other charge states. The now positive ${ }^{15} \mathrm{~N}^{9+}$ ions are accelerated in the second tandem stage towards the exit of the accelerator, where they attain an energy of $E_{i}=e\left(\left(V_{\mathrm{i}}+\right.\right.$ $\left.\left.V_{\mathrm{T}}\right)\left[m_{\mathrm{N}} /\left(m_{\mathrm{N}}+m_{\mathrm{C}}\right)\right]+q V_{\mathrm{T}}\right)$, where $V_{\mathrm{T}}$ is the terminal voltage, $e$ the elemental charge, $q$ the charge state of the ${ }^{15} \mathrm{~N}$ ion, and $m_{\mathrm{N}}$ and $m_{\mathrm{C}}$ are the masses of the ${ }^{15} \mathrm{~N}$ and ${ }^{12} \mathrm{C}$ atoms, respectively. The accelerated ions then pass through a mass and energy dispersive analyzer that extracts only a single selected ${ }^{15} \mathrm{~N}^{9+}$ charge state as the usable beam.

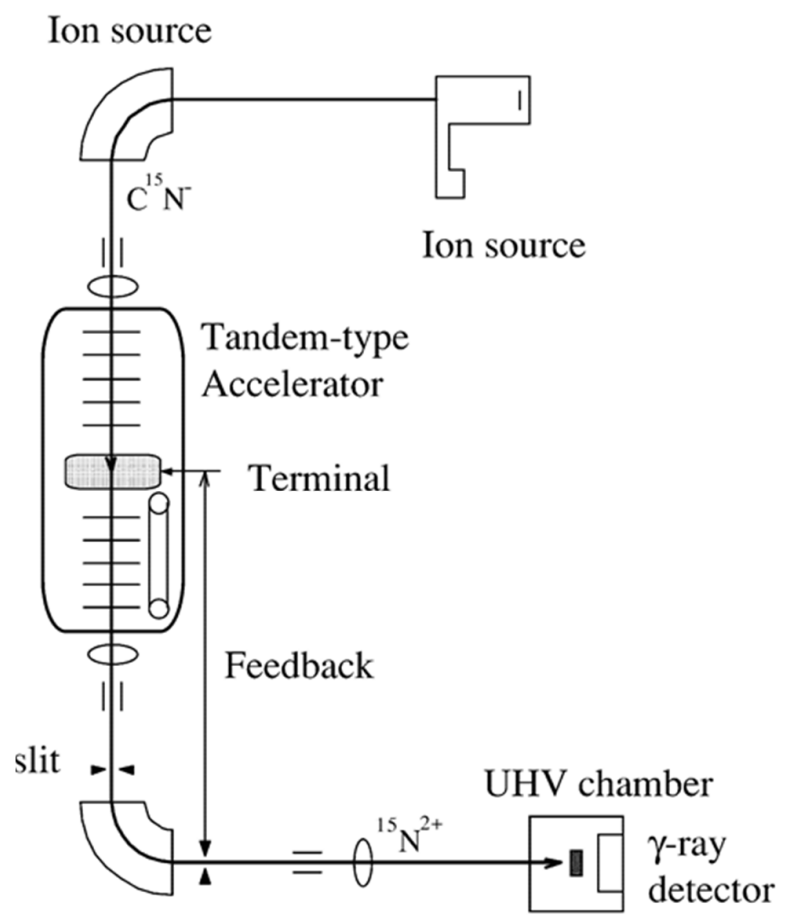

Energy analyzer

Fig 8. Schematic illustration of the MALT tandem accelerator system used for ${ }^{1} \mathrm{H}\left({ }^{15} \mathrm{~N}, \mathrm{ag}\right)^{12} \mathrm{C}$ NRA at the University of Tokyo. Reproduced from Ref. [57] with permission from Elsevier. 
This monochromator is a double focusing sector magnet in which for a given field $B$ only ions of a specific momentum/charge ratio pass on a trajectory along the center radius $r: B \cdot r=\left(2 m E / q^{2}\right)^{1 / 2}$. The analyzing magnet of the MALT system has adjustable entrance and exit slits and a large orbital radius of $r=1270 \mathrm{~mm}$ that achieves an excellent energy resolution of $\Delta E / E=\Delta s / 2 r=4.7 \times 10^{-4}$ at a $\Delta s=1.2 \mathrm{~mm}$ slit width setting, delivering a well-monochromatized $\left(\Delta E_{\mathrm{B}} \leq \sim 3 \mathrm{keV}\right){ }^{15} \mathrm{~N}^{2+}$ beam of 6.385 $\mathrm{MeV}$ (up to $40 \mathrm{nA}$ ) to the target in our UHV analysis chamber. The beam energy defining magnet field is measured with a high precision ${ }^{1} \mathrm{H}$ NMR probe placed between the magnet pole pieces and stabilized to within $10^{-5}$ with a feedback system, which warrants superior reproducibility of the beam energy.

The MALT system features a second feedback control for the terminal voltage, which senses the ion current on an upper (lower energy) and lower (high energy) probing slit (width $\pm 2.5 \mathrm{~mm}$ ) located just in front of the narrower energy defining exit slit of the analyzer, and regulates the accelerator terminal voltage such that the ions always pass through the center of the analyzer. This minimizes beam current fluctuations due to terminal voltage ripple and drift, thus providing excellent stability of the beam output.

Adjusting the analyzer magnet field with the accelerator in slit feedback mode enables a highly accurate setting of the beam energy and greatly facilitates energy scans for depth profiling. Alternative ways of beam energy scanning employ electrostatic deflectors behind the entrance and in front of the exit slits of the analyzer ('Amsel steerers') $[108,153,154,157,173]$, or impose a bias voltage to the target. These latter methods are faster and hysteresis-free but have only narrow 
scan ranges $(8-10 \%$ of the central analyzer pass energy $[153,154]$ or $~ 30 \mathrm{keV}$, respectively) before retuning of the analyzer and the terminal becomes necessary.

We routinely employ ${ }^{15} \mathrm{~N}^{2+}$ beams for ${ }^{1} \mathrm{H}\left({ }^{15} \mathrm{~N}, \alpha \gamma\right){ }^{12} \mathrm{C}$ measurements of surfacenear hydrogen distributions, allowing for energy scans from $6.385 \mathrm{MeV}$ up to 12.5 $\mathrm{MeV}$ by raising the terminal voltage towards the maximum of $5 \mathrm{MV}$. If necessary, higher beam energies up to $17.5 \mathrm{MeV}$ can be realized by selecting the ${ }^{15} \mathrm{~N}^{3+}$ charge state with the analyzing magnet.

\section{Ion Source}

The generation of the ${ }^{15} \mathrm{~N}$ ion beam for NRA with a tandem accelerator requires a negative ion in the first acceleration stage. This cannot be achieved with nitrogen alone since $\mathrm{N}^{-}$is unstable, leaving plasma sources (Alphatross, Duo-Plasmatron) to produce $\mathrm{NH}_{2}{ }^{-}$ions from gaseous $\mathrm{NH}_{3}$ or the SNICS type source (Source of Negative Ions by Cesium Sputtering, National Electrostatics Corp.) delivering $\mathrm{CN}^{-}$ions as viable alternatives. At MALT two multi-target SNICS ion sources are available, which allow exchanging exhausted cathodes quickly without disrupting the beam operation by remote-controlled rotation of a cathode wheel that accomodates up to 40 sputter targets. The SNICS ion source delivers a highly stable and intense output of $\mathrm{CN}^{-}$, routinely generating beams of $-2 \mu \mathrm{A}$ and more at the accelerator terminal. In the $\mathrm{Ar}$ stripper gas canal, straggling and fragmentation of $\mathrm{CN}^{-}$by Coulomb explosion after charge exchange induce considerable broadening to the ion energy distribution [178] (see below). Due to this energy broadening a relatively large fraction of the $\mathrm{N}^{++}$ beams is lost in subsequent energy analysis. In our case these losses are affordable since the efficient $\mathrm{CN}^{-}$output of the SNICS source provides for sufficient remaining 
${ }^{15} \mathrm{~N}$ ion beam current after monochromatization. At MALT, achieving stable monoenergetic beam output conditions with the gas ion source (Alphatros) to produce $\mathrm{NH}_{2}^{-}$ions was found to be difficult, so that we routinely employ $\mathrm{CN}^{-}$beams from the SNICS ion source.

\section{${ }^{15} \mathrm{~N}$ lon energy width without monochromatization}

The energy broadening through Coulomb explosion after charge exchange with the stripper gas is significantly smaller in case of $\mathrm{NH}^{-}$compared to $\mathrm{CN}^{-}$as the parent negative molecular ion. According to Hartmann et al. [178], the disintegration of the diatomic parent ion $(\mathrm{AB})$ into two fragments $\mathrm{A}$ and $\mathrm{B}$ of positive charges $q_{\mathrm{A}}$ and $q_{\mathrm{B}}$ superimposes a small randomly directed velocity $\left(\boldsymbol{v}_{\mathrm{D}}\right)$ component to the large terminal velocity $\left(\boldsymbol{v}_{T}\right)$ of the fragments (typically $10^{-3} \leq\left|v_{D} / v_{T}\right| \leq 10^{-2}$ ). This leads to satellite peaks in the fragments' energy distribution that are shifted to higher and lower values by a maximum of $\pm E_{\mathrm{s} 0}=\left(k E_{T} E_{\mathrm{D}}\right)^{1 / 2}$ against the center beam energy of neutrally dissociating $A B$ with $k=4 \mu /(1+\mu)^{2}$ and $\mu=m_{A} / m_{B}$. Here, $E_{T} \approx e V_{T}$ is the terminal energy of the parent molecular ion, and $E_{D}=q_{A} q_{B} e^{2} / 4 \pi \varepsilon_{0} d_{A B}$ the Coulomb energy due to molecular disintegration from an intial interatomic distance $d_{A B}$. The kinematic factor $k$ is symmetric in the atomic components $A$ and $B($ i.e., $k(\mu)=k(1 / \mu)$ ), so that the two fragments experience the same energy shift. For the nearly isobaric ${ }^{12} \mathrm{C}^{15} \mathrm{~N}$ molecule $k=0.988$, whereas in the highly asymmetric case of ${ }^{15} \mathrm{~N}^{1} \mathrm{H} k=0.234$ so that the Coulomb explosion related energy shift of $6.4 \mathrm{MeV}{ }^{15} \mathrm{~N}^{2+}$ ions $\left(E_{\mathrm{T}}=2.47\right.$ $\mathrm{MeV})$ produced from $\mathrm{NH}^{-}\left(E_{s 0}=3.8 \mathrm{keV}, q_{\mathrm{H}}=1, d_{\mathrm{NH}}=1 \AA\right)$ is only about half as large as that of those generated from $\mathrm{CN}^{-}\left(E_{\mathrm{s} 0}=7.8 \mathrm{keV}, q_{\mathrm{C}}=1\right.$ and $\left.d_{\mathrm{CN}}=1.158 \AA\right)$. For this reason $\mathrm{NH}_{2}^{-}$is often recommended in preference over $\mathrm{CN}^{-}$as the primary ion in 
tandem accelerators for the generation of $\mathrm{N}^{\mathrm{q}+}$ ion beams with small energy widths [52] [178].

All three peaks in the final ${ }^{15} \mathrm{~N}^{9+}$ ion energy distribution (the central $\left(E_{\mathrm{i}}\right)$ and the two symmetric satellites at $E_{\mathrm{i}} \pm E_{\mathrm{s} 0}$ ) have identical width, which is determined by terminal voltage ripple and by straggling in the stripper gas [178]. The intensity of the satellites relative to that of the center beam peak at $E_{i}$ also depends on the stripper gas density, since the latter influences the probability for Coulomb explosion, i.e., the likelihood that $\mathrm{CN}^{-}$(or $\mathrm{NH}^{-}$) become multiply ionized by charge exchange before collision-induced dissociation. Therefore the stripper gas pressure is an important parameter to optimize the yield of a desired $\mathrm{N}^{q^{+}}$charge state and to minimize the Coulomb explosion satellites. For ${ }^{15} \mathrm{~N}^{2+}$ of $6.4 \mathrm{MeV}$ generated from $\mathrm{CN}^{-}$Hartmann et al. found optimum conditions at a stripper gas $\left(\mathrm{N}_{2}\right)$ pressure around $10^{-3} \mathrm{mbar}$, at which the satellite peaks were almost completely reduced (to only $15 \%$ of the center peak intensity) and the straggling-dominated width of the center peak was in the order of $\sim 5 \mathrm{keV}$ (Figs. 8 and 10 in Ref. [178]).

We also routinely observe an energy width $\Delta E_{\mathrm{B}}$ (FWHM) of similar magnitude for the $6.385 \mathrm{MeV}{ }^{15} \mathrm{~N}^{2+}$ ion beam produced at MALT from $\mathrm{CN}^{-}$prior to its monochromatization (measured with a beam profile monitor located in front of the analyzer exit slit). Knowing this figure $\left(\Delta E_{B}\right)$ may help estimating the expectable nearsurface NRA depth resolution (from Eqs. (2.10) or (2.15)) or be useful for Doppler broadening analysis of surface hydrogen in cases where no monochromator is available. Note also that these $\Delta E_{\mathrm{B}}$ values are already smaller than $E_{\mathrm{s} 0}(7.8 \mathrm{keV})$ for 6.4 MeV ${ }^{15} \mathrm{~N}^{2+}$ produced from $\mathrm{CN}^{-}$, which suggests that a major additional resolution improvement by chosing $\mathrm{NH}_{2}^{-}$as the primary ion might no longer be expected if the 
satellite peaks due to Coulomb explosion are already sufficiently suppressed by suitable adjustment of the stripper gas pressure.

\subsubsection{Measurement apparatus and sample requirements}

\section{Measurement apparatus}

It has been recognized early on in the development of hydrogen depth analysis with ion beams that a very clean vacuum in the target chamber is highly important, since otherwise a gradual build-up of hydrocarbons on the sample surface will cause the depth scale as well as the surface hydrogen concentration to vary during the measurement $[71,162,179-181]$. A vacuum in the $10^{-7} \mathrm{~Pa}$ range is necessary to achieve stable conditions for hydrogen analysis at surfaces [179]. Accordingly, our UHV target chamber designated for NRA measurements at single crystal surfaces has a base pressure $<1 \times 10^{-8} \mathrm{~Pa}$. Fig. 10 shows a cross-sectional top view of the vacuum chamber in the plane that contains the NRA ion beam. Our $(\mathrm{ca} .4 \mathrm{~cm})$ Pb-shielded 4" BGO $\gamma$-detector is positioned outside the vacuum on the beam axis $30 \mathrm{~mm}$ behind the sample, which is mounted on a manipulator that enables its positioning along 3axes $(x, y, z)$ as well as rotation around the vertical chamber axis. The UHV chamber is equipped with all necessary facilities for in-situ preparation and characterization of single crystal targets, containing a sputter ion gun and 4-grid LEED optics with capability for AES analysis. 


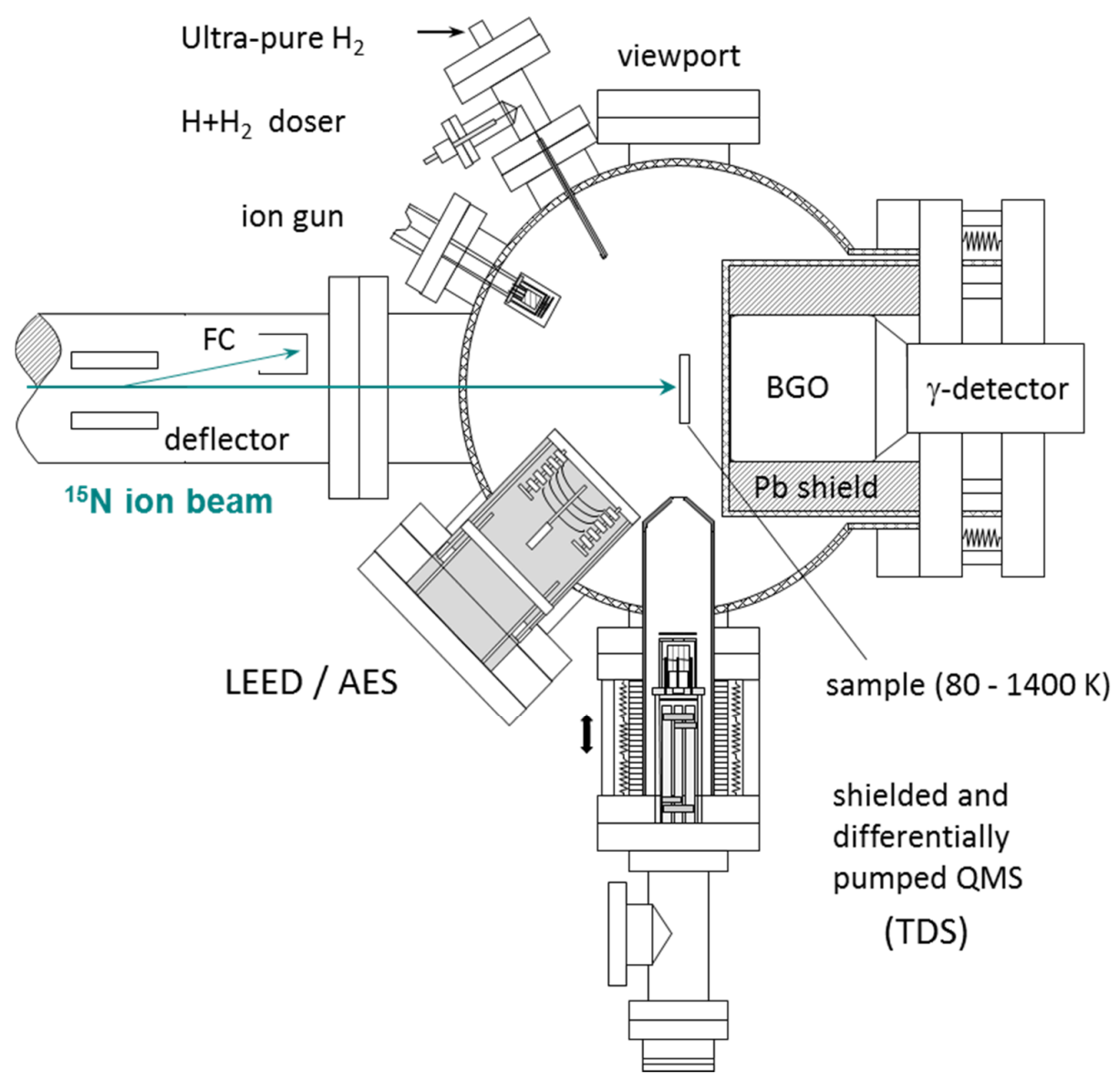

Fig. 9. UHV target chamber at the University of Tokyo's MALT tandem accelerator. The system has a base pressure $<1 \times 10^{-8} \mathrm{~Pa}$ and is equipped for resonant ${ }^{1} \mathrm{H}\left({ }^{15} \mathrm{~N}, \alpha \gamma\right){ }^{12} \mathrm{C}$ NRA measurements at in-situ prepared single crystal targets in combination with $\mathrm{H}_{2}$ TDS.

The sample can be cooled to $80 \mathrm{~K}$ with liquid nitrogen (optionally to $~ 20 \mathrm{~K}$ with liquid $\mathrm{He}$ ) and heated to $>1400 \mathrm{~K}$ by thermal radiation and electron bombardment from a tungsten filament mounted on its backside. Temperature control is achieved with a thermocouple directly spotwelded to the crystal side (in case of metal targets). The pressure is measured with a Bayard-Alpert ion gauge (not shown). A special feature of our apparatus is the shielded and differentially pumped quadrupole mass spectrometer (QMS, Balzers Prisma 200) that can be translated with aid of a linear motion device so that the small aperture in the tip of its shield can be approached as 
close as $0.5 \mathrm{~mm}$ to the sample surface. This equipment enables TDS measurements at hydrogen-exposed samples in combination with in-situ NRA analysis in the same system, which is particularly versatile in investigations of hydrogen adsorption and absorption processes at reactive metal surfaces as described in Sections 3.1 and 3.2. Ultrapure $\mathrm{H}_{2}(7 \mathrm{~N})$ from a PdAg membrane diffusion purifier is used for the hydrogen exposures in these experiments. If necessary, atomic $\mathrm{H}$ can be produced via partial thermal dissociation by passing the purified $\mathrm{H}_{2}$ gas through a Mo tube that contains a heated tungsten filament.

To exclude contamination of the sample surface by impurities introduced by the ion beam from the accelerator or the beam lines, the sample is positioned not in direct line of sight from the accelerator but slightly above the beam axis during the NRA measurements. An electrostatic deflector in the last differential pumping stage in front of the UHV chamber is used to steer only the ${ }^{15} \mathrm{~N}$ ion beam onto the sample, while any possible neutral contaminants are stopped by a Faraday cup mounted on the beam line axis. When the deflector state is off during the beam energy tuning intervals, the Faraday cup monitors the ion beam current, which also allows verifying proper beam transport to the UHV system from the accelerator's analyzer, where another Faraday cup monitors the monochromatized beam output. The ion beam is focused onto the target with set of magnetic quadrupole lenses on the beamline between the energy analyzer and the target chamber. In order to monitor the beam profile, the luminescent beam halo is observed on a sheet of sapphire or quartz mounted on the sample manipulator and brought into target position. The magnetic beam focusing projects the energy analyzer slit profile onto the target, i.e., the beam 
size is at least $\sim 1 \mathrm{~mm}$ in diameter for work at maximum depth resolution, but it can be defocused into virtually any shape if necessary, e.g., to reduce beam-induced $\mathrm{H}$ desorption from the target surface.

Accurate charge integration is an important requirement to obtain quantitative hydrogen concentration measurements (Section 2.2.6). Ideally, the entire target vacuum chamber is electrically insulated from the accelerator beamline and included in the charge integration system [50,51]. Alternatively, a Faraday-cup mounted target is recommended. In our system these conditions are not perfectly fulfilled as the facilities for sputtering and LEED/AES/TDS measurements prevent the electrical insulation of the vacuum chamber and also require access to the sample surface during its manipulation for preparation and characterization. We therefore apply a positive bias (on the order of $100 \mathrm{~V}$ ) to the sample in order to retain the low energy secondary electrons during the NRA measurement.

\section{Sample requirements}

${ }^{15} \mathrm{~N}$ NRA is highly versatile and almost universally applicable to a wide range of materials. Only a minimum of preconditions with regard to the sample material is required to allow for successful hydrogen depth profiling. Evidently the target must be a material that is stable in high vacuum and ideally shows a certain resistance against degeneration under the ion beam (cf. Section 2.2.5). The elemental composition of the material (except for the $\mathrm{H}$ content) and its density should be known as precisely as possible so that the stopping power can be calculated accurately. For a meaningful conversion of the ${ }^{15} \mathrm{~N}$ projectile energy loss into an analysis depth axis, the material should have a laterally uniform density (no pores) 
and, if possible, a flat surface. Surface roughness can affect the depth resolution as an additional broadening, since within the size of the ion beam diameter ( $>1 \mathrm{~mm}$ ) any height variations of the sample will be averaged in the measurement. While this poses no major concern in the case of a more or less continuous internal hydrogen distribution, it will smear out the locations of strongly localized $H$, such as at interface positions. The effects of surface roughness become increasingly pronounced at increasing projectile incidence angles, so very flat surfaces are required for measurements under strongly grazing ion incidence. Even steps of atomic height may lead to energy shifts in surface resonance profiles in such geometries (Section 3.3.2) [59].

\subsubsection{Depth resolution and range}

The depth resolution $\Delta d$ in $\mathrm{H}$ profiling by resonant NRA is determined by the energy width $\Delta E_{\text {tot }}$ of the instrumental function $F\left(E_{\mathrm{i}}, z\right)$ (Eq. 2.10) and the stopping power $S$ of the analyzed material:

$$
\Delta \mathrm{d}=\Delta E_{\mathrm{tot}} \cos \alpha_{\mathrm{i}} / S=\cos \alpha_{\mathrm{i}}\left(0.56 \Gamma+\left[8 \cdot \ln (2)\left(\delta_{\mathrm{B}}^{2}+\delta_{\mathrm{D}}^{2}+\left(\delta_{\mathrm{s}}(z)\right)^{2}\right)\right]^{1 / 2}\right) / S
$$

Owing to energy straggling of the projectile ions within the target the depth resolution is best at the surface but deteriorates with increasing probing depth: $\delta_{s}(z)$ $\propto(z)^{1 / 2}[106]$. We first discuss the ultimate resolution at the surface, where $\delta_{s}(z=0)$ $=0$. Eq. (2.15) shows that even at the surface the ion beam energy spread $\left(\delta_{B}\right)$ and, in particular, the Doppler Effect $\left(\delta_{D}\right)$ broaden the experimental resonance profiles considerably beyond the resonance width $(\Gamma)$. Especially in near-surface $\mathrm{H}$ profiling 
with the narrow ${ }^{1} \mathrm{H}\left({ }^{15} \mathrm{~N}, \alpha \gamma\right){ }^{12} \mathrm{C}$ resonance at $6.385 \mathrm{MeV}(\Gamma=1.8 \mathrm{keV})$ and a reasonably monochromatized ${ }^{15} \mathrm{~N}$ beam, Doppler broadening $\left(\delta_{D} \sim 4-5 \mathrm{keV}\right)$ is the major limitation of the energy resolution close to the surface, leading to total spectral widths $\left(\Delta E_{\text {tot }}\right)$ of $\sim 10-14 \mathrm{keV}$ for $\mathrm{H}$ on semiconductors and $\sim 8-10 \mathrm{keV}$ on metals (cf. Fig. 3). With $S=1-4 \mathrm{keV} / \mathrm{nm}$ for ${ }^{15} \mathrm{~N}$ at $6.4 \mathrm{MeV}$, this translates into nearsurface NRA depth resolutions of $\sim 2-10 \mathrm{~nm}$ if the analyzing ion beam impinges onto the target in the surface normal direction $\left(\alpha_{i}=0\right)$. The near-surface depth resolution can be increased to values around $1 \mathrm{~nm}$ by adopting a strongly grazing incidence geometry of the ion beam with the target surface $\left(\alpha_{i} \rightarrow 90^{\circ}\right)$, as the stoppinginduced energy loss to penetrate into a certain depth $d$ scales with $1 / \cos \alpha_{i}(\Delta E=$ $S d / \cos \alpha_{i}$, Fig. 2). Submonolayer thickness variations of a $\mathrm{Pb}$ film on a H-terminated $\mathrm{Si}(111)$ surface in the order of $0.2 \mathrm{~nm}$ have been distinguished in this way by analyzing the energy shift of the surface resonance curve [59], see Section 3.3.2. Caution must be executed in measurements at very large incidence angles $\left(\alpha_{i} \geq 80^{\circ}\right)$, as surface roughness can have disturbing effects, and for ion trajectory lengths $>\sim 10$ $\mathrm{nm}$ straggling will again become resolution limiting.

The maximum depth range $R$ in which hydrogen can be probed with NRA for a specific nuclear reaction resonance $E_{R}{ }^{(L)}$ is determined by the energy separation of this $E_{R}{ }^{(L)}$ from the next cross section resonance at higher energy $\left(E_{R}{ }^{(H)}\right)$ divided by the stopping power of the analyzed material $[49,86]$. It is easily verified that:

$$
R=\left(E_{R}^{(H)}-E_{R}^{(L)}\right) \cos \alpha_{i} / S
$$


For ${ }^{15} \mathrm{~N}$ NRA at $6.385 \mathrm{MeV}$ in normal incidence $\left(\alpha_{i}=0\right)$, roughly $7 \mathrm{MeV}$ separation to the $2^{\text {nd }}$ resonance at $13.35 \mathrm{MeV}$, and elemental ${ }^{15} \mathrm{~N}$ stopping powers between 1.5 and $4 \mathrm{keV} / \mathrm{nm}$, the usable $\mathrm{H}$ probing depth is typically around $2-4 \mu \mathrm{m}$.

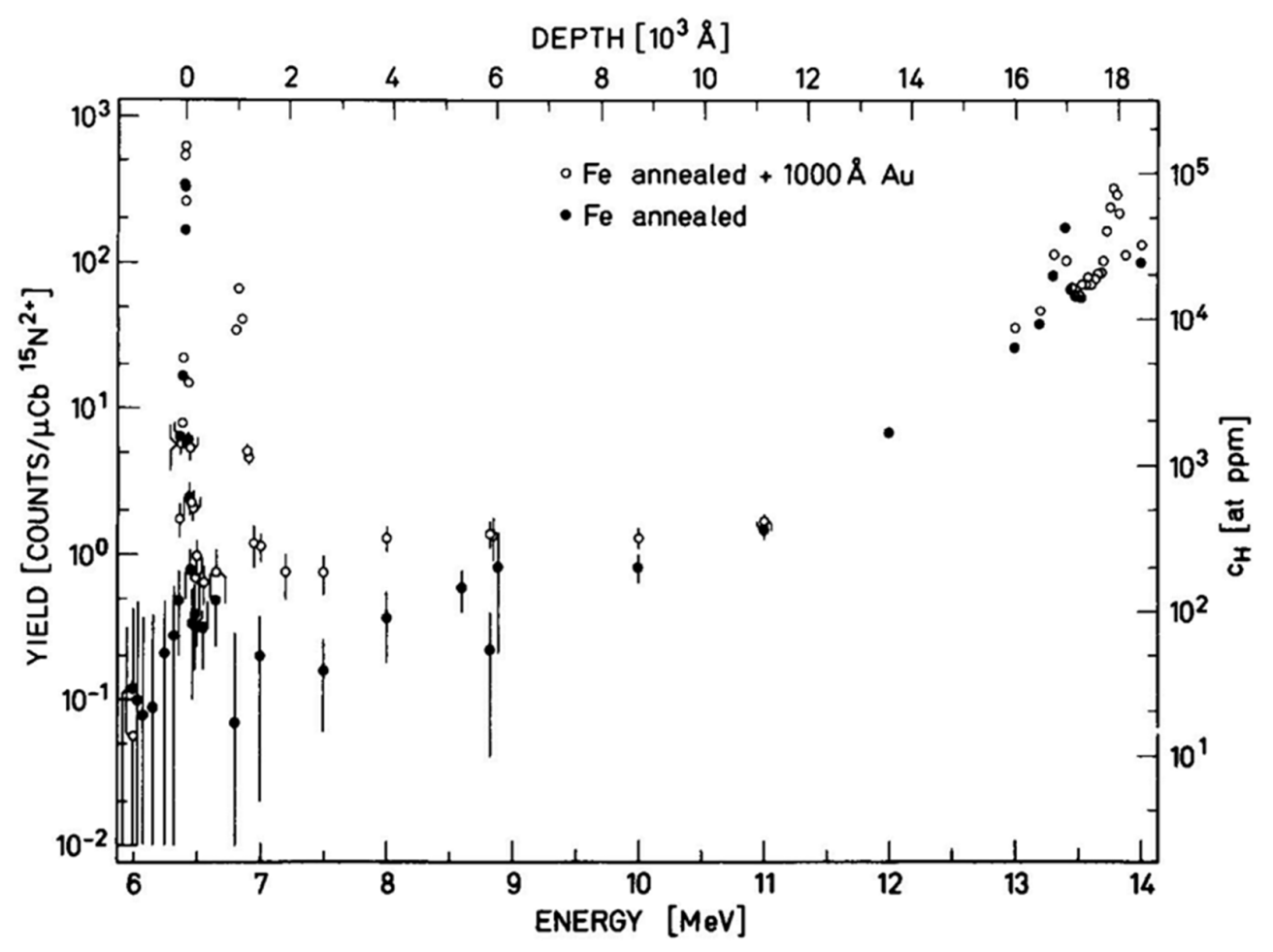

Fig. 10. $\gamma$-ray emission from the ${ }^{1} \mathrm{H}\left({ }^{15} \mathrm{~N}, \alpha \gamma\right){ }^{12} \mathrm{C}$ reaction obtained from 6 to $14 \mathrm{MeV}$ on a lowlevel detection system with an Fe target of immeasurably small $\mathrm{H}$ content in the bulk. $\mathrm{H}$ on the surface gives resonance peaks at 6.385 and $13.35 \mathrm{MeV}$. Additional Au/Fe interface peaks appear after a $1000 \AA$ Au film is deposited. Observe the strong increase of non-resonant reaction yield above $\sim 10 \mathrm{MeV}$. Adapted from Ref.[98] with permission from Elsevier.

Note, however, that $\mathrm{H}$ profiling with the ${ }^{1} \mathrm{H}\left({ }^{15} \mathrm{~N}, \alpha \gamma\right){ }^{12} \mathrm{C}$ reaction into extended depth regions may require caution in certain situations. If the target bears a $\mathrm{H}$-rich layer at the surface, such as ubiquitous hydrocarbon contaminations or hydroxyl in oxide skins common on air-exposed materials without in-situ surface cleaning, this surface hydrogen will interact with the nuclear reaction cross section between $E_{R}{ }^{(L)}$ 
and $E_{R}{ }^{(H)}$ to produce non-resonant $\gamma$-yield that bears no depth information but feigns non-existing bulk $\mathrm{H}$ content at the depth $d=\left(E_{\mathrm{i}}-E_{\mathrm{R}}{ }^{(\mathrm{L})}\right) \cos \alpha_{\mathrm{i}} / \mathrm{S}$. As an example, Fig. 10 displays the ${ }^{1} \mathrm{H}\left({ }^{15} \mathrm{~N}, \alpha \gamma\right){ }^{12} \mathrm{C} \gamma$-yield between 6 and $14 \mathrm{MeV}$ obtained from an annealed Fe sample before and after deposition of a $1000 \AA$ thick Au layer [98]. Hydrogen contamination on the $\mathrm{Fe}$ and $\mathrm{Au}$ surfaces gives rise to expected peaks at the 6.385 and 13.35 MeV resonances, and the additional features appearing at slightly higher energies after $\mathrm{Au}$ deposition suggest that some $\mathrm{H}$ was incorporated at the $\mathrm{Au} / \mathrm{Fe}$ interface. In the intermediate ${ }^{15} \mathrm{~N}$ energy region, observe that the $\gamma$-yield begins to increase markedly above $10 \mathrm{MeV}$ as the $13.35 \mathrm{MeV}$ resonance is approached. Since the $\mathrm{H}$ concentration in the Fe substrate was known to be immeasurably small, this yield must have resulted from non-resonant nuclear reactions with the surface (and $\mathrm{Au} / \mathrm{Fe}$ interface) hydrogen. Fig. 10 thus illustrates the fact that the off-resonant cross section for the $H\left({ }^{15} \mathrm{~N}, \alpha \gamma\right){ }^{12} \mathrm{C}$ reaction, which is small and usually negligible between 6 and $8.3 \mathrm{MeV}$ [100], increases by two orders of magnitude between 10 and $13 \mathrm{MeV}$ $[52,100,111]$. Hence, the non-resonant $\gamma$-yield at such ${ }^{15} \mathrm{~N}$ energies degrades the NRA sensitivity for $\mathrm{H}$ in large probing depths and may restrict the usuable depth range in which small $\mathrm{H}$ concentrations can be detected $[52,98-100,110,111]$, unless the non-resonant $\gamma$-yield is corrected for as explained in the following section.

\subsection{4 $\gamma$-detection, background, and sensitivity}

Here we describe the signal detection methods used in resonant NRA and explain the influence of various background sources on the sensitivity of $\mathrm{H}$ concentration measurements. For the $(p, \alpha \gamma)$ reactions listed in Table 1, detection of the characteristic $\gamma$-rays is usually preferred over the measurement of the product $\alpha$ - 
particles, which would also be possible in principle. The major advantage of $\gamma$ detection is that unlike any (solid state) detector for $\alpha$-particles, the appropriate $\gamma$ detectors do not require shielding from other energetic particles in the vicinity of the analyzed sample. Due to negligible attenuation of the $\mathrm{MeV} \gamma$-radiation in the thin stainless steel walls of vacuum chambers, the $\gamma$-detector can be placed conveniently on the atmospheric side of the analysis system. This arrangement elegantly circumvents the most intricate problems of alternative $\alpha$-particle detection, namely to avoid that the intense flux of scattered ${ }^{15} \mathrm{~N}$ ions and recoiled target atoms would overwhelm the faint $\alpha$-signal and possibly even damage the detector, and the additional analytical challenge to discriminate the $\alpha$-particles from target recoils (especially $\mathrm{H}$ ) and scattered beam projectiles.

It is apparent that collecting the characteristic $\gamma$-rays released in the nuclear reactions with a detector positioned outside the vacuum is the easiest and most efficient way to realize resonant $(p, \gamma)$ NRA measurements. The $\gamma$-rays produced in typical ${ }^{1} \mathrm{H}\left({ }^{15} \mathrm{~N}, \alpha \gamma\right){ }^{12} \mathrm{C}$ and ${ }^{1} \mathrm{H}\left({ }^{19} \mathrm{~F}, \alpha \gamma\right){ }^{16} \mathrm{O}$ NRA reactions (Table 1 ) are sufficiently more energetic than most natural radiation so that high signal-to-noise ratios can be achieved by $\gamma$-ray counting. For $\gamma$-ray detection $\mathrm{Nal}(\mathrm{TI})$ (thallium doped sodium iodide) and $\mathrm{BGO}$ (bismuth germanate, $\mathrm{Bi}_{4} \mathrm{Ge}_{3} \mathrm{O}_{12}$ ) scintillators of several inches in length and diameter are in common use. The scintillation crystals are coupled to photomultiplier tubes, so that the $\gamma$-ray energy is first converted into UV-VIS photons and then into an electron pulse of an amplitude proportional to the $\gamma$-ray energy. In addition to the signal at the incident $\gamma$-ray energy $E_{\gamma}$ (full-energy peak, $\mathrm{FE}$ ), the pulse height spectra also show so-called single-escape (SE) and double-escape (DE) peaks 
at the energies $E_{\gamma}-0.511 \mathrm{MeV}$ and $E_{\gamma}-1.022 \mathrm{MeV}$, repectively (Fig. 11). These features originate from $\gamma$-ray-induced electron-positron $\left(e^{-}-e^{+}\right)$pair production in the scintillation crystal. The positrons quickly annihilate upon collision with an electron, which releases two gamma rays of $h v_{\gamma}=m_{\mathrm{e}} c^{2}=0.511 \mathrm{MeV}$. The single and double-escape peaks then result from events when either one or both $0.511 \mathrm{MeV} \gamma$ rays leave the scintillation crystal without absorption and conversion into detectable UV-VIS photons.

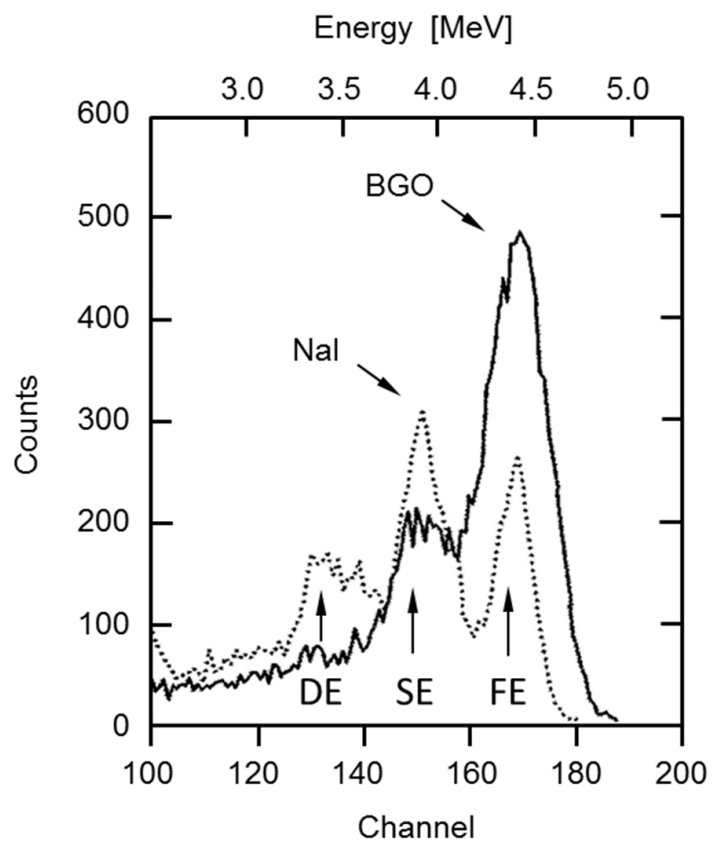

Fig. 11. Comparison of the gamma-ray spectra from the ${ }^{1} \mathrm{H}\left({ }^{15} \mathrm{~N}, \alpha \gamma\right){ }^{12} \mathrm{C}$ reaction recorded with 3 in. $x 3$ in. BGO and Nal detectors on the same target under the same conditions. Adapted from Ref. [182] with permission from Elsevier.

Due to its higher density BGO offers a superior $\gamma$-ray absorption coefficient compared to Nal [183] (on a transmitted length of 4 inches, BGO absorbs $~ 94 \%$ of 4 MeV $\gamma$-rays, Nal only $~ 73 \%$ [184]). This is advantageous since the rate of cosmic radiation background (CRB) increases roughly linearly with the volume of the 
scintillation crystal [185]; hence at the same sensitivity a smaller BGO detector has a lower CRB count rate than a larger Nal scintillator, which enhances the signal-tobackground ratio, cf. Eq. (2.17). BGO also has a 10 times smaller neutron absorption cross section than $\mathrm{Nal}[182,183]$, rendering BGO less susceptible to background in enviroments near thermal or radiative neutron sources.

Moreover, the signal content in the full-energy (FE) peak relative to that of the single- (SE) and double-escape (DE) features is higher in the energy spectrum of BGO due to the higher $\gamma$-ray attenuation coefficient of $B G O[182,185]$. This is seen in Fig. 11, which compares the $4.43 \mathrm{MeV} \gamma$-ray spectrum of a $\mathrm{H}$ containing target recorded under identical ${ }^{1} \mathrm{H}\left({ }^{15} \mathrm{~N}, \alpha \gamma\right){ }^{12} \mathrm{C}$ NRA conditions with Nal and BGO detectors. The Nal spectrum (dotted line) shows all three (FE, SE, DE) peaks, with an intensity ratio of about 1:2:1. In the BGO spectrum (solid line) the intensity ratio of the FE and SE peaks is about 3:1 and a DE peak is barely discernible. The intrinsic FE peak efficiency ratio of $\mathrm{BGO}$ and $\mathrm{Nal}$ is about $4: 1$; hence a $\mathrm{BGO}$ scintillator will provide a significantly higher signal-to-background ratio than an Nal detector [183]. If one tries to compensate for the weak FE peak of $\mathrm{Nal}$ by including the SE peak into the signal integration, one still recovers only $\sim 3 / 4$ of the $B G O F E$ peak intensity and the widened energy window will deteriorate the signal-to-noise ratio due to increased background detection.

The sensitivity for $\mathrm{H}$ concentration measurements with NRA is limited by the level of background signals in the $\gamma$-ray detection system. When the $\mathrm{H}$ concentration in the target is low, the small $\gamma$-signal will be obscured by fluctuations of the background. For a given acquisition time $t$, one has to accept a certain relative error 
$\Delta Y / Y$, which depends on the true $\gamma$-signal $(Y=\dot{Y} \cdot t)$ and the background $(U=\dot{U} \cdot t)$ $[98,107,185]:$

$$
\frac{\Delta Y}{Y}=\frac{(\dot{Y}+2 \dot{U})^{\frac{1}{2}}}{\dot{Y} t^{\frac{1}{2}}}
$$

In absence of background, the expression recovers the familiar $\gamma^{-1 / 2}$ dependence of counting statistics. At the detection limit, small signals prevail on a large background, i.e., $\dot{U} / \dot{Y} \gg>1$, and $\Delta Y / Y=(2 \dot{U} / \mathrm{t})^{1 / 2} / \dot{Y}$. The relative error can then be decreased through long data acquisition $\left(\Delta Y / Y \propto t^{-1 / 2}\right)$ and by applying high ion beam fluences $\left(\Delta Y / Y \propto \dot{Y}^{-1}\right)$. With unshielded detectors, ${ }^{15} \mathrm{~N}$ NRA usually achieves $\mathrm{H}$ sensitivities better than 1000 at. ppm $[107,182]$. To optimize the sensitivity, the signal rate $\dot{Y}$ must be maximized and that of the background $\dot{U}$ suppressed as far as possible. Since the sensitivity constant of a given $\gamma$-detector ( $K$ in Eq. (2.12)) writes as $K=\eta \Delta \Omega$ ( $\Delta \Omega$ is the subtended solid angle and $\eta$ the $\gamma$-absorption efficiency), the NRA signal is obviously maximized by providing an efficient $\gamma$-detector (large $\eta: \rightarrow$ BGO) placed as closely to the sample (large $\Delta \Omega$ ) as can be arranged without obstructing sample manipulations, and by performing the measurement with the largest possible ion beam current $\left(N_{\mathrm{i}}\right)$ that is tolerated by the target without deterioration.

Background signals in $\gamma$-spectroscopy arise mainly from cosmic radiation. Solar wind-induced nuclear reactions in the upper atmosphere generate a radiation background at sea level, which consists of a softer $\gamma$-component (on the order of $\sim 10$ $\mathrm{MeV}$ ) and a dominant (70-80\%) shower of hard muons up to $100 \mathrm{GeV}$ [98]. Some of these $\gamma$-rays may fall into the ROI for NRA and can be suppressed by $\gamma$-absorbing 
shields (concrete building walls or high density material $(\mathrm{Pb})$ detector enclosures). The highly penetrating muons cause signals by loosing a small portion of their energy upon passing through the scintillation crystal. This background can only be eliminated with anticoincidence techniques [98, 182, 185]. Horn and Lanford describe an efficient way to selectively protect the ROI of the $4.43 \mathrm{MeV} \gamma$-ray in ${ }^{1} \mathrm{H}\left({ }^{15} \mathrm{~N}, \alpha \gamma\right){ }^{12} \mathrm{C}$ NRA with an anticoincidence circuit and a single relatively small $(16 " \mathrm{x}$ $\left.16^{\prime \prime} \times 1 "\right)$ plastic scintillator sensitive to the cosmic radiation that covers a $(4 ") \mathrm{Pb}-$ shielded 3" × 3" BGO detector [182]. The resulting background suppression (Fig. 12) improves $\dot{U}$ in the NRA ROI from $\sim 0.8$ to $0.16 \mathrm{cts} / \mathrm{min}$, which allows for a $\mathrm{H}$ sensitivity of $10-100$ atomic ppm.

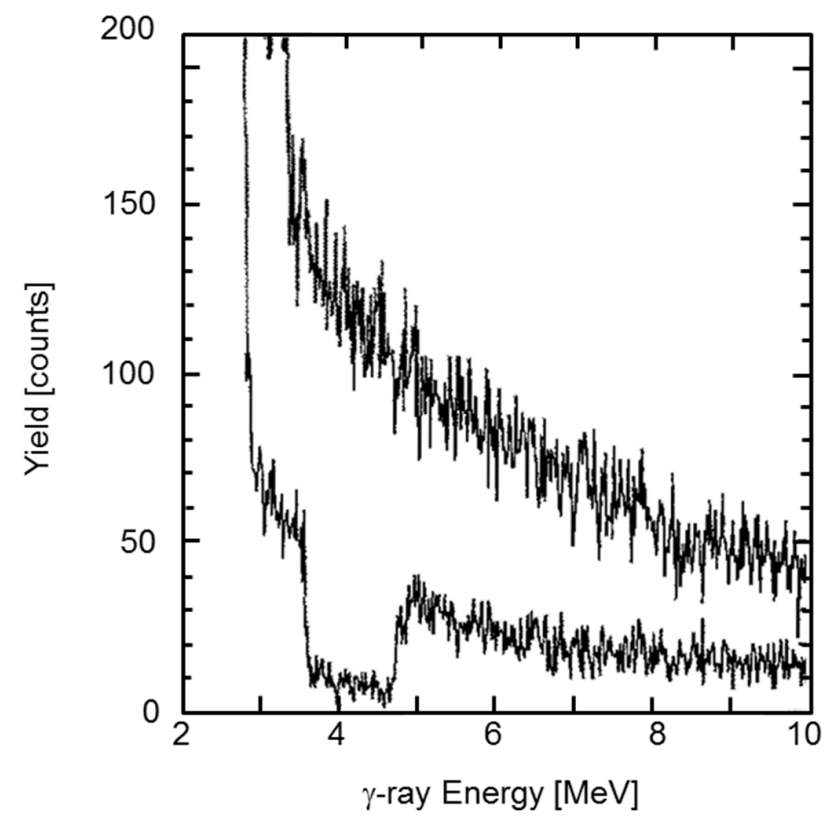

Fig. 12. Shielded and unshielded spectra using a 3 in. $x 3$ in. BGO detector. The "notch" extending from 3.5 to $4.75 \mathrm{MeV}$ corresponds to the region protected by the anticoincidence circuit. Reproduced from Ref. [182] with permission from Elsevier.

A similar low-level system described by Kuhn et al. [185] has a 3" $\times 3$ " BGO CRB rate of $0.55 \mathrm{cts} / \mathrm{min}$ in the $3.8-4.8 \mathrm{MeV}$ range and a detection efficiency for $4.4 \mathrm{MeV}$ 
$\gamma$-rays of $14 \%$, providing comparable $\mathrm{H}$ sensitivity $(<20$ at. ppm). These detection systems are relatively compact and allow easy sample access. This is more versatile than the highly effective but very large and heavily shielded detector case designed by Damjantschitsch et al., which achieves similar sensitivity (10-100 ppm) by reducing the background of a large $\left(10^{\prime \prime} \times 10^{\prime \prime}\right)$ Nal detector by a factor of $100[73$, 98], and can reduce the CRB rate of a 3 " $\times 3$ " BGO to $0.19 \mathrm{cts} / \mathrm{min}$ [185].

The NRA sensitivity can further be increased by maximizing the $\gamma$-detection efficiency with a large solid angle scintillation crystal that accommodates the target in a center hole, and by completely surrounding the $\gamma$-detector with passive and active (anticoincidence) shielding [186-189]. The highest reported $H$ sensitivity achieved in this way is better than $10 \mathrm{ppm}$ [186].

Beside the described natural backgrounds, there are also artificial sources of $\gamma$ radiation that may perturb NRA measurements. Possible external sources of artificial background radiation are $\gamma$-emitting nuclear reactions induced by neutron capture inside or near the detector, which may occur in laboratories located near neutron sources. Moreover, the NRA analysis itself may produce disturbing $\gamma$-radiation if applied to certain types of sample materials. The analyzing ${ }^{15} \mathrm{~N}$ ion beam may induce nuclear reactions with light elements in the target, such as $\mathrm{Li}, \mathrm{Be}$, and, most notably, D $[98,190]$. The likelihood of such undesired nuclear side reactions increases at high ${ }^{15} \mathrm{~N}$ energies due to the enhanced probability to overcome the Coulomb barrier with low $Z$ nuclei. In unfavorable cases the released $\gamma$-radiation (or its Compton continuum) falls into the ROI for the ${ }^{1} \mathrm{H}\left({ }^{15} \mathrm{~N}, \alpha \gamma\right){ }^{12} \mathrm{C}$ NRA $\gamma$-rays. 
A notable example for this pitfall is the $D\left({ }^{15} \mathrm{~N}, p\right){ }^{16} \mathrm{~N}$ reaction, which after betadecay of ${ }^{16} \mathrm{~N}$ to ${ }^{16} \mathrm{O}^{*}$ releases intense emission of 3.6, 6.1, and 7.1 MeV $\gamma$-rays and already has a sizeable cross section below the $6.385 \mathrm{MeV}$ resonance of ${ }^{1} \mathrm{H}\left({ }^{15} \mathrm{~N}, \alpha \gamma\right){ }^{12} \mathrm{C}$ $[191,192]$. The $D\left({ }^{15} \mathrm{~N}, \mathrm{p}\right){ }^{16} \mathrm{~N}$ reaction can very seriously disturb $\mathrm{H}$ profiling in samples that contain appreciable D concentrations $[98,193]$. For instance, it renders $\mathrm{H}$ profiling at $\mathrm{H}_{2} \mathrm{O} / \mathrm{D}_{2} \mathrm{O}$ sandwich structures for interdiffusion studies with ${ }^{15} \mathrm{~N}$ NRA completely fruitless by overwhelming the $4.4 \mathrm{MeV}$ ROI with $\gamma$-yield (an experience also the authors had to make). Unfortunately, also Li-based materials (which currently enjoy bright prospects as potential $\mathrm{H}$ storing media) are affected by similar problems due to a large variety of possible ${ }^{6} \mathrm{Li}+{ }^{15} \mathrm{~N}$ and ${ }^{7} \mathrm{Li}+{ }^{15} \mathrm{~N}$ reactions [190]. As long as the $\mathrm{Li}$ (or D) target concentrations are small (on the order of a few atomic \%), one may be able to correct for the resulting side reaction $\gamma$-yield after identifying and quantifying a characteristic $\gamma$-emission outside the ROI for ${ }^{15} \mathrm{~N}$ NRA [190]. Applying this to the $\gamma$-emission from $D\left({ }^{15} \mathrm{~N}, \mathrm{p}\right){ }^{16} \mathrm{~N}$ at 6.0 or $7.0 \mathrm{MeV}$ and quantifying the yield this reaction induces within the $4.43 \mathrm{MeV}{ }^{1} \mathrm{H}\left({ }^{15} \mathrm{~N}, \alpha \gamma\right){ }^{12} \mathrm{C}$ ROI might actually permit the simultaneous detection of $H$ and $D$ with the same ${ }^{15} \mathrm{~N}$ beam, although the $D$ signal will not be depth-resolved since the $D\left({ }^{15} N, p\right)^{16} \mathrm{~N}$ reaction is non-resonant.

Another source of possibly disturbing $\gamma$-radiation is the $4.4 \mathrm{MeV} \gamma$-yield produced by the interaction of hydrogen near the target surface with ${ }^{15} \mathrm{~N}$ at non-resonant energies above $\sim 10 \mathrm{MeV}$ described in the previous Section (Fig. 10). This nonresonant $\gamma$-yield may reduce the sensitivity for, e.g., small $\mathrm{H}$ quantities at deeply buried interfaces. Fortunately, the $\mathrm{H}\left({ }^{15} \mathrm{~N}, \alpha \gamma\right){ }^{12} \mathrm{C}$ cross section has been characterized over a wide energy region $[100,110,111]$, so that the non-resonant $\gamma$-yield can be 
corrected for. The non-resonant $\gamma$-yield $Y_{\text {off }}$ is proportional to the total $\mathrm{H}$ content in the surface layer, i.e., to the integrated $\gamma$-yield measured with the first resonance at 6.385 MeV, $I_{\text {on }}{ }^{(I)}$, and to the off-resonant cross section, $\sigma_{\text {off: }} Y_{\text {off }}=\sigma_{\text {off }} \times I_{\text {on }}{ }^{(1)}$. When $\sigma_{\text {off }}$ is known and the first-resonance $\gamma$-yield of surface- $\mathrm{H}\left(\mathrm{Ion}^{(I)}\right)$ has been determined from a shallow depth profile near $6.385 \mathrm{MeV}$, the non-resonant reaction yield can be estimated and, if necessary, subtracted from the raw $\gamma$-yield at high ${ }^{15} \mathrm{~N}$ energy to determine the $\mathrm{H}$ concentration at larger depths correctly $[100,110,111]$.

Evaluations of the energy-dependent off-resonant ${ }^{1} \mathrm{H}\left({ }^{15} \mathrm{~N}, \alpha \gamma\right){ }^{12} \mathrm{C}$ reaction cross section $\sigma_{\text {off }}(E)$ between 6.4 and $13.4 \mathrm{MeV}$ are available for the $\gamma$-detector positioned on $[98-100,111]$ and $90^{\circ}$ off the ion beam axis [110]. The distinction of the detector position is necessary because the $\gamma$-emission in the resonant ${ }^{1} \mathrm{H}\left({ }^{15} \mathrm{~N}, \alpha \gamma\right){ }^{12} \mathrm{C}$ reaction is slightly peaked in the forward direction [194], whereas the non-resonant $\gamma$-emission is nearly isotropic [100] (Fig. 13), making the relative contribution of the nonresonant yield somewhat higher in the $90^{\circ}$ detection geometry [110]. Through the above definition as $\sigma_{\text {off }}\left(E, \theta_{D}\right)=Y_{\text {off }}(E) / I_{\text {on }}{ }^{(1)}\left(\theta_{D}\right)$, the non-resonant ${ }^{1} \mathrm{H}\left({ }^{15} \mathrm{~N}, \alpha \gamma\right){ }^{12} \mathrm{C}$ cross section becomes also detection angle $\left(\theta_{D}\right)$ dependent. In the flat region between 6 and $8.3 \mathrm{MeV} \sigma_{\text {off }}$ amounts to $1.9 \times 10^{-5}$ with the detector in the $0^{\circ}$ position [100], and $\sigma_{\text {off }}=3.8 \times 10^{-5}$ for the detector $90^{\circ}$ off axis [110]. For $\sigma_{\text {off }}$ values at energies from 9 to 13.7 MeV, see Fig. 3 in Ref. [110]. The non-resonant $\gamma$-yield from surface $\mathrm{H}$ primarily affects measurements of deeply buried $\mathrm{H}$ concentrations probed at high ${ }^{15} \mathrm{~N}$ energies (> $10 \mathrm{MeV}$ ) and therefore does not present a major concern for $\mathrm{H}$ detection near surfaces and shallow interfaces reviewed here. 


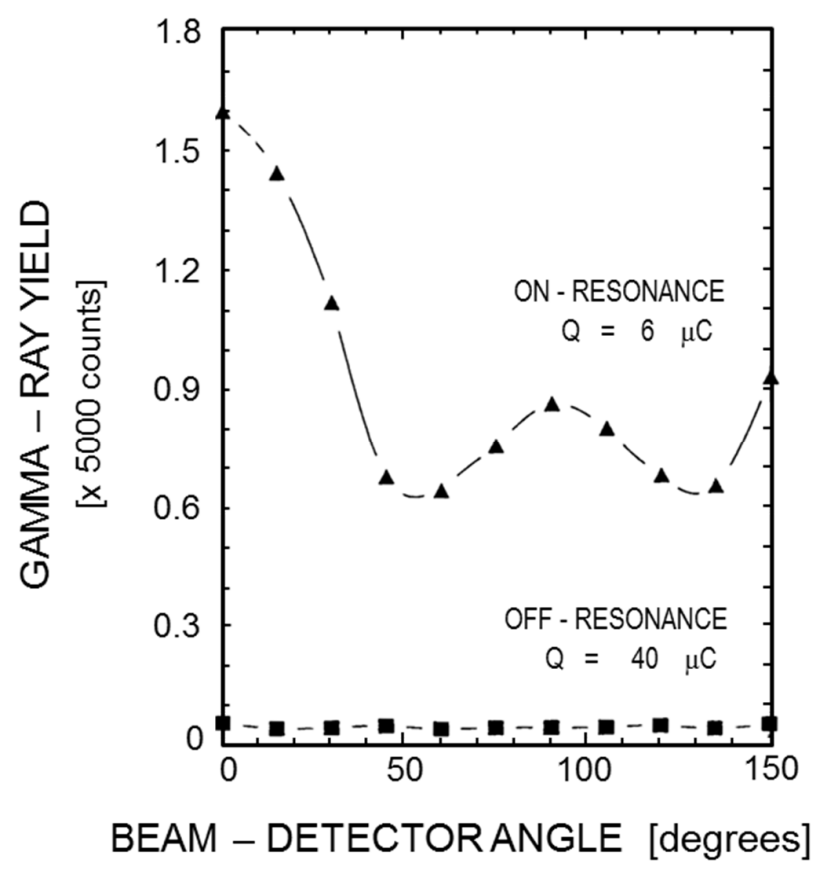

Fig. 13. Comparison of the angular distribution of resonant (6.385 MeV) and non-resonant $\gamma$ ray emission from the ${ }^{1} \mathrm{H}\left({ }^{15} \mathrm{~N}, \alpha \gamma\right){ }^{12} \mathrm{C}$ reaction. Reproduced from Ref. [100] with permission from Elsevier.

\subsubsection{Radiation effects}

NRA is widely regarded as a non-destructive technique of quantitative elemental analysis. While this is certainly justified in direct comparison to outright destructive methods of hydrogen detection such as SIMS, metallurgical vacuum extraction, or TDS, the influence of the analyzing ion beam cannot entirely be ignored. An important general concern in hydrogen depth profiling with energetic ion beams is the possibly irreversible alteration of the hydrogen distribution in the course of the analysis that may include redistribution and/or loss of hydrogen from the target. We describe in this section the origin, the correction for, and the analytical value of possible ${ }^{15} \mathrm{~N}$-induced alterations of the $\mathrm{H}$ distribution. 
The severity of radiation-induced effects strongly depends on the analyzed material as well as on the energy and nuclear charge of the ion projectiles. A general trend is that especially insulating low $Z$ materials such as organic polymers, carbon films, and $\mathrm{SiO}_{\mathrm{x}} \mathrm{N}_{\mathrm{y}}$ dielectrics are prone to suffer substantial losses of volatile light elements $(H, C, N, O)$ under energetic ion irradiation [180, 195-200], whereas metal and semiconductor targets appear to be much less vulnerable against beam-induced $H$ loss [201]. This difference can be understood by considering the basic interaction mechanism of energetic ions with the sample material. In the energy regime of the ${ }^{15} \mathrm{~N}$ ions used in ${ }^{1} \mathrm{H}\left({ }^{15} \mathrm{~N}, \alpha \gamma\right){ }^{12} \mathrm{C}$ NRA direct nuclear collisions that cause permanent damage by displacing target atoms contribute less than $1 \%$ to the total energy loss (Section 2.1.3), whereas the dominant interaction is electronic stopping that generates a large number of excited electrons for each incident ${ }^{15} \mathrm{~N}$ ion. In conducting materials, the excited electron energy is almost instantaneously dissipated, so that only the minor nuclear stopping leads to lasting damage in metals and semiconductors. In insulating materials, on the other hand, energetic electron impact causes localized bond cleavage that generates dangling bonds and free, diffusible radicals that may eventually leave the target. This explains the observations that the hydrogen depletion rate from insulating materials is many orders of magnitude larger than the nuclear scattering cross section and that it often scales with the electronic stopping power of the projectile ion $[195,197,198,200$, 202]. 
When the hydrogen concentration at a constant depth is monitored with NRA as a function of the incident ion beam fluence $\phi$ (ions $/ \mathrm{cm}^{2}$ ) one often observes a progressive damage pattern, in which an initially rapid $\mathrm{H}$ loss is followed by a slower decrease that may eventually saturate and even lead to a stable $\mathrm{H}$ concentration. As a typical example, Fig. 14 (a) shows the evolution of the $\mathrm{H}$ content in a thin film of hydrogenated amorphous silicon ( $a$-Si) deposited on a H-terminated $\mathrm{Si}(100)$ substrate as tracked with ${ }^{15} \mathrm{~N}$ NRA under UHV conditions [203]. Empirically, such $\gamma$ yield decay curves as a function of the incident beam fluence $Y(\phi)$ are often well reproduced by a double exponential form:

$$
Y(\phi)=n_{1} \cdot \exp \left(-\sigma_{1} \phi\right)+n_{2} \cdot \exp \left(-\sigma_{2} \phi\right)
$$

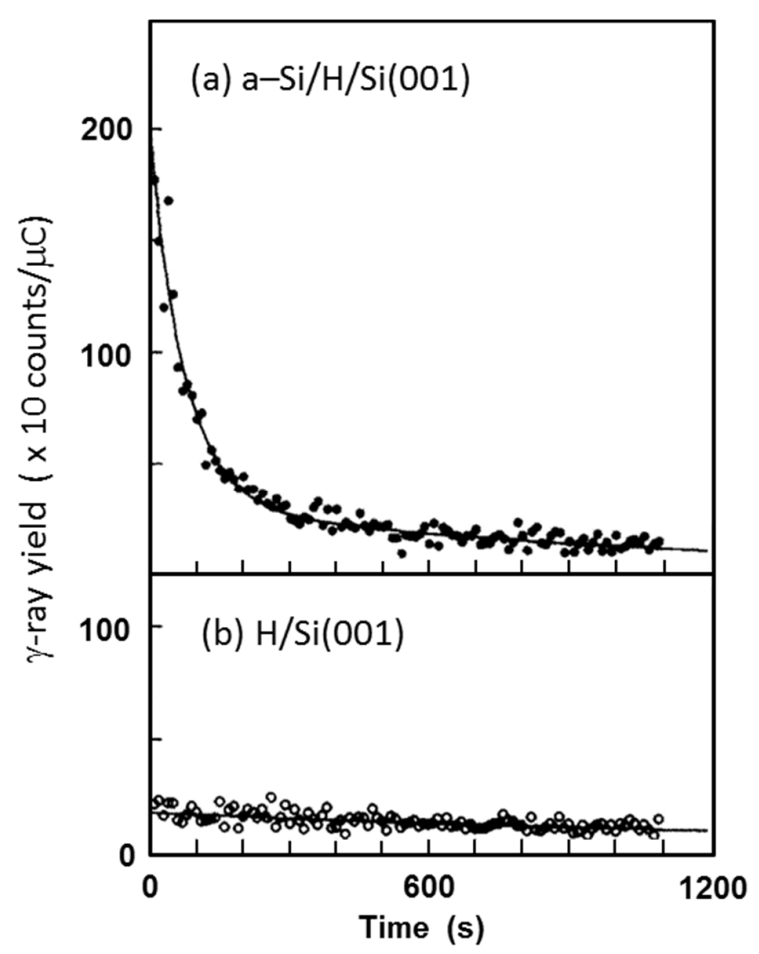

Fig. 14. Decay of the $\gamma$-yield from the resonant ${ }^{1} \mathrm{H}\left({ }^{15} \mathrm{~N}, \alpha \gamma\right){ }^{12} \mathrm{C}$ reaction as a function of irradiation time with a $20 \mathrm{nA}{ }^{15} \mathrm{~N}^{2+}$ beam. (a) $a-\mathrm{Si} / \mathrm{H} / \mathrm{Si}(001)$ and (b) H/Si(001). Solid lines are fits to a double (a) and (b) single exponential decay function. Reproduced from Ref. [203] with permission from Elsevier. 
where $\sigma_{1}$ and $\sigma_{2}$ are effective decay cross sections describing the initially fast and later more gradual decay channels, and $n_{1}+n_{2}$ represents the $\gamma$-yield corresponding to the initial $\mathrm{H}$ concentration, $Y(0)$. Retrieving the latter by extrapolating the $Y(\phi)$ dependence to zero ion exposure is important for the quantitative analysis of hydrogen distributions with NRA (Section 2.2.6). Further, with the parameters obtained from a fit of $Y(\phi)$ to Eq. (2.18), the relative $\mathrm{H}$ loss during NRA depth profiling can be calculated from the ion fluence accumulated at each data point of a measured excitation curve $Y\left(E_{\mathrm{i}}\right)$. These relative losses should be applied as weighting factors to the recorded yield curve data $Y\left(E_{i}\right)$ to compensate for the $\mathrm{H}$ loss during the analysis, so that unperturbed $\mathrm{H}$ depth distributions and surface resonance profiles can be determined.

The effective decay constants $\left(\sigma_{1}, \sigma_{2}\right)$ in Eq. (2.18) may bear physical significance, for instance, they may be specific to certain hydrogen species in the target. Such a strong material dependence of the beam-induced $\mathrm{H}$ decay kinetics is exemplified by Fig. 14 (b), which shows the decrease of the $\mathrm{H}$ coverage on H-terminated $\mathrm{Si}(100)$ surface in a single, slow depletion process that has the same cross section as the slower decay channel in Fig. 14 (a). In comparison, the data strongly suggest that this slow decay is characteristic of the H-termination layer on the Si(100) surface, whereas the rapid depletion of the initially high $\gamma$-yield in Fig. 14 (a) is related to the $\mathrm{H}$ content in the $a$-Si overlayer [203]. In the sense that the effective decay constants may reflect individual $\mathrm{H}$ species or be particular to a certain $\mathrm{H}$ transport property of the analyzed material, the ion beam-induced $\mathrm{H}$ decay is of analytical value, as it may 
help distinguish chemically different $\mathrm{H}$ species or be useful to evaluate, e.g., the hydrogen diffusion behavior underneath the surface. Examples for the assessment of surface conditions and interfacial $\mathrm{H}$ relocation phenomena with the aid of $\mathrm{H}$ decay kinetics measured by ${ }^{15} \mathrm{~N}$ NRA are presented in Sections 3.1.2 and 3.3.1, respectively.

\subsubsection{Quantitation and calibration}

In order to obtain absolute values for hydrogen surface coverages or volume concentrations from NRA measurements one has to know the sensitivity factor $K$ in Eqs. (2.11) and (2.12) that describes the efficiency of any given experimental setup to capture the $\gamma$-rays emitted in the nuclear reaction. $K$ contains the $\gamma$-ray absorption efficiency $\eta$ and the solid angle $\Delta \Omega$ subtended by the detector. The recorded $\gamma$-yield will also slightly depend on the detector position relative to the ion beam direction due to the angle variation of the $\gamma$-emission in the nuclear reaction (Fig. 13), over which the detection solid angle partially integrates. Hence, since the ${ }^{1} H\left({ }^{15} \mathrm{~N}, \alpha \gamma\right){ }^{12} \mathrm{C}$ resonance parameters $\left(\sigma_{R}, \Gamma\right)$ are well known, the relation of proportionality between the nuclear reaction $\gamma$-yield and the recorded NRA signal can in principle be calculated if $\Delta \Omega$ is evaluated carefully. In practice, it is common convenience to subsume all unknown factors except the parameters specific to the sample $(S)$ and the individual measurement $\left(N_{\mathrm{i}}, t\right)$ into an effective sensitivity constant $K_{\text {eff, }}$ which is characteristic for a given experimental setup with a particular detector in a defined position relative to the sample and a defined energy integration region (ROI) in the $\gamma$ spectrum, so that in acquisition time $(t)$ integrated form Eq. (2.12) gives the detected number of $\gamma$-counts $I\left(E_{\mathrm{i}}\right)$ at a given ion beam energy $E_{\mathrm{i}}$ as: 


$$
I\left(E_{i}\right)=\int_{0}^{t} Y\left(E_{i}\right) d \tau=K_{e f f} Q_{i} c_{b} / S
$$

where $Q_{i}=q \int_{0}^{t} N_{i} d \tau$ is the ion beam charge accumulated on the target ( $q$ is the charge state of the ${ }^{15} \mathrm{~N}$ projectile). The detection system is then calibrated by determining $K_{\text {eff }}$ with a reference sample of a precisely known volume $\mathrm{H}$ concentration $c_{\text {ref. }}$ With such a bulk standard of known stopping power $S_{\text {ref, }} K_{\text {eff }}$ is readily obtained from a single yield measurement in the calibration target. For the calibration measurement, any standard should be probed in a depth sufficiently far below the surface where not only $E_{\mathrm{i}}=E_{>}$(Fig. 4), but also the $\mathrm{H}$ content is certain to have attained the average bulk composition $c_{\text {ref, }}$ since near-surface deviations of the $\mathrm{H}$ concentration from the bulk value occur quite commonly on a scale of several 10 or even 100 nm (see 3.2.3.6-7 and, e.g., Fig. 3 in Ref. [204]).

Materials suitable as calibration standards for absolute $\mathrm{H}$ concentration measurements with NRA should satisfy several requirements [205]. $c_{\text {ref }}$ and the other elemental composition must be precisely known so that the stopping power $S_{\text {ref }}$ can be calculated accurately. The material must have a uniform lateral $\mathrm{H}$ distribution and density, and be stable in vacuum. Although not necessarily a strict requirement, convenient standards furthermore offer long-term stability, in particular under the ion beam, so that they can be used repeatedly.

A variety of materials that fulfill the above demands more or less ideally has been proposed as calibration standards, among them hydrogenated thin films of Ti [100] and Ta foil [204, 206], hydrogenated films of pyrolytic carbon [204], amorphous silicon [204, 207, 208], and silicon nitride [110], hydrogen-implanted Si [71, 207, 209], 
as well as the polymers polystyrene [210], polypropylene $\left(\mathrm{C}_{3} \mathrm{H}_{6}\right)_{n}$, polyester, Mylar $\left(\mathrm{C}_{10} \mathrm{H}_{8} \mathrm{O}_{4}\right)_{n}$, Kapton ${ }^{\circledR}\left(\mathrm{C}_{22} \mathrm{H}_{10} \mathrm{O}_{5} \mathrm{~N}_{2}\right)_{n}[205,211]$, as well as $\mathrm{H}_{2}$ gas [212].

The hydrogenated thin film materials have been confirmed to show quite favorable properties, such as long-term stability in UHV as well as under the ${ }^{15} \mathrm{~N}$ NRA ion irradiation, and sufficient conductivity for proper charge integration. Some of them, however, must be prepared by deposition and hydrogenation techniques or CVD methods that may not be available in most laboratories. Further, although $\mathrm{H}$ stability in these hydrided thin films or hydrogen-annealed metal (Ta, Ti) foils has been verified, the absolute $\mathrm{H}$ concentration in the obtained specimens still needs to be determined with another reference. The bulk polymers, on the other hand, have precisely known compositions and are readily available as thin foils, yet they suffer from rapid initial beam-induced $\mathrm{H}$ loss that introduces substantial uncertainty into the zero-fluence extrapolation of the decay curves (Eq. (2.18), Section 2.2.5). A once irradiated area of polymer foil must be discarded after acquisition of a single decay curve. To our experience and as also described in Ref. [211], Kapton (a polyimide) has a much better resistance against ${ }^{15} \mathrm{~N}$-induced $\mathrm{H}$ loss than most polymers, presumably due to its comparatively lower $\mathrm{H}$ content, to which the initial $\mathrm{H}$-loss rate is proportional $[195,198,199]$. To avoid charge up of the Kapton foils one may ideally deposit a thin metal layer onto their surface or alternatively clamp the foil under a thin wire mesh that is wide enough to accommodate the ion beam. The use of $\mathrm{H}_{2}$ for calibration ( $10^{4}-10^{5} \mathrm{~Pa}$ is convenient) by extracting the ${ }^{15} \mathrm{~N}$ ion beam into a gas cell, such as through a thin $(100 \mathrm{~nm}) \mathrm{SiN}$ membrane, requires a well-focused ion beam due to the necessarily small membrane size $\left(\sim 1 \times 1 \mathrm{~mm}^{2}\right)$ and a separate 
detector to measure the beam flux into the gas target, but is otherwise independent of any parameters except the $\mathrm{H}_{2}$ stopping power [212].

$\mathrm{H}$-implanted $\mathrm{Si}$ wafers are probably the most versatile (absolute) calibration standards, although B-doped p-Si should probably be avoided to exclude side nuclear reaction $\gamma$-yield. Implantation techniques are well established [213], and in preparing the standard, the $\mathrm{H}$ content can be known rather precisely if the charge deposited during the implantation process is measured accurately. $5 \times 10^{16} \mathrm{~cm}^{-2} \mathrm{H}^{+}$implanted at $6 \mathrm{keV}$ has produced quite practical results [209]. To prevent channeling effects in the typically single crystalline Si substrates, the implantation depth region is preferably amorphized with an auxiliary ion beam prior to $\mathrm{H}$ introduction. Alternatively, a small beam incidence angle against the surface normal can be adjusted during the calibration measurement to avoid ion incidence along a high-symmetry crystal axis. The implanted $\mathrm{H}$ is bound strongly at (implantation generated) dangling bond defects and thus well protected against thermal and irradiation-induced diffusion [207]. H-implanted Si standards thus promise good long-term stability, and influences from the surface (exposure to vacuum or atmosphere) on the implant are expected to be negligible as well. Hydrogen-implanted Si-standards have been used for calibration purposes in many of the quantitative NRA studies reviewed in Chapter 3. A recent international 'Round Robin' test by 6 different laboratories applying various forms of ERD and NRA to determine the $\mathrm{H}$ quantity implanted into an amorphized Si wafer achieved indeed a very high reproducibility (2.2\% relative error) that even exceeded the combined uncertainty estimates ( 6\%) of the participating laboratories [209]. 


\subsubsection{Data analysis and simulation}

In order to extract actual hydrogen concentration depth profiles from experimental $\gamma$-yield excitation curves in NRA one has to unfold the instrumental function from the data. Although solving Eq. (2.3) by numerical deconvolution would be the most direct method, this approach is rather cumbersome and has rarely been pursued. To our knowledge only one numerical deconvolution program for the analysis of experimental resonant NRA data has been developed, WinRNRA [214], which however, appears to have been applied only for ${ }^{15} \mathrm{~N}$ profiling with $\mathrm{H}^{+}$beams at the inverse laboratory resonance energy of the ${ }^{15} \mathrm{~N}\left({ }^{1} \mathrm{H}, \alpha \gamma\right){ }^{12} \mathrm{C}$ reaction at $429 \mathrm{keV}$ (in the center of mass system relevant to the nuclear reaction the collision energy of $429 \mathrm{keV}{ }^{1} \mathrm{H}$ and resting ${ }^{15} \mathrm{~N}$ is the same as for $6.385 \mathrm{MeV}{ }^{15} \mathrm{~N}$ impact on resting ${ }^{1} \mathrm{H}$ ).

The second, easier, and much more widely applied way [215-219] of interpreting the $\gamma$-yield excitation functions is to forward-convolve a trial $\mathrm{H}$ concentration profile with the NRA instrumental function (Section 2.1.1), to obtain a simulated excitation function that can be compared to the experiment. According to Eq. (2.3) a separate calculation of the expected $\gamma$-yield is necessary for each incidence energy value in the scan. The model $\mathrm{H}$ depth distribution is then refined within reasonable physical limits until good agreement of the simulated yield curve with the experimental data is achieved. This method is comparatively straightforward to implement from Eqs. (2.3) - (2.9), but the obtained simulations do not allow for an unambiguous determination of the actual $\mathrm{H}$ depth profile, as the assumed $\mathrm{H}$ model distribution is not necessarily unique. Good physical sense in constructing the model distribution is hence essential. 
The authors have implemented this approach for surface-near hydrogen profiling by following the ${ }^{15} \mathrm{~N}$ ion trajectory into the depth of the target, calculating the energy resolution function $F\left(E, E_{\mathrm{i}}, z\right)$ in trajectory length increments corresponding to atomic layer distances, to obtain a relative measure of the expected $\gamma$-yield from model $\mathrm{H}$ distributions [110, 220]. This simulation routine is a surface approximation (Section 2.1.3) for shallow depth profiling [220], which assumes a constant stopping power at all depths, and a Gaussian straggling distribution with a standard deviation based on Rud's formula [106]. Despite their limitations to uniquely identify hydrogen depth distributions, such simplified simulations can be helpful in judging which of several conceivable $\mathrm{H}$ distributions are compatible with a given set of experimental data and may thus aid the interpretation of measured excitation curves by at least excluding incompatible models [220].

More sophisticated ion beam analysis software has been devised that takes full account of the energy dependence of the stopping at larger depths (such as the DEPTH code $[221,222]$ and SRIM $[125,127])$ and offers choices between several theoretical frameworks to describe the straggling such as RUMP [223], RBX [224], and SIMNRA $[225,226]$. DataFurnace $[227,228]$ even provides the convenience of automated fitting in complex layered samples and the calculation of confidence limits. These programs are widely used in ion beam analysis with RBS, ERD, and nonresonant NRA and have recently been evaluated by IAEA [229-231]. These IBA techniques have in common that the material is analyzed at constant beam energy while the energy spectrum of the outgoing (scattered, recoiled, or non-resonantly produced) particles is measured to extract the depth information; hence the 
software codes are similar. Since this situation is notably different in narrow resonance profiling ( $r$-NRA) where the incident beam energy is scanned, the above programs have long excluded resonant NRA. Only recently special routines for analysis and simulation of PIXE and high-resolution resonant NRA data have been implemented into the DataFurnace [227] software [228].

\subsection{Comparison to ERDA}

Elastic recoil detection (analysis) (ERDA) is an alternative fast and quasi nondestructive method for hydrogen depth profiling that under certain provisions can achieve similar performance in the near surface region as NRA via ${ }^{1} \mathrm{H}\left({ }^{15} \mathrm{~N}, \alpha \gamma\right){ }^{12} \mathrm{C}$ in terms of sensitivity and depth resolution. For detailed descriptions of the ERD technique the interested reader is referred to original publications [51, 71, 232-239] and comprehensive reviews [193, 240-246]. A few essential principles of ERD that may help comprehending the following comparison with resonant ${ }^{15} \mathrm{~N}$ NRA are outlined in Appendix B.

\subsubsection{Performance (depth resolution, sensitivity, probing range)}

Owing to the variety of ERD configurations with respect to projectile masses and energies, recoil geometries, and detection schemes that are briefly described in Appendix B it is difficult to draw a general comparison of ERD to the NRA techniques for $\mathrm{H}$ and $\mathrm{D}$ detection. We have therefore compiled performance benchmarks of various ERD applications to hydrogen analysis in Table 2 for a case by case comparison with ${ }^{1} \mathrm{H}\left({ }^{15} \mathrm{~N}, \alpha \gamma\right){ }^{12} \mathrm{C}$ NRA at $6.385 \mathrm{MeV}$. Recall from Section 2.2 that the 
typical performance characteristics of the latter are a near surface depth resolution of 2-5 nm in normal and $<1 \mathrm{~nm}$ in grazing $\left(\alpha_{\mathrm{i}}=80^{\circ}\right)$ incidence, respectively, a probing depth range of $\sim 2-4 \mu \mathrm{m}$, and a sensitivity of $\sim 100-500 \mathrm{ppm}(0.05-0.01$ at.\%) for bulk $\mathrm{H}$ concentrations or $\mathrm{H}$ layer densities of $(1-5) \times 10^{13} \mathrm{~cm}^{-2}$ at the surface.

${ }^{15} \mathrm{~N} \mathrm{NRA}$ at $6.385 \mathrm{MeV}$ thus obviously outperforms conventional (range foil and SSBD) ERD with ${ }^{4} \mathrm{He}$ beams in terms of depth resolution, a slightly higher sensitivity, the ability to profile rough surfaces, and a larger probing depth range. On the other hand, ERD with ion projectiles heavier than ${ }^{4} \mathrm{He}$ (HIERD) offers considerably enhanced depth resolution $\left((\mathrm{d} E / \mathrm{d} z) \propto Z_{1}^{2}\right)$, improved sensitivity $\left(\sigma_{\mathrm{r}} \propto Z_{1}{ }^{2}\right)$, and in addition enables detection of other light target elements along with the hydrogen isotopes [247, 248], as long as the sample tolerates the heavy projectile beam.

With specialized high-resolution detection systems ERD achieves similarly good near-surface depth resolution as ${ }^{15} \mathrm{~N}$ NRA. Analyzers with large acceptance angles (such as kinematic spread corrected spectrometers) $[239,249,250]$ are by all means strongly preferable. If heavier ERD projectile beams can be provided and are safe to be applied to the given sample material from the viewpoint of radiation damage, the routinely achieved sensitivity of the two techniques is very similar, yet extended depth profiles can be obtained much faster with conventional ERD since no energy scanning is required [238]. High sensitivity measurements for small volume $\mathrm{H}$ concentrations in the order of a few atomic ppm have been demonstrated with both methods [186, 251]. 


\begin{tabular}{|c|c|c|c|c|c|c|c|}
\hline $\begin{array}{c}\text { Projectile } \\
\text { ERD }\end{array}$ & $\theta_{1}$ & $\theta_{\mathrm{r}}$ & Target & $\begin{array}{c}\text { Analysis } \\
\text { (Detection) }\end{array}$ & $\begin{array}{l}\text { Reso- } \\
\text { lution } \\
(\mathrm{nm})\end{array}$ & $\begin{array}{l}\text { Sensi- } \\
\text { tivity }\end{array}$ & Ref. \\
\hline $\begin{array}{c}2.4 \mathrm{MeV} \\
{ }^{4} \mathrm{He}^{+}\end{array}$ & $15^{\circ}$ & $30^{\circ}$ & $\begin{array}{l}\text { Silicon } \\
\text { nitride }\end{array}$ & $\begin{array}{l}\text { Conventional } \\
\text { (foil + SSBD) }\end{array}$ & 70 & $<0.1$ & {$[234]$} \\
\hline $\begin{array}{c}3 \mathrm{MeV} \\
{ }^{4} \mathrm{He}\end{array}$ & $\begin{array}{l}12.5^{\circ} \\
4.5^{\circ}\end{array}$ & $\begin{array}{l}23^{\circ} \\
15^{\circ}\end{array}$ & $a-\mathrm{Si}: \mathrm{H}$ & $\begin{array}{c}\text { Conventional } \\
\text { (foil + SSBD) }\end{array}$ & $\begin{array}{l}75 \\
22\end{array}$ & 0.1 & {$[235]$} \\
\hline $\begin{array}{l}\text { 4-6 MeV } \\
{ }^{4} \mathrm{He}\end{array}$ & $90^{\circ}$ & $0^{\circ}$ & Ni foil & $\begin{array}{l}\text { Transmission } \\
((\text { foil })+\text { SSD })\end{array}$ & - & 0.0001 & {$[251]$} \\
\hline $\begin{array}{c}220 \mathrm{keV} \\
\mathrm{Li}\end{array}$ & & $44.3^{\circ}$ & $\begin{array}{l}\text { HF- } \\
\text { etched } \\
\mathrm{Si}(111)\end{array}$ & $\begin{array}{c}\text { MEIS-ERD } \\
\text { TOF, (Toroidal } \\
\text { Electrostatic } \\
\text { Spectrometer) }\end{array}$ & 0.9 & - & {$[252]$} \\
\hline $\begin{array}{c}4.3 \mathrm{MeV} \\
{ }^{20} \mathrm{Ne}^{2+}\end{array}$ & $19^{\circ}$ & $38^{\circ}$ & $\mathrm{Cu}$ & $\begin{array}{l}\text { Electrostatic } \\
\text { Spectrometer } \\
\text { + PSD }\end{array}$ & $\begin{array}{l}1.8(\mathrm{H}) \\
1.5(\mathrm{D})\end{array}$ & - & $\begin{array}{l}{[238,} \\
253]\end{array}$ \\
\hline $\begin{array}{l}4 \mathrm{MeV} \\
{ }^{12} \mathrm{C}^{2+}\end{array}$ & $25^{\circ}$ & $35^{\circ}$ & $\begin{array}{l}\text { Polymer } \\
\text { film }\end{array}$ & $\begin{array}{c}\text { Magnetic } \\
\text { Spectrograph } \\
\text { Wien filter + } \\
\text { PSD }\end{array}$ & 4 & - & {$[254]$} \\
\hline $\begin{array}{c}500 \mathrm{keV} \\
{ }^{12} \mathrm{C}^{+}\end{array}$ & $15^{\circ}$ & $30^{\circ}$ & $\begin{array}{l}\text { CVD- } \\
\text { diamond } \\
\text { Si(001) }\end{array}$ & $\begin{array}{c}\text { Magnetic } \\
\text { Spectrometer } \\
+ \text { PSD }\end{array}$ & $\begin{array}{l}0.23 \\
0.28 \\
\end{array}$ & $\begin{array}{c}0.05 \\
<6.8 \times 10^{13} \\
\end{array}$ & $\begin{array}{l}{[249,} \\
255]\end{array}$ \\
\hline $\begin{array}{c}350 \mathrm{keV} \\
{ }^{4} \mathrm{He} \\
2.54 \mathrm{MeV} \\
15 \mathrm{~N}\end{array}$ & $15^{\circ}$ & $30^{\circ}$ & $\begin{array}{l}\mathrm{H} \text { and } \mathrm{D} \\
\text { implant } \\
\text { in Be }\end{array}$ & E $\times B$ filter & 3.3 & 0.2 & {$[256]$} \\
\hline $\begin{array}{c}3.8 \mathrm{MeV} \\
{ }^{4} \mathrm{He}\end{array}$ & $15^{\circ}$ & $30^{\circ}$ & $\begin{array}{l}\mathrm{Ti} \\
\mathrm{Er}\end{array}$ & $\begin{array}{c}\Delta \mathrm{E}-\mathrm{E} \\
\text { Telescope }\end{array}$ & - & $<0.0075$ & {$[257]$} \\
\hline $\begin{array}{c}2 \mathrm{MeV} \\
{ }^{4} \mathrm{He}\end{array}$ & $90^{\circ}$ & $0^{\circ}$ & $\mathrm{Pd}(100)$ & $\begin{array}{c}\text { Transmission } \\
\text { (foil + SSD) }\end{array}$ & - & - & {$[258]$} \\
\hline $\begin{array}{c}6 \mathrm{MeV} \\
\mathrm{F}^{3+}\end{array}$ & $20^{\circ}$ & $40^{\circ}$ & $\begin{array}{l}\text { Si(100) } \\
\text { Si(111) }\end{array}$ & $\begin{array}{c}\text { Conventional } \\
\text { (foil + SSD) }\end{array}$ & - & - & $\begin{array}{l}{[259]} \\
{[260]}\end{array}$ \\
\hline $2 \mathrm{keV}{ }^{4} \mathrm{He}$ & & $26^{\circ}$ & $\mathrm{SiC}(0001)$ & TOF-ERD & - & - & [261] \\
\hline \multicolumn{8}{|l|}{ NRA } \\
\hline $\begin{array}{l}6.4 \mathrm{MeV} \\
{ }^{15} \mathrm{~N}(\mathrm{H})\end{array}$ & $\begin{array}{l}90^{\circ} \\
10^{\circ}\end{array}$ & - & $\begin{array}{l}\text { Bulk } \\
\text { Surface }\end{array}$ & $\begin{array}{c}\gamma \text {-rays, BGO } \\
\text { or Nal }\end{array}$ & $\begin{array}{l}2-5 \\
<1\end{array}$ & $\begin{array}{l}0.004 \\
\sim 10^{13}\end{array}$ & $\begin{array}{l}{[61]} \\
{[59]}\end{array}$ \\
\hline $\begin{array}{l}700 \mathrm{keV} \\
{ }^{3} \mathrm{He} \text { (D) }\end{array}$ & any & - & $\begin{array}{l}\text { Bulk \& } \\
\text { surface D }\end{array}$ & $p, S S D$ & 500 & & {$[121]$} \\
\hline
\end{tabular}

Table 2. Performance benchmarks of various ERD applications in hydrogen and deuterium analysis compared to NRA. Depth resolution refers to the value near the surface. Sensitivities are given either in at.\% (general number) for bulk concentrations or in layer densities $\left(\mathrm{cm}^{-2}\right.$, scientific notation) for surface coverages. 
Several authors have directly compared the performance of ${ }^{15} \mathrm{~N}$ NRA and ERD in specific applications to hydrogen analysis [51, 71, 238, 262, 263]. An instructive comparison of the factors influencing the near-surface depth resolution of ${ }^{15} \mathrm{~N}$ NRA and high-resolution H-ERDA (with an electrostatic spectrometer) for the same 6.4 MeV ${ }^{15} \mathrm{~N}$ ion beam is given in Ref. [238]. The conclusion that the near-surface resolution achieved by H-ERDA is slightly better and may be further improved by using ${ }^{20} \mathrm{Ne}$ ions instead of ${ }^{15} \mathrm{~N}$ and apply grazing ion beam incidence is not entirely fair, as the latter important possibility for improvement has not been considered at all for ${ }^{15} \mathrm{~N}$ NRA (cf. Sections 3.2.1.8 and 3.3.2). Under grazing-incidence conditions, near-surface resolutions better than $1 \mathrm{~nm}$ have been demonstrated for both techniques $[59,255]$.

For the depth profiling analysis of $50 \mathrm{~nm}$ thick hydrocarbon layers that were sandwiched between multilayer structures of three metal films of $382 \mathrm{~nm} \mathrm{Al}, 193 \mathrm{~nm}$ $\mathrm{Cu}, 208 \mathrm{~nm} \mathrm{Ag}$, and $148 \mathrm{~nm}(\mathrm{Au})$ prepared on Si wafer backings (the film thicknesses give approximately equivalent energy losses), Wielunski et al. [263] concluded that normal incidence ${ }^{1} \mathrm{H}\left({ }^{15} \mathrm{~N}, \alpha \gamma\right){ }^{12} \mathrm{C}$ NRA (Fig. 15 (a)) offers the best depth resolution and sensitivity compared to conventional $4.5 \mathrm{MeV}{ }^{4} \mathrm{He}$ ERD (at $20^{\circ}$ and $30^{\circ}$ recoil angles, Fig. 15 (b)) and $30 \mathrm{MeV} \mathrm{F}^{6+} \mathrm{HIERD}$ (38 $8^{\circ}$ recoil angle). Conventional ERD with ${ }^{4} \mathrm{He}$ was found to suffer heavily from multiple scattering effects that degrade the depth resolution at larger analyzing depths [264, 265]. This is seen in Fig. 15 (b) as increased width, reduced height, and increased continuous background in the respective $\mathrm{H}$ profiles, and is particularly pronounced in the large $Z$ targets $(\mathrm{Ag}, \mathrm{Au})$ [263]. 

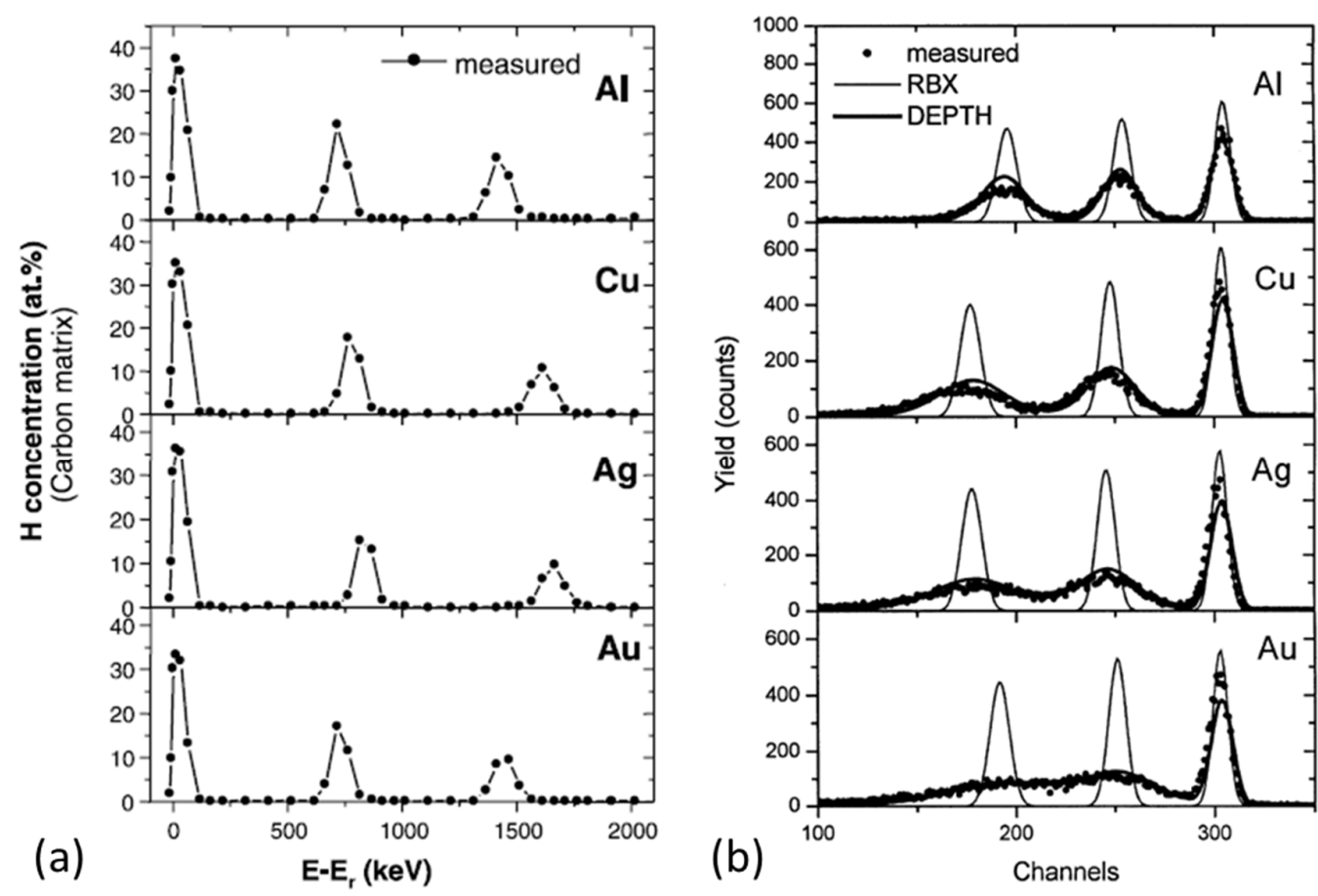

Fig. 15. (a) Excitation curves of the resonant $H\left({ }^{15} \mathrm{~N}, \alpha \gamma\right){ }^{12} \mathrm{C}$ nuclear reaction for all four multilayer structures. The energy straggling of primary ${ }^{15} \mathrm{~N}$ beam in sample material is responsible for the reduction of peak height and the increase in peak width as a function of increasing depth in the sample. (b) $4.5 \mathrm{MeV}{ }^{4} \mathrm{He}$ ERD spectra taken at tilt $75^{\circ}$ and recoil angle $30^{\circ}$ for all four multilayer samples. The thin line represents a simple (without multiple scattering effects) computer simulation with the RBX program. The thick line represents a DEPTH program simulation, which includes small angle multiple scattering effects. Reproduced from Ref. [263] with permission from Elsevier.

The comparison to simulated ERD spectra using RBX [224] and DEPTH [221, 222] simulation software, respectively, indicated that the spectral broadening was due to multiple scattering effects, which are not included in the RBX code. The latter thus severely overestimates the ERD depth resolution, which may lead to erroneous interpretations of ERD spectra. DEPTH, on the other hand, does take multiple scattering events into account and apparently reproduced the experimental data in Fig. 15 (b) rather adequately. In HIERD (not shown) the depth resolution shows a 
much smaller depth- and material- $(Z)$ dependence since heavier $F$ projectiles tend to be less affected by multiple scattering. For the same reason and due to the normal beam incidence resulting in a much shorter ion trajectory within the analyzed material, as well as due to the additional fact that in ${ }^{1} \mathrm{H}\left({ }^{15} \mathrm{~N}, \alpha \gamma\right){ }^{12} \mathrm{C}$ NRA only the incident ${ }^{15} \mathrm{~N}$ ion is subject to straggling, the NRA depth resolution was far superior in this particular application of $\mathrm{H}$ analysis in a metallic multilayer stack [263].

\subsubsection{Versatility}

In addressing practical differences between ERD and NRA for near-surface hydrogen analysis, we first note that there are extremely few variable experimental parameters in high resolution $\mathrm{H}$ depth profiling via ${ }^{1} \mathrm{H}\left({ }^{15} \mathrm{~N}, \alpha \gamma\right){ }^{12} \mathrm{C}$ NRA. Obviously, the

${ }^{15} \mathrm{~N}$ projectile and its energy, the size and energy width of the reaction cross section, the optimum choice of the detector for the $4.43 \mathrm{MeV} \gamma$-rays, etc. are rigidly defined by the characteristics of the nuclear reaction resonance at $6.385 \mathrm{MeV}$ (Table 1). Only the $\gamma$-detector's solid angle and background, the beam incidence angle on the target, and the beam energy spread remain 'adjustable' parameters that influence sensitivity and depth resolution. A sufficiently monochromatized ${ }^{15} \mathrm{~N}$ ion beam provided, the near-surface depth resolution is determined mainly by the vibrational Doppler broadening. Depth resolution and probing range are easily optimized by the angle of incidence. In $\mathrm{H}$ profiling with NRA, only straggling of the primary ${ }^{15} \mathrm{~N}$ ion needs consideration. Sensitivity is clearly maximized by increasing the detection solid angle and the beam flux, and by reducing the detector background. Once the detection system is calibrated only the ${ }^{15} \mathrm{~N}$ stopping power in the analyzed material 
(i.e., the target's chemical composition and density) is required to evaluate $\mathrm{H}$ concentrations quantitatively. This very manageable situation leads to ${ }^{15} \mathrm{~N}$ NRA measurements of great facility and straightforward data interpretations that are easily accomplished even by experimentalists without extensive experience in ion beam analysis.

For ERD, various detection schemes exist that require a certain understanding of ion optics, and the separation of projectiles and recoiled particles as well as the energy analysis of the latter is by no means trivial. Whereas in NRA the identification of the $4.43 \gamma$-rays of the ${ }^{1} \mathrm{H}\left({ }^{15} \mathrm{~N}, \alpha \gamma\right){ }^{12} \mathrm{C}$ reaction in the scintillation spectrum is simple and the sharp resonance at $6.385 \mathrm{MeV}$ may even be used to calibrate the accelerator energy, the channel-energy conversion in commonly used ERD detectors requires some attention [266, 267].

It might thus be stated that in routine ${ }^{15} \mathrm{~N}$ NRA the provision of an easily tunable, well-monochromatized ${ }^{15} \mathrm{~N}$ beam of $>6.4 \mathrm{MeV}$ is the instrumentally demanding part, whereas the actual measurements are simple to conduct and optimum conditions for the analysis of different sample systems are found easily. ERD, on the other hand, appears to be less demanding on the accelerator side (except to provide sufficient energy and stability), whereas the arrangement of the recoil experiment requires more thought (or experience). The recoil energy analysis is definitely more complex and requires specialized detection equipment for high resolution work (Appendix B).

ERD data analysis also appears to be somewhat more involved compared to ${ }^{15} \mathrm{~N}$ NRA, since also the stopping and straggling of the recoiled atoms on their path out of 
the target and through the range foil (if used) need to be accounted for in addition to the projectile trajectory into the sample. This requires detailed knowledge of the energy dependent stopping cross sections of the projectiles and recoils in the analyzed sample, which may not be available for novel materials at all energies, even though stopping and range databases are continuously expanded [126]. For quantitative ERD analysis the accurate knowledge of the hydrogen recoil cross section is a further crucial requirement. Non-Rutherford cross section effects and possible nuclear reaction backgrounds need careful attention in ERD with high energy (> $1 \mathrm{MeV} / \mathrm{amu}$ ) beams of ${ }^{4} \mathrm{He}$ projectiles on light targets (cf. Appendix B). In comparison, the ${ }^{1} \mathrm{H}\left({ }^{15} \mathrm{~N}, \alpha \gamma\right){ }^{12} \mathrm{C}$ cross section near the $6.385 \mathrm{MeV}$ resonance is very well-characterized and the off-resonance cross section is small and constant at least up to $9 \operatorname{MeV}[52,100,110,111]$ (Section 2.2.4).

In conclusion, ERD and ${ }^{15} \mathrm{~N}$ offer quite comparable performance in the analysis of near-surface hydrogen. Both have demonstrated ppm level sensitivity and subnanometer surface resolution under certain provisions. ${ }^{15} \mathrm{~N}$ NRA routinely provides good surface resolution in the order of a few $\mathrm{nm}$ and can easily switch between a high-resolution near-surface mode and standard measurements of extended depth profiles by a simple adjustment of the ion beam incidence angle. ERD, on the other hand, requires specialized spectrometers to achieve high resolution and then has a smaller probing depth range and likely non-maxiumum sensitivity, although this is apparently sufficient to detect sub-monolayer coverages of $H$ or D on a surface [255]. Sensitivity for bulk $\mathrm{H}$ or $\mathrm{D}$ is similar in both techniques. HIERD is an attractive option to achieve higher sensitivity and better depth resolution, provided the sample 
material withstands the more energetic irradiation. Depth profiling over an extended range with high-resolution spectrometers may require segmental measurements at readjusted analyzer pass energies. Simultaneous detection of several hydrogen isotopes $(H, D$, and perhaps even $T)$ with the same ion beam is only possible in conventional ERD with absorber foils and may require rather high projectile energies. Zero-point vibration measurements of surface $\mathrm{H}$ through Doppler width analysis are exclusive to ${ }^{15} \mathrm{NRA}$ at $6.385 \mathrm{MeV}$. 


\section{Applications}

\subsection{Surface hydrogen}

This chapter reviews quantitative NRA hydrogen coverage measurements at single crystal surfaces. Table 3 gives an overview of the studied systems. The chapter is organized into sections for metal, semiconductor, and insulator surfaces, with subdivisions for individual materials and surface orientations. Elements appear in order of their group in the periodic table. Single crystal surfaces of hydrogenabsorbing metals $\left(\mathrm{Ti}, \mathrm{Zr}, \mathrm{Pd}, \mathrm{Pd}_{70} \mathrm{Au}_{30}\right)$ are treated in Section 3.2.3. Since quite a few surface coverage data were obtained with the $D\left({ }^{3} \mathrm{He}, \mathrm{p}\right)^{4} \mathrm{He}$ reaction, 'hydrogen' will be used to synonymously represent both $\mathrm{H}$ and $\mathrm{D}$.

${ }^{15} \mathrm{~N}$ nuclear reaction analysis provides two important sources of information on the properties of surface-adsorbed $\mathrm{H}$ : (1) The $\mathrm{H}$ coverage is obtained quantitatively in a non-destructive fashion (as is D with $D\left({ }^{3} \mathrm{He}, \mathrm{p}\right)^{4} \mathrm{He} N R A$ ); (2) NRA via the narrow energy resonance of ${ }^{1} \mathrm{H}\left({ }^{15} \mathrm{~N}, \alpha \gamma\right){ }^{12} \mathrm{C}$ at $6.385 \mathrm{MeV}$ is the only experimental technique that can directly reveal the zero-point energy of the $\mathrm{H}$ vibrational motion on the surface, both in the perpendicular and in the parallel direction to the surface [67]. The latter property can be related to the mobility of $\mathrm{H}$ in surface diffusion and also to the possibility of $\mathrm{H}$ delocalization that has been under debate for $\mathrm{H}$ on a variety of metal surfaces (Cu(100), Ni, Pd, Pt) [68, 268, 269].

Table 3. Hydrogen and deuterium (saturation) coverages on single-crystal surfaces quantified with NRA and ERD techniques (materials in alphabetical order). 


\begin{tabular}{|c|c|c|c|c|c|c|}
\hline Surface & $\begin{array}{c}\text { ML density } \\
\text { (atoms } / \mathrm{cm}^{2} \text { ) }\end{array}$ & $\begin{array}{c}\theta_{\text {sat }} \\
(\mathrm{ML})\end{array}$ & $\begin{array}{c}\text { Structure } \\
\text { (LEED) }\end{array}$ & Method & $\begin{array}{c}\text { Preparation } \\
\text { Condition }\end{array}$ & Ref. \\
\hline $\mathrm{Ag}(111)$ & $1.38 \times 10^{15}$ & $0.6 \pm 0.1$ & $\begin{array}{c}(2 \times 2)+ \\
(3 \times 3)\end{array}$ & $\mathrm{D}\left({ }^{3} \mathrm{He}, \mathrm{p}\right)^{4} \mathrm{He}$ & $D_{\text {at }}$ (filament) & [270] \\
\hline $\mathrm{Cu}(111)$ & $1.77 \times 10^{15}$ & $0.67 \pm 0.03$ & $(3 \times 3)$ & $\mathrm{D}\left({ }^{3} \mathrm{He}, \mathrm{p}\right)^{4} \mathrm{He}$ & $\mathrm{D}_{\text {at }}$ (filament) & [271] \\
\hline $\mathrm{Cu}(100)$ & $1.54 \times 10^{15}$ & 1.0 & $\begin{array}{c}p(2 \times 2)- \\
p 4 g\end{array}$ & $\mathrm{D}\left({ }^{3} \mathrm{He}, \mathrm{p}\right)^{4} \mathrm{He}$ & $D_{a t}$ (filament) & {$[272,273]$} \\
\hline Diamond & $1.57 \times 10^{15}$ & $1.0 \pm 0.3$ & & HR-ERDA & Plasma CVD & [255] \\
\hline $\operatorname{Ir}(111)$ & $1.56 \times 10^{15}$ & $1.0 \pm 0.1$ & $(1 \times 1)$ & ${ }^{1} \mathrm{H}\left({ }^{15} \mathrm{~N}, \alpha \gamma\right){ }^{12} \mathrm{C}$ & $\begin{array}{l}1000 \mathrm{~L} \mathrm{H}_{2}, 80 \\
\mathrm{~K}\end{array}$ & {$[274]$} \\
\hline $\begin{array}{l}\operatorname{Mo}_{0.75} \operatorname{Re}_{0.25} \\
(100) \\
(110) \\
(111)\end{array}$ & & $\begin{array}{l}2.02 \pm 0.10 \\
0.99 \pm 0.05 \\
2.86 \pm 0.24 \\
\end{array}$ & $\begin{array}{l}(1 \times 1) \\
(1 \times 1) \\
(1 \times 1)\end{array}$ & $\mathrm{D}\left({ }^{3} \mathrm{He}, \mathrm{p}\right)^{4} \mathrm{He}$ & $\begin{array}{l}100 \mathrm{LD}_{2}, 100 \\
\mathrm{~K}\end{array}$ & [275] \\
\hline $\mathrm{Ni}(110)$ & & $\begin{array}{l}1.0 \\
1.5\end{array}$ & $\begin{array}{l}(2 \times 1) \\
(1 \times 2)\end{array}$ & $\mathrm{D}\left({ }^{3} \mathrm{He}, \mathrm{p}\right)^{4} \mathrm{He}$ & $\mathrm{D}_{2}, 175 \mathrm{~K}$ & {$[276,277]$} \\
\hline $\mathrm{Ni}(111)$ & $1.86 \times 10^{15}$ & $\begin{array}{c}0.5 \\
0.8 \\
1.0 \pm 0.1\end{array}$ & $\begin{array}{l}(2 \times 2) \\
(1 \times 1) \\
(1 \times 1)\end{array}$ & ERD & $\begin{array}{l}D_{2}, 120 \mathrm{~K} \\
D_{2}, 120 \mathrm{~K} \\
\mathrm{D}_{\mathrm{at}},>150 \mathrm{~K}\end{array}$ & [278] \\
\hline $\operatorname{Pd}(110)$ & $9.35 \times 10^{14}$ & $\begin{array}{c}0.5 \\
1.0 \pm 0.1 \\
1.0 \pm 0.1 \\
1.7 \pm 0.3\end{array}$ & $\begin{array}{l}c(2 \times 2) \\
(1 \times 1) \\
(1 \times 1) \\
(1 \times 2)\end{array}$ & $\begin{array}{c}\text { ERD } \\
{ }^{1} \mathrm{H}\left({ }^{15} \mathrm{~N}, \alpha \gamma\right){ }^{12} \mathrm{C} \\
{ }^{1} \mathrm{H}\left({ }^{15} \mathrm{~N}, \alpha \gamma\right){ }^{12} \mathrm{C}\end{array}$ & $\begin{array}{l}\mathrm{D}_{2},<120 \mathrm{~K} \\
\mathrm{H}_{2,}<160 \mathrm{~K} \\
1.3 \mathrm{~L} \mathrm{H}_{2}, 80 \mathrm{~K}\end{array}$ & $\begin{array}{l}{[258]} \\
{[61]} \\
{[279]}\end{array}$ \\
\hline $\operatorname{Pt}(111)$ & $1.5 \times 10^{15}$ & $1.0 \pm 0.1$ & $(1 \times 1)$ & ${ }^{1} \mathrm{H}\left({ }^{15} \mathrm{~N}, \alpha \gamma\right){ }^{12} \mathrm{C}$ & & [280] \\
\hline $\begin{array}{l}\mathrm{Pt}_{0.5} \mathrm{Ni}_{0.5} \\
(110) \\
(111)\end{array}$ & $\begin{array}{c}1.0 \times 10^{15} \\
1.64 \times 10^{15}\end{array}$ & $\begin{array}{l}1.0 \\
0.2 \\
\end{array}$ & $\begin{array}{l}(1 \times 1) \\
(1 \times 1)\end{array}$ & $\begin{array}{l}{ }^{1} \mathrm{H}\left({ }^{15} \mathrm{~N}, \alpha \gamma\right){ }^{12} \mathrm{C} \\
{ }^{1} \mathrm{H}\left({ }^{15} \mathrm{~N}, \alpha \gamma\right){ }^{12} \mathrm{C}\end{array}$ & & [281] \\
\hline $\mathrm{Rh}(111)$ & & $0.84 \pm 0.12$ & $(1 \times 1)$ & ${ }^{1} \mathrm{H}\left({ }^{15} \mathrm{~N}, \alpha \gamma\right){ }^{12} \mathrm{C}$ & $\begin{array}{l}1 \times 10^{-4} \mathrm{~Pa} \mathrm{H} \\
100 \mathrm{~K}\end{array}$ & [282] \\
\hline $\mathrm{Si}(100)$ & $6.78 \times 10^{14}$ & $\begin{array}{c}1.0 \\
1.5 \\
1.6 \\
1.6 \pm 0.1 \\
1.85 \pm 0.18 \\
1.3 \pm 0.1\end{array}$ & $\begin{array}{l}(2 \times 1) \\
(1 \times 1) \\
(1 \times 1) \\
(3 \times 1) \\
(1 \times 1) \\
-\end{array}$ & $\begin{array}{l}\mathrm{D}\left({ }^{3} \mathrm{He}, \mathrm{p}\right)^{4} \mathrm{He} \\
\mathrm{D}\left({ }^{3} \mathrm{He}, \mathrm{p}\right)^{4} \mathrm{He} \\
\left.\mathrm{D}\left({ }^{3} \mathrm{He}, \mathrm{p}\right)\right)^{4} \mathrm{He} \\
\mathrm{D}\left({ }^{3} \mathrm{He}, \mathrm{p}\right){ }^{4} \mathrm{He} \\
\text { ERD } \\
\left.{ }^{1} \mathrm{H}\left({ }^{15} \mathrm{~N}, \alpha \gamma\right)\right)^{12} \mathrm{C}\end{array}$ & $\begin{array}{l}\text { 'Monohydride' } \\
\text { 'Dihydride' } \\
400 \mathrm{~K} \\
\mathrm{D}_{\text {at, }} \text { RT } \\
\text { HF etched }\end{array}$ & $\begin{array}{l}{[283]} \\
{[283]} \\
{[284]} \\
{[285]} \\
{[259]} \\
{[286]}\end{array}$ \\
\hline $\mathrm{Si}(111)$ & $7.83 \times 10^{14}$ & $\begin{array}{c}1.25 \pm 0.13 \\
1.5 \pm 0.15 \\
1.11 \pm 0.1 \\
\end{array}$ & $\begin{array}{l}(7 \times 7) \\
(7 \times 7) \\
(1 \times 1)\end{array}$ & $\begin{array}{l}\mathrm{D}\left({ }^{3} \mathrm{He}, \mathrm{p}\right)^{4} \mathrm{He} \\
\text { ERD } \\
\text { MEIS-ERD }\end{array}$ & $\begin{array}{l}>1000 L^{\text {at }}, \\
\text { RT } \\
\mathrm{NH}_{4} \text { F-etched }\end{array}$ & $\begin{array}{l}{[287]} \\
{[260]} \\
{[288]}\end{array}$ \\
\hline $\mathrm{SiC}(0001)$ & & 1.7 & $\sqrt{3} x \sqrt{3}$ & TOF-ERD & $\mathrm{H}_{\mathrm{at}}, \mathrm{RT}$ & [261] \\
\hline $\mathrm{Si}_{0.7} \mathrm{Ge}_{0.3}$ & & $1.2 \pm 0.1$ & - & $\left.{ }^{1} \mathrm{H}\left({ }^{15} \mathrm{~N}, \alpha \gamma\right)\right)^{12} \mathrm{C}$ & HF etched & [286] \\
\hline $\mathrm{SrTiO}_{3}(001)$ & $6.6 \times 10^{14}$ & 0.47 & $(1 \times 1)$ & ${ }^{1} \mathrm{H}\left({ }^{15} \mathrm{~N}, \alpha \gamma\right){ }^{12} \mathrm{C}$ & $\mathrm{H}_{\mathrm{at}}, \mathrm{RT}$ & [289] \\
\hline$W(100)$ & $1.0 \times 10^{15}$ & 2.0 & $(1 \times 1)$ & $\mathrm{D}\left({ }^{3} \mathrm{He}, \mathrm{p}\right)^{4} \mathrm{He}$ & $120 L_{2}$ & [290] \\
\hline $\mathrm{Zn}(10 \overline{1} 0)$ & $5.9 \times 10^{14}$ & $\begin{array}{l}1.0 \\
2.0\end{array}$ & $(1 \times 1)$ & ${ }^{1} \mathrm{H}\left({ }^{15} \mathrm{~N}, \alpha \gamma\right){ }^{12} \mathrm{C}$ & $\begin{array}{l}\mathrm{H}_{\mathrm{at}}, 30-100^{\circ} \mathrm{C} \\
10^{-8} \mathrm{mbar} \mathrm{H}_{2} \mathrm{O} \\
\times 30 \mathrm{~min}, \mathrm{RT}\end{array}$ & [291] \\
\hline
\end{tabular}


Absolute hydrogen coverages are in general difficult to determine experimentally, e.g., by most frequently utilized thermal desorption spectroscopy, unless careful measures of calibration are taken $[292,293]$. In surface structure determinations through LEED I(V) analysis or He atom scattering, often the assumption is made that the $\mathrm{H}$ coverage is a rational fraction of a monolayer $(\mathrm{ML})$, which corresponds to a periodic arrangement of $\mathrm{H}$ atoms in symmetry-equivalent adsorption sites. These assumptions partially arise from the need to reduce the computational effort in the theoretical modeling of the surface structure that is required to analyze (i.e., simulate) diffraction patterns. Since the electron scattering cross section of $\mathrm{H}$ is very small, the validity of such assumptions can seldom be verified because LEED much more accurately locates the heavier substrate atoms rather than defining the $\mathrm{H}$ positions, especially when only $(1 \times 1)$ structures are observed or when several symmetry-equivalent site occupation patterns are conceivable. A similar problem is encountered in helium atom scattering (HAS). While the fractional loss of diffracted He beam intensity has certainly excellent sensitivity to $H$ on the topmost atomic layer (much superior to LEED), it is not necessarily directly proportional to the $\mathrm{H}$ coverage [294]. The difficulty to quantitatively correlate a measured signal with the $\mathrm{H}$ coverage is even more severe for work function changes, as the surface dipole is likely $\mathrm{H}$ coverage dependent, especially in presence of non-equivalent adsorption sites or due to $\mathrm{H}-\mathrm{H}$ interactions at high coverage. The quantitative information on the coverage of surface-adsorbed hydrogen provided by NRA is hence extremely valuable as a calibration reference for other $\mathrm{H}$-sensitive techniques that cannot directly deliver absolute coverage values. 


\subsubsection{Note on adsorption experiments with atomic hydrogen}

On a certain class of metals, $\mathrm{H}_{2}$ dissociates spontaneously and sticks on the surface without any activation barrier. In such cases, hydrogen adsorption can conveniently be studied after admission of molecular $\mathrm{H}_{2}$. On the other hand, several metal surfaces exist on which an activation barrier impedes molecular $\mathrm{H}_{2}$ dissociation, so that adsorption studies have been performed with atomic hydrogen in order to populate the chemisorbed $\mathrm{H}$ state(s). Atomic hydrogen can be generated by cracking $\mathrm{H}_{2}$ thermally or in a microwave plasma and thus bears excess energy $(1 / 2$ of the dissociation energy of a $\mathrm{H}_{2}$ molecule, $\left.\sim 2.23 \mathrm{eV}\right)$. This excess energy enables several other reactive processes of atomic hydrogen in addition to surface adsorption, among them penetration into subsurface sites. This can be effective even when the substrate does not exothermically absorb hydrogen, such as in the case of $\mathrm{Cu}(111)$, where the energy barrier for subsurface penetration amounts to at least $1.3 \mathrm{eV}$ [295].

The transfer of excess energy from the $\mathrm{H}$ atoms to the substrate via phonon coupling and electron hole pair excitation occurs on a picosecond timescale [295, 296]. Before their full accommodation either in surface adsorption or subsurface potentials, the $\mathrm{H}$ atoms may thus exist on (or below) the surface in a so-called 'hot atom' state for a finite lifetime, during which they remain highly mobile and exert multiple collisions with neighboring (substrate or hydrogen) atoms. On the surface this can lead to Langmuir-Hinshelwood-like abstraction of other adsorbed $\mathrm{H}$ atoms, in addition to Eley-Rideal-type $\mathrm{H}$ abstraction upon direct impact of $\mathrm{H}$ atoms from the gas phase [297, 298]. In subsurface sites, not fully accommodated H atoms may diffuse further into the bulk or return to the surface (as long as vacant sites are 
available there). At low temperatures the latter is the preferred diffusion direction, since in general the chemisorption potential minimum is energetically strongly favored over bulk interstitial sites [299] and separated from the subsurface potential by only a relatively small barrier [295, 300-304]. As a further possibility, also a direct stamp-in process of pre-adsorbed surface hydrogen into the subsurface by $\mathrm{H}$ atoms impinging from the gas phase has been reported for $\mathrm{H} / \mathrm{Pd}(100)$ [305].

Since several parallel reaction pathways (absorption, abstraction, desorption, and diffusion into the bulk) exist for energetic $\mathrm{H}$ atoms in addition to accommodation in the surface chemisorption potentials, the hydrogen coverage on an atomic $\mathrm{H}$-exposed surface will attain a stationary value that reflects a dynamic equilibrium between these processes. The sticking coefficient, the abstraction probability, and the likelihood of penetration into subsurface sites during atomic $\mathrm{H}$ exposure will depend on the energy of the impinging $\mathrm{H}$ beam, whereas sticking, desorption and bulk diffusion are strongly sensitive to the surface temperature. To our knowledge, the influence of these parameters has never been characterized systematically for any system, and it is likely that the atomic $\mathrm{H}$ energy has varied significantly among studies that employed different methods to supply dissociated $\mathrm{H}_{2}$ (microwave plasmas, heated tubes, or open filaments). These circumstances may partially explain the appreciable scatter among values of the $H$ 'saturation' coverage reported for certain atomic $\mathrm{H}$-exposed surfaces (see, e.g., Table 4 for $\mathrm{H} / \mathrm{Cu}(111)$ in Section 3.1.2). The point to be made here is that we consider such variations as likely to reflect different preparation conditions rather than errors in the coverage quantification with NRA. 


\subsubsection{Metals}

\section{W(100), W(110)}

The deuterium saturation coverage on $\mathrm{W}(100)$ was determined as $\theta_{\text {sat }}=2.0 \mathrm{ML}(1$ $\left.M L=1.0 \times 10^{15} \mathrm{~cm}^{-2}\right)$ by $D\left({ }^{3} \mathrm{He}, \mathrm{p}\right)^{4} \mathrm{He}$ NRA [290], confirming earlier results of flash desorption and flux-calibrated molecular beam experiments [306-308]. A similarly large group of authors $[292,309,310]$, however, states a smaller saturation coverage for $\mathrm{H} / \mathrm{W}(100)$ of $1.5 \times 10^{15} \mathrm{~cm}^{-2}$, including a carefully calibrated TDS measurement by Winkler [292]. As of today this discrepancy stands unresolved, which may exemplify that despite best efforts to reproduce a given adsorption system independently, experimental pitfalls may remain. Even when calibration and stability of the $\mathrm{H}$ detection method are guaranteed, differences in the surface preparation procedures may always introduce uncertainty, as the $\mathrm{H}$ coverage on metal surfaces tends to be strongly sensitive, e.g., to the presence of surface impurities.

The first ${ }^{1} \mathrm{H}\left({ }^{15} \mathrm{~N}, \alpha \gamma\right){ }^{12} \mathrm{C}$ NRA applications for the analysis of chemisorbed $\mathrm{H}$ layers on well-characterized single crystal surfaces were the measurements of hydrogen on $W(100)$ and $W(110)$ by Iwata et al. $[65,83]$. They observed the room temperature uptake and saturation of $\mathrm{H}$ on the $\mathrm{W}(100)$ surface during dissociative chemisorption under different $\mathrm{H}_{2}$ background pressures in the range of $6.0 \times 10^{-7}-1.2 \times 10^{-5} \mathrm{~Pa}$ (Fig. 16) and determined the initial $\mathrm{H}_{2}$ sticking probability as $\mathrm{s}_{0}=0.69 \pm 0.1$ in reasonable agreement with previous publications $[307,308]$. Although these early ${ }^{15} \mathrm{~N}$ NRA measurements of the $\mathrm{H}$ coverage were not quantified relative to a calibration standard and saturation at $2.0 \times 10^{15} \mathrm{~cm}^{-2}$ was merely assumed (in line with Refs. [290, 306-308]), the $\mathrm{H}_{2}$ pressure dependent changes of the adsorption rate and of the $\mathrm{H}$ 
coverage saturation levels in competition with ${ }^{15} \mathrm{~N}$ ion-induced desorption are clearly recognized (Fig. 16). Above $6.0 \times 10^{-6} \mathrm{~Pa}$ the ion beam-induced desorption is fully compensated and higher $\mathrm{H}_{2}$ pressures do not increase the $\mathrm{H}$ coverage beyond the saturation value. From the decay of the H-NRA signal in UHV (residual pressure: $6.7 \times 10^{-8} \mathrm{~Pa}$ ) the cross section for ${ }^{15} \mathrm{~N}$ ion-induced desorption was estimated as $2.4 \times 10^{-16} \mathrm{~cm}^{-2}$. Iwata et al. obtained similar results for $\mathrm{H} / \mathrm{W}(110)$, where the ioninduced desorption cross section was found to be the same and the $\mathrm{H}$ saturation coverage $(0.7 \pm 0.1)$ times smaller than that on $W(100)$ [83]. The latter observation is fairly consistent with earlier hydrogen adsorption studies on tungsten surfaces, which reported $\mathrm{H}$ saturation coverage ratios of $\mathrm{W}(110)$ relative to $W(100)$ between 0.7 (Ref. [311]) and 0.9 (Ref. [309]).

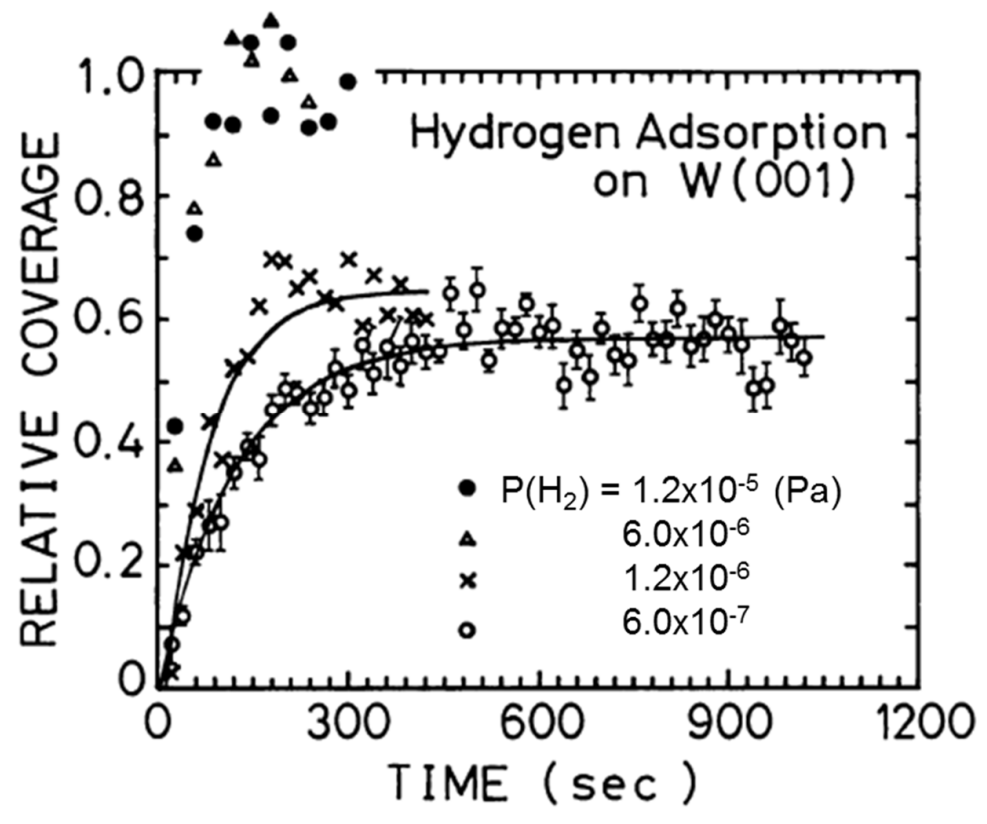

Fig. 16. Relative hydrogen coverage obtained from the $\gamma$-ray yields as a function of the time elapsed after introducing $\mathrm{H}_{2}$ at pressures of $1.2 \times 10^{-5}(\bullet), 6.0 \times 10^{-6}(\Delta), 1.2 \times 10^{-6}(\mathrm{x})$, and 6.0 $\times 10^{-7}$ (o) $\mathrm{Pa}$. Error bars are shown only at the lowest $\mathrm{H}_{2}$ pressure. The solid curves show fits to $s=s_{0}(1-a \theta)$. Adapted from Ref. [65] with permission. 
Iwata et al. also determined the zero-point energy of the $\mathrm{H}$-atom vibration perpendicular to the $\mathrm{W}(100)$ surface from the Doppler width of the nuclear reaction resonance profile measured with the ${ }^{15} \mathrm{~N}$ ions incident along the surface normal [65]. The obtained value of $E_{z}=64 \pm 16 \mathrm{meV}$ was in sound agreement with the zero-point energy expected in the framework of the harmonic oscillator model for the $v_{1} \mathrm{H}$ vibration mode (perpendicular to the surface) as measured by HREELS ( $65 \mathrm{meV}$ ) [312, 313] and RAIS (68 meV) [314] at saturation coverage.

\section{Rh(111)}

Hydrogen adsorption on the $\mathrm{Rh}(111)$ surface was investigated by ${ }^{1} \mathrm{H}\left({ }^{15} \mathrm{~N}, \alpha \gamma\right){ }^{12} \mathrm{C}$ NRA combined with dynamical LEED analysis [282] in order to clarify the adsorption site and vibrational properties of the adsorbed $\mathrm{H}$ atoms. LEED showed no indications for $\mathrm{H}$-induced surface reconstructions, the diffraction pattern remaining $(1 \times 1)$ up to $\mathrm{H}$-saturation. From the integrated $\gamma$-yield of the NRA surface resonance excitation profile (Fig. 17) the $\mathrm{H}$ saturation coverage at $\sim 100 \mathrm{~K}$ under $1 \times 10^{-4} \mathrm{~Pa} \mathrm{H}_{2}$ was determined to be $0.84 \pm 0.12 \mathrm{ML}$, suggesting that the local coverage is close to $1 \mathrm{ML}$ as previously assumed $[315,316]$. The small difference to the full monolayer coverage was rationalized by either unavoidable defects, repulsive interactions between large densely packed $(1 \times 1)$ domains in the $H$ adlayer, or possibly by hindrance of the $\mathrm{H}_{2}$ dissociation near coverage saturation [317]. Shifts in the LEED I(V) curves revealed that $H(D)$ adsorption lifts the first layer relaxation $(-1.2 \%$ contraction vs. the bulk interlayer distance) of the clean $\mathrm{Rh}(111)$ surface already at half saturation coverage, restoring a bulk-like spacing between the two outermost layers of Rh atoms. The $\mathrm{H}$ adsorption site was clearly identified as the fcc threefold 
hollow site in favor over mixed hollow site + bridge, pure hcp hollow, and on-top occupations [282].

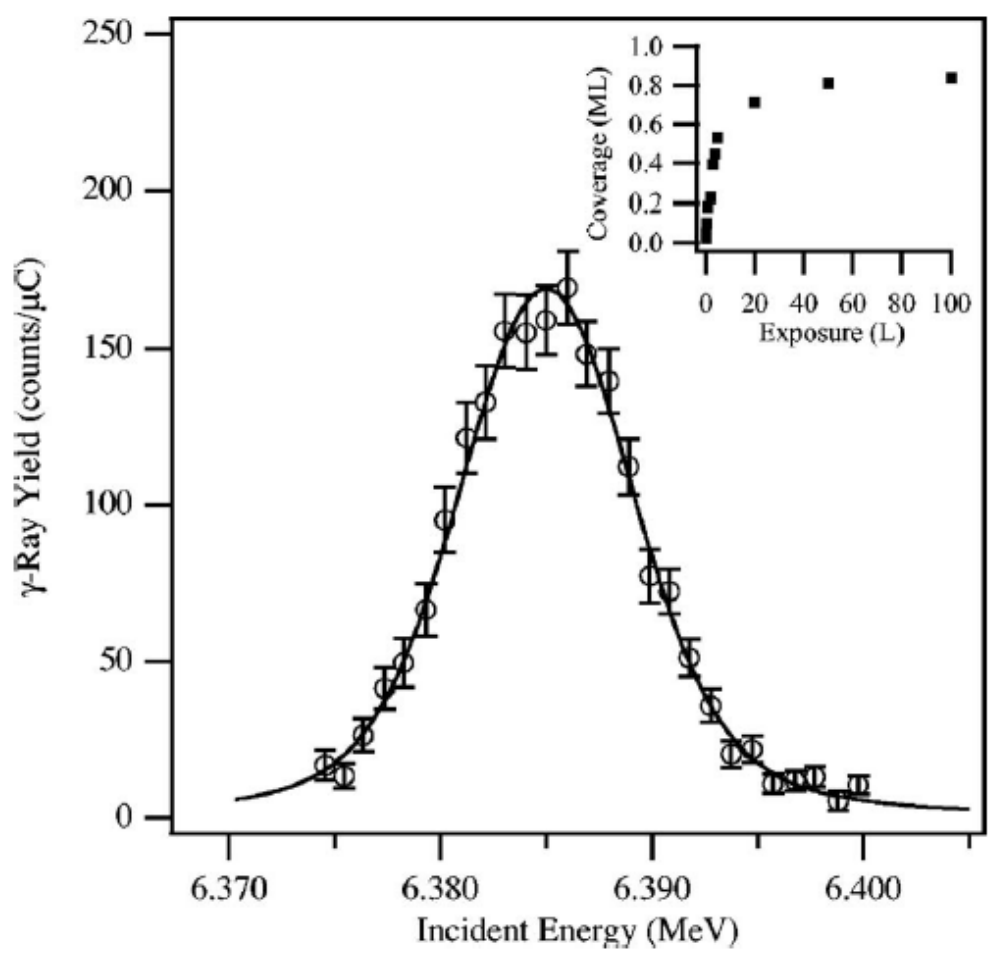

Fig. 17. NRA resonance profile of $\mathrm{H}$-saturated $\mathrm{Rh}(111)$ recorded at $\sim 100 \mathrm{~K}$ with normal incidence ${ }^{15} \mathrm{~N}$. Data are fitted to a Voigt profile (solid line). Inset: D uptake of $\mathrm{Rh}(111)$ under $\mathrm{D}_{2}$ exposure at $\sim 80 \mathrm{~K}$ determined by TDS. Reproduced with permission from Ref. [282].

The zero-point energy for the $\mathrm{H}$ vibration perpendicular to the $\mathrm{Rh}(111)$ surface was derived as $53.2 \pm 9.2 \mathrm{meV}$ from the Doppler width of the NRA surface resonance excitation profile (Fig. 17), measured under ${ }^{15} \mathrm{~N}$ ion incidence along the surfacenormal direction. This zero-point energy corresponds to a first vibrational excitation energy of $\sim 106$ meV within a harmonic oscillator model for the atomic $\mathrm{H}$ vibration against the surface. The NRA-derived value represents the average of all $\mathrm{H}$ vibrational motion components perpendicular to the surface. It is clearly smaller than the HREELS observed energy loss (136 meV) assigned to surface-perpendicular 
vibration excitation $[316,318]$ and also smaller than the theoretical prediction of a vibrational band with $A^{2}{ }_{1}$ symmetry and predominantly perpendicular character ( 150 meV) [319]. While the discrepancy could not be resolved conclusively, it was reasoned that the nature of the $\mathrm{H}$ wavefunction in the adsorbed state is too complex as to be reproduced in a simple harmonic oscillator model. Apparently the $\mathrm{H}$ atoms experience a strongly anharmonic potential, causing substantial mixing of the $\mathrm{H}$ motions perpendicular and parallel to the surface.

\section{$\operatorname{Ir}(111)$}

Matsumoto et al. investigated the $\mathrm{H}$-adsorption structure on the $\operatorname{Ir}(111)$ surface by means of dynamical LEED I(V) analysis [320]. Determining the $\mathrm{H}$ adsorption site on $\operatorname{Ir}(111)$ was of particular interest because this system is a rare case of an fcc (111) transition metal surface for which the on-top position has been proposed as the most likely $\mathrm{H}$ adsorption site. This conjecture was inferred from HREELS data revealing unusually high vibrational frequencies of the $\mathrm{H}$ adatom layer [269], and later corroborated by DFT total energy calculations [321]. Matsumoto et al. found no superstructures caused by hydrogen adsorption and the LEED pattern remained (1×1) up to saturation with $\mathrm{H}[320] .{ }^{1} \mathrm{H}\left({ }^{15} \mathrm{~N}, \alpha \gamma\right){ }^{12} \mathrm{C}$ NRA was used to confirm that the $\mathrm{H}$ coverage saturated at $1.0 \mathrm{ML}\left(1.56 \times 10^{15} \mathrm{~cm}^{-2}\right)$ after exposing the clean $\operatorname{Ir}(111)$ surface to $1000 \mathrm{~L}\left(2.66 \times 10^{-4} \mathrm{~Pa} \times 500 \mathrm{~s}\right) \mathrm{H}_{2}$ gas at $80 \mathrm{~K}$ [274]. The $\mathrm{H}$ saturation coverage and uptake behavior under $\mathrm{H}_{2}$ exposure was found to be in good agreement with a previous TDS investigation of $\mathrm{H}_{2}$ adsorption on $\operatorname{Ir}(111)$, which discovered a novel mechanism of isotope exchange reactions with preadsorbed $D$ driven by hot $\mathrm{H}$ adatoms that are generated in the dissociation process [322]. Based 
on the perfectly symmetric Gaussian shape of the NRA resonance excitation curve of $\mathrm{H} / \mathrm{Ir}(111)$ (Fig. 18), recorded with the ${ }^{15} \mathrm{~N}$ ion beam inclined by $60^{\circ}$ against the surface normal, it was concluded that it is not necessary to consider potential $\mathrm{H}$ absorption into the $\operatorname{Ir}(111)$ subsurface in modeling the $\mathrm{H}$ adsorption structure [274] (although partial first layer subsurface site occupation is principally indistinguishable, see Section 3.2.1). NRA further confirmed that the $H$ coverage was stable against desorption under UHV at $80 \mathrm{~K}$, the conditions under which the LEED I(V) measurements were performed [320].

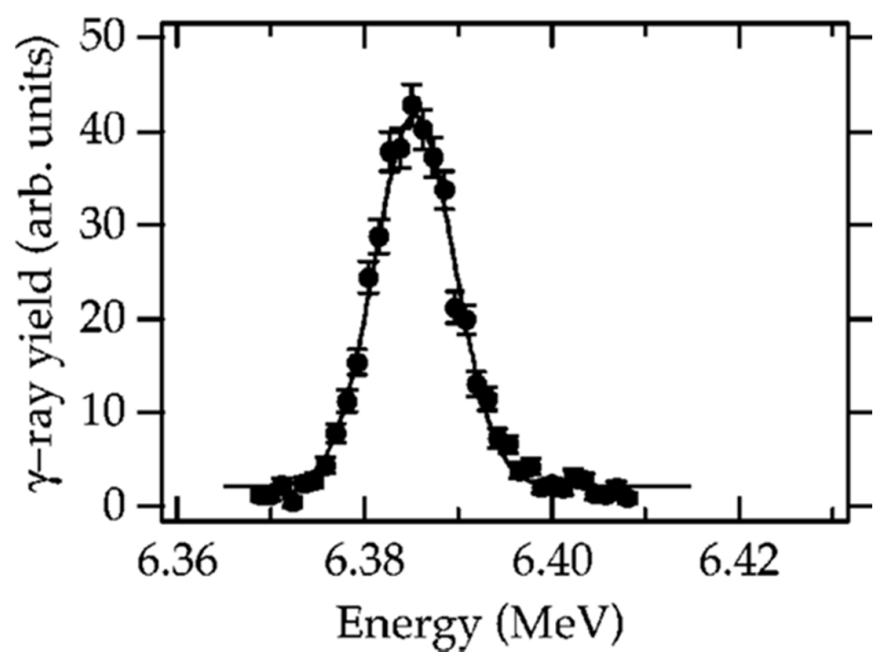

Fig. 18. Excitation curve of the ${ }^{1} \mathrm{H}\left({ }^{15} \mathrm{~N}, \alpha \gamma\right){ }^{12} \mathrm{C}$ nuclear reaction resonance from $0.5 \mathrm{ML}-\mathrm{H}$ covered $\operatorname{Ir}(111)$ recorded under a ${ }^{15} \mathrm{~N}$ incidence angle of $60^{\circ}$ from the surface normal. The solid curve is a fit to a Gaussian form. Reproduced with permission from Ref. [274].

The NRA-derived information on the $\mathrm{H}$ coverage and location provided the background for the structural analysis of H-saturated $\operatorname{Ir}(111)$ [320]. The dynamical LEED calculations, however, turned out to be rather insensitive towards modeling the $\mathrm{H}$ adsorption for either on-top, fcc hollow, or hcp hollow site occupation; the simulated I(V) curves reproducing the experimental data in the energy range of 100$400 \mathrm{eV}$ nearly equally well. This finding is possibly accounted for by the rather flat $\mathrm{H}$ 
adsorption potential on $\operatorname{Ir}(111)$, which according to DFT calculations shows only marginal corrugation with an only slightly deeper minimum at the on-top site and small energy barriers separating this site from similarly deep wells at neighboring high symmetry sites [321]. In addition, Nobuhara et al. found indications that the $\mathrm{H}$ nuclear wavefunction is partially delocalized around the on-top adsorption site in low-lying vibrationally excited states (mainly surface-parallel modes $\sim 50$ meV above the ground state) [321]. Although the $\mathrm{H}$ adsorption site could thus not be determined conclusively on the particular $\operatorname{Ir}(111)$ surface, the LEED analysis clearly revealed that $\mathrm{H}$ adsorption removes the small inward relaxation between the two outermost layers of Ir atoms (- $0.45 \%$ contracted vs. the bulk interlayer spacing) and that it instead produces a slight outward expansion of similar magnitude $(+\sim 0.45 \%)$ on the $\mathrm{H}$-saturated surface with $\mathrm{H}$ in threefold hollow sites (or $+0.52 \%$ with $\mathrm{H}$ on top, respectively) [320].

\section{Ni(110), Ni(111), Ni(100)}

\section{$\operatorname{Ni(110)}$}

Nickel surfaces readily dissociate molecular hydrogen [323]. The rather open and intensively studied $\mathrm{Ni}(110)$ surface shows a somewhat complex behavior in its hydrogen interactions, involving several structurally ordered phases developing under increasing $\mathrm{H}_{2}$ exposure. After small $\left(0.3 \sim 0.7\right.$ L) $\mathrm{H}_{2}$ exposures at low temperatures $(<200 \mathrm{~K})$ first a well-defined $(2 \times 1)$ LEED pattern with faint but clear half-order spots appears that subsequently $\left(\sim 2-3 \mathrm{~L} \mathrm{H}_{2}\right)$ transforms into a $\mathrm{H}$-induced $(1 \times 2)$ phase with intense diffraction spots indicative of substrate reconstruction [324, 
325]. Further ordered phases $(c(2 \times 6), c(2 \times 4)$, and $c(2 \times 6))$ of the $\mathrm{H}$ adatoms were discovered by helium diffraction in the sub-monolayer coverage regime [326-328]. Quantitative $D\left({ }^{3} \mathrm{He}, \mathrm{p}\right)^{4} \mathrm{He}$ NRA measurements by Norton's group established that the well-defined $(2 \times 1)$ structure corresponds to an ordered $\mathrm{H}$ adsorption layer of $1.0 \mathrm{ML}$ $\left(1.14 \times 10^{15} \mathrm{~cm}^{-2}\right)$ on an unreconstructed $\mathrm{Ni}(110)$ surface, whereas the $(1 \times 2)$ phase holds 1.5 ML H at saturation and involves a pairing row reconstruction of the two topmost $\mathrm{Ni}$ atom layers $[276,277,329,330]$. Fig. 19 shows respective models of the proposed $(1 \times 2)-2 \mathrm{H}$ and $(1 \times 2)-3 \mathrm{H}$ structures, which are supported by experiments (LEED, HAS) as well as by theory (DFT) [331, 332]. The DFT calculations additionally identified the most stable adsorption sites in the $(1 \times 2)$ pairing row reconstruction as the pseudo-threefold hollow and long-bridge sites. This information cannot be obtained from TDS due to overlapping desorption peaks and possible reconstruction lifting during the heating ramp [329, 331, 332].

The hydrogen coverages in the $(2 \times 1)$ and $(1 \times 2)$ phases had been debated for some time, but presently the respective values of 1.0 and $1.5 \mathrm{ML}$ are most widely accepted [276, 277, 333]. An earlier calibrated $D\left({ }^{3} \mathrm{He}, \mathrm{p}\right)^{4} \mathrm{He}$ NRA investigation notably determined the same coverage ratio of $(2: 3)$ for $D$ in the $(2 \times 1)$ and $(1 \times 2)$ phases of $\mathrm{H} / \mathrm{Ni}(110)$, but arrived at overall smaller saturation coverages of 0.67 and 1.0 ML, respectively [334]. It was speculated that either $\mathrm{H}_{2}$ impurities in the dosed $D_{2}$ gas or $H / D$ exchange on the D-exposed $\mathrm{Ni}(110)$ surface with $H_{2}$ in the UHV residual gas might have reduced the D coverage [276], pointing out a potential pitfall that might require attention in quantitative $D$ coverage evaluations with $D\left({ }^{3} \mathrm{He}, \mathrm{p}\right)^{4} \mathrm{He}$ 
NRA. Consequently, one may consider $\mathrm{H}$ coverage measurements with ${ }^{1} \mathrm{H}\left({ }^{15} \mathrm{~N}, \alpha \gamma\right){ }^{12} \mathrm{C}$ NRA more reliable, since residual $\mathrm{D}_{2}$ is usually negligible in UHV.

(a)

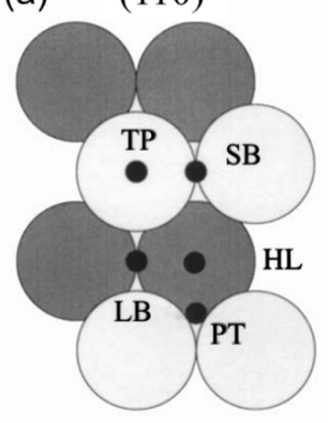

(b)

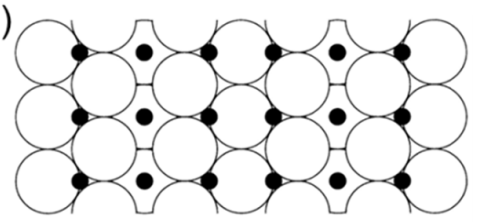

(c)

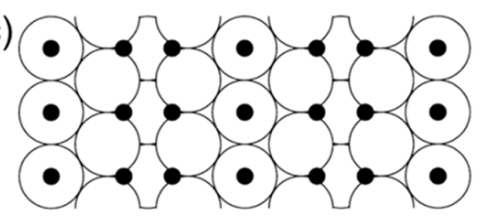

(d)

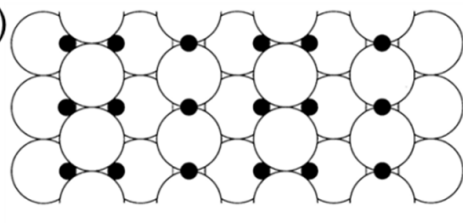

(e)

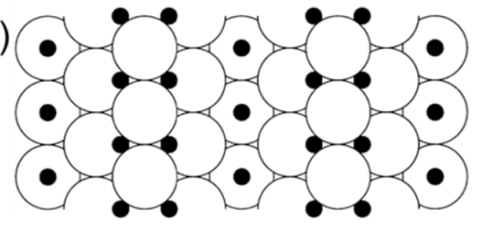

Fig. 19. (a) High-symmetry $\mathrm{H}$ adsorption sites on $\mathrm{Ni}(110)$. TP: top; LB: long bridge; SB: short bridge; HL: hollow; PT: pseudo-threefold. (b)-(e): Top views of the $\mathrm{H}$ adsorption structures on $\mathrm{Ni}(110)$ : (b) (2×1)-2H lattice gas structure (no reconstruction, $\mathrm{q}_{\mathrm{H}}=1.0 \mathrm{ML}$ ), (c) and (d) two models for the $(1 \times 2)-3 \mathrm{H}$ pairing-row reconstruction $\left(\mathrm{q}_{\mathrm{H}}=1.5 \mathrm{ML}\right)$, and $(\mathrm{e})(1 \times 2)-3 \mathrm{H}$ missing row reconstruction. Adapted from Ref. [331] with permission from Elsevier.

Warming the $(1 \times 2)-3 \mathrm{H}$ phase above $\sim 220 \mathrm{~K}$ or exposing a clean $\mathrm{Ni}(110)$ surface to $\mathrm{H}_{2}$ at room temperature produces another $(1 \times 2)$ phase that shows streaks along [001] in the LEED pattern [277, 325, 329, 334]. It has been suggested on the basis of first-principle computations (DFT) that the evolution of this streaky $(1 \times 2)$ phase is due to progressive transformation into a missing row structure [331]. This type of reconstruction involves substantial mass transport in the topmost $\mathrm{Ni}$ layers, hence the phase formation is expected to be thermally activated, consistent with the finding that it occurs only above $\sim 200 \mathrm{~K}[325,329]$. This interpretation might also explain differences in TD spectra and NRA-determined absolute coverages that depend on the exposure history of the sample. Winkler and Rendulic observed a coverage of $2.3 \mathrm{ML}$ at $140 \mathrm{~K}$ for the $\mathrm{H}_{2}$-saturated $(1 \times 2)$ phase with calibrated TDS, 
which showed a pronounced ' $\alpha$ ' feature at $~ 220 \mathrm{~K}$ [333]. Harrington and Norton note that cooling an annealed $\mathrm{Ni}(110)$ surface in $10^{-4} \mathrm{~Pa} \mathrm{D}_{2}$ from 350 to $170 \mathrm{~K}$ results in a larger $\mathrm{D}$ coverage $(1.7 \pm 0.05 \mathrm{ML})$ compared to the $1.5 \mathrm{ML}$ value obtained after saturation with $D_{2}$ at $170 \mathrm{~K}$, the former giving rise to a new ' $\beta_{3}$ ' desorption state at $435 \mathrm{~K}$ in TDS, whereas the low temperature ' $\alpha$ ' peak is missing [330]. No detailed explanations were offered, but one may speculate that different amounts and distributions of $\mathrm{H}$ absorbed below the surface and also different degrees of surface reconstruction might account for the observations. Owing to the activated and therefore strongly temperature sensitive trend to progressive reconstruction it is difficult to specify a saturation coverage for the streaky $(1 \times 2)$ phase.

\section{$\operatorname{Ni(100)}$}

Although no quantitative NRA measurements are reported for $\mathrm{Ni}(100)$, we note for the sake of completeness that the coverage is presumed to saturate at $1 \mathrm{ML}$ $\left(1.6 \times 10^{15} \mathrm{~cm}^{-2}\right)[331,335]$, where $\mathrm{H}$ occupies fourfold hollow sites in a $(1 \times 1)$ overlayer as concluded from HREELS [336] and HAS [337]. At half-saturation LEED indicates a faint $\mathrm{p}(2 \times 2)-\mathrm{H}$ adsorption structure [336]. The fourfold hollow adsorption site was confirmed through transmission channeling by Stensgaard and Jacobsen $[338,339]$, who also determined the height of the $\mathrm{H}$ atoms above the topmost $\mathrm{Ni}$ atom layer as $0.5 \pm 0.1 \AA$. The above structures and geometries were consistently reproduced in later theoretical treatments of the $\mathrm{H} / \mathrm{Ni}(100)$ adsorption system [331, $332,340]$. 


\section{$\operatorname{Ni(111)}$}

Exposure of $\mathrm{Ni}(111)$ to molecular $\mathrm{H}_{2}$ at $120-140 \mathrm{~K}$ leads to coverages of $\sim 0.8 \mathrm{ML}$ (1 $\left.\mathrm{ML}=1.86 \times 10^{15} \mathrm{~cm}^{-2}\right)[278,341]$, after which an activation barrier appears to prevent further $\mathrm{H}$ uptake. The true saturation coverage, $\theta_{\text {sat }}=1.0 \mathrm{ML}$, is only attained after atomic $\mathrm{H}$ exposure [278], see below. The dissociated $\mathrm{H}$ atoms chemisorb in two binding states $\left(\beta_{1}, \beta_{2}\right)$ with respective TDS maxima at 355 and $383 \mathrm{~K}$, which are filled sequentially and display equal populations after saturation [323, 333, 341]. Both desorption signals show the typical peak shift to lower temperature with increasing initial coverage characteristic of the second-order kinetics of $\mathrm{H}_{2}$ recombination.

The absolute D coverage was evaluated [278] through ${ }^{4} \mathrm{He}$ elastic recoiling, for which the cross-section is known quantitatively [342]. Up to $250 \mathrm{~K}$ and in a coverage range of $\sim 0.3$ and $0.6 \mathrm{ML}$ a clear $(2 \times 2)$ LEED pattern with weak (1-2\% of the fundamental diffraction spot intensity) half order spots appears, which is best developed at a $\mathrm{H}$ coverage of $0.5 \mathrm{ML}$, where the $\beta_{2}$ TDS signal saturates [341]. This $\mathrm{Ni}(111)(2 \times 2)-2 \mathrm{H}$ superstructure is of the honeycomb type, where the large $(2 \times 2)$ surface unit cell contains 2 non-equivalent $\mathrm{H}$ atoms in an hcp and in an fcc threefoldcoordinated site (Fig. 20) [341]. This structure model was confirmed by Helium scattering [343] and later refined with improved techniques of dynamical LEED I(V) analysis [344], which demonstrated that this $\mathrm{H}$-induced superstructure involves also a substrate reconstruction (the $\mathrm{H}$-coordinated $\mathrm{Ni}$ atoms are pulled out of the surface by $0.04 \AA$ to slightly buckle the top Ni layer). This $(2 \times 2)-2 \mathrm{H}$ structure is in excellent agreement with DFT calculations [331], just as the absolute $\mathrm{H}$ atom positions determined by ion scattering. Transmission channeling (TC) [278] and low energy 
recoil scattering (LERS) were used to independently confirm the simultaneous occupation of fcc and hcp sites [345, 346]. The height of the $H$ atoms above the surface was determined to $1.1-1.5 \AA$ by LERS, in reasonable agreement with the results of LEED ((1.15 $\pm 0.1 \AA ̊)$ [341] and $(0.98 \pm 0.08 \AA$ ) [344]), and HAS $(1.18 \pm 0.05 \AA$ ) [343]. The $D$ adsorption height evaluation by TC tends to be slightly smaller $(0.8 \pm 0.10$ Å) [278], possibly because the TC intensity mostly reflects channeling in the bulk of the thin film single crystal and may be less sensitive to the surface buckling and slight outward relaxations in the $\mathrm{H}$-covered outermost Ni layer [347].
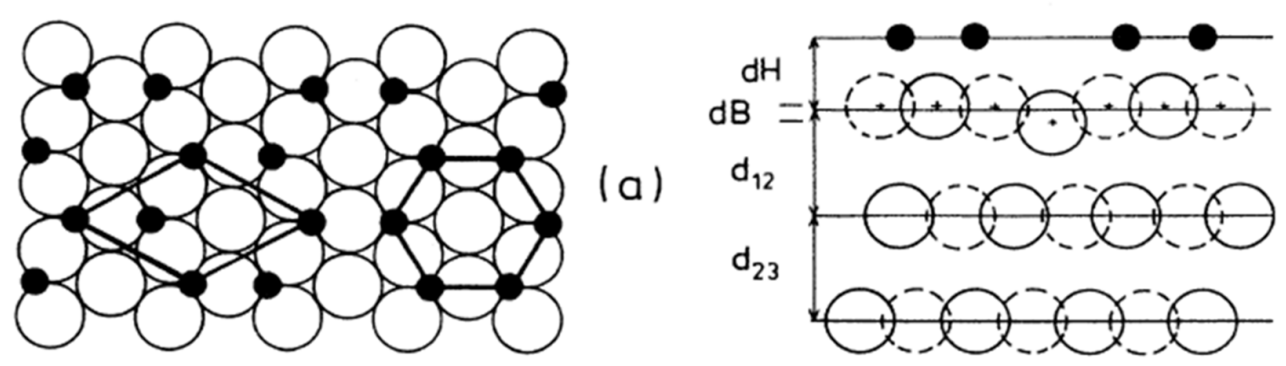

Fig. 20. Model for the $(2 \times 2)-2 \mathrm{H} / \mathrm{Ni}(111)$ honeycomb structure in the top (a) and side (b) views. Reproduced with permission from Ref. [344].

The hydrogen saturation coverage on the $\mathrm{Ni}(111)$ surface was quantified as 1.0 $\pm 0.1 \mathrm{ML}$, which was obtained by dosing atomic hydrogen (deuterium) at $\mathrm{T} \geq 150 \mathrm{~K}$ and resulted in the restoration of a (1×1) LEED pattern [278]. Atomic $\mathrm{H}$ exposure at surface temperatures below $150 \mathrm{~K}$ may cause hydrogen absorption, so that elevated exposures may produce a near-surface hydride phase $[48,348]$, in which $\mathrm{H}$ populates octahedral interstitial sites [278]. To avoid disturbances by absorbed D near the surface, Mortensen et al. prepared the D-saturated $(1 \times 1)$ surface phase by atomic $\mathrm{H}$ dosage at $190 \mathrm{~K}$, where subsurface $\mathrm{H}$ becomes unstable. Transmission channeling (TC) then revealed that D occupation of fcc sites dominates ( 90\%) on the D- 
saturated $(1 \times 1)$ surface [278]. Also a quite substantial lateral r.m.s. displacement $(0.25 \AA)$ of $D$ on the saturated $\mathrm{Ni}(111)$ surface was found [278], which was considered consistent with theoretical predictions that chemisorbed $\mathrm{H}$ may be delocalized along the surface [268, 349]. On the other hand, HREELS measurements could not conclusively confirm a quantum delocalization of the adsorbed $\mathrm{H}$ atom in the ground state wavefunction, although evidence for a delocalized nature of vibrationally excited states was found in the $\theta=0.5(2 \times 2)-2 \mathrm{H}$ phase [68]. In the high coverage $(1 \times 1)-1 \mathrm{H}$ phase at $\theta=1$, however, repulsive $\mathrm{H}-\mathrm{H}$ interactions again promote the localization of the mainly surface-parallel vibrational modes [350]. Quantum effects possibly play a role in the low-temperature $(<110 \mathrm{~K})$ surface diffusion behavior of $\mathrm{H}$ on $\mathrm{Ni}(111)$ [351].

The interaction of atomic hydrogen with Ni surfaces at temperatures below 140 $\mathrm{K}$ can lead to the population of subsurface sites, which is evidenced by a characteristic zero-order $(\alpha)$ TDS feature peaking at $180 \mathrm{~K}$ that may contain several $\mathrm{ML}$ of hydrogen after elevated dosages of atomic $\mathrm{H}[48,335,348]$. Notably, hydrogen in octahedral subsurface sites at $\mathrm{Ni}(111)$ is the only known case in which this species could be identified by means of HREELS [48, 352]. Much theoretical work has supported this assignment [331, 332, 340, 353-356] and confirmed that the firstlayer subsurface-absorbed $\mathrm{H}$ is metastable against desorption or returning to the energetically more favorable surface adsorption sites, thereby explaining the experimental observation that subsurface population only occurs below 150 K and not before all surface adsorption sites have been filled [335]. On $\mathrm{Ni}(100)$ the desorption of subsurface hydrogen involves recombination with surface-adsorbed $\mathrm{H}$ 
atoms [335] and for $\mathrm{H} / \mathrm{Ni}(111)$ the molecular desorption dynamics have been investigated with angle-resolved TDS [348] and quantum state resolving resonanceenhanced multiphoton ionization [357]. These studies revealed that desorbing subsurface hydrogen has a unique desorption temperature, translational energy, angular distribution, and vibrational frequency that differ distinctly from those of surface-adsorbed hydrogen. The results suggested that hydrogen does not directly recombine from subsurface sites but that diffusion on the surface is involved prior to recombination and molecular desorption.

Subsurface-absorbed hydrogen in $\mathrm{Ni}(111)$ exhibits a particular chemical reactivity with respect to hydrogen addition to simple co-adsorbed hydrocarbons, a reaction that notably would not occur with surface-adsorbed $\mathrm{H}$ atoms alone, and which is suspected to be of profound importance in the widespread application of nickel as a catalyst for hydrocarbon hydrogenation [48, 358-360]. The enhanced chemical reactivity of subsurface hydrogen has attracted considerable attention from theory [353-358, 361], yet the actual reaction pathways of subsurface hydrogen are still far from being fully understood. As of present, the behavior of subsurface $\mathrm{H}$ in $\mathrm{Ni}$ surfaces has not been addressed through NRA measurements, although such studies might add insight into the interesting problem of the subsurface hydrogen reactivity. The potential of ${ }^{15} \mathrm{~N}$ NRA to distinguish surface-adsorbed $\mathrm{H}$ species from subsurfaceabsorbed $\mathrm{H}$ at metal single crystal surfaces and nanoparticles is discussed in Section 3.2. 


\section{$\operatorname{Pt}(111)$}

As one of the most active materials for hydrogen dissociation platinum finds important industrial applications in heterogeneous catalysis of (de-)hydrogenation reactions, in electrochemistry, and in fuel cells. Hydrogen interactions with Pt surfaces have thus long been subject of intense research $[1,362,363]$, and even nowadays the complex $\mathrm{H}_{2}$ dissociation and surface diffusion dynamics continue to attract attention [69, 364, 365]. Several NRA and recoil scattering investigations unanimously agree on a $\mathrm{H}$ saturation coverage of $1.0 \mathrm{ML}\left(1 \mathrm{ML}=1.50 \times 10^{15} \mathrm{~cm}^{-2}\right)$ on $\operatorname{Pt}(111)[67,280]$. Nonetheless, the determination of the saturation coverage has turned out to be not as simple as one might expect. Although there is no activation barrier in the minimum reaction pathway for dissociative chemisorption of molecular hydrogen on $\mathrm{Pt}(111)$ [366-368], full saturation of the surface requires temperatures $<200 \mathrm{~K}[280,364]$ and rather large $\mathrm{H}_{2}$ exposures ( 1000 L) because the initial sticking coefficient does not exceed $s_{0}=0.1$ even on clean $\mathrm{Pt}(111)$ and further reduces substantially as the $H$ coverage increases $[364,367,369,370]$. The small sticking probability (especially on well-prepared surfaces with low defect densities [365] and its slight enhancement with increasing surface temperature [369], has been explained in terms of preferential $\mathrm{H}_{2}$ dissociation at step defects from a nonequilibrated mobile precursor [364, 365, 369]. This adsorption mechanism strongly dominates at low $\mathrm{H}$ coverage and small incident kinetic energies ( $\leq 60 \mathrm{meV}$ ) typical for conditions of $\mathrm{H}_{2}$ background gas exposure [365]. A second defect-related chemisorption channel operative at higher $\mathrm{H}$ coverages and at larger defect densities involves direct $\mathrm{H}_{2}$ dissociation at step sites with second-order Langmuir kinetics and 
an initial probability of $24 \%$ of the step density [364]. Molecular beam studies revealed that the dissociation probability at regular $\mathrm{Pt}(111)$ terrace sites increases strongly with the surface-normal kinetic energy component (50 meV $<\mathrm{E}_{\mathrm{T}}<550 \mathrm{meV}$ ) of the incident $\mathrm{H}_{2}$ molecules [367].

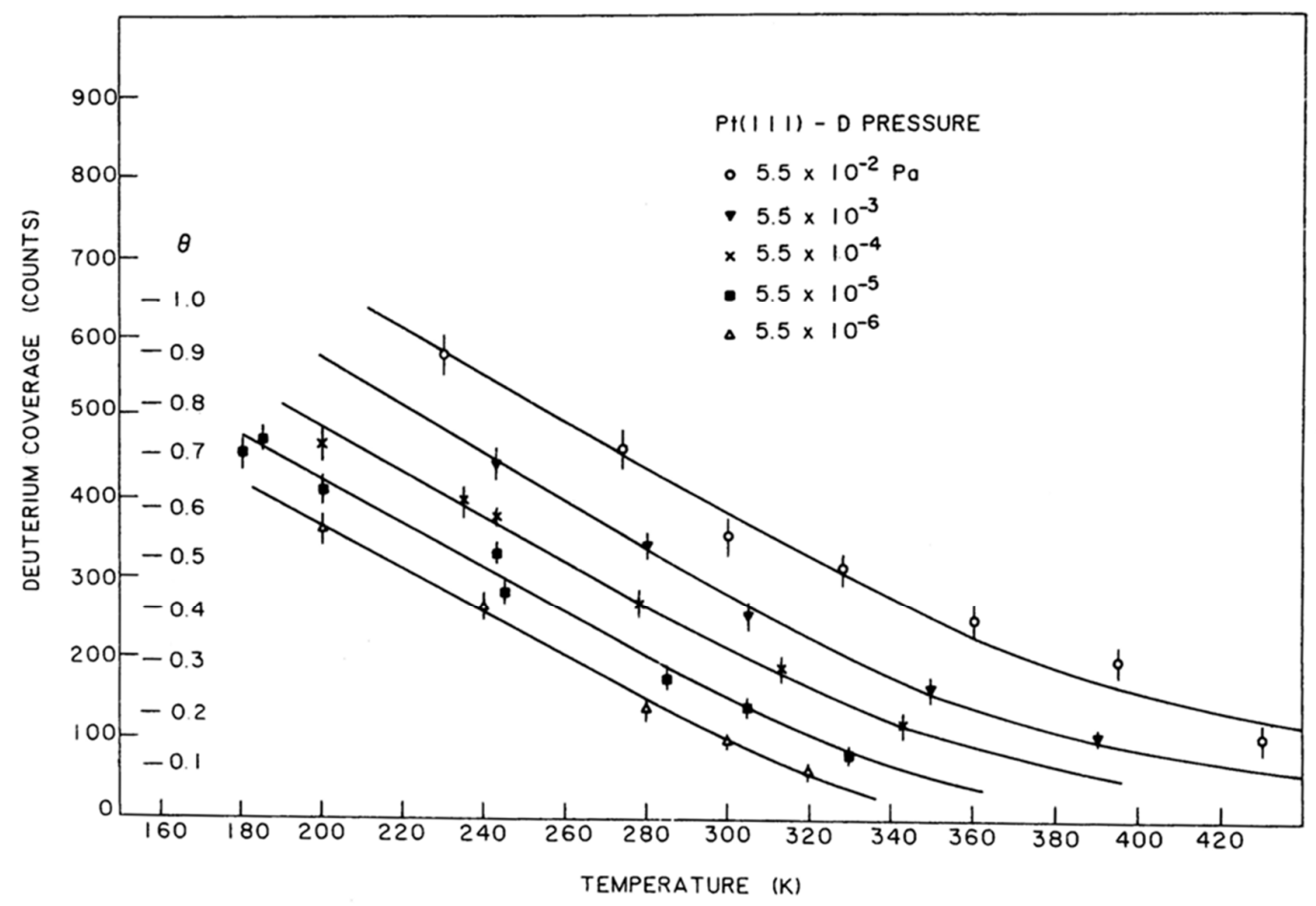

Fig. 21. Isobars for deuterium adsorbed on Pt(111) determined with $D\left({ }^{3} \mathrm{He}, \mathrm{p}\right)^{4} \mathrm{He} N R A \cdot \theta_{H}=$ 1.0 ML by definition at $1.5 \times 10^{15} \mathrm{D}$ atoms $\mathrm{cm}^{-2}$. Reproduced from Ref. [371] with permission from Elsevier.

The first absolute coverage measurement on $\mathrm{Pt}(111)$ with $\mathrm{D}\left({ }^{3} \mathrm{He}, \mathrm{p}\right)^{4} \mathrm{He}$ NRA was reported as early as 1980 by Davies and Norton, who found a coverage of $(1.10 \pm 0.05) \times 10^{15} \mathrm{D}$ atoms $\mathrm{cm}^{-2}(0.7 \mathrm{ML})$ after dosing $600 \mathrm{~L} \mathrm{D}_{2}\left(1.3 \times 10^{-4} \mathrm{~Pa}\right)$ at $185 \mathrm{~K}$ [123]. This exposure is insufficient to D-saturate the surface. The same group later performed a set of quantitative NRA equilibrium D coverage measurements on $\mathrm{Pt}(111)$ in the temperature and pressure ranges of $180 \leq \mathrm{T} \leq 440 \mathrm{~K}$ and $5 \times 10^{-6} \mathrm{~Pa} \leq \mathrm{P}$ 
$\leq 5 \times 10^{-2} \mathrm{~Pa} \mathrm{D}_{2}$ (Fig. 21) [371]. Converted into isosteres (families of $\ln (\mathrm{P})$ vs. $1 / T$ curves for $\theta=$ const., Fig. 22), these data allowed deriving the coverage dependence of the isosteric heat of adsorption ( $E_{a d s}$ ) for $D / P t(111)$. For $\theta \leq 0.3, E_{a d s}=67 \pm 7 \mathrm{~kJ} / \mathrm{mol}$ (Fig. 23). At coverages above $0.3 \mathrm{ML} E_{\mathrm{ads}}$ reduces to $34 \pm 3 \mathrm{~kJ} / \mathrm{mol}$ at $\theta=0.7$, presumably due to repulsive nearest neighbor interactions in the adsorbed $D$ layer ( $6-8 \mathrm{~kJ} / \mathrm{mol})$. The largest $D$ coverage observed in this study was $0.9 \mathrm{ML}\left(1.35 \times 10^{15} \mathrm{~cm}^{-2}\right)$ at $230 \mathrm{~K}$ under $5.5 \times 10^{-2} \mathrm{~Pa} \mathrm{D}_{2}$.

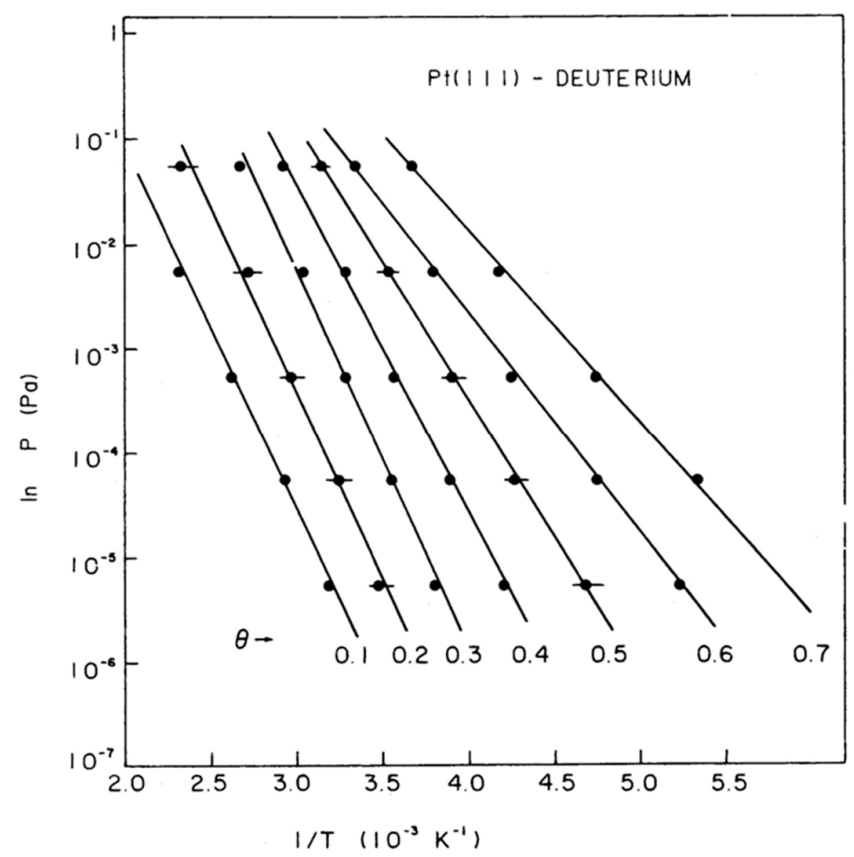

Fig. 22. Isosteres for deuterium adsorbed on Pt(111). Reproduced from Ref. [371] with permission from Elsevier.

The above described direct and non-invasive observation of the hydrogen adsorption/desorption equilibrium with NRA [371] further helped resolving an apparent discrepancy between $E_{\text {ads }}$ evaluations from TDS and helium atom scattering (77-85 kJ/mol) [372] and a considerably lower figure $(44 \mathrm{~kJ} / \mathrm{mol})$ deduced from the P$\mathrm{T}$ relationship at constant $\mathrm{H}$-induced work function changes [363]. The latter 
estimation had led to a speculation that particularly 'soft vibrational modes' might be involved in the $\mathrm{H}_{2}$ desorption process from $\mathrm{Pt}(111)$, since for $E_{\text {ads }}=44 \mathrm{~kJ} / \mathrm{mol}$ the pre-exponential factor $v$ in the second order rate equation for $\mathrm{H}_{2}$ recombination $\left[R_{\text {des }}\right.$ $=v \cdot\left(\theta_{\mathrm{H}} \cdot N_{\mathrm{S}}\right)^{2} \cdot \exp \left(-E_{\text {des }} / k_{\mathrm{B}} T\right) ; E_{\text {des }}=E_{\text {ads }}$ for non-activated adsorption] would have to be unusually small [373]. Norton's NRA-derived value for $E_{\text {ads }}(67 \pm 7 \mathrm{~kJ} / \mathrm{mol})$ [371], however, reconciled this problem by showing that $v$ values in the 'normal range' for second order desorption $\left(10^{-5}\right.$ to $10^{-2} \mathrm{~cm}^{2}$ atom $\left.{ }^{-1} \mathrm{~s}^{-1}\right)$ [374] were in fact well compatible with the available He scattering data [375].

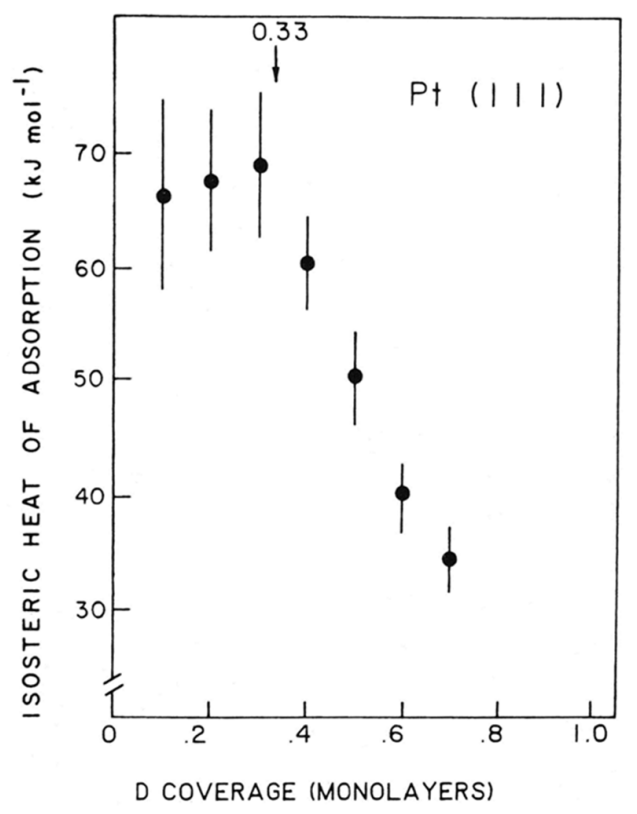

Fig. 23. Isosteric heat of adsorption of deuterium on Pt(111). Reproduced from Ref. [371] with permission from Elsevier.

The quantitative $\mathrm{D}$ coverage measurements on $\operatorname{Pt}(111)[280,371]$ further revealed independently that the $\mathrm{H}$ coverage reduces to $\sim 0.05 \mathrm{ML}$ when a $\mathrm{H}$-covered $\mathrm{Pt}$ (111) surface prepared by low temperature $\mathrm{H}_{2}$ admission is warmed to $270-300 \mathrm{~K}$ in UHV. This consistent observation invalidated an earlier interpretation of the 
disappearance of a $\mathrm{H}$-induced feature in angle-resolved valence band photoemission spectra around $270 \mathrm{~K}$ as the irreversible transition of surface-adsorbed hydrogen atoms into subsurface sites [376]. The latter conclusion was encouraged by the observation that at $270 \mathrm{~K}$ the work function remained strongly reduced relative to clean Pt(111), which seemingly suggested that $\mathrm{H}$ was still present on or very close below the surface. Mortensen et al., however, combined the NRA D coverage measurement with transmission channeling at thin Pt(111) single crystal films, which definitely ruled out D occupation of octahedral interstitial sites below the $\mathrm{Pt}(111)$ surface for the questionable temperature regime (110-300 K) [280]. In addition their channeling data specified the D adsorption position on $\mathrm{Pt}(111)$ as the fcc three-fold hollow site at a height of $0.58 \AA$ above the surface and thereby clearly ruled out hcp site occupation that also had been given consideration at the time. This result agreed with LAPW calculations that predicted a $0.2 \mathrm{eV}$ higher stability of fcc occupation over hcp [377]. Low energy recoil scattering experiments at $\mathrm{H} / \mathrm{Pt}(111)$ later confirmed the fcc adsorption site and the small initial $\mathrm{H}_{2}$ sticking coefficient $\left(\mathrm{s}_{0}=0.1\right)$ [370]. The $\mathrm{H}$ height above the surface (i.e., the center of mass position of the first layer Pt atoms) was reported as $1.2 \AA$.

The microscopic diffusion dynamics of $\mathrm{H}$ on $\mathrm{Pt}(111)$, possible quantum effects, and the vibrational properties of adsorbed $\mathrm{H}$ atoms have been receiving attention until very recently. $\mathrm{H}$ atoms on $\mathrm{Pt}(111)$ are highly mobile and the low coverage activation barrier for surface diffusion is exceptionally small for $\mathrm{H}$ on a transition metal [68]. To investigate the adsorption potential, Fukutani et al. measured the Doppler-width of the surface resonance excitation curve of $\mathrm{H}$ atoms in the saturated 
chemisorption layer on $\mathrm{Pt}(111)$ with ${ }^{1} \mathrm{H}\left({ }^{15} \mathrm{~N}, \alpha \gamma\right){ }^{12} \mathrm{C}$ NRA in surface normal and $45^{\circ}$ inclined ${ }^{15} \mathrm{~N}$ incidence geometries (Fig. 24) and extracted the zero-point energies for the $H$ vibrational motions perpendicular and parallel to the surface [67]. In comparison with HREELS [378], RAIRS [379], and computational studies [377], the zero-point energy for the $\mathrm{H}$ vibration perpendicular to the surface $\left(E_{z}=80.8 \pm 3.9\right.$ meV) was found to match well with approximately $1 / 2$ of the excitation energy of the surface stretching vibration (153 meV), supporting a harmonic oscillator model for this vibrational mode.

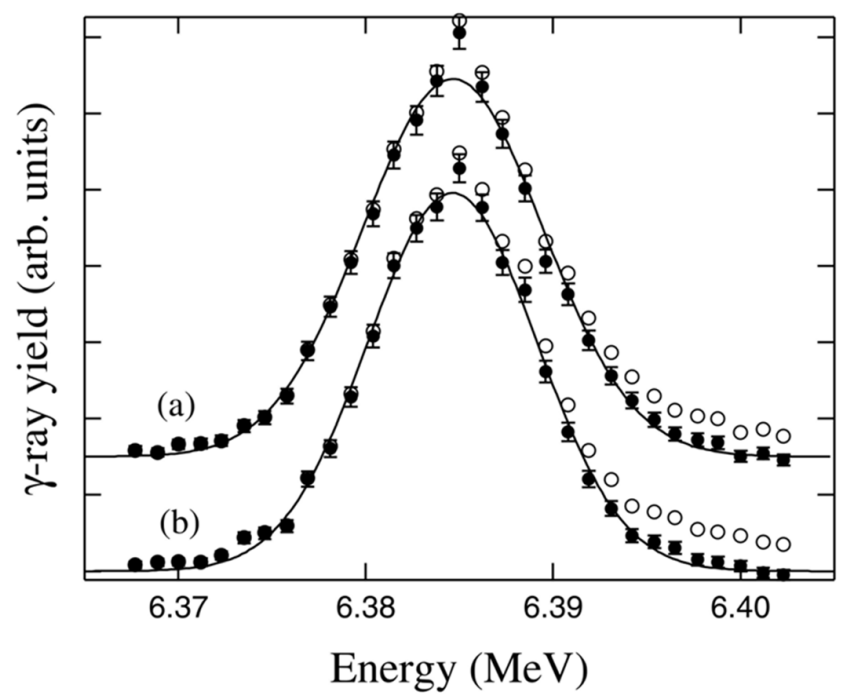

Fig. 24. Resonance excitation curves of the ${ }^{1} \mathrm{H}\left({ }^{15} \mathrm{~N}, \alpha \gamma\right){ }^{12} \mathrm{C}$ nuclear reaction for $\mathrm{Pt}(111)-\mathrm{H}$ recorded at ${ }^{15} \mathrm{~N}$ incidence angles of (a) $0^{\circ}$ and (b) $45^{\circ}$ with the surface normal. Open and filled circles, respectively, denote data before and after subtraction of subsurface- $\mathrm{H}$ induced background in form of a sigmoidal function. The solid curves are fits of the backgroundcorrected data to Gaussian forms. Reproduced with permission from Ref. [67].

On the other hand, the zero-point energy for the vibration parallel to the surface $\left(E_{||}=62.1 \pm 6.0 \mathrm{meV}\right)$ was found to be of similar magnitude as the full excitation energy of the vibration mode (68 meV) assigned to $H$ bending [378] and as the activation energy for $\mathrm{H}$ surface diffusion (68.5 $\pm 5 \mathrm{meV}$ ) [380]. The conclusion was 
that the potential for the largely surface-parallel bending vibration is apparently strongly anharmonic [67]; the first excited vibrational state already being close to the transition state for surface diffusion. The notion that the unusually large zero-point energy of the lateral $\mathrm{H}$ vibration relates to a strongly anharmonic potential found support by HREELS data and DFT calculations, which demonstrated that in the high coverage regime of Fukutani's NRA measurement $(\theta>0.75)$ a third vibrational excitation loss exists at $112 \mathrm{meV}$, in which the atomic $\mathrm{H}$ motions perpendicular and parallel to the surface are strongly coupled [381]. Roman et al. found significant amplitudes of the $\mathrm{H}$ wavefunction at neighboring hcp sites, suggesting a partially delocalized character of the first vibrational excited state [382]. Favored by the light $\mathrm{H}$ atom mass, quantum-mechanical tunneling was shown to dominate the surface diffusion of $\mathrm{H}$ on $\mathrm{Pt}(111)$ up to $140 \mathrm{~K}$ and to contribute largely even at surface temperatures as high as $250 \mathrm{~K}$ [69].

A final noteworthy result of the highly depth-resolving $\mathrm{H}$ detection with ${ }^{1} \mathrm{H}\left({ }^{15} \mathrm{~N}, \alpha \gamma\right){ }^{12} \mathrm{C}$ NRA in Ref. [67] is the observation of a small amount of $\mathrm{H}(0.29$ at. \%) distributed in a shallow region $(0 \sim 5 \mathrm{~nm})$ below the $\mathrm{Pt}(111)$ surface, which has been saturated with $1 \mathrm{ML} \mathrm{H}$ under $1 \times 10^{-4} \mathrm{~Pa} \mathrm{H}_{2}$ at $150-200 \mathrm{~K}$. This hydrogen gives rise to a faint but significant high energy tailing in the NRA surface resonance profiles of $\mathrm{H} / \mathrm{Pt}(111)$ (open symbols in Fig. 24), suggesting that this absorbed $\mathrm{H}$ is not confined only to first layer subsurface sites (cf. Section 3.2.1). Prior to the zero-point vibration analysis, the absorbed $\mathrm{H}$ signal component was modeled by a sigmoid function and subtracted from the raw $\gamma$-yield curves in order to recover the purely Gaussianshaped signal of surface-adsorbed H (cf. Fig. 4, Section 2.1.1). In light of the large 
endothermic $\mathrm{H}$ solution enthalpy in $\mathrm{Pt}\left(\Delta H_{\mathrm{s}}=+0.48 \mathrm{eV} / \mathrm{H}\right)$, such $\mathrm{H}$ absorption into the subsurface region of $\mathrm{Pt}(111)$ is remarkable since the equilibrium $\mathrm{H}$ concentration in bulk Pt would be immeasurably small under the conditions of the above experiment. Although the binding mechanism of the $\mathrm{H}$ atoms in the extended Pt subsurface region is unknown (it may, e.g., involve $\mathrm{H}$ interactions with vacancies, segregated impurities, or defects), at least the stability of $\mathrm{H}$ occupying first-layer subsurface sites beneath a $\mathrm{H}$-saturated $\mathrm{Pt}(111)$ surface exposed to $\mathrm{H}_{2}$ gas has recently been described theoretically [383], and the subsurface $\mathrm{H}$ concentration expected for the conditions of Fukutani's experiment [67] agrees quite favorably with the NRA observation. Legare [383] speculates that the presence of such subsurface hydrogen may have implications for certain Pt-catalyzed (de-)/hydrogenation reactions as well as for electrochemistry.

\section{$\mathrm{Cu}(111), \mathrm{Cu}(100), \mathrm{Cu}(110)$}

Copper surfaces have been studied as prime examples of noble metal surfaces for which the dissociative $\mathrm{H}_{2}$ adsorption is activated due to the lack of d-band holes near the Fermi energy [384]. With monoenergetic $\mathrm{H}_{2}$ beams Anger et al. [385] measured the translational energy $\left(E_{T}\right)$ dependence of the $\mathrm{H}_{2}$ sticking coefficient, clearly revealing the sizeable activation barrier for $\mathrm{H}_{2}$ dissociation on the (111), (100), and (110) surfaces of $\mathrm{Cu}$ (Fig. 25). To circumvent this dissociation barrier most adsorption studies on Cu surfaces have been conducted with atomic $\mathrm{H}$. This practice introduced considerable ambiguity into the determination of the hydrogen saturation coverage at $\mathrm{Cu}(111)$, as in addition to filling surface chemisorption sites, the atomic $\mathrm{H}$ exposure may cause population of subsurface sites as well as 
abstraction of already surface-adsorbed hydrogen atoms (cf. Section 3.1.1) [297, 335, $348,386]$. Possibly due to different methods of atomic $\mathrm{H}$ generation, the relative efficiencies of these three processes have likely been different in various published experiments, which might explain the substantial variation of the $H$ 'saturation' coverage values reported for $\mathrm{Cu}(111)$ (Table 4).

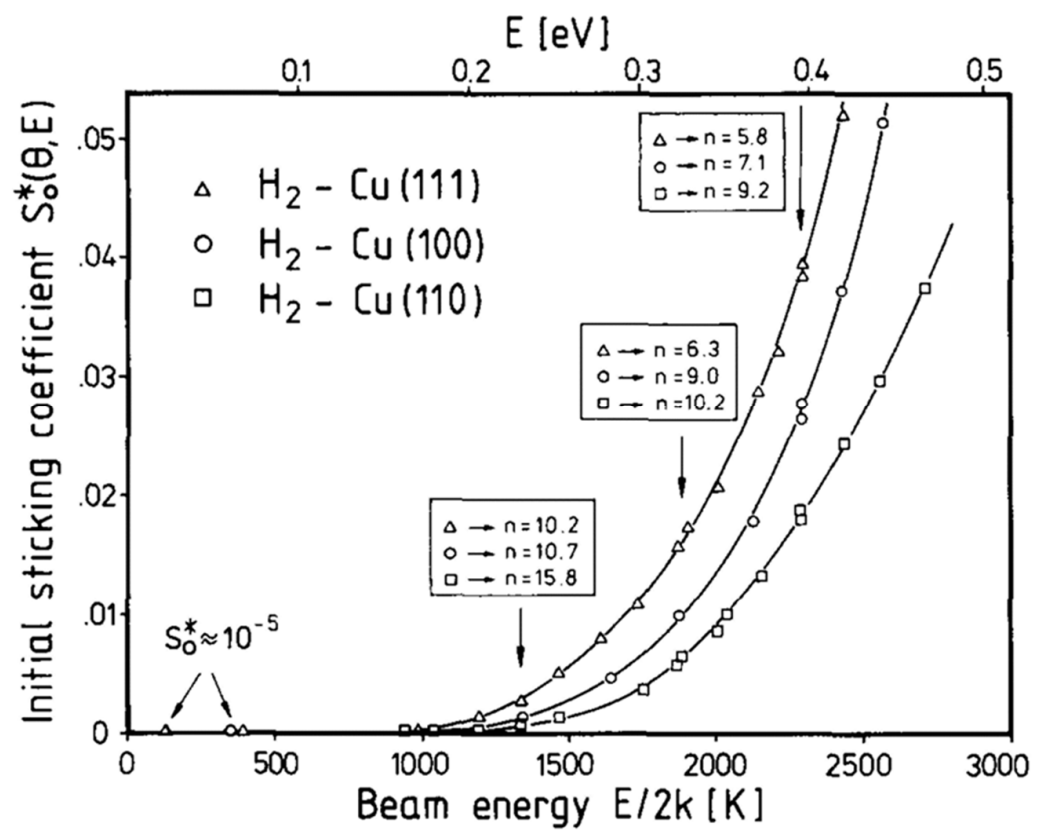

Fig. 25. Initial sticking coefficient $S_{0}(E, \theta)$ of monoenergetic $\mathrm{H}_{2}$ beams on $\mathrm{Cu}(111), \mathrm{Cu}(100)$ and $\mathrm{Cu}(110)$ surfaces as function of beam energy $(E)$ at a surface temperature of $190 \mathrm{~K}$. The values $n$ denote the empirical exponents characteristic of the incidence angular $(\theta)$ variation of the sticking coefficient: $S_{0}(E, q) / S_{0}\left(E, 0^{\circ}\right)=\cos ^{n} \theta$. Reproduced from Ref. [385] with permission from Elsevier.

\section{$\operatorname{Cu}(111)$}

Several studies assessed the $\mathrm{H}$ saturation coverage on $\mathrm{Cu}(111)$ with a variety of methods for atomic $\mathrm{H}$ generation (Table 4). Anger et al. [385] observed saturation of the $\mathrm{H}$ coverage at $0.5 \mathrm{ML}$ on all three lowest-index ((111), (110), and (100)) Cu surfaces with calibrated TDS [293] after dosing $\mathrm{H}_{2}$ with a molecular beam of $E_{T}=0.4$ 
eV translational energy. The obtained $\mathrm{H}_{2}$ desorption spectra from $\mathrm{Cu}(111)$ have symmetric shapes with maxima in the $300-360 \mathrm{~K}$ range and show low temperature shifts for increasing initial coverages typical for the second-order kinetics of $\mathrm{H}_{2}$ recombination.

\begin{tabular}{clcc}
\hline$\theta_{\text {sat }}[\mathrm{ML}]$ & Exposure condition & $\begin{array}{c}\text { TDS } \\
\text { peaks }\end{array}$ & Ref. \\
\hline 0.5 & $\mathrm{H}_{2}$ molecular beam $\left(E_{\mathrm{T}}=0.4 \mathrm{eV}\right), T_{\mathrm{s}}=190 \mathrm{~K}$ & $\beta_{2}$ & {$[385]$} \\
$0.67 \pm 0.03$ & $\mathrm{D}_{\mathrm{at}}$, open W-filament, $T_{\mathrm{s}}=100 \mathrm{~K}$ & $\beta_{2}, \beta_{1}$ & {$[271]$} \\
$0.34\left(\beta_{2}\right)$ & $\mathrm{H}_{\mathrm{at}}, \mathrm{W}$-tube $(2000 \mathrm{~K}), T_{\mathrm{s}}=79 \mathrm{~K}$ & $\beta_{2}, \beta_{1}, \alpha$ & {$[297]$} \\
$0.5^{*}$ & $\mathrm{H}_{\mathrm{at}}$, effusive W source, $2300 \mathrm{~K}$ & - & {$[387]$} \\
- & $\mathrm{H}_{\mathrm{at}}$, microwave source, $T_{\mathrm{s}}=100 \mathrm{~K}$ & $\alpha$ & {$[387]$} \\
& $\left(80 \%\right.$ dissociation, $\left.E_{\mathrm{T}}=70 \mathrm{meV}, 1 \mathrm{ML} / \mathrm{s}\right)$ & & \\
$0.5^{*}\left(\beta_{2}\right)$ & $\mathrm{H}_{\mathrm{at}}$, ion gun, 18 eV sample bias, $T_{\mathrm{s}}=98 \mathrm{~K}$ & $\beta_{2}, \beta_{1}, \alpha$ & {$[388]$} \\
\hline
\end{tabular}

Table 4. Hydrogen saturation coverages $\left(\theta_{\text {sat }}\right)$ on $\mathrm{Cu}(111)$ obtained with different methods of atomic $\mathrm{H}\left(\mathrm{H}_{\mathrm{at}}\right)$ or $\mathrm{D}\left(\mathrm{D}_{\mathrm{at}}\right)$ generation. $\mathrm{H}$ detection with calibrated TDS (only Ref. [271] used $D\left({ }^{3} \mathrm{He}, \mathrm{p}\right)^{4} \mathrm{He}$ NRA for calibration; the value of Ref. [387] $\left({ }^{*}\right)$ is only assumed). $1 \mathrm{ML}=$ $1.77 \times 10^{15} \mathrm{~cm}^{-2}$ for $\mathrm{Cu}(111)$. The TDS feature at $\sim 200 \mathrm{~K}(\alpha)$ is considered to be associated with desorption of bulk-absorbed $H . E_{T}$ : translational energy. $T_{s}$ : Surface temperature during hydrogen exposure.

Additional deuterium uptake to a saturation coverage of $0.67 \pm 0.03 \mathrm{ML}$ was found on atomic $\mathrm{D}$ exposed $\mathrm{Cu}(111)\left(1 \mathrm{ML}=1.77 \times 10^{15} \mathrm{~cm}^{-2}\right)$ by Lee et al. [271], who calibrated their TDS coverage-exposure relation by quantitative $D\left({ }^{3} \mathrm{He}, \mathrm{p}\right)^{4} \mathrm{He}$ NRA and could thereby assign the sequence of hydrogen-induced superstructure phases to absolute D coverage regimes (Fig. 26). A series of $(2 \times 2)$ and mixed $(2 \times 2)+(3 \times 3)$ LEED patterns appear with increasing $D$ coverage, finally converting into a $(3 \times 3)$ phase as the 
coverage saturates at $2 / 3 \mathrm{ML}$. The $\mathrm{D}$ coverage in the $(2 \times 2)$ phase on $\mathrm{Cu}(111)$ is 0.5 ML. In addition to the $2^{\text {nd }}$ order desorption peak at $300-340 \mathrm{~K}\left({ }^{\prime} \beta_{2}^{\prime}\right)$ saturating at 0.5 $\mathrm{ML}$, a low temperature peak at $\sim 230 \mathrm{~K}$ (denoted as ' $\beta_{1}$ ') develops in the TDS spectrum when the hydrogen coverage is increased from $0.5 \mathrm{ML}$ to $0.67 \mathrm{ML}$ at exposure temperatures below $200 \mathrm{~K}[271,297,389]$.

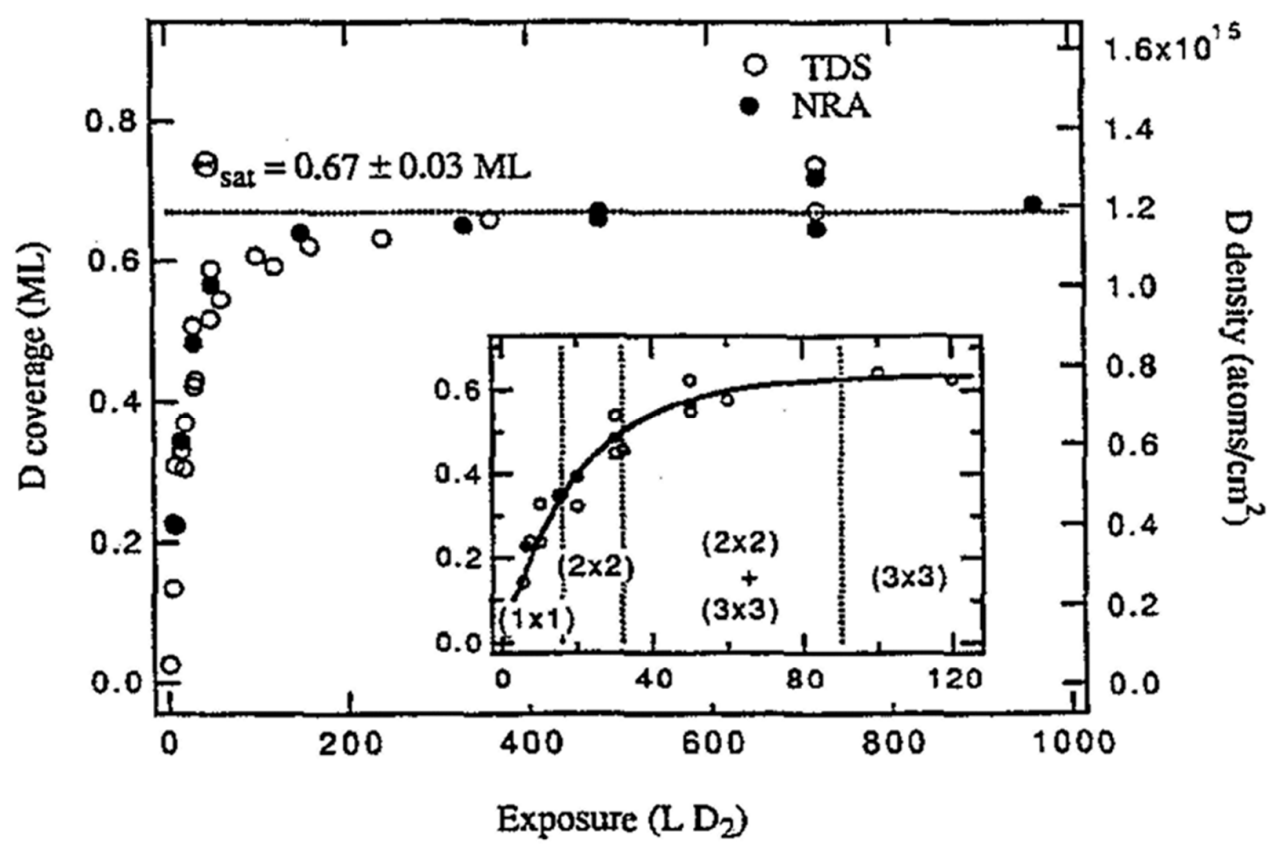

Fig. 26. Atomic deuterium coverage on $\mathrm{Cu}(111)$ held at $100 \mathrm{~K}$ and exposed to atomic $\mathrm{D}$ as a function of $D_{2}$ in Langmuir. Full and open circles correspond to NRA and TDS data, respectively. The inset is an expanded view of the low exposure range with the observed LEED patterns indicated (the line through the data is a guide for the eye). Reproduced from Ref. [271] with permission from Elsevier.

The hydrogen saturation coverage obtained by Lee et al. (0.67 ML) [271] appears to be most generally accepted, possibly because a $(3 \times 3)-2 \mathrm{H}$ superstructure is well compatible with the $(3 \times 3)$ LEED pattern observed at saturation, although here the fractional superstructure spots are quite strong, suggesting that the substrate reconstructs, i.e., that this periodicity may not necessarily reflect the arrangement of 
the $\mathrm{H}$ atoms [271]. Lee at al. furthermore has been the only group who observed a true saturation of the $\mathrm{H}$ uptake and simultaneously obtained the highest coverage under their particular atomic $\mathrm{H}$ exposure conditions (which, unfortunately, were not quantified), whereas several similar studies reported continued $\mathrm{H}$-uptake under atomic $\mathrm{H}$ exposure, presumably due to $\mathrm{H}$ absorption $[297,387,388,390]$. On the contrary, Kammler and Küppers [297], observed saturation of the $\beta_{2}$ TDS peak assigned to surface-adsorbed $\mathrm{H}$ at a coverage of only $0.34 \mathrm{ML}$ (Fig. 27) on atomic $\mathrm{H}$ exposed $\mathrm{Cu}(111)$, which is not only significantly smaller than Lee's value of $0.67 \mathrm{ML}$ [271] but even less than the $0.5 \mathrm{ML}$ found by Anger et al. after molecular $\mathrm{H}_{2}$ beam dosage [385].

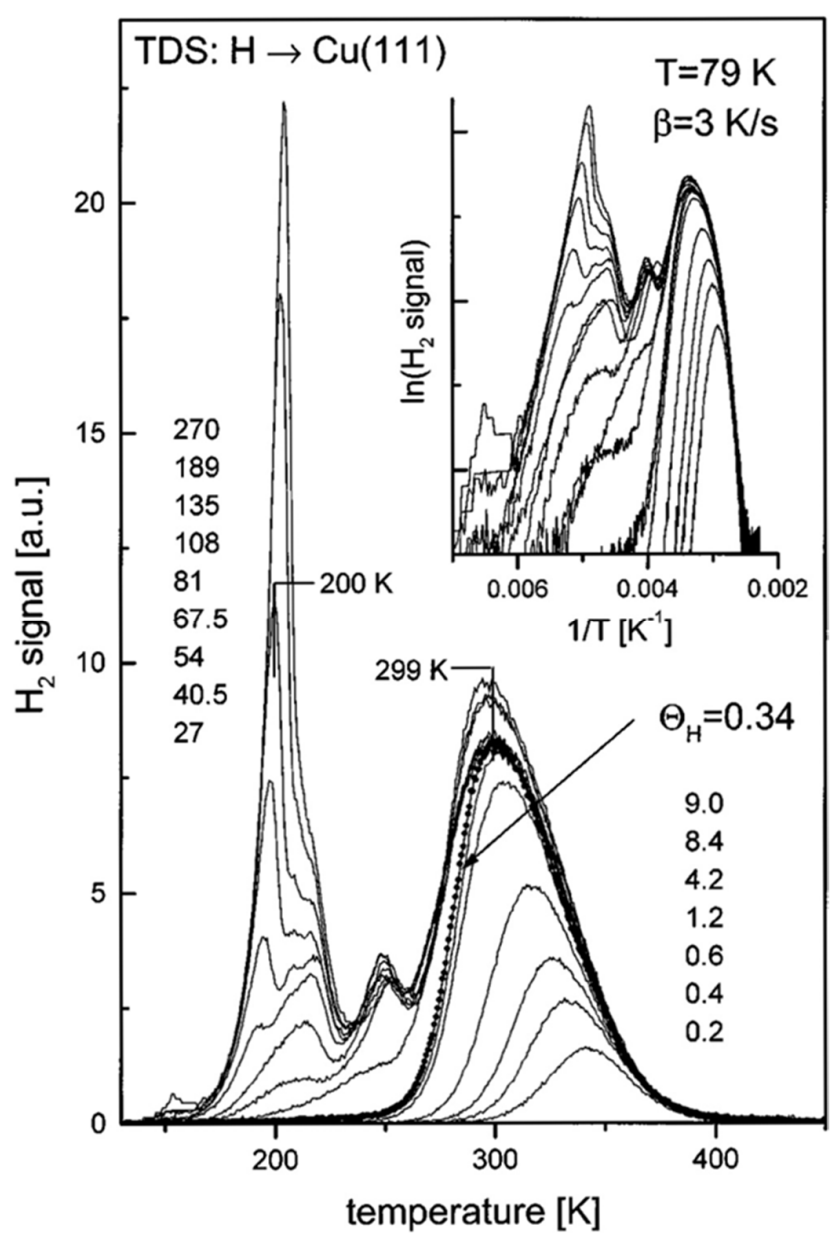

Fig. 27. Thermal desorption spectra of hydrogen measured after exposing clean $\mathrm{Cu}(111)$ surfaces to increasing exposures to $\mathrm{H}$ atoms at $79 \mathrm{~K}$. The spectra are stacked according to the applied $\mathrm{H}$ fluence (indicated by numbers). $1 \mathrm{ML}=1.77 \times 10^{15} \mathrm{~cm}^{-2}$. The desorption trace emphasized by small filled circles $\left(\theta_{\mathrm{H}}=0.34\right)$ illustrates the saturated adsorbed layer spectrum. The inset provides a logarithmic representation of the desorption rates. Reproduced with permission from Ref. [297]. 
A likely origin of this reduced saturation coverage under admission of atomic $\mathrm{H}$ is the abstraction of surface-adsorbed hydrogen by incoming $\mathrm{H}$ atoms in an EleyRideal-type mechanism [297, 386, 390]. For the particular exposure conditions [391] of Ref. [297], the probability to abstract surface-adsorbed hydrogen with gas phase atomic $H$ was found to be quite considerable (0.36), i.e., even larger than the initial sticking coefficient of atomic $\mathrm{H}$ on the clean $\mathrm{Cu}(111)$ surface $\left(\mathrm{s}_{0}=0.22\right)$.

Comparing the experimental conditions of the above studies suggests that hydrogen absorption can be enforced at Cu surfaces below $200 \mathrm{~K}$ through large atomic $H$ doses from high-flux sources [297, 387, 390] or mildly accelerated hydrogen ions (18 eV) [388]. Presumably this generates (near-surface) absorbed hydrogen, which desorbs in a characteristic zero-order, non-saturating (so-called ' $\alpha$ ') TDS peak at $200 \mathrm{~K}$ (Fig. 27). After elevated exposures the $\mathrm{H}$ quantity in this $\alpha$-peak may exceed the second-order desorption signal of surface-adsorbed $H\left(\right.$ ' $\beta_{2}{ }^{\prime}$ ) [297, $388,390]$, which saturates first after moderate atomic $H$ or $D$ exposures $[271,297$, 388]. From the population rate of the TDS $\alpha$ peak an absorption probability of $\leq 5 \%$ was deduced for atomic $\mathrm{H}$ generated with a microwave source [387]. The above interpretations (made in analogy to $\mathrm{Ni}$ and $\mathrm{Pd}$ surfaces) appear reasonable, although conclusive proof for the $\mathrm{H}$ locations assigned to the respective TDS features or for an extended depth range of the $\mathrm{H}$ state corresponding to the TDS $\alpha$ feature such as, e.g., for $\mathrm{H} / \mathrm{Pd}(100)$ and $\mathrm{H} / \mathrm{Pd}(110)$ with ${ }^{1} \mathrm{H}\left({ }^{15} \mathrm{~N}, \alpha \gamma\right){ }^{12} \mathrm{C}$ NRA $[61,279]$, has never been provided.

A somewhat surprising feature of the $\mathrm{H} / \mathrm{Cu}(111)$ system one would possibly not expect from this seemingly innocuous s-metal that does not absorb hydrogen in the 
bulk, is an open dispute concerning the preferred location of $\mathrm{H}$ atoms near the surface. Despite much theoretical and experimental effort, the relative stabilities of $\mathrm{H}$ in chemisorption sites on and in in first subsurface layer sites below $\mathrm{Cu}(111)$ remain a subject of debate, especially at low coverages [271, 302, 303, 389, 392-394]. All theoretical treatments of this problem find consistently that for $\mathrm{H}$ coverages $<1$ ML the subsurface-absorbed $H$ state is considerably less stable than surfacechemisorbed $\mathrm{H}$ [295, 300-303]. Hence, $\mathrm{H}$ trapping in $\mathrm{Cu}(111)$ subsurface sites should be possible only if the surface $\mathrm{H}$ layer has a coverage of $1 \mathrm{ML}$ with all energetically preferred fcc sites filled, whereas at coverages below $1 \mathrm{ML}$ the subsurface $\mathrm{H}$ would be unstable against returning to the surface [302]. A sizeable energy barrier ( $1 \mathrm{eV})$ furthermore prevents the penetration of chemisorbed $\mathrm{H}$ atoms into the subsurface of $\mathrm{Cu}(111)$ at all coverages up to the observed saturation at $0.67 \mathrm{ML}[295,300,303]$. Due to the difficulty to discriminate these two $\mathrm{H}$ states in closest vicinity of the surface, however, the situation has been discussed controversially from the experimental viewpoint.

An HREELS investigation of $\mathrm{H} / \mathrm{Cu}(111)$ by Lee and Plummer tentatively assigned an energy loss at $115 \mathrm{meV}$ appearing at coverages between 0.5 and $0.67 \mathrm{ML}$ to $\mathrm{H}$ in subsurface sites [392]. Due to the strong energetic preference $(0.5 \sim 0.68 \mathrm{eV})$ of $\mathrm{H}$ chemisorption sites at $\theta<1 \mathrm{ML}$, Nie et al. rejected this interpretation and proposed alternatively that the 115 meV HREELS feature reflects an asymmetric vibration mode of surface $\mathrm{H}$, which forms by degeneracy lifting of the parallel vibration mode due to $\mathrm{H}-\mathrm{H}$ interactions at coverages above $0.5 \mathrm{ML}$ [302]. 
The preferred stability of $\mathrm{H}$ atoms in chemisorption versus subsurface sites was challenged again by Luo et al., who monitored small $\mathrm{H}$ coverages $(\theta \sim 0.1 \mathrm{ML})$ on atomic $\mathrm{H}$-exposed $\mathrm{Cu}(111)$ at $210-245 \mathrm{~K}$ with in-situ HAS and observed that the $\mathrm{H}$ atoms seemed to disappear from the surface on a timescale of several 100 seconds as soon as the supply of atomic $H$ was terminated (dotted line in Fig. 28) [393, 394].

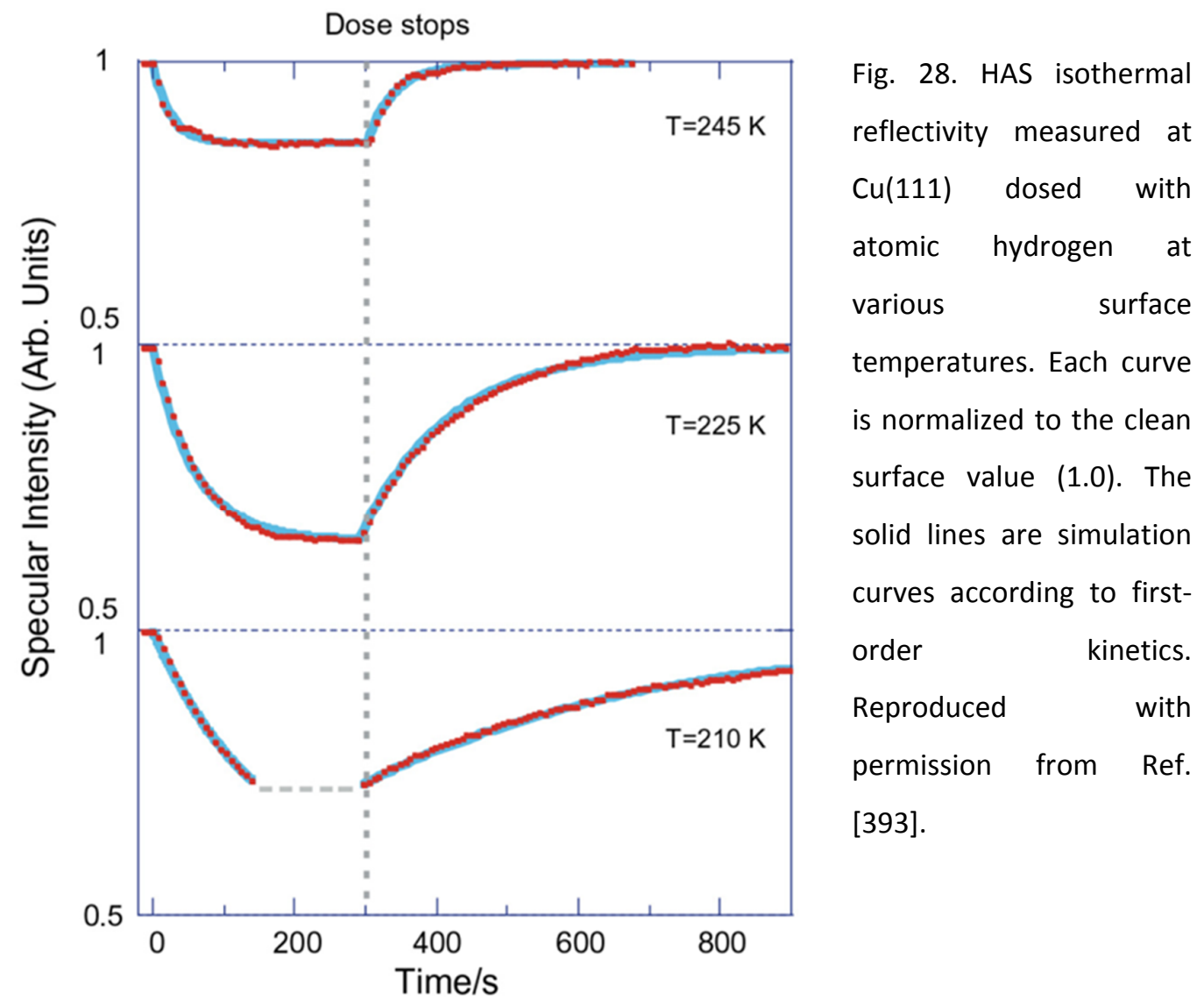

Eventually, the specular He reflection intensity of the clean $\mathrm{Cu}(111)$ surface recovered completely in a process of first-order kinetics, ruling out recombinative $\mathrm{H}_{2}$ desorption as the origin of the $\mathrm{H}$ disappearance [393]. TDS taken after complete restoration of the initial $\mathrm{He}$ reflectivity indicated that $\mathrm{H}$ was still present on or in the sample and that it desorbed above $250 \mathrm{~K}$ as the low coverage ' $\beta_{2}$ ' feature [394] (cf. 
Fig. 27). The temperature dependent kinetics of the He reflectivity restoration indicated that this process has a rather large activation barrier of $64.3 \pm 1.3 \mathrm{~kJ} / \mathrm{mol}$ [393], i.e., that it unlikely reflects, e.g., surface $\mathrm{H}$ diffusion and trapping at step edges. Luo et al. therefore attributed their observations to the transition of surfaceadsorbed $\mathrm{H}$ atoms into the subsurface.

If true, this would be quite remarkable as it implies that the subsurface sites rather than the adsorption sites on the $\mathrm{Cu}(111)$ surface should be the energetically more stable location for $\mathrm{H}$ atoms, in direct contradiction to the described theoretical results [295, 300-303]. Apart from the obvious question whether this inconsistency of the $\mathrm{H} / \mathrm{Cu}(111)$ system can be resolved in the near future, Luo's HAS data clearly highlight the potential merits of a nondestructive in-situ detection technique exclusively sensitive to surface hydrogen in elucidating its dynamic behavior.

\section{$\mathrm{Cu}(100)$}

The $\mathrm{Cu}(100)$ surface saturates at a coverage of $1.0 \mathrm{ML}\left(1.54 \times 10^{15} \mathrm{~cm}^{-2}\right)$ under exposure to atomic D. This was determined in two independent investigations via $\mathrm{D}\left({ }^{3} \mathrm{He}, \mathrm{p}\right)^{4} \mathrm{He}$ NRA $[272,273]$. Exposure to a molecular $\mathrm{H}_{2}$ beam $\left(\mathrm{E}_{\mathrm{T}}=0.4 \mathrm{eV}\right)$, on the other hand, only achieved an uptake of 0.5 ML [385]. In agreement with an earlier HREELS and LEED investigation [395], the atomic hydrogen-saturated $\mathrm{Cu}(100)$ surface $\left(\theta_{\text {sat }}=1.0 \mathrm{ML}\right)$ exhibits a rare $\mathrm{p}(2 \times 2)-\mathrm{p} 4 \mathrm{~g}$ reconstruction, in which the first layer $\mathrm{Cu}$ atoms are laterally displaced by $0.23 \AA$ (Fig. 29) [272]. 


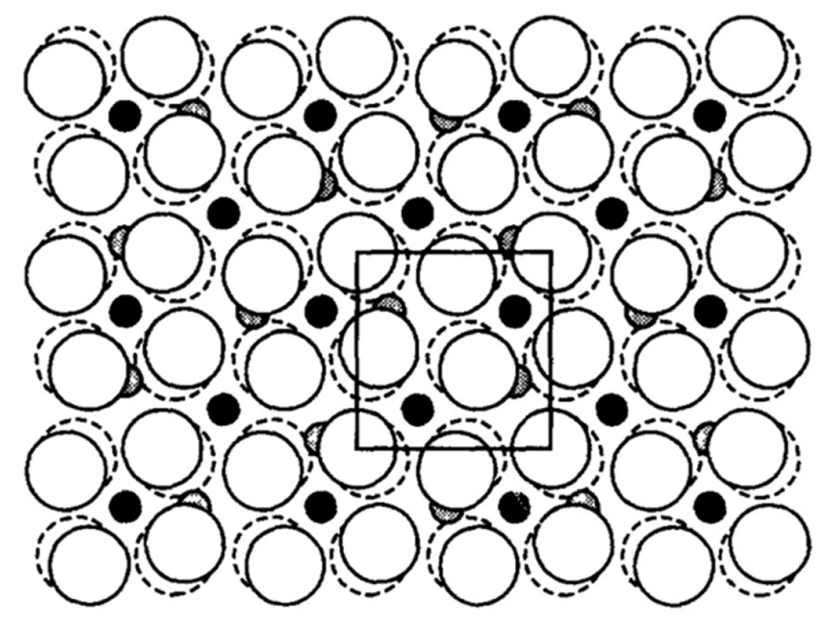

Fig. 29. Model for the $(2 \times 2) p 4 g-D$ structure on $\mathrm{Cu}(100)$. The clock reconstruction of the $\mathrm{Cu}$ lattice and a $(2 \times 2) \mathrm{Cu}$ unit cell are indicated. The small circles indicate $\mathrm{D}$ atoms adsorbed in four-fold-hollow sites (black) and displaced tetrahedral subsurface sites (grey). The experimental results do not reveal which of the displaced subsurface tetrahedral sites are populated with D. Reproduced from Ref. [272] with permission from Elsevier.

In this structure model, which relies on a robust determination of the $D$ adsorption positions by transmission channeling measurements, the first $\sim 0.5 \mathrm{ML}$ of D occupy the four-fold hollow sites on an unreconstructed $\mathrm{Cu}(100)$ lattice. Additional D atoms $(0.5<\theta<1.0 \mathrm{ML})$ then populate tetrahedral subsurface interstitial sites, which according to Foss et al. is the driving force for the $p(2 \times 2)-p 4 g$ reconstruction [272]. Walter et al. subsequently correlated as many as three additional hydrogeninduced surface reconstruction phases on $\mathrm{Cu}(100)$ to absolute $\mathrm{D}$ coverages (NRA) that appeared in the range between 0.45 and $1.0 \mathrm{ML}$ and $80-180 \mathrm{~K}$; among these a $(2 \sqrt{ } 2 \times 2 \sqrt{ } 2) R 45^{\circ}$, a $(4 \sqrt{ } 2 \times 2 \sqrt{ } 2) R 45^{\circ}$, and a particularly prominent superstructure of so far unidentified periodicity that maximizes at $\theta=0.65 \mathrm{ML}$ [273].

The above results [273] supported earlier speculations that the $\mathrm{Cu}(100)$ surface tends to reconstruct upon hydrogen adsorption, referring to peculiar anomalies in $\mathrm{H}_{2}$ 
TD spectra [385], [395]. D-induced changes of the surface morphology were also manifested by a high density of $\mu \mathrm{m}$-sized pits visible in SEM micrographs of Dexposed $\mathrm{Cu}(100)$, suggesting that high $\mathrm{D}$ coverages can apparently enhance the surface diffusion of $\mathrm{Cu}$ atoms and thus enable a substantial Cu mass transport [273].

\section{Cu(110)}

Although no NRA data of absolute $\mathrm{H}$ coverages are reported for $\mathrm{Cu}(110)$, we note here that the $\mathrm{H}$ coverage saturates at $0.5 \mathrm{ML}$ as determined by calibrated TDS [385, 390] and ion scattering [396, 397]. Atomic $\mathrm{H}$ adsorption on $\mathrm{Cu}(110)$ at $100 \mathrm{~K}$ induces first a $(1 \times 3)$ superstructure at $0.1<\theta<0.4 \mathrm{ML}$, which transforms into a $(1 \times 2)$ reconstruction as the $\mathrm{H}$ coverage increases to $0.5 \mathrm{ML}$ at saturation [398].

\section{$\operatorname{Ag}(111)$}

Owing to the completely filled d-band of the noble metal, a substantial activation barrier (1.1 eV) prevents gas phase hydrogen molecules from dissociating on $\mathrm{Ag}(111)$ surfaces at room temperature [399]. Lee et al. have therefore studied hydrogen chemisorption on $\mathrm{Ag}(111)$ after exposure to atomic $\mathrm{D}$, using LEED, HREELS, work function, TDS, and absolute coverage measurements with $D\left({ }^{3} \mathrm{He}, \mathrm{p}\right)^{4} \mathrm{He}$ NRA [270]. The $H(D)$ saturation coverage was determined to be $0.6 \pm 0.1 \mathrm{ML}$, where $1 \mathrm{ML}$ corresponds to an atomic density of $1.38 \times 10^{15} \mathrm{~cm}^{-2}$ on $\mathrm{Ag}(111)$. The quantitative NRA coverage was used to calibrate the D uptake curve measured by TDS (Fig. 30), which enabled assigning $D$ coverage ranges to the $D$-induced superstructures observed by LEED [400]. Lee's NRA value for the D saturation coverage is in excellent agreement with quantitative TDS data for $\mathrm{H} / \mathrm{Ag}(111)$ from Winkler's group ( $\theta_{\text {sat }}=0.65 \pm 0.05 \mathrm{ML}$ ) [401]. 


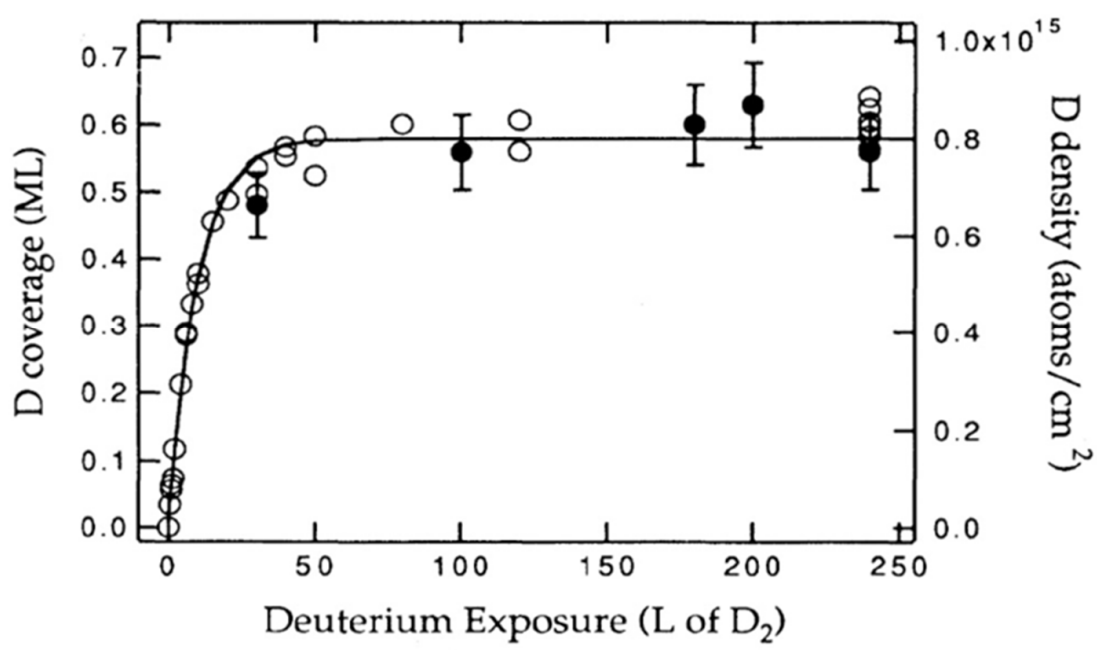

Fig. 30. Atomic deuterium coverage on $\mathrm{Ag}(111)$ as a function of $D_{2}$ exposure (the line is a guide for eyes). Solid circles $(\bullet)$ are from the coverage measurement using $D\left({ }^{3} \mathrm{He}, \mathrm{p}\right)^{4} \mathrm{He}$ NRA (Ref. [290]) and open circles (o) are from the integrated $D_{2}$ TDS signal calibrated to the absolute coverage. Reproduced from Ref. [417] with permission from APS.

$\mathrm{H}$ chemisorption on $\mathrm{Ag}(111)$ at $100 \mathrm{~K}$ first induces a sharp $(2 \times 2)$ superstructure in the coverage range of $0.25 \sim 0.5 \mathrm{ML}$, followed by a combined $(2 \times 2)+(3 \times 3)$ pattern as the coverage approaches saturation at $0.6 \mathrm{ML}[270]$, resembling the situation on $\mathrm{Cu}(111)[271]$. While the appearance of $\mathrm{H}$-induced superstructures is not uncommon for fcc (111) surfaces (e.g., $\mathrm{H} / \mathrm{Ni}(111)$ [341], $\mathrm{H} / \mathrm{Pd}(111)$ [402]), the lack of transformation into a $(1 \times 1)$ structure at saturation seems to be unique to $\mathrm{H}$ on $\mathrm{Ag}(111)$ and on $\mathrm{Cu}(111)[271,403]$. Lee et al. further note that the intensity of the extra diffraction spots in the $\mathrm{H}$-induced $(2 \times 2)$ and $(3 \times 3)$ superstructures are considerably more intense than those expected to arise solely from an ordered array of $\mathrm{H}$ atoms, which lets them consider that the $\mathrm{Ag}(111)$ surface may reconstruct upon $\mathrm{H}$ chemisorption. This argument is given support by the observation of positive work function changes in proportion to the $\mathrm{H}$ coverage, which reach $+0.32 \mathrm{eV}[270,400]$ at $\mathrm{H}$ saturation. According to Zhukov et al. who find $\Delta \phi=+0.24 \mathrm{eV}$ at $\theta_{\text {sat, }}$ this work 
function increase corresponds to dipole moments ( 0.07 Debye) that are unusually large for a $\mathrm{H}$ adsorption layer on a metal substrate and might hence be indicative of a surface reconstruction [401]. The latter two $\Delta \phi$ results are at variance with an earlier report of a work function decrease upon $\mathrm{H}$ adsorption [404].

The $\mathrm{H} / \mathrm{Ag}(111)$ system shows a peculiar and complex behavior with respect to recombinative $\mathrm{H}_{2}$ desorption, which occurs at $\sim 185-200 \mathrm{~K}$, corresponding to a desorption activation energy of $8.7 \mathrm{kcal} / \mathrm{mol}$ [270]. Lee's TD spectra show nearly symmetric desorption peak shapes indicative of second order kinetics, but the peak maximum shifts to higher temperatures as the initial $\mathrm{H}$ coverage increases, which is more common for a process of zero or fractional order kinetics. The presence of two $\mathrm{H}$ adsorption phases, i.e., a condensed $\mathrm{H}$ island structure in equilibrium with a two dimensional lattice gas (isolated $\mathrm{H}$ atoms diffusing on the $\mathrm{Ag}(111)$ terraces) has been proposed as an explanation [401]. The suggested formation of well-ordered $\mathrm{H}$ islands accompanied by surface reconstruction is consistent with the $\mathrm{H}$-induced superstructures observed by LEED, and the simultaneous presence of the lattice gas is compatible with the comparatively low saturation coverage $(0.6 \mathrm{ML})$ evidenced by NRA [270] and TDS [401]. Depending on the size and number of the dense $\mathrm{H}$ islands relative to the lattice gas area, i.e., on the microscopic surface roughness that is difficult to control (defects or contaminants provide nucleation sites for island formation), the desorption kinetics may either be dominated by desorption from the island rims (half order kinetics) or from the lattice gas (second order kinetics), or contain characteristics from both kinetic regimes in intermediate situations [401]. This argument by Zhukov et al. may reconcile the varying shapes and coverage 
dependences of the TD spectra that have been reported for the $\mathrm{H} / \mathrm{Ag}(111)$ system by different groups [270, 401, 404, 405].

\section{Ultrathin Films and (Surface) Alloys}

\section{$\mathrm{Au} / \operatorname{Ir}(111), \mathrm{Au} / \operatorname{Pt}(111), \mathrm{Ag} / \operatorname{Pt}(111)$}

A great deal of attention has been paid to the special physics and chemistry of ultra-thin noble metal films and nanostructures deposited on a variety of singlecrystalline substrates, because substantial differences in structural, electronic, and chemical properties clearly distinguish these artificial 'nanomaterials' from the respective bulk noble metals. Owing to the fully occupied d-band centered far below $\mathrm{E}_{\mathrm{F}}, \mathrm{H}_{2}$ dissociation on $\mathrm{Au}$ and $\mathrm{Ag}$ single crystal surfaces is inhibited by considerable activation barriers that prevent room temperature adsorption of $\mathrm{H}_{2}$ [384]. In striking contrast to this general expectation of surface 'nobility', Okada et al. reported a TDS study, which demonstrated that several $(\leq 8)$ monolayer thin Au films grown at $100 \mathrm{~K}$ on an $\operatorname{Ir}(111)$ substrate behave surprisingly reactive toward dissociating hydrogen $\left(D_{2}\right)$ molecules at temperatures as low as $100 \mathrm{~K}$ [406]. After depositing the Au onto D-precovered instead of clean $\operatorname{Ir}(111)$ surfaces, Okada et al. furthermore observed an additional low temperature ( $160 \mathrm{~K}) \mathrm{D}_{2}$ TDS feature which he assigned to a certain amount of $D$ that had become trapped at the $A u / I r$ interface. This suspect was subsequently corroborated by means of $\mathrm{H}$ depth profiling with ${ }^{1} \mathrm{H}\left({ }^{15} \mathrm{~N}, \alpha \gamma\right){ }^{12} \mathrm{C}$ NRA, which could clearly distinguish between the $\mathrm{H}$ adsorbed on the surface of the thin $\mathrm{Au}$ films and $\mathrm{H}$ located at the Au/Ir interface [274, 407, 408]. 


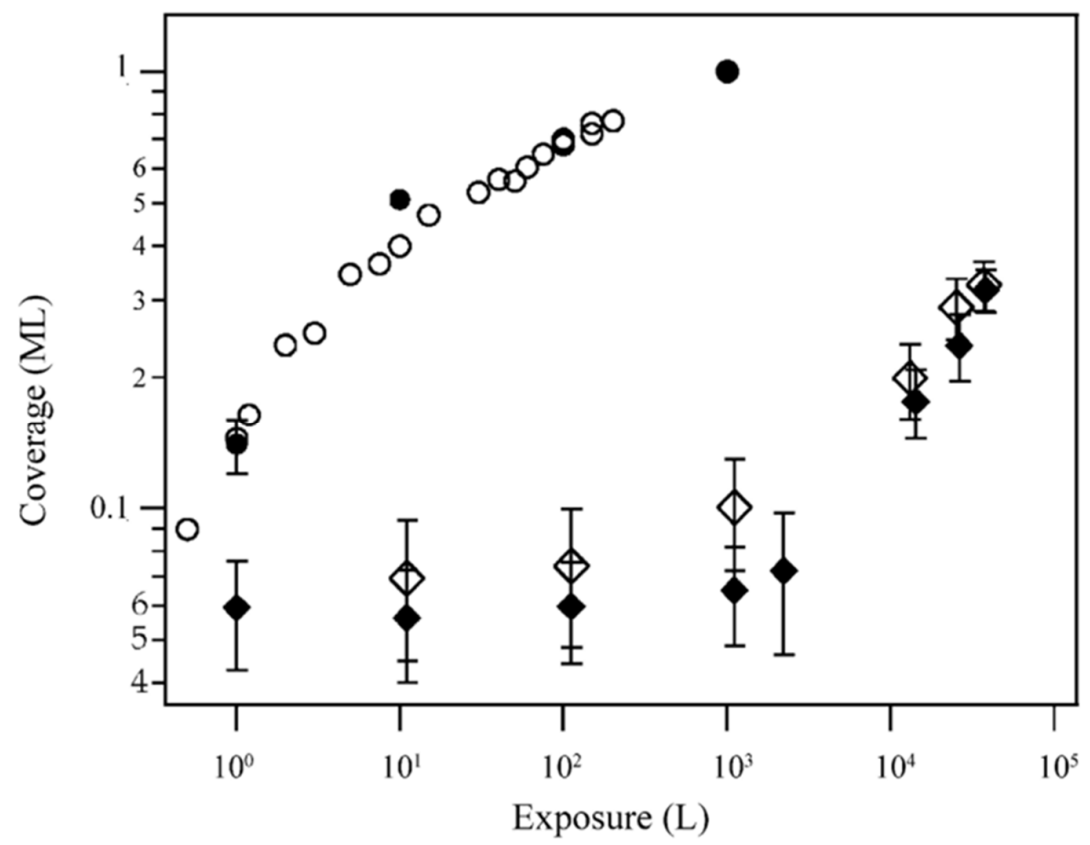

Fig. 31. H-uptake curves measured by NRA on the clean (full circles), 4-ML (full diamonds) and 10-ML (open diamonds) Au-covered $\operatorname{Ir}(111)$ surfaces under $\mathrm{H}_{2}$ at $60 \mathrm{~K}$. Au was deposited at $60 \mathrm{~K}$. Open circles denote a D-uptake curve for the $D_{2}$ exposure of $\operatorname{Ir}(111)$, measured with TDS and taken from Ref. [322]. Reproduced from Ref. [407] with permission from Elsevier.

Fig. 31 shows the NRA-determined $H$ uptake of the clean $\operatorname{Ir}(111)$ surface (consistent with earlier TDS data [322]) in comparison to 4 and $10 \mathrm{ML}$ thin Au films on $\operatorname{Ir}(111)$ under $\mathrm{H}_{2}$ exposure at $60 \mathrm{~K}$ [407]. Although the Au films are clearly less reactive than the clean $\operatorname{Ir}(111)$ substrate, they obviously do adsorb hydrogen after larger $\mathrm{H}_{2}$ exposures, with strikingly little influence of the Au thickness even up to 10 ML thick films. STM verified that these Au films cover the $\operatorname{Ir}(111)$ substrate completely [274, 407]. HD production in TPD after mixed $H_{2}$ and $D_{2}$ exposures identified the hydrogen adsorption process as dissociative [406]. The energy position, symmetric shape, and relatively narrow width of the high resolution NRA $\mathrm{H}$ depth profile for the $\mathrm{H}_{2}$ exposed $4 \mathrm{ML}$ Au film deposited onto clean $\operatorname{Ir}(111)$ in Fig. 32 (a) indicate that $\mathrm{H}$ resides on the Au surface, not inside the film volume. The small 
additional high energy peak in the $\mathrm{H}$ profile resulting from depositing the $4 \mathrm{ML} \mathrm{Au}$ film after pre-saturating the $\operatorname{Ir}(111)$ substrate with $1 \mathrm{ML} \mathrm{H}$ (Fig. 32 (b)) signifies that 0.2 ML H became trapped at the Au/Ir(111) interface [274]. Stopping and straggling of ${ }^{15} \mathrm{~N}$ in the Au film reasonably accounts for the energy shift and the width of the interface peak, consistent with the picture that $\mathrm{H}$ is located at an atomically sharp interface between Au and Ir; a plausible idea for a junction between two immiscible metals that both do not absorb $\mathrm{H}$ in the bulk.

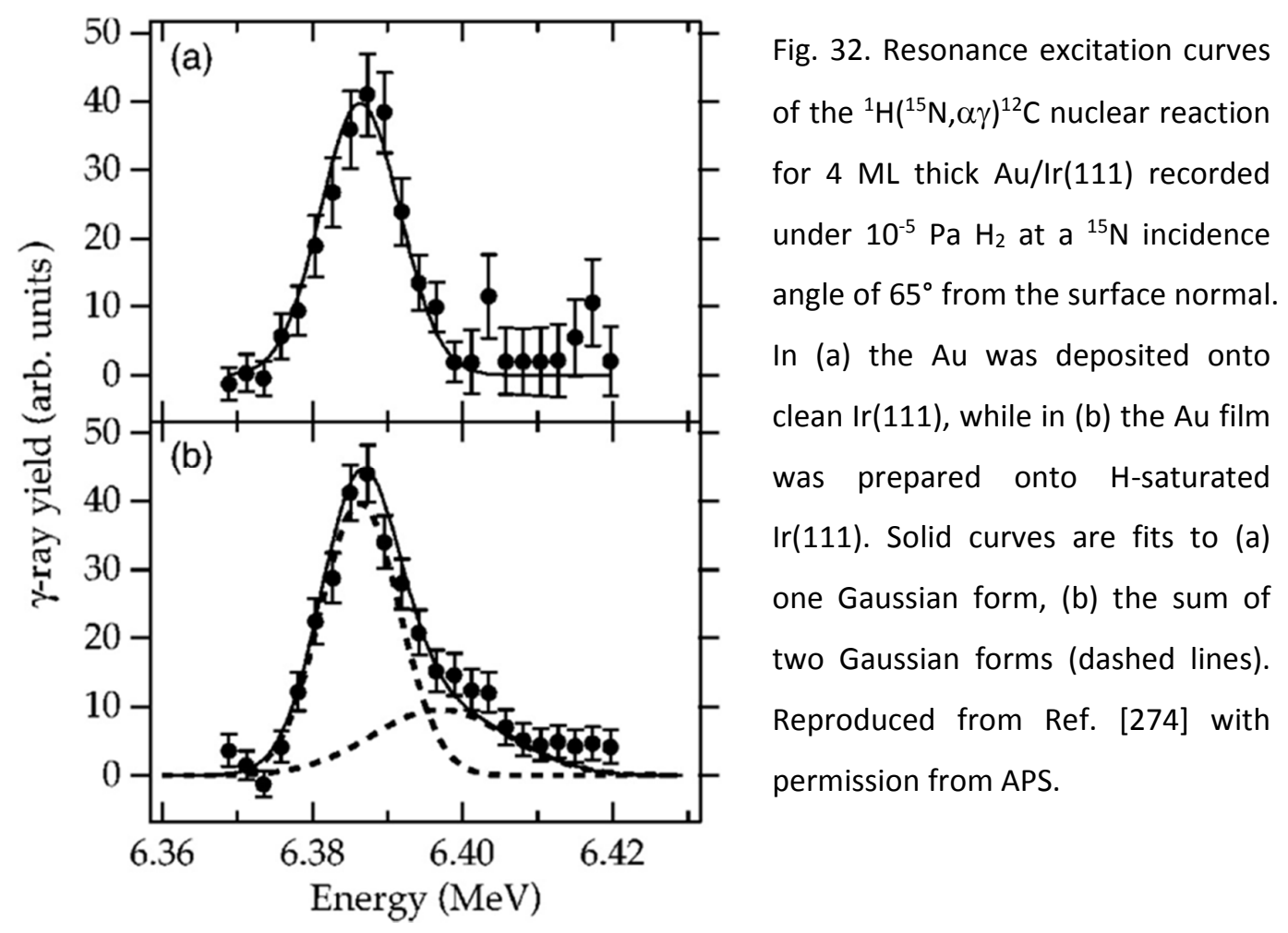

The possibility of metastable $\mathrm{H}$ binding in octahedral interstitial sites at the $\mathrm{Au} / \operatorname{Ir}(111)$ interface was further confirmed by DFT total energy calculations [274]. To our knowledge this NRA study currently is the only report of hydrogen trapping at a metal/metal interface. One may compare the situation, e.g., to Ag deposition onto $\mathrm{H}$-covered $\mathrm{W}(100)$, in the course of which $\mathrm{H}$ is completely desorbed as soon as the 
$\mathrm{Ag}$ film (which is presumed to grow in islands at room temperature) covers the entire surface [409].

The unusual $\mathrm{H}_{2}$ dissociation reactivity of the epitaxial Au films on $\operatorname{Ir}(111)$ was attributed to localized surface defects, where the half-occupied Au s-band centered at $E_{\mathrm{F}}$ is narrowed due to reduced coordination of the Au atoms [408]. STM images of the $\mathrm{Au} / \operatorname{Ir}(111)$ system clearly show the presence of localized defects such as $\mathrm{Au}$ island edges [274, 407]. In analogy to arguments of the d-band model [384], such localized s-band narrowing leads to reduced repulsion between electrons in the occupied bonding $\mathrm{H}_{2}\left(\sigma_{\mathrm{g}}\right)$ molecular orbital and Au states far below $E_{\mathrm{F}}$ and increases attraction between the unoccupied antibonding $\mathrm{H}_{2}\left(\sigma_{\mathrm{u}}\right)$ orbital and Au states near $E_{\mathrm{F}}$ [408]. The net effect is a smaller activation energy barrier for $\mathrm{H}_{2}$ dissociation. To illustrate this concept, DFT calculations at small $\mathrm{Au}_{2}-\mathrm{H}_{2}$ cluster models have been carried out, demonstrating that (at least in this vastly exaggerated model of a localized defect with obviously minimal s-band width) the $\mathrm{H}_{2}$ dissociation barrier may even vanish altogether [408]. It is worth mentioning that the d-band model itself is not able to explain the efficient $\mathrm{H}_{2}$ dissociation on the thin Au films on $\operatorname{Ir}(111)$, because paradoxically the $d$-band center of a Au monolayer on $\operatorname{Ir}(111)$ is located even $0.59 \mathrm{eV}$ farther below $\mathrm{E}_{\mathrm{F}}$ than in case of bulk $\mathrm{Au}(111)$, so that the model predicts an even higher activation barrier for $\mathrm{H}_{2}$ dissociation on $\mathrm{Au} / \operatorname{Ir}(111)$ [410].

In comparison to the highly reactive thin Au films on $\operatorname{Ir}(111)$, Ogura et al. tested Au and Ag monolayer films on $\mathrm{Pt}(111)$ for their activity towards $\mathrm{H}_{2}$ dissociation [411]. The NRA and TDS study revealed that in striking contrast to the Au/Ir(111) system, the $\mathrm{Pt}(111)$ surface became completely passivated for the dissociation of molecular 
$\mathrm{H}_{2}$ if covered with either a monolayer of $\mathrm{Au}$ or $\mathrm{Ag}$. $\mathrm{H}$ adsorption on these $\mathrm{Au} / \mathrm{Pt}(111)$ and $\mathrm{Ag} / \mathrm{Pt}(111)$ surfaces was hence possible only through admission of atomic $\mathrm{H}$, which desorbed associatively as $\mathrm{H}_{2}$ at $120 \mathrm{~K}$ from $\mathrm{Au} / \mathrm{Pt}(111)$ and at $160 \mathrm{~K}$ from $\mathrm{Ag} / \mathrm{Pt}(111)$, with a slight trend of increasing peak temperatures with $\mathrm{H}$ coverage for the latter, suggesting that a similar fractional order desorption process from rims of dense $\mathrm{H}$ islands is taking place as proposed for $\mathrm{H} / \mathrm{Ag}(111)$ [401].

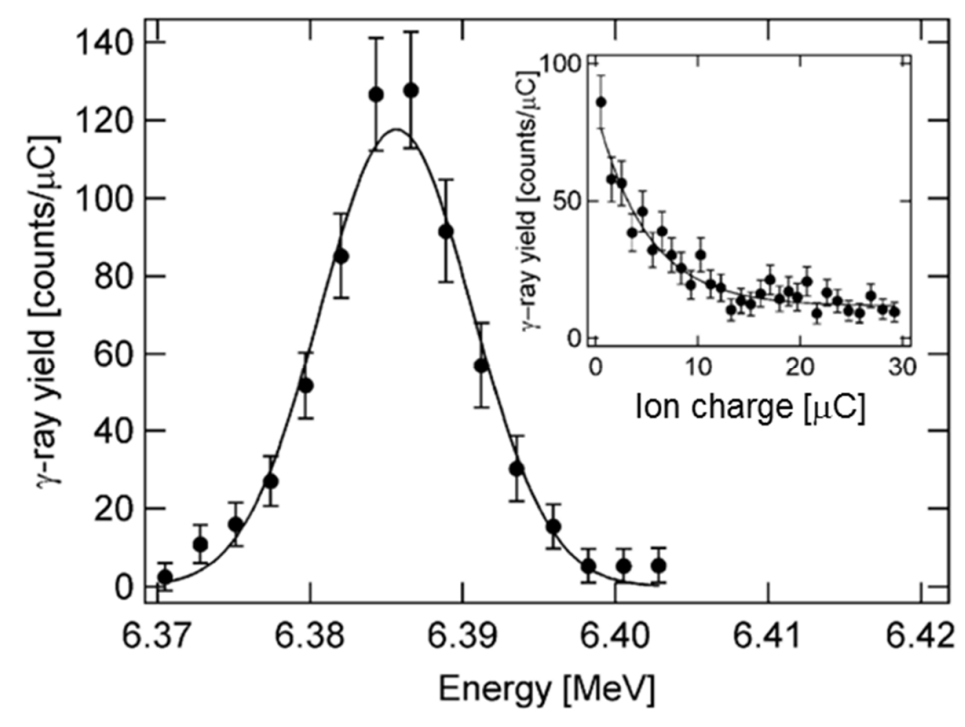

Fig. 33. ${ }^{1} \mathrm{H}\left({ }^{15} \mathrm{~N}, \alpha \gamma\right){ }^{12} \mathrm{C}$ surface resonance excitation curve for 1 $\mathrm{ML} \mathrm{Ag/Pt(111)} \mathrm{after}$ dosage of $1000 \mathrm{~L}$ atomic hydrogen. The inset shows the decay of the $\gamma$-ray yield as a function of accumulated ionbeam charge

incident on the sample. Reproduced from Ref. [411] with permission from Elsevier.

Fig. 33 shows the ${ }^{1} \mathrm{H}\left({ }^{15} \mathrm{~N}, \alpha \gamma\right){ }^{12} \mathrm{C}$ NRA surface resonance curve for the $1 \mathrm{ML}$ $\mathrm{Ag} / \mathrm{Pt}(111)$ surface after exposure to $1000 \mathrm{~L}$ of partially dissociated $\mathrm{H}_{2}$ at $90 \mathrm{~K}$. The $\mathrm{H}$ saturation coverage derived from the integrated profile area $(\gamma$-yield corrected for the decay through ion beam-induced $\mathrm{H}$ desorption shown in the inset) amounts to $0.9 \pm 0.1 \mathrm{ML}$ with respect to the $\mathrm{Pt}(111)$ substrate atomic density $\left(1 \mathrm{ML}=1.50 \times 10^{15}\right.$ $\mathrm{cm}^{-2}$ ). This result suggests that the $\mathrm{H}$ saturation coverage on the $\mathrm{Ag}$ monolayer on $\mathrm{Pt}(111)$ is considerably higher than on bulk $\mathrm{Ag}(111)$, where it amounts to only $0.6 \mathrm{ML}$ (here: $1 \mathrm{ML}=1.38 \times 10^{15} \mathrm{~cm}^{-2}$ ) [270], suggesting much higher $\mathrm{H}$ densities in the 
adsorption layer on the epitaxial Ag film. One may only speculate at this point whether the compressed lattice of the $\mathrm{Ag}$ monolayer on the $\mathrm{Pt}(111)$ substrate is responsible for this behavior. The slightly reduced $\mathrm{H}_{2}$ desorption temperature of $\mathrm{Ag} / \mathrm{Pt}(111)$ ( $160-180 \mathrm{~K})$ relative to that of $\mathrm{Ag}(111)$ single crystals ( $180-200 \mathrm{~K})$ might support the idea of a somewhat destabilized adsorption state owing to increasingly repulsive $\mathrm{H}-\mathrm{H}$ interactions in the much denser $\mathrm{H}$ islands.

On the $\mathrm{Au} / \mathrm{Pt}(111)$ surface the NRA evaluation of the $\mathrm{H}$ coverage produced a value of $0.7 \pm 0.1 \mathrm{ML}$, slightly smaller than the saturation coverage of the clean $\mathrm{Pt}$ (111) surface, determined to $(0.8 \pm 0.1 \mathrm{ML})$ in this study. As $\mathrm{H}$ adsorption on $\mathrm{Au}(111)$ is endothermic [384], no quantitative comparison to the $\mathrm{H}$ saturation coverage on bulk $\mathrm{Au}(111)$ can be made in this case. The TPD desorption peak temperature of $120 \mathrm{~K}$ matches reasonably with the one on $\mathrm{Au}(111)$ (Ref. 51 in [412]).

In their $\mathrm{H}_{2}$ adsorption/desorption properties the only one monolayer thin $\mathrm{Au}$ and Ag films on $\mathrm{Pt}(111)$ thus already resemble the (111) surfaces of the respective $\mathrm{Au}$ and $\mathrm{Ag}$ bulk single crystals [400, 404, 412-414] and in this respect behave indeed drastically differently compared to the Au films on $\operatorname{Ir}(111)$, which remain active for $\mathrm{H}_{2}$ dissociation up to Au thicknesses of at least $10 \mathrm{ML}$ [407]. The surface passivity of the $\mathrm{Au}$ and $\mathrm{Ag}$ monolayers on $\mathrm{Pt}(111)$ also contrasts the unique chemistry displayed by, e.g., the monolayer $\mathrm{Ni} / \mathrm{Pt}(111)$ surface with respect to cyclohexene (deJhydrogenation, which differs from both pure Pt(111) and thick Ni films, outlining the potential of bimetallic catalysis [415]. The low reactivity of the $\mathrm{Ag} / \mathrm{Pt}(111)$ and $\mathrm{Au} / \mathrm{Pt}(111)$ monolayer systems toward $\mathrm{H}_{2}$ dissociation observed by Ogura et al., however, is not completely unexpected but in line with predictions of the $d$-band 
model. According to this theory, the position of the metal d-band center with respect to the Fermi level $\left(E_{F}\right)$ is a good indicator of the chemical reactivity of a surface [384, 416]. As the d-band center shifts downward (upward), away from (closer to) $E_{F}$, the model predicts that the surface becomes less (more) reactive. The $d$-band center of $\mathrm{Au} / \mathrm{Pt}(111)$ is $0.08 \mathrm{eV}$ below that of $\mathrm{Au}(-3.59 \mathrm{eV})$, and the d-band center of $\mathrm{Ag} / \mathrm{Pt}(111)$ is $0.11 \mathrm{eV}$ above that of $\mathrm{Ag}(-4.30 \mathrm{eV})$ but still below that of $\mathrm{Au}$ [410], so that $\mathrm{H}_{2}$ molecules are neither expected to dissociate readily on $\mathrm{Ag} / \mathrm{Pt}(111)$ nor on $\mathrm{Au} / \mathrm{Pt}(111)$.

\section{$\operatorname{Pt}(111)-\operatorname{Sn}(2 \times 2), \operatorname{Pt}(111)-\operatorname{Sn}(\sqrt{3} \times \sqrt{3})$}

Motivated by the question whether a chemical modification by substituting surface atoms with heteroelements might alter the $\mathrm{H}$ adsorption state on $\mathrm{Pt}(111)$, for which delocalization of the $\mathrm{H}$ nuclear wavefunction has been discussed $[67,68$, 268, 382], Fukutani et al. investigated the $\mathrm{H}$ adsorption potential on Pt-Sn surface alloys by means of zero-point vibrational analysis using ${ }^{1} \mathrm{H}\left({ }^{15} \mathrm{~N}, \alpha \gamma\right){ }^{12} \mathrm{C}$ NRA Doppler spectroscopy [417]. Well-ordered Pt-Sn surface alloys of $(2 \times 2)$ and $(\sqrt{ } 3 \times \sqrt{ } 3)$ structures that involve only the topmost surface layer and correspond to Sn coverages of $1 / 4$ and $1 / 3 \mathrm{ML}$, respectively, can be prepared in a controlled fashion by $\mathrm{Sn}$ deposition onto $\mathrm{Pt}(111)$ and annealing at $1000 \mathrm{~K}$ [418]. As illustrated in Fig. 34, such alloying substitutes $75 \%$ or even all 3 -fold hollow adsorption sites that are coordinated by three $\mathrm{Pt}$ atoms on clean $\mathrm{Pt}(111)$ with $\mathrm{Pt}_{2} \mathrm{Sn}$ environments in the $\mathrm{Pt}(111)-\mathrm{Sn}(2 \times 2)$ and $\mathrm{Pt}(111)-\mathrm{Sn}(\sqrt{3} \times \sqrt{3})$ structures, respectively. As a consequence of Sn-allyoing, $\mathrm{H}_{2}$ 
dissocation was found to be strongly suppressed so that the surface alloys had to be exposed to atomic $\mathrm{H}$ from a partial thermal $\mathrm{H}_{2}$ dissociation doser in order to create the $\mathrm{H}$ adsorption layers [417].
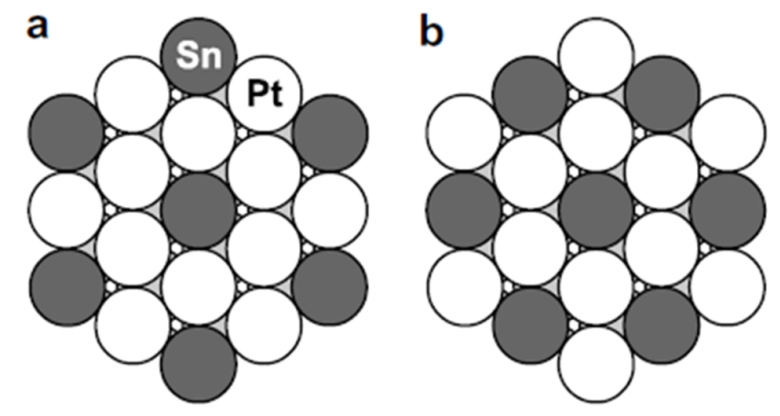

Fig. 34. Structure models of wellordered Pt-Sn surface alloys: (a) $\operatorname{Pt}(111)-\operatorname{Sn}(2 \times 2)$ and (b) $\mathrm{Pt}(111)-$ $\operatorname{Sn}(\sqrt{ } 3 \times \sqrt{3})$. Adapted with permission from Ref. [417].

NRA indicated that the $\mathrm{H}$ saturation coverage is greatly reduced from $1.0 \mathrm{ML}$ on clean Pt(111) [67] to $0.40 \mathrm{ML}$ on the Pt(111)-Sn(2×2) and $0.42 \mathrm{ML}$ on the Pt(111)$\mathrm{Sn}(\sqrt{3} \times \sqrt{3})$ alloys [417]. From the Doppler widths of the nuclear reaction resonance excitation curves measured in surface-normal and $45^{\circ}$ inclined incidence, the zeropoint energies of the $\mathrm{H}$ vibrations in the vertical and in-plane directions were estimated to be $78.2 \pm 3.7$ and $53.6 \pm 5.8 \mathrm{meV}$ on $\mathrm{Pt}(111)-\mathrm{Sn}(2 \times 2)$, and $72.5 \pm 3.7$ and $58.5 \pm 6.7$ meV on $\mathrm{Pt}(111)-\mathrm{Sn}(\sqrt{ } 3 \times \sqrt{ } 3)$, respectively. Compared to the respective zeropoint energies of $80.8 \pm 3.9$ and $62.1 \pm 6.0 \mathrm{meV}$ for $\mathrm{H}$ on clean $\mathrm{Pt}(111)$ these values are only slightly smaller, suggesting that the local $\mathrm{H}$ adsorption potential on the Pt-Sn surface alloys does not differ substantially from the one on clean Pt(111). It was concluded that alloying with Sn apparently does not weaken the H-substrate interaction to a large degree, and that the predominant effect of Sn appears to be the introduction of a considerable activation barrier for $\mathrm{H}_{2}$ dissociation. 
$M_{0.75} \operatorname{Re}_{0.25}$

Surfaces of transition metal alloys are interesting as their elemental distribution may differ considerably from the bulk composition owing to segregation phenomena. Absolute D coverage measurements on the (100), (110), and (111) surfaces of the bcc $\mathrm{Mo}_{0.75} \mathrm{Re}_{0.25}$ alloy were performed with $\mathrm{D}\left({ }^{3} \mathrm{He}, \mathrm{p}\right)^{4} \mathrm{He}$ NRA by Okada et al. [275]. Due to different tendencies for elemental segregation, the relative atomic composition of the outermost layers on these alloy surfaces was found to be orientation dependent. While the (100) and (111) surfaces are enriched in Mo, the (110) surface has a bulk-like composition. Only (1×1) LEED patterns were observed on all surfaces upon $D_{2}$ exposure, showing no indications for adsorption-induced superstructures at any D coverage. The D saturation coverage on the alloy surfaces was determined to be $2.02 \pm 0.10 \mathrm{ML}$ for the (100), $0.99 \pm 0.05 \mathrm{ML}$ for the (110), and $2.86 \pm 0.24 \mathrm{ML}$ for the (111) orientation. Exposure to atomic $\mathrm{D}$ did neither result in a significant increase of the NRA nor of the TDS intensity, so that the possibility of D absorption into the alloy bulk could be dismissed.

The (100) alloy surface contains nearly $100 \%$ Mo atoms, and similar to Mo(100) the $\mathrm{D}$ saturation coverage of $2 \mathrm{ML}$ is well consistent with an adsorption structure model based on HREELS [419] and LEED I(V) [420,421] results, in which D atoms occupy the bridge sites (Fig. 35 (a)). A $c(7 \sqrt{ } 2 \times \sqrt{ } 2) R 45^{\circ}$ reconstruction $[422,423]$ as well as a variety of hydrogen-induced superstructures that form on pure $\mathrm{Mo}(100)$ $[424,425]$, however, do not appear on the $\mathrm{Mo}_{0.75} \mathrm{Re}_{0.25}(100)$ alloy surface [275] 
(a)

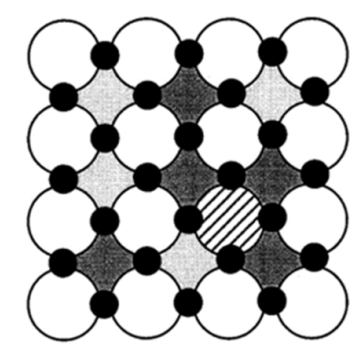

(b)

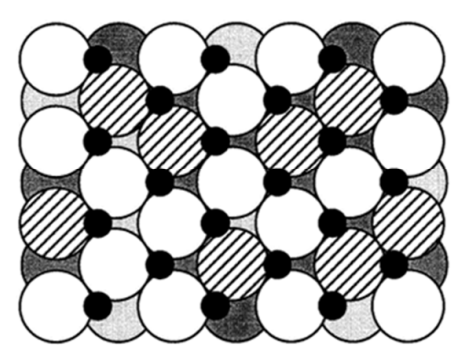

(c)

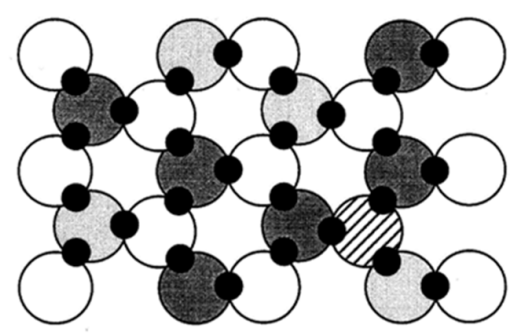

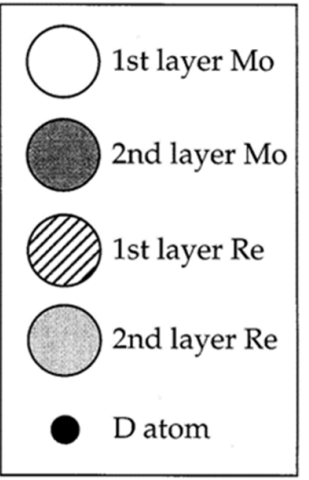

Fig. 35. Structural models for $\mathrm{Mo}_{0.75} \mathrm{Re}_{0.25}$ (a) (100), (b) (110), and (c) (111) surfaces saturated by $D$ at about 100 K. Saturation coverages correspond to 2,1 , and $3 \mathrm{ML}$, respectively. Re positions are represented randomly. Adapted from Ref. [275] with permission from Elsevier.

On the $\mathrm{Mo}_{0.75} \mathrm{Re}_{0.25}$ (110) surface the $\mathrm{D}$ saturation coverage of $0.99 \mathrm{ML}$ and the (1×1) LEED pattern are the same as the proposed coverage and the observed LEED patterns for $\mathrm{H}$ adsorption on the pure $\mathrm{Mo}(110)[426]$ and $\mathrm{W}(110)[427,428]$ surfaces. The proposed structural model with D in quasi-trigonal sites is depicted in Fig. 35 (b). $\mathrm{H} / \mathrm{W}(110)$ has not been evaluated quantitatively, but Iwata et al. observed with ${ }^{15} \mathrm{~N}$ NRA that the $\mathrm{H}$ coverage on $\mathrm{W}(110)$ was $(0.7 \pm 0.1)$ times smaller than that on $\mathrm{H}$ saturated $\mathrm{W}(100)$. Whether the $\mathrm{H}$ coverage on $\mathrm{W}(100)$ saturates at $1.5 \mathrm{ML}$ or $2.0 \mathrm{ML}$ is subject of some debate (see W section above), and Iwata et al. themselves favored the 2.0 ML hypothesis [83], but if it in fact saturated at $1.5 \mathrm{ML}$, then Okada's suggestion that the $\mathrm{D}$ layer on $\mathrm{Mo}_{0.75} \mathrm{Re}_{0.25}$ (110) at $0.99 \mathrm{ML}$ is isostructural to $\mathrm{H}$ saturated $\mathrm{W}(110)$ would be quite consistent $[(0.7 \pm 0.1) \times 1.5 \mathrm{ML} \approx 1 \mathrm{ML}]$. 
On the $\mathrm{Mo}_{0.75} \mathrm{Re}_{0.25}(111)$ surface the first and second atomic layers are enriched in Mo (nearly $100 \%$ ) and Re, respectively. The D saturation coverage of $2.86 \mathrm{ML}$ and the $(1 \times 1)$ LEED pattern are similar to the situation proposed for the isostructural $W(111)$ surface $[428,429]$, with $3 \mathrm{ML} D$ in bridge sites where they make contact with first layer Mo and second layer Re atoms (Fig. 35 (c)). This structural model is in good agreement with the dominating TPD signal at $233 \mathrm{~K}$ that also appears (yet at $205 \mathrm{~K}$ and with reduced intensity) on $\mathrm{Mo}_{0.75} \mathrm{Re}_{0.25}(100)$ and has been assigned to Re-rich (i.e., Mo-Re bridge) sites. D desorption from Mo-Mo sites give rise to a TPD signal at a higher temperature (295 K on the (111) and $274 \mathrm{~K}$ on the (100) surface) [275].

\section{$\mathrm{Pt}_{0.5} \mathrm{Ni}_{0.5}$}

Hydrogen adsorption on the (110) and (111) surfaces of PtNi alloy was investigated with quantitative ${ }^{1} \mathrm{H}\left({ }^{15} \mathrm{~N}, \alpha \gamma\right){ }^{12} \mathrm{C}$ NRA coverage measurements by Fallavier et al. [281]. The PtNi alloy shows remarkably different catalytic properties, e.g. with respect to olefin hydrogenation reactions, than the constituting elements [430]. Similar to the above $\mathrm{Mo}_{0.75} \mathrm{Re}_{0.25}$ case, the elemental composition of the $\mathrm{Pt}_{0.5} \mathrm{Ni}_{0.5}$ alloy surfaces is orientation dependent: The (110) surface is enriched with $100 \% \mathrm{Ni}$ atoms, while the (111) surface contains 88 at.\% Pt [281]. The elemental enrichment alternates in the topmost three atomic layers and approaches the bulk composition of the alloy in a slightly damped oscillating fashion. The $\mathrm{H}$ coverage on the alloys surfaces was evaluated in the pressure range from $10^{-6}-10^{-2} \mathrm{~Pa} \mathrm{H}_{2}$ for temperatures between 115 and $400 \mathrm{~K}$. For temperatures above $200 \mathrm{~K}$ the coverage dependence exhibited Sievert behavior ( $\theta$ being proportional to $p\left(H_{2}\right)^{1 / 2}$ ), indicating dissociative adsorption at identical surface sites and the absence of phase transitions. 
Below $200 \mathrm{~K}$ the coverage dependence on the temperature indicated an ordered overlayer, evidenced by coverage saturation of under $6 \times 10^{-8} \mathrm{mbar}_{2}$ at $1 / 3 \mathrm{ML}(1$ $\mathrm{ML}=1.0 \times 10^{15} \mathrm{~cm}^{-2}$ ) on the Ni-terminated (110) alloy surface, which was assigned to a quasi-(2×6) $\mathrm{H}$ phase in analogy to pure $\mathrm{Ni}(110)[431]$.

In contrast to $\mathrm{Ni}(110)$, however, where the $\mathrm{H}$ coverage saturates at $1.5 \mathrm{ML}$ on a (1×2) reconstruction [276, 329], the maximum $\mathrm{H}$ coverage on $\mathrm{Pt}_{0.5} \mathrm{Ni}_{0.5}(110)$ is only 1.0 ML, suggesting that the $\mathrm{H}$-induced pairing-row $(1 \times 2)$ surface reconstruction of $\mathrm{Ni}(110)$ may not occur on the PtNi alloy. This situation is reminiscent of the above $\mathrm{Mo}_{0.75} \mathrm{Re}_{0.25}$ case, where alloying with Re apparently suppressed the formation of an intrinsic and a variety of $\mathrm{H}$-induced superstructures that are observed, e.g., on pure Mo(100) [275]. On the strongly Pt-enriched (111) surface of the PtNi alloy the $\mathrm{H}$ saturation coverage is only $0.2 \mathrm{ML}\left(1 \mathrm{ML}=1.64 \times 10^{15} \mathrm{~cm}^{-2}\right)$, i.e. much smaller than on $\operatorname{Pt}(111)\left(1.0 \mathrm{ML}=1.50 \times 10^{15} \mathrm{~cm}^{-2}\right)[67,280]$. This strong coverage reduction may be related to the compressed PtNi alloy surface lattice constant relative to $\mathrm{Pt}(111)$ (1 $M L)$, which may change the electronic surface properties with respect to $\mathrm{H}$ and $\mathrm{CO}$ adsorption [281]. Alternatively, Ref. [281] speculates that $\mathrm{H}$ adsorption on $\mathrm{Pt}_{0.5} \mathrm{Ni}_{0.5}$ may require surface $\mathrm{Ni}$ atoms, the number of which being small ( 12\%) on the (111) surface due to predominant segregation of Pt.

\subsubsection{Semiconductors}

\section{Diamond}

To our knowledge only a single ${ }^{15} \mathrm{~N}$ NRA investigation of hydrogen on diamond single crystal surfaces has been published. Jans et al. [432] prepared (100), (110), 
and (111) diamond surfaces by chemically treating single crystals in a $\mathrm{HNO}_{3}, \mathrm{HCl}$, and $\mathrm{H}_{2} \mathrm{SO}_{4}$ mixture. After transfer into vacuum and evacuation to $10^{-7} \mathrm{mbar}$, large coverages (several $\mathrm{ML}$ ) of excess $\mathrm{H}$ species were observed on these surfaces. This initial $\mathrm{H}$ coverage quickly desorbed under the ${ }^{15} \mathrm{~N}$ ion beam to yield more stable $\mathrm{H}$ layers of coverages very close to the values expected for ideal mono-hydride termination $\left(H_{t h}\right)$ of the individual diamond crystallographic planes, i.e., $3.16 \times 10^{15}$ $\mathrm{cm}^{-2}\left(0.95 \mathrm{H}_{\mathrm{th}}\right)$ on $(100), 2.23 \times 10^{15} \mathrm{~cm}^{-2}\left(0.98 \mathrm{H}_{\mathrm{th}}\right)$ on $(110)$, and $1.82 \times 10^{15} \mathrm{~cm}^{-2}(1.06$ $H_{\text {th }}$ ) on the (111) surface, respectively. Despite these nearly-ideal termination values, the 'stable' $\mathrm{H}$ coverage was found to vary with the ion beam current density and increased after the ion beam was blanked for a period of time, suggesting a dynamic equilibrium of beam-induced $\mathrm{H}$ desorption and readsorption from the residual gas.

Jans et al. intended to demonstrate the dependence of the nuclear reaction resonance Doppler width on the orientation of the $\mathrm{C}-\mathrm{H}$ bonds, which are inclined against the ${ }^{15} \mathrm{~N}$ ion beam incident along the surface normal direction with angles of $55^{\circ}$ for the $(100), 35^{\circ}$ for the (110), and $0^{\circ}$ for the (100) surface, respectively. The measured Doppler width of $14.0 \pm 0.2 \mathrm{keV}$, however, did not show any significant variation with the crystal orientation and was larger even than the expected value for the fully aligned (111) H surface (13.6 keV). This unfortunate result suggests that the diamond surfaces in Ref. [432] might not have attained their anticipated atomically controlled structures, probably as a consequence of less than ideal surface preparation and/or vacuum conditions during the NRA measurements. The principal possibility to observe the orientation dependent Doppler width of the nuclear reaction resonance for $\mathrm{H}$ bound covalently to spatially aligned dangling 
bonds on semiconductor surfaces has later been demonstrated by ${ }^{15} \mathrm{~N}$ NRA measurements at in-situ prepared and UHV-maintained H-terminated Si(111)(1x1)-H surfaces, which are described in the next subsection [67].

A larger body of literature treats $\mathrm{H}$ at surfaces of natural and synthetic diamond, which is of great relevance in diamond production by chemical vapor deposition (CVD), as well as for common surface processes such as H-plasma annealing or proton implantation $[6,433]$. Since in such situations $H$ is often also found to be absorbed in the diamond bulk or in the subsurface region, this is (briefly) described in Section 3.2.4.

\section{Silicon}

The interaction of hydrogen with silicon surfaces has been characterized extensively by a manifold of techniques, driven by the crucial importance of $\mathrm{H}$ terminated silicon surfaces in the fabrication of semiconductor devices and in their reliability physics, as well as out of fundamental interest in covalent $\mathrm{H}$ chemisorption on and desorption from semiconductor surfaces. Clean silicon surfaces generally reconstruct in order to lower their surface energy by minimizing the number of dangling bonds that exist in the bulk-truncated structure. Chemisorption bonds with hydrogen atoms remove or rearrange these Si dangling bond states, rendering $\mathrm{H}$ terminated Si surfaces with structures closer to the bulk truncation and with far less reactive properties.

Three characteristics of $\mathrm{H}$-terminated $\mathrm{Si}$ surfaces have attracted particular technological and scientific interest: (1) the passivating effect of the hydrophobic $\mathrm{H}$ 
adlayer that helps preventing the contamination of etch-cleaned Si wafers during handling in ambient conditions between successive stages of semiconductor device production, (2) the strong impact of surface hydrogen on the structure and epitaxial growth behavior of metals [434-436] and semiconductors [437-440] on silicon substrates (surfactant effect), and (3) a giant isotope effect of $D$ vs. $H$ with respect to hot electron-stimulated desorption [441, 442]. For a comprehensive discussion of the latter two phenomena as well as for a detailed presentation of the formation and thermal decomposition mechanisms of the hydrogen adsorption phases on the most stable silicon surface reconstructions consult the excellent review by Oura et al. [2].

The above work shall not be duplicated here and we confine ourselves to pointing out that by quantifying the $H(D)$ coverage NRA and ERDA have contributed substantially to establishing our current microscopic understanding of the hydrogen adsorption phases on silicon surfaces. The clarification of the complex $\mathrm{Si}(111)(7 \times 7)$ reconstruction [443], the development of surface imaging by STM [444], and the introduction of novel optical high-resolution surface vibrational spectroscopy (Multiple internal reflection (MIR)-FTIR [445]) coincided with early applications of ion beam techniques, i.e., $D\left({ }^{3} \mathrm{He}, \mathrm{p}\right)^{4} \mathrm{He}$ NRA $[283,290]$, ERDA $[252,259,260]$, and ${ }^{1} \mathrm{H}\left({ }^{15} \mathrm{~N}, \alpha \gamma\right){ }^{12} \mathrm{C}$ NRA $[65,83,203,446]$ to the (quantitative) analysis of hydrogen on well-defined single crystalline surfaces. Although $D\left({ }^{3} \mathrm{He}, \mathrm{p}\right)^{4} \mathrm{He}$ NRA provides no notable depth resolution, its application to quantify the D coverage on Si surfaces is justified by the negligible hydrogen solubility in bulk Si [109]. After the TOF-ERD technique was refined to allow combination with MEIS by Copel and Tromp at IBM 
$[252,288,437]$, quantitative and highly depth resolved $H$ coverage measurements became also possible with ERD.

The absolute hydrogen coverage values combined with the identification of $\mathrm{Si}$ mono-, di-, and trihydride units (see Fig. 37 (a) for their structures on Si(100)) by vibrational fingerprinting with IRAS [447-449] and STM surface imaging [450-453] have provided the key information to characterize the respective adsorption phases. This has been instrumental in assessing the quality of the various $\mathrm{H}$-termination layers on $\mathrm{Si}$ surfaces that are obtained by different methods of preparation. Principally, there are two main routes leading to clean and nearly ideally $\mathrm{H}$ terminated Si surfaces: (a) ex-situ wet chemical oxidation $\left(\mathrm{H}_{2} \mathrm{O}_{2}\right)$ and subsequent etching in aquous HF solutions [286, 288, 446, 447, 454, 455] and (b) in-situ exposure of UHV-cleaned Si surfaces to atomic H (or D) [259, 260, 283, 285, 287, 448-450, 452, $453,456]$.

Si(111)

According to IRAS and STM characterizations, ideally smooth and only monohydride-terminated surfaces can be prepared on $\mathrm{Si}(111)$ by wet chemical etching in $\mathrm{NH}_{4}$ buffered $\mathrm{HF}$ solutions [454, 457]. The $\mathrm{pH}$ of the etching solution is essential to achieve the homogeneous monohydride termination, as treatment in unbuffered dilute HF yields the Si(111) surfaces covered with a microscopically rough layer that contains a mixture of $\mathrm{SiH}$ monohydride, $\mathrm{SiH}_{2}$ dihydride and $\mathrm{SiH}_{3}$ trihydride species [447]. Alternatively, a monohydride-terminated $\mathrm{Si}(111)(1 \times 1)-\mathrm{H}$ surface can be produced in-situ through atomic $\mathrm{H}$ exposure of UHV-cleaned $\mathrm{Si}(111)(7 \times 7)$ at $380^{\circ} \mathrm{C}$, 
which yields a surface condition with less than $5 \%$ remaining stacking faults, $1 \%$ of point defects, and less than $0.5 \%$ contamination $[452,453]$.

The Si-H bonds on the uniformly monohydride-terminated $\mathrm{Si}(111)(1 \times 1)$ are oriented perpendicular to the surface, and subsurface hydrogen is not expected since the $\mathrm{H}$ solubility in $\mathrm{Si}$ is immeasurably small [109]. The Si(111)(1x1)-H system thus presents an ideal reference system to demonstrate the possibility of zero-point vibration spectroscopy with ${ }^{15} \mathrm{~N}-\mathrm{H}$ NRA, in which the sensitivity of the Doppler broadening component in the surface resonance excitation curve to the nature of the chemical bonding state of the surface $H$ atoms is exploited $[63,64,66]$. Fukutani et al. measured the ${ }^{1} \mathrm{H}\left({ }^{15} \mathrm{~N}, \alpha \gamma\right){ }^{12} \mathrm{C}$ resonance excitation curves on a $\mathrm{H}$-terminated Si(111) target (prepared in-situ by atomic $\mathrm{H}$ exposure) using the surface normal (dashed line) and a $40^{\circ}$ inclined (solid line) ${ }^{15} \mathrm{~N}$ ion incidence geometry (Fig. 36) [67]. For clarity, the normal incidence profile is represented only by its Gaussian fit curve (dashed line in Fig. 36; the original data can be seen in Fig. 3).

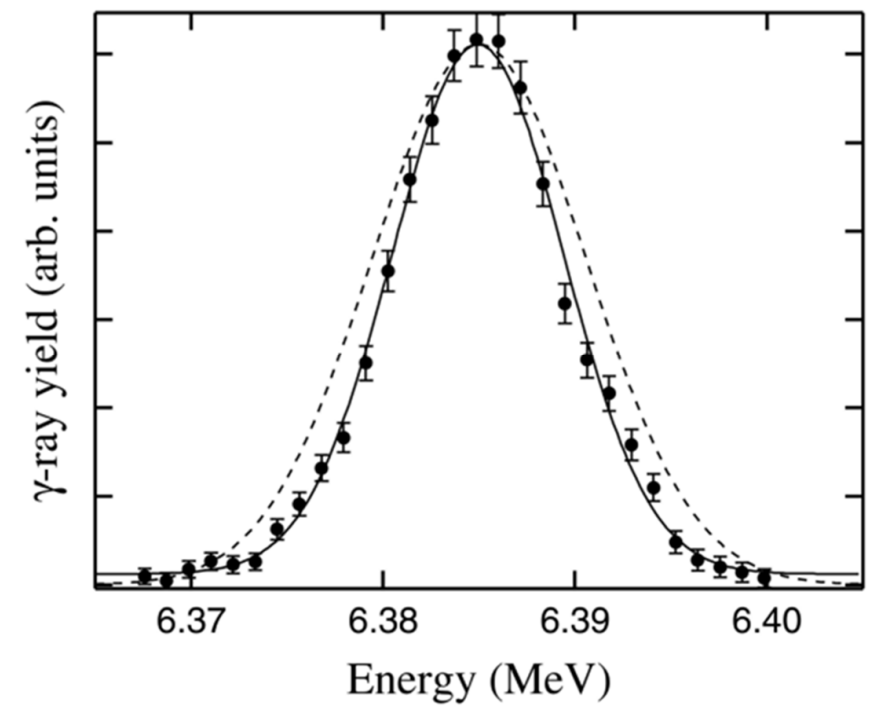

Fig. 36. ${ }^{1} \mathrm{H}\left({ }^{15} \mathrm{~N}, \alpha \gamma\right){ }^{12} \mathrm{C}$ surface resonance excitation curve for $\mathrm{Si}(111)-(1 \times 1)-\mathrm{H}$ taken at an incidence angle of $\alpha_{i}=40^{\circ}$ with the surface normal (data points and solid line, Gaussian fit). For comparison the Gaussian fit profile recorded at $0^{\circ}$ incidence (data in Fig. 3) is shown as the dashed curve. Adapted from Ref. [67]. 
By deconvoluting the ion beam energy spread ( $3 \mathrm{keV})$ and the NR resonance as described in Section 2.1, Fukutani et al. obtained the Doppler width parameter $\delta_{D}$ in Eq. (2.7), from which the zero-point vibrational energy of the $\mathrm{H}$ atoms in the surface normal direction $E_{\mathrm{H}, \mathrm{Z}}$ could be determined with Eq. (2.8) as $123.4 \pm 4.6 \mathrm{meV}$ [67]. This value of the zero-point vibrational energy amounts to almost exactly one half of the first excitation energy of the surface-normal Si-H stretching vibration known from IRAS and HREELS experiments (258 meV) [454], suggesting that the Si-H bond potential in this vibrational mode is reasonably well described by a harmonic oscillator model. As seen in Fig. 36, the resonance curve recorded under the incidence angle of $\alpha_{1}=40^{\circ}$ is significantly narrower compared to the data at normal incidence. The physical reason for this effect is that the vibrational $\mathrm{H}$ motion effective for the NR Doppler broadening is the projection of all $\mathrm{H}$ vibrational modes in the ion beam incidence direction [64]. At an incidence angle inclined against the surface normal $\left(\alpha_{i}\right)$ the beam thus also probes the $\mathrm{H}$ bending vibration mode parallel to the surface ('frustrated translation'), which is known to be softer than the vertical $\mathrm{Si}-\mathrm{H}$ stretch (corresponding to a first excitation energy of $78 \mathrm{meV}$ for $\mathrm{Si}(111)(1 \times 1)-\mathrm{H}$ $[458,459])$. With $E_{H, x}$ as the zero-point energy of the surface-parallel vibrational mode, the effective variance of the Doppler broadening distribution becomes [67]:

$$
\delta_{D}=\sqrt{\frac{2 m_{N} E_{\mathrm{Res}}}{m_{H}}\left(\frac{\sin ^{2} \alpha_{i}}{E_{H, X}}+\frac{\cos ^{2} \alpha_{i}}{E_{H, Z}}\right)^{-1}} .
$$

By performing an analog analysis of the $40^{\circ}$ incidence resonance curve as described above and using the $E_{\mathrm{H}, \mathrm{z}}$ value determined there, Fukutani et al. obtained $E_{H, x}$ with Eq. (3.1) as $44.6 \pm 6.2 \mathrm{meV}$. This value is again reasonably close to half the 
vibrational excitation energy of the $\mathrm{Si}-\mathrm{H}$ bending mode, suggesting that also this (mainly) surface-parallel motion occurs within a dominantly harmonic potential, as one might expect from this covalently bound surface $\mathrm{H}$ adsorption system.

The above study [67] of $\mathrm{Si}(111)(1 \times 1)-\mathrm{H}$ so far demonstrated the applicability of zero-point vibrational spectroscopy with ${ }^{15} \mathrm{~N}-\mathrm{H}$ NRA at a well-aligned $\mathrm{H}$ adsorption system most clearly. Earlier attempts to do this [446] had apparently failed, from a retrospective viewpoint presumably due to experimental reasons. In Ref. [446] the HF-etched H-terminated $\mathrm{Si}(111)$ surfaces were not annealed in vacuum prior to NRA after transferring the samples into the analysis system. NRA then found large quantitites $(3.5 \mathrm{ML})$ of volatile $\mathrm{H}$ species on the sample surfaces in addition to the expected monohydride termination layer. These weakly adsorbed $\mathrm{H}$-bearing compounds were desorbed by ion beam impact under NRA observation until a stable $\mathrm{H}$ coverge of ca. $1 \mathrm{ML}$ was generated in a dynamic equilibrium with $\mathrm{H}$ readsorption from the UHV residual gas ( $10^{-9}$ mbar range). In order to deplete the $\mathrm{H}$-bearing adsorbates, rather large ${ }^{15} \mathrm{~N}$ ion fluences of $5 \times 10^{16} \mathrm{~cm}^{-2}$ had to be administered to the samples before the resonance excitation curves could be recorded on the stable H monolayer. Hartmann et al. then observed NRA Doppler widths that were characteristic of $\mathrm{Si}-\mathrm{H}$ bonds in a disordered layer (with random beam-bond orientations) and showed no dependence at all on the ${ }^{15} \mathrm{~N}$ ion incidence angle onto the surface [446]. Although it is not clear whether this disordered Si-H layer was a consequence of the sample preparation or excessive ${ }^{15} \mathrm{~N}$ ion irradiation, the results may emphasize again the statements of Section 2.2.5 that great care has to be taken to exclude irradiation damage and contamination effects in the NRA analysis of 
especially sensitive surface $\mathrm{H}$ systems. In comparison, the ${ }^{15} \mathrm{~N}$ ion fluence to record one entire resonance excitation curve from the H-terminated Si(111) prepared insitu by Fukutani et al. [67] was only $2 \times 10^{15} \mathrm{~cm}^{-2}$ and the analysis was performed in an UHV system with a base pressure $<1 \times 10^{-10}$ mbar (Section 2.2.2).

\section{Si(100)}

Addressing atomic hydrogen exposed $\mathrm{Si}(100)$ surfaces first, a series of investigations has shown that the $\mathrm{Si}(100)(2 \times 1)-\mathrm{H}$ monohydride phase is obtainable either by moderate atomic $H(D)$ exposures of clean $\mathrm{Si}(100)$ surfaces at room temperature, $[259,285,450]$ by heating the $\mathrm{H}$-saturated $\mathrm{Si}(100)-(2 \times 1)$ surface from RT to $600 \mathrm{~K}[283,449]$, or by applying a $\mathrm{H}$ saturation dose at $650 \mathrm{~K}[450]$. These 'recipes' are based on the fact that the dihydride $\left(\mathrm{SiH}_{2}\right)$ and trihydride $\left(\mathrm{SiH}_{3}\right)$ species (Fig. 45 (a)), which form at larger exposures after completion of the monohydride phase, desorb above $\sim 600 \mathrm{~K}\left(\beta_{3}\right.$ and $\beta_{2}$ TDS peaks at $\sim 450 \mathrm{~K}$ and at $\sim 650 \mathrm{~K}$, respectively), whereas the monohydride phase is stable up to $\sim 730 \mathrm{~K}$ ( $\beta_{1}$ TDS peak at $\sim 760 \mathrm{~K}$ in Fig. 37 (b)) [460].

In the monohydride phase at room temperature the original dimer row reconstruction and corresponding $(2 \times 1)$ periodicity are conserved and each of the two Si dimer dangling bonds are terminated by a single $\mathrm{H}$ atom (Fig. 37 (a)), which corresponds to a $\mathrm{H}$ coverage of $1.0 \mathrm{ML}$ [450]. Jiang et al. measured the evolution of the deuterium coverage on initially clean $\mathrm{Si}(100)(2 \times 1)$ under atomic $D$ exposure at $400 \mathrm{~K}$ with $\mathrm{D}\left({ }^{3} \mathrm{He}, \mathrm{p}\right)^{4} \mathrm{He}$ NRA and characterized the corresponding adsorption phases with LEED (Fig. 38). Subsequent to completion of the monohydride phase the $\mathrm{H}$ uptake slows considerably $[259,285]$ and the surface gradually attains a $(1 \times 1)$ 
structure, with exposure and temperature dependent 'saturation' coverages (Table 3) reported as 1.5-1.6 ML [283, 284], 1.7 $\pm 0.285 \mathrm{ML}(\mathrm{RT})$ [456], $1.6 \pm 0.1 \mathrm{ML}(400 \mathrm{~K})$ [285], 1.7 ML $\left(100^{\circ} \mathrm{C}\right)$ [259], $1.85 \pm 0.18 \mathrm{ML}(\mathrm{RT})$ [259], and $1.9 \mathrm{ML}\left(\mathrm{T}_{\mathrm{e}}=210 \mathrm{~K}, \mathrm{TDS}\right.$, containing some $\mathrm{SiH}_{3}$ ) [460]. These coverages are slightly smaller than the $2 \mathrm{ML}$ expected for the ideal bulklike (1x1) 'dihydride' structure depicted in Fig. 37 (a), in which the original Si dimer bonds are removed and each surface Si atom holds two H atoms.

(a)
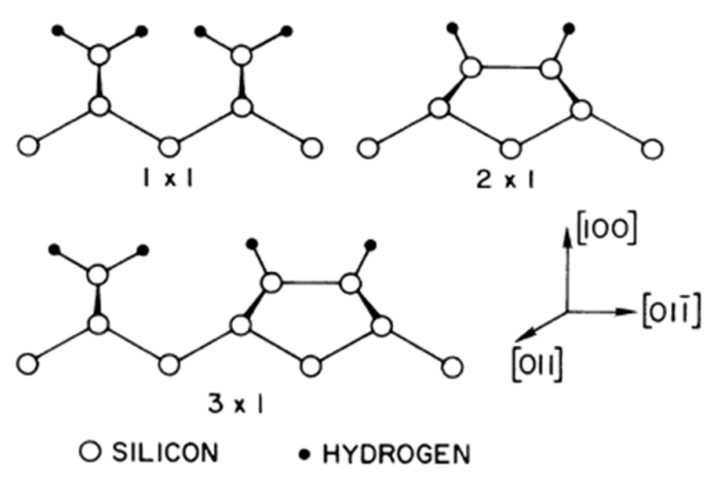

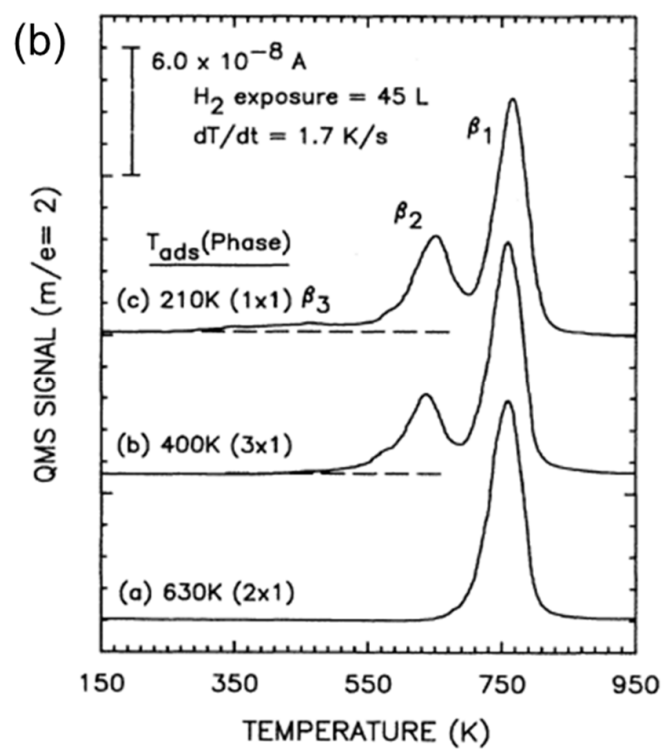

Fig. 37. (a) Hydrogen adsorption structures on $\mathrm{Si}(100)$ : Dihydride-terminated $1 \times 1$ phase, monohydride-terminated $2 \times 1$ phase, and mono- and dihydride terminated $3 \times 1$ phase. Adapted from Ref. [450]. (b) $\mathrm{H}_{2}$ TDS spectra from $\mathrm{Si}(100)$ saturated with atomic $\mathrm{H}$ at (a) $630 \mathrm{~K}$, (b) $400 \mathrm{~K}$, and (c) $210 \mathrm{~K}$. Adapted from Ref. [460] with permission from APS.

At $370-400 \mathrm{~K}$ an intermediate $(3 \times 1)$ structure evolves from the $(2 \times 1)$ monohydride phase $[285,448,450]$, which consists of alternating monohydride and dihydride units (Fig. 37 (a)) and thus corresponds to a $\mathrm{H}$ coverage of $1.33 \mathrm{ML}$. This (3x1) phase, however, does not represent the saturated surface condition even at 
$400 \mathrm{~K}$ as originally suggested on the basis of IR spectroscopy [448]. Instead, Jiang's data in Fig. 38 show that the intermediate $(3 \times 1)$ phase degrades under further $D$ uptake and slowly converts into the $(1 \times 1)$ structure upon prolonged $D$ exposure [285].

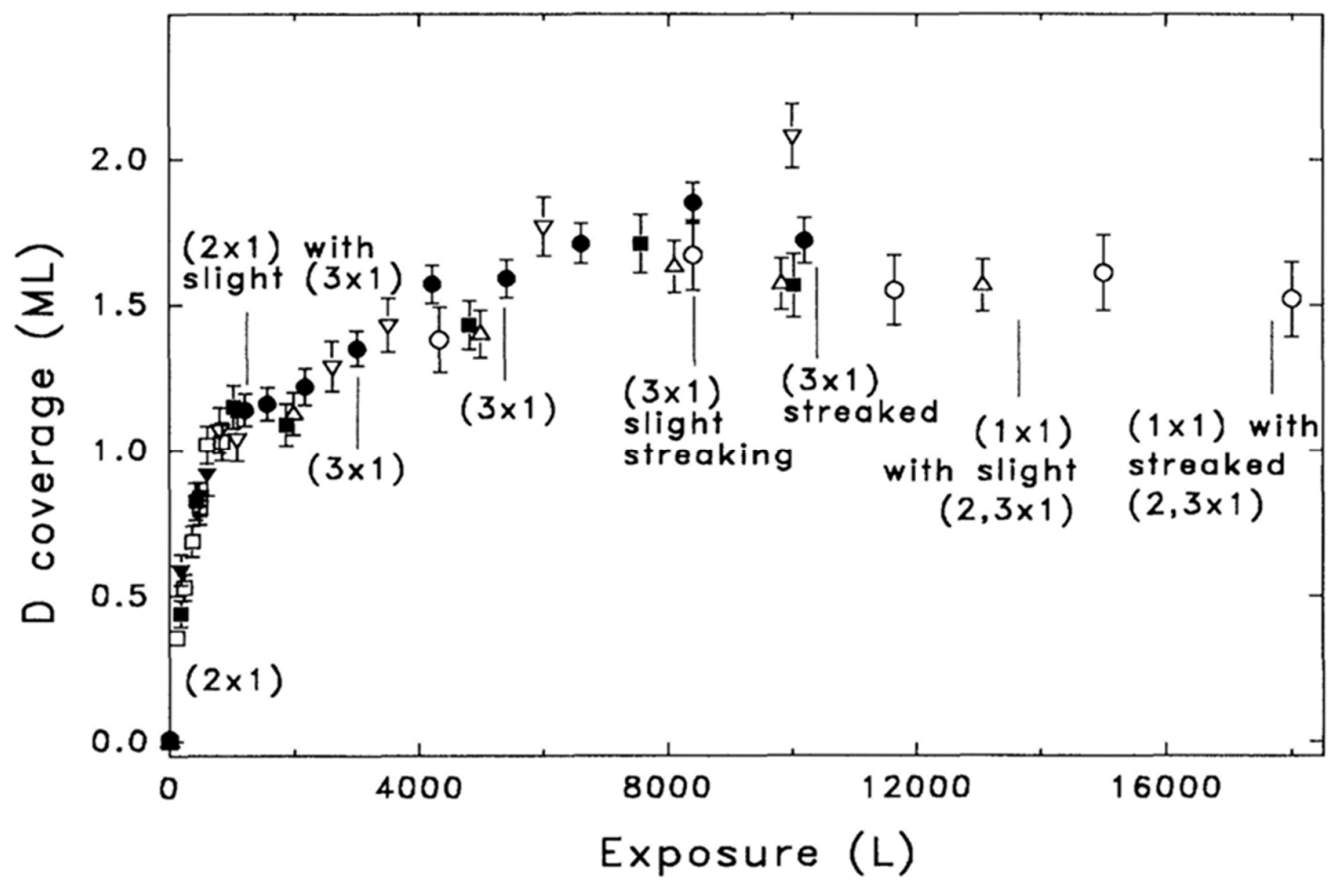

Fig. 38. Evolution of the absolute deuterium coverage measured with ${ }^{3} \mathrm{He}(\mathrm{D}, \mathrm{p})^{4} \mathrm{He}$ NRA and the corresponding adsorption phases (LEED) on $\mathrm{Si}(100)$ under atomic $\mathrm{D}$ exposure in $2 \times 10^{-6}$ Torr $\mathrm{D}_{2}$ gas at $400 \mathrm{~K}$. Adapted from Ref. [304] with permission from APS.

As noted above, the $\mathrm{H}$ saturation coverage in the $(1 \times 1)$ phase was found to be consistently larger than $1.33 \mathrm{ML}$ by several groups, indicating that the $(3 \times 1) \rightarrow(1 \times 1)$ transformation is not due to a simple increase of disorder in the (3x1) phase [448] but that it reflects a continued surface etching process, in which the number of $\mathrm{SiH}_{2}$ species increases gradually as the coverage approaches the ideal dihydride termination $[285,450,460]$. Since the formation of higher hydride species involves cleavage of the Si-Si dimer bonds in the $2 \times 1$ reconstruction, their presence 
introduces a form of microscopic surface roughness on monohydride-terminated Si(100)-(2x1) that can disrupt the homoepitaxial growth of Si on Si(100) subtrates when the $\mathrm{H}$ coverage exeeds one monolayer [437].

The tendency to form higher hydrides as well as the pronouncedly larger etching rate in the (111) direction relative to (100) also affect the quality of wet chemically etched $\mathrm{Si}(100)$ surfaces. Whereas etching in $\mathrm{NH}_{4} \mathrm{~F}$ buffered $\mathrm{HF}$ solutions produces ideally smooth and only monohydride-terminated surfaces on Si(111) [454], the same treatment renders $\mathrm{Si}(100)$ surfaces covered by several hydride species $(\mathrm{SiH}$, $\mathrm{SiH}_{2}$, and $\left.\mathrm{SiH}_{3}\right)[288,447]$. Nonetheless, this mixed hydride termination layer is hydrophobic and effectively protects $\mathrm{Si}(100)$ wafers from recontamination in ambient conditions; therefore the HF etching procedure is widely applied in semiconductor industry [461]. The resulting absolute $\mathrm{H}$ coverage in the passivating H-termination layer on HF-etched $\mathrm{Si}(100)$ had nonetheless remained obscure for quite some time until Wilde et al. determined it as $1.3 \pm 0.1 \mathrm{ML}$ with ${ }^{1} \mathrm{H}\left({ }^{15} \mathrm{~N}, \alpha \gamma\right){ }^{12} \mathrm{C}$ NRA [286] (cf. Fig. 39 (a) in the next subsection). The value is remnant of the $\mathrm{H}-(3 \times 1)$ phase described above and quite consistent with Chabal's IRAS observation [447] that predominantly $\mathrm{SiH}$ mono- and $\mathrm{SiH}_{2}$ dihydrides form under the $\mathrm{NH}_{4} \mathrm{~F}$-buffered $\mathrm{HF}$ etching with only a minor fraction of $\mathrm{SiH}_{3}$ units. 


\section{$S i_{1-x} G e_{x}(100)$}

In analogy to $\mathrm{Si}(100)$, HF etching treatments are also commonly applied procedures to hydrogen-passivate SiGe alloy surfaces in the fabrication of high performance SiGe/Ge/SiGe heterostructure devices through epitaxial growth techniques [462]. The resulting $\mathrm{H}$ coverages and the stability of the $\mathrm{H}$-terminating layers on $\mathrm{Si}_{1-x} \mathrm{Ge}_{x}$ alloys in ambient conditions as a function of the Ge content were characterized with ${ }^{1} \mathrm{H}\left({ }^{15} \mathrm{~N}, \alpha \gamma\right){ }^{12} \mathrm{C}$ NRA after HF-etching and air-to-vacuum transfer [286]. The hydrogen coverages on HF-etched $\mathrm{Si}_{1-x} \mathrm{Ge}_{\mathrm{x}}$ surfaces $(\mathrm{x}=0,0.30,0.60,0.82)$ were determined from the integrated NRA surface resonance excitation curves after correcting the data for the ion irradiation-induced $\mathrm{H}$ decay, which showed different kinetics for the individual samples. The results are summarized in Fig. 39.
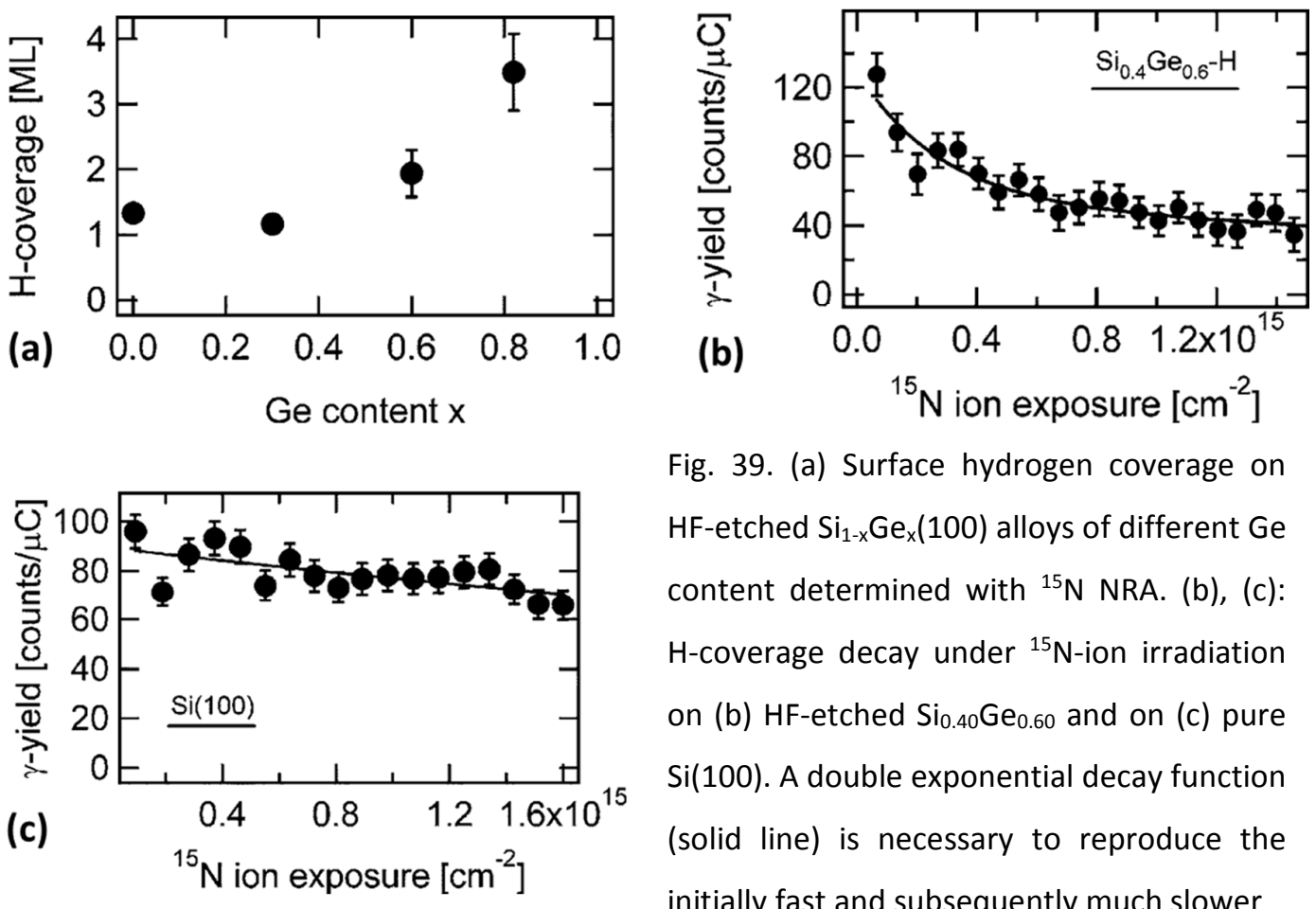

Fig. 39. (a) Surface hydrogen coverage on HF-etched $\mathrm{Si}_{1-x} \mathrm{Ge}_{\mathrm{x}}(100)$ alloys of different $\mathrm{Ge}$ content determined with ${ }^{15} \mathrm{~N}$ NRA. (b), (c): $\mathrm{H}$-coverage decay under ${ }^{15} \mathrm{~N}$-ion irradiation on (b) HF-etched $\mathrm{Si}_{0.40} \mathrm{Ge}_{0.60}$ and on (c) pure $\mathrm{Si}(100)$. A double exponential decay function (solid line) is necessary to reproduce the initially fast and subsequently much slower

$\mathrm{H}$ depletion on the $\mathrm{Si}_{0.40} \mathrm{Ge}_{0.60}$ alloy (b), whereas a single exponential decay is observed on $\mathrm{Si}(100)$ (c). Adapted with permission from Ref. [286]. 
As-etched $\mathrm{Si}_{0.7} \mathrm{Ge}_{0.3}$ was found to expose a similar initial H-coverage $(1.2 \pm 0.1$ $\mathrm{ML})$ as pure $\mathrm{Si}(100)(1.3 \pm 0.1 \mathrm{ML})$, but the alloys of higher Ge contents had strongly increased $\mathrm{H}$ coverages that approached $2 \mathrm{ML}$ at $\mathrm{x}=0.60$ and even exceeded $3 \mathrm{ML}$ at $x=0.82$ (Fig. 39 (a)). In addition to these large $\mathrm{H}$ coverages on the Ge-rich SiGe alloys, the Doppler width of their respective surface resonance profiles was significantly broadened and the ${ }^{15} \mathrm{~N}$-ion-induced desorption kinetics of the surface $\mathrm{H}$ atoms showed a second, initially rapid decay channel (Fig. 39 (b)) that was neither observed on pure $\mathrm{Si}(100)$ (Fig. 39 (c)) nor on $\mathrm{Si}_{0.7} \mathrm{Ge}_{0.3}$.

Together, these observations were interpreted in terms of $\mathrm{H}$-rich contaminants (most likely hydrocarbons from the laboratory air) that repopulate the surfaces of the Ge-rich alloys $(x=0.60,0.82)$ within minutes after removal from the HF etching solution and thus increase the NRA Doppler broadening width owing to their stiffer $(\mathrm{C}-\mathrm{H}, \mathrm{O}-\mathrm{H})$ vibrational stretching modes compared to those of the $\mathrm{Si}-\mathrm{H}_{\mathrm{n}}$ surface species [286]. The observation that the resistance of HF-etched $\mathrm{Si}_{1-x} \mathrm{Ge}_{x}$ alloys against recontamination in air appearently reduces drastically at higher Ge contents was in agreement with the practical experience from MBE production that the $\mathrm{H}$ passivation layers become increasingly unstable at Ge contents above $60 \%$. Although the $\mathrm{H}$-coverage on the $\mathrm{Si}_{0.7} \mathrm{Ge}_{0.3}$ alloy was similar as on pure $\mathrm{Si}(100)$ right after the $\mathrm{HF}$ etching, it gradually increased over a duration of one week in air from 1.2 to $1.6 \mathrm{ML}$ and also the vibrational Doppler width increased from initially 9.8 to $11.2 \mathrm{keV}$, suggesting that a slight recontamination of the $\mathrm{Si}_{0.7} \mathrm{Ge}_{0.3}$ surface occurs during air storage [286]. On HF-etched pure $\mathrm{Si}(100)$, on the other hand, no significant changes of the $\mathrm{H}$ coverage nor of the Doppler width were observed during 167 hours in air 
within the experimental accuracy, consistent with the well-known rather stable properties of $\mathrm{H}$-terminated $\mathrm{Si}(100)$. One should note, however, that despite the apparent integrity of the $\mathrm{H}$-termination layer a complete passivation of the surface in air cannot be deduced from the NRA data alone, as a very slow but nonetheless noticeable process of surface oxidation is known to occur, which inserts $\mathrm{O}$ atoms into Si-backbonds but leaves the terminating H-layer intact [455].

The above study exemplifies that although the ${ }^{15} \mathrm{~N}-\mathrm{H}$ NRA technique is principally insensitive to the chemical oxidation state of ${ }^{1} \mathrm{H}$, a distinction of well-passivated SiGe surfaces terminated exclusively by hydrides of Si and Ge from those partially covered by contaminating adsorbates can be made if the information on the total $\mathrm{H}$ coverage, on the $\mathrm{H}$ zero-point vibrational width, and on the ${ }^{15} \mathrm{~N}$ ion-induced $\mathrm{H}$-decay kinetics are considered in combination. The fact that the Doppler broadening of the surface resonance curves in NRA reflects the vibrational nature of the surface $\mathrm{H}$ species and thus bears a certain degree of chemical information was pointed out as early as 1986 by Zinke-Allmang et al. $[63,64]$. Presumably due to the scarcity of well-defined $\mathrm{H}$ surface target systems and the rather limited resolution with respect to differences in vibrational frequency $\left(\omega_{\text {vib }}\right)$, however, this potential NRA application has rarely found analytical use $[66,67,286,446]$.

By applying Eq. (2.8) in the harmonic approximation $\left(E_{H} \approx 1 / 2 \hbar \omega_{\text {vib }}\right)$ the Doppler broadening parameter $\left(\delta_{D}\right)$ for different chemically bound $H$ species can be estimated from respective vibrational excitation energies, and the resulting width (FWHM) of the NRA surface resonance profiles $\left(\Delta E_{\text {tot }}\right)$ follows from Eq. (2.10) for $z=$ 0 . Assuming that the vibrational amplitude is fully aligned in the ${ }^{15} \mathrm{~N}$ ion incidence 
direction (for a disordered surface layer a reducing factor of $\sim 0.7(=1 / \sqrt{ } 2)$ enters from integration over all possible bond-beam angles between 0 and $90^{\circ}$ ), $\Delta E_{\text {tot }}$ values of $13.1 \mathrm{keV}$ for $\mathrm{Si}-\mathrm{H}\left(\hbar \omega_{\text {vib }}=2100 \mathrm{~cm}^{-1}\right), 14.7 \mathrm{keV}$ for $\mathrm{C}-\mathrm{H}\left(\hbar \omega_{\text {vib }}=\sim 2700 \mathrm{~cm}^{-1}\right)$, and 16.5 keV for $\mathrm{O}-\mathrm{H}\left(\hbar \omega_{\text {vib }}=\sim 3500 \mathrm{~cm}^{-1}\right)$ species result for an incident ion beam energy spread of $\Delta E_{\mathrm{B}}=3 \mathrm{keV}$. Experimentally $\Delta E_{\text {tot }}$ is easily determined with an accuracy of $\sim<0.5 \mathrm{keV}$, thus in the present example [286] the $\mathrm{Si}-\mathrm{H}_{\mathrm{n}}$ surface species were clearly distinguishable from $\mathrm{C}-\mathrm{H}$ or $\mathrm{O}-\mathrm{H}$ contaminants.

\subsubsection{Insulators}

Owing to the electrostatic charging problem that affects bulk samples of insulating materials under ion or electron probing beams, the preparation and characterization of single crystalline insulator surfaces is inherently difficult with standard techniques of surface analysis. Much progress in the Surface Science of metal oxides has been achieved after methods of charge compensation for bulk samples were introduced [463]. Later, especially the establishment of preparation routes for thin epitaxial oxide films on metal substrates has very significantly expanded the range of well-ordered metal oxides that can be studied by Surface Science techniques, which propelled the field much further [464]. Some oxides $\left(\mathrm{TiO}_{2}\right.$, $\mathrm{ZnO}, \mathrm{SrTiO}_{3}$, etc.) also develop a certain bulk or surface conductivity in presence of impurities (hydrogen being discussed as one of them [38]) or oxygen vacancies that form by annealing in UHV, and hence permit surface analysis with charged particle probes. The interaction with hydrogen or water can rather dramatically change the chemical and physical properties of oxide surfaces; surface hydroxylation, reconstruction, metallization, hydrophilicity, hydrogen incorporation into the bulk or 
subsurface region, and induction of electrical conductivity are prominent examples of the possible effects. These phenomena have tremendous technological importance for heterogeneous catalysis, photocatalysis, self-cleaning and antifogging coating materials, electronic and photonic devices, and sensor applications. Accordingly, the subject has received considerable attention from the Surface Science community and is documented in excellent reviews [3, 294, 465, 466].

Nevertheless, compared to the wealth of knowledge on the hydrogen behavior on metal and semiconductor surfaces described in the preceding sections, there is still a striking lack of quantitative information on the hydrogen coverage on single crystalline oxide surfaces under well-defined conditions in the literature. Most of the existing knowledge on surface hydroxyl on oxides stems from vibrational spectroscopy (HREELS, IRAS) and XPS, where $\mathrm{OH}$ is identified by a characteristic shoulder in the bulk oxide $02 \mathrm{p}$ signal at a $~ 1.5 \mathrm{eV}$ higher binding energy. To our best knowledge, only two quantitative ${ }^{15} \mathrm{~N}$ NRA evaluations of hydrogen interactions with single crystalline oxide surfaces have been published so far.

\section{$\operatorname{ZnO}(10 \overline{1} 0)$}

The hydrogen content on the surface, in the subsurface region, and in the bulk of hydrothermally grown ZnO single crystals of different surface orientations (pure Znterminated polar $\mathrm{Zn}(0001)$, pure O-terminated polar $\mathrm{ZnO}(000 \overline{1})$, and mixed ( $\mathrm{Zn}$ and O) terminated non-polar $\mathrm{ZnO}(10 \overline{1} 0)$ ) was studied with ${ }^{15} \mathrm{~N}$ NRA, comparing pristine samples to those after in-situ exposure to atomic hydrogen or water vapor [291]. The structural details of these $\mathrm{ZnO}$ surfaces and their behavior towards $\mathrm{H}$ adsorption are comprehensively reviewed by Wöll [294]. Traeger et al. determined native bulk H 
concentrations of $(0.02-0.04)$ at.\% in the above $\mathrm{ZnO}$ samples, which did not depend on their surface orientation [291]. A roughly 10 times larger $\mathrm{H}$ amount (up to 0.17 at. \%) was detected in the $\mathrm{ZnO}$ subsurface region in $10 \mathrm{~nm}$ depth. A fraction of this subsurface $\mathrm{H}$ could reversibly be removed by heating to $550{ }^{\circ} \mathrm{C}$ and subsequently be reloaded with atomic $\mathrm{H}$ dosed at room temperature, yet 0.1 at.\% always stably remained (heating above $550^{\circ} \mathrm{C}$ was avoided to prevent formation of O-vacancies).

For the mixed-terminated nonpolar $\mathrm{ZnO}(10 \overline{1} 0)$ surface the $\mathrm{H}$ coverage and $\mathrm{H}$ uptake into the near surface region under atomic $\mathrm{H}$ dosage was studied in more detail. The same saturation $\mathrm{H}$ coverage was found to result reproducibly on $\mathrm{ZnO}(10 \overline{1} 0)$ either from surface hydroxylation with a dose of $1-2 \times 10^{-8} \mathrm{mbar} \mathrm{H}_{2} \mathrm{O}$ vapor at $130-200^{\circ} \mathrm{C}$ or by atomic $\mathrm{H}$ exposure at $30-100^{\circ} \mathrm{C}$. After applying the same $\mathrm{H}_{2} \mathrm{O}$

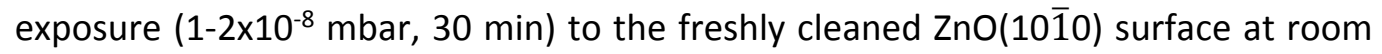
temperature $\left(25^{\circ} \mathrm{C}\right)$, the saturated amount of surface $\mathrm{H}$ was found to be twice as large (Fig. 40 (a)). Using a calibrated $\gamma$-detection system, these discrete $\mathrm{H}$ coverage conditions were found to correspond to about $80 \%$ of the $\mathrm{H}$ densities expected for ideal termination layers with $\mathrm{H}$ equivalents of 1 and $2 \mathrm{ML}$, respectively [291]. The 1 $M L$ structure (Fig. 40 (b)) contains one $\mathrm{H}$ atom per surface unit cell $\left(5.9 \times 10^{14} \mathrm{~cm}^{-2}\right)$ bound in an $\mathrm{OH}$ group and is metallic [467]). The $2 \mathrm{ML} \mathrm{H}$ structure (Fig. 40 (c)) holds an equivalent of one partially dissociated $\mathrm{H}_{2} \mathrm{O}$ molecule per surface unit cell, i.e., one $\mathrm{OH}$ group and an additional $\mathrm{Zn}-\mathrm{H}$ unit, which are stabilized by intralayer hydrogen bonds [468]]. 
(a)

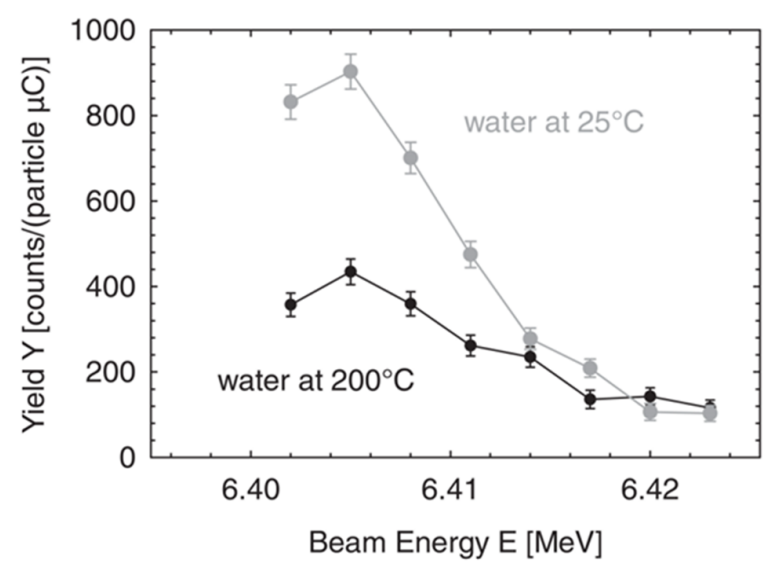

(b)

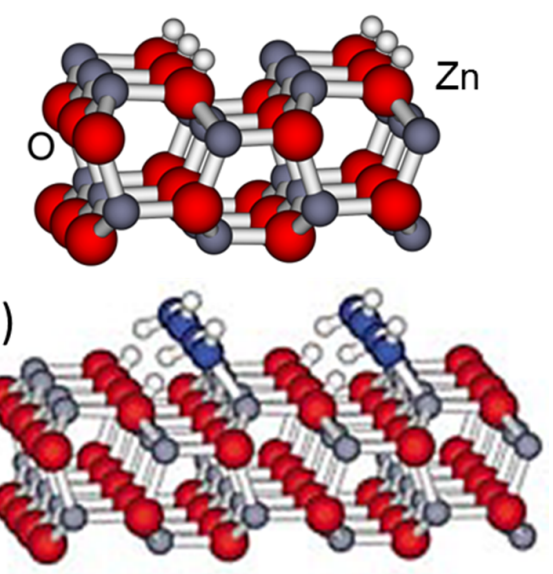

Fig. 40. (a) ${ }^{1} \mathrm{H}\left({ }^{15} \mathrm{~N}, \alpha \gamma\right)^{12} \mathrm{C}$ resonance excitation curves of $\mathrm{ZnO}(10 \overline{1} 0)$ surfaces saturated with $\mathrm{H}_{2} \mathrm{O}$ at $25^{\circ} \mathrm{C}$ (gray) and $200{ }^{\circ} \mathrm{C}$ (black). (b) Structure model of the $\mathrm{ZnO}(10 \overline{1} 0)-\mathrm{H}$ surface [467]. (c) Structure model for the half-dissociated $\mathrm{H}_{2} \mathrm{O} / \mathrm{ZnO}(10 \overline{1} 0)$ surface. Adapted from Refs. [291, $467,468]$.

Traeger et al. rationalized the significant deficiency of the ${ }^{15} \mathrm{~N}$ NRA-observed $\mathrm{H}$ coverages relative to the ideal $1 \mathrm{ML}$ or $2 \mathrm{ML}$ termination layers on $\mathrm{Zn}(10 \overline{1} 0)$ by crystal imperfections (evidenced by a less than $10 \mathrm{~nm}$ small coherent scattering domain size in HAS) [291], which may account for a surface defect density around $\sim 10 \%$. Although no further comments were offered, the $\gamma$-yield curves in Ref. [291] appear to be uncorrected for ion beam-induced $\mathrm{H}$-loss that is expected to occur to some degree even under the low ${ }^{15} \mathrm{~N}$ beam fluxes applied $\left(<20 \mathrm{nA}\right.$ of ${ }^{15} \mathrm{~N}^{2+}$ in a $\sim 3.5$ $\mathrm{mm}^{2}$ beam spot), which might also in part explain smaller than ideal $\mathrm{H}$ coverages derived from the yield curve integrals (cf. Section 2.2.5).

The saturation of the surface $\mathrm{H}$ peak under atomic $\mathrm{H}$ admission above room temperature was evidenced in Fig. 41 (a) by the identical height of the $\gamma$-yield curves. The high energy tail in the surface resonance peak developing under increasing atomic $\mathrm{H}$ dosage indicated a gradual $\mathrm{H}$ incorporation into the $\mathrm{ZnO}(10 \overline{1} 0)$ subsurface 
region. A close inspection of the NRA profiles in Fig. 41 (a) also revealed that the surface peak maximum continuously shifted to higher energy (up to $4 \mathrm{keV}$ after 50 min $\mathrm{H}$-loading). In order to separate the signal contributions from $\mathrm{H}$ on the surface and in the subsurface region, Traeger et al. convolved parameterized error function $\mathrm{H}$ concentration profiles (Fig. 41 (b)) (assuming an in-diffusion model under constant $\mathrm{H}$-supply through the $\mathrm{H}$-saturated surface) with the NRA instrumental function (a total width of $17 \mathrm{keV}$ was derived from the surface peak of the sample before $\mathrm{H}$ loading), until the excitation curves (solid lines in Fig. 41 (a)) were adequately reproduced. The analysis revealed that $\mathrm{H}$-loading for $20 \mathrm{~min}$ and $50 \mathrm{~min}$ increased the $\mathrm{H}$ content in the first $\mathrm{ZnO}$ double layer below the surface relative to the $\mathrm{H}$ surface layer density from the native content ( $1 \%)$ to $2.7 \%$ and $4.7 \%$, respectively.
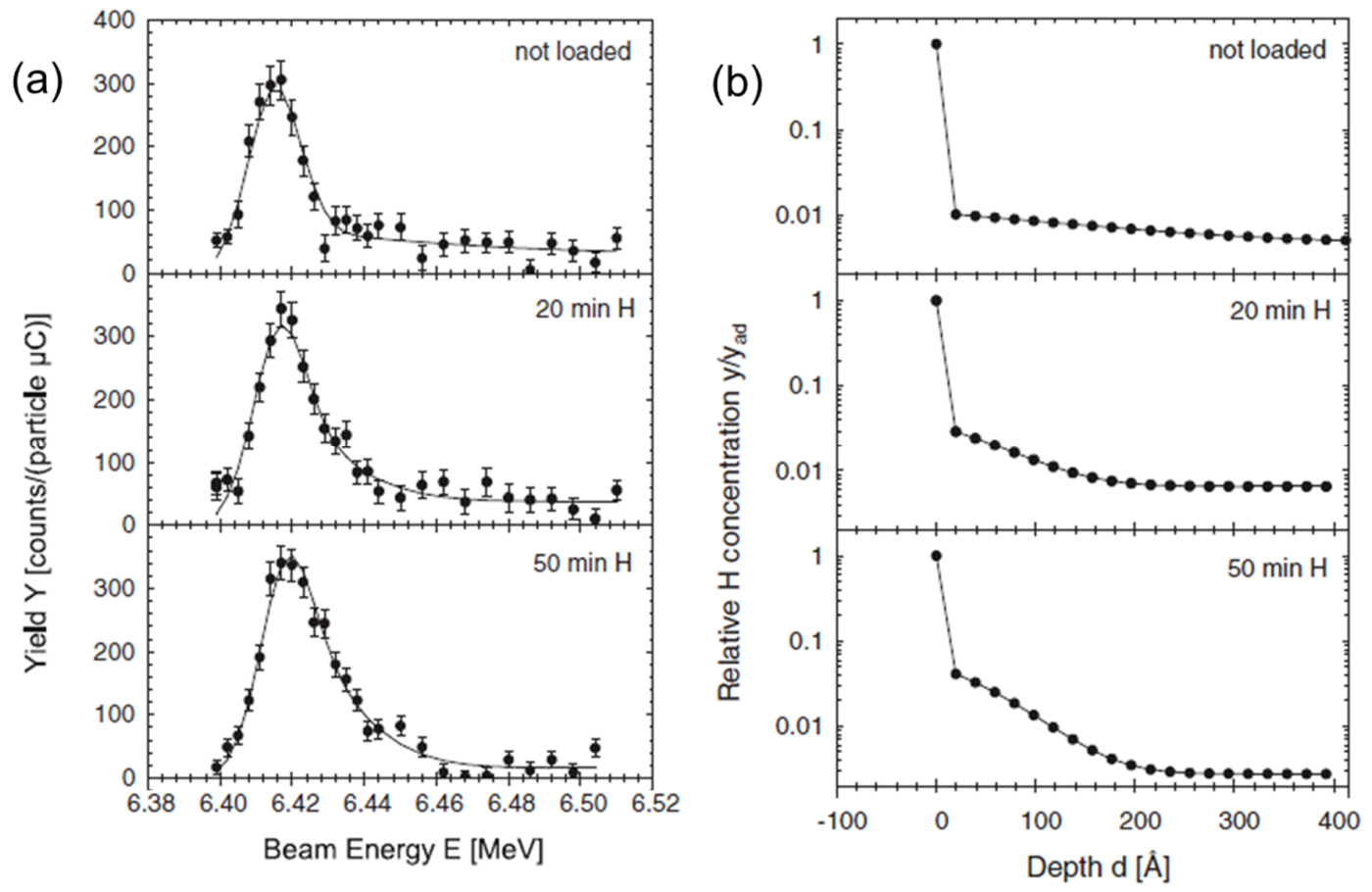

Fig. 41. (a) ${ }^{1} \mathrm{H}\left({ }^{15} \mathrm{~N}, \alpha \gamma\right)^{12} \mathrm{C}$ excitation curves for $\mathrm{ZnO}(10 \overline{1} 0)$ surfaces exposed to various doses of atomic $\mathrm{H}$ above room temperature. (b) Model $\mathrm{H}$ depth distributions that reproduce the data (solid lines in (a)) by convolution with the NRA instrumental function. Adapted from Ref. [291] with permission from APS. 
The study highlights the potential of ${ }^{15} \mathrm{~N}$ NRA to reveal details of the $\mathrm{H}$ behavior in closest vicinity of oxide surfaces that are of high potential value, e.g., for the elucidation of many open questions regarding the role of hydrogen in sensor action and catalytic reactivity of $\mathrm{ZnO}$ surfaces in contact with $\mathrm{H}_{2}$ gas or water (vapor). At the same time the present case also illustrates the difficulties associated with the quantification of the surface $H$ coverage when the subsurface region contains significant amounts of hydrogen, since these two $\mathrm{H}$-states cannot be resolved within the surface resonance peak width. This problem resembles the situation of $\mathrm{H}$ near surfaces of $\mathrm{H}$-absorbing metals, which is discussed in Section 3.2.

The case of zinc oxide is special in this repect, since appreciable amounts hydrogen have also be found in the bulk of crystalline $\mathrm{ZnO}$. The relation of these hydrogen impurities to the electrical and optical properties of zinc oxide $(\mathrm{ZnO})$ has attracted considerable interest, because $\mathrm{H}$ could be demonstrated to be a source of n-conductivity in $\mathrm{ZnO}$ [38-40, 469-472], which precludes its application, e.g. as a dieletric in electronic devices. Since IR-active as well as 'invisible' (i.e., Raman-active) hydrogen states were found to coexist in $\mathrm{ZnO}$ [39], the total hydrogen concentration could not be revealed by either of the optical techniques alone (cf. Section 3.2.5.1). This has motivated several further ion beam studies including NRA $[470,473,474]$ and positron annihilation spectroscopy (PAS) at $\mathrm{ZnO}$ nanowires [473] and electrochemically hydrogen-charged single crystals [474].

As a final remark, we notice a substantial shift $(\sim 30 \mathrm{keV})$ of the surface peak positions in the NRA excitation curves of Figs. 40 (a) and 41 (a) from the ${ }^{1} \mathrm{H}\left({ }^{15} \mathrm{~N}, \alpha \gamma\right){ }^{12} \mathrm{C}$ resonance energy (6.385 MeV), which Ref. [291] left uncommented. It 
is not clear to us, whether this shift results from a slightly miscalibrated accelerator energy, a floating potential sample stage (the entire sample compartment appears to be electrically insulated from the accelerator for the convenience of accurate ion beam charge integration in the NRA apparatus of Ref. [291]), or whether it might possibly reflect a more general complication for the NRA analysis of single crystalline bulk insulators (of which there are still only very few to date) due to charging of the target under the ion beam.

\section{$\mathrm{SrTiO}_{3}(001)$}

Takeyasu et al. recently applied ${ }^{1} \mathrm{H}\left({ }^{15} \mathrm{~N}, \alpha \gamma\right){ }^{12} \mathrm{C}$ NRA to investigate the interaction of hydrogen with $\mathrm{SrTiO}_{3}(001)$ prepared in two different controlled conditions with respect to the surface electron density [289]. The group had previously demonstrated the possibility to deliberately manipulate the $\operatorname{SrTiO}_{3}(001)$ surface electron density by selectively introducing oxygen-vacancies through energetic electron irradiation [475] (high-temperature vacuum annealing also generates Ovacancies on $\mathrm{SrTiO}_{3}$ surfaces but affects the bulk as well). O-vacancies modify the surface electronic structure of $\mathrm{SrTiO}_{3}(001)$ by introducing a $\mathrm{Ti} 3 \mathrm{~d}$ state in the band gap (induced gap state, IGS), which is observable at $1.3 \mathrm{eV}$ below $E_{\mathrm{F}}$ in the UV photoemission spectrum. Oxygen $\left(\mathrm{O}_{2}\right)$ adsorption ( $10 \mathrm{~L}$ at room temperature) on an oxygen-deficient (OD) surface, on the other hand, was shown to passivate these vacancies to produce nearly-vacancy-free (NVF) $\mathrm{SrTiO}_{3}(001)$, where the Ti 3d state (IGS) is completely quenched.

In their ${ }^{15} \mathrm{~N}$ NRA study [289], Takeyasu et al. quantified the amount of hydrogen on such (NVF) and (OD) $\mathrm{SrTiO}_{3}(001)$ surfaces that adsorbed upon room temperature 
exposures to either only molecular $\left(\mathrm{H}_{2}\right)$ or to a mixture of molecular and atomic hydrogen $\left(\mathrm{H}+\mathrm{H}_{2}\right)$ and characterized the surface electronic structure alterations concomitant with hydrogen adsorption by UPS. Fig. 42 depicts the ${ }^{1} \mathrm{H}\left({ }^{15} \mathrm{~N}, \alpha \gamma\right){ }^{12} \mathrm{C}$ NRA hydrogen profiles obtained from the NVF and OD surfaces after applying large hydrogen exposures $\left(5 \times 10^{4} \sim 1 \times 10^{5} \mathrm{~L}\right)$ that saturated the $\mathrm{H}$-induced changes in the UP spectra (described below).

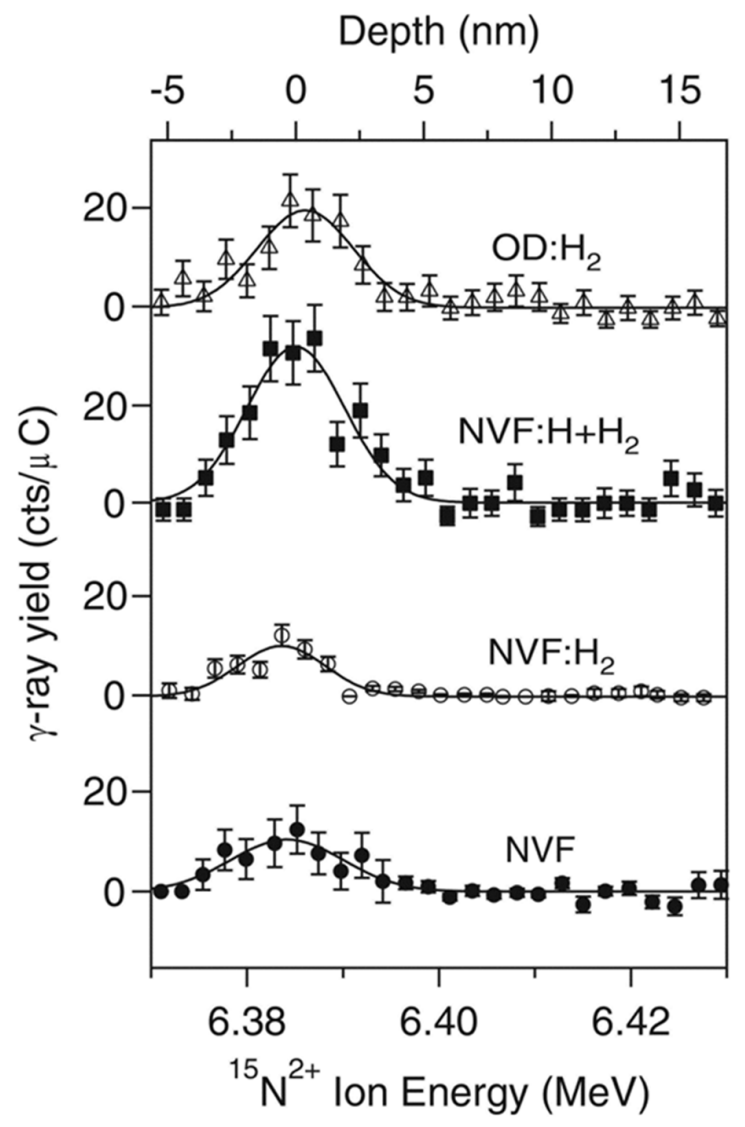

Fig. 42. ${ }^{1} \mathrm{H}\left({ }^{15} \mathrm{~N}, \alpha \gamma\right){ }^{12} \mathrm{C}$ nuclear reaction profiles taken at room temperature for the nearly-vacancyfree (NVF) $\mathrm{SrTiO}_{3}(001)$ surface, the NVF surface exposed to $5 \times 10^{4} \mathrm{~L} \mathrm{H}_{2}$ $\left(\mathrm{NVF}: \mathrm{H}_{2}\right)$, the NVF surface exposed to $1 \times 10^{5} \mathrm{~L} \mathrm{H}+\mathrm{H}_{2}\left(\mathrm{NVF}: \mathrm{H}+\mathrm{H}_{2}\right)$, and the oxygen-deficient $\mathrm{SrTiO}_{3}(001)$ surface exposed to $1 \times 10^{5} \mathrm{~L} \mathrm{H}_{2}$ (OD: $\mathrm{H}_{2}$ ). Reproduced from Ref. [289] with permission from AIP.

As seen in Fig. 42, all NRA profiles maintained Gaussian-shapes centered at $E_{R}=$ 6.385 MeV, i.e., neither before nor after the hydrogen exposures did the subsurface region of $\mathrm{SrTiO}_{3}(001)$ contain any measureable $\mathrm{H}$ concentrations, so that the respective surface $\mathrm{H}$ coverages could readily be evaluated from the resonance curve 
integrals. The NVF surface held a small initial $\mathrm{H}$ coverage of $(2.2 \pm 0.4) \times 10^{14} \mathrm{~cm}^{-2}$. The source and nature of this $\mathrm{H}$ remained unclear, but it was suspected that $\mathrm{H}_{2}$ residual gas adsorbed and bound strongly to defects. The NRA data in Fig. 42 showed that molecular $\mathrm{H}_{2}$ evidently did not adsorb on the near-stoichiometric $\mathrm{SrTiO}_{3}(001)$ surface without O-vacancies (NVF: $\mathrm{H}_{2}$ ), whereas the partially dissociated $\mathrm{H}+\mathrm{H}_{2}$ gas caused a substantial increase of the $\mathrm{H}$ coverage by $(3.1 \pm 0.8) \times 10^{14} \mathrm{~cm}^{-2}\left(\mathrm{NVF}: \mathrm{H}+\mathrm{H}_{2}\right)$. On the OD surface, molecular $\mathrm{H}_{2}$ led to a moderate $\mathrm{H}$ coverage increase by $(0.9 \pm 0.7)$ $x 10^{14} \mathrm{~cm}^{-2}\left(\mathrm{OD}: \mathrm{H}_{2}\right)$.

Complementary UPS data evidenced that the $\mathrm{H}+\mathrm{H}_{2}$ adsorption on the NVF surface induced novel Ti 3d gap state density, which corresponded to about one third of the initial IGS intensity of the OD surface. The latter had an estimated Ovacancy density of $1 \times 10^{14} \mathrm{~cm}^{-2}$, and was produced by irradiating NVF $\mathrm{SrTiO}_{3}(001)$ with an electron dose of $5 \times 10^{18} \mathrm{~cm}^{-2}$ at an energy of $1500 \mathrm{eV}$ [289]. Contrary to the effect of atomic $\mathrm{H}$ adsorption on the NVF surface to generate new IGS, $\mathrm{H}_{2}$ adsorption on $\mathrm{OD} \mathrm{SrTiO}_{3}(001)$ partially reduced the IGS density on that surface to about half of its initial value. By taking the absolute $\mathrm{H}$ coverage, the in-gap state photoemission intensity, and the $\mathrm{H}$-induced work function changes into account, Takeyasu et al. estimated that hydrogen is positively $\left(\mathrm{H}^{\sim 0.3+}\right)$ and negatively $\left(\mathrm{H}^{-}\right)$charged on the nearly-vacancy-free and oxygen-deficient surfaces, respectively, clearly demonstrating its amphoteric character. 


\subsection{Absorbed Hydrogen}

Prior to reviewing NRA applications to hydrogen absorbed in the near-surface region of (mainly single crystalline) materials, we provide a minimum physical background on the hydrogen absorption phenomenon at metal surfaces, describe briefly a few common experimental practices in hydrogen absorption studies, and introduce the type and benefit of the information that NRA can contribute therein. This will allow describing results from individual materials more consisely in the following Sections (3.2.3.1-4).

\subsubsection{General aspects of hydrogen absorption at metal surfaces}

a) Energetics of surface and absorbed $H$ states: In hydrogen-absorbing metals, one can distinguish three characteristic hydrogen atom locations that differ in terms of the metal-hydrogen interaction strength: sites on the surface, in the first subsurface layer, and in the bulk. On metal surfaces, hollow sites are often (but not always, cf. Section 3.1) the most stable locations for chemisorbed hydrogen. Bulkdissoved $\mathrm{H}$ atoms usually prefer octahedral interstitial sites in fcc and hcp metals, and tetrahedral interstitials in bcc metals [27]. In general, hydrogen atoms are considerably more strongly chemisorbed $\left(E_{\text {ads }}\right)$ at the relatively low-coordinated surface metal atoms than they are dissolved in bulk interstitial sites ( $\mathrm{H}$ solution enthalpy $\Delta H_{\mathrm{s}} \gg E_{\mathrm{ads}}$ ) [299]. First-layer subsurface sites provide a special environment for absorbed $\mathrm{H}$ atoms that differs both from the surface chemisorption as well as from the bulk interstitial sites [301, 304, 476, 477]. In metals, Thomas-Fermi screening by conduction electrons rapidly restores the bulk electronic structure 
within a very short distance from the surface, yet at least the first one or two atomic layers are distinguished from surface and from bulk sites, both electronically (by reduced bandwidth and slight d-band center shifts) as well as geometrically (by particular interlayer separations (relaxations) that deviate from the bulk lattice parameter) $[476,478-480]$. As a result, hydrogen in first-layer subsurface sites often assumes an energetically intermediate position $\left(E_{s s}\right)$ between the most stable chemisorbed and the much more weakly bound bulk dissolved state [299], i.e., $\Delta H_{s}>$ $E_{\mathrm{ss}}>E_{\text {ads. }}$. This principal energetic position of $\mathrm{H}$ in first-layer subsurface sites prevails regardless of whether $\mathrm{H}$ absorption in the metal is exothermic or endothermic [ 301 , 304]. Even for certain metals with endothermic $\mathrm{H}$ dissolution in the bulk (such as $\mathrm{Cu}(111))$, the possibility of $\mathrm{H}$ stabilization in first-layer subsurface sites through strong repulsive interactions with surface hydrogen at large coverages has been suggested theoretically [303].

The content of bulk-dissolved hydrogen in $\mathrm{H}$-absorbing metals can vary widely over a large concentration range and even nominally clean single crystals may contain residual $\mathrm{H}$ impurities in the bulk. At low concentrations, $\mathrm{H}$ atoms exist within the host metal lattice in solid solution. At elevated $\mathrm{H}$ levels, a phase separation in certain metals causes precipitation of hydride grains in the solid solution matrix when the concentration limit of the latter phase is exceeded. The $\mathrm{H}_{2}$ pressure- and temperature-dependent equilibrium $\mathrm{H}$ contents in the dilute solid solution and in the concentrated hydride are described by material-specific metal-hydrogen phase diagrams [26]. Within the two-phase coexistence regime the average bulk $\mathrm{H}$ content corresponds to $c_{\text {avg }}=V_{\alpha} c_{\alpha}{ }^{*}+V_{\beta} c_{\beta}{ }^{*}$, where $c_{\alpha}{ }^{*}$ and $c_{\beta}{ }^{*}$ are the phase boundary 
concentrations and $V_{\alpha}$ and $V_{\beta}$ the volume fractions of the solid solution $(\alpha)$ and hydride $(\beta)$ phases, respectively.

b) Equilibrium hydrogen depth distribution and temperature-induced changes: The hydrogen depth distribution in the near-surface region of $\mathrm{H}$-absorbing metals depends sensitively on the particular experimental history of any given sample, especially on the conditions ( $\mathrm{H}_{2}$ pressure and temperature) during preceding $\mathrm{H}_{2}$ gas exposures and/or annealing treatments. Before addressing the outcome of typical absorption experiments we describe the equilibrium $\mathrm{H}$ distribution in a $\mathrm{H}$-containing single crystal in UHV, where additional $\mathrm{H}$ uptake from the gas phase is negligible.

For $\mathrm{p}\left(\mathrm{H}_{2}\right) \approx 0$, the relative thermal equilibrum population of surface, subsurface, and bulk sites by $\mathrm{H}$ atoms, i.e., their depth distribution, is a readily predictable function of the sample temperature. At low temperatures the sizeable enthalpy difference $\left(\Delta H_{\mathrm{s}}^{\mathrm{b}}=\Delta H_{\mathrm{s}}-E_{\mathrm{ads}} \gg>0\right)$ between the surface chemisorption and bulk dissolution states stabilizes hydrogen on the surface (analog arguments apply to the surface/subsurface and subsurface/bulk site population ratios, for which separate equilibria can be considered on the basis of $E_{\mathrm{ads}}, E_{\mathrm{ss}}$, and $\left.\Delta H_{\mathrm{s}}\right)$. We describe only the surface/bulk case here. When the temperature is raised, the gain in configurational entropy, $\Delta \mathrm{S}_{\mathrm{s}}{ }^{\mathrm{b}}=k_{\mathrm{B}} \ln [b ! /(n !(b-n) !)] \approx k_{\mathrm{B}} \ln (b / n)$, associated with distributing $n$ surface (or analog: subsurface) $\mathrm{H}$ atoms into a much larger number of bulk interstitial sites (b) will eventually turn the free energy difference between $\mathrm{H}$ in bulk and surface sites, $\Delta G_{\mathrm{s}}^{\mathrm{b}}=\Delta H_{\mathrm{s}}{ }^{\mathrm{b}}-T \Delta S_{\mathrm{s}}{ }^{\mathrm{B}}$ negative, so that initially surface-adsorbed $\mathrm{H}$ may migrate into the bulk through diffusion. When this change of sign in $\Delta G_{\mathrm{s}}^{\mathrm{b}}$ occurs at a temperature prior to the onset of thermal desorption, bulk dissolution of surface $\mathrm{H}$ is 
the result of heating a H-covered crystal in $\operatorname{UHV}[62,481]$. This is the case for metals with large negative $\mathrm{H}$ solution enthalpies such as $\mathrm{Ti}\left(\Delta \mathrm{H}_{\mathrm{s}}=-0.47 \mathrm{eV} / \mathrm{H}[26]\right)$ and $\mathrm{Zr}$ $\left(\Delta \mathrm{H}_{\mathrm{s}}=-0.567 \mathrm{eV} / \mathrm{H}[482], \alpha-\right.$ phase values $)$. Here, the strong tendency of surfaceadsorbed hydrogen to dissolve into the bulk upon heating lets the standard TDS experiment devised to reveal the $\mathrm{H}$ chemisorption energy fail completely due to lacking $\mathrm{H}_{2}$ desorption $[62,483,484]$. Care is then required not to mistake such observations for the absence of surface hydrogen. On the other hand, if $\mathrm{H}$ absorption is only weakly exothermic (such as in $\mathrm{Pd}, \Delta \mathrm{H}_{\mathrm{s}}=-0.1 \mathrm{eV} / \mathrm{H}, \alpha$-phase [26]), the free energy contribution $T \Delta \mathrm{S}_{\mathrm{s}}$ from the even larger entropy gain by releasing the surface $\mathrm{H}$ atoms into the vacuum rather than into the metal bulk (the entropy difference between these two final states corresponds to the entropy of $\mathrm{H}$ dissolution (e.g., $\Delta \mathrm{S}_{\mathrm{s}}$ $=-7 k_{\mathrm{B}}$ for $\left.\left.\mathrm{Pd}[27]\right)\right)$ dominates over the small negative $\mathrm{H}$ solution enthalpy, thus favoring desorption over bulk absorption. For this reason the TDS technique is successfully applied to $\mathrm{Pd}$ as well as to the many transition metals that absorb $\mathrm{H}$ endothermically (positive $\Delta H_{\mathrm{s}}$ ).

c) Hydrogen absorption epxeriments: In typical Surface Science hydrogen absorption studies, initially clean single crystals are exposed to $\mathrm{H}_{2}$ gas at relatively low (exposure) temperatures $\left(T_{\mathrm{e}}\right)$ so that dissociative $\mathrm{H}$ adsorption more or less spontaneously saturates the energetically strongly favored chemisorption sites, before $\mathrm{H}$ incorporation into the metal interior takes place on a slower timescale. The detailed microscopic $\mathrm{H}_{2}$ absorption mechanism at such $\mathrm{H}$-saturated surfaces is still subject of ongoing research [279] and its discussion goes beyond the scope of this review. In general, the result of such low-temperature $\mathrm{H}_{2}$ exposures is the 
simultaneous presence of surface-adsorbed and (potentially multiple) absorbed hydrogen states, where $\mathrm{H}$ may populate subsurface sites and/or bulk interstitials, the latter potentially in solid solution as well as in hydride phases. The amount of absorbed hydrogen and its depth-dependent distribution among these absorbed states are very difficult to predict quantitatively, and have been found to vary strongly with the metal and the $\mathrm{H}_{2}$ exposure conditions $\left(\mathrm{H}_{2}\right.$ pressure, time, and temperature, $\left.T_{\mathrm{e}}\right)[61,62,279,485]$. Often the $\mathrm{H}_{2}$ exposures and hence the absorbed $\mathrm{H}$ quantitites are too small to fully establish the equilibrium between the applied $\mathrm{H}_{2}$ gas pressure and bulk-absorbed $\mathrm{H}$ throughout the entire single crystal (i.e., the $\mathrm{H}_{2}$ dosage is terminated before the $\mathrm{H}$-uptake saturates), so that $\mathrm{H}$ concentration gradients can remain between the near-surface region and the deeper bulk. A common observation is that the near-surface $\mathrm{H}$ depth distribution extends into larger depths when $T_{\mathrm{e}}$ is increased, which can be qualitatively understood because the diffusion of absorbed $\mathrm{H}$ into the bulk is thermally activated and becomes increasingly favored entropically at elevated temperatures. Upon $\mathrm{H}$ absorption at low $T_{\mathrm{e}}$, hydride may nucleate just underneath the surface, producing a near-surface hydride with steep concentration gradients into the bulk. If thermodynamically favorable, hydride may also nucleate homogeneously in the metal bulk.

\subsubsection{NRA observation of absorbed hydrogen}

The main experimental strategies to characterize adsorbed and absorbed hydrogen states with ${ }^{15} \mathrm{~N}$ NRA can be classified into two procedures: (i) The $\mathrm{H}$ probing depth is scanned at a constant target temperature. If perfomed at sufficiently low temperatures where neither thermal $H$ diffusion nor the NRA 
measurement perturb the hydrogen profile, this delivers 'static' visualizations of the $\mathrm{H}$ depth distribution. (ii) The ${ }^{15} \mathrm{~N}$ energy is fixed to selectively observe $\mathrm{H}$ at a particular probing depth while either the target temperature is varied or the evolution of the $\mathrm{H}$ concentration in the selected depth is monitored as a function of time. This allows capturing 'dynamical' aspects of the $\mathrm{H}$ behavior, such as thermally driven $\mathrm{H}$ redistribution processes between the surface and the bulk, or $\mathrm{H}$ uptake kinetics on or underneath $\mathrm{H}_{2}$-exposed surfaces in absorption experiments.

(i) In $\mathrm{H}$-depth profiling at constant temperature, surface hydrogen (always present at low temperatures) gives rise to the nearly-Gaussian shaped $\gamma$-peak at $E_{R}$ (Fig. 4 (a) and Section 3.1). The NRA measurement of bulk-absorbed hydrogen at $E_{\mathrm{i}} \geq$ $E_{>}$(Fig. 4 (b)) will assess the areal average bulk $\mathrm{H}$ content $\left(c_{\text {avg }}\right.$ in the hydride/solid solution coexistence region) at the depth selected by $E_{\mathrm{i}}$, provided that the hydride grains are much smaller than the analyzing ${ }^{15} \mathrm{~N}$ ion beam spot on the target surface. The outstanding advantage of ${ }^{1} \mathrm{H}\left({ }^{15} \mathrm{~N}, \alpha \gamma\right){ }^{12} \mathrm{C}$ NRA in hydrogen absorption studies lies in its unique ability to reveal the hydrogen concentration-depth distribution and thereby to identify the absorbed $\mathrm{H}$ states, i.e., $\mathrm{H}$ in first layer subsurface sites, in bulk solid solution, and in bulk or near-surface hydrides). With reference to metal- $\mathrm{H}$ phase diagram data, NRA thus allows for direct observations of hydride nucleation phenomena in the near-surface region (Sections 3.2.3.1-4).

In addition to low-temperature hydrogen absorption studies at metal single crystals, non-uniform near-surface $\mathrm{H}$ depth distributions may also prevail in a variety of other pure and homogeneous host materials, such as in the case of the gradual hydration of mineral surfaces (Section 3.2.5.1), where the bulk diffusion of absorbed 
hydrogen species is rather slow. $\mathrm{H}$-enriched or $\mathrm{H}$-depleted near-surface layers may further arise from the interaction of absorbed hydrogen with lattice imperfections and impurities that result, e.g., from a modification of the near-surface region through an external treatment such as ion implantation or polishing (cf. Sections 3.2.2.5 and 3.2.2.6). Here, H-profiling with ${ }^{15} \mathrm{~N}$ NRA can be useful to evaluate the depth extension of the affected region.

Hydrogen in first-layer subsurface sites is located in a very shallow depth $(d)$ not more than about $1 \AA$ beneath the substrate surface atoms. Thus, in surface-normal ${ }^{15} \mathrm{~N}$ incidence the NRA signal of $\mathrm{H}$ in first-layer subsurface sites is shifted relative to $E_{\mathrm{R}}$ by only a very small energy increment $\Delta E=d \cdot S$, which amounts to $\sim 0.2 \mathrm{keV}$ for $\mathrm{Ti}$ $(S=2.335 \mathrm{keV} / \mathrm{nm})$ and $\sim 0.4 \mathrm{keV}$ for $\mathrm{Pd}(S=3.90 \mathrm{keV} / \mathrm{nm})$. Since on otherwise clean metal surfaces $\mathrm{H}$ can stably occupy first-layer subsurface sites only at relatively low temperatures in coexistence with a larger quantity of surface chemisorbed hydrogen $\left(E_{s s}>E_{\text {ads }}\right)$, the $\mathrm{H}$ occupation of subsurface sites will merely add surplus $\gamma$-yield to the resonance profile of surface $H$ so close to $E_{R}$, that neither the center position nor the width of the excitation curve change markedly within the experimental accuracy. The sizeable width of the Doppler-broadened surface resonance (ca. 8-10 keV on metals) and the finite ${ }^{15} \mathrm{~N}$ stopping power $\left(2^{\sim} 4 \mathrm{keV} / \mathrm{nm}\right)$ thus principally make the resolution of $\mathrm{H}$ in first-layer subsurface-sites from surface hydrogen very difficult, even for ${ }^{15} \mathrm{~N}$ NRA at $6.385 \mathrm{MeV}$. Adopting strongly grazing ${ }^{15} \mathrm{~N}$ ion incidence geometries is an effective way to increase the near-surface NRA depth resolution in the top 1-2 nm, as demonstrated for $\mathrm{H}$ in $\mathrm{Pd}$ nanoparticles (Sect. 3.2.3.8) and at ultra-shallow metal/Si(111) interfaces (Sect. 3.3.2). So far, however, the ultimate distinction 
between surface-adsorbed and coexisting $\mathrm{H}$ absorbed in first-layer subsurface sites by means of ${ }^{15} \mathrm{~N}$ NRA has remained an unaccomplished challenge.

(ii) Through its unique ability to detect hydrogen at a selected depth while the temperature of a previously $\mathrm{H}$-exposed sample is varied, ${ }^{15} \mathrm{~N}$ NRA can evaluate the thermal stability of surface and absorbed hydrogen states against redistribution by diffusion and/or desorption. This is suited in particular to observe in-situ thermally induced $\mathrm{H}$ redistribution between surface-adsorbed and bulk-dissolved states, as the free energy balance for $\mathrm{H}$ occupation of surface and bulk sites shifts as a function of temperature. As noted in Section 3.2.1, diffusion of surface-adsorbed $\mathrm{H}$ into the bulk may occur when $\mathrm{H}$-covered surfaces of $\mathrm{H}$-absorbing materials are heated in vacuum (such as in common TDS measurements), or, conversely, residual bulk-dissolved hydrogen can segregate onto the (initially $\mathrm{H}$-depleted) surface when a $\mathrm{H}$-containing crystal is quenched from a high annealing temperature in vacuum (Sections 3.2.3.12) $[62,486]$.

The same type of ${ }^{15} \mathrm{~N}$ NRA measurement (monitoring $\mathrm{H}$ at a fixed depth while $\mathrm{T}$ is varied) is also useful in hydrogen absorption studies in combination with TDS. When surface-chemisorbed and absorbed hydrogen species coexist and give rise to multiple TDS states, it may not be immediately apparent which TDS signals originate from surface hydrogen and which represent(s) the absorbed $\mathrm{H}$ state(s). As illustrated at the example of $\mathrm{Pd}(100)$ in Sect. $3.2 .3 .3,{ }^{15} \mathrm{~N}$ NRA achieves unambiguous identifications of individual TDS features by correlating the selectively monitored temperature-dependent signatures of surface and absorbed $\mathrm{H}$ species with the TDS trace $[61,62,279]$. 
Examples for the monitoring of surface hydrogen uptake processes at $\mathrm{H}_{2}$-exposed metals have been described in Section 3.1 (Figs. 16, 26, 30). The H-uptake into the entire near-surface region of $\operatorname{Zr}(10 \overline{1} 0)$ has been studied with $D\left({ }^{3} \mathrm{He}, \mathrm{p}\right)^{4} \mathrm{He}$ NRA (Fig. 49). With ${ }^{15} \mathrm{~N}$ NRA it is furthermore possible to reveal the uptake kinetics at $\mathrm{H}_{2}$ exposed single crystal surfaces in a selected narrow depth region, although no systematic studies have been published yet to our knowledge.

We would like to add one comment on terminology. Most experimental Surface Science studies related to absorbed hydrogen have been conducted without depth resolving detection methods so that the actual $\mathrm{H}$ distribution below the surface often remained unclarified. This situation has produced the vague but frequently encountered term 'subsurface hydrogen', which has been used synonymously to address $\mathrm{H}$ in first layer subsurface sites as well as to denote $\mathrm{H}$ that is distributed over a wider region below the surface, although these circumstances are evidently rather different (Section 3.2.1). We will avoid the ambiguous 'subsurface hydrogen' label here althogether and prefer to speak more generally of absorbed hydrogen whenever its location below the surface cannot be specified, as no potentially unjustified surface vicinity is implied. Since ${ }^{15} \mathrm{~N}$ NRA at $6.385 \mathrm{MeV}$ can quantify and locate absorbed $\mathrm{H}$ with a precision of a few $\mathrm{nm}$ beneath the surface, we will use the more specific definitions of ' $\mathrm{H}$ in first-layer subsurface sites' and 'bulk-absorbed $\mathrm{H}$ states', which include ' $\mathrm{H}$ in solid solution' as well as 'near-surface' and 'bulk hydrides'.

\subsubsection{Metals}




\subsubsection{Titanium (0001)}

a) As-cleaned Ti(0001)

As an example for the distinction between surface-adsorbed and bulk-absorbed hydrogen with ${ }^{1} \mathrm{H}\left({ }^{15} \mathrm{~N}, \alpha \gamma\right){ }^{12} \mathrm{C}$ NRA we present the excitation curve from a $\mathrm{Ti}(0001)$ single crystal that had been cleaned by sputtering and annealing at $970 \mathrm{~K}$ and was allowed to cool to room temperature in UHV (Fig. 43) [62]. The perfectly symmetric Gaussian resonance profile centered at $6.385 \mathrm{MeV}$ was indicative of surface $\mathrm{H}$ and the small and constant $\gamma$-yield at ${ }^{15} \mathrm{~N}$ energies above $6.40 \mathrm{MeV}$ showed the presence of $\mathrm{H}$ below the surface that exhibited no $\mathrm{H}$ concentration gradient within the investigated depth.

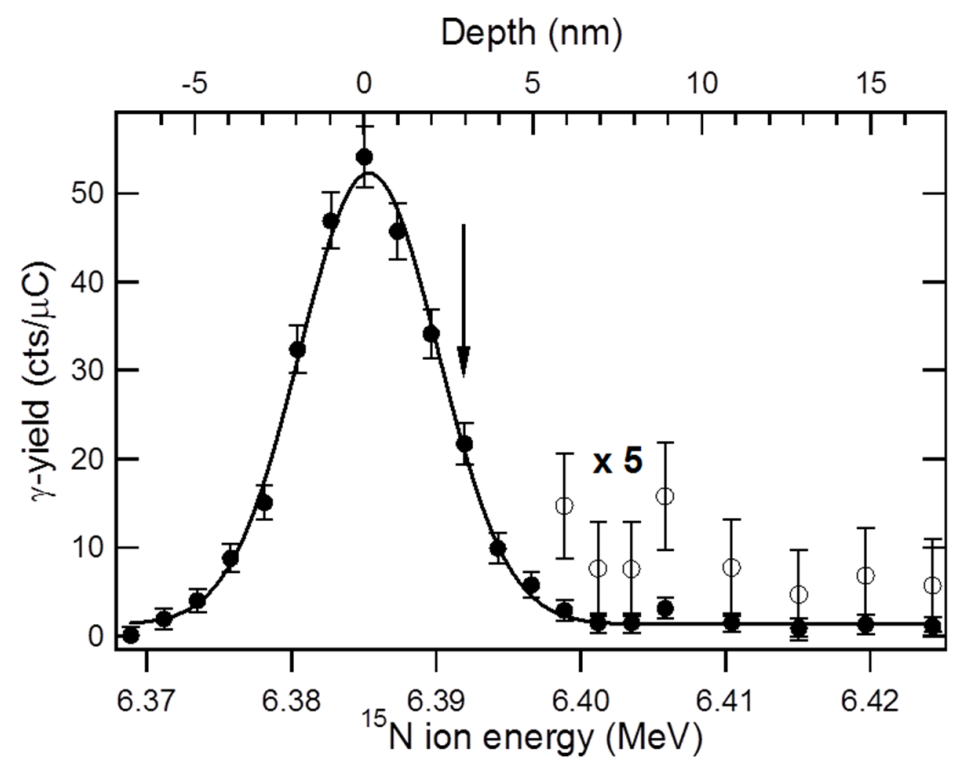

Fig. 43. NRA $\gamma$-yield curve of $\mathrm{Ti}(0001)$ single crystal recorded in UHV at $\mathrm{T}=300$ $\mathrm{K}$ after cleaning by sputtering/annealing. The line through the experimental data points is a fit to a Gaussian and an error function-like step profile representing hydrogen on the surface and in the bulk, respectively. The $\gamma$-yield averaged between $7-17 \mathrm{~nm}$ depth $(1.4 \mathrm{cts} / \mu \mathrm{C})$ corresponds to a bulk $\mathrm{H}$ concentration of $500 \mathrm{ppm}$. Arrow: lon energy used for probing surface- $\mathrm{H}$ in the experiments of Figs. 44 and 45. Adapted from Ref. [62] .

The resonance peak integral quantified the surface $\mathrm{H}$ coverage to $0.5 \mathrm{ML}(1 \mathrm{ML}=$ $1.33 \times 10^{15} \mathrm{~cm}^{-2}$ on $\left.\mathrm{Ti}(0001)\right)$ and the low $\gamma$-yield plateau of $1.39 \pm 0.35 \mathrm{cts} / \mathrm{C}$ reflected 
a $\mathrm{H}$ impurity concentration of $\sim 500 \mathrm{ppm}$ in the topmost $17 \mathrm{~nm}$. Although only a shallow region was analyzed, it could be concluded that this $\mathrm{H}$ impurity content extended homogeneously throughout the entire crystal specimen, since it had been annealed repeatedly at $970 \mathrm{~K}$ in UHV so that any remaining concentration gradients of absorbed $\mathrm{H}$ were deemed unlikely as they should have leveled out by diffusion. The existence of a large reservoir of bulk-absorbed $\mathrm{H}$ was corroborated by intense $\mathrm{H}_{2}$ desorption that evolved during UHV annealing of the single crystal. The TD spectrum in Fig. 44 evidenced this bulk-dissolved $\mathrm{H}$ release by the exponentially growing $\mathrm{H}_{2}$ desorption rate above $700 \mathrm{~K}$, which (at $900 \mathrm{~K}$ ) exceeded the intensity expected for a monolayer of surface-adsorbed $\mathrm{H}$ by several orders of magnitude. From the temperature dependence of this $\mathrm{H}_{2}$ release, an activation energy equivalent to the $\mathrm{H}$ solution enthalpy in $\alpha-\mathrm{Ti}\left(\Delta \mathrm{H}_{\mathrm{s}}=-0.47 \mathrm{eV} / \mathrm{H}\right.$ [26]) was deduced [62].

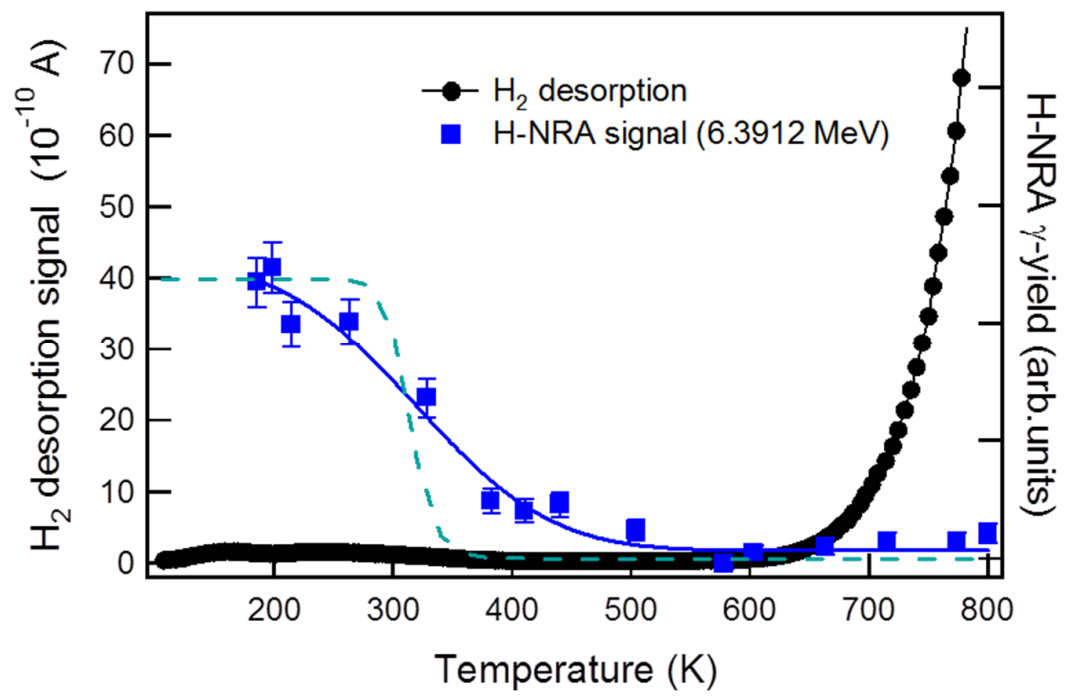

Fig. 44. $\mathrm{H}_{2}$ thermal desorption spectrum of a $\mathrm{H}$-saturated Ti(0001) single crystal $(12000 \mathrm{~L} \mathrm{H}$ at $120 \mathrm{~K}$ ) compared to the temperature dependence of the NRA signal of surface hydrogen $\left(\theta_{H}=0.5 \mathrm{ML}\right.$ at $\left.200 \mathrm{~K}\right)$. The dashed line indicates the $\mathrm{H}$ surface occupation expected for thermal equilibrium with the Ti bulk for an adsorption energy of $-1.05 \mathrm{eV} / \mathrm{H}$. Adapted from Ref. [62]. 
Remarkably, the TDS trace in Fig. 44 detected only spurious $\mathrm{H}_{2}$ desorption signals below $600 \mathrm{~K}$, although the Ti(0001) surface was prepared for the TDS measurement with a large $\mathrm{H}$ coverage (saturated with $\theta=1.3 \mathrm{ML} \mathrm{H}$ at $120 \mathrm{~K}$, including some $\mathrm{H}$ in the subsurface region, cf. Fig. 46). For comparison, Fig. 44 shows the temperaturedependent ${ }^{15} \mathrm{~N}$ NRA $\gamma$-yield (blue squares) obtained in a separate experiment by directly probing the surface $\mathrm{H}$ coverage (at a slightly off-resonant ${ }^{15} \mathrm{~N}$ energy of 6.391 $\mathrm{MeV}$, arrow in Fig. 43), while the sample prepared with an initial surface $\mathrm{H}$ coverage of 0.5 ML was heated incrementally in UHV [62]. The surface $\mathrm{H}$ was found to be approximately stable between $200 \mathrm{~K}$ and $300 \mathrm{~K}$, above which it vanished gradually in a broad decay up to $500 \mathrm{~K}$. These combined TDS/NRA data clearly demonstrate the tendency of chemisorbed $\mathrm{H}$ atoms to penetrate below the Ti(0001) surface upon heating in vacuum - rather than to desorb -, and to escape out of the NRA depth detection window by diffusing into the deeper bulk.

Selective hydrogen surface coverage monitoring with ${ }^{15} \mathrm{~N}$ NRA was also applied to observe the segregation of the bulk-dissolved hydrogen to the strongly binding sites on the Ti(0001) surface, while the Ti single crystal (containing 500 ppm $\mathrm{H}$ in the bulk) was rapidly quenched to below room temperature after flash-heating to $>900 \mathrm{~K}$ in UHV (Fig. 45). This 'resurfacing' of bulk-dissolved $\mathrm{H}$ was considered to be the major source of surface hydrogen detected in the NRA H-profile of Fig. 43, as even under different UHV background pressures a very reproducible $\mathrm{H}$ coverage of $0.5 \mathrm{ML}$ was observed under the particular heating/quenching conditions and the bulk $\mathrm{H}$ content of the Ti(0001) specimen of Ref. [62], which could not be explained only by $\mathrm{H}_{2}$ readsorption from the residual gas. As described in Sect. 3.2.1, this $\mathrm{H}$ 
redistribution between the bulk and the surface results from a shift in the free energy balance that favors hydrogen surface site occupation over bulk-dissolution at low temperatures.

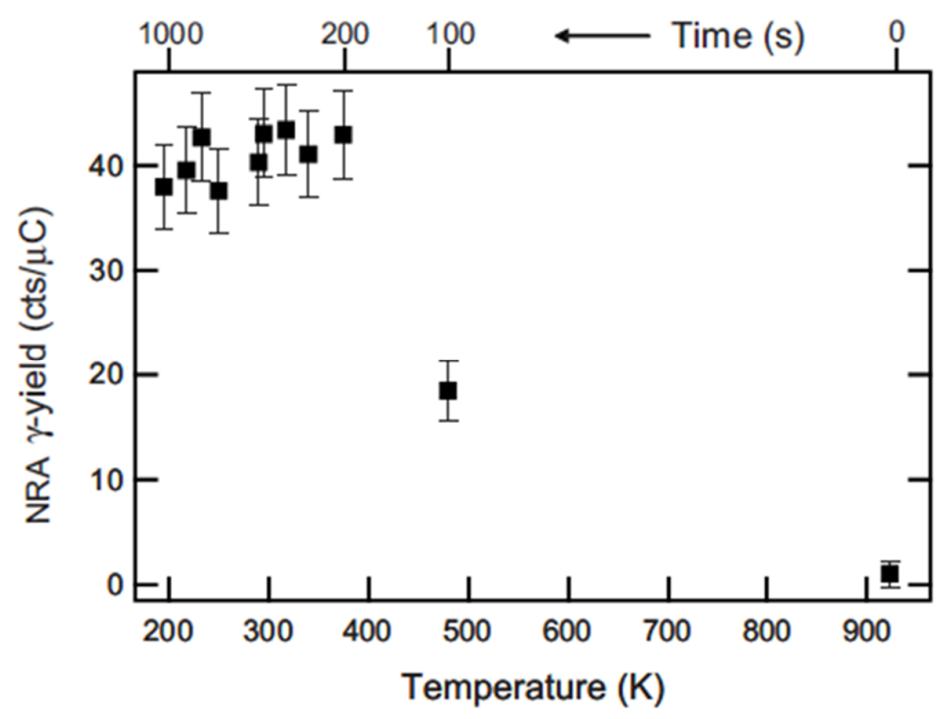

Fig. 45. Rapid recovery of the H-NRA signal on the Ti(0001) surface in UHV upon cooling a Ti single crystal containing $500 \mathrm{ppm} \mathrm{H}$ in the bulk after flash-annealing to $923 \mathrm{~K}$. The acquisition time for each data point is $100 \mathrm{~s}$. Within the first $200 \mathrm{~s}$, the $\mathrm{H}$ coverage rapidly saturates at $0.5 \mathrm{ML}(40 \mathrm{cts} / \mu \mathrm{C}$ at $6.3912 \mathrm{MeV}$, arrow in Fig. 43). Reproduced with permission from Ref. [62].

The verification of this 'resurfacing' effect with NRA showcased that remaining traces of bulk-dissolved hydrogen will pose serious impediments to the preparation of ideally clean $\mathrm{Ti}$ surfaces by UHV sputtering/annealing cycles unless all $\mathrm{H}$ is completely removed from the bulk. This is an important concern for accurate geometric structure determinations of Ti surfaces, because adsorbed $\mathrm{H}$ is known to very strongly affect the interlayer relaxations at the Ti(0001) surface $[487,488]$. Thus, together with the possibility of $\mathrm{H}_{2}$ adsorption from the UHV residual gas on the highly reactive clean $\mathrm{Ti}(0001)$ surface, the segregation tendency of bulk-dissolved $\mathrm{H}$ aggravates the difficulty to perform, e.g., LEED I(V) measurements under well- 
controlled (especially zero) $\mathrm{H}$ coverage conditions. Since the $\mathrm{H}$ coverage on Ti cannot be evaluated by TDS due to the described propensity of surface $\mathrm{H}$ to diffuse into the bulk upon heating, ${ }^{15} \mathrm{~N}$ NRA in combination with LEED may be the only experimental routes available to establish a quantitative correlation between the $\mathrm{H}$ coverage and the Ti(0001) surface interlayer relaxations.

b) $\mathrm{H}_{2}$-exposed $\mathrm{Ti}(0001)$

${ }^{1} \mathrm{H}\left({ }^{15} \mathrm{~N}, \alpha \gamma\right){ }^{12} \mathrm{C}$ NRA has also been applied to investigate low-temperature $\mathrm{H}_{2}$ adsorption and absorption at Ti(0001). In Fig. 46 we present the $\gamma$-yield curve of a $\mathrm{Ti}(0001)$ single crystal surface that was exposed to large dose of $12000 \mathrm{~L} \mathrm{H}$ at $120 \mathrm{~K}$, which saturated the $\mathrm{H}$ uptake [62]. The profile shows a clear asymmetric broadening on the high energy side and the resonance peak center is shifted from the surface position to a depth of about $1 \mathrm{~nm}$, indicating that in addition to $\mathrm{H}$-saturating the surface sites the large $\mathrm{H}_{2}$ exposure had caused $\mathrm{H}$ absorption into shallow depths $(<$ $10 \mathrm{~nm}$ ) below the surface. The total hydrogen uptake deduced from the profile integral was (1.3 $\pm 0.1 \mathrm{ML})$. Obviously, the absorbed $\mathrm{H}$ is not resolved from the surface hydrogen signal, so that neither the surface saturation coverage nor the amount of absorbed $\mathrm{H}$ atoms could be specified with certainty in this situation. The shifted peak maximum and the asymmetry of the $\mathrm{H}$ profile, however, indicate that the absorbed $\mathrm{H}$ atoms were clearly not confined only to first-layer subsurface sites, but that they must have been distributed over several tens of atomic layers below the surface, likely with a concentration gradient into the depth.

This somewhat extended $\mathrm{H}$ depth distribution is remarkable because at $120 \mathrm{~K}$ the thermal diffusion of $\mathrm{H}$ in bulk titanium is essentially frozen $\left(D=D_{0} \cdot \exp \left(-E_{\text {diff }} / k_{\mathrm{B}} T\right)\right.$ with 
$D_{0}=3.64 \times 10^{-7} \mathrm{~m}^{2} \mathrm{~s}^{-1}, E_{\text {diff }}=0.53 \mathrm{eV}, \mathrm{L}_{D}=(D \cdot \Delta t)^{1 / 2}=1.4 \times 10^{-4} \mathrm{~nm}$ in $\left.\Delta t=1000 \mathrm{~s}\right)$, so that any absorbed $\mathrm{H}$ is expected to remain in closest vicinity of the single crystal surface, i.e., not to escape from the first few subsurface layers. The origin of the observed slight low-temperature in-diffusion has remained unclear. It was verified that the NRA measurement did not perturb the $\mathrm{H}$ depth profile, leaving tunneling diffusion, altered $\mathrm{H}$ diffusion properties in the near-surface region, or energy dissipation in the absorption and potential hydride nucleation processes as possible tentative explanations. Fig. 46 thus illustrates the unique possibility to study such so far rarely investigated phenomena with highly depth-resolving ${ }^{15} \mathrm{~N}$ NRA.

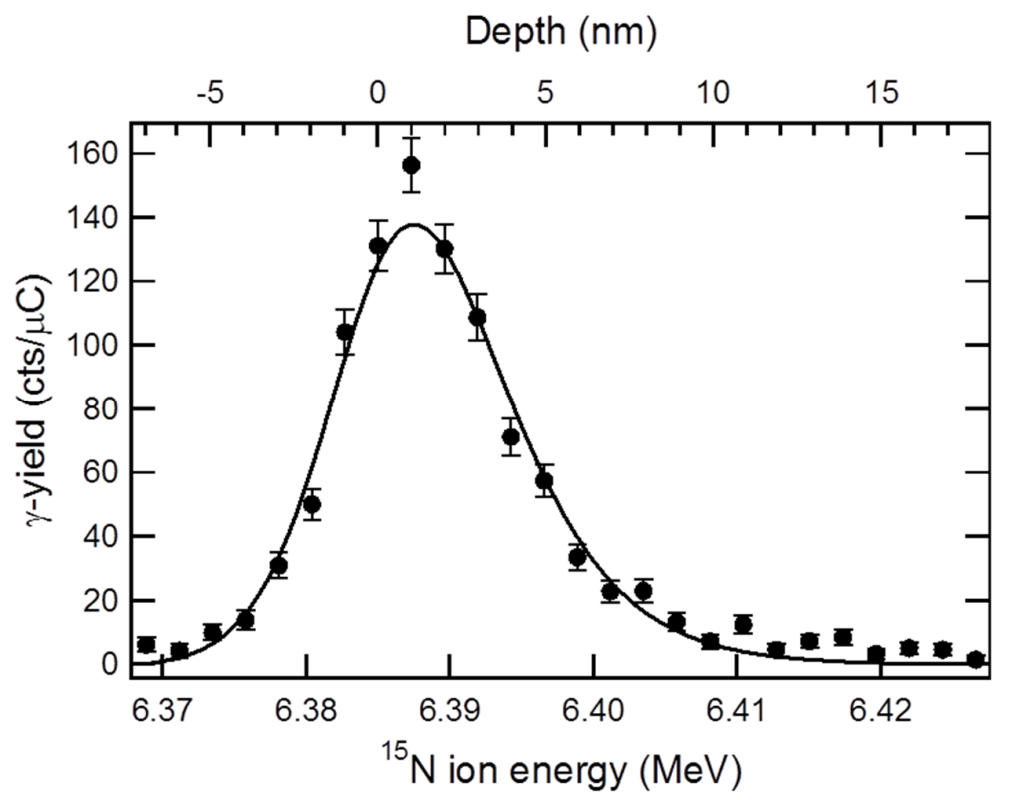

Fig. $46 .{ }^{15} \mathrm{~N}$ NRA $\gamma$-yield curve obtained from a $\mathrm{H}$-saturated Ti(0001) single crystal surface (exposed to $12000 \mathrm{~L} \mathrm{H}_{2}$ at $120 \mathrm{~K}$ ). The solid line is a guide to the eye. Reproduced from Ref. [62].

The potential of ${ }^{15} \mathrm{~N}$ NRA to reveal temperature-dependent hydride nucleation processes in the near-surface region with nanometer resolution is exemplified in Fig. 47, which displays two $\mathrm{H}$ profiles of a Ti(0001) single crystal that had been exposed 
to a large dose $(12000 \mathrm{~L})$ of partially dissociated hydrogen gas $\left(\mathrm{H}+\mathrm{H}_{2}\right)$ at $200 \mathrm{~K}$ and $250 \mathrm{~K}$ [84]. A thin ( $10 \mathrm{~nm}) \mathrm{H}$-rich layer with an average $\mathrm{H}$-concentration of about 10 atomic $\%$ was found below the Ti(0001) surface after the exposure at $200 \mathrm{~K}$, whereas in addition to some $\mathrm{H}$ accumulation below the surface a wider $\mathrm{H}$ plateau reaching more than $40 \mathrm{~nm}$ deep into the bulk resulted at $250 \mathrm{~K}$. This is clearly indicative of a temperature dependent transition from near-surface to bulk hydride nucleation. In Ti the low concentration $(<<1 \% \mathrm{H})$ solid solution $\alpha$-phase has hcp structure and is in equilibrium with $\gamma$-Ti below room temperature, a phase of fcc structure and a composition close to Ti-dihydride $\left(\mathrm{TiH}_{2}\right)$ [26]. In notable contrast to Pd (Sect. 3.2.3.3), the $\mathrm{H}_{2}$ exposure at $200 \mathrm{~K}$ saturated the $\mathrm{H}$ uptake into the only $\sim 10 \mathrm{~nm}$ wide nearsurface layer of Ti(0001). This suggests that the near-surface hydride of Ti exhibits self-limiting growth behavior at low temperatures. Apparently this limitation is lifted at $250 \mathrm{~K}$, presumably due to a higher diffusion mobility of the absorbed $\mathrm{H}$.

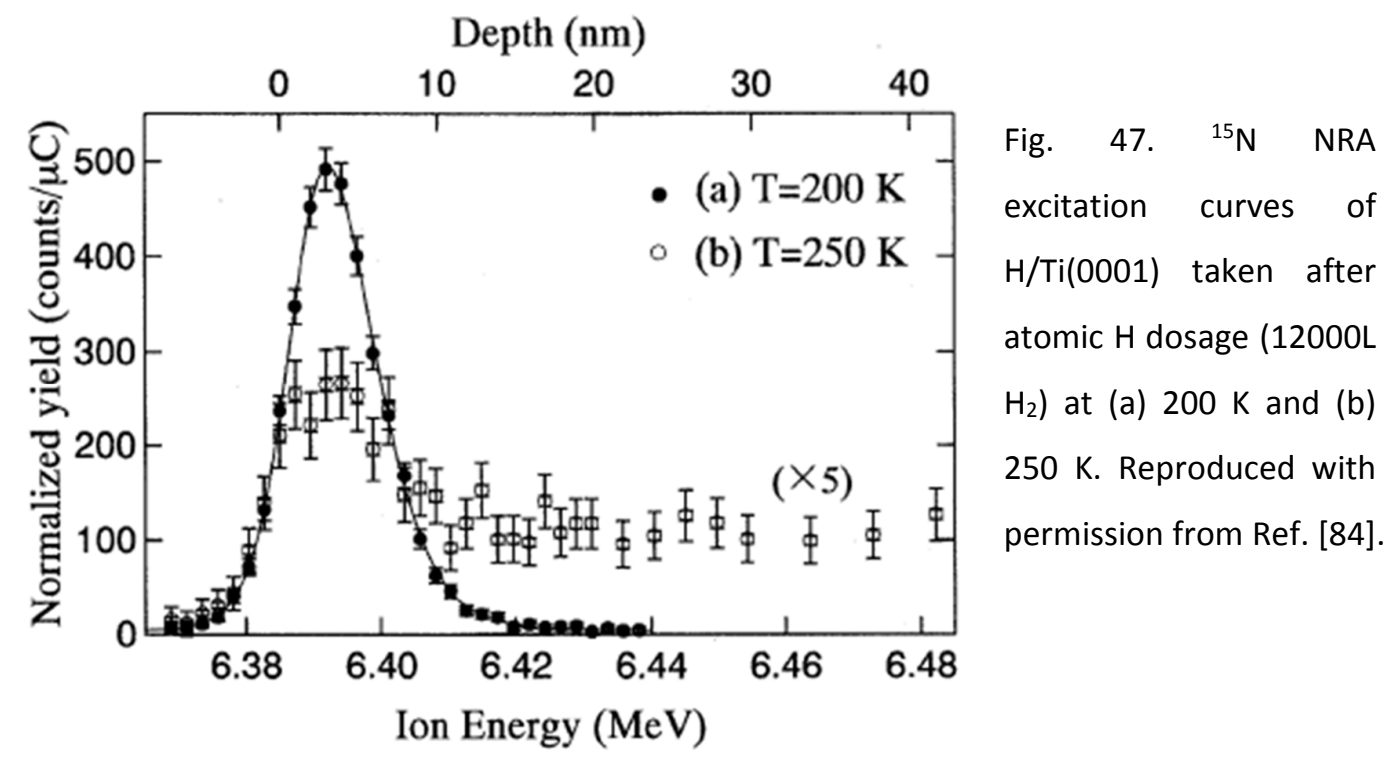




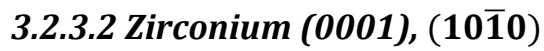

Hydrogen absorption in $\mathrm{Zr}$ is even more exothermic $\left.\left(\Delta \mathrm{H}_{\mathrm{s}}=-0.567 \mathrm{eV} / \mathrm{H}\right)[482]\right)$ than in $\mathrm{Ti}$, and the tendency to dissolve surface-adsorbed hydrogen into the bulk (Sect. 3.2.2) is so strong, that even under the extreme heating rates $\left(>10^{10} \mathrm{~K} / \mathrm{s}\right)$ of laser-induced thermal desorption experiments only a tiny fraction ( $1 \%)$ of an initially adsorbed $\mathrm{H}$ coverage desorbs, wheras the dominant part migrates below the surface [484, 489-491]. This precludes meaningful TDS measurements at Zr surfaces $[62,483,484]$ and thus hampers determining, e.g., the $\mathrm{H}$ chemisorption energy and also the verification of the $\mathrm{H}_{2}$ exposure that saturates the surface $\mathrm{H}$ coverage under any given temperature and $\mathrm{H}_{2}$ pressure conditions, an otherwise trivial task for TDS.

A possible solution for this problem has been proposed by Norton and coworkers $[483,484,486,492,493]$. They measured the D-uptake into the entire near-surface region of a $\mathrm{D}_{2}$-exposed $\operatorname{Zr}(10 \overline{1} 0)$ single crystal with $\mathrm{D}\left({ }^{3} \mathrm{He}, \mathrm{p}\right)^{4} \mathrm{He}$ NRA (no depth resolution in the top $500 \mathrm{~nm}$ ) and correlated the obtained uptake curve (Fig. 48) with corresponding SSIMS data, which exclusively monitor the surface hydrogen [484]. This figure shows that for proper relative axis scaling, the SSIMS $\mathrm{H}^{-}$yield and the NRA D signal are strictly proportional in the initial $H_{2}\left(D_{2}\right)$ exposure regime up to the saturation point of the SSIMS data, so that the quantitative NRA information on the D coverage can be used to calibrate the SSIMS signal. The SSIMS $\mathrm{H}^{-}$yield uptake terminates after a $\mathrm{H}_{2}$ exposure of $\sim 1 \mathrm{~L}$, indicating saturation of the surface chemisorption layer, whereas the NRA D signal grows further due to continued Duptake into the $\mathrm{Zr}$ bulk [484]. The combined data thus rather accurately identify the exposure regime where surface-adsorption saturates and quantitatively reveal the 
saturation coverage. These experiments permitted determining the initial sticking coefficients $\left(s_{0}=0.38 \pm 0.16\right.$ for $\operatorname{Zr}(0001)$ and $s_{0}=1.0,0.71$, and 0.51 at $100 \mathrm{~K}, 293 \mathrm{~K}$, and $370 \mathrm{~K}$, respectively, for $\operatorname{Zr}(10 \overline{1} 0))$ and that the hydrogen saturation coverages on these surfaces are 0.77 ML for $\operatorname{Zr}(0001)$ and $1.0 \mathrm{ML}$ for $\operatorname{Zr}(10 \overline{1} 0))$ [483, 484]. This information had remained entirely elusive in a previous study [494], where the tendency of surface hydrogen to dissolve into the bulk during the TDS heating ramp was not realized and the resulting small desorption signals were misinterpreted as a low sticking probability [483].

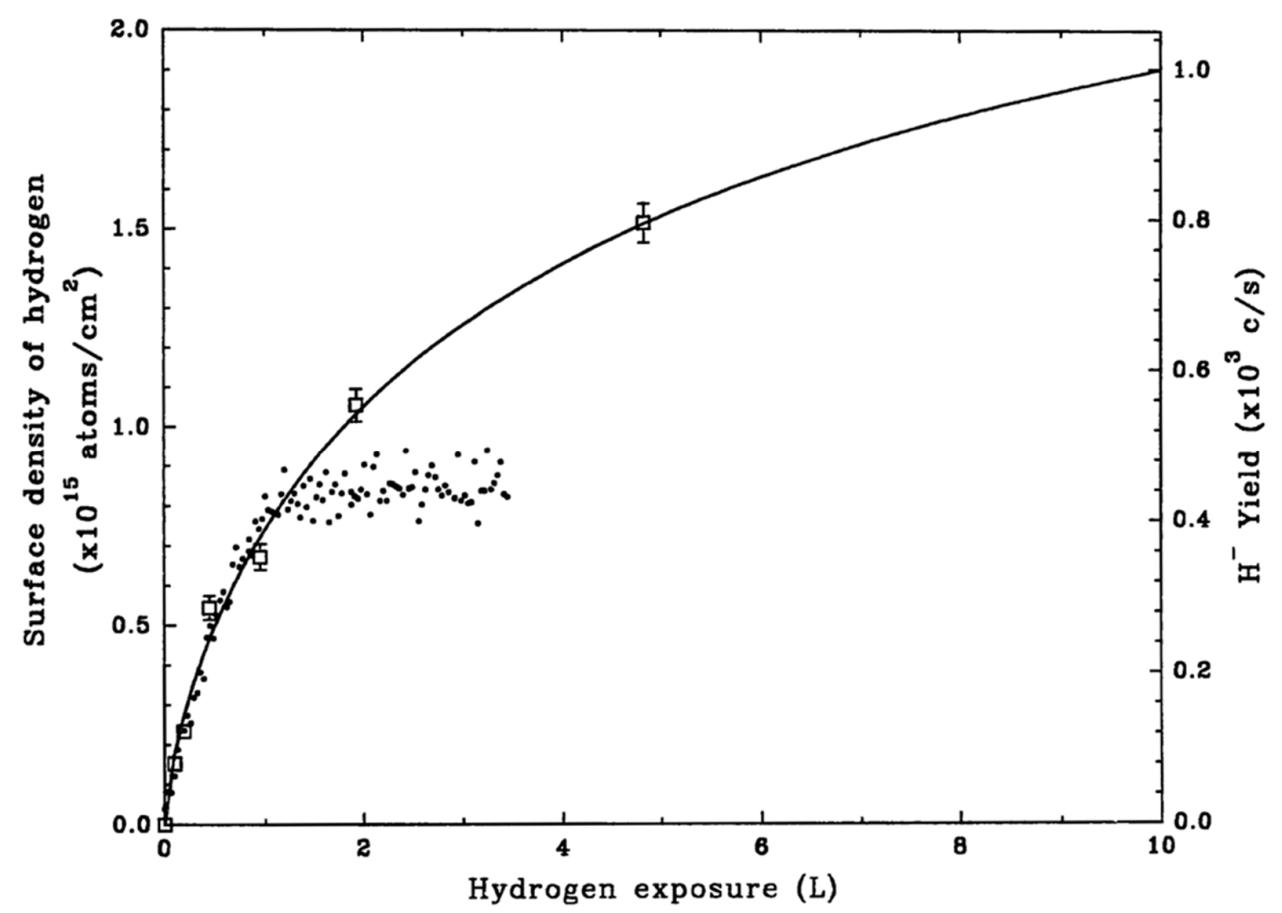

Fig. 48. Comparison of the hydrogen uptake at the $\operatorname{Zr}(\mathbf{1 0} \overline{10})$ surface measured by $\mathrm{D}\left({ }^{3} \mathrm{He}, \mathrm{p}\right)^{4} \mathrm{He}$ NRA (square symbols and fitting curve) and SSIMS (small circles) at $293 \mathrm{~K}$. Reproduced from Ref. [484].

Norton's group also established a similar 'calibration' of work function changes against absolute coverages via $\mathrm{D}\left({ }^{3} \mathrm{He}, \mathrm{p}\right)^{4} \mathrm{He}$ NRA during adsorption and $\mathrm{H}$-induced reconstruction of $\mathrm{H}(\mathrm{D})$ on $\mathrm{Pd}(110)$, and thereby contributed significantly to the 
clarification of the surface adsorption structure. These work function measurements identified the $\mathrm{H}_{2}$ TDS ' $\alpha_{2}$ ' desorption feature at $220 \mathrm{~K}$ related to the lifting of the $\mathrm{H}$ induced (1x2) pairing-row reconstruction of $\mathrm{Pd}(110)$ as a surface-adsorbed species $[492,493]$.

Norton et al. also investigated the exposure temperature dependence of $D$ adsorption and absorption at Zr surfaces with $D\left({ }^{3} \mathrm{He}, \mathrm{p}\right)^{4} \mathrm{He}$ NRA. Fig. 49 shows the initial $D$ uptake on a $D_{2}$ exposed $\operatorname{Zr}(10 \overline{1} 0)$ surface [484] at different temperatures. Due to the limited depth resolution, the data quantify the total $D$ uptake within the top $\sim 500 \mathrm{~nm}$ of the sample.

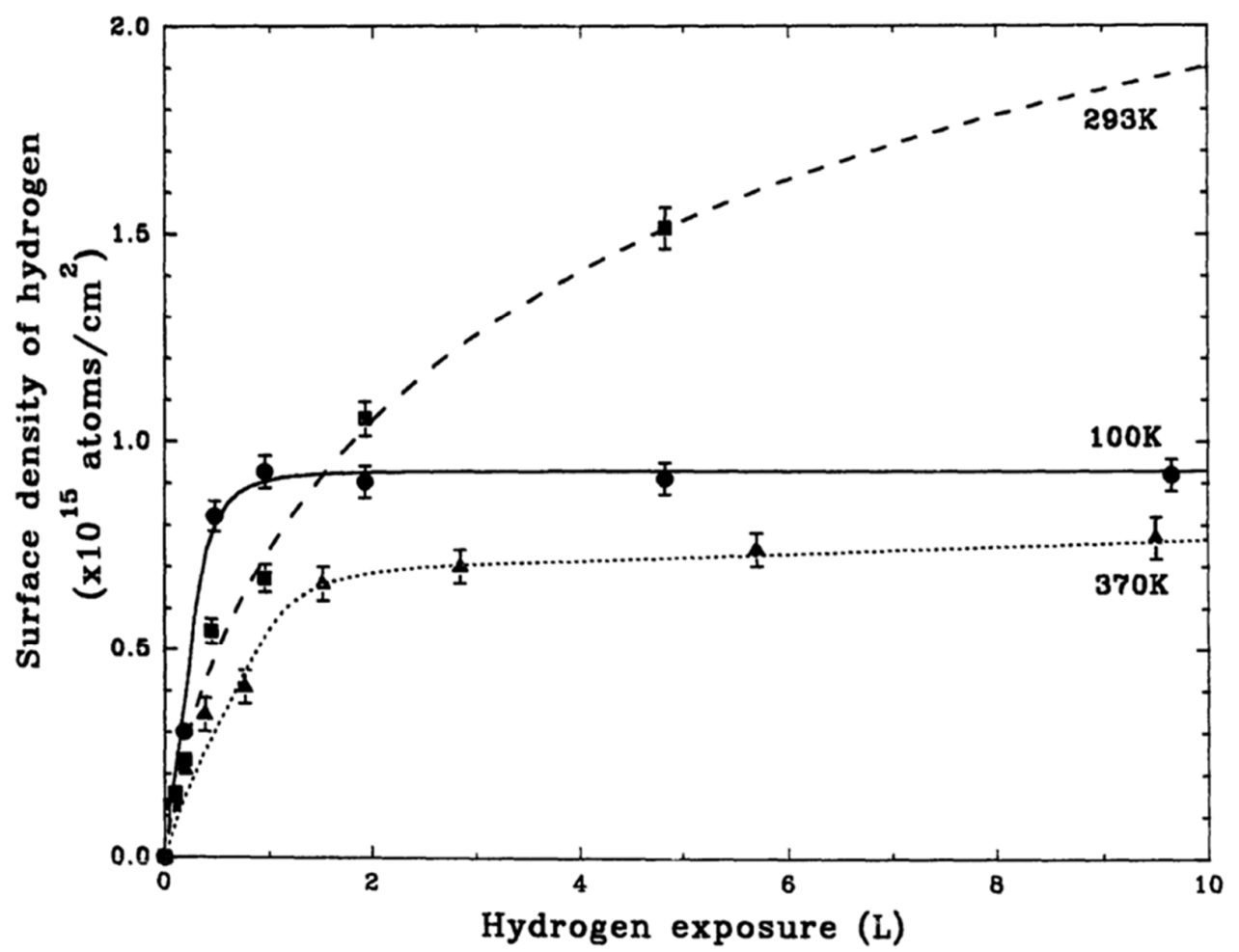

Fig. 49. Uptake of hydrogen at the $\operatorname{Zr}(\mathbf{1 0} \overline{10}))$ surface measured by $D\left({ }^{3} \mathrm{He}, \mathrm{p}\right)^{4} \mathrm{H} N R A$ at $100 \mathrm{~K}$ (circles), $293 \mathrm{~K}$ (squares) and $370 \mathrm{~K}$ (triangles). The lines through the data points are polynomial fitting curves. Reproduced with permission from Ref. [484]. 
At $100 \mathrm{~K}$ the $\mathrm{D}$ uptake saturates rapidly at a density of $9.1 \times 10^{14} \mathrm{~cm}^{-2}$ corresponding to a surface coverage of $1 \mathrm{ML}\left(9.04 \times 10^{14} \mathrm{~cm}^{-2}\right)$, suggesting that the energy barrier for the penetration into the bulk cannot be overcome at the low temperature, so that $D$ remains confined to the deep chemisorption potentials on the surface [484]. At $293 \mathrm{~K}$ a continuous D uptake exceeding the monolayer equivalent is observed during the first $10 \mathrm{~L}$ of exposure, the excess being most likely due to the build-up of a D concentration below the surface. At $370 \mathrm{~K}$, the D diffusion into the bulk ( $L_{D}=11 \mu \mathrm{m}$ in $\left.100 \mathrm{~s}\right)$ has apparently become so effective that a reduced stationary surface $D$ coverage and a D concentration in the $D\left({ }^{3} \mathrm{He}, \mathrm{p}\right)^{4} \mathrm{He}$ detection region result, which even combined are smaller than the monolayer density. These $\mathrm{D}\left({ }^{3} \mathrm{He}, \mathrm{p}\right)^{4} \mathrm{He}$ NRA data demonstrate the strong effect of temperature on the $\mathrm{D}_{2}$ transport across the $\mathrm{Zr}$ surface during absorption, yet no statements about the relative $D$ concentrations on the surface and in the subsurface region can be made due to the lack of depth resolution. Effective $D_{2}$ 'sticking coefficients' on the $\operatorname{Zr}(10 \overline{1} 0)$ surface were determined from the slopes of the uptake curves in the initial exposure range [484]. Note that for $293 \mathrm{~K}$ and $370 \mathrm{~K}$ such coefficients make no distinction between $\mathrm{D}$ surface adsorption and absorption into a $\sim 500 \mathrm{~nm}$ deep region.

Norton's group also presented a detailed analysis of segregation kinetics of bulkdissolved hydrogen onto $\operatorname{Zr}(0001)$ and $\operatorname{Zr}(10 \overline{1} 0)$ surfaces, again in an experiment that monitored the surface $H$ coverage with NRA-calibrated SSIMS [486]. Fig. 50 (a) shows the evolution of hydrogen on these surfaces as the single crystals containing a few ppm of $\mathrm{H}$ in the bulk were heated between room temperature and $400^{\circ} \mathrm{C}$ at 1 ${ }^{\circ} \mathrm{C} / \mathrm{s}$. At first, a non-equilibrium $\mathrm{H}$ distribution with an almost empty surface was 
prepared by rapidly quenching the heated crystals from $650^{\circ} \mathrm{C}$ to room temperature. Upon slow heating, the $\mathrm{H}$ coverage increased first as the bulk diffusion rate rose exponentially, allowing $\mathrm{H}$ segregation onto the surface up to $~ 0.2-0.4 \mathrm{ML}$ (depending on the bulk $\mathrm{H}$ content). At higher temperatures desegregation diminished the surface coverage again, as entropy drove the $\mathrm{H}$ atoms back into the bulk, so that the curves ran through a maximum. The onset of segregation at $80^{\circ} \mathrm{C}$ on $\operatorname{Zr}(10 \overline{1} 0)$ was lower than that on $\operatorname{Zr}(0001)\left(150{ }^{\circ} \mathrm{C}\right)$, since the $\mathrm{H}$ bulk diffusion along the $\langle 10 \overline{1} 0\rangle$ direction is faster than along $\langle 0001\rangle$, presumably as a consequence of the almost two-fold reduced atomic density in these layers relative to the close packed $\operatorname{Zr}(0001)$ basal plane [486].

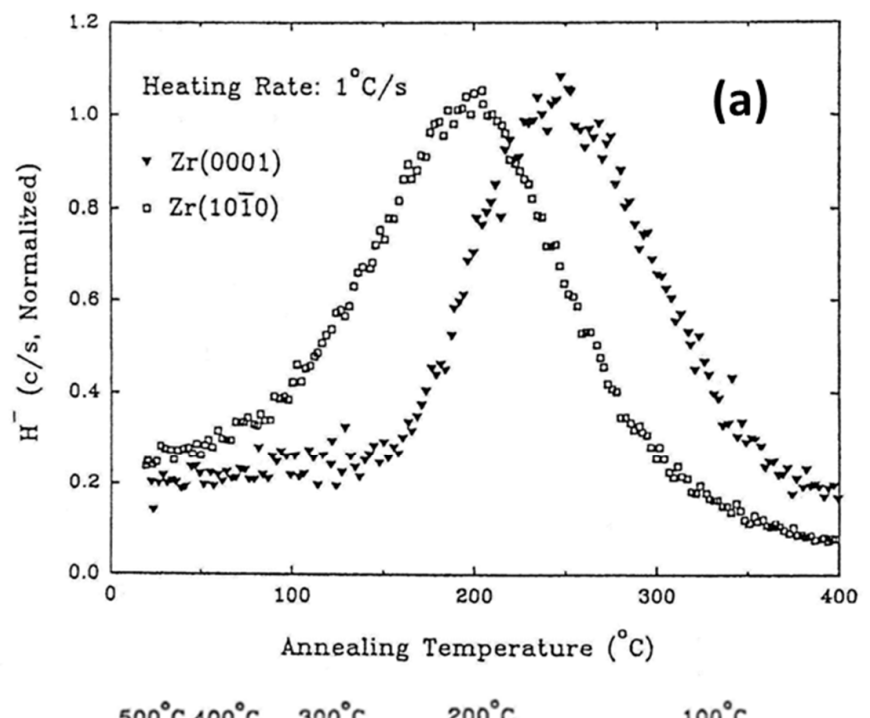

Fig. 50. (a) Surface segregation of hydrogen on $\operatorname{Zr}(0001)$ and $\operatorname{Zr}(\mathbf{1 0} \overline{10})$ measured with NRAcalibrated SSIMS during linear heating $\left(1^{\circ} \mathrm{C} \mathrm{s}^{-1}\right)$. (b) Arrhenius plots of the $\mathrm{H}$ - yield vs. $1 / \mathrm{T}$ on $\operatorname{Zr}(0001)$ and $\operatorname{Zr}(\mathbf{1 0} \overline{\mathbf{1}} \mathbf{0})$. A linear relationship is observed from 300 to $410^{\circ} \mathrm{C}$ on $\operatorname{Zr}(0001)$ and from 260 to $380^{\circ} \mathrm{C}$ on $\operatorname{Zr}(\mathbf{1 0} \overline{10})$. The heats of segregation were derived from these linear portions. Reproduced with permission from Ref. [486]. 
The above experiment was performed at a slow heating rate so that establishment of thermal equilibrium between the surface and bulk sites could be confirmed in the high temperature decay part of Fig. 50 (a), where bulk diffusion was sufficiently fast as not to kinetically control the $\mathrm{H}$ redistribution. The Arrhenius plot of the high temperature coverage decay shown in Fig. 50 (b) yielded the expected linear relationship, from which the energy difference between sites in the bulk and on the surfaces, i.e., the heat of $\mathrm{H}$ segregation, could be determined as $10.1 \mathrm{kcal} / \mathrm{mol}$ for $\operatorname{Zr}(0001)$ and $10.7 \mathrm{kcal} / \mathrm{mol}$ for $\operatorname{Zr}(10 \overline{1} 0)$, respectively [486]. Thus, with reference to the known $\Delta \mathrm{H}_{\mathrm{s}}$, such NRA-calibrated SSIMS measurements permit the experimental determination of the $\mathrm{H}$ chemisorption energy on strongly exothermic $\mathrm{H}$ absorbing metals, which cannot be achieved with TDS. In principle, this should also be possible through a careful evaluation of the surface/bulk occupation equilibrium with ${ }^{15} \mathrm{~N}$ NRA, as suggested by the experiments in Figs. 44 and 45 for H/Ti(0001) [62].

\subsubsection{Palladium(100)}

To demonstrate the unambiguous identification of $\mathrm{H}_{2}$ TDS features in the case of the simultaneous presence of surface-chemisorbed and absorbed hydrogen species with ${ }^{15} \mathrm{~N}$ NRA we consider the $\mathrm{H}_{2}$ TDS trace in Fig. 51, measured at a $\mathrm{Pd}(100)$ surface after dosing a mixture of molecular and atomic hydrogen at $100 \mathrm{~K}[61,62]$. (Atomic $\mathrm{H}$ was used as a means to accelerate the $\mathrm{H}$ uptake below the surface since the $\mathrm{H}_{2}$ absorption probability at $\mathrm{Pd}(100)$ is rather small $\left(\sim 10^{-3}\right)$ at $100 \mathrm{~K}$ [495].) Two $\mathrm{H}_{2}$ desorption signals with peak temperatures of $185 \mathrm{~K}$ and $330 \mathrm{~K}$ were seen. The characteristically sharp low-temperature (so-called $\alpha_{1}$ ) desorption feature at $185 \mathrm{~K}$ 
has been observed in numerous previous TDS studies of low-index Pd surfaces [61, $279,402,495-499$ ] and was attributed to 'subsurface` hydrogen [495], on the basis of its peculiar desorption kinetics (close to zero or fractional orders at not too large populations) and due to the facts that its integrated intensity may exceed the desorption signal of surface-adsorbed $\mathrm{H}$ (the rapidly saturating feature at $330 \mathrm{~K}$ ) after large exposures but does nonetheless not saturate, and that it does not induce notable work function changes. While all this certainly has been compelling indirect evidence for an absorbed $\mathrm{H}$ species, its nature and location below the surface could not be assessed any further.

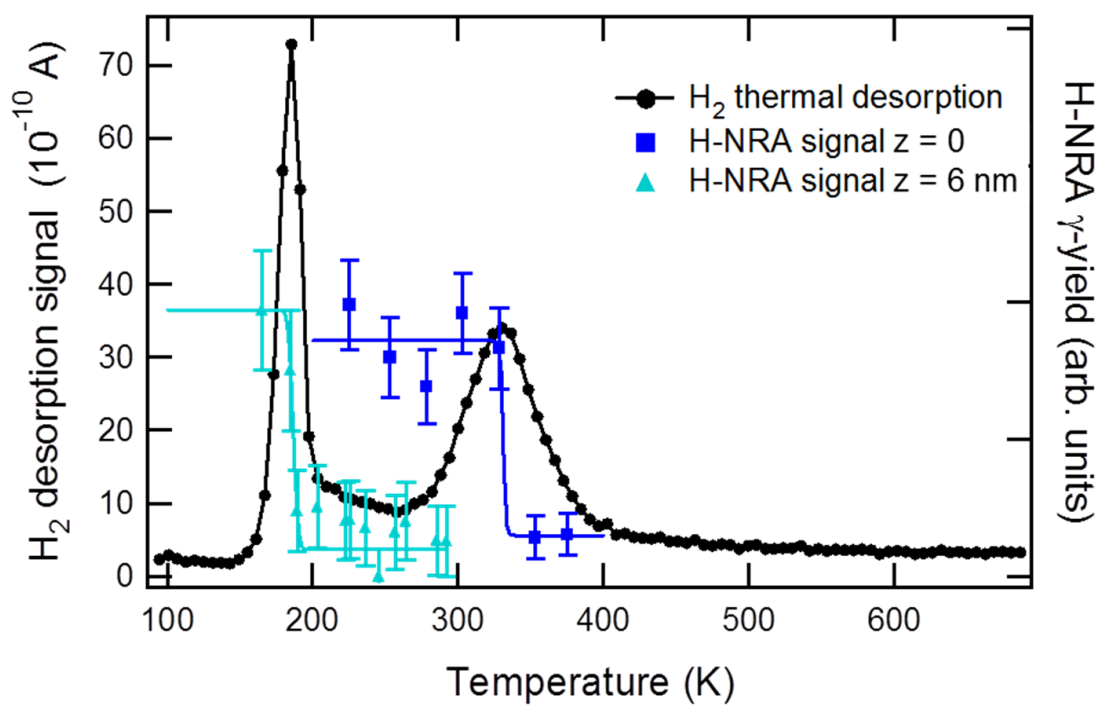

Fig. 51. $\mathrm{H}_{2}$ thermal-desorption spectrum of $\mathrm{Pd}(100)$ exposed to $300 \mathrm{~L} \mathrm{H}+\mathrm{H}_{2}$ at $100 \mathrm{~K}$ compared with temperature-dependent NRA signals of surface $(z=0 \mathrm{~nm})$ and near-surface absorbed ( $z=6 \mathrm{~nm}$ ) hydrogen on/in $\mathrm{Pd}(100)$. Reproduced from Ref. [62].

The NRA $\gamma$-yield curve of $\operatorname{Pd}(100)$ obtained after identical exposure conditions as in the above TDS experiment (Fig. 52) exhibited the dominant Gaussian peak at 6.385 $\mathrm{MeV}$ characteristic of surface-adsorbed hydrogen with an integrated area corresponding to a full monolayer of $\mathrm{H}$ atoms $\left(\mathrm{Pd}(100): 1 \mathrm{ML}=1.32 \times 10^{15} \mathrm{~cm}^{-2}\right)$. 
Saturation at 1.0 ML met expectations from the large exposure (300 $\mathrm{L} \mathrm{H}_{2}$ ) [499], and this $\mathrm{H}$ coverage agreed quantitatively with an earlier ERD determination calibrated against $\mathrm{D}\left({ }^{3} \mathrm{He}, \mathrm{p}\right)^{4} \mathrm{He}$ NRA [258]. In the saturated $(1 \times 1)$ phase on $\mathrm{Pd}(100)$ hydrogen chemisorbs in fourfold hollow sites at a height of $0.3 \AA$ above the Pd surface layer as clarified by transmission ion channeling [258] and confirmed by low energy recoil scattering [500], which directly probed the hydrogen-exposed surface and thus avoided possible errors in transmission channeling due to surface relaxations. The latter possibility had been a non-trivial concern for $\mathrm{Pd}(100)$ since $\mathrm{H}$ adsorption gives rise to a large (4.7\%) first layer outward expansion [501], attributed to strong s-d hybridization between $\mathrm{H}$ and surface $\mathrm{Pd}$ atoms that weakens the $\mathrm{Pd}$ back bonds to the second layer $[476,479,502]$.

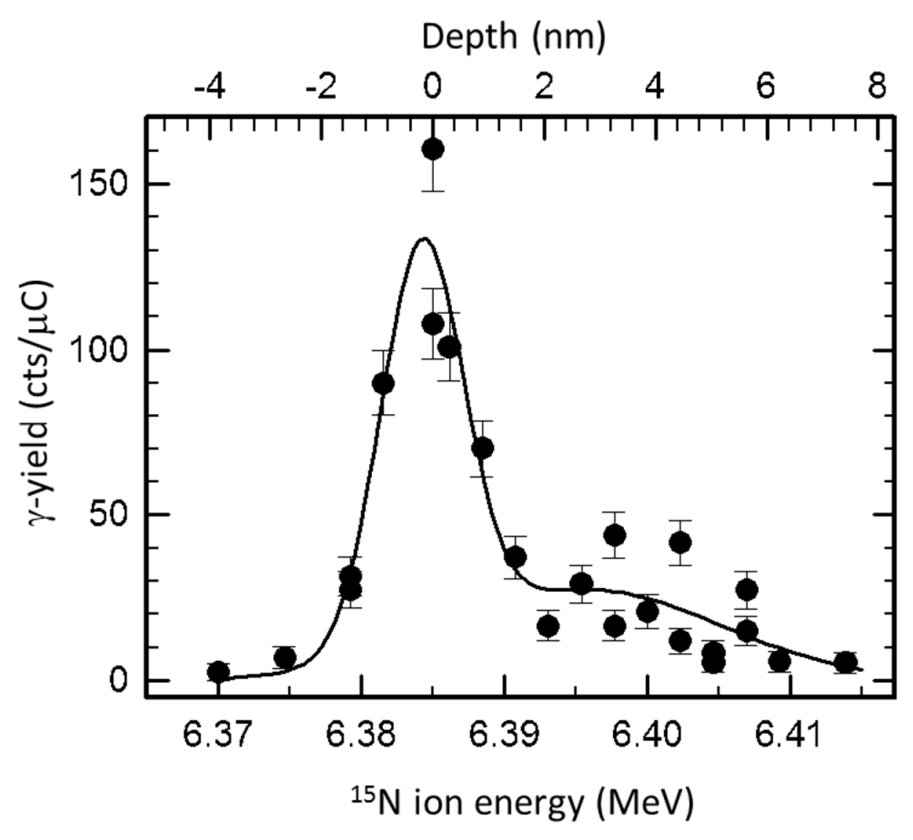

Fig. 52. NRA $\gamma$-yield curve of $\operatorname{Pd}(100)$ after exposure to $300 \mathrm{~L} \mathrm{H}+\mathrm{H}_{2}$ at $100 \mathrm{~K}$. Sample temperature during NRA: $<150 \mathrm{~K}$. The line through the data is a guide for the eye. The incident ${ }^{15} \mathrm{~N}$ ion energy is converted into a probing depth scale (upper abscissa) with the stopping power of $6.4 \mathrm{MeV}{ }^{15} \mathrm{~N}$ in $\mathrm{Pd}$ $(\mathrm{dE} / \mathrm{dz}=3.90 \mathrm{keV} / \mathrm{nm})$. Reproduced from Ref. [62].

In addition to the surface peak of chemisorbed hydrogen the NRA profile in Fig. 52 also showed a broad $\gamma$-yield distribution between 6.39 and $6.41 \mathrm{MeV}$. This 
revealed the presence of absorbed hydrogen, which was distributed within a few nanometer wide region below the $\operatorname{Pd}(100)$ surface [61]. Quantification of the absorbed $\mathrm{H}$ content yielded an areal average of $2.6 \%$ of the $\mathrm{Pd}$ atomic density. This suggested that the very small $\mathrm{H}$ solubility in the solid solution phase of the $\mathrm{Pd}-\mathrm{H}$ system (the terminal $\mathrm{H}$ concentration is only $12.3 \mathrm{ppm}$ at $100 \mathrm{~K}$ ) [503] was exceeded and that a few grains of Pd hydride (containing 65 at. \% H) [504-506] had precipitated in a shallow region below the surface. Hence, the NRA H profile revealed that the absorbed hydrogen here again was clearly not confined only to the first layer subsurface sites but that it rather corresponded to a near-surface hydride phase $[61,279]$. Its assignment to the TDS $\alpha_{1}$ state is described in the following.

By measuring the NRA signals at fixed ${ }^{15} \mathrm{~N}$ ion energies that selectively probed the near-surface hydride and surface-adsorbed $H$ at depths of $6 \mathrm{~nm}$ and $0 \mathrm{~nm}$, respectively, while incrementally raising the sample temperature, the thermal stability of the absorbed and the chemisorbed $\mathrm{H}$ species were evaluated individually. The obtained NRA signals were superimposed with the TDS trace of the hydrogenexposed $\mathrm{Pd}(100)$ surface in Fig. 51, where they are seen to decay sharply at temperatures that coincide exactly with the peak positions of the two dominant $\mathrm{H}_{2}$ desorption features at $185 \mathrm{~K}$ and $330 \mathrm{~K}$ [62]. This experiment convincingly assigned the former $\left(\alpha_{1}\right)$ feature to thermal decomposition of the near-surface hydride and the latter to recombinative desorption of chemisorbed surface hydrogen. This direct and unambiguous identification of TDS desorption signals by non-destructive, highly depth-resolved, and temperature-selective monitoring of the $\mathrm{H}$ density on or below the surface represents one of the strongest advantages of ${ }^{15} \mathrm{~N}$ NRA in the 
characterization of $\mathrm{H}$ states in the near-surface region of hydrogen-absorbing materials.

The revalation of the $\mathrm{H}_{2}$ exposure temperature $\left(T_{\mathrm{e}}\right)$ dependence of near-surface hydrogen profiles through ${ }^{15} \mathrm{~N}$ NRA is exemplified by comparing the excitation curves of Fig. 52 and Fig. 53 (a). For the latter, $300 \mathrm{~L} \mathrm{H}_{2}$ were dosed onto the $\mathrm{Pd}(100)$ surface at $160 \mathrm{~K}$ (at $3 \times 10^{-6} \mathrm{~Pa}$ ). The $\gamma$-yield profile in Fig. 53 (a) displays the saturated resonance peak due to surface-adsorbed $\mathrm{H}$ (1 $\mathrm{ML}$ coverage) and an additional intensity plateau of $(28 \pm 1) \mathrm{cts} / \mu \mathrm{C}$ height extending to at least $16 \mathrm{~nm}$ below the surface, indicative of an absorbed $\mathrm{H}$ concentration of $7.1 \times 10^{20} \mathrm{~cm}^{-3}$, or $1 \%$ of the $\mathrm{Pd}$ atomic density. Together with the associated TDS spectrum (Fig. 53 (b), this NRA H profile corroborated a previous tentative assignment of the TDS $\alpha_{2}$ feature to bulkabsorbed hydrogen. The TDS $\alpha_{2}$ feature is clearly different from the $\alpha_{1}$ peak in Fig. 51 as it shows a long high temperature tail and a higher peak maximum position, which, moreover, shifted to even higher temperatures when the $T_{\mathrm{e}}$ was raised. This suggested bulk diffusion-controlled $\mathrm{H}_{2}$ desorption [495], a conjecture here clearly confirmed by ${ }^{15} \mathrm{~N}$ NRA through the larger depth extension of the $\mathrm{H}$ distribution in Fig. 53 (a) relative to that in Fig. 52. 

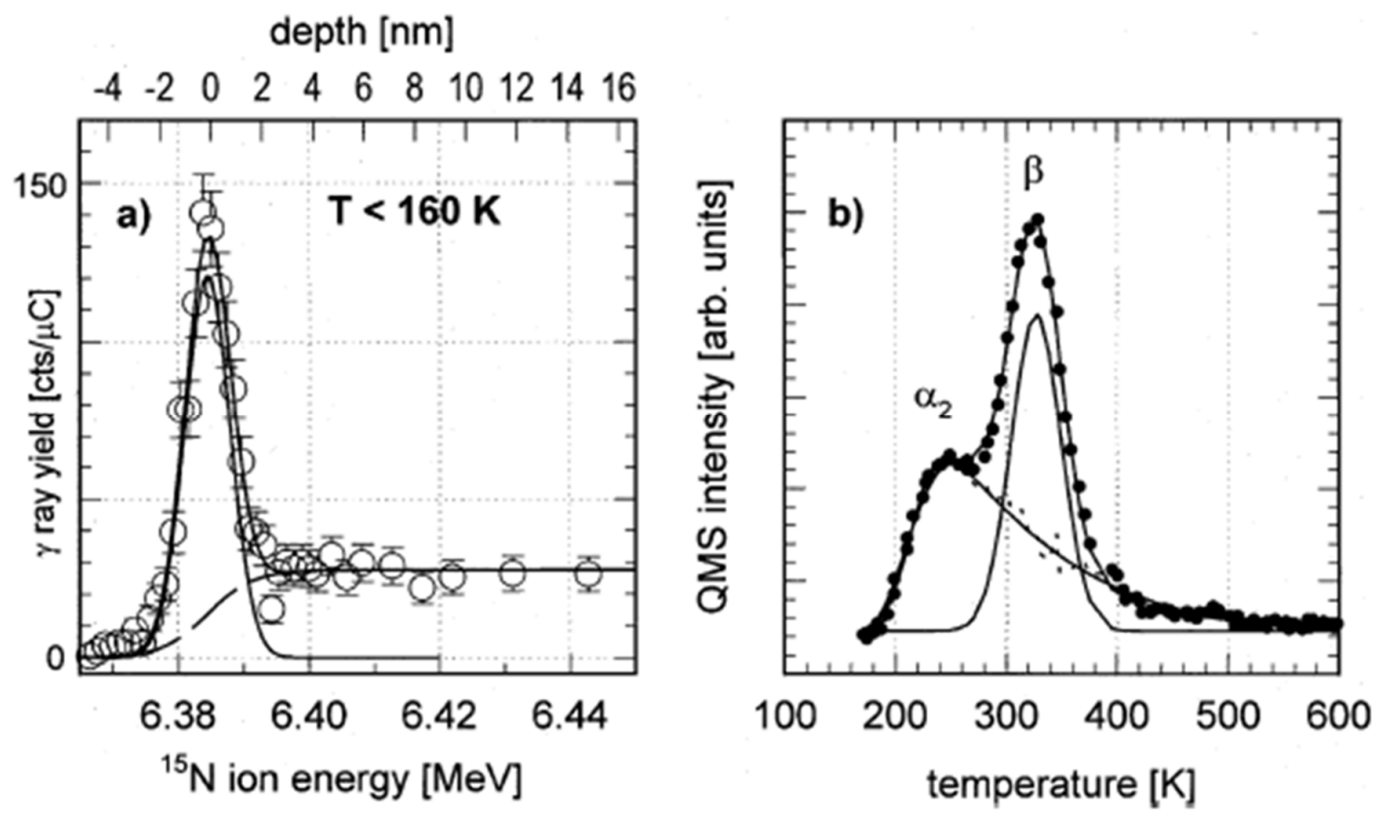

Fig. 53. (a) NRA depth profile of $\mathrm{H} / \mathrm{Pd}(100)$ at $\mathrm{T}<160 \mathrm{~K}$ under $3 \times 10^{-6} \mathrm{~Pa} \mathrm{H}_{2}$. (b) Post-NRA $\mathrm{H}_{2}$ TDS (linear heating rate $2 \mathrm{~K} / \mathrm{s}$ ). Total $\mathrm{H}_{2}$ dosage: $300 \mathrm{~L}$. Reproduced with permission from Ref. [61].

By extrapolating the $\mathrm{H}_{2}$ plateau pressures in the solid-solution/hydride coexistence region of the $\mathrm{Pd}-\mathrm{H}$ phase diagram to low temperatures and comparing with isotherm data at the Pd solid solution phase boundary [503], one realizes that the absorbed $\mathrm{H}$ concentration in the near-surface NRA depth profiles ( $\sim 1-2.6$ at. \%) in Figs. 52 and 53 exceed the $\mathrm{H}$ solubility limits of the Pd solid solution phase (12 ppm at $100 \mathrm{~K}$ and $570 \mathrm{ppm}$ at $160 \mathrm{~K}$ ). This indicates that $\mathrm{Pd}$ hydride has precipitated in the solid solution phase matrix in both cases. According to the Pd-H phase diagram [505] Pd hydride contains $\sim 65$ at. $\% \mathrm{H}$ under the low temperature conditions of the present experiments, whereas the terminal solid solution phase concentrations (12 and $570 \mathrm{ppm}$ at $100 \mathrm{~K}$ and $160 \mathrm{~K}$, respectively) do not or just barely exceed the NRA sensitivity ( $400 \mathrm{ppm}$ for $\mathrm{Pd})$. This suggests that almost all (98\%) absorbed $\mathrm{H}$ atoms detected in the NRA profiles of Figs. 52 and 53 are contained in hydride grains. 
In the $\mathrm{Pd}$ solid solution phase the $\mathrm{H}$ diffusion coefficient $D=D_{0} \cdot \exp \left(E_{\text {diff }} / k_{\mathrm{B}} T\right)\left(D_{0}=\right.$ $42.9 \times 10^{-9} \mathrm{~m}^{2} \mathrm{~s}^{-1} ; E_{\text {diff }}=0.13 \mathrm{eV}$ at $\left.T<220 \mathrm{~K}\right)$ [507] translates into diffusion lengths ( $L_{\mathrm{D}}$ $\left.=(D \Delta t)^{1 / 2} ; \Delta t=100 \mathrm{~s}\right)$ of $340 \mathrm{~nm}$ at $100 \mathrm{~K}$ and $5 \mu \mathrm{m}$ at $160 \mathrm{~K}$. Compared to this, the depth extensions of the hydride-containing regions in Figs. 52 and 53 are considerably shorter $[28,29,61,508]$. In Pd hydride the fcc lattice is expanded by 3.3\% from 3.89 to $4.02 \AA$, which slows the $\mathrm{H}$ diffusion as the jump distance between adjacent octahedral interstitial sites elongates. Also the number of vacant interstitial sites reduces strongly due to the much larger $\mathrm{H}$ content in the hydride $[25,28,29]$. The elastic $\mathrm{H}-\mathrm{H}$ interaction, which is repulsive in the solid solution phase but attractive in the expanded lattice of Pd hydride, further promotes localization of absorbed $\mathrm{H}$ atoms in growing hydride grains $[27,28]$. These factors may provide a qualitative explanation for the reduced extension of the observed hydride distributions compared to the $\mathrm{H}$ diffusion length in the solid solution phase [61]. The depth extension of the hydride distribution obtained by $\mathrm{H}_{2}$ exposure of $\mathrm{Pd}(100)$ at $160 \mathrm{~K}$ (Fig. 53) is evidently larger than that formed in the absorption experiment at $100 \mathrm{~K}$ (Fig. 52). Similar as in recent observations of $\mathrm{H}_{2}$-absorption at $\mathrm{Pd}(110)$ [279], this may be ascribed qualitatively to strongly temperature sensitive hydride nucleation underneath the surface, as the absorbed $\mathrm{H}$ can diffuse larger distances at higher temperatures and may give rise to hydride nucleation in more extended regions below the surface.

\subsubsection{4 $P d_{70} A u_{30}(110)$}

Ogura et al. reported a combined NRA and TDS investigation of the lowtemperature $\mathrm{H}_{2}$ absorption at a single crystalline $\mathrm{Pd}_{70} \mathrm{Au}_{30}(110)$ alloy surface [485]. 
Due to their expanded lattice compared to pure $\mathrm{Pd}, \mathrm{Pd}-\mathrm{Au}$ alloys exhibit increased dilute phase $\mathrm{H}$ solubility at a given $\mathrm{H}_{2}$ pressure (a more negative $\Delta \mathrm{H}_{s}$ ) and they do not precipitate a hydride phase above $298 \mathrm{~K}$ if the Au content exceeds $\sim 17 \%[509$, 510]. As mentioned in Sect. 3.1.2, alloys are interesting also because their surface elemental composition may deviate significantly from the bulk stoichiometry due to segregation effects. Ogura et al. found that through variation of the UHV annealing temperature $(400-900 \mathrm{~K})$ for the ion sputter-cleaned single crystal, the surface layer composition of $\mathrm{Pd}_{70} \mathrm{Au}_{30}(110)$ can be adjusted to contain between 40 and $90 \%$ $\mathrm{Au}$, and that a surface Au coverage of $\sim 90 \%$ is obtained by annealing at $700 \mathrm{~K}$ [485]. $\mathrm{H}_{2}$ TD spectra of such a $700 \mathrm{~K}$-annealed strongly Au-enriched $\mathrm{Pd}_{70} \mathrm{Au}_{30}(110)$ surface are shown in Fig. 54 (a) after exposure to $100 \mathrm{~L} \mathrm{H}_{2}$ at various temperatures between 105 and $180 \mathrm{~K}$. All $\mathrm{H}_{2}$ TDS traces showed a broad main desorption feature at $240 \mathrm{~K}$ with a small shoulder at $360 \mathrm{~K}$. While the amount of desorbed hydrogen at $105 \mathrm{~K}$ was very small (corresponding to a coverage of $0.1 \mathrm{ML}\left(1 \mathrm{ML}=8.8 \times 10^{14} \mathrm{~cm}^{-2}\right)$ with reference to the $\mathrm{H}_{2}$ TDS intensity of a $\mathrm{H}$-saturated $\mathrm{Pd}(110)$ surface $\left.[279,511]\right)$, it increased steadily with the temperature during $\mathrm{H}_{2}$ exposure above $120 \mathrm{~K}$ and assumed quite substantial values of several (>8) MLs at $180 \mathrm{~K}$ as shown in the inset. This suggested that at $105 \mathrm{~K} \mathrm{H}$ adsorbed only on the few remaining Pd atoms in the surface layer ( 10\%), whereas larger $H$ quantitites appeared to have been incorporated into the alloy interior at temperatures above $120 \mathrm{~K}$. 

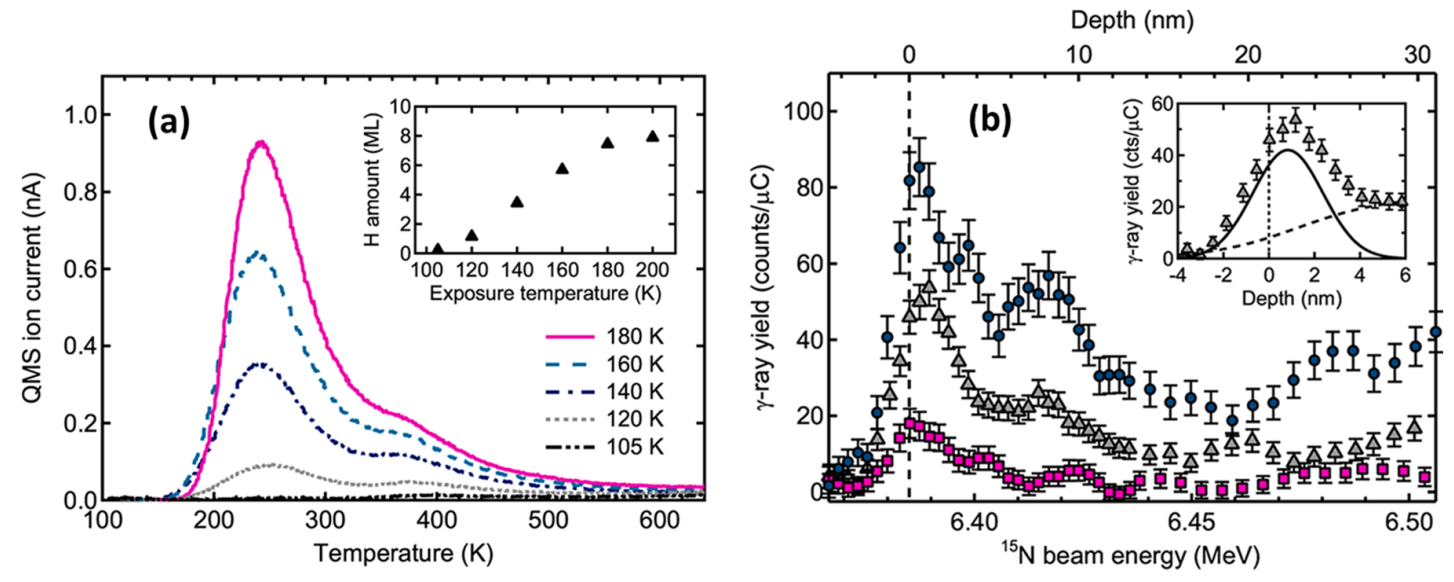

Fig. 54. (a) TD spectra from a $\mathrm{Pd}_{70} \mathrm{Au}_{30}(110)$ surface annealed at $700 \mathrm{~K}$ exposed to $100 \mathrm{~L} \mathrm{H}_{2}$ at various temperatures. The inset shows the amount of desorbed hydrogen in ML units as a function of sample temperature during $\mathrm{H}_{2}$ exposure. (b) NRA yield curves from $\mathrm{Pd}_{70} \mathrm{Au}_{30}(110)$ after exposure to $1000 \mathrm{~L} \mathrm{H}_{2}$ at 120 (circles), 140 (triangles), and $180 \mathrm{~K}$ (squares). The inset shows the $\gamma$-yield curve of $140 \mathrm{~K}$ enlarged near the surface. Solid and dashed lines are Gaussian and sigmoid components, respectively. Reproduced with permission from Ref. [485].

This conjecture was corroborated by the ${ }^{15} \mathrm{~N}$ NRA $\mathrm{H}$ profiles in Fig. 54 (b), obtained after exposing the alloy surface of the same composition to $1000 \mathrm{~L} \mathrm{H}_{2}$ at 120,140 , and $180 \mathrm{~K}$. The NRA profiles clearly confirmed that hydrogen absorption had occurred in the probed region $<30 \mathrm{~nm}$ deep below the surface, evidencing accumulation regions in 0-12 $\mathrm{nm}$ and plateau-like features at larger depths. All profiles showed maximum $\mathrm{H}$ content near but not right at the surface. The decomposition of the $140 \mathrm{~K}$ profile into a Gaussian peak and a sigmoid component reflecting absorbed $\mathrm{H}$ shown in the inset revealed that the $\mathrm{H}$ distribution peaked at a depth of $(0.8 \pm 0.2) \mathrm{nm}$, cleary indicating $\mathrm{H}$ accumulation below the surface. $\mathrm{A}$ surface $\mathrm{H}$ coverage smaller than $0.1 \mathrm{ML}$ was deduced from the data, quite consistent with the quantity of TDS-detected $\mathrm{H}_{2}$ after exposure at $105 \mathrm{~K}$, supporting the interpretation that the surface-adsorption of hydrogen remains rather limited. 
Contrary to the increasing amount of $\mathrm{H}_{2}$ desorbing in TDS (Fig. 54 (a)), the average concentration of $\mathrm{H}$ absorbed in the $12-30 \mathrm{~nm}$ deep alloy region evaluated with ${ }^{15} \mathrm{~N}$ NRA decreased at higher exposure temperatures, from an average of 2.7 at. $\%$ at $120 \mathrm{~K}$, to 1.2 at.\% at $140 \mathrm{~K}$, and 0.3 at.\% at $180 \mathrm{~K}$. This apparent contradiction was qualitatively reconciled by the fact that the depth distribution of the absorbed $\mathrm{H}$ is expected to extend into increasingly larger depths by entropydriven diffusion into the bulk at higher $\mathrm{H}_{2}$-exposure temperatures [485]. The noticeably non-uniform depth distribution of the absorbed hydrogen was tentatively attributed to possible Pd-Au alloy composition fluctuations below the surface, since the Au segregation process creates Pd-enriched layers below the surface [512], and the $\mathrm{H}$ solubility in Pd-Au alloy is known to vary with the alloy composition [510, 513, 514].

\subsubsection{Hydrogen trapping at internal defects and surfaces}

The research reviewed so far has focused on (low-temperature) $\mathrm{H}_{2}$ absorption studies at (more or less perfect) metal single crystal surfaces and on the direct and depth-resolved ${ }^{15} \mathrm{~N}$ NRA observation of variations in the hydrogen gas/surface/bulk population equilibrium elicited by changes of the thermodynamic boundary conditions (temperature or $\mathrm{H}_{2}$ pressure). Methodically closely related are NRA investigations of the thermally induced redistribution of bulk-absorbed hydrogen between various kinds of $\mathrm{H}$ trapping sites thay may exist in the interior of metals. Such studies aim at evaluating the bonding strength of hydrogen as it interacts with immobilizing entities in the metal host lattice such as implanted heteroatoms, 
radiation defects, vacancies, vacancy-solute complexes, crystalline precipitates, and in particular with the interior surfaces of gas $\left(\mathrm{He}, \mathrm{D}_{2}, \mathrm{H}_{2}\right)$ bubbles (voids) that are suspected as a cause of blistering and embrittlement [122, 515-518].

In order to experimentally distinguish internal hydrogen redistribution processes from desorption through the surface, initial hydrogen and trap site distributions that differ largely in their depth location (on the order of several micrometers) can be prepared by energy-controlled ion implantation, so that even the very limited depth resolution of $D\left({ }^{3} \mathrm{He}, \mathrm{p}\right)^{4} \mathrm{He}$ NRA suffices to provide information on the hydrogen relocation behavior [122, 517, 518]. This technique is illustrated in Fig. 55 (a), which shows the $D\left({ }^{3} \mathrm{He}, \mathrm{p}\right)^{4} \mathrm{He}$ nuclear reaction cross section as a function of depth in beryllium for incident $500 \mathrm{keV}{ }^{3} \mathrm{He}$ together with the TRIM-calculated implantation ranges of $10 \mathrm{keV} \mathrm{H}$ and $15 \mathrm{keV} \mathrm{He}$ ions, which were used in high doses to generate gas bubbles as traps for $D$ in about $0.1 \mu \mathrm{m}$ depth below the surface [122]. After implanting $60 \mathrm{keV} \mathrm{D}$ into a deeper range at $20^{\circ} \mathrm{C}$ (a smaller dose that does not create bubbles), the NRA proton yield from the $D\left({ }^{3} \mathrm{He}, \mathrm{p}\right)^{4} \mathrm{He}$ reaction was monitored while the sample temperature was ramped linearly to $600^{\circ} \mathrm{C}$.

It is seen in Fig. 55 (b) that between 440 and $480{ }^{\circ} \mathrm{C}$ the $\mathrm{D}$ was removed by desorption from the Be sample implanted with $\mathrm{D}$ only. When $\mathrm{H}$ or He had been implanted in addition, the NRA yield increased instead as soon as the implanted D became mobile when it detached from its own implantation-induced defects near $400{ }^{\circ} \mathrm{C}$, because the $\mathrm{D}$ relocated to the bubble defects near the surface where the $\mathrm{D}\left({ }^{3} \mathrm{He}, \mathrm{p}\right)^{4} \mathrm{He}$ cross section is larger. At yet higher temperatures of $550{ }^{\circ} \mathrm{C}$ (preimplanted $\mathrm{H}$ ) or even $600^{\circ} \mathrm{C}$ (pre-implanted He), D was then thermally released from 
the near-surface traps and desorbed. These data demonstrated a significantly stronger interaction of $\mathrm{D}$ with the gas bubbles than with the lattice defects generated by its own implantation. The interaction energy difference between $\mathrm{H}$ bound to He bubbles and $\mathrm{H}$ at $\mathrm{D}$-implantation-induced defects can be extracted from the NRA redistribution data $[122,517]$.

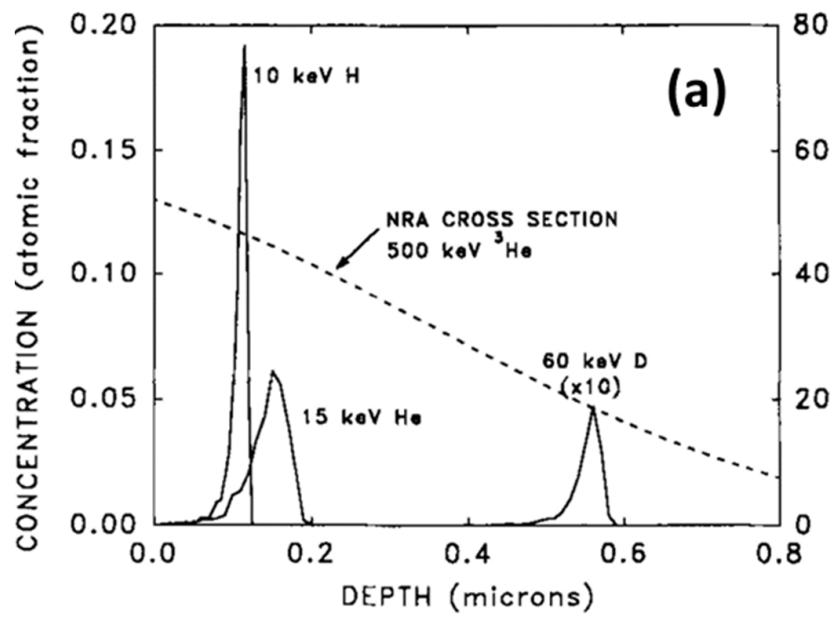

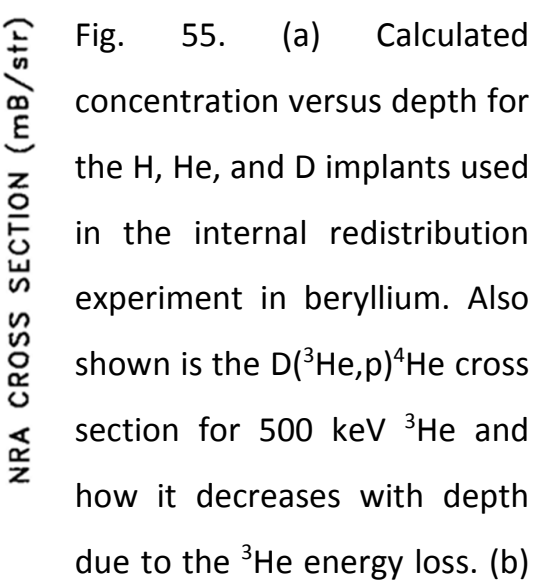

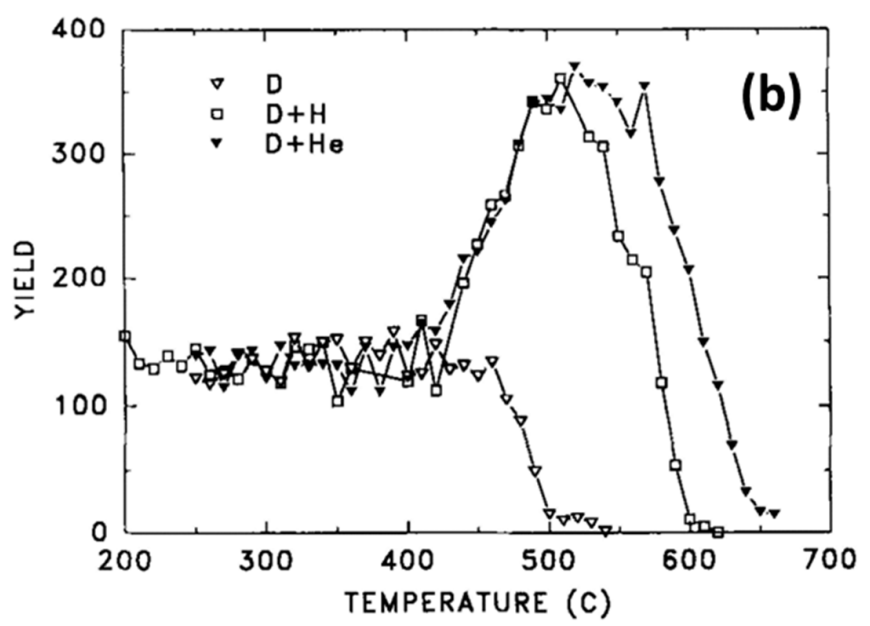

NRA yield during temperature ramping at $2^{\circ} \mathrm{C} / \mathrm{min}$. The yield increase near $450^{\circ} \mathrm{C}$ occurs when D migrates from its implanted depth to the $\mathrm{H}$ or He nearer the surface where the NRA cross section is larger. Reproduced with permission from Ref. [122].

Similar investigations of $D$ interaction with implanted He have been performed for a series of metals including Al, Fe, Ni, Mo, Pd, Ta, stainless steel, and Inconel [516, 517]. Including evaluations of near-surface $H(D)$ depth distributions by NRA and ERDA $[519,520]$, this line of research has remained highly active to the present day, in part motivated by the importance to understand the mechanisms of deuterium 
retention and tritium inventory in plasma facing components of fusion-energy devices (JT-60, JET, ITER, etc.), where the proper material selection is a top engineering priority. To give just one example of an important result, a substantial suppression of near-surface $D$ retention in tungsten under fusion-relevant conditions by small additions of He to the plasma has recently been discovered by $D\left({ }^{3} \mathrm{He}, \mathrm{p}\right)^{4} \mathrm{He}$ NRA and TDS measurements [515]. In conjunction with microstructure characterization by TEM [521] it was suggested that this effect of He results from reduced blistering and the formation of a network of He nanobubbles underneath the surface that may effectively interrupt the $D$ transport from the surface region into the bulk [522].

Hydrogen trap generation by ion implantation in combination with NRA analysis has also been used to investigate the $\mathrm{H}$-absorption and release kinetics between the metal interior and the gas phase, which has enormous fundamental importance for hydrogen storage, purification, sensors, and catalysis [508, 516, 523-526]. Similar to penetration studies [527-529], such experiments aimed to extract information on the relative energy levels of $\mathrm{H}$ atoms in the metal interior, on the surface, in the gas phase, and on the kinetic barriers between them. A presentation of the extensive research in this area and of the proposed kinetic models is beyond the scope of the present article. For earlier reviews of the NRA contribution to this field in conjunction with the implantation technique see Myers et al. [517, 518].

As a representative example we refer to the deuterium uptake/release kinetics at a clean (polycrystalline) palladium surface monitored by means of $D\left({ }^{3} \mathrm{He}, \mathrm{p}\right)^{4} \mathrm{He}$ NRA after introducing bubble traps into a shallow average depth (56 $\mathrm{nm}$ ) below the 
surface by implantating a large dose $\left(4 \times 10^{16} \mathrm{~cm}^{-2}\right)$ of $15 \mathrm{keV} \mathrm{He}[516]$. The He traps confined the major amount of absorbed $D$ to a shallow depth region where the $\mathrm{D}\left({ }^{3} \mathrm{He}, \mathrm{p}\right)^{4} \mathrm{He}$ nuclear reaction cross section is approximately constant, and thus allowed observing the equilibration kinetics of $D$ between these He traps and the gas phase during $D_{2}\left(10^{-9}-10^{-5}\right.$ Torr, 1 Torr $\left.=133 \mathrm{~Pa}\right)$ absorption as well as during $D_{2}$ release upon evacuation.

Such data are shown in Fig. 56 as (a) D uptake and (c) D release curves. An analysis of the initial uptake and release rates as well as of the equilibrium trap populations determined the energetic positions of $D$ bound to the He traps $(-0.29 \mathrm{eV}$ relative to $D$ in solid solution) and in recombination sites (at least $0.15 \mathrm{eV}$ less strongly bound than D in low coverage chemisorption sites) [516]. Besides ensuring full visibility to $D\left({ }^{3} \mathrm{He}, \mathrm{p}\right)^{4} \mathrm{He} N R A$, a further advantage of restricting the major $D$ uptake to a shallow depth region was that the distance of the He traps from the surface was short enough to exclude that the uptake/release kinetics were limited by bulk diffusion. This was verified by Fig. 56 (b), which shows that the experimental D uptake rate was much smaller than an upper ('bulk permeation rate') limit estimated from the $\mathrm{H}$ diffusion rate in the Pd solid solution phase, and also by Fig. 56 (c), where the D-release is much slower than calculated for bulk-diffusion limited desorption. Thus, the kinetics of the D uptake into and of the release from the near-surface He traps were concluded to be limited by surface processes, and indeed showed the expected linear dependence on the $D_{2}$ pressure (Fig. 56 (b)). Owing to the $\sim 10^{4}$ times shorter diffusion length involved, this result was no contradiction to the fact that the macroscopic equilibration kinetics of $\mathrm{H}_{2}$ gas with clean $\mathrm{Pd}$ single crystals were 
observed to be controlled by bulk diffusion, showing a characteristic $p^{1 / 2}$ dependence of the initial uptake rate $[508,516]$.

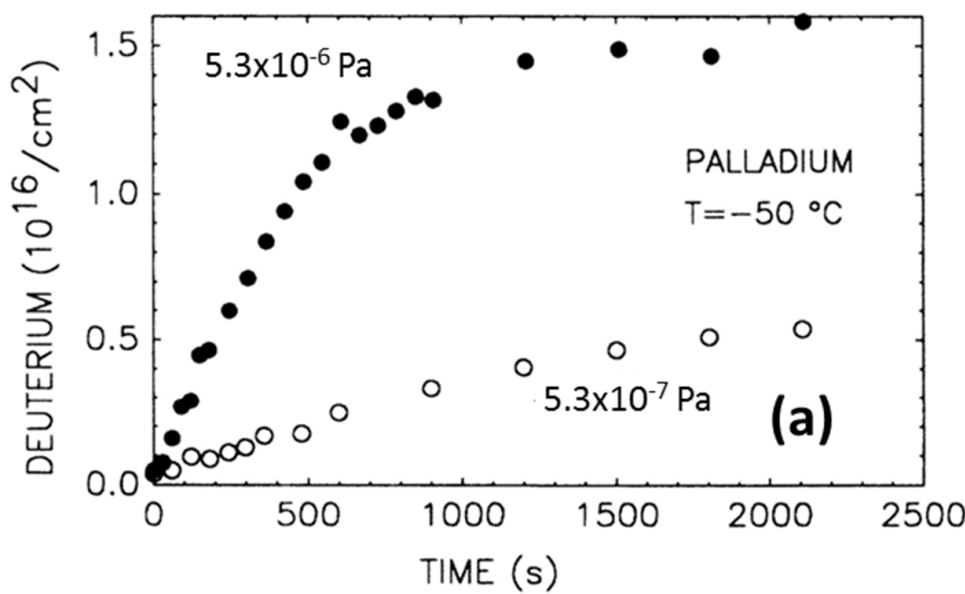

Fig. 56. (a) Uptake of $D$ into $\mathrm{Pd}$ monitored by $\mathrm{D}\left({ }^{3} \mathrm{He}, \mathrm{p}\right)^{4} \mathrm{He}$ NRA after $\mathrm{D}_{2}$ enters the chamber at $\mathrm{t}=$ 0 . The closed and open circles show data for $D_{2}$ gas pressures of $5.3 \times 10^{-6}$ and $\quad 5.3 \times 10^{-7} \quad \mathrm{~Pa}$, respectively.

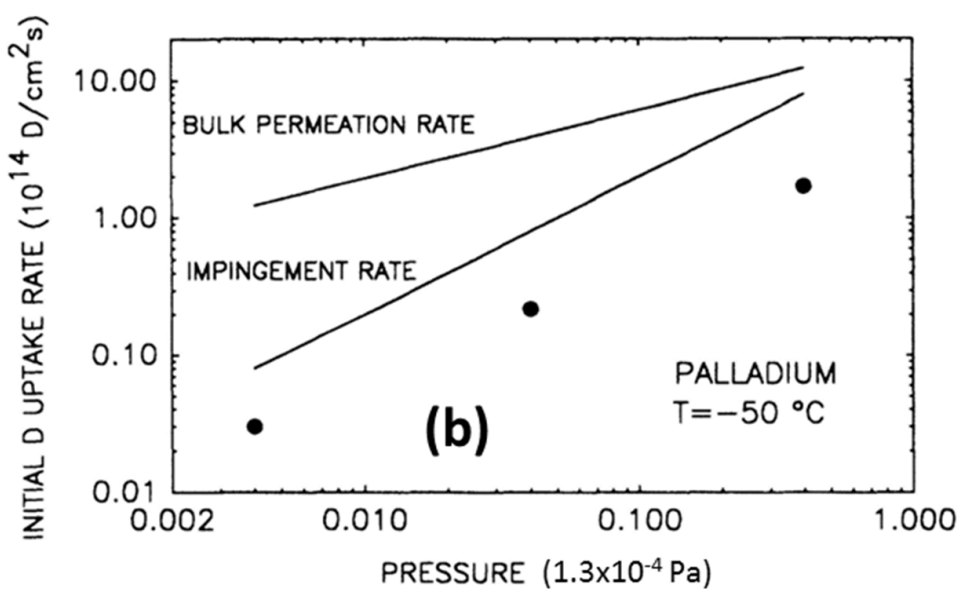

(b) Initial D-uptake rate vs. gas pressure (closed circles). The rate of impingement on the surface from the $D_{2}$ gas and the bulk transportlimited uptake rate are shown by the two solid lines.

(c) Areal density of D vs.

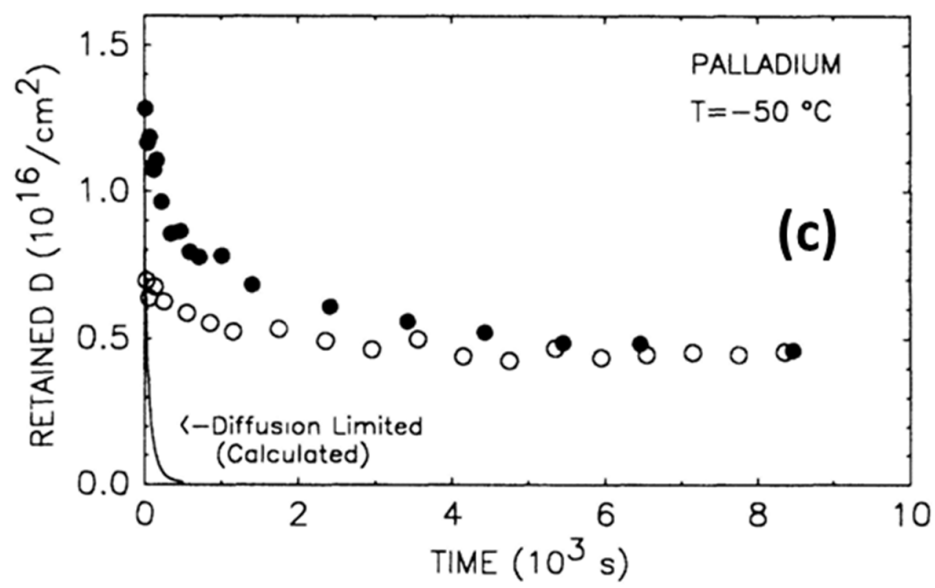
time during release after equilibrating at pressures of $5.3 \times 10^{-7} \quad \mathrm{~Pa} \quad$ (open circles) and $5.3 \times 10^{-5} \mathrm{~Pa}$ (closed circles). The curve shows the calculated $D$ release when surface processes are not rate limiting. The time scale begins when the $D_{2}$ gas was pumped from the chamber. Adapted from Ref. [516]. 
Fig. 56 (b) also showed that the population of the He traps by gas phase $D_{2}$ proceeded quite efficiently with a sticking probability of 0.2-0.4 (compare the Duptake rate (closed circles) with the impingement rate). This probability is several orders of magnitude higher than reported in typical low pressure $\mathrm{H}_{2}$ absorption studies at Pd single crystals $\left(\sim 10^{-3}-10^{-2}\right)[61,279,495,496]$. Since surface defects such as steps and vacancies are well known to enhance $\mathrm{H}_{2}$ dissociation on metal surfaces $[364,365,530,531]$ and are suspected to be sites of high absorption rate $[279,402,495]$ this observation hints at the possibility that the generality of the results in Ref. [516] regarding the energy position of the recombination sites is limited to surfaces damaged by ion implantation.

\subsubsection{Modified surface layers}

Hydrogen uptake and release kinetics into and from bare metals [525, 526] are extremely sensitive to surface modifications, and much research effort has been devoted to clarify the influence of native oxides [166, 532], hydrides [533], metallic overlayers [534, 535], implanted heteroatoms [516, 517, 532] (including hydrogen itself $[515,536,537])$, as well as surface processing (e.g., polishing) [537]. A prominent application of NRA has been to reveal the near-surface $\mathrm{H}$-depth distributions resulting from either the modification process itself or after hydrogen uptake through the modified surface. Similarly, near-surface hydrogen depletion layers resulting from vacuum annealing of titanium hydrides have been analyzed by ERD [538]. The thin native oxide layers formed on metal surfaces in air are frequently 
found to suppress the hydrogen uptake into the bulk material. On $\mathrm{H}$-loaded samples this is manifested as a depletion layer near the surface, where the absorbed $\mathrm{H}$ concentration shows a depression that relates to the content of dissolved oxygen, as exemplified by ${ }^{15} \mathrm{~N}$ H profiles from polycrystalline $\mathrm{Nb}$ foils [166]. Rauch and coworkers demonstrated by $\mathrm{H}$ profiling with ${ }^{15} \mathrm{~N}$ NRA that such inhibition of $\mathrm{H}$ absorption from $\mathrm{H}_{2}$ gas at oxide-passivated Ti surfaces can be circumvented even without removing the surface oxide layer through the implantation of low energy $(<5$ keV) Pd ions [532]. Enhancements of the $\mathrm{H}$ uptake in $\mathrm{Nb}$ and Ta foils by coating with $\mathrm{Pd}$ are also reported [534, 535]. Here, the origin of the improved $\mathrm{H}$ uptake is threefold, as Pd (1) prevents oxidation of the surface, (2) enables efficient $\mathrm{H}_{2}$ dissociation, and (3) lowers the energy of the surface adsorption state and thereby reduces the energy barrier for the transition of $\mathrm{H}$ atoms into the bulk $[525,534,535]$. Owing to this unique property combination, thin Pd films are widely employed as capping layers in oxidation-sensitive hydrogen absorbing metallic thin film devices such as metal gas sensors and switchable mirrors [32, 33, 539-543].

Recent developments in ion beam analysis include the application of strongly focused beams that allow for the detection of lateral elemental concentration differences within the surface plane of a specimen. Combined with the high depthresolving powers of ${ }^{15} \mathrm{~N}$ NRA or ERD these micro-beam techniques enable threedimensional evaluations of the $\mathrm{H}$ concentration distribution in the surface-near region on a micrometer scale [537, 544-553]. An example is given in Figs. 57-58, where the laterally and in-depth $\mathrm{H}$ distribution in mechanically polished and $\mathrm{H}$ implanted $\mathrm{Ti}$ samples is revealed by combined ${ }^{15} \mathrm{~N}$ NRA and micro-ERD 
measurements [537]. The conventional near-surface ${ }^{15} \mathrm{~N}$ NRA H profile in Fig. 57 shows the areal averaged in-depth $\mathrm{H}$ distribution. A $\sim 70 \mathrm{~nm}$ wide layer in $\sim 220 \mathrm{~nm}$ depth holds a high $\mathrm{H}$ concentration ( $~ 50$ a.\%) as a result of the implantation ( $6 \times 10^{17}$ $\mathrm{H}_{2}{ }^{+} / \mathrm{cm}^{2}$ at $25 \mathrm{keV} / \mathrm{amu}$ ). Furthermore a broad $\mathrm{H}$ distribution is seen in which the concentration gradually decreases from close to 60 at.\% near the surface to below 10 at.\% in the bulk at depths above $330 \mathrm{~nm}$. This broad structure originates from $\mathrm{H}$ incorporation during the polishing process, which is in good agreement with earlier ERD observations of Mizuno et al. [554].

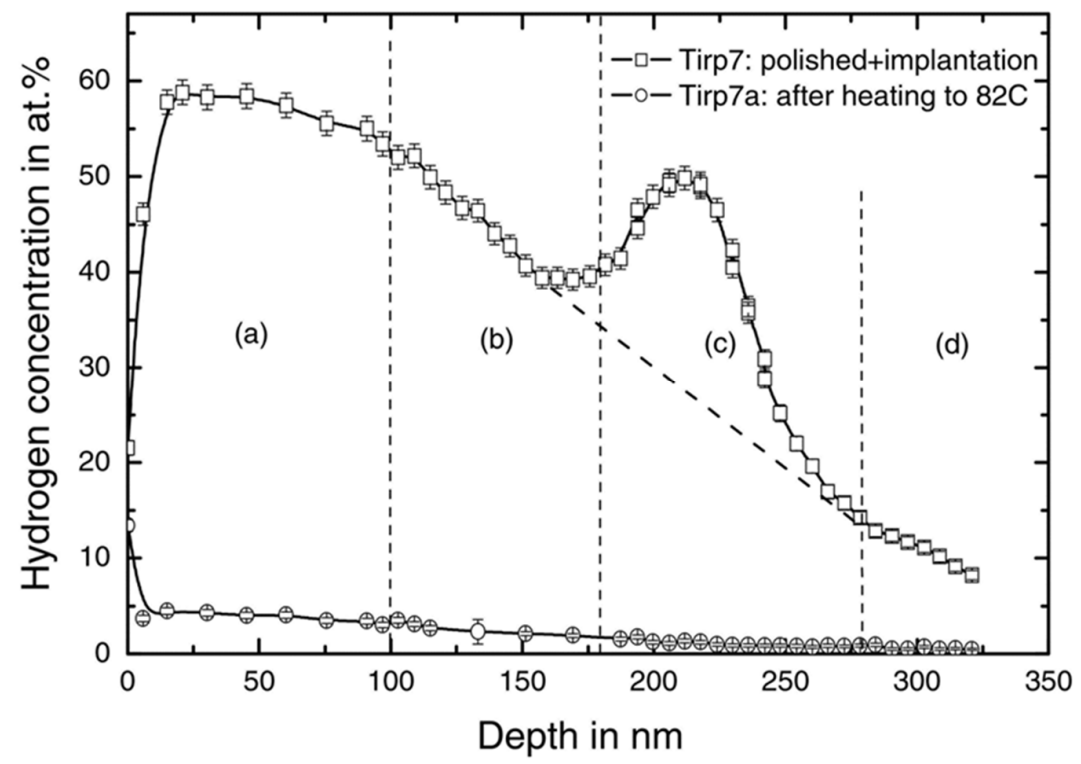

Fig. $57 .{ }^{15} \mathrm{~N}$ NRA depth profile of $\mathrm{H}$ in a mechanically polished Ti sample implanted with a fluence of $6 \times 10^{17} \mathrm{H} / \mathrm{cm}^{2}$. An implantation peak at $\sim 230 \mathrm{~nm}$ is seen on a broad $\mathrm{H}$ distribution in a near-surface $\mathrm{H}$-enriched layer. Reproduced from Ref. [537].

As indicated in Fig. 57, four depth regions ((a): 0-100 nm, (b): 101-180 nm, (c): 181-280 nm, (d): >281 nm) were assigned, for which in-plane $\mathrm{H}$ concentration distribution maps were obtained with micro-ERD by scanning a focused $16 \mathrm{MeV} \mathrm{Si}^{5+}$ ion beam of $3 \mu \mathrm{m}$ diameter across the sample at an incidence angle of $70^{\circ}$ relative to the surface normal. This resulted in a lateral resolution of $10 \mu \mathrm{m}(\mathrm{X}$, beam direction) 
X $3 \mu \mathrm{m}(\mathrm{Y})$ sized pixels [537]. In each depth region the lateral $\mathrm{H}$ concentration distribution was evaluated in $32 \times 64$ pixel maps, corresponding to a scan area of $\sim 300 \times 250 \mu \mathrm{m}^{2}$. The obtained concentration maps in Fig. 58 (implantation dose: $1 \mathrm{x}$ $10^{17} \mathrm{~cm}^{-2}$ ) clearly reveal lateral concentration gradients in the in-plane $\mathrm{H}$ distributions. The inhomogeneity is particularly pronounced near the depth region of the hydrogen implant [537]. Similar laterally inhomogeneous $\mathrm{H}$ distributions were previously observed at surfaces of a $\mathrm{H}$-implanted titanium alloy [536] and of a titanium sheet under bending stress [555], the latter revealing the redistribution of implanted hydrogen due to the mechanical deformation (Gorsky effect). Complementary X-ray diffraction data and SEM images gave evidence that the laterally inhomogeneous $\mathrm{H}$ distributions arise from the non-uniform nucleation of titanium hydride $[536,537]$. 


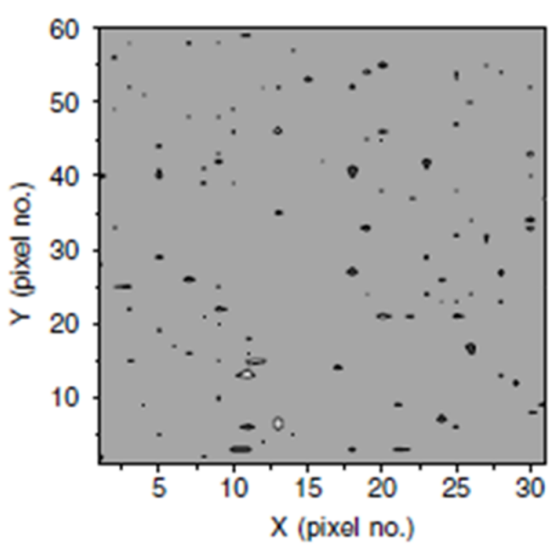

(a) $0-100 \mathrm{~nm}$

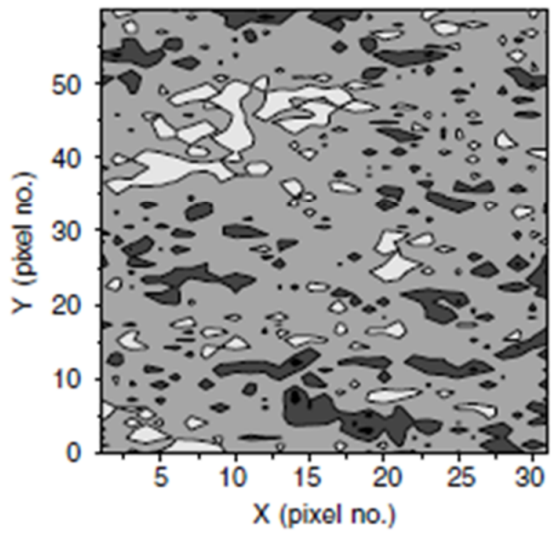

(c) $181-280 \mathrm{~nm}$

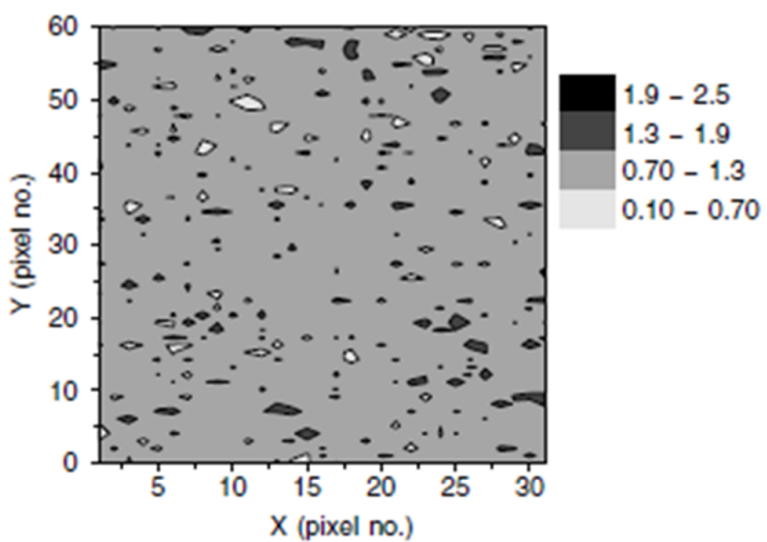

(b) $101-180 \mathrm{~nm}$

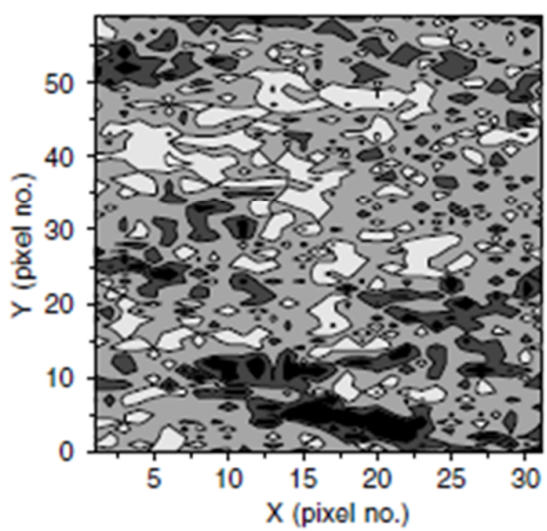

(d) $281-350 \mathrm{~nm}$

Fig. 58. Depth dependent in-homogeneities of the in-plane $\mathrm{H}$ distribution on a mechanically polished and $\mathrm{H}$-implanted $\left(1 \times 10^{17} \mathrm{H} / \mathrm{cm}^{2}\right)$ Ti surface determined by micro-ERD. $\mathrm{H}$-distribution maps in depths of (a) $0-100 \mathrm{~nm}$; (b) $101-180 \mathrm{~nm}$; (c) $181-280 \mathrm{~nm}$ and (d) $>280 \mathrm{~nm}$. Reproduced from Ref. [537].

A recent two-dimensional and depth-resolved analysis of $\mathrm{H}$ distributions in the plane of fracture surfaces of amorphous glassy alloys by ${ }^{15} \mathrm{~N}$ NRA is also reported [551]. In contrast to ordinary crystalline alloys, where hydrogen bubble formation at grain boundaries may cause embrittlement, the fatigue mechanism of ductile $\mathrm{Zr}$ - and Pd-based bulk glassy alloys (BGAs) is discussed as being due to extreme hardening and embrittlement at the tip of fatigue cracks [556]. Since the alloys retain their glassy structure without nucleating a crystalline phase near the fracture tip, 
hardening by hydrogen was considered a possible explanation. Previous $\mathrm{H}$ depth profiling measurements by conventional ${ }^{15} \mathrm{~N}$ NRA near the crack tip of a fatigue fractured BGA surface showed a slight enhancement of the $\mathrm{H}$ concentration in comparison to an impact-fractured surface (which displays the average bulk composition that includes 1 at. $\% \mathrm{H}$ ), supporting the hypothesis of hydrogen involvement in the fracture mechanism [556]. The conclusion remained preliminary though, as the ${ }^{15} \mathrm{~N}$ ion beam ( $3 \mathrm{~mm}$ diameter) irradiated both fatigue fractured and impact fractured area simultaneously.

A verification of the result at higher spatial resolution succeeded recently by utilizing a focused ${ }^{15} \mathrm{~N}$ ion beam (rectangular profile of $17 \times 30 \mu \mathrm{m}^{2}$ area) in conjunction with optical microscopy, which could selectively confine the NRA analysis region to either the fatigue fractured tip or the impact fractured surface area [551]. The thumbnail pattern corresponding to the fatigue-fractured region is seen in the upper left corner of the $\mathrm{Zr}_{50} \mathrm{Cu}_{37} \mathrm{Al}_{10} \mathrm{Pd}_{3}$ fracture surface as indicated by the white dashed line in the optical micrograph (Fig. 59 (a)). The in-plane hydrogen concentration distribution was evaluated by scanning the sample under the focused ${ }^{15} \mathrm{~N}$ ion beam in $150 \mu \mathrm{m}$ increments within the analyzed $3 \times 3 \mathrm{~mm}^{2}$ area (white square in (a)) and recording the NRA $\gamma$-yield at each position. For a shallow probing depth of $2.5 \mathrm{~nm}$ below the surface, Fig. 59 (b) shows that the $\mathrm{H}$ concentration in the fatigue-fractured region (A) is very substantial (exceeding 50 at.\%) and significantly higher than in the central bulk region (C). 


\section{$\mathrm{Zr}_{50} \mathrm{Cu}{ }_{37} \mathrm{Al}_{10} \mathrm{Pd}{ }_{3}$}
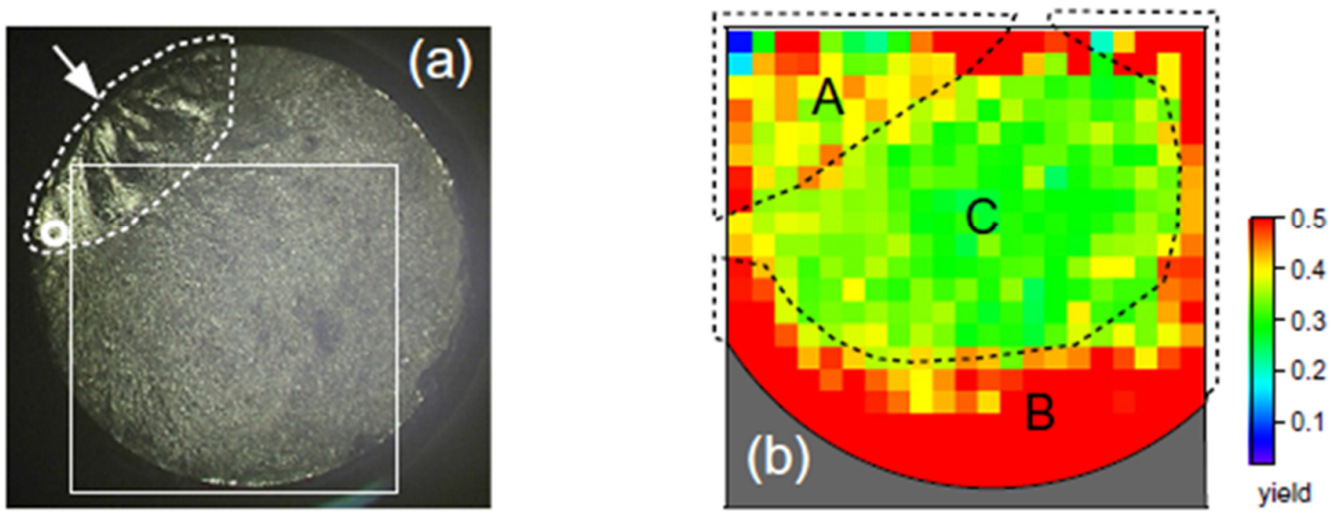

Fig. 59. (a) Optical microscope image and (b) ${ }^{15} \mathrm{~N}$ NRA-determined in-plane $\mathrm{H}$ distribution in $2.5 \mathrm{~nm}$ depth on a fatigue-fractured surface of $\mathrm{Zr}_{50} \mathrm{Cu}_{37} \mathrm{Al}_{10} \mathrm{Pd}_{3}$ glassy alloy. The fatiguefractured region is encircled by a white dashed line in (a). The NRA H-distribution map (b) shows that it corresponds to a region of increased $\mathrm{H}$ content closely below the surface (region A). Reproduced from Ref. [551].

In a probing depth of $8 \mathrm{~nm}$ still a similar relative in-plane $\mathrm{H}$ concentration contrast was observed, while in $20 \mathrm{~nm}$ depth the $\mathrm{H}$ concentration was nearly uniform, which is consistent with an conventional area-integrated $\mathrm{H}$ depth profile showing that the $\mathrm{H}$ enrichment over the bulk concentration ( 1 at. \%) is limited to only the top $20 \mathrm{~nm}$ [551]. These spatially-resolved H profiling results thus support the suspect that the fatigue fracture of the glassy alloys correlates with high local $\mathrm{H}$ concentrations near the crack tips. Several open questions, such as the origin of the hydrogen accumulation in the vicinity of the crack tip and in the outer circumference region (B), as well as the distribution of other elements, however, remain subject to further investigation. 


\subsubsection{Thin films and superlattices}

Several groups have invested substantial effort in elucidating the effects of finite size and interfacial strain on the hydrogen behavior in thin metallic films [540, 557560] and metal/metal superlattices [561-572]. Pundt and Kirchheim offer a comprehensive review of the subject [573]. With respect to the influence of hydrogen absorption on the host metal's material properties, $\mathrm{H}$-concentration dependent variations of the magnetic coupling in metallic superlattices [562, 567, 568] as well as $\mathrm{H}$-driven metal/insulator transitions allowing to fabricate thin films with switchable optical properties [32, 33, 539-543, 574-577] have been in the center of attention. To highlight the contribution of ${ }^{15} \mathrm{~N}$ NRA in this line of research, we illustrate examples of $\mathrm{H}$ concentration and distribution measurements in thin film systems, going from simple metallic layers on rigid substrates to more complex clamped thin films and finally to metallic superlattices. From the viewpoint of hydrogen, the most frequently encountered phenomenon is a pronounced variation of the $\mathrm{H}$ solubility in the near interfacial regions of the thin films, giving rise to notable changes in the pressure-composition phase diagrams compared to the respective bulk metals $[557,558,567,570,571,578]$.

Fig. 60 (a) displays ${ }^{15} \mathrm{~N}$ NRA hydrogen depth profiles of a thin (140 nm) Pd-capped $\mathrm{Nb}$ film grown by $\mathrm{MBE}$ onto an $\mathrm{Al}_{2} \mathrm{O}_{3}$ substrate after hydrogen loading at $200^{\circ} \mathrm{C}$ under different external $H_{2}$ pressures [557]. The $H$ concentration information obtained from the central $\mathrm{Nb}$ plateau region of the profiles was used to construct the equilibrium phase diagram for the thin film system. Open and filled circle symbols denote data points of two consecutive ${ }^{15} \mathrm{~N}$ energy scans, verifying that the 
system had reached equilibrium conditions. The main observation was that the film showed considerably reduced hydrogen solubility compared to bulk Nb. Further characteristic changes in the pressure-concentration (H/Nb) phase diagram (Fig. 60 (b)) compared to that of bulk $\mathrm{Nb}$ were sloped isotherms in the solid solution/hydride coexistence region and increased solubility in the low concentration solid solution phase of the thin film. This behavior has been attributed to a reduction of the attractive $\mathrm{H}-\mathrm{H}$ interaction in the concentrated $\mathrm{Nb}$ hydride phase due to the epitaxial clamping of the films by the substrate, since the rigid interfacial locking to the $\mathrm{Al}_{2} \mathrm{O}_{3}$ hinders the lattice expansion of the $\mathrm{Nb}$ film upon hydrogen absorption $[557,558]$.
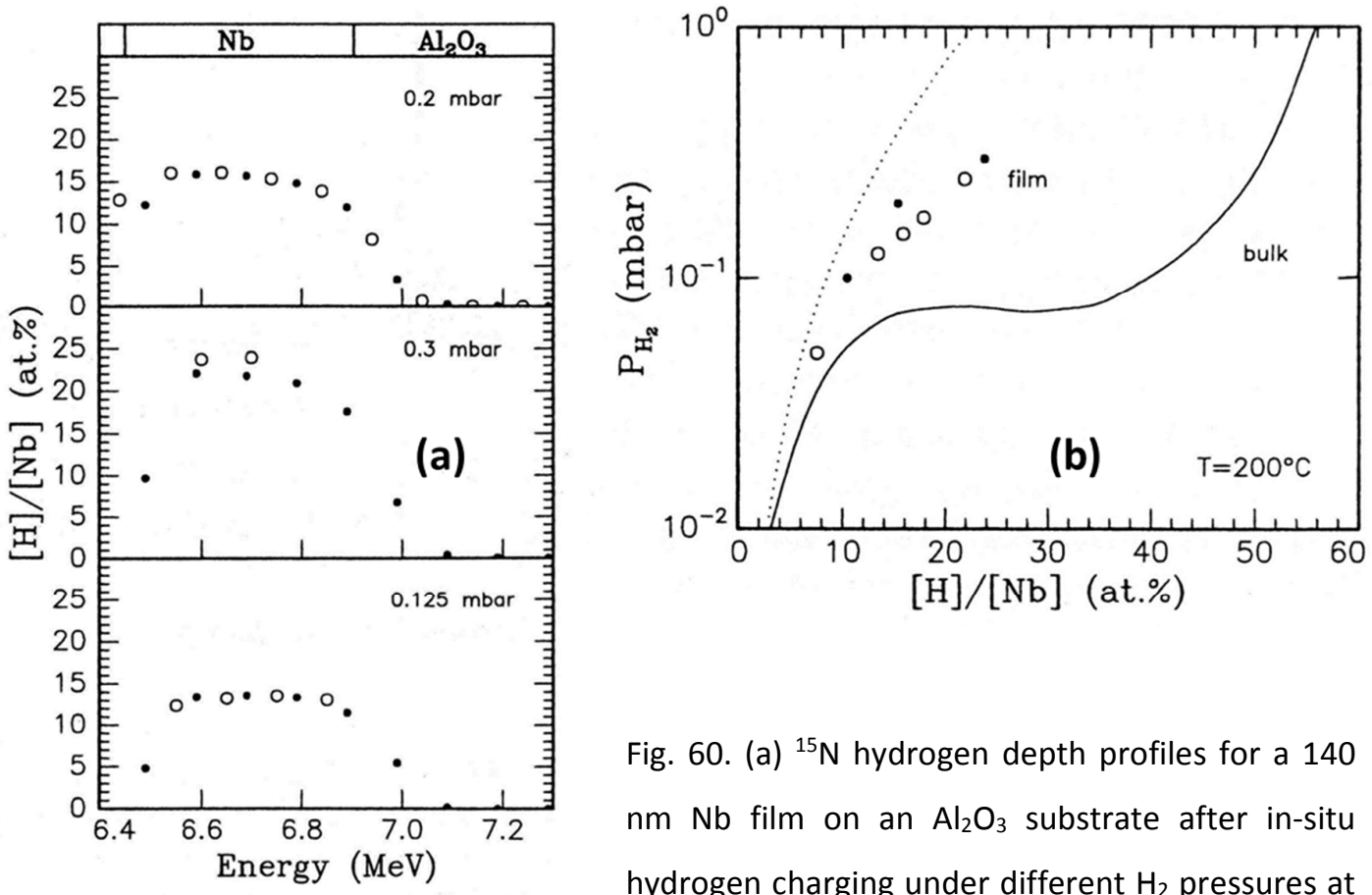

Fig. 60. (a) ${ }^{15} \mathrm{~N}$ hydrogen depth profiles for a 140 $\mathrm{nm} \mathrm{Nb}$ film on an $\mathrm{Al}_{2} \mathrm{O}_{3}$ substrate after in-situ hydrogen charging under different $\mathrm{H}_{2}$ pressures at a temperature of $200^{\circ} \mathrm{C}$. The film structure is illustrated on top of the figure. The small rectangle on the left represents a Pd cap layer. (b) Solubility curve for the $\mathrm{Nb}$ film (circles) at $\mathrm{T}=200^{\circ} \mathrm{C}$ compared with those of bulk Nb (solid line) and an extrapolation of Sievert's law to higher H-concentrations (dotted line). Reproduced from Ref. [557]. 
As an example of mutual interfacial strain effects in stacked metal layers on the $\mathrm{H}$ solubility we show in Fig. $61{ }^{15} \mathrm{~N}$ NRA H depth profiles of a $45 \mathrm{~nm}$ thin $\mathrm{Hf}$ sheet sandwiched between two epitaxial $\mathrm{Nb}$ films before and after $\mathrm{H}$ charging [579]. In this system the clamped $\mathrm{Hf}$ layer represents the component of higher hydrogen affinity, i.e., the more negative $\mathrm{H}$ solution enthalpy [580]. In addition to a thin $\mathrm{H}$ rich surface layer, the depth profiles reveal $\mathrm{H}$ accumulation at the upper metal/metal interface and a small native $\mathrm{H}$ concentration in the $\mathrm{Nb}$ layer, presumably due to incorporation of $\mathrm{H}$ surface impurities during layer deposition. LEED investigations of the Hf lattice structure at early stages of the deposition show a high amount of distortion in the first $5 \mathrm{~nm}$ from the interface presumably due to the misfit between the $\mathrm{Nb}$ (bcc) and the $H f(h c p)$ lattices, which may explain the interfacial $H$ accumulation by trapping at the lattice distortions.

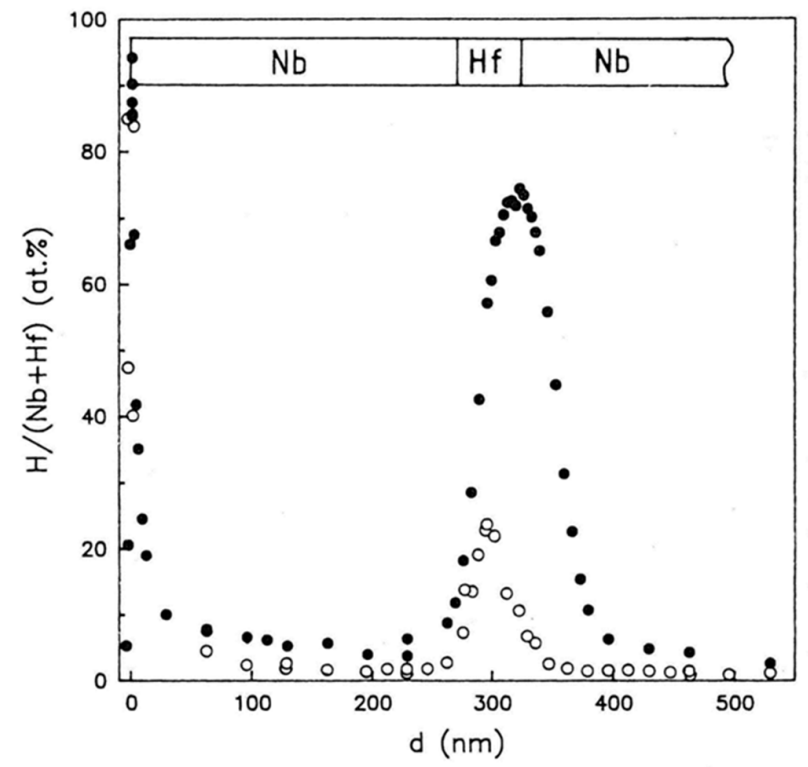

Fig. $61 .{ }^{15} \mathrm{~N}$ NRA H depth profiles of a $\mathrm{Nb}-\mathrm{Hf}-\mathrm{Nb}$ multilayer before (open circles) and after (filled circles) hydrogen charging. The thickness of the Hf layer is $45 \mathrm{~nm}$. Reproduced from Ref. [579].

Most obvious, however, is the strong increase of the $\mathrm{H}$ concentration in the $\mathrm{Hf}$ layer after electrolytic hydrogen charging. Despite this substantial hydrogenation, the atomic $\mathrm{H} / \mathrm{Hf}$ ratio in the clamped $\mathrm{Hf}$ layer does not attain the value of 1.63 
expected for the bulk $\mathrm{Hf}-\delta$ phase, indicating again a reduced $\mathrm{H}$ solubility compared to the bulk material. Furthermore, the $\mathrm{H}$ distribution is seen to extend below the bottom $\mathrm{Hf} / \mathrm{Nb}$ interface, i.e. the $\mathrm{H}$ solubility in the $\mathrm{Nb}$ is locally strongly increased over the bulk value near the interface. Both effects are ascribed to mutual interfacial strain exerted between the two metals due to their interface-locked lattices. Clamping of the $\mathrm{Hf}$ film to the rigid $\mathrm{Nb}$ substrate causes compressive strain in the $\mathrm{Hf}$ layer that hinders its lattice expansion during $\mathrm{H}$ absorption, resulting in overall reduced $\mathrm{H}$ solubility in the $\mathrm{Hf}$ layer $[557,558]$. On the other hand, upon $\mathrm{H}$ loading the expanding $\mathrm{Hf}$ lattice exerts tensile stress on the $\mathrm{Nb}$ layers adjacent to the interface, expanding the $\mathrm{Nb}$ lattice locally, which in turn increases the $\mathrm{H}$ solubility in the $\mathrm{Nb}$ substrate near the interfaces $[558,579]$. Similar clamping-related alterations of the $H$ solubility in interfacial regions revealed by ${ }^{15} \mathrm{~N}$ NRA measurements are also reported for $\mathrm{Nb}$ layers sandwiched between $\mathrm{Cu}$ films $[571,581]$ as well as in thin films of $\mathrm{Nb}$ and $V$ due to interfacial interactions with the respective substrates $[560,569,582$, 583] and for Pd-capped Mg films [578].

The groups of Hjörvarsson and Zabel extended these investigations to bimetallic superlattices, which are open to structural analysis by X-ray diffraction $[167,563,565$, $566,572]$. In such multilayer stacks of two alternating metals the hydrogen potential (i.e., $\Delta H_{s}$ ) is modulated periodically. The amplitude of the modulation depends on the hydrogen solubility difference between the two metals and the period is determined by the respective layer thicknesses $[563,567]$. Analog to the interfacial clamping effects described above, the average hydrogen solubility can thus be adjusted by the selection of the metal combination in the superlattice and by 
variation of the respective layer thicknesses, which may be as small as only a few atomic distances. A well-studied weakly modulated system both in terms of the $\mathrm{H}$ solubility difference and similar lattice parameters is $\mathrm{Nb}-\mathrm{Ta}$. On the other hand, sandwiching, e.g., vanadium into either $\mathrm{Fe} / \mathrm{V}$ or Mo/V superlattices achieves a very strong modulation of the $\mathrm{H}$ potential (the $\Delta \mathrm{H}_{\mathrm{s}}$ difference is as large as $\sim 0.6 \mathrm{eV} / \mathrm{H}$ ), and the resulting interfacial strain can be switched from compressive (Fe/V) to tensile (Mo/V) [167, 562-564, 569].

${ }^{15} \mathrm{~N}$ NRA $\mathrm{H}$ profiles of $\mathrm{H}$-loaded Fe/V systems of different periodicities are shown in Fig. 62 (a). It is seen that the $\mathrm{H}$ concentration reduced strongly at shorter periods $\left(\Lambda_{1}\right.$, i.e., thinner layers) of the superlattice, where the proportion of the $\mathrm{Fe} / \mathrm{V}$ interfacial region in the respective V layers was large [564]. As illustrated in Fig. 62 (b), the H-depletion was explained by destabilization of hydrogen in the interfacial regions of the $\mathrm{V}$ layers adjacent to the Fe interlayers owing to lattice strain and interfacial charge transfer $[167,563,564]$. Clarifications of the interfacial structure in the $\mathrm{V}$ layers after $\mathrm{H}$ loading to $\mathrm{H} / \mathrm{V}=0.5$ by $\mathrm{XRD}$ (avg. lattice expansion $4.5 \%$ ) [565] and EXAFS (5.1\%) [561] were in good agreement. A similar interfacial charge transfer effect deduced from average ${ }^{15} \mathrm{~N}$ NRA $\mathrm{H}$ concentration measurements was also reported for $\mathrm{Mo}_{x} \mathrm{~V}_{1-\mathrm{x}} / \mathrm{V}$ superlattices $[167,584]$. Contrary to Hjörvarsson and Zabel's reasoning that interfacial strain and charge transfer are the origin of the reduced $\mathrm{H}$ solubility $[167,563,567]$, Pundt et al. suggested based on 3-dimensional atom probe measurements that the modulation of the near-interfacial $\mathrm{H}$ potential results from a partial intermixing of the two metals near the interfaces. [585] 

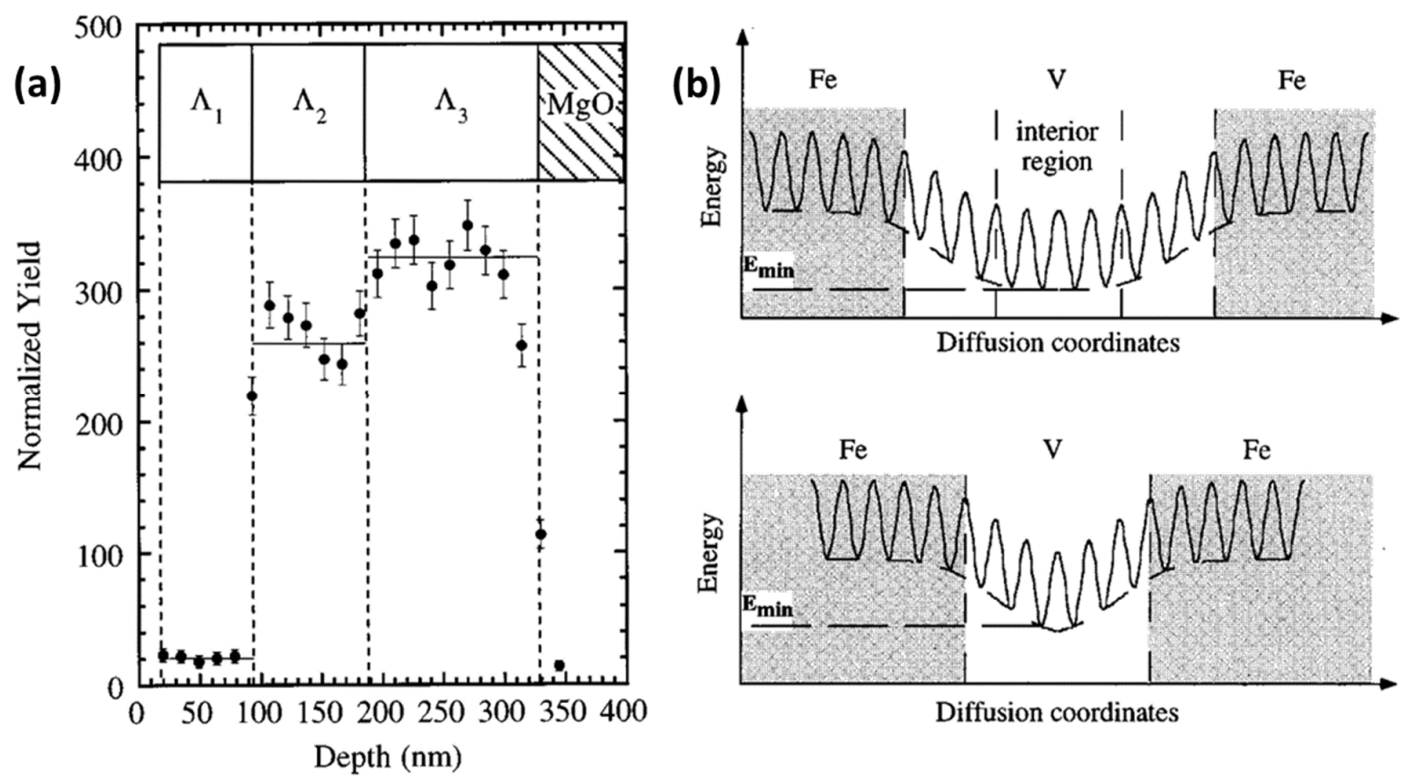

Diffusion coordinates

Fig. 62. (a) ${ }^{15} \mathrm{~N}$ NRA hydrogen depth profile of a Fe/ $\mathrm{V}$ multilayer superlattice on an MgO substrate hydrogenated at $150^{\circ} \mathrm{C}$ in 2100 Torr $\mathrm{H}_{2}$. The superlattice periodicities are $\Lambda_{1}=1.8$ $\mathrm{nm}, \Lambda_{2}=3.1 \mathrm{~nm}$, and $\Lambda_{3}=4.4 \mathrm{~nm}$. The normalized yield is proportional to the $\mathrm{H} / \mathrm{V}$ ratio. (b) Schematic view of the potential seen by a diffusing $\mathrm{H}$ atom in the Fe/ $\mathrm{V}$ superlattice. The shadowed areas are the iron layers surrounding the vanadium, which is $10 \mathrm{ML}$ in the upper and $6 \mathrm{ML}$ thick in the lower panel of (b), respectively. In the upper panel of (b) the energy difference between monolayers 3 and 4 from the interface is 97(5) meV. $E_{\min }$ in the lower panel is $87(6)$ meV higher than in the upper panel. Reproduced from Ref. [564].

Much attention has been paid to the drastic optical property changes that certain rare-earth metal thin films display during their reversible metal-to-insulator phase transitions upon hydrogenation, which may find applications as energy-saving smart windows that change between reflective and transparent properties on demand. Griessen and coworkers demonstrated first that the optically reflective (metallic $\alpha$ $\mathrm{YH}_{\mathrm{x}}$ and $\mathrm{YH}_{2}$ ) or transparent (insulating $\mathrm{YH}_{3}$ trihydride) phases of thin yttrium films on glass substrates can reversibly be interchanged by controlling the external $\mathrm{H}_{2}$ pressure $[32,33,539,541] .{ }^{15} \mathrm{~N}$ NRA depth profiling was used here to verify that the optical transmission variations indeed correlated to changes of the $\mathrm{H}$ concentration 
in the thin films, for which the $\mathrm{YH}_{\mathrm{x}}$ phase transition points apparently showed little differences to those of bulk yttrium [586]. These results were later substantiated by spatially resolved ERD measurements at $Y$ films to which $\mathrm{H}$ was only locally supplied through Pd caps that were separated by a $\mathrm{H}_{2}$-impermeable $\mathrm{Y}_{2} \mathrm{O}_{3}$ surface oxide layer as illustrated in Fig. 63 (a) $[587,588]$. After hydrogenation the $\mathrm{H}$ concentrations in lateral sections of different optical transparency were analyzed by ERD using a 0.1$\mathrm{mm}$ diameter beam, which again confirmed that these regions corresponded to the respective $\mathrm{YH}_{\mathrm{x}}$ phases of the bulk $\mathrm{Y}-\mathrm{H}$ system and that the boundaries between them were comparatively sharp (Fig. 63 (b)) [587, 588].

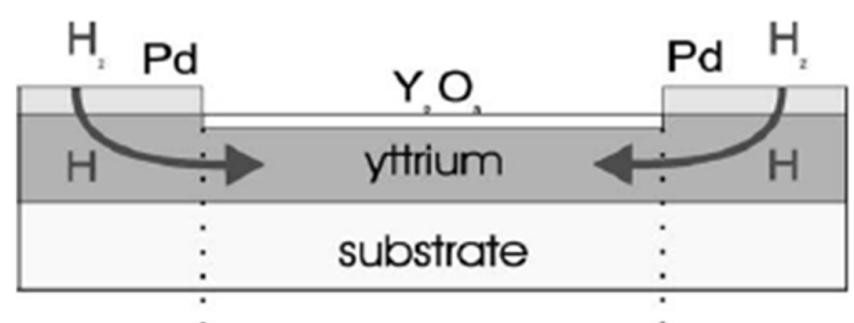

$\mathrm{Pd}: \begin{array}{llllll:}\gamma & \beta & \alpha & \beta & \gamma: \mathrm{Pd}\end{array}$

(a)

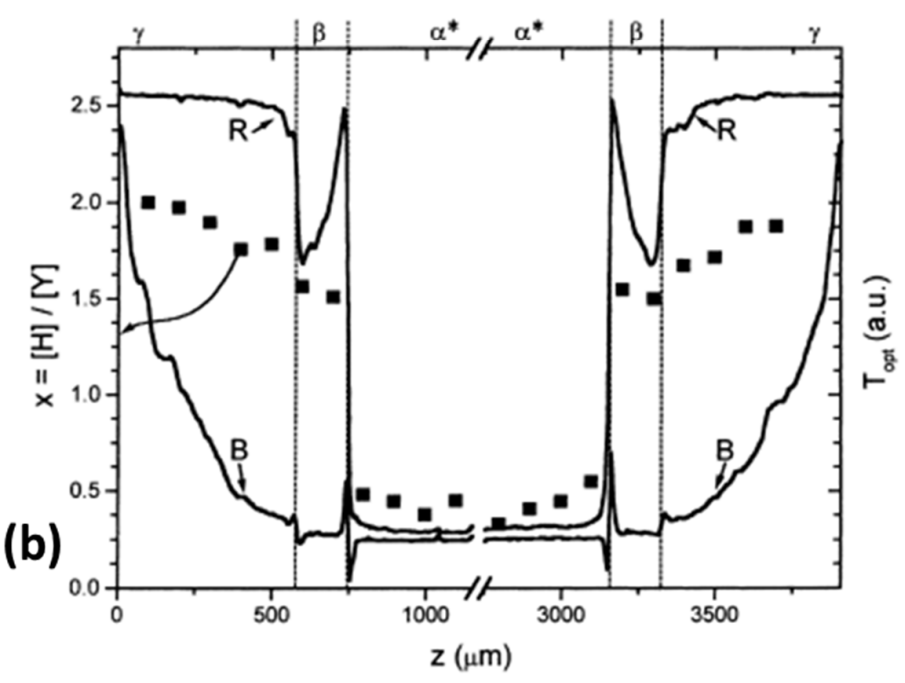

Fig. $63 . \quad$ (a) Schematic illustration of the Y-film on a glass substrate as well as an optical photograph of the sample after a few weeks of $\mathrm{H}$ loading at $373 \mathrm{~K}$. The different $\mathrm{YH}_{\mathrm{x}}$ phases $(\alpha, \beta, \gamma)$ can be recognized in the photograph. Adapted from Ref.[601].

(b) Results of an ERD line scan (squares) on a H-loaded double diffusion sample ( $Y$ film on glass; region between the $\mathrm{Pd}$ caps in (a). The solid lines represent an optical transmission measurement along the same line. Both the red $(R)$ and blue (B) signals of the used 3-CCD camera are shown. Adapted from Ref. [602]. 
A few years later Mg alloys [542, 543] were proposed for hydrogen storage and switchable mirror applications operable at room temperature. Pure $\mathrm{Mg}$ has a very favorable $\mathrm{H}$ storage capacity of 7.6 mass \% in its ionic hydride $\mathrm{MgH}_{2}$, however, the strongly exothermic $H$ bonding $\left(\Delta H_{s}=-0.37 \mathrm{eV} / \mathrm{H}\right)[27,589]$ implies an impracticably low equilibrium $\mathrm{H}_{2}$ pressure at room temperature $\left(10^{-3} \mathrm{mbar}\right)$ that precludes its application in devices relying on quick reversible $\mathrm{H}$ loading and release. $\mathrm{As} \mathrm{MgH}_{2}$ is in addition almost impermeable to $\mathrm{H}_{2}$, it can passivate the metal against further $\mathrm{H}$ uptake near the $\mathrm{H}_{2}$ exposed surface $[533,590]$. Hydrogen destabilization through alloying [542, 543, 576, 591] and/or elastic effects due to interfacial clamping [578] was shown to be effective in improving the hydrogenation equilibrium, yet $\mathrm{Pd}$ capping layers are still required to prevent surface oxidation and to catalyze $\mathrm{H}_{2}$ dissociation. Currently, efforts are undertaken to improve the reproducibility of the mirror switching process $[592,593]$ and the durability of the film systems in ambient conditions [594-596]. The amplitude of the transmittance change of the switchable layers degrades during hydrogenation/dehydrogenation cycling and the devices finally attain an irreversible half-mirror state [574, 592]. The degradation apparently relates to the tendency of alloy formation between $\mathrm{Pd}$ and $\mathrm{Mg}$ at the cap layer/alloy interface, causing interdiffusion of the two metals that enables oxidation of $\mathrm{Mg}$ at the surface [574].

A recent ion beam analysis study combining RBS, ERD, and ${ }^{15} \mathrm{~N}$ NRA has provided insight into the degradation mechanism of such a switchable mirror alloy film system consisting of $\mathrm{Pd}$-capped $\mathrm{Mg}$-rich $\mathrm{Mg}_{3.3} \mathrm{Ni}$ alloy on a diamond-like carbon (DLC)/glass substrate [597]. RBS data demonstrated clearly that Mg segregates into the 
interface between the Pd cap and the Mg-Ni alloy, and the total number of atoms above the DLC substrate increased after many switching repetitions when the system approached the half mirror state near the end of its life cycle (Fig. 64 (a)) [597]. The NRA depth profile obtained in this investigation (Fig. 64 (b)) evidenced that this effect is due to strong permanent $\mathrm{H}$ incorporation into the $\mathrm{Pd} / \mathrm{Mg}$ interfacial region [597]. Together, the data support the conclusion that the formation of $\mathrm{Mg}$ hydride and oxide compounds into the $\mathrm{Mg}$ enriched region at the $\mathrm{Pd}$ cap/alloy interface increasingly impedes the switching performance by blocking the hydrogen transport.

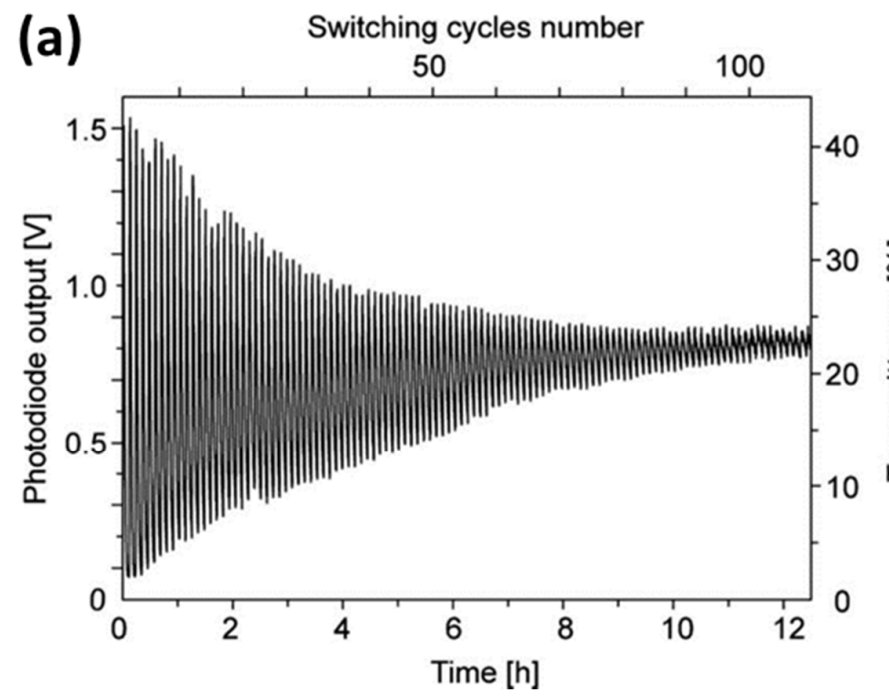

Fig. 64. (a) Light $(\lambda=670 \mathrm{~nm})$ switching behavior of a $\mathrm{Pd}$ capped $\mathrm{Mg}_{3.3} \mathrm{Ni}$ thin film as a function of the switching cycles by alternating hydrogenation and dehydrogenation.

\section{(b)}

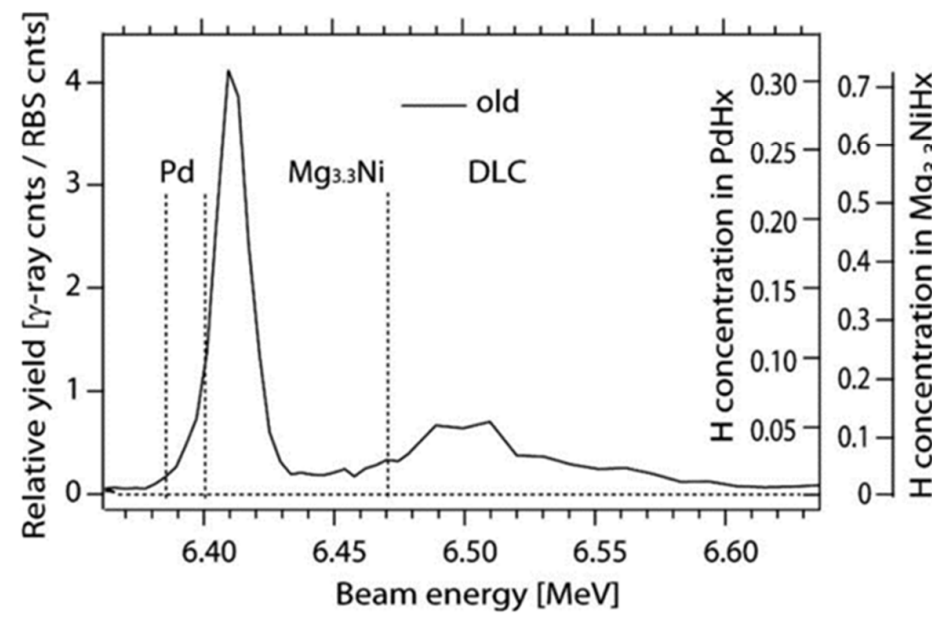

(b) ${ }^{15} \mathrm{~N}$ NRA H profile taken on an 'old' Pd-capped $\mathrm{Mg}_{3.3} \mathrm{Ni}$ film (after many switching cycles). The corresponding $\mathrm{H}$ concentrations in $\mathrm{Pd}$ and in $\mathrm{Mg}_{3.3} \mathrm{Ni}$ are indicated on the right hand side. Adapted from Ref. [597]. 
Qualitatively similar conclusions on the hindering effect of $\mathrm{MgH}_{2}$ layers in the $\mathrm{Pd} / \mathrm{Mg}$ interfacial region on the hydrogenation kinetics of $\mathrm{Pd}$-capped $\mathrm{Mg}$-based multilayers intended for hydrogen storing applications were recently drawn from combined by X-ray diffraction and neutron reflectivity measurements [598]. The above degradation problem in Mg-based switchable mirrors is closely related to the strong tendency for interdiffusion at interfaces between $\mathrm{Pd}$ and $\mathrm{Mg}$, which readily form alloys. Interfacial alloying has very recently been pointed out as the dominant factor responsible for the considerable destabilization of hydrogen in Pd-capped $\mathrm{MgH}_{2}$ [599], rather than the elastic strain effects due to interfacial clamping assumed previously [578]. The above examples clearly illustrate that in order to understand the behavior of hydrogen in multilayer systems it is necessary to reveal the in-depth distribution of all constituting elements, including that of hydrogen, and therefore highlight the valuable contribution of non-destructive $\mathrm{H}$ profiling by ${ }^{15} \mathrm{~N}$ NRA.

\subsubsection{Nanoparticles}

Nanoparticles may exhibit size-dependent properties that can differ drastically from that of the corresponding bulk metals. A common observation with respect to hydrogen absorption in $\mathrm{Pd}$ nanoparticles made by volumetric pressure-composition analysis is a narrowed coexistence region of the solid solution and hydride phases in the H-Pd phase diagram, implying increased solubility in the low concentration solid solution phase and reduced solubility in the hydride, as well as a lowering of the critical temperature $[573,600-602]$. In addition, the pressure-composition isotherms show sloped intervals instead of the flat equilibrium pressure plateau characteristic for the bulk metal, and a strong hysteresis upon absorption/desorption cycling is 
observed [603]. Although the phenomena are still not completely understood [573], the most widely accepted explanation has been the hypothesis that $\mathrm{H}$ binds into particular subsurface sites that are presumed to stabilize hydrogen more strongly than regular bulk interstitial sites (cf. Section 3.2.1) [602]. Owing to the large surface to volume ratio of nanoparticles, surface and subsurface sites can comprise very significant atomic fractions of the $\mathrm{H}$-binding sites in these particles, amounting to, e.g., 40 or even $75 \%$ of all atoms in the particle if the size is decreased from $5 \mathrm{~nm}$ to $2 \mathrm{~nm}$ [602].

Finely dispersed palladium particles on oxide supports are widely utilized industrial catalysts for olefin hydrogenation reactions [604], and Pd-absorbed hydrogen has long been suspected to play a role in the catalytic reaction. Model catalysts [605] consisting of UHV deposited nanometer-sized Pd crystallites on a thin alumina film $[606,607]$ have shown increased reactivity for ethene hydrogenation compared to $\mathrm{Pd}(111)$ single crystals $[608,609]$. This was tentatively attributed to particle-absorbed hydrogen as TDS gave evidence for a weakly bound $\mathrm{H}$ state that only existed in the latter but not in the single crystal $[610,611]$. The unambiguous confirmation that low-pressure $\left(<2 \times 10^{-3} \mathrm{~Pa}\right) \mathrm{H}$ absorption inside such oxidesupported Pd nanocrystals does indeed take place was achieved via high resolution depth profiling with ${ }^{1} \mathrm{H}\left({ }^{15} \mathrm{~N}, \alpha \gamma\right){ }^{12} \mathrm{C}$ NRA in grazing ion incidence $\left(75^{\circ}\right.$ relative to the surface normal) $[59,612]$, which matched the 3.9-fold enhanced depth resolution (Eq. (2.15)) to the small size (5-10 nm width and $2 \mathrm{~nm}$ height) of the Pd nanocrystals $[607,613]$ and thereby succeeded in a straightforward discrimination between surface-adsorbed and volume-absorbed hydrogen in the nano-sized particles [60]. 


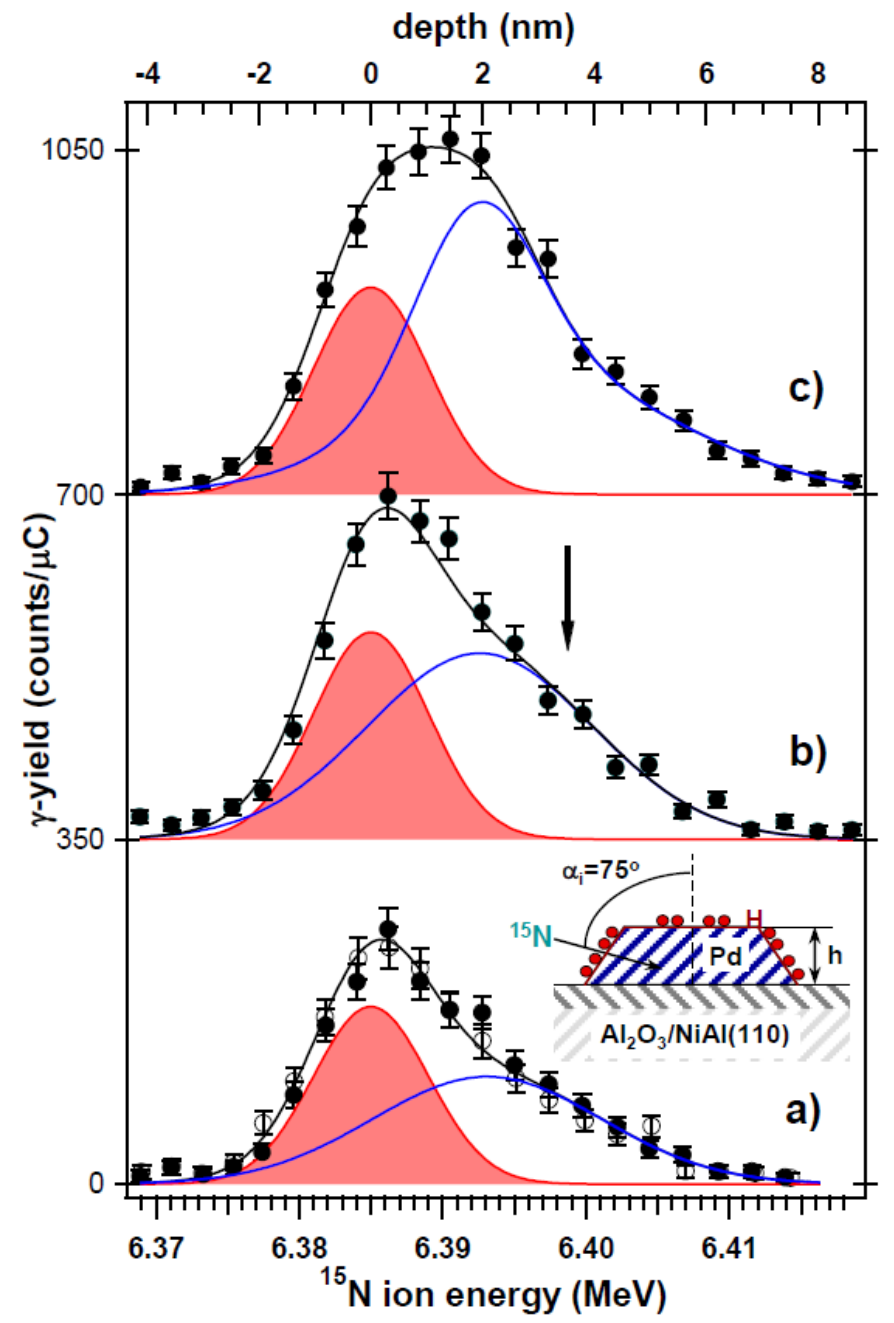

Fig. 65. NRA $\gamma$-yield curves of $5.85 \AA \mathrm{Pd} / \mathrm{Al}_{2} \mathrm{O}_{3} / \mathrm{NiAl}(110)$ at $90 \mathrm{~K}$ for $\mathrm{H}_{2}$ pressures of a) $2 \times 10^{-5} \mathrm{~Pa}$, b) $\left.6 \times 10^{-4} \mathrm{~Pa}, \mathrm{c}\right)$ $2 \times 10^{-3} \mathrm{~Pa}$ at $75^{\circ}$ grazing ion incidence. Profile (a) shows data of two consecutive scans (filled and open circles) demonstrating the reproducibility of the measurement. The filled Gaussian curves indicate surface-adsorbed $\mathrm{H}$. The open curves symbolize $\gamma$-yield from $\mathrm{H}$ in the interior of the $\mathrm{Pd}$ particles. Inset: grazing incidence geometry and $\mathrm{Pd}$ nanocrystal morphology (schematic). Reproduced from Ref. [60].

Fig. 65 schematically shows the particle morphology, the adopted NRA geometry, and three hydrogen depth profiles obtained for the $\mathrm{Pd}$ nanocrystals under $\mathrm{H}_{2}$ pressures between $2 \times 10^{-5}$ and $2 \times 10^{-3} \mathrm{~Pa}$ at $90 \mathrm{~K}$. The profiles are asymmetrically broadened on the high energy side in addition to the symmetric $\sim 10 \mathrm{keV}$ wide signal of surface hydrogen (shaded Gaussian shapes centered at $E_{R}$ ) $[61,62,67]$, clearly revealing a substantial amount of absorbed $\mathrm{H}$ (open Gaussian-shaped curves) that increases with the applied $\mathrm{H}_{2}$ pressure. Near the resonance energy the height of the profiles saturates quickly below $2 \times 10^{-4} \mathrm{~Pa}$ reflecting the saturation of the surfaceadsorbed hydrogen states on the nanocrystals, whereas the absorbed $\mathrm{H}$ species 
remains pressure-dependent. For the given particle size, a rather large average $\mathrm{H} / \mathrm{Pd}$ ratio of up to 0.8 was obtained, consistent with previous TDS results [610]. Panel (a) of Fig. 65 contains two data sets of consecutively recorded depth profiles, demonstrating that the NRA measurement is non-destructive and reproducible. The data also show that equilibration of the $\mathrm{H}$-concentration inside the nanocrystals with the surrounding $\mathrm{H}_{2}$ gas phase has taken place; an observation in full agreement with recent synchrotron XAFS results, which demonstrated that the Pd lattice expansion upon $\mathrm{H}$ uptake saturates very rapidly (on a microsecond time scale at room temperature) [614].

The dominant advantage of the highly depth-resolved analysis is the ability to separate the NRA signal of the particle-absorbed $\mathrm{H}$ from that of the surface hydrogen. At ${ }^{15} \mathrm{~N}$ energies above $6.399 \mathrm{MeV}$ (arrow in Panel (b) of Fig. 65), surface $\mathrm{H}$ remains excluded from detection, allowing for the selective monitoring of the $\mathrm{H}$ content in the interior of the particles. In turn, the distinction of surface- $\mathrm{H}$ from absorbed $\mathrm{H}$ at the resonance energy is not quite as strict, since the NRA $\gamma$-yield also contains a significant contribution from $\mathrm{H}$ absorbed closely below the surface, although the surface hydrogen signal still dominates at lower $\mathrm{H}_{2}$ pressures. An individual evaluation of the thermal stabilities of the particle-absorbed hydrogen and of the (predominantly) surface-adsorbed $\mathrm{H}$ could hence be performed by monitoring the respective NRA signals at $6.399 \mathrm{MeV}$ and $6.385 \mathrm{MeV}$ in a constant $\mathrm{H}_{2}$ background pressure while the sample was heated incrementally. The results in Fig. 66 demonstrate that the particle-absorbed $\mathrm{H}$ is more weakly bound (the signal decays at lower temperatures, i.e., 200-300 K) than the surface-adsorbed $\mathrm{H}$ (decay between 
$300-450 \mathrm{~K}$ ), thereby confirming general expectations (surface- $\mathrm{H}$ is the most strongly bound $\mathrm{H}$ species, cf. Section 3.2.1.2) and the interpretation by Morkel et al. that the catalytically reactive low-temperature TDS states $(200-300 \mathrm{~K})$ indeed represent nanoparticle-absorbed hydrogen $[610,611]$.

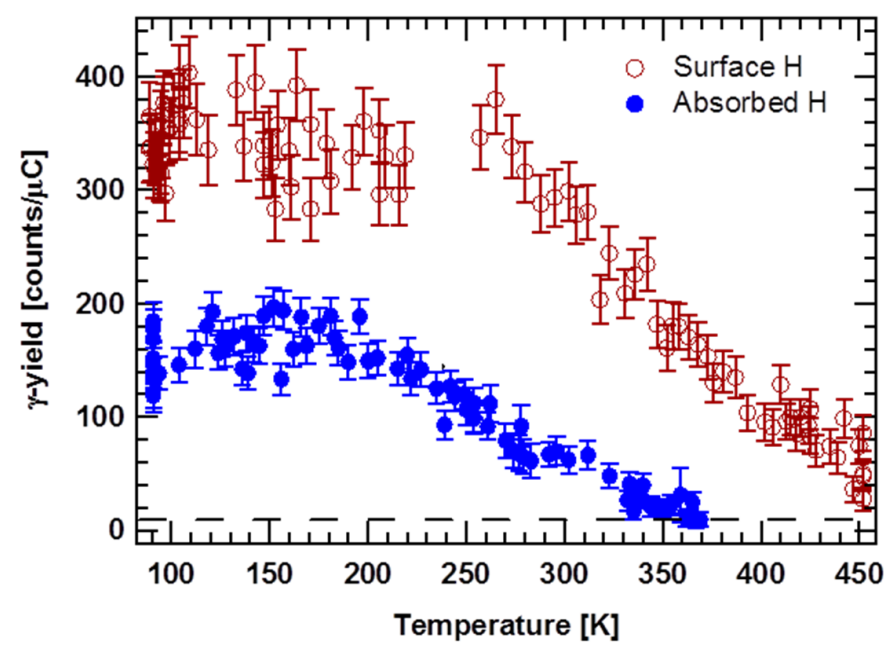

Fig. 66. Temperature dependent NRA signals of surface $\left(E=6.385 \mathrm{MeV}, 1.5 \times 10^{-3} \mathrm{~Pa} \mathrm{H}_{2}\right)$ and Pd-absorbed $\mathrm{H}\left(E=6.399 \mathrm{MeV}, 9 \times 10^{-5} \mathrm{~Pa} \mathrm{H}_{2}\right)$ in alumina-supported $\mathrm{Pd}$ crystallites. The dashed line indicates the small background $(10 \mathrm{cts} / \mu \mathrm{C}$ ) at $E=6.399 \mathrm{MeV}$ (arrow in Fig. 65) due to energy-shifted surface H signal. Reproduced from Ref. [60].

The van't Hoff analysis of the temperature-dependent equilibrium $H$ concentration inside the $\mathrm{Pd}$ nanocrystals for the dilute regime of $\mathrm{H} / \mathrm{Pd}<0.20$ revealed that the $\mathrm{H}$ solution enthalpy of $(-0.28 \pm 0.02) \mathrm{eV} / \mathrm{H}[60]$, is $2-3$ fold increased relative to bulk Pd [27]. This result is quite consistent with the extended $\mathrm{H}$ concentration range of the solid solution phase in nanoparticles as observed by volumetric pressure-composition analysis [573, 600-602] and X-ray diffraction studies [603, 614616]. The depth-resolved NRA analysis, on the other hand, indicates that this particular property of the nanocrystals is not a consequence of preferential hydrogen binding into first-layer subsurface sites [573, 600-602], which even at the 
enhanced depth resolution of the above experiment remain almost indistinguishable from surface sites, so that predominant subsurface site occupation should result in much narrower NRA profiles than those seen in Fig. 65. A more or less homogeneous $\mathrm{H}$ distribution within the interior of the nanoparticles appears to be most consistent with the observed depth profiles [60], which is also in line with X-ray diffraction [616] and X-ray absorption data that show a continuous lattice expansion throughout the entire particles [614]. These results suggest that the volume of the small crystallites rather than their immediate surface vicinity provides enhanced $\mathrm{H}$ solubility compared to bulk Pd. It is speculated that this effect is due to free boundary surfaces of the nanoparticles, as unlike agglomerated grains in macroscopic bulk material they do not experience a spatial obstruction of their lattice expansion upon $\mathrm{H}$ absorption $[60,616]$.

The question of possible catalytic activity of the nanoparticle-absorbed hydrogen suggested by evidence from TDS [608-611] was subsequently addressed by combining ${ }^{15} \mathrm{~N}$ NRA measurements of $\mathrm{H}$-absorption at alumina-supported $\mathrm{Pd}$ nanocrystals with molecular beam reactive scattering experiments that monitored the hydrogenation of butene $\left(\mathrm{C}_{4} \mathrm{H}_{8}\right)$ to butane $\left(\mathrm{C}_{4} \mathrm{H}_{10}\right)$ at comparable $\mathrm{Pd} / \mathrm{Fe}_{3} \mathrm{O}_{4}$ model catalysts under identical pressure/temperature conditions [617]. A very similar $\mathrm{H}_{2}$ pressure dependence of the nanoparticle-absorbed hydrogen state occupation and the butane production rate was observed. NRA thus helped substantiating the longstanding suspect that hydrogen absorbed inside the Pd-nanocrystals is indeed an essential ingredient for the catalytic olefin hydrogenation reaction on oxide supported nanoparticles [617]. 


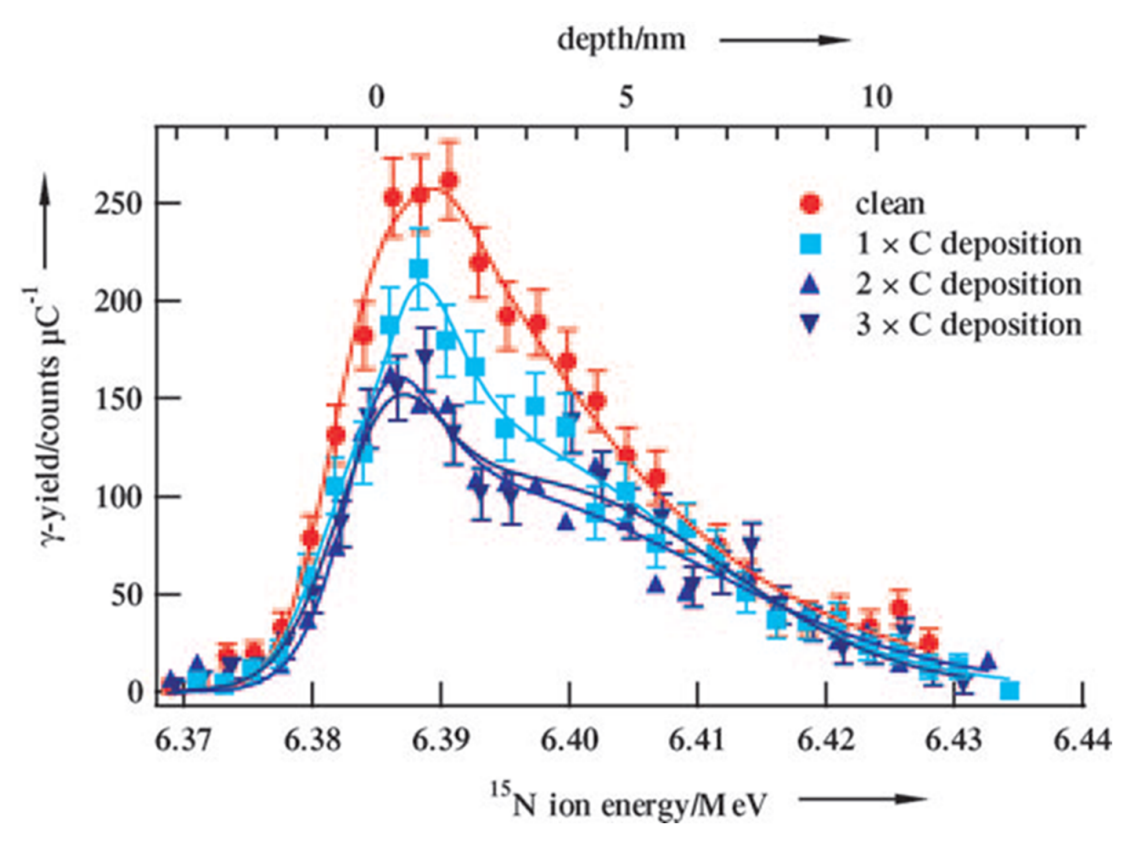

Fig. 67. NRA $\gamma$-yield curves demonstrating the change of the $\mathrm{H}$ distribution in the $\mathrm{Pd} / \mathrm{Al}_{2} \mathrm{O}_{3} / \mathrm{NiAl}(110)$ model catalyst under the cis-2-butene conversion conditions $(260 \mathrm{~K}$, $4 \times 10^{-6} \mathrm{mbar}_{2}$ ) induced by successive carbon depositions. Reproduced from Ref. [617].

Interestingly, the hydrogenation reactivity was found to be unstable on clean $\mathrm{Pd}$ nanoparticles, whereas slightly carbon-contaminated Pd crystallites showed sustained catalytic hydrogenation reactivity. This promotion of hydrogenation reactivity was striking in the sense that carbon is commonly known as a catalyst poison. The observation could be explained qualitatively, however, by an enhancement of the replenishing rate of $\mathrm{Pd}$-absorbed hydrogen through the carbon impurities, which are mainly located underneath the nanoparticle surface near the crystal edges. Rather than blocking $\mathrm{H}$ adsorption sites on the large crystal facets, carbon only energetically destabilizes the surface-adsorbed hydrogen state and thereby reduces the energy barrier for its transition from the surface into the interior of the nanocrystals, which accelerates the $\mathrm{H}$ uptake. This interpretation was based on ${ }^{15} \mathrm{~N}$ NRA H profiles recorded under hydrogenation reaction conditions (260 
$\mathrm{K}, 4 \times 10^{-6} \mathrm{mbar}_{2}$ ) for clean and carbon-contaminated Pd nanocrystals, on which the deposited C coverage was stepwise increased (Fig. 67) [617]. The data demonstrate the $\mathrm{H}$ coverage depletion on the nanoparticle surface as a consequence of the carbon deposition at the reaction temperature. At $90 \mathrm{~K}$, however, the $\mathrm{H}$ surface coverage on the C-contaminted Pd nanocrystals was found to be fully restored, in line with the hypothesis that carbon only destabilizes but does not block surface-H. The proposed facilitation of hydrogen absorption into Pd nanocrystals in presence of subsurface carbon has meanwhile found theoretical confirmation [618-620].

\subsubsection{Semiconductors: $H$ in thin films}

Pure and crystalline semiconductors generally have only very little hydrogen solubility [109]. However, the microelectronic industry fabricates semiconductor thin films for integrated circuit devices, optoelectronics, solar cells, sensors, displays, etc. on an enormous scale, and these materials are often not single crystalline. Widely applied film growth techniques such as chemical vapor deposition (CVD) employ Hcontaining gaseous precursors such as $\mathrm{H}_{2}$, methane $\left(\mathrm{CH}_{4}\right)$, acetylene $\left(\mathrm{C}_{2} \mathrm{H}_{2}\right)$, silanes $\left(\mathrm{SiH}_{4}\right.$ and $\left.\mathrm{Si}_{2} \mathrm{H}_{6}\right)$, and ammonia $\left(\mathrm{NH}_{3}\right)$. Residual hydrogen is therefore a very common observation in the produced films. Here, $\mathrm{H}$ interacts dominantly with bulk defects, such as vacancies, grain boundaries, heteroatoms (dopants and impurities), and surfaces. Since the purity, defect concentration, and microstructure of the films depend sensitively on the synthesis conditions, their $\mathrm{H}$ content can vary widely in a range from a few ppm to several atomic percent [621]. The incorporated hydrogen strongly influences the electrical and optical properties of the materials and therefore their performance in devices. In amorphous silicon, for example, hydrogen 
is introduced deliberately to passivate dangling bonds, which eliminates electrically active states from the band gap and thereby enables application of the material in solid state detectors and solar cells.

The characterization of the $\mathrm{H}$ content in thin semiconducting films is thus of high technological interest, and ${ }^{15} \mathrm{~N}$ NRA (as well as ERD) has found wide applications to materials such as hydrogenated amorphous silicon [203, 622-629], silicon carbide [630-632], silicon nitride [110, 633-636], BCN [637], CVD diamond [255, 621, 638, 639] and diamond-like carbon films [634]. Notable phenomena include, e.g., the sensitivity of the $\mathrm{H}$ bonding states in amorphous silicon to elastic stress [622]. Myers et al. studied hydrogen interactions with dopants and radiation-induced defects in Mg-doped p-type gallium nitride $(\mathrm{GaN})$ with $\mathrm{D}\left({ }^{3} \mathrm{He}, \mathrm{p}\right)^{4} \mathrm{He}$ NRA by monitoring the kinetics of isotopic exchange against $\mathrm{H}$ after $\mathrm{D}$ incorporation [640]. In this context most ${ }^{15} \mathrm{~N}$ NRA investigations focused on determining the average $\mathrm{H}$ content in the semiconductor films in order to draw indirect conclusions on the microstructure. Since these studies seldom had explicit relation to hydrogen at surfaces or interfaces, we only provide the above references here and refrain from detailed descriptions.

We would like to mention, however, the important role of NRA as a calibration reference for the optical hydrogen analysis of thin film semiconductor materials (as well as of insulators, cf. Sect. 3.2.5.1) with vibrational spectroscopy. Infrared absorption and Raman spectroscopy readily identify $\mathrm{H}$ species in different chemical environments through their specific vibrational fingerprints. In many IR-transparent materials linear correlations have been found between the IR absorption or Raman scattering intensities of $\mathrm{H}$-related vibrational bands and the absolute volume 
hydrogen concentrations that were determined with NRA $[55,622]$. With NRA as a reference, calibration relations could thus be established that allowed measuring average bulk $\mathrm{H}$ concentrations quantitatively with the highly convenient optical techniques [622, 640-642].

To illustrate that also the depth-resolving powers of ${ }^{15} \mathrm{~N}$ NRA can be beneficial in the hydrogen analysis of thin semiconducting films, Fig. 68 displays $\mathrm{H}$ depth profiles of CVD grown diamond films of various thicknesses [643]. In addition to the large $\mathrm{H}$ concentration $(7.5-8 \%)$ in the film volume evidenced by the plateau region of the thickest film, a H-rich surface layer was observed on all samples.

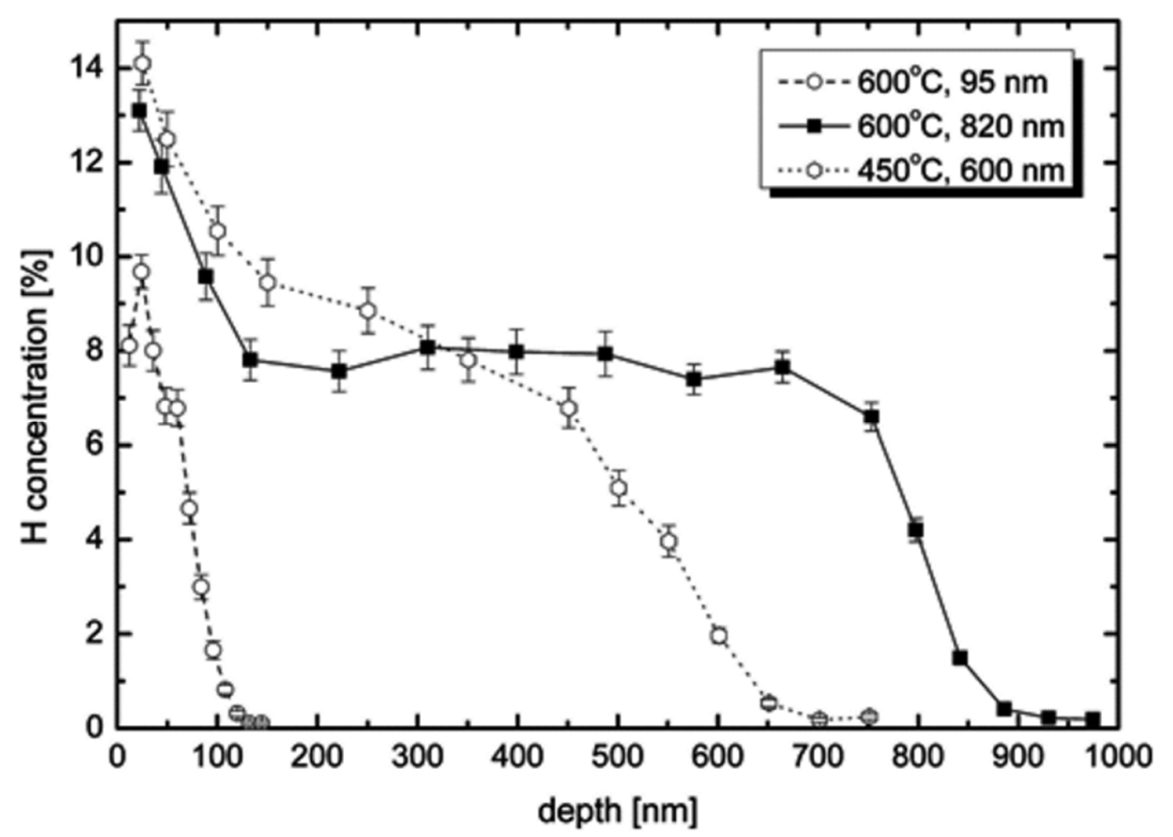

Fig. $68 .{ }^{15} \mathrm{~N}$ NRA hydrogen depth profiles of the three samples of ultra-nanocrystalline diamond films of 95, 600, and $820 \mathrm{~nm}$ thickness on Si(100) substrates. From Ref. [643] .

Apparently this layer formed already at the beginning of the deposition process and grew outward as the film thickness increased. The authors revealed with vibrational spectroscopy (FT-IRAS) that this surface layer contained a large fraction of $\mathrm{sp}^{3}$ 
hybridized $\mathrm{CH}_{2}$ groups and accordingly concluded that surface stabilization by hydrogen is an important factor in the CVD growth of diamond. The large $\mathrm{H}$ concentration in the film volume was attributed to the formation of an amorphous carbon matrix that embeds diamond nanocrystals [643].

The surface interaction of crystalline diamond with hydrogen and its possible induction of electrical conductivity has also been investigated with a variety of ion beam analysis techniques that include ${ }^{15} \mathrm{~N}$ NRA $[432,433,644,645]$ and highresolution ERD $[255,638,639]$. Kimura et. al addressed the debated possibility of $\mathrm{H}$ subsurface site occupation at CVD diamond surfaces with high-resolution ERD H profiling and could indeed confirm a small amount of $\mathrm{H}$ buried in a region less than 1 $\mathrm{nm}$ deep below the surface [255].

\subsubsection{Insulators}

\subsubsection{H in near-surface regions of oxides and minerals}

${ }^{15} \mathrm{~N}$ NRA has found numerous applications in the analysis of water and hydroxyl $(\mathrm{OH})$ in the near-surface region of oxides and silicates. Central research interests encompass the chemical modification of glass surfaces by leaching in aqueous environments [646-648], related to moist air and pollution-induced weathering of glass that causes, e.g., the decay of historic stained glass windows in European churches $[649,650]$, the non-destructive chemical analysis of archaeological and historical artifacts [78, 79, 651-654], the surface hydration kinetics of silicates in Portland cement relevant to concrete technology [655-657], as well as the quantitation of the water content in minerals and gemstones that are important 
parameters in the geochemistry of the Earth's mantle materials [641, 642, 658-663]. According to Doremus' theory [664-666] water penetrates into the surface of alumino-silicates and replaces network modifying $\mathrm{Na}, \mathrm{Ca}$, and $\mathrm{Al}$ ions against protons in exchange processes such as:

$$
\left.\mathrm{Na}^{+} \text {(glass) }+\mathrm{H}_{2} \mathrm{O} \rightleftarrows \mathrm{H}^{+} \text {(glass }\right)+\mathrm{NaOH}
$$

A common observation in leached glasses and minerals thus is a near-surface region that is depleted in $\mathrm{Na}, \mathrm{Ca}$, etc., but enriched with $\mathrm{H}$. Through revealing the hydrogen depth distribution, ${ }^{15} \mathrm{~N}$ NRA hydrogen profiling allows assessing the thickness of the erosion layer. Fig. 69 shows exemplarily a typical ${ }^{15} \mathrm{~N}$ NRA depth profile of a alumino-silicate exposed to air and water vapor [649] that evidences hydration up to a depth of $\sim 0.4 \mu \mathrm{m}$ with an average $\mathrm{H}$ concentration of $1.2 \times 10^{22} \mathrm{~cm}^{-}$ 3 , as well as a much thinner hydrogen-rich surface layer. Trocellier et al. applied three resonant nuclear reactions $\left({ }^{1} \mathrm{H}\left({ }^{15} \mathrm{~N}, \alpha \gamma\right)\right)^{12} \mathrm{C},{ }^{23} \mathrm{Na}(p, \alpha \gamma){ }^{20} \mathrm{Ne}$, and $\left.{ }^{27} \mathrm{Al}(\mathrm{p}, \gamma){ }^{28} \mathrm{Si}\right)$ to study the leaching of lime-soda glasses by deionized water through monitoring $\mathrm{Na}, \mathrm{Al}$ and $H$ [646]. Mäder et al. analyzed the corrosion of a potash-lime glass of medieval composition ( $60 \mathrm{wt} \% \mathrm{SiO}_{2}, 25 \mathrm{wt} \% \mathrm{CaO}, 15 \mathrm{wt} \% \mathrm{~K}_{2} \mathrm{O}$ ) leached in $0.1 \mathrm{~N} \mathrm{HCl}$ at room temperature and cross-correlated the depth extension of the hydrated layer derived from $\mathrm{H}$ profiling with ${ }^{15} \mathrm{~N}$ NRA to the $\mathrm{Ca} / \mathrm{K} / \mathrm{Si}$ composition information obtained from PIXE and PIGE measurements with a $4 \mathrm{MeV}$ proton beam. The authors observed that the ratio of the Si PIXE/PIGE signals increased linearly with the thickness of the leached layer in the range of 0-5 $\mu \mathrm{m}$ but saturated at thicker corrosion layers. They consequently suggested to use this information as a rough indicator for the presence 
of an eroded surface layer $[653,654]$, which enables a quick distinction between a thin surface-hydration layer from a deeply bulk corroded sample condition in objects of historical value by a simple non-destructive measurement.

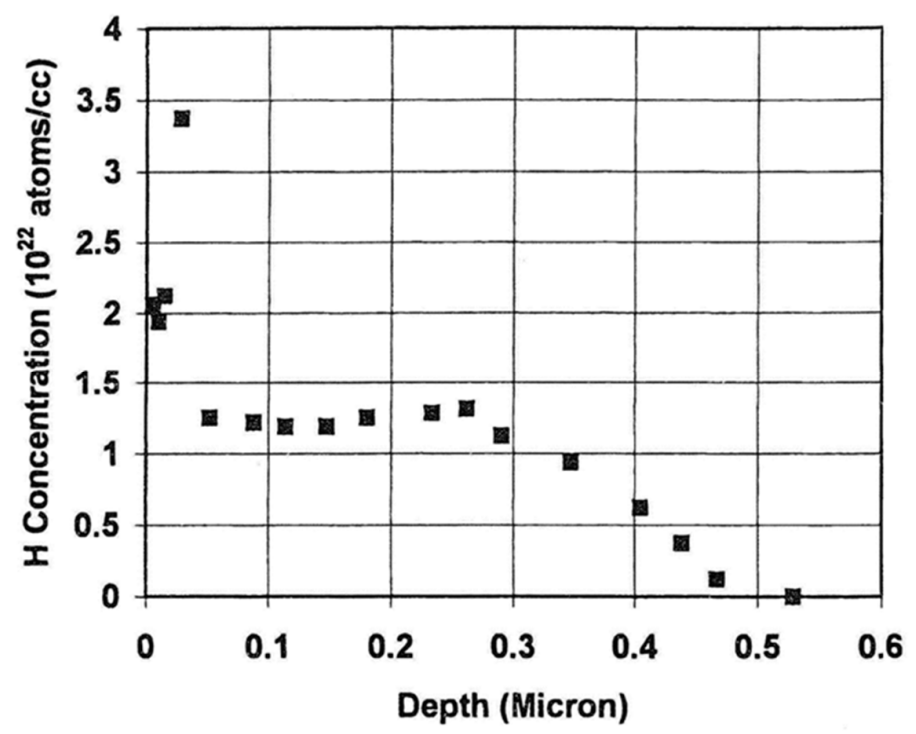

Fig. 69. A typical NRA hydrogen concentration profile measured with ${ }^{15} \mathrm{~N}$ NRA in an alumino-silicate sample exposed to air and water vapor. Characteristics of the profile include the hydration depth $(0.4 \mathrm{~mm})$, the equilibrium hydration concentration $\quad\left(1.2 \times 10^{22}\right.$

atoms $\mathrm{cm}^{-3}$ ) and a H-rich surface layer. From Ref. [649].

Compared to leaching of amorphous glass, the water diffusion in crystalline quartz is many orders of magnitude slower and studies of the hydration process had long been limited to temperatures above $500^{\circ} \mathrm{C}$. Dersch and Rauch extended the observation regime to a lower temperature range of 60 to $200^{\circ} \mathrm{C}$ by using hydrothermal treatments, i.e., by heating the quartz samples with a certain quantity of $\mathrm{H}_{2} \mathrm{O}$ (or $\mathrm{H}_{2}{ }^{18} \mathrm{O}$ ) in an autoclave for several days, and analyzed the resulting nearsurface $\mathrm{H}$ and ${ }^{18} \mathrm{O}$ diffusion profiles with the ${ }^{1} \mathrm{H}\left({ }^{15} \mathrm{~N}, \alpha \gamma\right){ }^{12} \mathrm{C}$ and ${ }^{18} \mathrm{O}(\mathrm{p}, \alpha){ }^{15} \mathrm{~N}$ nuclear reactions $[78,79,652]$. Examples are shown in Fig. 70. The observed $\mathrm{H}$ depth profiles were well reproduced by error function shaped concentration distributions:

$$
c(x, t)=c_{0} \cdot \operatorname{erfc}\left(\frac{x}{2 \sqrt{D_{e f f} t}}\right)+c_{i},
$$


where $x$ : depth, $c_{0}$ : concentration at depth zero, $c_{i}$ : intrinsic (bulk) concentration, $t$ : treatment time. Note that $D_{\text {eff }}$ is an effective diffusion-reaction rate (not merely a diffusion coefficient), since the hydration process includes the reaction of $\mathrm{H}_{2} \mathrm{O}$ with the $\mathrm{SiO}_{2}$ lattice, according to [664]:

$$
\mathrm{SiO}_{2}+\mathrm{H}_{2} \mathrm{O} \rightleftarrows 2 \mathrm{SiOH}
$$

From the temperature dependence of $D_{\text {eff }}$ Dersch and Rauch determined the activation energy for the hydration process and obtained a common value of 60 $\mathrm{kJ} / \mathrm{mol}$ for various samples of natural and synthetic quartz [652]. The absolute $D_{\text {eff }}$ value was found to depend also on the bulk $\mathrm{H}$ concentration $c_{i}$ and on the angle of the surface with the c-axis of the quartz crystals, indicating an orientation dependence of the diffusion process. The bulk concentrations were found to vary between 10 and 100 at. ppm for synthetic and up to 500 at. ppm for natural quartz specimen [79]. On the basis of the common activation energy for synthetic and prehistoric quartz artifacts, a universal hydration mechanism was suggested [651]. Indeed, modeling H-diffusion profiles with Eq. (3.3) for an average ambient temperature of $10^{\circ} \mathrm{C}$ gave good agreement with NRA $\mathrm{H}$ profiles of hydration layers obtained from natural, age-dated quartz specimen, confirming that the same form of quartz hydration kinetics also applies in natural environments [652]. The evaluation of the hydration layer thickness (defined as the depth where the $\mathrm{H}$ concentration attains half the value of depth zero) was thus proposed as a versatile and nondestructive method for the age determination of quartz crystals in geological material or in historical artifacts [79]. Based on the water mobility in $\mathrm{SiO}_{2}$ and the 
NRA-accessible probing depth range, this ${ }^{15} \mathrm{~N}$ NRA 'quartz hydration dating' technique allows for the specification of hydration periods between 100 to more than 10,000 years with an accuracy of $35 \%$ to $20 \%$ depending on the sample material $[79,80]$.

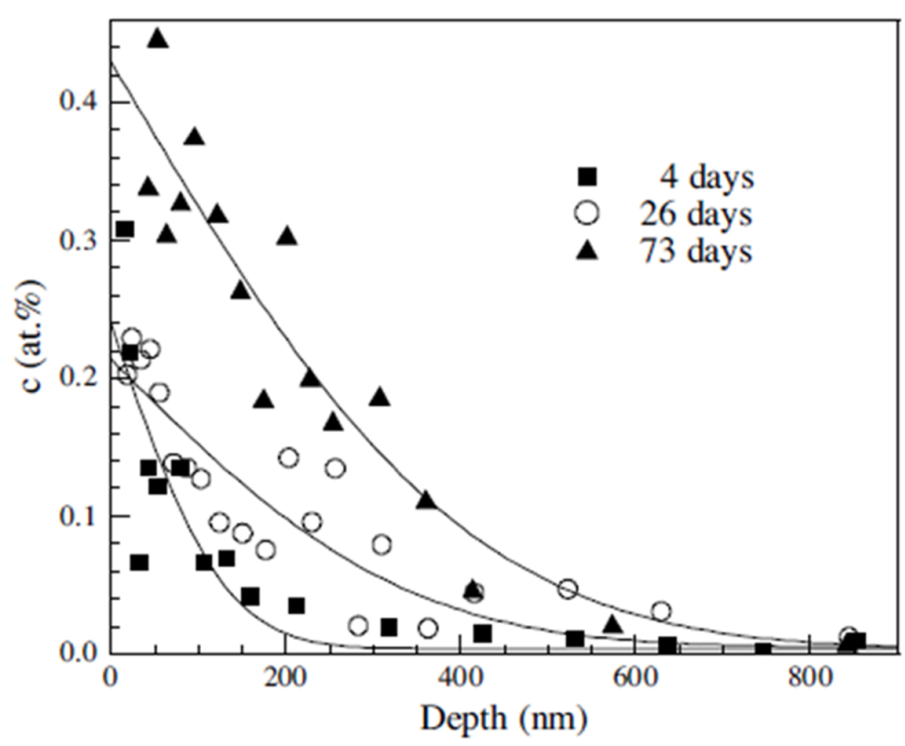

Fig. 70. Comparison of ${ }^{15} \mathrm{~N}$ NRA $H$ profiles of quartz samples exposed for different times to a hydrothermal treatment at $200{ }^{\circ} \mathrm{C}$ in $1.56 \mathrm{MPa} \mathrm{H}_{2} \mathrm{O}$ vapor showing progressive hydration of the nearsurface region. From Ref. [79].

Similarly, observing hydrated surface layers that form on tricalcium silicate with time-resolved ${ }^{15} \mathrm{~N}$ NRA was pointed out as a possibility to investigate the hydration kinetics of Portland cement at a suitable model system [655-657]. The study established that during an induction period in the first few hours, the hydrogen diffusion is controlled by an only $10-20 \mathrm{~nm}$ thin surface layer, which poses high demands on the ${ }^{15} \mathrm{~N}$ beam energy resolution (on the order of $10 \mathrm{keV}$ or less) to be observed [656]. Lanford's group studied the fracture mechanism of glass under mechanical stress and moisture exposure by monitoring the diffusion of water into silica glass with ${ }^{15} \mathrm{~N}$ NRA while simultaneously applying a hydrothermal treatment at several temperatures and mechanical stress [650]. It was found that at low temperatures the stress affected the kinetics of the water uptake, while at high 
temperatures the applied stress influences the glass-water reaction equilibrium [650]. The above examples illustrate the versatility of NRA to investigate the response of a given sample material in terms of its internal $\mathrm{H}$ distribution to changes of external conditions, based on the non-destructive nature of the measurement.

\subsubsection{Hydrogen redistribution in electrochromic multilayers}

Wagner et al. applied ${ }^{15} \mathrm{~N}$ NRA to monitor in-situ the proton transport occurring under different operational conditions in electrochromic multilayer systems [81, 82]. These optical devices are all-solid-state film structures on glass substrates, in which an active layer of electrochromic $\mathrm{WO}_{3}$, a storage layer $\left(\mathrm{WO}_{3}\right)$ or anodically coloring electrochromic material $\left(\mathrm{NiO}_{x}\right)$, and a solid electrolyte (here: $\mathrm{Ta}_{2} \mathrm{O}_{5}$ ) placed between these two layers are sandwiched between two electrodes, such as illustrated in Fig. 71.

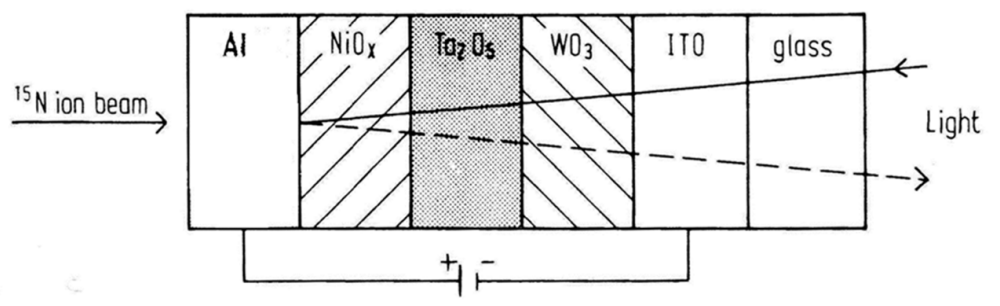

Fig. 71. Configuration of the electrochromic mirror system. Reproduced from Ref. [81].

Indium tin oxide (ITO) is used as a transparent and conductive electrode adjacent to the glass. The outermost Al layer serves simultaneously as the counter electrode (anode) and as the metallic reflector. The reflectance, absorbance, or transmittance of the optical device can thus be controlled by applying an external voltage to the outer electrodes, typically in the order of $1 \mathrm{~V}$. Reflectance measurements with a HeNe laser and a photodiode detector established that the reflectivity of the above 
system changes from $10 \%$ to $70 \%$ when the external voltage is varied between +2 to $-1.5 \mathrm{~V}$. To the human eye, the system appears dark blue in the low-reflectance region, whereas at $\mathrm{U} \leq 0 \mathrm{~V}$ it has a metallic luster.

The absorbance change results from the electrochromic effect that has been discussed in relation to the following red/ox reactions: $\mathrm{WO}_{3}$ is a cathodically coloring electrochromic material which varies between transparent and blue during the transition of the $\mathrm{W}^{6+}$ (transparent) oxidation state to $\mathrm{W}^{5+}$ (blue) [667] in the reaction [668]:

$$
\mathrm{WO}_{3}+x \mathrm{H}^{+}+x \mathrm{e}^{-} \rightleftarrows \mathrm{H}_{\mathrm{x}} \mathrm{WO}_{3}
$$

The electrochromism of the anodic coloration of $\mathrm{NiO}_{\mathrm{x}}$ is due to the reaction of $\mathrm{Ni}^{2+}$ (transparent) to $\mathrm{Ni}^{3+}$ (colored) according to [669]:

$$
\mathrm{Ni}(\mathrm{OH})_{2} \rightleftarrows \mathrm{NiOOH}+\mathrm{H}^{+}+\mathrm{e}^{-}
$$

Wagner et al. visualized the actual proton transport involved in these reactions by means of NRA via ${ }^{1} \mathrm{H}\left({ }^{15} \mathrm{~N}, \alpha \gamma\right){ }^{12} \mathrm{C}$. Figs. 72 (a) and (b) show $\mathrm{H}$ profiles of the above optical system (Fig. 71) under application of different voltages, with the $0 \mathrm{~V}$ profile displayed as a reference. For simplicity, only the H/W ratio is given on the ordinate. No $\mathrm{H}$ is detected in the Al layer (except for a surface peak) and the slight $\mathrm{H}$ concentration at the ITO/glass substrate boundary is due to a thin $\mathrm{SiO}_{2}$ layer on the glass serving as an alkali diffusion barrier. The $\mathrm{H}$ content in the $\mathrm{NiO}_{\mathrm{x}}$ is smaller than in $\mathrm{WO}_{3}$ and corresponds to $\mathrm{x}=1.5$ as judged from RBS [81]. The $\mathrm{H}$ concentration in $\mathrm{Ta}_{2} \mathrm{O}_{5} / \mathrm{WO}_{3}$ is very high, and exceeds that of single $\mathrm{Ta}_{2} \mathrm{O}_{5}$ or $\mathrm{WO}_{3}$ layers without the NiOx/Al overlayer structure [670]. 

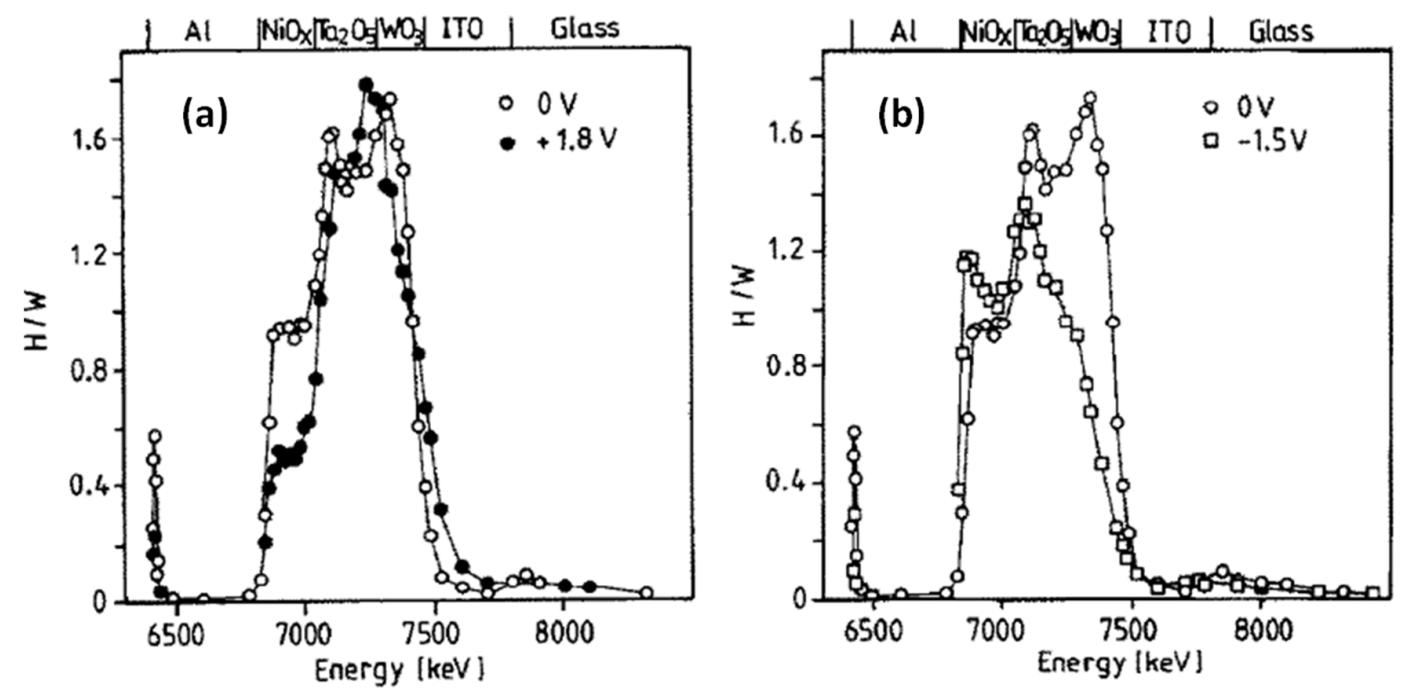

Fig. 72. (a) ${ }^{15} \mathrm{~N}$ NRA $\mathrm{H}$ concentration profile of the electrochromic system at $\mathrm{U}=+1.8 \mathrm{~V}$ (Reflectivity state: dark blue) and the reference profile. (b) $\mathrm{H}$ concentration profile of the electrochromic system at $\mathrm{U}=-1.5 \mathrm{~V}$ (Reflectivity state: strongly bleached) and the reference profile. Reproduced From Ref. [81].

As seen in Fig. 72 (a), a voltage of $+1.8 \mathrm{~V}$ applied to the Al electrode (corresponding to the dark state of the system) causes a strong depletion of the $\mathrm{H}$ concentration $\left(\mathrm{C}_{\mathrm{H}}\right)$ in the NiOx layer and enhances $\mathrm{C}_{\mathrm{H}}$ in the $\mathrm{Ta}_{2} \mathrm{O}_{5}$ near the $\mathrm{Ta}_{2} \mathrm{O}_{5} / \mathrm{WO}_{3}$ interface. In addition the $\mathrm{H}$ concentration is decreased in the $\mathrm{WO}_{3}$ and increased in the ITO layer. The integral amount of $\mathrm{H}$ in the system has not changed, and the direction of the $\mathrm{H}$ transport is in accordance with the expectation that the $\mathrm{H}^{+}$ ions are shifted towards the negative ITO electrode. NRA also verified that shortcircuiting $(0 \mathrm{~V})$ the device reversibly restores the original $\mathrm{H}$ distribution without hysteresis in the $\mathrm{H}$ transport and no $\mathrm{H}$ loss from the system. For negative voltages (1.5 V, Fig. $72(b))$, the $\mathrm{H}$ distribution is drastically altered in the opposite direction, i.e., $\mathrm{C}_{\mathrm{H}}$ increases in the $\mathrm{NiO}_{\mathrm{x}}$ layer at the outer interface, while it decreases in the $\mathrm{Ta}_{2} \mathrm{O}_{5}$ layer and very strongly in the $\mathrm{WO}_{3}$ and ITO layers. As expected, the $\mathrm{H}$ displacement is directed towards the (negative) Al electrode. A loss of $\mathrm{H}$ is also 
noticed in Fig. 72 (b), which is attributed to the large negative voltage that has been outside the permitted range for this electrochromic system and is suspected to cause $\mathrm{H}$ desorption through micro-pores of the Al film [81].

By demonstrating that the $\mathrm{H}$ concentration in $\mathrm{NiO}_{\mathrm{x}}$ is smaller in the colored state than in the bleached condition, the NRA results confirmed that the optical changes in the electrochromic system agree with reaction (3.6). Apparently, the $\mathrm{H}^{+}$ions are sufficiently mobile to be noticeably redistributed between the $\mathrm{NiO}_{x}, \mathrm{Ta}_{2} \mathrm{O}_{5}, \mathrm{WO}_{3}$, and ITO layers through external electric field application. An increase of the $\mathrm{H} / \mathrm{W}$ ratio associated with the colored state of the $\mathrm{WO}_{3}$ layer according to reaction (3.5), however, could not be observed [81].

\subsubsection{Hydrogen in ultrathin dielectric films on silicon}

The detection of residual hydrogen in ultrathin dielectric films of metal-oxidesemiconductor (MOS) capacitors and gate insulating layers of field-effect transistors (MOSFETs) is one of the most prominent ${ }^{15} \mathrm{~N}$ NRA applications in industry-related research. In view of the nanometer scale of the thin layers and the small $\mathrm{H}$ impurity concentrations in MOS devices, these NRA applications are challenging with respect to depth resolution and sensitivity. The dominant motivation to investigate hydrogen in stacked dielectric layers with NRA is that the $\mathrm{H}$ interaction with the respective interfaces is believed to very significantly affect the electrical device characteristics. Especially the problem of potentially hydrogen-related degradation of the $\mathrm{SiO}_{2} / \mathrm{Si}$ interface is recognized as a highly relevant reliability concern in semiconductor device technology, which will be given particular attention in Section 3.3.1. Here, we first introduce general aspects of ${ }^{1} \mathrm{H}\left({ }^{15} \mathrm{~N}, \alpha \gamma\right){ }^{12} \mathrm{C}$ NRA hydrogen analysis in ultrathin 
dielectric films on Si substrates, focusing primarily on the $\mathrm{H}$ concentration and its distribution in various materials, on the influence of the NRA measurement on this distribution and the analytical value of this effect, and on the possibility to study insitu the impact of processing treatments on the $\mathrm{H}$ distribution by exploiting the nondestructive virtues of NRA. The literature contains a sizeable body of NRA investigations of hydrogen in the traditional silicon oxide on silicon $\left(\mathrm{SiO}_{2} / \mathrm{Si}\right)[107$, $160,161,671-676]$ [677-682], in buried $\mathrm{SiO}_{2}$ layers in $\mathrm{Si} / \mathrm{SiO}_{2} / \mathrm{Si}$ structures $[683,684]$, in silicon nitride $(\mathrm{SiN})[634,636,685,686]$, and in silicon oxynitrides $\left(\mathrm{SiO}_{\mathrm{x}} \mathrm{N}_{\mathrm{y}}\right)[687-$ 691].

\section{a) Hydrogen distribution in $\mathrm{SiO}_{2} / \mathrm{Si}$}

The hydrogen (deuterium) content $[677,692]$ and distribution [107] [161, 678, 680] in $\mathrm{SiO}_{2}$ films of various thickness on $\mathrm{Si}(100)$ wafers has been studied by several groups. $\mathrm{H}$ concentrations in the order of $\left(10^{18}-10^{19} \mathrm{~cm}^{-3}\right)$ in the volume of the $\mathrm{SiO}_{2}$ layers have been found, corresponding to areal $\mathrm{H}$ densities in the order of $\sim 10^{13-14}$ $\mathrm{cm}^{-2}$ if the concentration is integrated over the oxide thickness (typically several tens of nanometers). Similar $\mathrm{H}$ concentrations were detected in buried $\mathrm{SiO}_{2}$ layers of $\mathrm{Si} / \mathrm{SiO}_{2} / \mathrm{Si}$ structures [684]. The depth-resolving ${ }^{15} \mathrm{~N}$ NRA measurements revealed in addition that the $\mathrm{H}$ distribution within the oxide is not necessarily uniform, but that hydrogen tends to accumulate not exactly at but in a region extending over a few $\mathrm{nm}$ of the oxide in front of the interface $[107,160,161,671,673,675-678,680,681]$. Fig. 73 (a) illustrates this situation at the example of three ${ }^{15} \mathrm{~N}$ NRA H depth profiles of wet oxidized $\mathrm{Si}(100)$ specimens bearing $\mathrm{SiO}_{2}$ films with thicknesses of 19.0, 30.0, and $41.5 \mathrm{~nm}[680]$. 
(a)
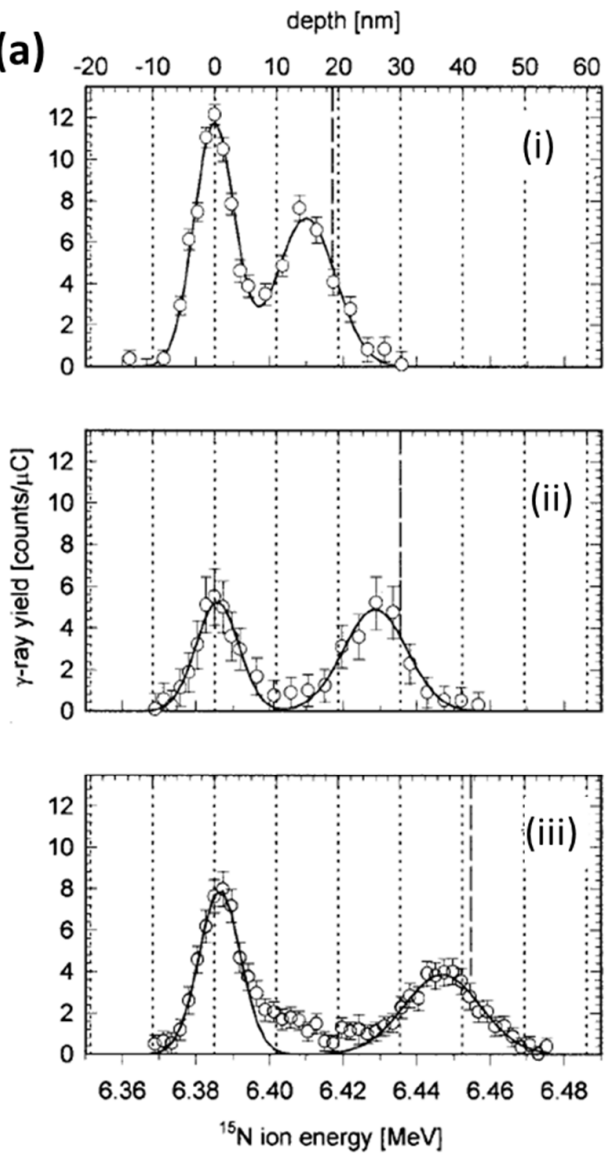

(b)

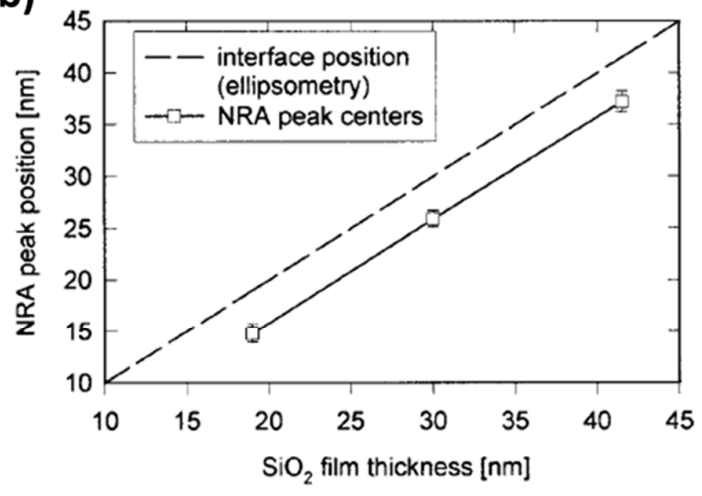

Fig. 73. (a) NRA H-profiles for three $\mathrm{SiO}_{2}$ films on $\mathrm{Si}(100)$ with thicknesses of (i) $19.0 \mathrm{~nm}$, (ii) $30.0 \mathrm{~nm}$, and (iii) $41.5 \mathrm{~nm}$. Interface positions are indicated by dashed vertical lines. (b) Center positions of the NRAdetermined near $\mathrm{SiO}_{2} / \mathrm{Si}$ interfacial $\mathrm{H}$ distributions compared to the optically obtained $\mathrm{SiO}_{2}$ film thickness. From Ref. [680].

Fig. 73 (b) indicates that the centroid of this peak follows the interface position but is separated from it by a small offset of a few $\mathrm{nm}$. This $\mathrm{H}$ localization behavior has been attributed to the near-interfacial $\mathrm{SiO}_{2}$ structure, where a large number of strain and sub-oxide [693] related defects provide preferential binding sites for hydrogen species [694]. The strained interfacial region results from the reduction of the $\mathrm{Si}$ atom number density by a factor of $\sim 2$ in the abrupt transition from crystalline $\mathrm{Si}$ to amorphous $\mathrm{SiO}_{2}$.

\section{b) ${ }^{15} \mathrm{~N}$-induced $\mathrm{H}$ accumulation near the $\mathrm{SiO}_{2} / \mathrm{Si}$ interface}

It is necessary at this point to recognize a characteristic influence of the NRA depth profiling on the $\mathrm{H}$ distribution in thin dielectric layers on Si substrates. As first 
reported by Briere et al. [160] and later observed in many similar studies [636, 680, $681,685,686,695-697]$, the ${ }^{15} \mathrm{~N}$ ion irradiation can cause a noticeable redistribution of hydrogen in the analyzed targets that leads to a pile-up of $\mathrm{H}$ in the near-interfacial $\left(\mathrm{SiO}_{2} / \mathrm{Si}\right)$ accumulation region. As the $\mathrm{Si}$ substrate is generally $\mathrm{H}$-free, the relocating hydrogen can originate from any region above the $\mathrm{SiO}_{2} / \mathrm{Si}$ interface that contains $\mathrm{H}$, such as the silicon oxide (or oxynitride) film volume, hydroxylated or H-contaminated surface layers on uncapped dielectric films, an interface layer formed with a capping electrode material (such as in $\mathrm{Al} / \mathrm{SiO}_{2} / \mathrm{Si}$ )[698], or the volume of a $\mathrm{H}$-rich material covering the oxide (such as in $\mathrm{SiN} / \mathrm{SiO}_{2} / \mathrm{Si}$ [686], or $\mathrm{SiO}_{2} / a-\mathrm{Si}: \mathrm{H} / \mathrm{SiO}_{2} / \mathrm{Si}$ [697]) stacks.

As illustrated in Fig. 74, the redistribution phenomenon is imagined to result from $\mathrm{H}$ release in such $\mathrm{H}$-rich layers due to local $\mathrm{H}$-bond cleavage by ion beam generated secondary electrons (and holes), followed by diffusion of the mobilized $\mathrm{H}$ species through the dielectric towards the $\mathrm{SiO}_{2} / \mathrm{Si}$ interfacial region, where a fraction of them binds to the available $\mathrm{H}$ trap sites. Many details of the process (the bondbreaking mechanism, the chemical identity and charge state of the diffusing $\mathrm{H}$ species, the nature of the near-interfacial trap sites, etc.) are still unknown. Nonetheless, the effective $\mathrm{H}$ accumulation cross section (determined through NRA measurements of the near-interfacial pile-up kinetics described below) is in the order of $10^{-16} \sim 10^{-15} \mathrm{~cm}^{2}$ which points towards an electron-induced rather than nuclear collision-related origin of the relocation $[680,686]$. 


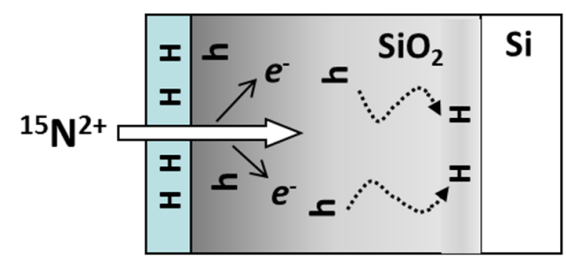

$$
\begin{array}{lll}
\hline \mathrm{H} \mathrm{H} & : \text { H-rich layer } \\
\cline { 1 - 2 }{ }^{15} \mathrm{~N}^{2+} & \text { : NRA ion beam } \\
\mathrm{e}^{-} & \text {: Secondary electron } \\
\mathrm{h} & \text { : Migrating H-species } \\
\mathrm{H} & \text { : Trapped H-species }
\end{array}
$$

Fig. 74. Schematic illustration of the $H$ relocation process under the NRA ion beam. Interaction of beam-generated secondary electrons with $\mathrm{H}$ bound in surface-near layers releases mobile $\mathrm{H}$ species that migrate through the MOS structure and may accumulate in near $\mathrm{SiO}_{2} / \mathrm{Si}$ interfacial trap sites.

The $\mathrm{H}$ redistribution is observed at room temperature as well as in liquid nitrogen cooled targets [107]. The phenomenon is furthermore not specific to ${ }^{15} \mathrm{~N}$ NRA. High-resolution ERD measurements with $400 \mathrm{keV} \mathrm{C}+$ ions at ultrathin $\mathrm{SiO}_{2}$ films on Si(100) have reported a similar near-interfacial H accumulation effect [699], supporting the notion that the process is driven by secondary electrons, which are universally released upon energetic ion beam impact on insulating materials (cf. Section 2.2.5). Hydrogen accumulation at $\mathrm{SiO}_{2} / \mathrm{Si}$ interfaces as a consequence of electron injection into $\mathrm{Al} / \mathrm{SiO}_{2} / \mathrm{Si}$ MOS structures has also been reported in SIMS studies $[700,701]$, but due to the destructive nature of the latter technique no assessement can be made as how far the observed $\mathrm{H}$ distributions are characteristic of the native samples and how strongly they may be distorted by artifacts from the sputtering analysis.

Due to the ion beam-induced $\mathrm{H}$ relocation the NRA depth profiles such as those in Fig. 73 do not reflect the initial $\mathrm{H}$ distribution of the unperturbed sample but display some additional hydrogen in the near-interfacial accumulation region that has piled up during acquisition of the profile. The $\mathrm{H}$ accumulation in the nearinterfacial region typically saturates after application of moderate $\left(<10^{16} \mathrm{~cm}^{-2}\right){ }^{15} \mathrm{~N}$ 
ion doses (cf. Fig. 75 (b)) [702]. Prior to the state of pileup saturation the application of different ${ }^{15} \mathrm{~N}$ ion exposures will lead to variations in the near-interfacial accumulation peak amplitude. Fig. 75 (a) exemplarily shows two $\mathrm{H}$ profiles recorded subsequently with the beam in the same sampling area of a $25 \mathrm{~nm} \mathrm{SiO} / 2 \mathrm{Si}$ stack capped by a thin (10 nm) H-rich SiN layer [686], thus comparing an early stage of the $\mathrm{H}$ redistribution process with the situation after $\mathrm{H}$ uptake saturation. The initially smaller $\mathrm{H}$ content in the $\mathrm{SiO}_{2} / \mathrm{Si}$ interfacial region compared to the one obtained after saturation is evident. In addition, the $\mathrm{H}$ concentration in the SiN layer clearly reduces in the course of the measurements. Apparently, the $\mathrm{H}$ loss in the SiN film is larger than the $\mathrm{H}$ gain in the $\mathrm{SiO}_{2} / \mathrm{Si}$ interfacial region and in the $\mathrm{SiO}_{2}$ volume combined, suggesting that besides $\mathrm{H}$ relocation from $\mathrm{SiN}$ towards the $\mathrm{SiO}_{2} / \mathrm{Si}$ interface the NRA ion irradiation also causes desorption of mobilized $\mathrm{H}$ species from this near-surface layer.
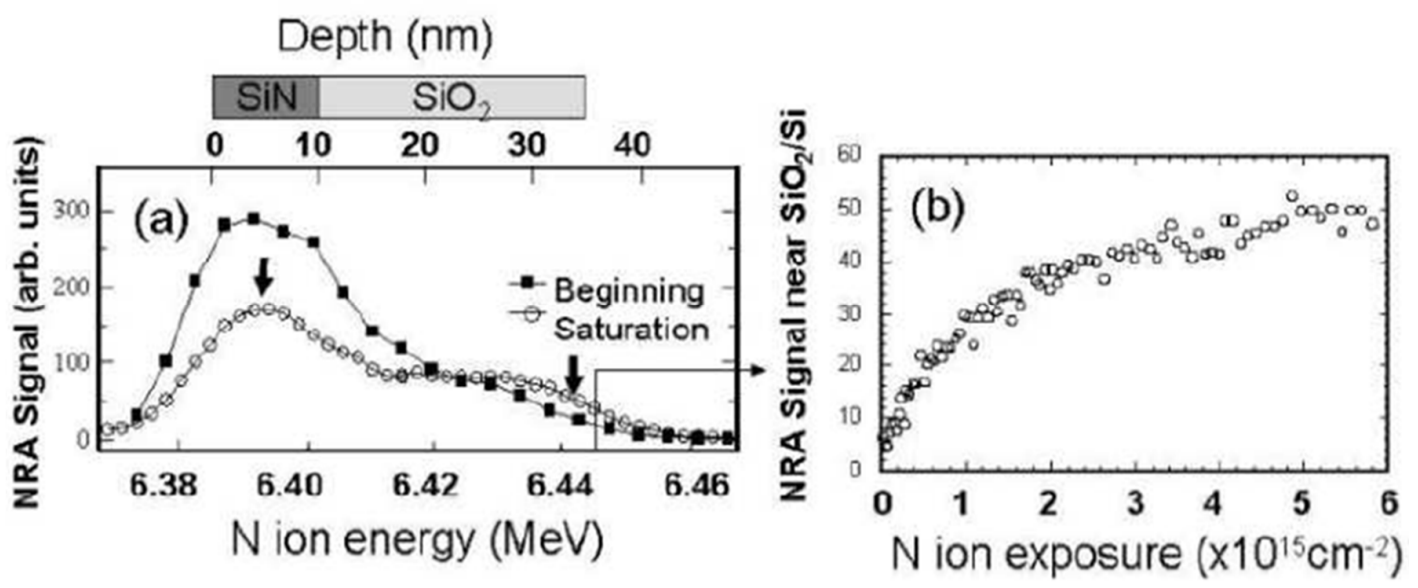

Fig. 75. (a) NRA H depth profiles of $\mathrm{SiN} / \mathrm{SiO}_{2}$ stacks in an early stage and after saturation of the beam-induced $\mathrm{H}$-redistribution. (b) $\mathrm{H}$-uptake as a function of the cumulative ${ }^{15} \mathrm{~N}$ ion dose observed in the near $\mathrm{SiO}_{2} / \mathrm{Si}$ interfacial accumulation region (probing depth: $32 \mathrm{~nm}$, arrow in (a)). From Ref. [46] with permission from ECS. 
Depth-resolving ${ }^{1} \mathrm{H}\left({ }^{15} \mathrm{~N}, \alpha \gamma\right){ }^{12} \mathrm{C}$ NRA can directly visualize the progressing $\mathrm{H}$ accumulation in the near-interfacial region of $\mathrm{SiO}_{2} / \mathrm{Si}$. To this end the evolution of the near-interfacial $\mathrm{H}$ concentration is probed selectively as a function of the cumulative ${ }^{15} \mathrm{~N}$ ion dose with the incident ${ }^{15} \mathrm{~N}$ ion energy fixed to the maximum of the interfacial pile-up peak position. This procedure is exemplified in Fig. 75 (b), which shows the $\mathrm{H}$ uptake in the $\mathrm{SiO}_{2} / \mathrm{Si}$ interfacial region of the above $\mathrm{SiN} / \mathrm{SiO}_{2} / \mathrm{Si}(100)$ stack in a probing depth of $\sim 32 \mathrm{~nm}$ (arrow in Fig. 75 (a)). The data indicate that the near-interfacial $\mathrm{H}$ concentration is initially very small and that the uptake tends to saturate at a ${ }^{15} \mathrm{~N}$ ion fluence well below $10^{16} \mathrm{~cm}^{-2}$.

The authors have found empirically in a large number of investigations of different dielectric materials that the ion beam-induced $\mathrm{H}$ uptake in the $\mathrm{SiO}_{2} / \mathrm{Si}$ interfacial region is rather well described by a function of the type:

$$
[H]\left(\Phi_{N}\right)=\left[H_{0}\right]+\left[H_{\infty}\right]^{*}\left[1-\exp \left(-\sigma_{p} \Phi_{N}\right)\right]
$$

Here, $[H]\left(\Phi_{N}\right)$ is the NRA $\gamma$-signal proportional to the near-interfacial $\mathrm{H}$ concentration after a given ${ }^{15} \mathrm{~N}$ ion fluence $\left(\Phi_{N}\right)\left[\right.$ ions $\left./ \mathrm{cm}^{2}\right]$ has impinged on the sample, $\left[H_{0}\right]$ is its initial value, $\left[H_{\infty}\right]$ is the signal increase due to $\mathrm{H}$ relocation after uptake saturation, and $\sigma_{p}$ is an effective $\mathrm{H}$ redistribution ('pile-up') cross section that characterizes the uptake rate.

Evaluating the near-interfacial $\mathrm{H}$ accumulation kinetics by fitting experimental NRA pile-up curves to relation (3.7) provides highly useful analytical information as the specific $\mathrm{H}$ uptake parameters $\left(\left[H_{0}\right],\left[H_{\infty}\right]\right.$, and $\left.\sigma_{p}\right)$ can depend rather sensitively on the analyzed material. Evidently, by performing pile-up measurements in 
different depth locations one can establish the original $\mathrm{H}$ distribution in the $\mathrm{SiO}_{2}$ layer from the obtained $\left[H_{0}\right]$ values. If the $\mathrm{H}$ uptake in the accumulation region is not limited by the supply from the H-rich 'reservoir' layer above the dielecric film, the saturation value $\left(\left[\mathrm{H}_{0}\right]+\left[H_{\infty}\right]\right)$ may be considered to reflect the density of available $\mathrm{H}$ trapping sites in the near-interfacial oxide region, which can hence be evaluated. Finally, $\sigma_{p}$, the effective kinetic rate constant of the near-interfacial $H$ pile up provides information on the mobility of hydrogen species diffusing in the MOS structure. Examples for the evaluation of the material-specific mobility of $\mathrm{H}$ impurities by means of NRA in conjunction with electrical measurements as a highly versatile analytical tool in device reliability research will be presented in Section 3.3.1.

c) Effect of $\mathrm{H}_{2} \mathrm{FGA}$

Although the $\mathrm{SiO}_{2} / \mathrm{Si}$ interface is atomically sharp, a small number of Si dangling bonds (trivalent $\mathrm{Si}$ defects backbonded to the $\mathrm{Si}$ crystal, $\mathrm{Si}_{3} \equiv \mathrm{Si} \cdot$, so-called $\mathrm{P}_{\mathrm{b}}$ centers) remains, which due to their radical character act as electrical charge traps and are thus highly undesirable for the device performance and reliability. In order to $\mathrm{H}$ passivate these interfacial $\mathrm{P}_{\mathrm{b}}$ centers the semiconductor industry deliberately introduces hydrogen into the MOS structures by subjecting the fabricated devices to a so-called forming gas annealing (FGA) process in a $\mathrm{H}_{2}$ containing atmosphere (typical are several $\% \mathrm{H}_{2}$ diluted in $\mathrm{N}_{2}$ and temperatures around $400^{\circ} \mathrm{C}$ ). This treatment is known to reduce the interface state density by $1-2$ orders of magnitude and dramatically improves the electrical device characteristics [703-705]. Electron 
spin resonance (ESR) has confirmed that $\mathrm{H}_{2}$ FGA eliminates the paramagnetic $\mathrm{P}_{\mathrm{b}}$ states according to reaction (3.8) [703, 705]:

$$
\mathrm{Si}_{3} \equiv \mathrm{Si} \cdot+\mathrm{H} \rightarrow \mathrm{Si}_{3} \equiv \mathrm{Si}-\mathrm{H} .
$$

The influence of $\mathrm{H}_{2}$ forming gas annealing on the hydrogen distribution near $\mathrm{SiO}_{2} / \mathrm{Si}$ interfaces was also studied by in $\operatorname{situ}{ }^{1} \mathrm{H}\left({ }^{15} \mathrm{~N}, \alpha \gamma\right){ }^{12} \mathrm{C}$ nuclear reaction analysis [680]. The results are summarized in Fig. 76.

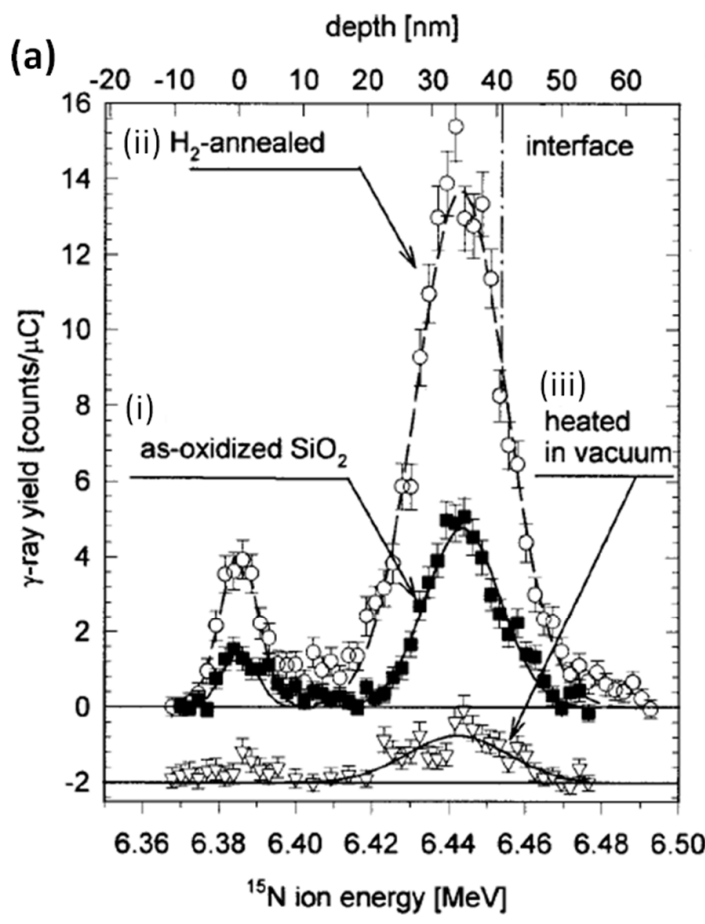

(b)

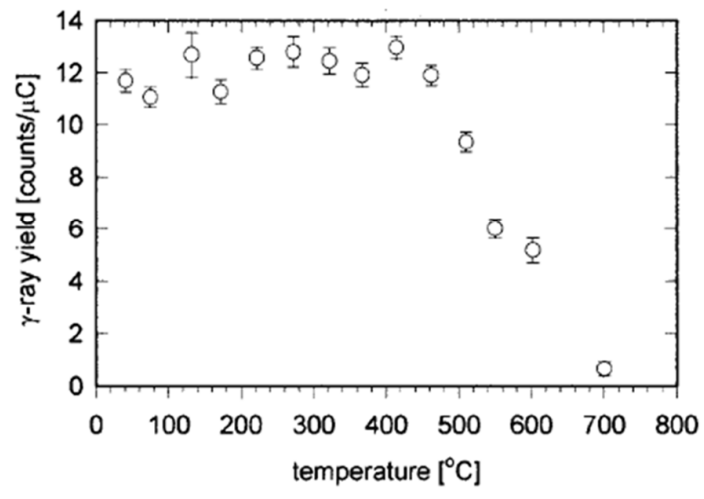

Fig. 76. (a) Effect of $\mathrm{H}_{2}$ annealing (FGA) on the $\mathrm{H}$ distribution in 41.5-nm-thick $\mathrm{SiO}_{2}$ on $\mathrm{Si}(100)$. (i) As-oxidized sample prior to FGA, (ii) after annealing in $260 \mathrm{mbar}_{2}$ at $400{ }^{\circ} \mathrm{C}$ for $40 \mathrm{~min}$, and (iii) after heating the FGA-treated sample in vacuum to $540{ }^{\circ} \mathrm{C}$. For clarity, trace (iii) is vertically shifted by an offset of -2 cts $/ \mu \mathrm{C}$. (b) Thermal stability of the $\mathrm{H}_{2}$ FGA-introduced $\mathrm{H}$ in the near-interfacial region upon heating in vacuum for $60 \mathrm{~s}$ to the given temperatures. From Ref. [680] with permission from AIP.

The $\mathrm{H}$ depth profile (ii) in Fig. 76 (a) evidences a dramatically enhanced $\mathrm{H}$ content in a $41.5 \mathrm{~nm}$ thick thermal $\mathrm{SiO}_{2}$ film on $\mathrm{Si}(100)$ after the sample was annealed inside the NRA vacuum system for $40 \mathrm{~min}$ in $260 \mathrm{mbar} \mathrm{H}_{2}$ gas at $400^{\circ} \mathrm{C}$. After this anneal the 
sample had no air contact prior to the subsequent NRA analysis. The total amount, average areal density, and volume concentration of $\mathrm{H}$ incorporated into the ca. $8 \mathrm{~nm}$ wide near-interfacial oxide region were found in the orders of $(1-3) \times 10^{14} \mathrm{~cm}^{-2}$, $>5 \times 10^{12} \mathrm{~cm}^{-2}$, and $\sim 1.2 \times 10^{20} \mathrm{~cm}^{-3}$, respectively. These figures greatly exceeded the interfacial $\mathrm{P}_{\mathrm{b}}$ center density $\left(1 \times 10^{12} \mathrm{~cm}^{-2}\right)$ and were also far larger than the native $\mathrm{H}$ concentration in the bulk $\left(1.5 \times 10^{19} \mathrm{~cm}^{-3}\right)$ and in the interfacial region of the wetgrown $\mathrm{SiO}_{2}$ dielectric.

The result was interpreted as to demonstrate that the near-interfacial oxide apparently contained a very substantial number of hydrogen trap sites that became populated during the FGA process [680]. By selectively probing the $\mathrm{H}$ content in the near-interfacial region it was verified that the in-situ FGA treatment incorporated hydrogen directly into the accumulation region near the $\mathrm{SiO}_{2} / \mathrm{Si}$ interface. Any ion beam induced $\mathrm{H}$ redistribution that would cause further $\mathrm{H}$ uptake in this region as initially observed in the as-oxidized $\mathrm{SiO}_{2}$ film sample without FGA (profile (i) in Fig. 76 (a)) did not take place. This suggested that all available trap sites in the nearinterfacial $\mathrm{SiO}_{2}$ had been $\mathrm{H}$-saturated during the FGA treatment. Fig. 76 (b) illustrates the selective NRA monitoring of the $\mathrm{H}$ content in the interfacial region of the FGAtreated sample while the specimen was heated incrementally in vacuum. This experiment established that the FGA-introduced $\mathrm{H}$ was rather stable and did not tend to desorb below $430^{\circ} \mathrm{C}$. The depth profile taken after the heating step to $540^{\circ} \mathrm{C}$ ((iii) in Fig. $76(a))$ indicated that the small quantity of remaining $\mathrm{H}$ in the interfacial region was even stronger bound than $\mathrm{H}$ on the surface of the $\mathrm{SiO}_{2}$ film, which was almost completely removed at this temperature. 


\section{d) High-k dielectrics}

The profound interest in hydrogen impurities in insulating thin films extends further to the rapidly developing field of novel gate dielectrics [706]. The aggressive down-scaling of device dimensions in Si-based MOSFET technology has spurred an evolution of alternative gate dielectric materials as a replacement for the conventional $\mathrm{SiO}_{2}$. As the gate dielectric thicknesses approached the order of $1 \mathrm{~nm}$ or less, excessive leakage current by direct electron tunneling through the $\mathrm{SiO}_{2}$ gate oxide reached untolerable levels. Novel high dielectric constant (high-k) materials were devised to overcome this limitation by allowing for physically thicker films that reduce the leakage current, while still maintaining the necessary MOSFET capacity. Thus hafnium oxide $\left(\mathrm{HfO}_{2}\right)$ [707], hafnium silicates $\left(\left(\mathrm{SiO}_{2}\right)_{x}\left(\mathrm{HfO}_{2}\right)_{(1-x)}\right)[210,708-710]$, and other high- $\mathrm{k}$ materials (such as $\mathrm{Al}_{2} \mathrm{O}_{3}$ [711]) have been proposed as alternative gate dielectrics, and their $\mathrm{H}$ content was assessed with NRA (among other techniques).

Hafnium silicate films ('HfSiO') were found to contain much higher $\mathrm{H}$ impurity levels than the bulk regions of thermally grown $\mathrm{SiO}_{2}$, exceeding the $\mathrm{H}$ concentration of the latter $\left(10^{18}-10^{19} \mathrm{~cm}^{-3}\right)[675,676,680,692]$ by more than 2 orders of magnitude $\left(10^{21}-10^{22} \mathrm{~cm}^{-3}\right)[210,709]$. Driemeier and coworkers observed with $\mathrm{D}\left({ }^{3} \mathrm{He}, \mathrm{p}\right)^{4} \mathrm{He}$ NRA that the total D content in HfSiO dielectrics on Si increased linearly with the film thickness [708], concluding that the hydrogen is preferentially built into the bulk of the HfSiO films. This finding was corroborated directly by depth-resolved $\mathrm{H}$ profiling via ${ }^{1} \mathrm{H}\left({ }^{15} \mathrm{~N}, \alpha \gamma\right){ }^{12} \mathrm{C}$ NRA [210]. The $\mathrm{H}$ distribution in $\mathrm{HfSiO}$ thus differs from that in $\mathrm{HfO}_{2} / \mathrm{Si}$ thin film structures, where $\mathrm{H}$ mainly incorporates close to the $\mathrm{HfO}_{2}$ 
surfaces $[707,708,712]$, and also contrasts $\mathrm{SiO}_{2} / \mathrm{Si}$ structures, where $\mathrm{H}$ accumulates mainly in the $\mathrm{SiO}_{2} / \mathrm{Si}$ interfacial region as described above (Fig. 73) $[677,680]$.

Based on a strongly reduced $D$ uptake during $D_{2}$ FGA after a pre-annealing treatment in $\mathrm{O}_{2}$ gas and backed by DFT calculations which demonstrated that oxygen defect sites in hafnium silicates can stabilize hydrogen, it was concluded that $\mathrm{H}$ mainly interacts with $\mathrm{O}$ deficiencies in the $\mathrm{HfSiO}$ films, which were assumed to result from the MOCVD deposition process [210, 708, 709, 712]. Presumably related to similar fabrication techniques such as reactive sputtering and CVD from $\mathrm{H}$-containing precursors, much larger $\mathrm{H}$ impurity levels than in thermal $\mathrm{SiO}_{2}$ layers have also been found in other novel dielectric films, such as CVD-silica grown from $\mathrm{SiH}_{4}$ and $\mathrm{O}_{2}$ [713]. As also revealed through NRA, the ion implantation-induced damage of amorphous $\mathrm{SiO}_{2}$ films (used, e.g., for the synthesis of $\mathrm{Si}, \mathrm{Ge}$, or $\mathrm{Sn}$ nanocrystals in an $\mathrm{SiO}_{2}$ matrix) and the subsequent interaction of the generated defects with atmospheric moisture can present a major source of hydrogen impurities in dielectric layers [714].

While the above investigations demonstrate the versatility of ${ }^{1} \mathrm{H}\left({ }^{15} \mathrm{~N}, \alpha \gamma\right)^{12} \mathrm{C}$ NRA to characterize the $\mathrm{H}$ content and distribution in novel insulating thin film materials, a clear correlation between the $\mathrm{H}$ impurity concentration and the electrical characteristics of MOSFET devices employing these high-k dielectrics has yet to be established. This appears to present a highly challenging task, given that the very large $\mathrm{H}$ concentrations found in these films outnumber the intrinsic $\mathrm{SiO}_{2} / \mathrm{Si}$ interfacial defect density by yet two additional orders of magnitude compared to devices based on traditional silicon oxide. At least for the conventional $\mathrm{SiO}_{2}$ dielectric, 
${ }^{1} \mathrm{H}\left({ }^{15} \mathrm{~N}, \alpha \gamma\right){ }^{12} \mathrm{C}$ NRA has contributed substantially to clarifying several influences that hydrogen impurities in MOS structures can take on the device reliability $[46,715$, 716], which is discussed in the following chapter (Section 3.3.1). 


\subsection{Interfacial $\mathrm{H}$}

\subsubsection{H at MOS interfaces and its relation to device reliability}

The previous section has introduced hydrogen as a common impurity in the oxide dielectrics of MOS structures. Electrical device characterization has lead to the suspicion that such residual hydrogen can critically affect the device reliability when it interacts with the $\mathrm{SiO}_{2} / \mathrm{Si}$ interface, where it appears to play an ambivalent role in the passivation and regeneration of electrically active defects [703-705, 717-721]. On one hand, hydrogen has the described beneficial effect to passivate the $\mathrm{SiO}_{2} / \mathrm{Si}$ interfacial $\mathrm{P}_{\mathrm{b}}$ centers $[694,703,705,717]$ and is thus deliberately introduced in device fabrication through FGA (reaction (3.8)). On the other hand, diffusible $\mathrm{H}$ species in the MOS structure are suspected to cause $\mathrm{SiO}_{2} / \mathrm{Si}$ interface degradation by extracting the terminating $\mathrm{H}$ atoms from the interfacial Si dangling bonds, which reactivates the charge trapping $\mathrm{P}_{b}$ centers. This notion grounds on electrical evidence for interface degradation under conditions that favor the production of diffusible atomic $\mathrm{H}$ species: heat (which reverses reaction (3.8)) [704], exposure to atomic $\mathrm{H}$ [718], $\mathrm{H}_{2} \mathrm{FGA}$ at elevated temperatures [719], as well as injection of hot carriers into the gate oxide such as those generated by ionizing radiation under bias $[698,700,701,722,723]$ or through high electric fields (> $6-20 \mathrm{MV} / \mathrm{cm}$ ) [724] that cause Fowler-Nordheim (F-N) tunneling current through the dielectric.

A second device degradation phenomenon that involves $\mathrm{P}_{\mathrm{b}}$ center depassivation under the alleged influence of diffusible 'hydrogenous' species near the $\mathrm{Si} / \mathrm{SiO}_{2}$ interface occurs under low electric fields $(<6 \mathrm{MeV} / \mathrm{cm})$ and is known as negative bias temperature instability (NBTI) $[45,720,721,724,725]$. NBTI poses a serious 
reliability concern and primarily affects p-channel MOSFETs stressed at negative gate voltages and typical temperatures of $100-250^{\circ} \mathrm{C}$. The degradation is manifested through interface trap $\left(D_{\mathrm{it}}\right)$ generation and the build up of fixed (positive) charges in the gate oxide, which reduce the transconductance and the absolute drain current and detrimentally increase the threshold voltage $\left(V_{T}\right)$. Also here the suspect that hydrogen is involved has initially been based primarily on circumstantial evidence, such as the greater NBTI susceptibility of wet as opposed to dry gate oxides (i.e., $\mathrm{SiO}_{2}$ films grown by $\mathrm{Si}$ oxidation with steam instead of $\mathrm{O}_{2}$ as oxidant) [725], or the generally enhanced NBTI affinity of silicon oxynitride $\left(\mathrm{SiN}_{x} \mathrm{O}_{y}\right)$ based devices, since nitrogen incorporation into $\mathrm{SiO}_{2}$ increases the $\mathrm{H}$ content in the dielectric [720].

The first direct experimental support for the hypothesis that the attack of the $\mathrm{H}$ passivated $\mathrm{SiO}_{2} / \mathrm{Si}$ interface by diffusible $\mathrm{H}$ species in the gate dielectric is the cause of the electrical degradation has been provided through ${ }^{15} \mathrm{~N}$ NRA investigations in conjunction with electrical device characterization, which evidenced a clear correlation between $\mathrm{H}$ impurity redistribution in MOS structures and electrical device degradation $[45,681,685,686,689,691,698,715]$.

Buchanan et al. were the first to apply ${ }^{15} \mathrm{~N}$ NRA $\mathrm{H}$ depth profiling together with electrical measurements to prove that electrical degradation upon hot-carrier injection into MOS capacitors, which generates interfacial defects, is linked to the redistribution of hydrogen impurities in the device [698]. Internal photoemission under applied electric fields between 0 and $6.5 \mathrm{MV} / \mathrm{cm}$ was used to inject hot electrons from the $\mathrm{Al}$ cathode $\left(14 \mathrm{~nm}\right.$ thick) into the $\mathrm{SiO}_{2}$ dielectric $(\sim 90 \mathrm{~nm})$ of large Al-gate capacitors. As evidenced by the NRA H depth profiles in Fig. 77 (a), a 
hydrogen-rich layer $\left(\sim 10^{15}\right.$ atoms $\left./ \mathrm{cm}^{2}\right)$ at the $\mathrm{Al} / \mathrm{SiO}_{2}$ interface was found to serve as the source of diffusive hydrogen during the hot-electron stress. For large electron fluences a small fraction of the hydrogen released from this layer was found to be retrapped near the $\mathrm{Si} / \mathrm{SiO}_{2}$ interface, indicated by the characteristic $\mathrm{H}$ accumulation peak in $\sim 1000 \AA$ depth. The buildup of hydrogen at the $\mathrm{Si} / \mathrm{SiO}_{2}$ interface exhibited an electric field threshold at $-1 \mathrm{MV} / \mathrm{cm}$, consistent with the onset of electron heating in $\mathrm{SiO}_{2}$. The electron fluence dependent $\mathrm{H}$ buildup at the $\mathrm{Si} / \mathrm{SiO}_{2}$ interface is shown in Fig. 77 (b) for both positive $\left(V_{G}{ }^{+}\right)$and negative $\left(V_{G}{ }^{-}\right)$gate voltages in dry and wet oxide dielectrics.
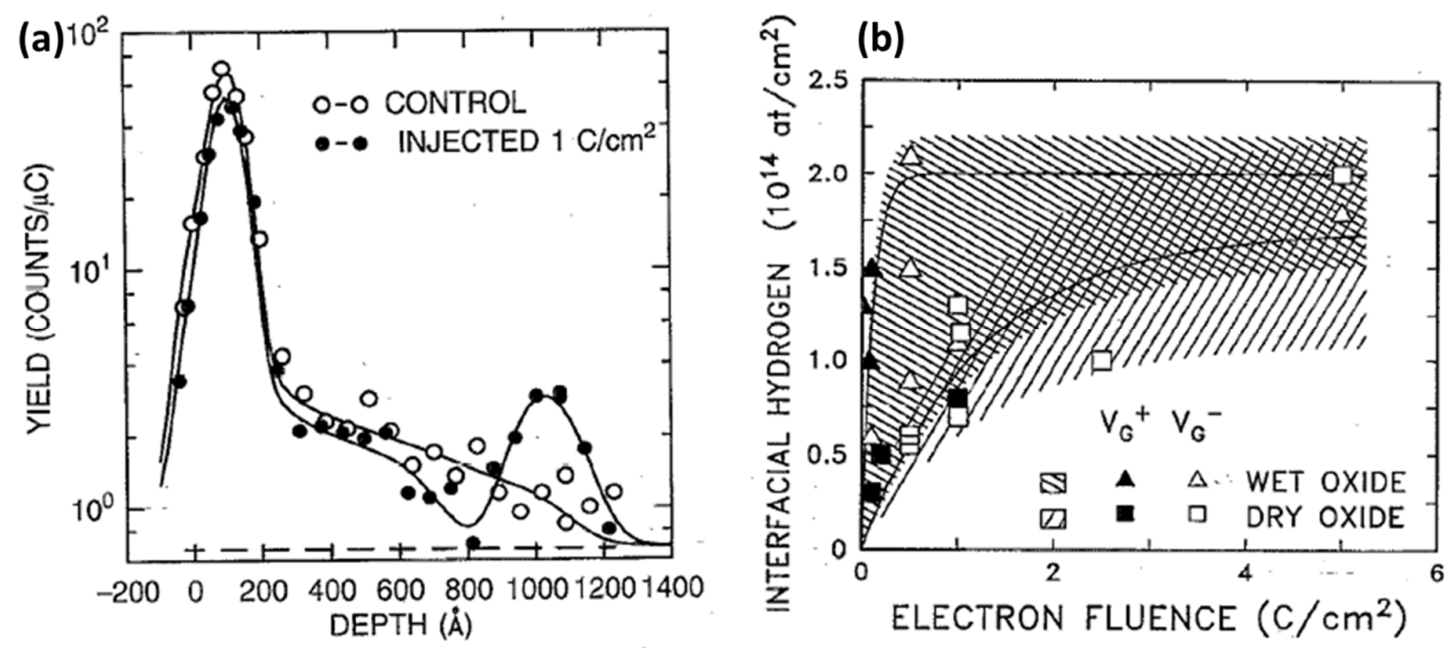

Fig. 77. (a) NRA H depth profiles of $\mathrm{Al}(140 \AA) / \mathrm{SiO}_{2}(900 \AA) / \mathrm{Si}$ MOS capacitors prior to and after injection of an electron fluence of $1 \mathrm{C} / \mathrm{cm}^{2}$ using internal photoemission from the $\mathrm{Al}$ gate at a field of $\sim-4.2 \mathrm{MV} / \mathrm{cm}$. In the injected sample note the decrease in the hydrogen amount at the $\mathrm{Al} / \mathrm{SiO}_{2}$ interface, and the appearance of a peak at a depth of $1020 \AA$, corresponding to $\mathrm{H}$ at the $\mathrm{Si} / \mathrm{SiO}_{2}$ interface. (b) Dependence of $\mathrm{Si} / \mathrm{SiO}_{2}$ interface hydrogen on the injected electron fluence at oxide fields $>3 \mathrm{MV} / \mathrm{cm}$. Data are shown for both positive $V_{\mathrm{g}}{ }^{+}$ and negative $V_{\mathrm{g}}^{-}$gate bias for both wet and dry oxides. Reproduced from Ref. [698] with permission from AIP. 
In "wet" $\mathrm{SiO}_{2}$ films with deliberately introduced excess hydrogen (through $\mathrm{H}_{2} \mathrm{O}$ exposure), the $\mathrm{H}$ buildup rate was seen to be significantly greater than that found in the "dry" (as oxidized) films. During electron injection, hydrogen redistribution was also confirmed via the deactivation of boron dopant in the silicon substrate, suggesting that the interface-attacking species was atomic $\mathrm{H}$, since molecular $\mathrm{H}_{2}$ did not show such reactive behavior [698]. The generation rates of interface states, neutral electron traps, and anomalous positive charge were found to increase with increasing $\mathrm{H}$ buildup in the substrate and with the initial $\mathrm{H}$ concentration in the film. This allowed for the conclusion that the generation of interfacial defects is preceded by the hot-electron-induced release and transport of atomic hydrogen and that the chemical reaction of this species within the metal-oxide-semiconductor structure generates electrically active defects.

Also with respect to NBTI degradation, the combination of ${ }^{15} \mathrm{~N}$ NRA and electrical characterization succeeded in confirming that the application of negative bias temperature stress (NBTS) on poly-Si/SiO $/ / \mathrm{Si}$ MOS diodes causes increased $\mathrm{H}$ densities near the $\mathrm{SiO}_{2} / \mathrm{Si}$ interface $[45,681]$. Liu et al. measured the $\mathrm{H}$-concentration in the near-interfacial region of the $25 \mathrm{~nm} \mathrm{SiO} 2$ dielectric of MOS diodes before and after application of NBTS at $-5 \mathrm{MV} / \mathrm{cm}$ and zero-bias temperature stress (ZBTS) at 0 $\mathrm{MV} / \mathrm{cm}$. Some samples received an additional POA treatment (post-oxidation anneal at $1000^{\circ} \mathrm{C}$ in an $\mathrm{N}_{2}$ ambient).

The electrical capacitance-voltage (CV) data (Fig. 78 (a)) indicated that only NBTS caused $C V$ curve distortions characterstic of interface state generation and positive oxide-fixed charge, whereas the ZBTS-treated and pristine samples exhibited ideal 
MOS capacitor CV curves, which emphasized the critical importance of bias rather than temperature for the degradation. The POA treatment obviously alleviated the detrimental NBTS effects. Fig. 78 (b) shows corresponding NRA evaluations of the $\mathrm{SiO}_{2} / \mathrm{Si}$ near-interfacial $\mathrm{H}$ content. Evidently, the near-interfacial $\mathrm{H}$ concentrations followed the same trend as the degree of stress-induced electrical degradation, which is pronounced for NBTS but insignificant after ZBTS. POA was seen to strongly suppress the local $\mathrm{H}$ accumulation under NBTS and to slightly reduce the $\mathrm{H}$ content near the $\mathrm{SiO}_{2} / \mathrm{Si}$ interface in the pristine diodes.

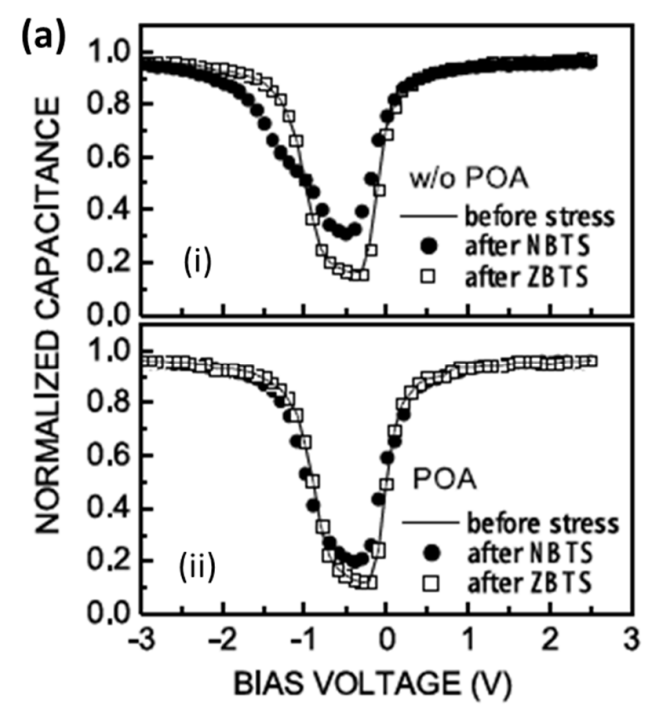

(b)

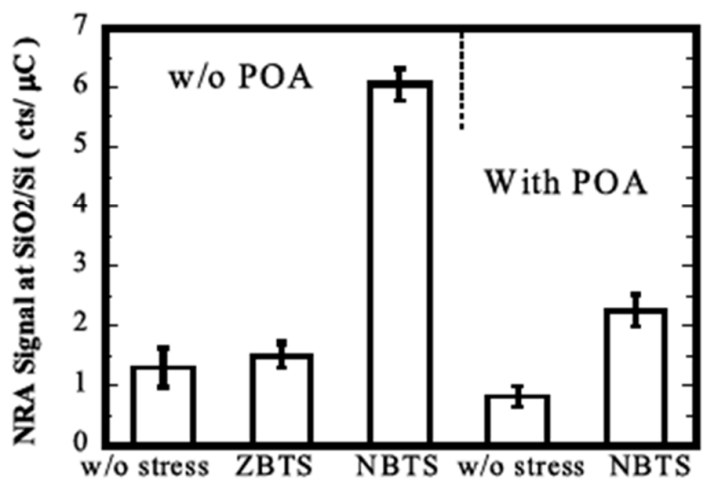

Fig. 78. (a) $\mathrm{CV}$ curves of poly-Si/SiO $/ \mathrm{S}_{2} / \mathrm{Si}$ diodes before and after application of ZBTS (0 $\mathrm{MV} / \mathrm{cm})$ and NBTS $(-5 \mathrm{MV} / \mathrm{cm})$ at $360^{\circ} \mathrm{C}$ for $6 \mathrm{~h}$ in vacuum. Panels ii) and i) refer to samples with and without a POA (post-oxidation anneal at $1000^{\circ} \mathrm{C}$ in $\mathrm{N}_{2}$ ) treatment of the oxide layer, respectively. (b) Comparison of the NRA-determined $\mathrm{H}$ content in the $\mathrm{SiO}_{2} / \mathrm{Si}$ near-interfacial region of the samples with and without POA for different stress conditions). Reproduced from Ref.[681].

The above investigation strongly corroborated the correlation between NBTI and $\mathrm{H}$ in the $\mathrm{SiO}_{2} / \mathrm{Si}$ near-interfacial region and suggested further that the enhanced stability versus NBTS of POA-treated diodes may be connected to a reduced $\mathrm{H}$ 
content in this critical region of the device structure $[45,681]$. A number of related ${ }^{15} \mathrm{~N}$ NRA H profiling studies combined with electrical characterizations performed by the authors' group clearly linked the hydrogen content and its mobility to reliability degradation in several types of MOS devices $[45,685,686,689,691,715]$. The consistently observed failure pattern was that mobile $\mathrm{H}$ species originating in upper sections of the device structures approach the $\mathrm{SiO}_{2} / \mathrm{Si}$ interface, where their accumulation may cause electrical degradation [46]. Liu et al. hence suggested that achieving control of the $\mathrm{H}$ impurity transport in MOS structures is an important key to improve the device reliability $[46,690,691]$, for which the visualization of $\mathrm{H}$ relocation processes with ${ }^{15} \mathrm{~N}$ NRA is an essential and highly effective tool.

Fig. 79 exemplifies the NRA evaluation of the $\mathrm{H}$ impurity mobility in two different $\mathrm{SiN}$ layers on $\mathrm{SiO}_{2} / \mathrm{Si}$, which model the liner material in split-gate type FLASH EEPROMs (Electrically Erasable Programmable Read-Only Memory, a type of nonvolatile memory). Their structure is illustrated in Fig. 79 (a) [686] (the NRA sample structure and a corresponding $\mathrm{H}$ depth profile are depicted in Fig. 75 (a)). Panel (i) of Fig. 79 (b) displays the ${ }^{15} \mathrm{~N}$ ion beam-induced reduction of the $\mathrm{H}$ content in the $\mathrm{H}$-rich SiN layers due to $\mathrm{H}$ relocation and desorption. The associated $\mathrm{H}$ pile-up kinetics in the $\mathrm{SiO}_{2} / \mathrm{Si}$ interfacial regions are shown in Panel (ii). Obviously both the $\mathrm{H}$ decay in the SiN layer and the near-interfacial $\mathrm{H}$ uptake were considerably faster for P-SiN (produced by plasma-deposition) than in LP-SiN (fabricated by low pressure CVD). Solid lines in Panel (b) of Fig. 79 are fits of the data to Eq. (3.7), which revealed that the effective interfacial $\mathrm{H}$ pile-up cross section $\sigma_{p}$ was 5 times larger in the oxide dielectric covered by P-SiN than in that underneath LP-SiN [686]. 
(a)

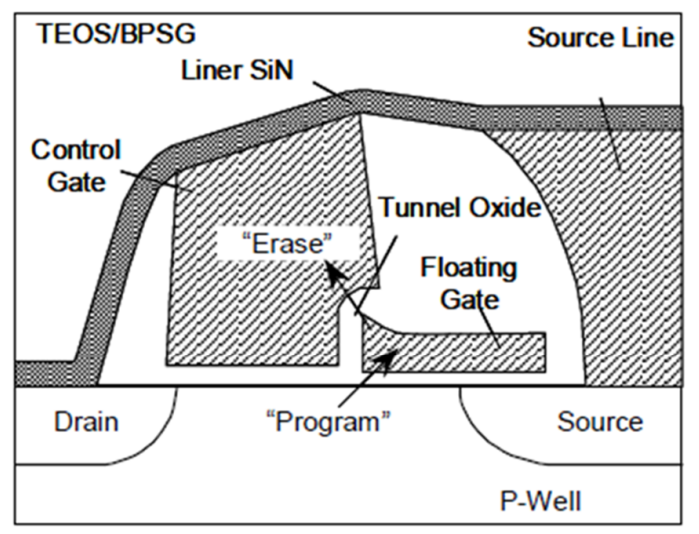

Fig. 79. (a) Schematic illustration of the split-gate type FLASH EEPROM memory cell structure. Hydrogen impurities are considered to diffuse from the BPSG region through the SiN liner film into the tunnel oxide dielectric. (b) NRA ion beam-induced H-decay in the $\mathrm{SiN}$ films (i) and concomitant $\mathrm{H}$-uptake in the $\mathrm{SiO}_{2} / \mathrm{Si}$ near-interfacial region (ii) of $\mathrm{SiN}(10 \mathrm{~nm}) / \mathrm{SiO}_{2}(25 \mathrm{~nm}) / \mathrm{Si}$ model structures for two different types of SiN. Reproduced from Ref. [686].

Since the initial $\mathrm{H}$ content in the SiN films and hence the hydrogen mobilization probability as well as the oxide below the two films were identical, the $\sigma_{p}$ values could be regarded to reflect the respective $\mathrm{H}$ permeabilities of the SiN materials. Electrical characterization revealed that the memory cells using LP-SiN liner exhibited an overall drastically enhanced performance compared to those under P-SiN liner, manifested as increased program/erase $(\mathrm{P} / \mathrm{E})$ endurance, higher resistance against $\mathrm{F}$ $\mathrm{N}$ stress, and strongly reduced interface state density generation upon charge injection. This ${ }^{15} \mathrm{~N}$ NRA study demonstrated that the P/E endurance of split-gate type FLASH memory cells is strongly influenced by the hydrogen permeability of the liner SiN, and hence suggested a possible route to improve the device reliability by 
choosing a liner material that can reduce the diffusion of $\mathrm{H}$-impurities into the tunnel dielectric [686].

The secondary electron generation in $\mathrm{SiO}_{2}$ films under ${ }^{15} \mathrm{~N}$ ion irradiation resembles the hot carrier injection conditions encountered in MOS dielectrics. The evaluation of the trend to NRA-induced redistribution of hydrogen impurities can therefore also provide valuable information on the tendency of $\mathrm{H}$ species in a given device structure to potentially relocate to the $\mathrm{SiO}_{2} / \mathrm{Si}$ interfacial region and concomitantly cause electrical degradation. This concept has been followed in a series of ${ }^{15} \mathrm{~N}$ NRA studies by Liu et al. [636, 685, 689-691, 715], which among other findings led to the discovery of an ultrathin $(<1 \mathrm{~nm})$ silicon oxynitride layer of $\mathrm{Si}_{2} \mathrm{~N}_{2} \mathrm{O}$-like stoichiometry on the surface of $\mathrm{N}_{2}$-annealed $\mathrm{SiN}$ films that can bind hydrogen very stably with respect to thermal desorption (above $1050^{\circ} \mathrm{C}$ ) and to ion beam-induced relocation [636]. Liu et al. consequently suggested incorporating an ideal $\mathrm{Si}_{2} \mathrm{~N}_{2} \mathrm{O}$-like layer (without mobile $\mathrm{H}$ ) as a hydrogen diffusion barrier in front of the $\mathrm{SiO}_{2} / \mathrm{Si}$ interface as a structural means to enhance the MOS device reliability, since the trapping of diffusing $\mathrm{H}$ species in this $\mathrm{H}$-retaining layer can be expected to protect the interface from the approach by mobile $\mathrm{H}$ species $[46,715,716]$.

\subsubsection{H at metal/silicon interfaces}

Hydrogen situated at interfaces between metals and semiconductors has been discussed as the origin of Schottky barrier height variations in metal/silicon contacts [726-729]. Mönch and coworkers observed a significant alteration of the Schottky barrier height in lead contacts fabricated by $\mathrm{Pb}$ deposition onto $\mathrm{H}$-terminated 
$\mathrm{Si}(111)(1 \times 1)$ surfaces in contrast to the $\mathrm{Pb}$ contacts formed with bare $\mathrm{Si}(111)(7 \times 7)$ surfaces. The effect was attributed to an interfacial electric dipole layer constituted by the slightly polarized covalent $\mathrm{Si}-\mathrm{H}$ bonds that were assumed to remain intact at the $\mathrm{Pb} / \mathrm{Si}$ interface $[726,728]$. Consistent with this model, the different orientation of the interfacial $\mathrm{C}-\mathrm{H}$ bond dipole was found to induce barrier height changes of the opposite sign in metal contacts on H-terminated diamond [727, 730]. On the contrary, no difference in the Schottky barrier heights resulted from the room temperature formation of $\mathrm{Ag}$ contacts on bare and $\mathrm{H}$-terminated $\mathrm{Si}(111)$ surfaces [730], which was ascribed to hydrogen loss from the $\mathrm{Si}(111)$ surface upon $\mathrm{Ag}$ deposition. Hydrogen is nonetheless known to strongly influence the morphology of $\mathrm{Ag}$ overlayers grown on this substrate [435, 731-733], and the possibility of $\mathrm{H}$ existing at $\mathrm{Ag}-\mathrm{Si}(111)$ interfaces has been discussed controversially [734-737]. Similarly, the epitaxial growth mode of Au on the H-terminated $\mathrm{Si}(111)(1 \times 1)$ surface as opposed to bare $\mathrm{Au} / \mathrm{Si}(111)(7 \times 7)$ has been subject of some debate, although both groups assumed that $\mathrm{H}$ would be desorbed upon $\mathrm{Au}$ deposition [738, 739]. These issues prompted Fukutani and coworkers to investigate the possible existence of hydrogen atoms at a variety of metal/silicon interfaces $(\mathrm{Cu} / \mathrm{Si}(111)$ and $\mathrm{Ag} / \mathrm{Si}(111)$ $[42,43], \mathrm{Pb} / \mathrm{Si}(111)[42,43,59]$, and $\mathrm{Au} / \mathrm{Si}(111)[612])$ by means of ${ }^{1} \mathrm{H}\left({ }^{15} \mathrm{~N}, \alpha \gamma\right){ }^{12} \mathrm{C}$ NRA, exploiting the non-destructive character and the high depth resolution advantage of the technique.

The first papers $[42,43]$ addressed the stability problem of interfacial hydrogen in various metal/Si systems and investigated the temperature-dependent growth mode of the metals on the $\mathrm{H}$-terminated $\mathrm{Si}(111)$ surface. Fig. 80 (a) shows NRA 
excitation curves for the H-terminated $\mathrm{Si}(111)(1 \times 1)$ surface (in-situ prepared by atomic $\mathrm{H}$ exposure of $\mathrm{Si}(111)(7 \times 7)$ at $380^{\circ} \mathrm{C}$ and desorbing excessive di- and trihydride species $\left(\mathrm{SiH}_{2}, \mathrm{SiH}_{3}\right)$ at $430^{\circ} \mathrm{C}$ in UHV [453]) before (trace (i)) and after deposition of $45 \AA \mathrm{Pb}$ at $360 \mathrm{~K}$ (trace (ii)) and at $110 \mathrm{~K}$ (trace (iii)), respectively. The latter two NRA profiles maintained the integrated $\gamma$-yield of the bare $\mathrm{H}$-terminated $\mathrm{Si}(111)(1 \times 1)$ substrate, indicating that the initial $\mathrm{H}$ coverage was fully conserved in the system after $\mathrm{Pb}$ deposition at both temperatures. Trace (iii) has the shape of a single Gaussian feature with a broadened width (FWHM $=21.3 \pm 1.2 \mathrm{keV}$ ) and its center is shifted by $10.3 \pm 0.4 \mathrm{keV}$ relative to the resonance energy. Judged from the ${ }^{15} \mathrm{~N}$ stopping power in $\mathrm{Pb}$ at $6.385 \mathrm{MeV}$ and the ion beam incidence along the surface normal, this shift corresponds to a mean $\mathrm{H}$ atom depth of $44.4 \AA$.

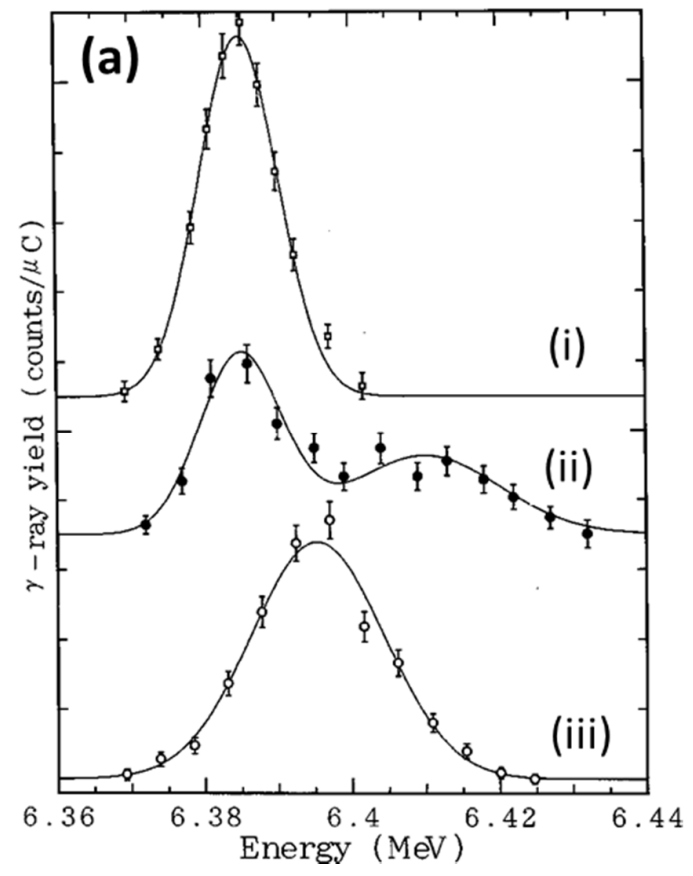

(b)
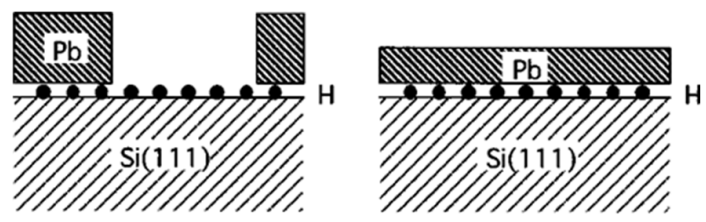

Fig. 80. (a) $\gamma$-ray yield profiles from the ${ }^{1} \mathrm{H}\left({ }^{15} \mathrm{~N}, \alpha \gamma\right){ }^{12} \mathrm{C}$ nuclear reaction as a function of the incident ${ }^{15} \mathrm{~N}$ beam energy taken after $\mathrm{Pb}$ deposition onto $\mathrm{Si}(111)(1 \times 1)-\mathrm{H}$. (i) $\mathrm{Si}(111)(1 \times 1)-\mathrm{H}$ before $\mathrm{Pb}$ deposition, (ii) $\mathrm{Pb}$ deposition of $45 \AA$ at $360 \mathrm{~K}$, and (iii) $\mathrm{Pb}$

deposition of $45 \AA$ at $110 \mathrm{~K}$. Solid curves are fits to a Gaussian form [a sum of two Gaussian forms for (ii)]. (b) Schematic figures of $\mathrm{Pb}$ growth on $\mathrm{Si}(111)(1 \times 1)-\mathrm{H}$ at substrate temperatures of $360 \mathrm{~K}$ (left panel) and $110 \mathrm{~K}$ (right panel). Black dots denote $\mathrm{H}$ atoms. Adapted from Ref. [760] with permission. 
The results are therefore fully consistent with the interpretation that at $110 \mathrm{~K} \mathrm{~Pb}$ grows in a uniform overlayer on the $\mathrm{H}$-terminated $\mathrm{Si}(111)(1 \times 1)$ substrate without intermixing with $\mathrm{Si}$ or disrupting the interfacial $\mathrm{H}$ atoms, which are considered to be confined in an atomically sharp interface layer as illustrated in the right panel of Fig. 80 (b). For Pb deposition above room temperature (360 K, trace (ii)), the NRA profile splits into two Gaussian-shaped components; a surface peak containing $0.63 \pm 0.1 \mathrm{ML}$ $\mathrm{H}$ and a broadened $(\mathrm{FWHM}=24.0 \pm 8.5 \mathrm{keV})$ and energy shifted $\left(23.5 \pm 3.5 \mathrm{keV}\right.$ vs. $\left.E_{\mathrm{R}}\right)$ peak, containing $0.41 \pm 0.17 \mathrm{ML} \mathrm{H}$. The energy shift of the latter feature corresponds to an average $\mathrm{H}$ depth of $101 \AA$, suggesting that $\sim 40 \%$ of the $\mathrm{H}$ is buried under $\sim 10$ $\mathrm{nm}$ high $\mathrm{Pb}$ islands, between which $\sim 60 \%$ of the open substrate surface is exposed to the vacuum as schematically depicted in the left panel of Fig. 80 (b).

The NRA results in Fig. 80 thus positively confirmed the conjecture from the Schottky barrier measurements $[726,728]$ on the existence of stable $H$ atoms at the $\mathrm{Pb} / \mathrm{H} / \mathrm{Si}(111)(1 \times 1)$ interface and revealed in addition that the $\mathrm{Pb}$ growth mode on $\mathrm{H}$ terminated $\mathrm{Si}(111)(1 \times 1)$ apparently changes from a layer-by-layer fashion at $110 \mathrm{~K}$ to an island forming mode at $360 \mathrm{~K}$. The latter result may be related to the unique tendency of $\mathrm{Pb}$ to form islands with flat tops, uniform height, and high aspect ratios on $\mathrm{Si}(111)$ surfaces that are driven by quantum effects in their electronic structure, although most of such observations have been made for $\mathrm{Pb}$ films grown onto bare Si(111)(7x7) substrates [740-743]. A comparison to the island structure that would be obtained on $\mathrm{H}$-terminated $\mathrm{Si}(111)(1 \times 1)$ or $\mathrm{Si}(111)(7 \times 7)$ appears to be an interesting task for the future. 
Similarly, a more recent evaluation of the current-voltage characteristics of Mg/p-Si(111)-H Schottky diodes grown at $190 \mathrm{~K}$ on H-terminated Si(111) [729] revealed that the barrier height in the as-deposited condition exceeded the value predicted from the concepts of electronegativity and metal induced gap states (MIGS) for $\mathrm{Mg}$ and $\mathrm{Si}$, by an amount $(\sim 0.36 \mathrm{eV})$ in very close agreement to the change of the homogeneous Schottky barrier height that is expected to result when a monolayer of $\mathrm{H}$ atoms is present at the interface $(0.35 \mathrm{eV})$ [728]. Annealing to room temperature reduced this initially increased barrier height by $0.15 \mathrm{eV}$ and increased the inhomogeneity of the interface, suggesting that the $\mathrm{H}$ atoms initially residing at the metal/Si interface were desorbed upon warming and that formation of a $\mathrm{Mg}_{2} \mathrm{Si}$ silicide interface layer was taking place [729]. The authors invited the verification of these hypotheses by means of NRA.

The NRA study of Fig. $80[42,43]$ clearly proved the possibility that $\mathrm{H}$ interfacial atoms can prevail at least up to $360 \mathrm{~K}$ after $\mathrm{Pb}$ depositions onto the $\mathrm{H}$-terminated $\mathrm{Si}(111)(1 \times 1)$ surface, yet the interface structure still remained obscure. In order to shed light into this matter, Fukutani and coworkers investigated in detail the initial stages of low-temperature $\mathrm{Pb}$ growth on $\mathrm{Si}(111)(1 \times 1)-\mathrm{H}$, using a strongly grazing ion incidence geometry for the detection of the interfacial hydrogen, and thereby achieved the best resolved distinction of $\mathrm{Pb}$ film thickness variations (in the submonolayer regime) that has presently been realized with ${ }^{1} \mathrm{H}\left({ }^{15} \mathrm{~N}, \alpha \gamma\right){ }^{12} \mathrm{C}$ NRA [59]. The NRA profiles shown in Fig. 81 (a) were recorded at a ${ }^{15} \mathrm{~N}$ incidence angle of $\alpha_{i}=80^{\circ}$ with respect to the surface normal. It is seen that the center positions of the resonance peaks due to the $\mathrm{Si}(111)$-terminating $\mathrm{H}$ layer shifted to higher energies 
and that the profiles broadened continuously as the amount of lead (deposited at $110 \mathrm{~K})$ was increased, whereas at all $\mathrm{Pb}$ coverages the original $\mathrm{H}$-coverage (1.01 \pm $0.07 \mathrm{ML}$ ) was fully maintained. As discussed in the previous examples, the peak center shifts reflect the energy loss of the ${ }^{15} \mathrm{~N}$ ions as they intersect the respective $\mathrm{Pb}$ overlayers before reaching the $\mathrm{H}$ buried at the $\mathrm{Pb} / \mathrm{H} / \mathrm{Si}(111)$ interface.
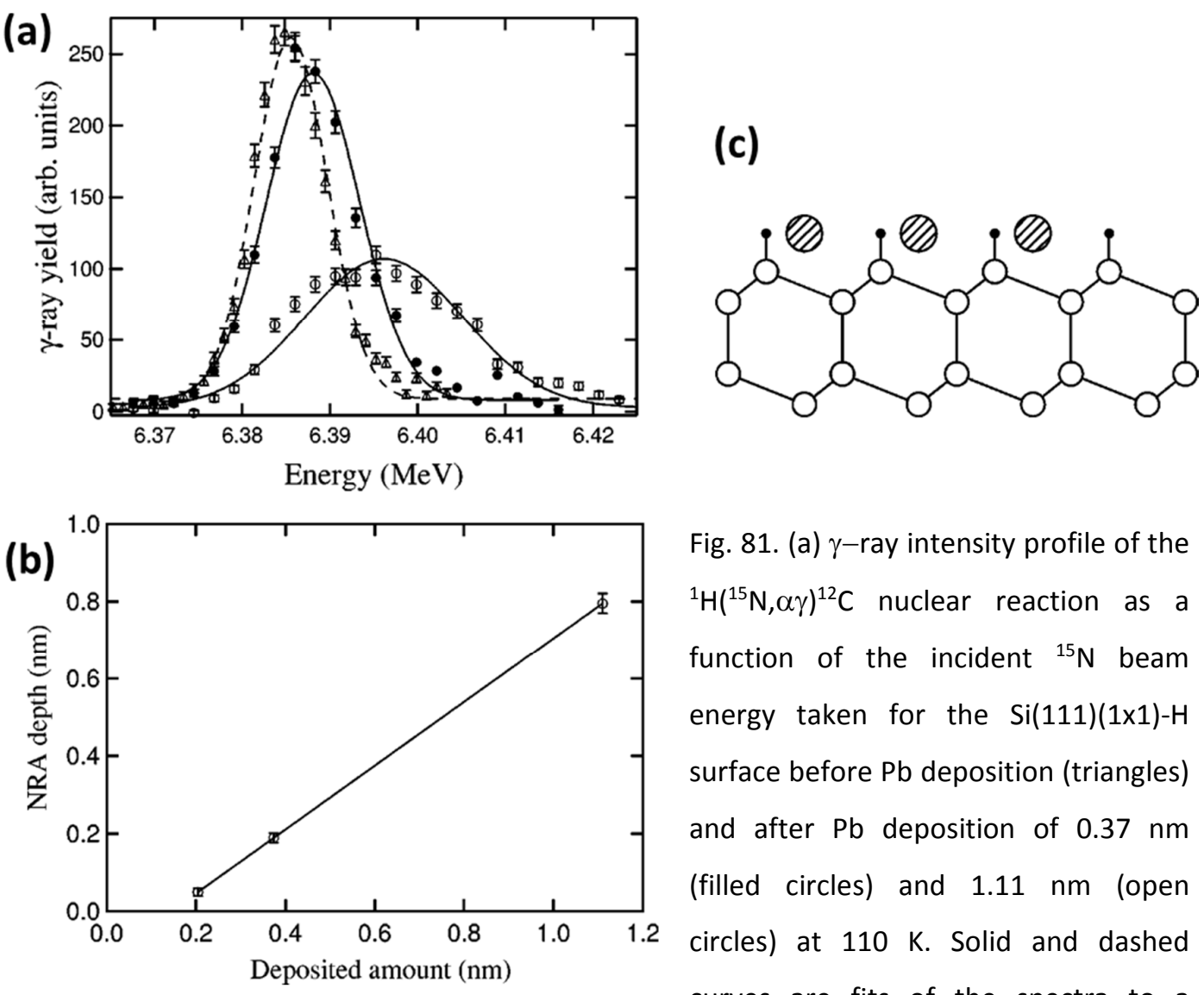

Fig. 81. (a) $\gamma$-ray intensity profile of the ${ }^{1} \mathrm{H}\left({ }^{15} \mathrm{~N}, \alpha \gamma\right){ }^{12} \mathrm{C}$ nuclear reaction as a function of the incident ${ }^{15} \mathrm{~N}$ beam energy taken for the $\mathrm{Si}(111)(1 \times 1)-\mathrm{H}$ surface before $\mathrm{Pb}$ deposition (triangles) and after $\mathrm{Pb}$ deposition of $0.37 \mathrm{~nm}$ (filled circles) and $1.11 \mathrm{~nm}$ (open circles) at 110 K. Solid and dashed curves are fits of the spectra to a

Gaussian form. (b) Relation between the $\mathrm{Pb}$ thickness estimated from the shift of the NRA spectra (including additional data for $0.2 \mathrm{~nm} \mathrm{~Pb}$ ) and the deposited amount measured by the quartz oscillator. Solid line is a fit to a linear relation. (c) Side view of the schematic model for the initial adsorption of $\mathrm{Pb}$ on $\mathrm{Si}(111)(1 \times 1)-\mathrm{H}$ at $110 \mathrm{~K}$. Filled, open, and shaded circles denote $\mathrm{H}, \mathrm{Si}$, and $\mathrm{Pb}$ atoms, respectively. Reproduced from Ref. [48] with permission from AIP. 
The linear dependence of this energy shift on the deposited $\mathrm{Pb}$ thickness is displayed in Fig. 81 (b). Clearly, even submonolayer changes of the Pb layer thickness as small as $0.1 \mathrm{~nm}$ are discernible by distinctly different energy shifts, highlighting the excellent depth resolving capability of ${ }^{1} \mathrm{H}\left({ }^{15} \mathrm{~N}, \alpha \gamma\right){ }^{12} \mathrm{C}$ NRA in grazing incidence [59]. It can further be observed in Fig. 81 (b) that the first $~ 0.1 \mathrm{~nm}$ of deposited $\mathrm{Pb}$ did not lead to any appreciable shift of the NRA profile, which the authors tentatively attributed to an interface structure in which the $\mathrm{Pb}$ atoms partially reside at a similar height within the plane of the $\mathrm{H}$-adlayer on the Si substrate, so that the first $\sim 0.5 \mathrm{ML}$ of $\mathrm{Pb}$ atoms have a reduced shading effect on the ${ }^{15} \mathrm{~N}$ ion beam at the grazing incidence. The suggested interfacial model structure is schematically illustrated in Fig. 81 (c). Fukutani et al. demonstrated further that when $\mathrm{H}$ exists only at the metal overlayer/Si interface or on openly exposed $\mathrm{H}$-terminated Si-substrate surface, the energy shift $\left(\Delta E_{c}\right)$ of the experimental NRA profile center will always be strictly proportional to the average thickness $(h)$ of the deposited $\mathrm{Pb}$ layer (i.e., $\Delta E_{\mathrm{c}}=$ $S \cdot h / \cos \alpha_{i}$, even if the film grows in form of three-dimensional islands (S: stopping power) [59].

As a final result, the spectral width of the NRA profiles for the Pb-covered samples (circles in Fig. 81 (a)) was observed to consistently exceed the broadening obtained from convolving the profile of the $\mathrm{H}$-terminated $\mathrm{Si}(111)$ surface before $\mathrm{Pb}$ deposition (triangles in Fig. 81 (a)), which contained all experimental energy broadenings, i.e., resonance width, beam spread, Doppler broadening, and any possible roughness of the $\mathrm{Si}(111)$ surface) with an additional Gaussian component to reflect the ${ }^{15} \mathrm{~N}$ ion straggling in the $\mathrm{Pb}$ overlayers, when this latter component was 
based on an estimation of the straggling cross section derived by Rud et al. [106]. The deviation increased with the $\mathrm{Pb}$ thickness and could thus neither be accounted for by assuming morphological roughness of the $\mathrm{Pb} / \mathrm{Si}$ interface (intermixing of $\mathrm{Pb}$ and $\mathrm{Si}$ was further excluded by the low experimental temperature) nor height variations of the overlayer (ascribing the experimental spectral widths to the latter would have required the unreasonable assumption of surface roughness in excess of the actual Pb film thickness). As a byproduct, this high-resolution study thus pointed out the possibility that the Rud theory might underestimate the straggling cross section of 6.4 MeV ${ }^{15} \mathrm{~N}$ in $\mathrm{Pb}$ [59] (cf. Section 2.1.4).

It should be noted that the extremely acute sensitivity to overlayer thickness variations in the work above became possible by virtue of three combined favorable properties of the particular $\mathrm{Pb} / \mathrm{H} / \mathrm{Si}(111)$ system: Apparently $\mathrm{H}$ is much more stable at the $\mathrm{Pb} / \mathrm{Si}(111)$ interface rather than (1) either on the surface of the top $\mathrm{Pb}$ layer or (2) in the volume of the $\mathrm{Pb}$ film, and (3) the $\mathrm{Pb}-\mathrm{Si}$ interaction is significantly weaker than the $\mathrm{Si}-\mathrm{H}$ bond. These conditions ensured that the original density and location of the Si-terminating $\mathrm{H}$-adlayer is preserved during the overgrowth by the $\mathrm{Pb}$ film and that assuming the $\mathrm{H}$ distribution to remain confined to a single layer at an atomically sharp $\mathrm{Pb} / \mathrm{H} / \mathrm{Si}(111)$ interface was justified. The position shifts of the NRA profile centers could thus be determined with sufficient numerical accuracy and be interpreted as the effective $\mathrm{Pb}$ overlayer thickness retarding the ${ }^{15} \mathrm{~N}$ ion beam. Any possible solution of $\mathrm{H}$ in the metal film or in potentially forming metal-silicide compounds, any degradation of the abrupt interface structure by reactive metalsilicon interdiffusion, or $\mathrm{H}$ adsorption on the surface of the growing overlayer that 
may occur in other metal/Si systems would seriously hamper a straightforward analysis such as the one above. An example for such a more complicated (yet far more common) situation is presented by the NRA profiles measured in normal incidence at a thin (35 $\AA$ ) amorphous $\mathrm{Si}$ film deposited onto $\mathrm{H}$-terminated $\mathrm{Si}(001)$ [203]. Here, the energy-shifted signal component of the interfacial hydrogen could not be resolved from the simultaneously present signal of $\mathrm{H}$ adsorbed on the surface of the $a$-Si layer (and from possibly remaining $\mathrm{H}$ traces within the $a$-Si film), and the experimental energy shift of the unresolved profile amounted to only about one half of the value expected from the $a$-Si film thickess for the case that $\mathrm{H}$ existed only at the $a-\mathrm{Si} / \mathrm{H}: \mathrm{Si}(001)$ interface [203].

Addressing now the $\mathrm{Ag} / \mathrm{Si}(111)(1 \times 1)-\mathrm{H}$ system, Fig. 82 (a) shows NRA excitation profiles for H-terminated $\mathrm{Si}(111)(1 \times 1)$ surfaces covered by Ag deposits of $39 \AA$ (trace (i)) and $70 \AA$ (trace (ii)) prepared at $360 \mathrm{~K}$ [43]. Compared to the original resonance profile of the $\mathrm{H}$-terminated substrate (dashed curve), the $\mathrm{H}$ coverage was obviously strongly reduced, i.e. to $0.25 \pm 0.03 \mathrm{ML}$ and $0.15 \pm 0.02 \mathrm{ML}$ for the 39 and $70 \AA$ thick $\mathrm{Ag}$ films, respectively. As $\mathrm{H}$ desorbs from $\mathrm{Ag}(111)$ surfaces at $190 \mathrm{~K}$ [270], the remaining $\mathrm{H}$ was concluded to be present on uncovered patches of the Si substrate between $\mathrm{Ag}$ islands rather than on the surface of these islands. This situation is modeled in the left panel of Fig. 82 (c). The inferred islanding growth mode of $\mathrm{Ag}$ on $\mathrm{H}$-terminated $\operatorname{Si}(111)(1 \times 1)$ and the estimated island height were in good agreement with results obtained with various surface imaging techniques [435, 731-733]. To explain the remaining $\mathrm{H}$ on the open $\mathrm{Si}$ substrate surface between the $\mathrm{Ag}$ islands Fukutani et al. conjectured that isolated $\mathrm{Ag}$ atoms diffused over the $\mathrm{H}$-termination layer without 
disrupting it, causing $\mathrm{H}$ detachment from the interface only upon aggregation into $\mathrm{Ag}$ islands. Exactly this scenario has recently been confirmed by first-principles calculations [734, 735] and synchrotron based photoemission spectroscopy that evidenced the formation of direct Ag-Si chemical interactions and a concomitant decrease of a Si-H bond related spectral feature [736]. In line with the unaltered Schottky barrier height of $\mathrm{Ag} / \mathrm{Si}$ contacts on bare and $\mathrm{H}$-terminated $\mathrm{Si}(111)$ surfaces [730], the above results thus consistently support the hypothesis that the preadsorbed Si-terminating $\mathrm{H}$ atoms are replaced by deposited $\mathrm{Ag}$ at room temperature.
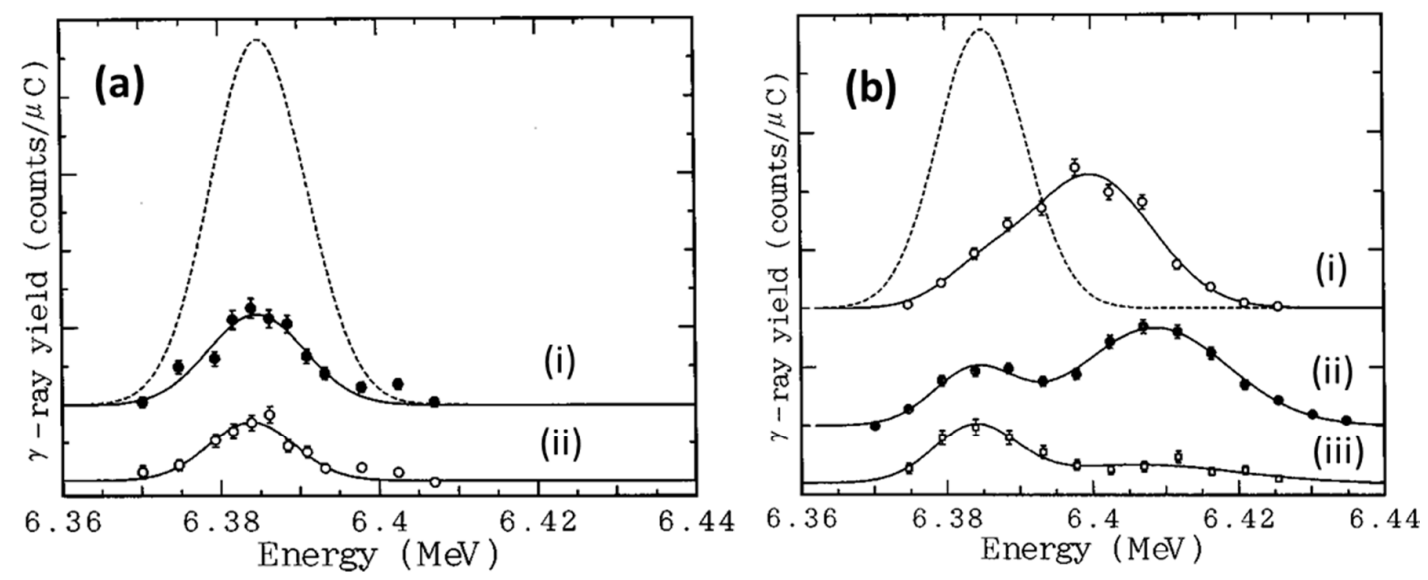

(c)
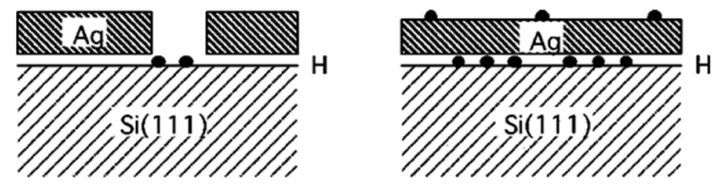

Fig. 82. (a) $\gamma$-ray yield profiles from the ${ }^{1} \mathrm{H}\left({ }^{15} \mathrm{~N}, \alpha \gamma\right){ }^{12} \mathrm{C}$ nuclear reaction as a function of the incident ${ }^{15} \mathrm{~N}$ beam energy taken for Ag deposition onto $\mathrm{Si}(111)(1 \times 1)-\mathrm{H}$ with coverages of (i) $39 \AA$ and (ii) $70 \AA$ at a sample temperature of $360 \mathrm{~K}$. Solid curves are fits to a Gaussian form and the broken curve denotes the profile for $\mathrm{Si}(111)(1 \times 1)-\mathrm{H}$. (b) $\gamma$-ray yield profiles from the ${ }^{1} \mathrm{H}\left({ }^{15} \mathrm{~N}, \alpha \gamma\right)^{12} \mathrm{C}$ nuclear reaction as a function of the incident ${ }^{15} \mathrm{~N}$ beam energy taken for $\mathrm{Ag}$ deposition onto $\mathrm{Si}(111)(1 \times 1)-H$ with coverages of (i) $33 \AA$ and (ii) $67 \AA$ at a sample temperature of $110 \mathrm{~K}$. (iii) Intensity profile taken after annealing the sample corresponding to (ii) at $300 \mathrm{~K}$. Solid curves are fits to a sum of two Gaussian forms and the broken curve denotes the profile for 
$\mathrm{Si}(111)(1 \times 1)-\mathrm{H}$. (c) Schematic figures of Ag growth on $\mathrm{Si}(111)(1 \times 1)-\mathrm{H}$ at temperatures of 360 $\mathrm{K}$ (left) and $110 \mathrm{~K}$ (right). Black dots denote $\mathrm{H}$ atoms. Adopted from Ref. [760].

Analog NRA measurements after Ag deposition onto $\mathrm{Si}(111)(1 \times 1)-\mathrm{H}$ at $110 \mathrm{~K}$ demonstrated that this behavior changes at low temperature [43]. As seen in Fig. 82 (b), the single surface peak in the NRA profile of the H-terminated Si(111) substrate decreased strongly as before, but at the same time a second component emerged that intensified and separated farther from the surface $\mathrm{H}$ signal as the $\mathrm{Ag}$ coverage was increased from $33 \AA$ (profile (i)) to $67 \AA$ (profile (ii)), clearly suggesting the presence of $\mathrm{H}$ at the $\mathrm{Ag} / \mathrm{Si}$ interface. The intitial $\mathrm{H}$ coverage was almost completely preserved in this condition. The center positions of the interface components coincided quite precisely with the respective deposited Ag thicknesses, suggesting that closed Ag films had formed at $\mathrm{T}=110 \mathrm{~K}$, with some $\mathrm{H}$ (ca. $0.25 \mathrm{ML}$ ) residing on their surface, as illustrated on the right hand side of Fig. 82 (c). The NRA profile (iii) measured after warming to $300 \mathrm{~K}$ indicated by the almost complete loss of the higher energy signal component that the interfacial $\mathrm{H}$ was only stable at low temperatures, whereas the surface- $\mathrm{H}$ appeared to remain. Although no definite conclusion could be reached on which surface the $\mathrm{H}$ actually resided, it was suggested that the originally closed Ag film had transformed into an island structure upon annealing, which partially re-exposed the still H-covered Si surface, whereas $\mathrm{H}$ was expected to desorb from the Ag surface at $300 \mathrm{~K}$ [43].

Qualitatively similar results as for $\mathrm{Ag} / \mathrm{Si}(111)(1 \times 1)-\mathrm{H}$ were obtained also for $\mathrm{Cu}$ deposition onto $\mathrm{Si}(111)(1 \times 1)-\mathrm{H}$ [43]. $\mathrm{Cu}$ deposition at $360 \mathrm{~K}$ resulted in a very substantial reduction of the $\mathrm{H}$ coverage without any evidence for a buried $\mathrm{H}$ 
component (only $0.13 \mathrm{ML}$ remained for either $19 \AA$ or $31 \AA \mathrm{Cu}$ ), whereas 0.19 $\mathrm{ML} \pm 0.04$ of interfacial $\mathrm{H}$ could be observed at $110 \mathrm{~K}$ underneath an apparently closed Cu film of $60 \AA$ in addition to $\sim 0.29 \pm 0.04 \mathrm{ML}$ of surface hydrogen. Nonetheless, about one half of the orginal $\mathrm{H}$-coverage was found to be desorbed after $\mathrm{Cu}$ deposition even at $110 \mathrm{~K}$. In summarizing the chemical trend of the $\mathrm{H}$ atom stability at metal/Si(111)(1x1) surfaces revealed in this NRA study, Fukutani et al. noted that the $\mathrm{Pb} / \mathrm{Si}(111)$ interface apparently provides a stable environment for interfacial hydrogen even at room temperature, whereas $\mathrm{Ag}$ and $\mathrm{Cu}$ interact increasingly strongly with the Si substrate, expelling $\mathrm{H}$ from the metal/Si bonds at room temperature and only weakly stabilizing them at $110 \mathrm{~K}$.

Even stronger interfacial metal/Si interactions occur during the growth of Au on Si(111) surfaces. At room temperature, Au-silicide surface layers are produced by the out-diffusion of Si atoms onto the surface of the Au film, when the Au film thickness exceeds 0.5 ML $[744,745]$. This reactive Si segregation is substantially suppressed at low temperatures (173 K), where closed Au films grow on both $\mathrm{Si}(111)(7 \times 7)$ and $\mathrm{Si}(100)$ [746]. H-termination of $\mathrm{Si}(111)$ has been proposed to change the room temperature growth of $\mathrm{Au}$ from a layer-by-layer fashion on bare $\mathrm{Si}(111)(7 \times 7)$ to island formation on $\mathrm{H}$-terminated $\mathrm{Si}(111)(1 \times 1)$. In contrast, even at thicknesses as low as $2 \AA$ closed Au films were observed by STM on Si(111)(1x1)-H [747]. On the basis of the known reactive $\mathrm{Au} / \mathrm{Si}$ interaction, both studies assumed but could not prove with the available methods that desorption of $\mathrm{H}$ occurred upon $\mathrm{Au}$ deposition. On the other hand, other authors claimed the possibility of $\mathrm{H}$ prevalence at $\mathrm{Au} / \mathrm{Si}$ 
interfaces such as those formed on amorphous $\mathrm{Si}$ [748] or on H-terminated $\mathrm{Si}(100)$ [738].

Wilde and Fukutani therefore addressed the controversial hydrogen stability and the growth mode during the low-temperature $\mathrm{Au} / \mathrm{Si}$ interface formation on $\mathrm{H}$ terminated $\mathrm{Si}(111)(1 \mathrm{x} 1)$ by means of high-resolution ${ }^{1} \mathrm{H}\left({ }^{15} \mathrm{~N}, \alpha \gamma\right){ }^{12} \mathrm{C}$ NRA [612]. Fig. 83 displays the NRA resonance excitation curve measured in strongly grazing ion incidence $\left(\alpha_{i}=80^{\circ}\right)$ at the $\mathrm{H}$-terminated $\mathrm{Si}(111)(1 \times 1)$ surface before and after the deposition of $4.1 \AA \mathrm{Au}$ at a low temperature of $110 \mathrm{~K}$, where the reactive Au-Si segregation could safely be ruled out.

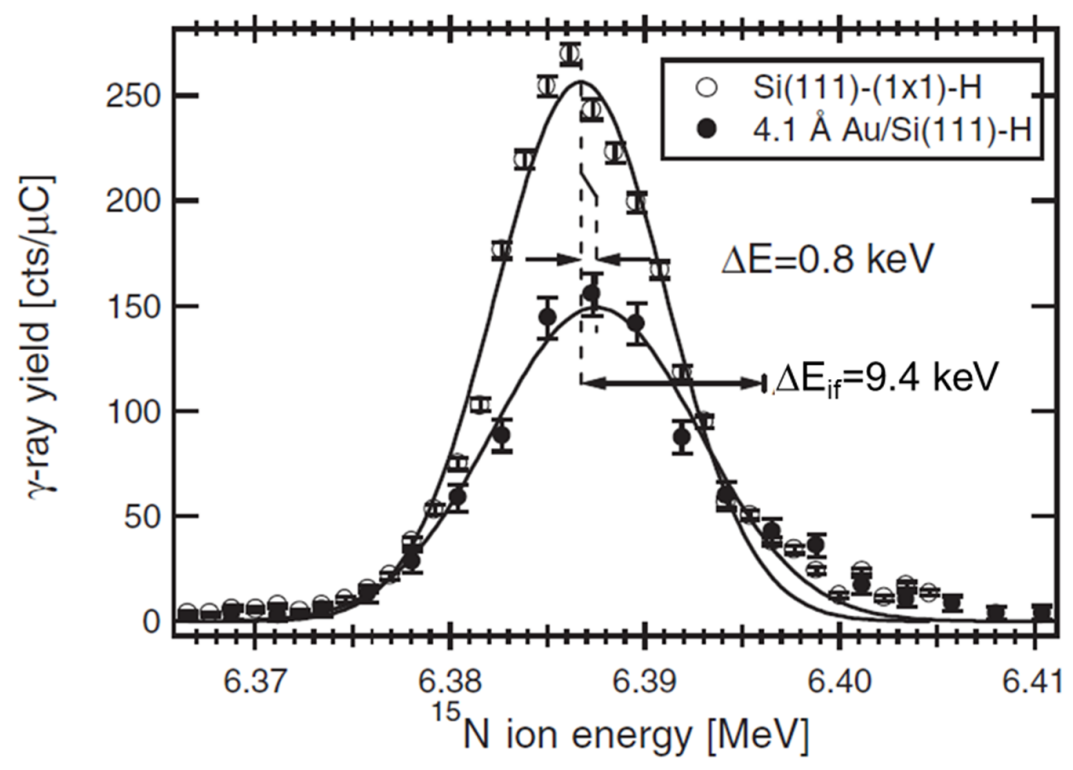

Fig. 83. Grazing-incidence ${ }^{15} \mathrm{~N}$ NRA profiles of the $\mathrm{Si}(111)(1 \times 1)-\mathrm{H}$ surface before and after deposition of $4.1 \AA \mathrm{Au}$ at $110 \mathrm{~K}$. The energy shift $\Delta E_{\mathrm{if}}=9.4 \mathrm{keV}$ expected for $\mathrm{H}$ located the $\mathrm{Au} / \mathrm{Si}$ interface is indicated. Reproduced from Ref. [612].

The reduced integral $\gamma$-yield of the post-deposition NRA profile indicated that evidently a fraction of the H-termination layer was lost upon arrival of the Au atoms, yet $\sim 70 \%$ of the original $\mathrm{H}$ coverage was still preserved. Relative to the excitation 
curve of the bare $\mathrm{H}$-terminated $\mathrm{Si}(111)$-substrate the peak maximum of the postdeposition NRA profile was shifted by $(\Delta E=0.8 \pm 0.3) \mathrm{keV}$ to higher energy and the spectral width had increased from $(10.3 \pm 0.3) \mathrm{keV}$ to $(12.5 \pm 0.6) \mathrm{keV}$ due to energy loss and straggling of the ${ }^{15} \mathrm{~N}$ ions transecting the $\mathrm{Au}$ deposit before their reaction with $\mathrm{H}$ atoms. This energy shift was substantially smaller than the energy loss expected for the hypothetical situation that the remaining $\mathrm{H}$ was located in a single atomic layer at the Au/Si interface, where it would appear shaded by the $\mathrm{Au}$ overlayer (thickness $h=4.1 \AA$ ) by an effective ${ }^{15} \mathrm{~N}$ ion trajectory length $z=h / \cos \alpha_{i}=$ $2.41 \mathrm{~nm}$ and should thus give rise to an energy shift of $\Delta E_{\text {if }}=S \cdot h / \cos \alpha_{i}=9.4 \mathrm{keV}$ (cf. Fig. 2). Dominant hydrogen prevalence at the $\mathrm{Au} / \mathrm{Si}$ interface after Au deposition onto H-terminated $\mathrm{Si}(111)(1 \times 1)$ could therefore conclusively be ruled out even for the low deposition temperature of $110 \mathrm{~K}$ [612].

In order to further elucidate the low-temperature growth mode of $\mathrm{Au}$ on $\mathrm{H}$ terminated $\mathrm{Si}(111)(1 \times 1)$, the authors performed simulations of the NR resonance excitation curve for all conceivable hydrogen depth distributions and Au overlayer morphologies that were compatible with the deposited Au film thickness and the observed $\mathrm{H}$ coverage, and compared the resulting energy shift in the simulated NRA profile with the experimentally observed value [612]. For $\mathrm{H}$ on top of a layer-by-layer growing film (Frank-van-der-Merwe growth mode: $4.1 \AA \mathrm{Au}$ (1.78ML in the lattice spacing of $\mathrm{Au}(111))$ correspond to a closed $\mathrm{Au}$ monolayer with flat terraces of monoatomic step height nucleating in the second layer) resulted in an energy shift (0.4 keV) even smaller than the experimental one $(0.8 \mathrm{keV})$. As such a surface morphology thus apparently did not offer sufficient surface roughness to account for 
the experimental energy shift, a layer-by-layer growth mode of Au on $\mathrm{H}$-terminated $\mathrm{Si}(111)(1 \times 1)$ at $110 \mathrm{~K}$ could be excluded. On the other hand, Stranski-Krastanov-type (SK) nucleation of $\sim 6 \mathrm{~nm}$ small 3-dimensional islands on top of a H-covered closed Au wetting layer appeared equally compatible with the measured NRA profile as did Vollmer-Weber (VW) growth of 5-8 layers high and 7-15 nm wide Au islands with undisrupted $\mathrm{H}$-terminated $\mathrm{Si}(111)(1 \times 1)$ substrate patches between them.

The information on the $\mathrm{H}$ location in addition to the total coverage gained from grazing incidence NRA further allowed the authors to discuss interesting aspects of the $\mathrm{H}$ behavior during the Au film growth in both of the two conceivable epitaxial scenarios. In case of SK growth, $\mathrm{H}$ remaining on the Au wetting layer would imply a stabilization of $\mathrm{H}$ atoms on a Au surface at $110 \mathrm{~K}$, which is not observed for $\mathrm{Au}(111)$ single crystals. The situation of $\mathrm{Au} / \mathrm{Si}(111)$ might thus resemble the peculiar hydrogen adsorption properties of thin Au films such as those on $\operatorname{Ir}(111)$ substrates $[274,407,408]$ (cf. Section 3.1.2). On the other hand, if VW island growth left the Hpassivation layer on the Si surface between the 3-dimensional islands intact, the $\mathrm{Au}$ atoms impinging on the open terraces should be able to diffuse over the terminating $\mathrm{H}$ layer without disrupting the $\mathrm{Si}-\mathrm{H}$ bonds, whereas $\mathrm{H}$ would be desorbed upon merging of the Au adatoms into islands [612].

Exactly this latter interpretation of the above NRA measurements has recently received support from first principles calculations of Au atom diffusion dynamics on H-terminated $\mathrm{Si}(111)(1 \times 1)$ surfaces. Lee and Hwang found that at moderate temperatures far below the onset of $\mathrm{H}$ desorption from $\mathrm{Si}\left(450^{\circ} \mathrm{C}\right)$, isolated $\mathrm{Au}$ atoms can diffuse on the H-terminated Si surface by reversibly inserting into and emerging 
from the $\mathrm{Si}-\mathrm{H}$ bonds without disrupting the latter, forming intermediate linear $\mathrm{Si}-\mathrm{Au}-$ $\mathrm{H}$ units [749]. The overall activation energy for this diffusion-reaction pathway was predicted to be $0.5 \mathrm{eV}$, implying that the Au adatom mobility is much smaller than, e.g., that of $\mathrm{Ag}$ adatoms $(0.14 \mathrm{eV})[734,735]$ on $\mathrm{Si}(111)(1 \times 1)-\mathrm{H}$, which Lee and Hwang presume to diffuse above the $\mathrm{H}$-termination layer without insertion into the $\mathrm{Si}-\mathrm{H}$ bonds [749]. Similar to the case of $\mathrm{Ag} / \mathrm{Si}(111)$ [735], their calculations also predicted the liberation of $\mathrm{H}$ atoms from the $\mathrm{Au} / \mathrm{Si}(111)$ interface upon agglomeration of the Au atoms into clusters [749]. In agreement with the above NRA results [612], the $\mathrm{H}$ atoms were shown to be energetically more stable on the Au cluster surface rather than at the $\mathrm{Au} / \mathrm{Si}$ interface. The $\mathrm{Au}$-surface $\mathrm{H}$ bonding interaction varies as a function of the Au cluster size and $\mathrm{H}$ coverage but is generally very weak, so that the $\mathrm{H}$ atoms easily recombine and desorb without a significant activation barrier [749], which is consistent with the NRA-observed substantial $\mathrm{H}$ loss caused by Au island formation on $\mathrm{Si}(111)(1 \times 1)-\mathrm{H}$ even at $110 \mathrm{~K}[612]$.

\subsubsection{H at metal-insulator interfaces:}

$\mathrm{Cr}_{2} \mathrm{O}_{3} /(\mathrm{Cr} 110)$

The investigations reviewed so far have already showcased the versatility of ${ }^{15} \mathrm{~N}$ NRA to observe hydrogen at buried interfaces. For hydrogen trapped at metal/silicon interfaces, the interpretation of the NRA profiles has greatly been aided by the fact that the $\mathrm{H}$ solubility in the $\mathrm{Si}$ substrate as well as in the metals selected for deposition was extremely small, i.e., well below the NRA detection limit. Thus, only $\mathrm{H}$ at the metal/Si interface and on the sample surface had to be considered. In the 
case of metal/oxide interfaces this situation can be more complicated. As shown in Section 3.2, certain metals are prone to dissolve $\mathrm{H}$ in the bulk and many metal oxides can contain sizeable proton concentrations. In principle, hydrogen may thus be located directly at as well as in the material volume on either side of the interface. The following ${ }^{15} \mathrm{~N}$ NRA studies of $\mathrm{H}$ distributions near metal/oxide interfaces will exemplify that the actual $\mathrm{H}$ behavior is not always easily anticipated, and that one may encounter actually $\mathrm{H}$-free interfaces, $\mathrm{H}$-trapping interfaces, as well as interfaces where $\mathrm{H}$ accumulates on either the oxide or on the metal side. Among the phenomena that make $\mathrm{H}$ localization at metal/oxide interfaces potentially interesting we mention the possibility that interfacial hydrogen may destabilize the metal/oxide adherence and thus lead to loss of surface protecting films, or the role that hydroxyl impurities appear to play in reducing the strength of passivating oxide films to resist metal corrosion [750, 751].

Hjörvarsson et al. investigated the possibility of $\mathrm{H}$ trapping at a structurally highly-defined metal/insulator interface between a layer of $\mathrm{Cr}$ metal of excellent crystalline quality and its oxide, $\alpha-\mathrm{Cr}_{2} \mathrm{O}_{3}$, grown epitaxially by in-situ oxidation under control with X-ray reflectivity measurements [752]. This $\alpha-\mathrm{Cr}_{2} \mathrm{O}_{3}(0001) / \mathrm{Cr}(110)$ interface was almost atomically flat; its roughness (rms) did not exceed $0.2-0.3 \mathrm{~nm}$. A single-crystal like $\mathrm{Nb}$ film on a sapphire $\left(\mathrm{Al}_{2} \mathrm{O}_{3}\right)$ substrate was chosen to serve simultaneously as a high quality epitaxial buffer layer for the $\operatorname{Cr}(110)$ film growth and as an indicator for the successful $\mathrm{H}$ uptake by the system upon later hydrogenation. The $\alpha-\mathrm{Cr}_{2} \mathrm{O}_{3}(0001)$ layer was further protected from hydroxylation in ambient atmosphere through an additional thin $\mathrm{Cr}$ overlayer, which was allowed to oxidize 
naturally as to provide a passivating surface film. Thickness and crystalline quality of all layers were carefully characterized with RBS and X-ray reflectivity measurements. The hydrogen affinity at the lower $\alpha-\mathrm{Cr}_{2} \mathrm{O}_{3} / \mathrm{Cr}(110)$ metal/oxide interface in the resulting $\mathrm{Cr}_{2} \mathrm{O}_{3}(0001) / \mathrm{Cr}(110) / \mathrm{Cr}_{2} \mathrm{O}_{3}(0001) / \mathrm{Cr}(110) / \mathrm{Nb}(110) / \mathrm{Al}_{2} \mathrm{O}_{3}(11 \overline{2} 0)$ multilayer structure was finally evaluated by measuring the $\mathrm{H}$ distribution with ${ }^{15} \mathrm{~N}$ NRA after hydrogenation under $10^{5} \mathrm{~Pa}$ of purified $\mathrm{H}_{2}$ at $573 \mathrm{~K}$ [752].

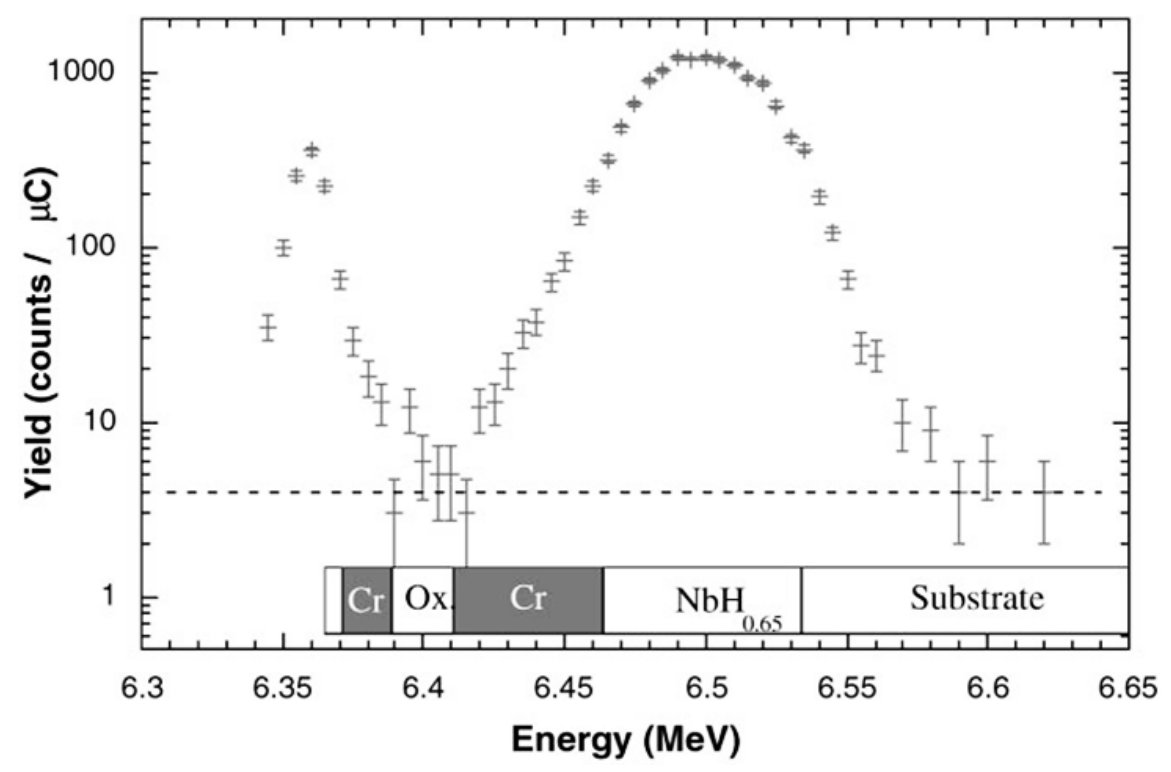

Fig. 84. Normalized $\gamma$-yield from the ${ }^{1} \mathrm{H}\left({ }^{15} \mathrm{~N}, \alpha \gamma\right){ }^{12} \mathrm{C}$ reaction as a function of the incident ${ }^{15} \mathrm{~N}$ ion energy. The hydrogen concentration is proportional to the normalized yield, and corresponds to a $\mathrm{H} / \mathrm{Nb}$ atomic ratio of $0.65 \pm 0.03$. A schematic illustration of the sample structure is included for clarity. The dotted horizontal line is the natural background, giving the definition of zero concentration in the measurements. Ox denotes the chromium oxide region. Reproduced with permission from Ref. [752].

The obtained $\mathrm{H}$ depth profile is shown together with the schematic sample structure in Fig. 84. The large and confirmedly saturated $\mathrm{H}$ content in the $\mathrm{Nb}$ film corresponded to the atomic ratio of $x=0.65 \pm 0.03$ as in the ordered $\beta$-phase of $\mathrm{NbH}_{\mathrm{x}}$ indicating that hydrogen had successfully penetrated all layers of the sample system, 
i.e., that the $\mathrm{Cr}_{2} \mathrm{O}_{3} / \mathrm{Cr}$ layers did not present $\mathrm{H}$ diffusion barriers. The integrated intensity of the smaller $\mathrm{H}$ peak in the surface region corresponded to roughly one monolayer of $\mathrm{H}$ equivalent [752]. The dashed line at 4 counts $/ \mu \mathrm{C}$ marks the natural background of the $\gamma$-detection system, defining the sensitivity limit of the measurement. The $\mathrm{H}$ concentration at the lower $\mathrm{Cr}_{2} \mathrm{O}_{3} / \mathrm{Cr}$ interface did not exceed this detection limit and was thus estimated to be smaller than $1 \%$ of a monolayer. The authors hence concluded that the highly crystalline $\alpha-\mathrm{Cr}_{2} \mathrm{O}_{3} / \mathrm{Cr}$ metal/oxide interface did apparently not provide a suitable (electronic) structure to stabilize hydrogen, but noted that structurally less coherent interfaces with higher defect densities may offer energetically favorable trapping sites for hydrogen [752].

\section{$\mathrm{Al}_{2} \mathrm{O}_{3} / \mathrm{Al}_{0.065} \mathrm{~W}_{0.935}$-alloy}

The question of possible $\mathrm{H}$ binding into metal-insulator interfaces raised above is closely related to the distribution and behavior of hydrogen impurities in thin alumina films formed by anodic oxidation of aluminum and its alloys. Water penetration into the oxide films had long been suspected to be the cause for the loss of dielectric strength of such barrier layers. In one of the first $\mathrm{H}$ profiling applications of ${ }^{1} \mathrm{H}\left({ }^{15} \mathrm{~N}, \alpha \gamma\right){ }^{12} \mathrm{C}$ NRA, Lanford indeed proved as early as 1980 the hydrogen inclusion into anodic alumina films [753]. A more recent NRA investigation of the $\mathrm{H}$ impurity behavior in Al-6.5 at.\% W alloy during anodic oxidation reported increased $\mathrm{H}$ concentrations near the alloy/anodic oxide interface, amounting to $\sim 2 \times 10^{15} \mathrm{H}$ atoms $\mathrm{cm}^{-2}$, whereas the bulk of the anodic oxide films contained $\sim 0.1-0.3$ at. $\%$ hydrogen [754]. This was the first report of $\mathrm{H}$ accumulation at an aluminumalloy/anodic film interface. Accidentally, the magnetron sputtering produced Al-W 
alloys used in this study contained up to 0.5 at. $\% \mathrm{H}$. This raised the question whether the source of the incorporated $\mathrm{H}$ in the oxide films and of that accumulated at the alloy/oxide interfaces might originate from the aquous electrolyte or from $\mathrm{H}$ impurities that diffused from the bulk of the alloys to the interface during the anodic oxidation [754].

\section{Anodically oxidized Zr-Nb alloy}

The possibility that metal-dissolved hydrogen diffuses and localizes near metal-oxide interfaces during anodic oxidation was elegantly demonstrated with ${ }^{15} \mathrm{~N}$ NRA by Khatamian for the anodization of $\mathrm{Zr}-\mathrm{Nb}$ alloys [755]. This is exemplified by the $\mathrm{H}$ profiles of Zr-2.5Nb alloy (2.5 wt. \% NB 10 wt. ppm H) in Fig. 85, which clearly display hydrogen accumulation peaks on the metal side of the respective interfaces to oxide layers of various thicknesses that were grown by different anodizing potentials.

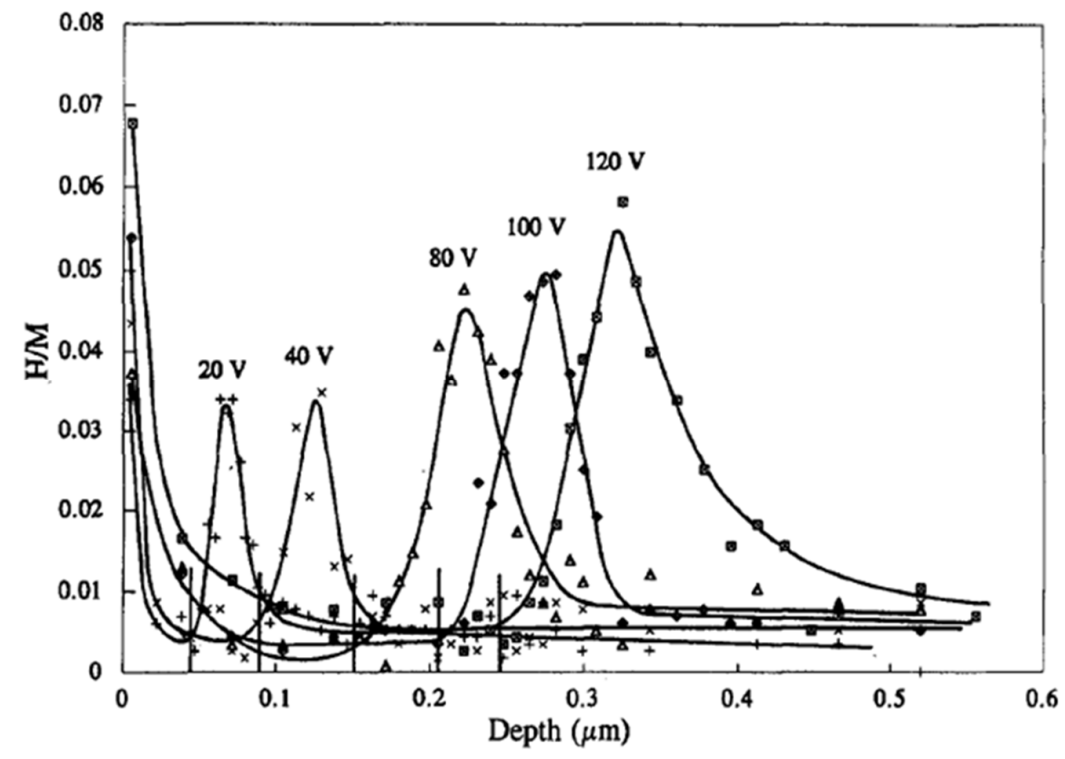

Fig. 85. Hydrogen profiles of a set of low-H $\quad \mathrm{Zr}-2.5 \mathrm{Nb}$ samples anodized in $\mathrm{KOH}$ at different applied potentials and $1 \mathrm{~mA} \mathrm{~cm}$ current density. The vertical lines mark the depth at which the oxygen signal starts

decreasing from a plateau level in the oxide measured by SAM (Scanning Auger Microprobe). From Ref. [755]. 
The source of the accumulated hydrogen was identified as bulk-dissolved $\mathrm{H}$ in the alloy rather than $\mathrm{H}$ from the electrolyte [756]. The $\mathrm{H}$ peak position below the oxide and the accumulated $\mathrm{H}$ amount followed the interface position in a strictly linear fashion. This behavior was the same for low (10 wt. ppm) as well as higher (150 wt. ppm) $\mathrm{H}$ concentrations in the alloy bulk. The $\mathrm{H}$ quantity below the metal/oxide interface increased somewhat with the $\mathrm{H}$ content in the alloy bulk but was clearly not proportional to it. The amount of $\mathrm{H}$ trapped below the interface also increased with the applied anodizing potential, i.e., with the longer time required to grow thicker oxides. The sub-interfacial $\mathrm{H}$ profiles were very stable at room temperature but first increased further in height and width (towards the alloy bulk) upon moderate annealing of the anodized specimen at 400-500 K. Upon prolonged annealing the $\mathrm{H}$ peaks first saturated and then started to decrease, and became entirely depleted by annealing at higher temperatures (e.g., $673 \mathrm{~K}$ ) for short periods ( $\sim 5 \mathrm{~min}$ ). Khatamian attributed his observations to trapping of hydrogen in the strained sub-interfacial region, where the metal lattice had been expanded due to the presence of the superficial oxide [755]. He assumed that $\mathrm{H}$ in the bulk of the alloy became mobile during moderate annealing and started populating the trap sites in the stressed sub-oxide region until all sites were filled. He reasoned further that as the heating process continued, the stressed lattice beneath the oxide layer gradually annealed, so that the hydrogen in the region moved back into the alloy. The disappearance of the $\mathrm{H}$ peak upon annealing at elevated temperatures was ascribed to much faster stress relief in the region. 
To test the alternative hypothesis that $\mathrm{H}$ may have been trapped at alloydissolved oxygen (which had been the tentative explanation for the sub-interfacial $\mathrm{H}$ accumulation prior to the study [756]), Khatamian implanted first oxygen ( $\sim 6$ at.\% O) and then hydrogen into the same depth region $(0.32 \mu \mathrm{m})$ of unoxidized $\mathrm{Zr}-2.5 \mathrm{Nb}$ alloy. $\mathrm{Zr}$ and its $\mathrm{Nb}$-alloys have a high solubility for oxygen, so that oxide precipitates would only form above an $\mathrm{O}$ concentration of 30 at. \% [757]. The expected (TRIMcalculated) depth distributions of the implanted oxygen and hydrogen are compared to the actually measured ${ }^{15} \mathrm{~N}$ NRA H profile in Fig. 86.

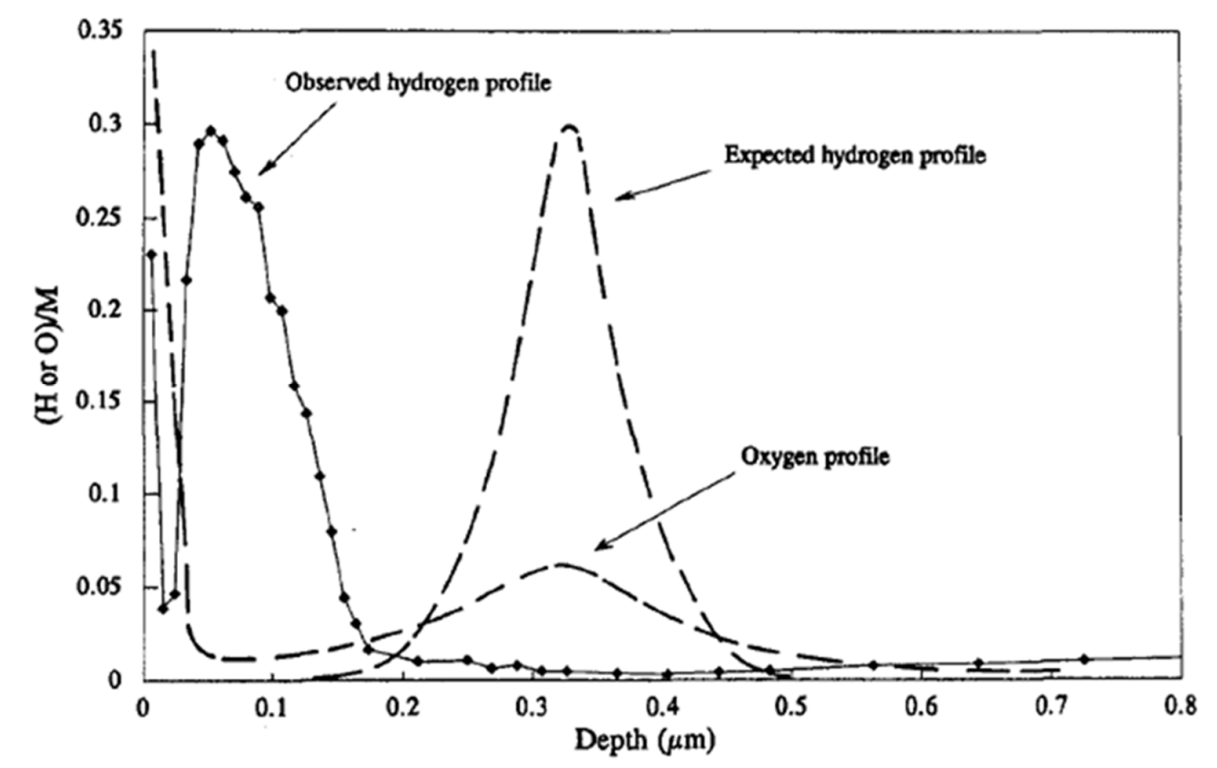

Fig. 86. Oxygen and hydrogen profiles of a low-H Zr-2.5Nb sample implanted with oxygen and subsequently implanted with hydrogen at room temperature. The position of the expected hydrogen profile, based on previous hydrogen implantations at lower temperatures, is also shown. Reproduced from Ref. [755].

Contrary to the calculation, the actual hydrogen distribution showed accumulation just beneath the (naturally oxidized) surface at a shallow depth of $0.06 \mu \mathrm{m}$, but a minimum $(0.08$ at. $\% \mathrm{H}$ ) in the 0 -implanted region (around $0.32 \mu \mathrm{m})$, suggesting that the $\mathrm{H}$ interaction with dissolved oxygen in the $\mathrm{Zr}$ - $\mathrm{Nb}$ alloys was in fact repulsive [755]. 
Through this experiment Khatamian showed convincingly that dissolved $\mathrm{O}$ was not responsible for the $\mathrm{H}$ trapping beneath the $\mathrm{Zr}$ - $\mathrm{Nb}$ alloy-oxide interfaces seen in Fig. 85. The apparently repulsive $\mathrm{O}-\mathrm{H}$ interaction observed here for the $\mathrm{Zr}-\mathrm{Nb}$ alloys thus closely resembles the reduced $\mathrm{H}$ solubility found in surface layers of naturally oxidized Ti and Ti alloys [166, 532] (Section 3.2.3.6). 


\section{Conclusions}

We hope to have presented a clear picture of the potential of ${ }^{1} \mathrm{H}\left({ }^{15} \mathrm{~N}, \alpha \gamma\right){ }^{12} \mathrm{C}$ NRA to contribute invaluably to the analysis of hydrogen at surfaces and interfaces. Due to its virtues of detecting hydrogen quantitatively, highly depth-resolved, and nondestructively, ${ }^{1} \mathrm{H}\left({ }^{15} \mathrm{~N}, \alpha \gamma\right){ }^{12} \mathrm{C}$ NRA has proven instrumental in elucidating the static and dynamical behavior of hydrogen at surfaces and interfaces in a wide variety of processes and materials.

Resonant ${ }^{1} \mathrm{H}\left({ }^{15} \mathrm{~N}, \alpha \gamma\right){ }^{12} \mathrm{C}$ NRA at $6.385 \mathrm{MeV}$ determines surface $\mathrm{H}$ coverages with a sensitivity in the order of $\sim 10^{13} \mathrm{~cm}^{-2}(\sim 1 \%$ of a monolayer) and $\mathrm{H}$ volume concentrations with a typical detection limit of $\sim 10^{18} \mathrm{~cm}^{-3}(\sim 100$ at. ppm). For surface-normal ${ }^{15} \mathrm{~N}$ ion incidence on the analyzed target the near-surface depth resolution is $2 \sim 5 \mathrm{~nm}$, which can easily be enhanced to below $1 \mathrm{~nm}$ by adopting a surface-grazing incidence geometry. Especially in combination with standard surface science techniques for in-situ preparation and characterization ${ }^{1} \mathrm{H}\left({ }^{15} \mathrm{~N}, \alpha \gamma\right){ }^{12} \mathrm{C}$ NRA is very well suited to hydrogen analysis at atomically controlled surfaces and nanostructured interfaces.

By quantifying the hydrogen coverage NRA has helped substantially to establish our current microscopic understanding of the hydrogen behavior in adsorption phases on an appreciable number of single crystal surfaces (Section 3.1, Table 2). ${ }^{1} \mathrm{H}\left({ }^{15} \mathrm{~N}, \alpha \gamma\right){ }^{12} \mathrm{C}$ NRA is the only experimental technique to determine the zero-point vibrational energy of surface-adsorbed $\mathrm{H}$ atoms as a function of the azimuthal angle [67], i.e., it delivers the real space vibrational amplitude of the adsorbed $\mathrm{H}$ atoms. 
The nanometer-scale discrimination between surface-adsorbed and absorbed $\mathrm{H}$ is a particularly important capability of ${ }^{1} \mathrm{H}\left({ }^{15} \mathrm{~N}, \alpha \gamma\right){ }^{12} \mathrm{C}$ NRA that can provide valuable insight, e.g., into hydrogen ingress processes into materials, relevant to mineral hydration dating [79] or the observation of hydride nucleation in the near-surface region of $\mathrm{H}$-absorbing metals (Section 3.2.3) [61, 84, 279]. In high-resolution mode the potential to detect submonolyer thickness variations of adlayers [59] and for the distinction of surface-adsorbed from volume-absorbed hydrogen in nanocrystalline materials have been demonstrated [60]. The combination with TDS allows for unambiguous identifications of thermal desorption features and for the depthresolved assessement of the thermal stability of adsorbed and absorbed hydrogen states against desorption and diffusion [61, 62, 279].

Its nondestructive nature and high depth resolution also make ${ }^{1} \mathrm{H}\left({ }^{15} \mathrm{~N}, \alpha \gamma\right){ }^{12} \mathrm{C}$ NRA the ideal method to detect hydrogen buried at intact interfaces, allowing to study hydrogen trapping at metal/semiconductor interfaces (Section 3.3.2), to track hydrogen transport phenomena in stacked thin film systems [81], to reveal nonhomogeneous hydrogen distributions and interface accumulation in clamped metal thin films (Section 3.2.3.7), etc. In directly visualizing hydrogen redistribution processes between interfaces of MOS structures under conditions relevant to electrical degradation (Section 3.3.1) ${ }^{1} \mathrm{H}\left({ }^{15} \mathrm{~N}, \alpha \gamma\right){ }^{12} \mathrm{C}$ NRA has made invaluable contributions to device reliability research [46].

Recent developments include micro-beam applications for the three-dimensional mapping of hydrogen distributions in the surface region of materials $[551,552]$ as well as NRA measurements under atmospheric pressure conditions [212]. As future 
perspectives we mention the remaining challenge to achieve the ultimate distinction of hydrogen in surface chemisorption and first-layer subsurface sites. This may be realized by applying ${ }^{1} \mathrm{H}\left({ }^{15} \mathrm{~N}, \alpha \gamma\right){ }^{12} \mathrm{C}$ NRA in a strongly grazing incidence geometry on large single crystal targets. Such measurements may help clarifying the peculiar chemical reactivity of subsurface hydrogen in catalytic hydrogenation reactions of co-adsorbed hydrocarbon molecules [48, 358-360] or finally resolving the longstandig controversy about the exact location preference of $\mathrm{H}$ atoms at the $\mathrm{Cu}(111)$ surface $[302,393,394]$. Also the quantification of the hydroxyl coverage at singlecrystalline oxide surfaces is worth exploring further.

\section{Acknowledgements}

The authors thank Y. Murata for many valuable discussions, helpful advice, and careful reading of the manuscript. Financial support through the Ministry of Education, Sports, and Science of Japan, NEDO, and CREST-JST is gratefully acknowledged. We are also greatly indepted to H. Matsuzaki and C. Nakano at Tokyo University for many years of support in operating the MALT tandem accelerator. 


\section{Appendix A}

\section{Stopping cross sections for ${ }^{15} \mathrm{~N}$ in elemental targets}

Note that two stopping cross section notations are in equally common use:

(1) $\varepsilon=1 / N(\mathrm{~d} E / \mathrm{dz})\left[\mathrm{eV} \mathrm{cm} / 10^{15}\right.$ atoms], where $N$ is the number density of the material in [atoms $/ \mathrm{cm}^{3}$ ]. This notation is convenient to calculate the energy loss in a layer of target atoms. The other definition is:

(2) $\varepsilon=1 / \rho(\mathrm{d} E / \mathrm{dz})\left[\mathrm{MeV} /\left(\mathrm{mg} / \mathrm{cm}^{2}\right)\right]$, with $\rho$ being the mass density $\left[\mathrm{g} / \mathrm{cm}^{3}\right]$ of the material.

To calculate the stopping power $S=(\mathrm{d} E / \mathrm{d} z)$ in $[\mathrm{keV} / \mathrm{nm}]$ from the above stopping cross sections, either

- multiply $\varepsilon\left[\mathrm{eV} \mathrm{cm} \mathrm{cm}^{2} / 10^{15}\right.$ atoms] by the number density $N\left[\mathrm{~cm}^{-3}\right]$ and divide by $10^{25}$, or

- multiply $\varepsilon\left[\mathrm{MeV} /\left(\mathrm{mg} / \mathrm{cm}^{2}\right)\right]$ by the mass density $\rho\left[\mathrm{g} / \mathrm{cm}^{3}\right]$ and divide by 10 .

Table A-1. Stopping cross sections $(\varepsilon)$ of ${ }^{15} \mathrm{~N}$ in elemental targets at 6.385 and 13.35 MeV. Data reproduced from Ref. [124].

\begin{tabular}{lcccc}
\hline Element & $\begin{array}{c}\text { Atomic } \\
\text { number }\end{array}$ & $\begin{array}{c}\text { Density } \\
{\left[\mathrm{g} \mathrm{cm}^{-3}\right]}\end{array}$ & $\begin{array}{c}\varepsilon \text { at } 6.385 \mathrm{MeV} \\
{\left[\mathrm{MeV} /\left(\mathrm{mg} \mathrm{cm}^{-2}\right)\right]}\end{array}$ & $\begin{array}{c}\varepsilon \text { at 13.35 MeV } \\
{\left[\mathrm{MeV} /\left(\mathbf{m g ~ c m}^{-2}\right)\right]}\end{array}$ \\
\hline $\mathrm{H}$ & 1 & & 28.38 & 21.66 \\
$\mathrm{He}$ & 2 & & 11.48 & 9.233 \\
$\mathrm{Li}$ & 3 & 0.54 & 8.514 & 7.399 \\
$\mathrm{Be}$ & 4 & 1.82 & 7.693 & 7.055 \\
$\mathrm{~B}$ & 5 & 2.47 & 8.307 & 7.227 \\
$\mathrm{C}$ & 6 & 3.52 & 8.453 & 7.397 \\
$\mathrm{~N}$ & 7 & & 8.537 & 7.112 \\
$\mathrm{O}$ & 8 & & 7.965 & 6.923 \\
$\mathrm{~F}$ & 9 & & 6.915 & 6.266 \\
$\mathrm{Ne}$ & 10 & & 6.747 & 6.198 \\
$\mathrm{Na}$ & 11 & 1.01 & 6.987 & 6.442
\end{tabular}




\begin{tabular}{|c|c|c|c|c|}
\hline Element & $\begin{array}{l}\text { Atomic } \\
\text { number }\end{array}$ & $\begin{array}{l}\text { Density } \\
{\left[\mathrm{g} \mathrm{cm}^{-3}\right]}\end{array}$ & $\begin{array}{c}\varepsilon \text { at } 6.385 \mathrm{MeV} \\
{\left[\mathrm{MeV} /\left(\mathrm{mg} \mathrm{cm}^{-2}\right)\right]}\end{array}$ & $\begin{array}{c}\varepsilon \text { at } 13.35 \mathrm{MeV} \\
{\left[\mathrm{MeV} /\left(\mathrm{mg} \mathrm{cm}^{-2}\right)\right]}\end{array}$ \\
\hline $\mathrm{Mg}$ & 12 & 1.74 & 6.535 & 6.022 \\
\hline $\mathrm{Al}$ & 13 & 2.70 & 5.858 & 5.556 \\
\hline $\mathrm{Si}$ & 14 & 2.33 & 6.242 & 5.677 \\
\hline $\mathrm{P}$ & 15 & 1.82 & 6.055 & 5.458 \\
\hline$S$ & 16 & 2.07 & 5.820 & 4.924 \\
\hline $\mathrm{Cl}$ & 17 & & 6.162 & 5.222 \\
\hline $\mathrm{Ar}$ & 18 & & 5.416 & 4.834 \\
\hline K & 19 & 0.91 & 6.133 & 5.456 \\
\hline $\mathrm{Ca}$ & 20 & 1.53 & 5.978 & 5.354 \\
\hline Sc & 21 & 2.99 & 5.613 & 4.872 \\
\hline $\mathrm{Ti}$ & 22 & 4.51 & 5.178 & 4.737 \\
\hline V & 23 & 6.09 & 5.263 & 4.566 \\
\hline $\mathrm{Cr}$ & 24 & 7.19 & 4.825 & 4.477 \\
\hline $\mathrm{Mn}$ & 25 & 7.47 & 4.550 & 4.264 \\
\hline $\mathrm{Fe}$ & 26 & 7.87 & 4.531 & 4.261 \\
\hline Co & 27 & 8.9 & 4.243 & 4.037 \\
\hline $\mathrm{Ni}$ & 28 & 8.91 & 4.160 & 4.116 \\
\hline $\mathrm{Cu}$ & 29 & 8.93 & 3.776 & 3.814 \\
\hline $\mathrm{Zn}$ & 30 & 7.13 & 3.797 & 3.783 \\
\hline $\mathrm{Ga}$ & 31 & 5.91 & 3.606 & 3.537 \\
\hline $\mathrm{Ge}$ & 32 & 5.32 & 3.547 & 3.593 \\
\hline As & 33 & 5.77 & 3.501 & 3.417 \\
\hline $\mathrm{Se}$ & 34 & 4.81 & 3.321 & 3.319 \\
\hline $\mathrm{Br}$ & 35 & & 3.422 & 3.646 \\
\hline $\mathrm{Kr}$ & 36 & & 3.434 & 3.340 \\
\hline $\mathrm{Rb}$ & 37 & 1.63 & 3.783 & 3.570 \\
\hline $\mathrm{Sr}$ & 38 & 2.58 & 3.749 & 3.431 \\
\hline$Y$ & 39 & 4.48 & 3.669 & 3.404 \\
\hline $\mathrm{Zr}$ & 40 & 6.51 & 3.704 & 3.405 \\
\hline $\mathrm{Nb}$ & 41 & 8.58 & 3.828 & 3.601 \\
\hline No & 42 & 10.22 & 3.440 & 3.249 \\
\hline Tc & 43 & 11.5 & 3.455 & 3.302 \\
\hline $\mathrm{Ru}$ & 44 & 12.36 & 3.431 & 3.271 \\
\hline $\mathrm{Rh}$ & 45 & 12.42 & 3.332 & 3.211 \\
\hline $\mathrm{Pd}$ & 46 & 12.00 & 3.244 & 3.169 \\
\hline $\mathrm{Ag}$ & 47 & 10.5 & 3.187 & 3.032 \\
\hline $\mathrm{Cd}$ & 48 & 8.65 & 3.082 & 2.998 \\
\hline In & 49 & 7.29 & 3.066 & 2.984 \\
\hline Sn & 50 & 5.76 & 2.985 & 2.855 \\
\hline $\mathrm{Sb}$ & 51 & 6.69 & 2.955 & 2.866 \\
\hline $\mathrm{Te}$ & 52 & 6.25 & 2.932 & 2.782 \\
\hline 1 & 53 & & 2.947 & 2.859 \\
\hline Xe & 54 & & 2.894 & 2.825 \\
\hline Cs & 55 & 1.99 & 3.151 & 2.994 \\
\hline $\mathrm{Ba}$ & 56 & 3.59 & 3.037 & 2.891 \\
\hline La & 57 & 6.17 & 3.083 & 2.866 \\
\hline
\end{tabular}




\begin{tabular}{|c|c|c|c|c|}
\hline Element & $\begin{array}{l}\text { Atomic } \\
\text { number }\end{array}$ & $\begin{array}{l}\text { Density } \\
{\left[\mathrm{g} \mathrm{cm}^{-3}\right]}\end{array}$ & $\begin{array}{c}\varepsilon \text { at } 6.385 \mathrm{MeV} \\
{\left[\mathrm{MeV} /\left(\mathrm{mg} \mathrm{cm}^{-2}\right)\right]}\end{array}$ & $\begin{array}{c}\varepsilon \text { at } 13.35 \mathrm{MeV} \\
{\left[\mathrm{MeV} /\left(\mathrm{mg} \mathrm{cm}^{-2}\right)\right]}\end{array}$ \\
\hline $\mathrm{Ce}$ & 58 & 6.77 & 2.729 & 2.732 \\
\hline $\operatorname{Pr}$ & 59 & 6.78 & 2.859 & 2.828 \\
\hline $\mathrm{Nd}$ & 60 & 7.00 & 2.753 & 2.731 \\
\hline $\mathrm{Pm}$ & 61 & & 2.755 & 2.709 \\
\hline $\mathrm{Sm}$ & 62 & 7.54 & 2.844 & 2.809 \\
\hline $\mathrm{Eu}$ & 63 & 5.25 & 2.523 & 2.613 \\
\hline $\mathrm{Gd}$ & 64 & 7.89 & 2.541 & 2.541 \\
\hline $\mathrm{Tb}$ & 65 & 8.27 & 2.441 & 2.503 \\
\hline Dy & 66 & 8.53 & 2.405 & 2.477 \\
\hline Ho & 67 & 8.80 & 2.181 & 2.308 \\
\hline $\mathrm{Er}$ & 68 & 9.04 & 2.327 & 2.413 \\
\hline $\mathrm{Tm}$ & 69 & 9.32 & 2.165 & 2.276 \\
\hline $\mathrm{Yb}$ & 70 & 6.97 & 2.164 & 2.207 \\
\hline Lu & 71 & 9.84 & 2.094 & 2.184 \\
\hline $\mathrm{Hf}$ & 72 & 13.2 & 2.060 & 2.157 \\
\hline Ta & 73 & 16.66 & 2.092 & 2.191 \\
\hline w & 74 & 19.25 & 2.083 & 2.083 \\
\hline $\operatorname{Re}$ & 75 & 21.03 & 2.062 & 2.137 \\
\hline Os & 76 & 22.58 & 2.025 & 2.101 \\
\hline Ir & 77 & 22.55 & 1.981 & 2.082 \\
\hline $\mathrm{Pt}$ & 78 & 21.47 & 1.917 & 2.015 \\
\hline $\mathrm{Au}$ & 79 & 19.28 & 2.022 & 2.043 \\
\hline $\mathrm{Hg}$ & 80 & 14.26 & 1.949 & 2.000 \\
\hline $\mathrm{Tl}$ & 81 & 11.87 & 1.984 & 2.017 \\
\hline $\mathrm{Pb}$ & 82 & 11.34 & 2.042 & 2.022 \\
\hline $\mathrm{Bi}$ & 83 & 9.8 & 2.134 & 2.077 \\
\hline Po & 84 & 9.24 & 2.097 & 2.090 \\
\hline At & 85 & & 2.122 & 2.113 \\
\hline $\mathrm{Rn}$ & 86 & & 2.030 & 2.025 \\
\hline $\mathrm{Fr}$ & 87 & & 2.088 & 2.021 \\
\hline $\mathrm{Ra}$ & 88 & 5 & 2.129 & 2.040 \\
\hline$A c$ & 89 & & 2.217 & 2.110 \\
\hline Th & 90 & 11.2 & 2.117 & 2.035 \\
\hline $\mathrm{Pa}$ & 91 & 15.4 & 2.207 & 2.090 \\
\hline U & 92 & 18.7 & 1.948 & 1.962 \\
\hline
\end{tabular}




\section{Appendix B}

\section{Elastic recoil detection (ERD)}

\section{a. Principles}

The most basic and conventional arrangement for ERD analysis of thick solid targets is the reflection geometry illustrated in Fig. B-1. A primary beam of projectile ions (mass $M_{1}$, energy $E_{1}$ ) incident at a small (near-grazing) angle with the target surface $\left(\theta_{1}\right)$ recoils atoms out of the sample into a laboratory angle $\theta_{\mathrm{r}}<90^{\circ}$. According to the kinematics of elastic nuclear collisions (energy and momentum conservation), the energy of the recoiled particles $\left(E_{\mathrm{r}}\right)$ depends as follows on $M_{1}, E_{1}$, and $\theta_{r}$, and thus provides information on their atomic masses $\left(M_{r}\right)$ :

$$
E_{r}=E_{1} \cdot\left[\frac{4 M_{1} M_{r}}{\left(M_{1}+M_{r}\right)^{2}} \cos ^{2} \theta_{r}\right]=E_{1} \cdot K_{r}
$$

$K_{\mathrm{r}}$ is called the kinematic factor for recoiling. A silicon surface barrier detector (SSBD) is typically used to analyze the energy distribution of these recoiled elements. A thin absorber (or range) foil (often a few $\mu \mathrm{m}$ of Mylar or rolled metal) placed in front of the detector lets hydrogen isotopes penetrate due to their small stopping powers, but fully retains the scattered primary ions and heavier recoils. Target tilt angles (measured between the surface normal and the beam direction) of $75^{\circ}$ or larger $\left(\theta_{1}<15^{\circ}\right)$ and recoil angles $\left(\theta_{r}\right)$ around $30^{\circ}$ are the most common settings.

The energy spectra of the recoiled particles are converted into respective depth profiles by taking into account the stopping energy losses of both the incident 
projectile ion and of the recoiled atoms along their trajectories into and out of the target, respectively. At a depth $d$ normal from the surface, the incident projectile energy has decreased from $E_{1}$ to $E_{1}{ }^{\prime}$ and recoiling transfers the energy $E_{2}^{\prime}=K_{\mathrm{r}} E_{1}{ }^{\prime}$ to the recoiled target atom, which reduces to $E_{2}$ on the recoil's way to the surface (Fig. B-1). For recoiling in shallow depths, the principally energy-dependent stopping powers of the projectile $\left(S_{1}\right)$ and the recoils $\left(S_{r}\right)$ can reasonably be assumed as constants (surface approximation), which combine into an effective target stopping power $S_{\text {eff }}=K_{\mathrm{r}} \cdot S_{1}\left(E_{1}\right) / \sin \theta_{1}+S_{\mathrm{r}}\left(K_{\mathrm{r}} \cdot E_{1}\right) / \sin \theta_{2}$, so that $E_{2}(d)=K_{\mathrm{r}} \cdot E_{1}-d \cdot S_{\text {eff. }}$ Obtaining the final detection energy $\left(E_{\mathrm{d}}\right)$ requires calculating the additional energy loss of the recoils in the range foil by means of a slab analysis, in which the foil is divided into a set of equally thin layers for which the stopping power of the recoils is reasonably assumed as constant at their respective energy [193, 240].

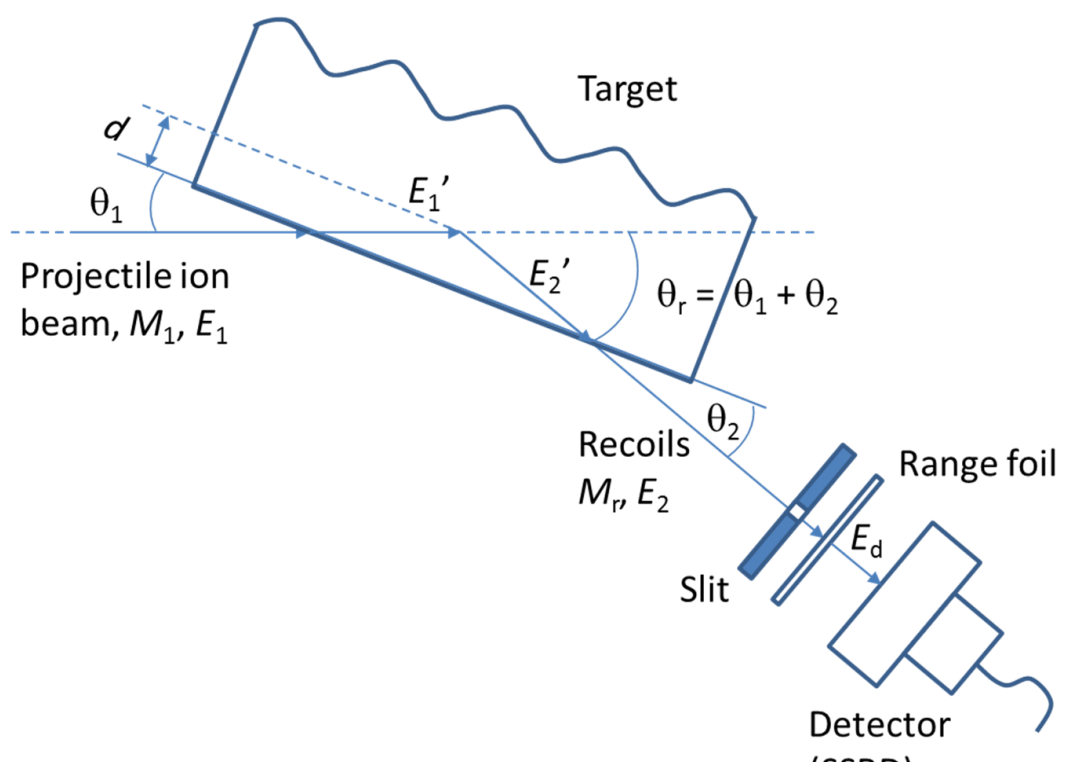

(SSBD)

Fig. B-1. Schematic illustration of the conventional ERDA recoiling and detection geometry, defining the laboratory angles of recoil $\left(\theta_{r}\right)$, incidence $\left(\theta_{1}\right)$, and exit $\left(\theta_{2}\right)$, as well as the 
projectile $\left(M_{1}\right)$ and recoil particle $\left(M_{r}\right)$ energies $\left(E_{1}, E_{2}\right.$, respectively). Only the light hydrogen isotopes $(H$ and $D)$ can pass through the range foil and are counted by the SSBD with a final detection energy $E_{\mathrm{d}}$.

\section{b. Sensitivity}

The sensitivity of ERD measurements for a given target element is principally determined by the differential recoil cross section, $\sigma_{r}\left[\right.$ barn $\left.\mathrm{sr}^{-1}\right]\left(1\right.$ barn $\left.=10^{-24} \mathrm{~cm}^{2}\right)$. In the laboratory frame, this recoil cross section is again only a function of the recoil angle $\left(\theta_{\mathrm{r}}\right)$, of the projectile energy $\left(E_{1}\right)$, and of the atomic numbers and masses of the projectile $\left(Z_{1}, M_{1}\right)$ and the recoiled target atoms $\left(Z_{r}, M_{r}\right)$ :

$$
\sigma_{r}=\left(\frac{Z_{1} Z_{r} e^{2}}{2 E_{1}}\right)^{2}\left(\frac{M_{1}}{M_{r}}+1\right)^{2} \frac{1}{\cos ^{3} \theta_{r}}
$$

This Rutherford recoil cross section applies as long as the repulsive interaction between the colliding nuclei is adequately described by a pure Coulomb potential. This is a reasonable assumption for projectile velocities in the oder of ( 1-2 $\mathrm{MeV} / \mathrm{amu})^{1 / 2}$, which are therefore frequently employed conditions for quantitative and standard-free ERD measurements. In routinely used ERD of $\mathrm{H}$ and $\mathrm{D}$ with ${ }^{4} \mathrm{He}$ beams, significant deviations of the ${ }^{1} \mathrm{H}\left({ }^{4} \mathrm{He},{ }^{1} \mathrm{H}\right){ }^{4} \mathrm{He}[235,262,758-763]$ and $\mathrm{D}\left({ }^{4} \mathrm{He}, \mathrm{D}\right)^{4} \mathrm{He}[235,342,758,759,763,764]$ scattering cross sections from the Rutherford values require attention at ${ }^{4} \mathrm{He}$ energies already above $1 \mathrm{MeV}$. The $\mathrm{D}\left({ }^{4} \mathrm{He}, \mathrm{D}\right)^{4} \mathrm{He}$ cross section furthermore has a $\sim 60-70 \mathrm{keV}$ wide (FWHM) and strongly $\theta_{r}$-dependent $[193,235,342,759,764]$ resonance at $2.135 \pm 0.01 \mathrm{MeV}$, which corresponds to the first excited nuclear level of ${ }^{6} \mathrm{Li}[758]$. 
The recoil cross section decreases strongly with the projectile's energy $\left(\sigma_{\mathrm{r}} \sim\right.$ $\left.1 / E_{1}{ }^{2}\right)$ and shows a promiment dependence on the nuclear charge of the latter $\left(Z_{1}\right.$ in Eq. (B. 2)). For hydrogen recoiling by heavy projectiles $\left(M_{1} \gg M_{r}\right)$ at a constant $E_{1}$ and in the approximation $M_{1} \approx 2 Z_{1}, \sigma_{r}$ increases very significantly with $Z_{1}\left(\sigma_{r} \sim Z_{1}^{4}\right)$, which promises substantial gains in sensitivity through the use of large $Z$ projectiles. This is exploited as one major advantage in ERD with heavy ion projectiles (HIERD). In practice, the ratio $\left(E_{1} / M_{1}\right)$ is often kept approximately constant in the $\sim 1-2 \mathrm{MeV} / \mathrm{amu}$ range to ensure Rutherford scattering behavior, so effectively a $Z_{1}^{2}$ dependence of $\sigma_{r}$ applies when different projectiles are to be compared. The $Z_{r} / M_{r}$ ratio is nearly the same $(\sim 1 / 2)$ for all elements except $H$, where it is unity. Hence HIERD offers an approximately constant sensitivity for all recoiled elements with the exception of $\mathrm{H}$, for which it is relatively enhanced by a factor of 4 . The requirements, advantages, and limitations of hydrogen analysis by HIERD are discussed in detail by Dollinger et al. $[765,766]$ and Davies et al. [200, 248].

\section{c. Depth resolution}

The depth resolution in ERDA is generally expressed as $\Delta d=\Delta E_{\mathrm{d}} / S_{\text {eff, }}$ where $\Delta E_{\mathrm{d}}$ is the total spread in the detected recoil energy. As explained in Refs. [193, 222, 238, 767-770], several factors enter $\Delta E_{\mathrm{d}}$ : (1) Energy (typically $\Delta E_{1} / E_{1} \sim 10^{-3}$ ) and angular spread of the projectiles (negligible for well-collimated beams), (2) geometrical spread caused by the finite beam spot and detector solid angle ('kinematic broadening') , (3) straggling and multiple scattering in the target [221, 222, 263, 771], (4) Doppler broadening (negligible), (5) energy straggling in and inhomogeneities of the absorber foil, and (6) energy resolution of the detection system. 
Straggling and multiscattering of projectile and recoil in the target are sizeable contributions to $\Delta E_{\mathrm{d}}$. Small incidence angles with the target surface $\left(\theta_{1}\right)$ make better use of the higher stopping power of the heavier projectile relative to that of the lighter recoils, so that the ERD depth resolution can be optimized by adjusting the recoil geometry within the limit $\theta_{r}=\theta_{1}+\theta_{2}$ (Fig. B-1) $[771,772]$. The near-surface resolution of $\mathrm{H} E R D$, e.g. in amorphous $\mathrm{Si}$ with a $3 \mathrm{MeV}{ }^{4} \mathrm{He}$ beam, thus improves significantly from 75 to $22 \mathrm{~nm}$ when $\theta_{1}$ and $\theta_{\mathrm{r}}$ are decreased from $12.5^{\circ}$ and $23^{\circ}$ to $4.5^{\circ}$ and $15^{\circ}$, respectively [235] (Table 2 ). The small angles crucially demand high precision with respect to the sample position and recoil geometry (on the order of $0.1 \mathrm{~mm}$ and $0.1^{\circ}$, respectively) and samples with smooth surfaces are a strict requirement $[266,267]$.

The geometric recoil energy spread, also addressed as 'kinematic broadening', is another sizeable and inevitable resolution limiting factor in ERD. The size of the beam spot on the target and the detector slit dimensions define the recoil geometry less than ideally and allow that target atoms recoiled into slightly different directions $\Delta \theta_{r}$ reach the detector, which according to Eq. (B.1) implies a difference in recoil energy. The resulting geometric energy spread $\Delta E_{\mathrm{r}}$ can be approximated as $\Delta \mathrm{E}_{\mathrm{r}} / \mathrm{E}_{\mathrm{r}}=$ $2 \tan \theta_{r} \Delta \theta_{r}[235,238,250,254,765,768,769]$.

In conventional range foil/SSBD setups the dominating resolution-limiting factors are the energy straggling of the recoil in the (typically several $\mu \mathrm{m}$ thick) range foil and the energy resolution of the detector itself ( $\sim 15 \mathrm{keV}$ for standard silicon surfacebarrier detectors). Hence, detector quality and the thickness, homogeneity, and uniformity of the range foil are highly important. Mylar and other organic polymers 
are popular absorber foils because films with uniform thickness free of pinholes can easily be otbained and straggling is relatively weak in these low $Z$ materials. Conventional ERD setups with absorber foils typically achieve some $10 \mathrm{~nm}$ depth resolution near the surface and a sensitivity $\sim 0.1$ at.\% in $\mathrm{H}$ depth profiling (Table 2) $[234,235,758,772]$. Mainly due to elimination of the range foil and better timingresolution compared to the energy resolution of standard SSBD's, about one order of increased depth resolution is realized in TOF-ERD [193, 244, 773, 774]. In high resolution ERD applications with magnetic or electrostatic spectrometers that avoid the most prominent energy spreads (namely range foil straggling and SSBD detector resolution) of the conventional ERD setup altogether, the geometric energy spread prevails as the dominant resolution-limiting factor, and it is then essential to correct for the kinematic broadening with focusing ion optics in order to realize high depth resolution [238, 249, 250, 765].

\section{d. Advanced detection systems}

Several improved detection systems have been developed to enhance the ERD depth resolution and to become able to distinguish recoiled elements of very small mass differences, such as $\mathrm{H}, \mathrm{D}, \mathrm{B} C, \mathrm{~N}$, and $\mathrm{O}$. One such device is the $\Delta \mathrm{E}-\mathrm{E}$ telescope, in which the recoils deposit only a small portion of their energy $(\Delta E)$ upon transmitting a first thin absorber foil, before they are fully stopped in a second detector that measures their remaining energy $[768,769,775,776]$. The sum of the two energies gives the total recoil energy and provides the depth information. $\Delta \mathrm{E}$ is proportional to the stopping power of the transmitting particle, so that different recoil species can be identified through their mass (more precisely $Z_{R}$ ) even if they 
have the same kinetic energy. Since their principle is still based on energy loss in transmitted foils, $\Delta \mathrm{E}-\mathrm{E}$ detectors do not offer a particularly enhanced depth resolution, but they tolerate large acceptance angles, so that high sensitivity measurements can be performed, and several recoil species can be detected simultaneously $[257,776]$. This is particularly useful for the ERD analysis of hydrogen isotopes, when several of these species are present in a target at the same time [257, 776-778].

Also time-of-flight (TOF) spectrometers [244, 773, 774], E x B filters [256, 779$781]$, or sophisticated electrostatic $[238,253,782]$ and magnetic $[239,247,249,250$, 254, 783-785] spectrographs with position sensitive detectors (PSDs) in the analyzer focal plane have been devised for the purposes of rejecting scattered projectiles and highly resolved mass-dispersive energy analysis of the recoils. These instruments identify recoils of individual masses by virtue of measuring either their velocity, momentum, electric or magnetic rigidity, respectively. In addition to determining the total energy, i.e., providing depth information, these advanced detection systems can achieve sub-nanometer depth resolution for $\mathrm{H}$ isotopes near surfaces, provided that kinematical broadening is corrected for [238, 247, 249, 253, 255]. 


\section{References}

[1] K. Christmann, Interaction of Hydrogen with Solid-Surfaces, Surf. Sci. Rep. 9 (1988) 1-163. [2] K. Oura, V.G. Lifshits, A.A. Saranin, A.V. Zotov, M. Katayama, Hydrogen interaction with clean and modified silicon surfaces, Surf. Sci. Rep. 35 (1999) 1-69.

[3] M.A. Henderson, The interaction of water with solid surfaces: fundamental aspects revisited, Surf. Sci. Rep. 46 (2002) 1-308.

[4] K. Christmann, 3.4.1 Adsorbate properties of hydrogen on solid surfaces, in: H.P. Bonzel (Ed.) Adsorbed Layers on Surfaces. Part 5: Adsorption of molecules on metal, semiconductor and oxide surfaces, Springer Berlin Heidelberg, 2006, pp. 1-130.

[5] M. Dürr, U. Höfer, Dissociative adsorption of molecular hydrogen on silicon surfaces, Surf. Sci. Rep. 61 (2006) 465-526.

[6] H. Kawarada, Hydrogen-terminated diamond surfaces and interfaces, Surf. Sci. Rep. 26 (1996) 205-259.

[7] S. Nannarone, M. Pedio, Hydrogen chemisorption on III-V semiconductor surfaces, Surf. Sci. Rep. 51 (2003) 1-149.

[8] P.A. Thiel, T.E. Madey, The interaction of water with solid surfaces: Fundamental aspects, Surf. Sci. Rep. 7 (1987) 211-385.

[9] E. Ilisca, Ortho-para conversion of hydrogen molecules physisorbed on surfaces, Prog. Surf. Sci. 41 (1992) 217-335.

[10] H. Kasai, W.A. Diño, R. Muhida, Surface science-based reaction design: increasing the ortho-para hydrogen conversion yield via molecular orientation, a case study, Prog. Surf. Sci. 72 (2003) 53-86.

[11] K. Fukutani, K. Niki, T. Ito, H. Tashiro, M. Matsumoto, M. Wilde, T. Okano, W.A. Diño, H. Kasai, Photodesorption of hydrogen molecules. physisorbed on Ag: Isotope dependence of translational-energy distribution, Surf. Sci. 593 (2005) 229-234.

[12] K. Niki, T. Kawauchi, M. Matsumoto, K. Fukutani, T. Okano, Mechanism of the orthopara conversion of hydrogen on Ag surfaces, Phys. Rev. B 77 (2008) 201404.

[13] K. Niki, S. Ogura, M. Matsumoto, T. Okano, K. Fukutani, Effects of coadsorbed O(2) on hydrogen ortho-para conversion on Ag surfaces, Phys. Rev. B 79 (2009) 085408.

[14] T. Sugimoto, K. Fukutani, Electric-field-induced nuclear-spin flips mediated by enhanced spin-orbit coupling, Nature Physics 7 (2011) 307-310.

[15] K. Fukutani, K. Yoshida, M. Wilde, W.A. Diño, M. Matsumoto, T. Okano, Photostimulated desorption and ortho-para conversion of H-2 on Ag surfaces, Phys. Rev. Lett. 90 (2003) 096103.

[16] K. Fukutani, T. Sugimoto, Physisorption and ortho-para conversion of molecular hydrogen on solid surfaces, Prog. Surf. Sci. 88 (2013) 279-348.

[17] F. Ding, B. Yakobson, Challenges in hydrogen adsorptions: from physisorption to chemisorption, Front. Phys., 6 (2011) 142-150.

[18] A. Züttel, Hydrogen storage methods, Naturwissenschaften, 91 (2004) 157-172.

[19] K. Christmann, Some General-Aspects of Hydrogen Chemisorption on Metal-Surfaces, Prog. Surf. Sci. 48 (1995) 15-26.

[20] A. Gross, Reactions at surfaces studied by ab initio dynamics calculations, Surf. Sci. Rep. 32 (1998) 291-340.

[21] G.-J. Kroes, Six-dimensional quantum dynamics of dissociative chemisorption of $\mathrm{H} 2$ on metal surfaces, Prog. Surf. Sci. 60 (1999) 1-85.

[22] W.A. Diño, H. Kasai, A. Okiji, Orientational effects in dissociative adsorption/associative desorption dynamics of H2(D2) on Cu and Pd, Prog. Surf. Sci. 63 (2000) 63-134. 
[23] G.J. Kroes, A. Gross, E.J. Baerends, M. Scheffler, D.A. McCormack, Quantum theory of dissociative chemisorption on metal surfaces, Acc. Chem. Res. 35 (2002) 193-200.

[24] D. Farias, R. Miranda, Diffraction of molecular hydrogen from metal surfaces, Prog. Surf. Sci. 86 (2011) 222-254.

[25] D.P. Smith, Hydrogen in Metals, Univ. of Chicago Press, Chicago, IL., 1948.

[26] W.M. Mueller, J.P. Blackledge, G.G. Lipowitz, Metal Hydrides, Academic Press, New York, 1968.

[27] Y. Fukai, The Metal Hydrogen System, Springer, Berlin, New York, 2005.

[28] G. Alefeld, J.E. Völkl, Hydrogen in Metals I, Springer Berlin Heidelberg, 1978.

[29] G. Alefeld, J.E. Völkl, Hydrogen in Metals II, Springer Berlin Heidelberg, 1978.

[30] L. Schlapbach, Introduction, in: L. Schlapbach (Ed.) Hydrogen in Intermetallic

Compounds I, Springer Berlin Heidelberg, 1988, pp. 1-10.

[31] L. Schlapbach, J. Osterwalder, T. Riesterer, Recent experimental results on the electronic structure of binary and ternary hydrides, J. Less Common Metals 103 (1984) 295-307.

[32] J.N. Huiberts, R. Griessen, J.H. Rector, R.J. Wijngaarden, J.P. Dekker, D.G. de Groot, N.J. Koeman, Yttrium and lanthanum hydride films with switchable optical properties, Nature, 380 (1996) 231-234.

[33] R. Griessen, J.N. Huiberts, M. Kremers, A.T.M. van Gogh, N.J. Koeman, J.P. Dekker, P.H.L. Notten, Yttrium and lanthanum hydride films with switchable optical properties, J. Alloys Compds. 253-254 (1997) 44-50.

[34] R.A. Oriani, Hydrogen embrittlement of steels, Annu. Rev. Mater. Sci. 8 (1978) 327-357.

[35] V. Nemanic, J. Setina, Outgassing in thin wall stainless steel cells, J. Vac. Sci. Technol. A, 17 (1999) 1040-1046.

[36] Y. Ishikawa, V. Nemanic, An overview of methods to suppress hydrogen outgassing rate from austenitic stainless steel with reference to UHV and EXV, Vacuum 69 (2003) 501-512.

[37] S.N. Paglieri, J.D. Way, Innovations in palladium membrane research, Separation and Purification Methods 31 (2002) 1-169.

[38] C.G. Van de Walle, Hydrogen as a Cause of Doping in Zinc Oxide, Phys. Rev. Lett. 85 (2000) 1012-1015.

[39] E.V. Lavrov, F. Herklotz, J. Weber, Identification of Hydrogen Molecules in ZnO, Phys. Rev. Lett. 102 (2009) 185502.

[40] C.G. Van de Walle, J. Neugebauer, Hydrogen in semiconductors, Annu. Rev. Mater. Res. 36 (2006) 179-198.

[41] W. Mönch, Hydrogen-Modification of Electronic Surface, Bulk, and Interface Properties of Si, physica status solidi (a) 159 (1997) 25-37.

[42] K. Fukutani, H. Iwai, H. Yamashita, Y. Murata, S. Hatori, K. Kobayashi, Interface hydrogen between a $\mathrm{Pb}$ overlayer and $\mathrm{H}$-saturated $\mathrm{Si}(111)$ studied by a resonant nuclear reaction, Surf. Sci. 377 (1997) 1010-1014.

[43] K. Fukutani, H. Iwai, Y. Murata, H. Yamashita, Hydrogen at the surface and interface of metals on Si(111), Phys. Rev. B 59 (1999) 13020-13025.

[44] E.H. Poindexter, Physical chemistry of hydrogeneous species in the $\mathrm{Si}^{-\mathrm{SiO}_{2}}$ system, Z. Naturforschung A 50 (1995) 653.

[45] S. Fujieda, M. Terai, M. Saitoh, A. Toda, Y. Miura, Z. Liu, Y. Teraoka, A. Yoshigoe, M. Wilde, K. Fukutani, Bias Temperature Instability Characterization of Advanced Gate Stacks, ECS Transactions 6 (2007) 185-202.

[46] Z. Liu, S. Fujieda, H. Ishigaki, M. Wilde, K. Fukutani, Current Understanding of the Transport Behavior of Hydrogen Species in MOS Stacks and Their Relation to Reliability Degradation, ECS Transactions 35 (2011) 55-72.

[47] A single notable exception is the identification of a $\mathrm{H}$ subsurface state vibration in $\mathrm{Ni}(111)$ by HREELS (Ref. [48]). 
[48] A.D. Johnson, K.J. Maynard, S.P. Daley, Q.Y. Yang, S.T. Ceyer, Hydrogen embedded in Ni: Production by incident atomic hydrogen and detection by high-resolution electron energy loss, Phys. Rev. Lett. 67 (1991) 927-930.

[49] W.A. Lanford, H.P. Trautvetter, J.F. Ziegler, J. Keller, New precision technique for measuring concentration versus depth of hydrogen in solids, Appl. Phys. Lett. 28 (1976) 566568.

[50] W.A. Lanford, N-15 Hydrogen profiling - Scientific applications, Nucl. Instrum. Methods 149 (1978) 1-8.

[51] W.A. Lanford, Analysis for hydrogen by nuclear-reaction and energy recoil detection, Nucl. Instrum. Methods Phys. Res. B 66 (1992) 65-82.

[52] W.A. Lanford, Nuclear Reactions for Hydrogen Analysis, in: J.R. Tesmer, M. Nastasi (Eds.) Handbook of Modern Ion Beam Materials Analysis, Materials Research Society, Pittsburgh, PA, 1995, pp. 193.

[53] P. Trocellier, C. Engelmann, Hydrogen depth profile measurement using resonant nuclear reaction: An over view, J. Radioanalytical Nucl. Chem. 100 (1986) 117-127.

[54] R.C. Ross, I.S.T. Tsong, R. Messier, W.A. Lanford, C. Burman, Quantification of hydrogen in $a$-Si-H films by IR spectrometry, ${ }^{15} \mathrm{~N}$ nuclear-reaction, and SIMS, J. Vac. Sci. Technol. 20 (1982) 406-409.

[55] T. Suzuki, J. Konishi, K. Yamamoto, S. Ogura, K. Fukutani, Practical IR extinction coefficients of water in soda lime aluminosilicate glasses determined by nuclear reaction analysis, J. Non-Crystalline Solids 382 (2013) 66-69.

[56] K. Fukutani, M. Wilde, M. Matsumoto, Y. Murata, H. Yamashita, Study of hydrogen in the near-surface region by resonant nuclear reaction analysis, Kotai Butsuri 36 (2001) 353.

[57] K. Fukutani, Below-surface behavior of hydrogen studied by nuclear reaction analysis, Curr. Opin. Solid State Mater. Sci. 6 (2002) 153-161.

[58] K. Fukutani, M. Wilde, Depth profiling of hydrogen with nuclear reaction analysis, Kotai Butsuri 41 (2006) 719.

[59] K. Fukutani, M. Wilde, M. Matsumoto, Nuclear-reaction analysis of $\mathrm{H}$ at the $\mathrm{Pb} / \mathrm{Si}(111)$ interface: Monolayer depth distinction and interface structure, Phys. Rev. B 64 (2001) 245411.

[60] M. Wilde, K. Fukutani, M. Naschitzki, H.J. Freund, Hydrogen absorption in oxidesupported palladium nanocrystals, Phys. Rev. B 77 (2008) 113412.

[61] M. Wilde, M. Matsumoto, K. Fukutani, T. Aruga, Depth-resolved analysis of subsurface hydrogen absorbed by Pd(100), Surf. Sci. 482 (2001) 346-352.

[62] M. Wilde, K. Fukutani, Penetration mechanisms of surface-adsorbed hydrogen atoms into bulk metals: Experiment and model, Phys. Rev. B 78 (2008) 115411.

[63] M. Zinke-Allmang, S. Kalbitzer, A novel method to determine vibrational energy states of atomic systems, Z. Physik A 323 (1986) 251-252.

[64] M. Zinke-Allmang, S. Kalbitzer, M. Weiser, Nuclear reaction spectroscopy of vibrational modes of solids, Z. Physik A 325 (1986) 183-191.

[65] Y. Iwata, F. Fujimoto, E. Vilalta, A. Ootuka, K.-i. Komaki, K. Kobayashi, H. Yamashita, Y. Murata, Detection of Adsorbed Hydrogen on W(001) by Using ${ }^{1} \mathrm{H}\left({ }^{15} \mathrm{~N}, \alpha \gamma\right)^{12} \mathrm{C}$ Reaction, Jpn. J. Appl. Phys. 26 (1987) L1026-L1028.

[66] K.M. Horn, W.A. Lanford, Observation of the bond-dependent Doppler broadening of the $p\left({ }^{15} \mathrm{~N}, \alpha \gamma\right){ }^{12} \mathrm{C}$ nuclear reaction, Nucl. Instrum. Methods Phys. Res. B 29 (1988) 609-620.

[67] K. Fukutani, A. Itoh, M. Wilde, M. Matsumoto, Zero-Point Vibration of Hydrogen Adsorbed on Si and Pt Surfaces, Phys. Rev. Lett. 88 (2002) 116101.

[68] M. Nishijima, H. Okuyama, N. Takagi, T. Aruga, W. Brenig, Quantum delocalization of hydrogen on metal surfaces, Surf. Sci. Rep. 57 (2005) 113-156. 
[69] A.P. Jardine, E.Y.M. Lee, D.J. Ward, G. Alexandrowicz, H. Hedgeland, W. Allison, J. Ellis, E. Pollak, Determination of the Quantum Contribution to the Activated Motion of Hydrogen on a Metal Surface: H/Pt(111), Phys. Rev. Lett. 105 (2010) 136101.

[70] M. Wilde, K. Fukutani, S. Koh, K. Sawano, Y. Shiraki, Quantitative coverage and stability of hydrogen-passivation layers on HF-etched $\mathrm{Si}_{(1-x)} \mathrm{Ge}_{x}$ surfaces, J. Appl. Phys. 98 (2005) 023503.

[71] J.F. Ziegler, C.P. Wu, P. Williams, C.W. White, B. Terreault, B.M.U. Scherzer, R.L. Schulte, E.J. Schneid, C.W. Magee, E. Ligeon, J. Lecuyer, W.A. Lanford, F.J. Kuehne, E.A. Kamykowski, W.O. Hofer, A. Guivarch, C.H. Filleux, V.R. Deline, C.A. Evans, B.L. Cohen, G.J. Clark, W.K. Chu, C. Brassard, R.S. Blewer, R. Behrisch, B.R. Appleton, D.D. Allred, Profiling hydrogen in materials using ion-beams, Nucl. Instrum. Methods 149 (1978) 19-39.

[72] C. Engelmann, A complementary technique to PIXE: The nuclear interaction analysis method - advantages, possibilities and typical application examples, Nucl. Instrum. Methods Phys. Res. B 49 (1990) 33-38.

[73] G. Frech, G.K. Wolf, H. Damjantschitsch, M. Weiser, S. Kalbitzer, Defect trapping of hydrogen in iron studied by the ${ }^{1} \mathrm{H}\left({ }^{15} \mathrm{~N}, \alpha \gamma\right){ }^{12} \mathrm{C}$ reaction, Nucl. Instrum. Methods Phys. Res. 218 (1983) 500-503.

[74] D. Grambole, F. Herrmann, R. Klabes, W. Hauffe, R. Behrisch, Investigation of the deuterium distribution in a JET limiter using the nuclear microprobe and ion beam slope cutting, Nucl. Instrum. Methods Phys. Res. B 68 (1992) 154-157.

[75] J. Küppers, The hydrogen surface chemistry of carbon as a plasma facing material, Surf. Sci. Rep. 22 (1995) 249-321.

[76] D. Grambole, F. Herrmann, R. Behrisch, W. Hauffe, Hydrogen and deuterium depth profiling in divertor tiles of a fusion experiment by micro-ERDA, Nucl. Instrum. Methods Phys. Res. B 158 (1999) 647-652.

[77] P. March, F. Rauch, Hydration of soda-lime glasses studied by ion-induced nuclear reactions, Nucl. Instrum. Methods Phys. Res. B 15 (1986) 516-519.

[78] O. Dersch, A. Zouine, F. Rauch, J.E. Ericson, Investigation of water diffusion into quartz using ion beam analysis techniques, Fresenius' J. Analytical Chem. 358 (1997) 217-219.

[79] J.E. Ericson, O. Dersch, F. Rauch, Quartz hydration dating, J. Archaeol. Sci. 31 (2004) 883902.

[80] J.E. Ericson, F. Rauch, O. Dersch, Quartz hydration dating: A new mineral geochronological technique, Geochimica Et Cosmochimica Acta 69 (2005) A323-A323.

[81] W. Wagner, F. Rauch, C. Ottermann, K. Bange, Hydrogen dynamics in electrochromic multilayer systems investigated by the N-15 technique, Nucl. Instrum. Methods Phys. Res. B 50 (1990) 27-30.

[82] F. Rauch, W. Wagner, K. Bange, Nuclear reaction analysis of hydrogen in optically active coatings on glass, Nucl. Instrum. Methods Phys. Res. B 42 (1989) 264-267.

[83] Y. Iwata, F. Fujimoto, E. Vilalta, A. Ootuka, K.-i. Komaki, K. Kobayashi, H. Yamashita, Y. Murata, Detection of hydrogen adsorbed on tungsten surfaces using the ${ }^{1} \mathrm{H}\left({ }^{15} \mathrm{~N}, \alpha \gamma\right){ }^{12} \mathrm{C}$ reaction, Nucl. Instrum. Methods Phys. Res. B 33 (1988) 574-577.

[84] M. Wilde, M. Matsumoto, K. Fukutani, T. Okano, Y. Mizuno, T. Homma, Hydrogen sorption by Ti(0001) single crystal surfaces, J. Vac. Soc. Jpn. 45 (2002) 458-462.

[85] S.T. Picraux, Analyzing hydrogen with nuclear reactions, Physics Today 30 (1977) 42-51.

[86] W. Rudolph, C. Bauer, P. Gippner, D. Grambole, C. Heiser, F. Herrmann, H.J. Thomas, Hydrogen determination by means of the ${ }^{1} \mathrm{H}\left({ }^{19} \mathrm{~F}, \alpha \gamma\right){ }^{16} \mathrm{O}$ and ${ }^{1} \mathrm{H}\left({ }^{15} \mathrm{~N}, \alpha \gamma\right){ }^{12} \mathrm{C}$ resonance reactions, J. Radioanalytical Nucl. Chem. 83 (1984) 99-105.

[87] M. Matsunami, Nuclear reaction analysis for hydrogen isotopes near surface, Oyo Butsuri 54 (1985) 471. 
[88] G. Amsel, C. Cohen, B. Maurel, A new measurement of the $429 \mathrm{keV}{ }^{15} \mathrm{~N}(\mathrm{p}, \alpha \gamma)^{12} \mathrm{C}$ resonance. Applications of the very narrow width found to ${ }^{15} \mathrm{~N}$ and ${ }^{1} \mathrm{H}$ depth location: II. Applications, Nucl. Instrum. Methods Phys. Res. B 14 (1986) 226-233.

[89] X. Fulin, F. Rauch, S. Chengru, Z. Zhuying, R.P. Livi, T.A. Tombrello, Hydrogen depth profiling in solids: A comparison of several resonant nuclear reaction techniques, Nucl. Instrum. Methods Phys. Res. B 27 (1987) 432-441.

[90] S.W. Russell, T.E. Levine, A.E. Bair, T.L. Alford, Guidelines to the application of nuclear resonance to quantitative thin film analysis, Nucl. Instrum. Methods Phys. Res. B 118 (1996) 201-205.

[91] H. Iwai, M. Okada, K. Fukutani, Y. Murata, Chlorine-induced de-reconstruction on $\mathrm{Au}(001)$ and $\mathrm{Cl}$-adsorbed layers, J. Phys.: Condensed Matter 7 (1995) 5163-5176.

[92] F. Ajzenberg, T. Lauritsen, Energy Levels of Light Nuclei. IV, Rev. Mod. Phys. 24 (1952) 321-402.

[93] F. Ajzenberg, T. Lauritsen, Energy Levels of Light Nuclei. V, Rev. Mod. Phys. 27 (1955) 77166.

[94] Compilation series "Energy Levels of Light Nuclei, A = 3 - 20" by F. Ajzenberg-Selove, published in Nuclear Physics A (1959-1991), available at http://www.tunl.duke.edu/nucldata/index.shtml (last accessed: August 5, 2014)

[95] M. Zinke-Allmang, S. Kalbitzer, M. Weiser, Vibrational Doppler-broadening of inverse $p$, $\gamma$ reactions on ${ }^{1} \mathrm{H}$ bearing targets, Z. Physik A 320 (1985) 697-698.

[96] G. Amsel, B. Maurel, High resolution techniques for nuclear reaction narrow resonance width measurements and for shallow depth profiling, Nucl. Instrum. Methods Phys. Res. 218 (1983) 183-196.

[97] B. Maurel, G. Amsel, A new measurement of the $429 \mathrm{keV}^{15} \mathrm{~N}(\mathrm{p}, \alpha \gamma){ }^{12} \mathrm{C}$ resonance. Applications of the very narrow width found to ${ }^{15} \mathrm{~N}$ and ${ }^{1} \mathrm{H}$ depth location: I. Resonance width measurement, Nucl. Instrum. Methods Phys. Res. 218 (1983) 159-164.

[98] H. Damjantschitsch, M. Weiser, G. Heusser, S. Kalbitzer, H. Mannsperger, An in-beamline low-level system for nuclear reaction $\gamma$-rays, Nucl. Instrum. Methods Phys. Res. 218 (1983) 129-140.

[99] P. Trocellier, C. Engelmann, Determination de la fonction d'excitation de la reaction nucleaire ${ }^{1} \mathrm{H}\left({ }^{15} \mathrm{~N}, \alpha \gamma\right){ }^{12} \mathrm{C}$ entre 6 et $14 \mathrm{MeV}$, J. Radioanal. Chem. 77 (1983) 149-157.

[100] K.M. Horn, W.A. Lanford, Measurement of the off-resonance cross section of the 6.4 $\mathrm{MeV}{ }^{1} \mathrm{H}\left({ }^{15} \mathrm{~N}, \alpha \gamma\right){ }^{12} \mathrm{C}$ nuclear reaction, Nucl. Instrum. Methods Phys. Res. B 34 (1988) 1-8.

[101] D.R. Tilley, H.R. Weller, C.M. Cheves, Energy levels of light nuclei $A=16-17$, Nucl. Phys. A 564 (1993) 1-183.

[102] F. Ajzenberg-Selove, Energy levels of light nuclei A = 11-12, Nucl. Phys. A 506 (1990) 1158.

[103] J.F. Ziegler, J.P. Biersack, U. Littmark, Stopping and Ranges of lons in Solids, Pergamon, New York, 1985.

[104] J.F. Ziegler, Handbook of Stopping Cross-Sections for Energetic lons in All Elements Pergamon, New York, 1980.

[105] N. Bohr, K. Dan. Vidensk. Selsk. Mat.-Fys. Medd. 18 (1948).

[106] N. Rud, J. Bøttiger, P.S. Jensen, Measurements of energy-loss distributions for $6.5 \mathrm{MeV}$ ${ }^{15} \mathrm{~N}$ ions in solids, Nucl. Instrum. Methods 151 (1978) 247-252.

[107] M.A. Briere, D. Bräunig, A quantitative investigation of hydrogen in the metal-oxidesilicon system using NRA, IEEE Transactions on Nuclear Science 37 (1990) 1658-1669. [108] B. Hartmann, S. Kalbitzer, C. Klatt, Energy widths of ion beams from a tandem accelerator, Nucl. Instrum. Methods Phys. Res. B 113 (1996) 50-53.

[109] A. Van Wieringen, N. Warmoltz, On the permeation of hydrogen and helium in single crystal silicon and germanium at elevated temperatures, Physica 22 (1956) 849-865. 
[110] M. Wilde, K. Fukutani, Evaluation of non-resonant background in hydrogen depth profiling via ${ }^{1} \mathrm{H}\left({ }^{15} \mathrm{~N}, \alpha \gamma\right){ }^{12} \mathrm{C}$ nuclear reaction analysis near $13.35 \mathrm{MeV}$, Nucl. Instrum. Methods Phys. Res. B 232 (2005) 280-284.

[111] B. Hjörvarsson, J. Rydén, Off-resonance and straggling measurements using the ${ }^{1} \mathrm{H}\left({ }^{15} \mathrm{~N}\right.$, $\alpha$ p) ${ }^{12} \mathrm{C}$ reaction, Nucl. Instrum. Methods Phys. Res. B 45 (1990) 36-40.

[112] G.J. Clark, C.W. White, D.D. Allred, B.R. Appleton, F.B. Koch, C.W. Magee, Application of nuclear-reactions for quantitative hydrogen analysis in a variety of different materials problems, Nucl. Instrum. Methods 149 (1978) 9-18.

[113] K. Umezawa, A. Kitamura, S. Yano, Hydrogen depth profiling using the $6.46 \mathrm{MeV}^{1} \mathrm{H}\left({ }^{19} \mathrm{~F}\right.$, $\alpha \gamma)^{16} \mathrm{O}$ resonance by detection of alpha particles, Nucl. Instrum. Methods Phys. Res. B 28 (1987) 377-384.

[114] K. Umezawa, T. Kuroi, J. Yamane, F. Shoji, K. Oura, T. Hanawa, S. Yano, Quantitative hydrogen analysis by simultaneous detection of ${ }^{1} \mathrm{H}\left({ }^{19} \mathrm{~F}, \alpha \gamma\right){ }^{16} \mathrm{O}$ at $6.46 \mathrm{MeV}$ and ${ }^{19} \mathrm{~F}$-ERDA, Nucl. Instrum. Methods Phys. Res. B 33 (1988) 634-637.

[115] C. Ascheron, D. Lehmann, C. Neelmeijer, A. Schindler, F. Bigl, Hydrogen depth profiling in bevelled proton-implanted semiconductors by ${ }^{4} \mathrm{He}$ ERDA and ${ }^{19} \mathrm{~F}$ NRA, Nucl. Instrum.

Methods Phys. Res. B 63 (1992) 412-420.

[116] K. Sasa, H. Ohshima, Y. Yamato, T. Komatsubara, T. Katabuchi, K. Shima, K. Furuno, M. Kurosawa, N. Yanagisawa, Hydrogen analysis of mineral samples at University of Tsukuba, Nucl. Instrum. Methods Phys. Res. B 190 (2002) 287-290.

[117] D.A. Leich, T.A. Tombrello, A technique for measuring hydrogen concentration versus depth in solid samples, Nucl. Instrum. Methods 108 (1973) 67-71.

[118] H.W. Becker, M. Bahr, M. Berheide, L. Borucki, M. Buschmann, C. Rolfs, G. Roters, S. Schmidt, W.H. Schulte, G.E. Mitchell, J.S. Schweitzer, Hydrogen depth profiling using ${ }^{18} \mathrm{O}$ ions, Z. Physik A 351 (1995) 453-465.

[119] P.P. Pronko, J.G. Pronko, Depth profiling of ${ }^{3} \mathrm{He}$ and ${ }^{2} \mathrm{H}$ in solids using the ${ }^{3} \mathrm{He}(\mathrm{d}, \mathrm{p})^{4} \mathrm{He}$ resonance, Phys. Rev. B 9 (1974) 2870-2878.

[120] D. Dieumegard, D. Dubreuil, G. Amsel, Analysis and depth profiling of deuterium with the $\mathrm{D}\left({ }^{3} \mathrm{He}, \mathrm{p}\right)^{4} \mathrm{He}$ reaction by detecting the protons at backward angles, Nucl. Instrum.

Methods 166 (1979) 431-445.

[121] W. Möller, F. Besenbacher, A note on the ${ }^{3} \mathrm{He}+\mathrm{D}$ nuclear-reaction cross section, Nucl. Instrum. Methods 168 (1980) 111-114.

[122] W.R. Wampler, Trapping of deuterium in beryllium, J. Nucl. Mater. 196-198 (1992) 981-985.

[123] J.A. Davies, P.R. Norton, Absolute coverage measurement of adsorbed $C O$ and $D_{2}$ on platinum, Nucl. Instrum. Methods 168 (1980) 611-615.

[124] W.A. Lanford, Hydrogen Nuclear Reaction Data, in: J.R. Tesmer, M. Nastasi (Eds.) Handbook of Modern Ion Beam Materials Analysis, Materials Research Society, Pittsburgh, PA, 1995, pp. 615-618.

[125] J.F. Ziegler, SRIM-2003, Nucl. Instrum. Methods Phys. Res. B 219-220 (2004) 10271036.

[126] J.F. Ziegler, M.D. Ziegler, J.P. Biersack, SRIM - The stopping and range of ions in matter (2010), Nucl. Instrum. Methods Phys. Res. B 268 (2010) 1818-1823.

[127] J. Ziegler, SRIM-2008.

[128] See www.SRIM.org (last accessed: August 5, 2014).

[129] H. Paul, Stopping Power for Light and Heavier lons,

http://www.exphys.jku.at/stopping/ (last accessed: August 5, 2014).

[130] E. Rauhala, Energy Loss, in: J.R. Tesmer, M. Nastasi (Eds.) Handbook of Modern Ion Beam Materials Analysis, Materials Research Society, Pittsburgh, PA, 1995, p. 3.

[131] N. Bohr, II. On the theory of the decrease of velocity of moving electrified particles on passing through matter, Philos. Mag. 25 (1913) 10-31. 
[132] N. Bohr, LX. On the decrease of velocity of swiftly moving electrified particles in passing through matter, Philos. Mag. 30 (1915) 581-612.

[133] H. Bethe, Zur Theorie des Durchgangs schneller Korpuskularstrahlen durch Materie, Annalen der Physik 397 (1930) 325-400.

[134] F. Bloch, Bremsvermögen von Atomen mit mehreren Elektronen, Z. Physik 81 (1933) 363-376.

[135] F. Bloch, Zur Bremsung rasch bewegter Teilchen beim Durchgang durch Materie, Annalen der Physik 408 (1933) 285-320.

[136] J. Lindhard, M. Scharff, H.E. Schiott, Kgl. Danske Vid. Selsk. Mat.-Fys. Medd. 33 (1963) 1.

[137] O.B. Firsov, Zh. Eksp. Teor. Fiz. 36 (1959) 1517.

[138] S. Jans, P. Oberschachtsiek, S. Kalbitzer, M. Behar, Experimental evidence for ${ }^{15} \mathrm{~N}$ charge-change processes at a low energy regime, Nucl. Instrum. Methods Phys. Res. B 83 (1993) 1-4.

[139] W.H. Bragg, R. Kleeman, XXXIX. On the $\alpha$ particles of radium, and their loss of range in passing through various atoms and molecules, Philos. Mag. 10 (1905) 318-340.

[140] J.F. Ziegler, J.M. Manoyan, The stopping of ions in compounds, Nucl. Instrum. Methods Phys. Res. B 35 (1988) 215-228.

[141] D.I. Thwaites, Departures from Bragg's rule of stopping power additivity for ions in dosimetric and related materials, Nucl. Instrum. Methods Phys. Res. B 69 (1992) 53-63.

[142] L. Leblanc, G.G. Ross, P.A. Gollier, P. Bertrand, Measured stopping cross sections for helium in a-Si $\mathrm{C}_{1-\mathrm{x}}: \mathrm{H}$ compounds near their maximum values, Nucl. Instrum. Methods Phys. Res. B 111 (1996) 17-21.

[143] H. Paul, A. Schinner, Statistical analysis of stopping data for protons and alphas in compounds, Nucl. Instrum. Methods Phys. Res. B 249 (2006) 1-5.

[144] H. Paul, New developments in stopping power for fast ions, Nucl. Instrum. Methods Phys. Res. B 261 (2007) 1176-1179.

[145] H. Paul, The solid-gas difference in stopping powers, and statistical analysis of stopping power data, Nucl. Instrum. Methods Phys. Res. B 267 (2009) 9-14.

[146] M.S. Livingston, H.A. Bethe, Rev. Mod. Phys. 9 (1937) 245-390.

[147] J. Lindhard, M. Scharff, Kgl. Danske Vid. Selsk. Mat.-Fys. Medd. 27 (1953) 1.

[148] P.V. Vavilov, Sov. Phys. JETP 5(1957) 749.

[149] P. Hvelplund, Kgl. Danske Vid. Selsk. Mat.-Fys. Medd, 38 (1971) 1.

[150] M.A. Briere, J.P. Biersack, Energy loss straggling of $\mathrm{MeV}$ ions in thin solid films, Nucl. Instrum. Methods Phys. Res. B 64 (1992) 693-700.

[151] P. Goppelt-Langer, S. Yamamoto, Y. Aoki, H. Takeshita, H. Naramoto, Stopping-powers and straggling of ${ }^{15} \mathrm{~N}$ ions for nuclear reaction analysis at $6.385 \mathrm{MeV}$, Nucl. Instrum. Methods Phys. Res. B 118 (1996) 7-10.

[152] G. Amsel, J.P. Nadai, E. D'Artemare, D. David, E. Girard, J. Moulin, Microanalysis by the direct observation of nuclear reactions using a $2 \mathrm{MeV}$ Van de Graaff, Nucl. Instrum. Methods 92 (1971) 481-498.

[153] G. Amsel, E. d'Artemare, E. Girard, A simple, digitally controlled, automatic, hysteresis free, high precision energy scanning system for Van de Graaff type accelerators: Part I: Principle, results and applications, Nucl. Instrum. Methods Phys. Res. 205 (1983) 5-26.

[154] G. Amsel, E. d'Artemare, G. Battistig, E. Girard, L.G. Gosset, P. Révész, Narrow nuclear resonance position or cross section shape measurements with a high precision computer controlled beam energy scanning system, Nucl. Instrum. Methods Phys. Res. B 136-138 (1998) 545-550.

[155] E. Brauer, R. Doerr, R. Gruner, F. Rauch, A nuclear-physics method for the determination of hydrogen diffusion-coefficients, Corrosion Sci. 21 (1981) 449-457. 
[156] E. Brauer, R. Gruner, F. Rauch, Kinetics and Mechanism of Hydrogen Diffusion in Hydrides of Titanium, Zirconium and TiNio.5, Ber. Bunsenges. Phys. Chem. 87 (1983) 341-345. [157] M. Zinke-Allmang, V. Kössler, S. Kalbitzer, Improved nuclear reaction analysis for solid state research by automatic energy scanning, Nucl. Instrum. Methods Phys. Res. B 15 (1986) 563-568.

[158] P. Oberschachtsiek, M. Weiser, S. Kalbitzer, A 3-MV Pelletron for materials research at Heidelberg, Nucl. Instrum. Methods Phys. Res. B 56-57 (1991) 1010-1013.

[159] F. Herrmann, D. Grambole, The new Rossendorf nuclear microprobe, Nucl. Instrum. Methods Phys. Res. B 104 (1995) 26-30.

[160] M.A. Briere, F. Wulf, D. Bräunig, Measurements of the accumulation of hydrogen at the silicon-silicon-dioxide interface using nuclear reaction analysis, Nucl. Instrum. Methods Phys. Res. B 45 (1990) 45-48.

[161] J. Krauser, F. Wulf, D. Bräunig, Measurement and analysis of hydrogen depth profiles in MOS-structures by using the ${ }^{15} \mathrm{~N}$ nuclear reaction method, J. Non-Crystalline Solids 187 (1995) 264-269.

[162] M. Fallavier, M.Y. Chartoire, J.P. Thomas, UHV equipment for in situ studies of hydrogen interaction with materials based on NRA, Nucl. Instrum. Methods Phys. Res. B 15 (1986) 712-717.

[163] J.C. Davis, The LLNL multi-user tandem laboratory, Nucl. Instrum. Methods Phys. Res. B 40-41 (1989) 705-708.

[164] M.L. Roberts, T.L. Moore, R.S. Hornady, J.C. Davis, Distributed computer control at the LLNL tandem, Nucl. Instrum. Methods Phys. Res. B 54 (1991) 1-6.

[165] A. Polman, A.M. Vredenberg, W.H. Urbanus, P.J. van Deenen, H. Alberda, H. Krop, I. Attema, E. De Haas, H. Kersten, S. Doorn, J. Derks, J. Ter Beek, S. Roorda, R. Schreutelkamp, J.G. Bannenberg, F.W. Saris, A MeV facility for materials research, Nucl. Instrum. Methods Phys. Res. B 37-38 (1989) 935-940.

[166] M.A. Pick, A. Hanson, K.W. Jones, A.N. Goland, Depth-concentration profile of hydrogen in niobium, Phys. Rev. B 26 (1982) 2900-2906.

[167] B. Hjörvarsson, J. Rydén, E. Karlsson, J. Birch, J.E. Sundgren, Interface effects of hydrogen uptake in Mo/V single-crystal superlattices, Phys. Rev. B 43 (1991) 6440-6445. [168] H.H. Andersen, P. Hornsh øj, L. Højsholt-poulsen, H. Knudsen, B.R. Nielsen, R. Stensgaard, A simple energy-calibration procedure for electrostatic accelerators, Nucl. Instrum. Methods 136 (1976) 119-124.

[169] M. Berheide, H.W. Becker, M. Buschmann, N. Piel, C. Rolfs, W.H. Schulte, G.E. Mitchell, J.S. Schweitzer, A new UHV setup for investigations of narrow nuclear resonances with high energy resolution ion beams, Nucl. Instrum. Methods Phys. Res. B 99 (1995) 289-292.

[170] S. Kubsky, L. Borucki, M. Berheide, S. Baier, H.W. Becker, F. Gorris, C. Grunwald, T. Gutt, G. Krüger, M. Mehrhoff, N. Piel, W.H. Schulte, C. Rolfs, Concept and status of the new sample preparation and analyzing facility at Bochum, Nucl. Instrum. Methods Phys. Res. B 113 (1996) 63-66.

[171] S. Kubsky, L. Borucki, F. Gorris, H.W. Becker, C. Rolfs, W.H. Schulte, I.J.R. Baumvol, F.C. Stedile, Interconnected UHV facilities for materials preparation and analysis, Nucl. Instrum. Methods Phys. Res. A 435 (1999) 514-522.

[172] M. Schwickert, F. Harbsmeier, H. Schebela, M. Uhrmacher, E. Carpene, P. Schaaf, K.P. Lieb, Nitrogen and hydrogen depth profiling with MaRPel, Surf. Coat. Technol. 151-152 (2002) 222-226.

[173] M. Uhrmacher, M. Schwickert, H. Schebela, K.P. Lieb, Miss MaRPel - a 3 MV pelletron accelerator for hydrogen depth profiling, J. Alloys Compds. 404-406 (2005) 307-311.

[174] S. Yamamoto, P. Goppelt-Langer, H. Naramoto, Y. Aoki, H. Takeshita, Analysis of hydrogen in Nb/Cu multilayers using ion beams, J. Alloys Compds. 231 (1995) 310-314. 
[175] I.J.R. Baumvol, Atomic transport during growth of ultrathin dielectrics on silicon, Surf. Sci. Rep. 36 (1999) 1-166.

[176] R.M.C. de Almeida, I.J.R. Baumvol, Reaction-diffusion in high-k dielectrics on Si, Surf. Sci. Rep. 49 (2003) 1-114.

[177] MALT, http://malt.n.t.u-tokyo.ac.jp/index.html (last accessed: August 5, 2014).

[178] B. Hartmann, S. Kalbitzer, C. Klatt, Energy spread of ion beams passing a gas stripper, Nucl. Instrum. Methods Phys. Res. B 124 (1997) 490-499.

[179] J.P. Bugeat, E. Ligeon, Influence of ion beam bombardment in hydrogen surface layer analysis, Nucl. Instrum. Methods 159 (1979) 117-124.

[180] J.P. Thomas, M. Fallavier, C. Pijolat, J. Tousset, A model for chemical effects induced by $\mathrm{MeV}$ ion beams on hydrogenated species at the surface of solid materials, Radiat. Effects 61 (1982) 207-214.

[181] W.H. Schulte, H. Ebbing, H.W. Becker, M. Berheide, M. Buschmann, C. Angulo, C. Rolfs, G. Amsel, I. Trimaille, G. Battistig, G.E. Mitchell, J.S. Schweitzer, High resolution depth profiling in near-surface regions of solids by narrow nuclear reaction resonances below 0.5 MeV with low energy spread proton beams, Vacuum 44 (1993) 185-190.

[182] K.M. Horn, W.A. Lanford, Suppression of background radiation in BGO and Nal detectors used in nuclear reaction analysis, Nucl. Instrum. Methods Phys. Res. B 45 (1990) 256-259.

[183] Z.H. Cho, M.R. Farukhi, Bismuth Germanate as a Potential Scintillation Detector in Positron Cameras, J. Nucl. Med. 18 (1977) 840-844.

[184] Saint Gobain Crystals, Efficiency Calculations for Selected Scintillators, 2014-06, http://www.crystals.saint-gobain.com/Scintillation_Materials.aspx (last accessed: August 5, 2014).

[185] D. Kuhn, F. Rauch, H. Baumann, A low-background detection system using a BGO detector for sensitive hydrogen analysis with the ${ }^{1} \mathrm{H}\left({ }^{15} \mathrm{~N}, \alpha \gamma\right){ }^{12} \mathrm{C}$ reaction, Nucl. Instrum. Methods Phys. Res. B 45 (1990) 252-255.

[186] D. Endisch, H. Sturm, F. Rauch, Nuclear reaction analysis of hydrogen at levels below 10 at. ppm, Nucl. Instrum. Methods Phys. Res. B 84 (1994) 380-392.

[187] N. Piel, W.H. Schulte, M. Berheide, H.W. Becker, L. Borucki, C. Grama, M. Mehrhoff, C. Rolfs, Efficient $p$-ray detection in ion beam analysis, Nucl. Instrum. Methods Phys. Res. B 118 (1996) 186-189.

[188] R.L. Zimmerman, D. Ila, J.K. Hirvonen, Optimized system for hydrogen detection, Nucl. Instrum. Methods Phys. Res. B 118 (1996) 206-208.

[189] M. Mehrhoff, M. Aliotta, I.J.R. Baumvol, H.W. Becker, M. Berheide, L. Borucki, J. Domke, F. Gorris, S. Kubsky, N. Piel, G. Roters, C. Rolfs, W.H. Schulte, Gamma-ray detection with a $4 \pi \mathrm{Nal}$ spectrometer for material analysis, Nucl. Instrum. Methods Phys. Res. B 132 (1997) 671-684.

[190] O. Dersch, M. Laube, F. Rauch, Nuclear reaction analysis of hydrogen in Li-containing material, Nucl. Instrum. Methods Phys. Res. B 118 (1996) 180-185.

[191] N.A. Bostrom, E.L. Hudspeth, I.L. Morgan, Deuteron-Induced Reactions Leading to $\mathrm{N}^{16}$, Phys. Rev. 105 (1957) 1545-1548.

[192] F. Ajzenberg-Selove, Energy levels of light nuclei A = 16-17, Nuclear Physics A, 166 (1971) 1-139.

[193] J.C. Barbour, B.L. Doyle, Elastic Recoil Detection: ERD, in: J.R. Tesmer, M. Nastasi (Eds.) Handbook of Modern Ion Beam Materials Analysis, Materials Research Society, Pittsburgh, PA, 1995, p. 83.

[194] A.A. Kraus, Jr., A.P. French, W.A. Fowler, C.C. Lauritsen, Angular Distribution of Gamma-Rays and Short-Range Alpha-Particles from N ${ }^{15}(p, \alpha \gamma) C^{12}$, Phys. Rev. 89 (1953) 299301. 
[195] M.E. Adel, O. Amir, R. Kalish, L.C. Feldman, lon-beam-induced hydrogen release from a-C:H: A bulk molecular recombination model, J. Appl. Phys. 66 (1989) 3248-3251.

[196] J. Tirira, P. Trocellier, M. Mosbah, N. Metrich, Study of hydrogen content in solids by ERDA and radiation-induced damage, Nucl. Instrum. Methods Phys. Res. B 56-57 (1991) 839842.

[197] S. Jans, S. Kalbitzer, P. Oberschachtsiek, M. Behar, Radiolytic effects in organic compounds induced and analyzed by a ${ }^{15} \mathrm{~N}$ beam, J. Mater. Res. 9 (1994) 1596-1603. [198] F. Abel, V. Quillet, M. Schott, Degradation of polystyrene thin films under d, ${ }^{4} \mathrm{He}$ and ${ }^{12} \mathrm{C}$ irradiation studied by ion beam analysis: effects of energy loss, sample thickness and isotopic content, Nucl. Instrum. Methods Phys. Res. B 105 (1995) 86-90.

[199] M.P.d. Jong, A.J.H. Maas, L.J.v. Ijzendoorn, S.S. Klein, M.J.A.d. Voigt, A model for ionirradiation induced hydrogen loss from organic materials, J. Appl. Phys. 82 (1997) 1058-1064. [200] S.R. Walker, J.A. Davies, J.S. Forster, S.G. Wallace, A.C. Kockelkoren, Radiation damage during heavy ion elastic recoil detection analysis of insulating materials, Nucl. Instrum. Methods Phys. Res. B 136-138 (1998) 707-712.

[201] G.G. Ross, I. Richard, Influence of the ion beam induced desorption on the quantitative depth profiling of hydrogen in a variety of materials, Nucl. Instrum. Methods Phys. Res. B 64 (1992) 603-607.

[202] E. Balanzat, S. Bouffard, A. Bouquerel, J. Devy, C. Gaté, Swift heavy ion irradiation of polystyrene, Nucl. Instrum. Methods Phys. Res. B 116 (1996) 159-163.

[203] K. Fukutani, M. Tsunoda, Y. Murata, H. Yamashita, K. Komaki, K. Kobayashi, Highresolution depth analysis of hydrogen by a resonance nuclear reaction: $a-\mathrm{Si} / \mathrm{H} / \mathrm{Si}(001)$, Surf. Sci. 283 (1993) 447-451.

[204] L. Westerberg, L.E. Svensson, E. Karlsson, M.W. Richardson, K. Lundström, Stable absolute calibration standards for hydrogen profile analysis using nuclear resonance techniques, Nucl. Instrum. Methods Phys. Res. B 9 (1985) 49-54.

[205] J.P. Hirvonen, R. Lappalainen, Particle-Gamma-Data, in: J.R. Tesmer, M. Nastasi (Eds.) Handbook of Modern Ion Beam Materials Analysis, Materials Research Society, Pittsburgh, PA, 1995, p. 590.

[206] B. Hjörvarsson, J. Rydén, T. Ericsson, E. Karlsson, Hydrogenated tantalum: A convenient calibration substance for hydrogen profile analysis using nuclear resonance reactions, Nucl. Instrum. Methods Phys. Res. B 42 (1989) 257-263.

[207] H.J. Whitlow, J. Keinonen, M. Hautala, A. Hautojärvi, Thermal- and radiation-stability of hydrogen-implanted silicon standards for ion-beam analysis, Nucl. Instrum. Methods Phys.

Res. B 5 (1984) 505-510.

[208] U. Reinholz, W. Bremser, K.-W. Brzezinka, E. Strub, H.-P. Weise, S. Merchel, A thinlayer reference material for hydrogen analysis, Nucl. Instrum. Methods Phys. Res. B 266 (2008) 2418-2423.

[209] G. Boudreault, R.G. Elliman, R. Grötzschel, S.C. Gujrathi, C. Jeynes, W.N. Lennard, E. Rauhala, T. Sajavaara, H. Timmers, Y.Q. Wang, T.D.M. Weijers, Round Robin: measurement of $\mathrm{H}$ implantation distributions in Si by elastic recoil detection, Nucl. Instrum. Methods Phys. Res. B 222 (2004) 547-566.

[210] C. Driemeier, R.P. Pezzi, I.J.R. Baumvol, Nuclear reaction analysis of ${ }^{1} \mathrm{H}$ and ${ }^{2} \mathrm{H}$ in hafnium silicate films on Si, Nucl. Instrum. Methods Phys. Res. B 266 (2008) 1824-1827. [211] W. Rudolph, C. Bauer, K. Brankoff, D. Grambole, R. Grötzschel, C. Heiser, F. Herrmann, Plastic foils as primary hydrogen standards for nuclear reaction analysis, Nucl. Instrum. Methods Phys. Res. B 15 (1986) 508-511.

[212] H. Yonemura, Y. Kitaoka, D. Sekiba, H. Matsuzaki, S. Ogura, M. Matsumoto, Y. Iwamura, T. Ito, T. Narusawa, K. Fukutani, Depth profiling of hydrogen under an atmospheric pressure, Nucl. Instrum. Methods Phys. Res. B 269 (2011) 632-635.

[213] J. Ziegler, Ion Implantation Technology, Elsevier, Amsterdam, 1992. 
[214] F. Landry, P. Schaaf, Simulation and deconvolution program WinRNRA for depth profiling of light elements via nuclear resonance reactions, Nucl. Instrum. Methods Phys. Res. B 179 (2001) 262-266.

[215] B. Maurel, G. Amsel, J.P. Nadai, Depth profiling with narrow resonances of nuclear reactions: Theory and experimental use, Nucl. Instrum. Methods Phys. Res. 197 (1982) 1-13. [216] G. Vizkelethy, Simulation and evaluation of nuclear reaction spectra, Nucl. Instrum. Methods Phys. Res. B 45 (1990) 1-5.

[217] I. Vickridge, G. Amsel, SPACES: A PC implementation of the stochastic theory of energy loss for narrow-resonance depth profiling, Nucl. Instrum. Methods Phys. Res. B 45 (1990) 611.

[218] G. Amsel, I. Vickridge, Analytic calculation for some useful depth profiles of the linear expansion coefficients used in SPACES, Nucl. Instrum. Methods Phys. Res. B 45 (1990) 12-15. [219] A.D. Marwick, J.C. Liu, T.H. Zabel, J.P. Doyle, Use of simulations of ${ }^{15} \mathrm{~N}$ profiling in studying hydrogen segregation at interfaces, Nucl. Instrum. Methods Phys. Res. B 64 (1992) 408-412.

[220] M. Wilde, K. Fukutani, Low-temperature growth of Au on H-terminated Si(111): Instability of hydrogen at the $\mathrm{Au} / \mathrm{Si}$ interface revealed by non-destructive ultra-shallow $\mathrm{H}$ depth profiling, Jpn. J. Appl. Phys. 42 (2003) 4650-4654.

[221] E. Szilágyi, F. Pászti, Theoretical calculation of the depth resolution of IBA methods, Nucl. Instrum. Methods Phys. Res. B 85 (1994) 616-620.

[222] E. Szilágyi, F. Pászti, G. Amsel, Theoretical approximations for depth resolution calculations in IBA methods, Nucl. Instrum. Methods Phys. Res. B 100 (1995) 103-121.

[223] L.R. Doolittle, Algorithms for the rapid simulation of Rutherford backscattering spectra, Nucl. Instrum. Methods Phys. Res. B 9 (1985) 344-351.

[224] E. Kótai, Computer methods for analysis and simulation of RBS and ERDA spectra, Nucl. Instrum. Methods Phys. Res. B 85 (1994) 588-596.

[225] M. Mayer, SIMNRA, a Simulation Program for the Analysis of NRA, RBS and ERDA, in: J.L. Duggan, I.L. Morgan (Eds.) 15th International Conference on the Application of Accelerators in Research and Industry, American Institute of Physics, 1999, p. 541.

[226] M. Mayer, SIMNRA User's Guide, Tech. Rep., Max-Planck-Institut für Plasmaphysik, Garching, 1997, http://home.rzg.mpg.de/ mam/ (last accessed: August 5, 2014).

[227] C. Jeynes, N.P. Barradas, P.K. Marriott, G. Boudreault, M. Jenkin, E. Wendler, R.P. Webb, Elemental thin film depth profiles by ion beam analysis using simulated annealing - a new tool, J. Phys. D 36 (2003) R97.

[228] N.P. Barradas, R. Mateus, M. Fonseca, M.A. Reis, K. Lorenz, I. Vickridge, Thin film depth profiling using simultaneous particle backscattering and nuclear resonance profiling, Nucl. Instrum. Methods Phys. Res. B 268 (2010) 1829-1832.

[229] E. Rauhala, N.P. Barradas, S. Fazinic, M. Mayer, E. Szilágyi, M. Thompson, Status of ion beam data analysis and simulation software, Nucl. Instrum. Methods Phys. Res. B 244 (2006) 436-456.

[230] N.P. Barradas, K. Arstila, G. Battistig, M. Bianconi, N. Dytlewski, C. Jeynes, E. Kótai, G. Lulli, M. Mayer, E. Rauhala, E. Szilágyi, M. Thompson, International Atomic Energy Agency intercomparison of ion beam analysis software, Nucl. Instrum. Methods Phys. Res. B 262 (2007) 281-303.

[231] N.P. Barradas, K. Arstila, G. Battistig, M. Bianconi, N. Dytlewski, C. Jeynes, E. Kótai, G. Lulli, M. Mayer, E. Rauhala, E. Szilágyi, M. Thompson, Summary of "IAEA intercomparison of IBA software", Nucl. Instrum. Methods Phys. Res. B 266 (2008) 1338-1342.

[232] B.L. Cohen, C.L. Fink, J.H. Degnan, Nondestructive Analysis for Trace Amounts of Hydrogen, J. Appl. Phys. 43 (1972) 19-25. 
[233] J. L'Ecuyer, C. Brassard, C. Cardinal, J. Chabbal, L. Deschenes, J.P. Labrie, B. Terreault, J.G. Martel, R. St.-Jacques, An accurate and sensitive method for the determination of the depth distribution of light elements in heavy materials, J. Appl. Phys. 47 (1976) 381-382. [234] B.L. Doyle, P.S. Peercy, Technique for profiling ${ }^{1} \mathrm{H}$ with $2.5-\mathrm{MeV}$ Van de Graaff accelerators, Appl. Phys. Lett. 34 (1979) 811-813.

[235] F. Pászti, E. Kótai, G. Mezey, A. Manuaba, L. Pócs, D. Hildebrandt, H. Strusny, Hydrogen and deuterium measurements by elastic recoil detection using alpha particles, Nucl. Instrum. Methods Phys. Res. B 15 (1986) 486-491.

[236] M. Naitoh, H. Morioka, F. Shoji, K. Oura, Coadsorption of hydrogen and deuterium on Si(100) surfaces studied by elastic recoil detection analysis, Surf. Sci. 297 (1993) 135-140. [237] O. Kenjiro, N. Masamichi, M. Hajime, W. Michio, S. Fumiya, Elastic recoil detection analysis of coadsorption of hydrogen and deuterium on clean Si surfaces, Nucl. Instrum. Methods Phys. Res. B 85 (1994) 344-346.

[238] O. Kruse, H.D. Carstanjen, High depth resolution ERDA of $H$ and $D$ by means of an electrostatic spectrometer, Nucl. Instrum. Methods Phys. Res. B 89 (1994) 191-199.

[239] K. Kimura, K. Nakajima, M. Mannami, Some applications of high-resolution RBS and ERD using a magnetic spectrometer, Nucl. Instrum. Methods Phys. Res. B 136 (1998) 11961202.

[240] B.L. Doyle, D.K. Brice, The analysis of elastic recoil detection data, Nucl. Instrum. Methods Phys. Res. B 35 (1988) 301-308.

[241] D. David, New trends in ion-beam analysis, Surf. Sci. Rep. 16 (1992) 333-375.

[242] F.H.P.M. Habraken, Light element depth profiling using elastic recoil detection, Nucl. Instrum. Methods Phys. Res. B 68 (1992) 181-189.

[243] W.M. Arnoldbik, C.H.M. Marée, A.J.H. Maas, M.J. van den Boogaard, F.H.P.M. Habraken, A.E.T. Kuiper, Dynamic behavior of hydrogen in silicon nitride and oxynitride films made by low-pressure chemical vapor deposition, Phys. Rev. B 48 (1993) 5444-5456.

[244] D.K. Avasthi, Developments in nuclear techniques for hydrogen depth profiling, Bulletin Mater. Sci. 19 (1996) 3.

[245] J. Tirira, Y. Serruys, P. Trocellier, in: Forward Recoil Spectrometry, Plenum Press, New York, NY, 1996, p. 1.

[246] R.J. Composto, R.M. Walters, J. Genzer, Application of ion scattering techniques to characterize polymer surfaces and interfaces, Mater. Sci. Eng. R 38 (2002) 107-180.

[247] G. Dollinger, T. Faestermann, P. Maier-Komor, High resolution depth profiling of light elements, Nucl. Instrum. Methods Phys. Res. B 64 (1992) 422-427.

[248] J.A. Davies, J.S. Forster, S.R. Walker, Elastic recoil detection analysis with heavy ion beams, Nucl. Instrum. Methods Phys. Res. B 136-138 (1998) 594-602.

[249] K. Kimura, K. Nakajima, H. Imura, Hydrogen depth profiling with sub-nm resolution in high-resolution ERD, Nucl. Instrum. Methods Phys. Res. B 140 (1998) 397-401.

[250] G. Dollinger, Elastic recoil detection analysis with atomic depth resolution, Nucl. Instrum. Methods Phys. Res. B 79 (1993) 513-517.

[251] L. Wielunski, R. Benenson, K. Horn, W.A. Lanford, High-sensitivity hydrogen analysis using elastic recoil, Nucl. Instrum. Methods Phys. Res. B 15 (1986) 469-474.

[252] M. Copel, R.M. Tromp, Elastic recoil detection for medium-energy ion scattering, Rev. Sci. Instrum. 64 (1993) 3147-3152.

[253] H.D. Carstanjen, Ion beam analysis with monolayer depth resolution, Nucl. Instrum. Methods Phys. Res. B 136-138 (1998) 1183-1190.

[254] W.M. Arnoldbik, W. Wolfswinkel, D.K. Inia, V.C.G. Verleun, S. Lobner, J.A. Reinders, F. Labohm, D.O. Boerma, A high resolution magnetic spectrograph for ion beam analysis, Nucl. Instrum. Methods Phys. Res. B 118 (1996) 566-572. 
[255] K. Kimura, K. Nakajima, S. Yamanaka, M. Hasegawa, H. Okushi, Hydrogen depthprofiling in chemical-vapor-deposited diamond films by high-resolution elastic recoil detection, Appl. Phys. Lett. 78 (2001) 1679-1681.

[256] F. Schiettekatte, A. Chevarier, N. Chevarier, A. Plantier, G.G. Ross, Quantitative depth profiling of light elements by means of the ERD E $\times$ B technique, Nucl. Instrum. Methods Phys. Res. B 118 (1996) 307-311.

[257] R.J. Sweeney, V.M. Prozesky, C.L. Churms, J. Padayachee, K. Springhorn, Application of a $\triangle E-E$ telescope for sensitive ERDA measurement of hydrogen, Nucl. Instrum. Methods Phys. Res. B 136-138 (1998) 685-688.

[258] F. Besenbacher, I. Stensgaard, K. Mortensen, Adsorption position of deuterium on the $\mathrm{Pd}(100)$ surface determined with transmission channeling, Surf. Sci. 191 (1987) 288-301. [259] K. Oura, J. Yamane, K. Umezawa, M. Naitoh, F. Shoji, T. Hanawa, Hydrogen adsorption on Si(100)-2x1 surfaces studied by elastic recoil detection analysis, Phys. Rev. B 41 (1990) 1200-1203.

[260] K. Oura, M. Naitoh, F. Shoji, J. Yamane, K. Umezawa, T. Hanawa, Elastic recoil detection analysis of hydrogen adsorbed on solid-surfaces, Nucl. Instrum. Methods Phys. Res. B 45 (1990) 199-202.

[261] T. Fujino, T. Fuse, J.T. Ryu, K. Inudzuka, Y. Yamazaki, M. Katayama, K. Oura, Observation of hydrogen adsorption on 6H-SiC(0001) surface, Appl. Surf. Sci. 169 (2001) 113116.

[262] L.S. Wielunski, R.E. Benenson, W.A. Lanford, Helium-induced hydrogen recoil analysis for metallurgical applications, Nucl. Instrum. Methods Phys. Res. 218 (1983) 120-124.

[263] L.S. Wielunski, D. Grambole, U. Kreissig, R. Grötzschel, G. Harding, E. Szilágyi, Hydrogen depth resolution in multilayer metal structures, comparison of elastic recoil detection and resonant nuclear reaction method, Nucl. Instrum. Methods Phys. Res. B 190 (2002) 693-698. [264] L.S. Wielunski, Multiple and double scattering contributions to depth resolution and low energy background in hydrogen elastic recoil detection, Nucl. Instrum. Methods Phys. Res. B 118 (1996) 256-261.

[265] L.S. Wielunski, E. Szilágyi, G.L. Harding, Multiple scattering effects in depth resolution of elastic recoil detection, Nucl. Instrum. Methods Phys. Res. B 136-138 (1998) 713-718.

[266] R.D. Verda, J.R. Tesmer, C.J. Maggiore, M. Nastasi, R.W. Bower, Geometric considerations relevant to hydrogen depth profiling by reflection elastic recoil detection analysis, Nucl. Instrum. Methods Phys. Res. B 183 (2001) 391-400.

[267] R.D. Verda, J.R. Tesmer, M. Nastasi, R.W. Bower, An energy spread correction for depth profiling by elastic recoil detection analysis, Nucl. Instrum. Methods Phys. Res. B 187 (2002) 383-392.

[268] M.J. Puska, R.M. Nieminen, M. Manninen, B. Chakraborty, S. Holloway, J.K. Nørskov, Quantum Motion of Chemisorbed Hydrogen on Ni Surfaces, Phys. Rev. Lett. 51 (1983) 10811084.

[269] C.J. Hagedorn, M.J. Weiss, W.H. Weinberg, Dissociative chemisorption of hydrogen on Ir(111): Evidence for terminal site adsorption, Phys. Rev. B 60 (1999) R14016-R14018.

[270] G. Lee, P.T. Sprunger, M. Okada, D.B. Poker, D.M. Zehner, E.W. Plummer, Chemisorption of hydrogen on the Ag(111) surface, J. Vac. Sci. Technol. A 12 (1994) 21192123.

[271] G. Lee, D.B. Poker, D.M. Zehner, E.W. Plummer, Coverage and structure of deuterium on Cu(111), Surf. Sci. 357-358 (1996) 717-720.

[272] M. Foss, F. Besenbacher, C. Klink, I. Stensgaard, Deuterium-induced restructuring of $\mathrm{Cu}(100)$, Chem. Phys. Lett. 215 (1993) 535-540.

[273] C.F. Walters, D.B. Poker, D.M. Zehner, E.W. Plummer, The deuterium-induced reconstruction of $\mathrm{Cu}(100)$ : Correlation of surface structures with absolute coverage, Surf. Sci. 312 (1994) L759-L766. 
[274] M. Okada, K. Moritani, T. Kasai, W.A. Diño, H. Kasai, S. Ogura, M. Wilde, K. Fukutani, Trapping hydrogen with a bimetallic interface, Phys. Rev. B 71 (2005) 033408.

[275] M. Okada, D.B. Poker, D.M. Zehner, Absolute deuterium coverage determination for the $\mathrm{Mo}_{0.75} \operatorname{Re}_{0.25}(100),(110)$, and (111) surfaces, Surf. Sci. 417 (1998) 1-8.

[276] T.E. Jackman, J.A. Davies, P.R. Norton, W.N. Unertl, K. Griffiths, Saturation coverage of deuterium on Ni(110), Surf. Sci. 141 (1984) L313-L316.

[277] K. Griffiths, P.R. Norton, J.A. Davies, W.N. Unertl, T.E. Jackman, Deuterium adsorption on $\mathrm{Ni}(110)$ : Reconstructed and un-reconstructed phases, Surf. Sci. 152-153 (1985) 374-381.

[278] K. Mortensen, F. Besenbacher, I. Stensgaard, W.R. Wampler, Deuterium on the Ni(111) surface: An adsorption-position determination by transmission channeling, Surf. Sci. 205 (1988) 433-446.

[279] S. Ohno, M. Wilde, K. Fukutani, Novel insight into the hydrogen absorption mechanism at the $\operatorname{Pd}(110)$ surface, J. Chem. Phys. 140 (2014) 134705.

[280] K. Mortensen, F. Besenbacher, I. Stensgaard, C. Klink, Adsorption position of deuterium on the $\mathrm{Pt}(111)$ surface determined with transmission channeling, Surf. Sci. 211-212 (1989) 813-818.

[281] M. Fallavier, B. Hjörvarsson, M. Benmansour, J.P. Thomas, Determination of hydrogen surface coverage of $\mathrm{Pt}_{0.5} \mathrm{Ni}_{0.5}$ single crystals by NRA, Nucl. Instrum. Methods Phys. Res. B 64 (1992) 83-87.

[282] M. Fukuoka, M. Okada, M. Matsumoto, S. Ogura, K. Fukutani, T. Kasai, Location of hydrogen adsorbed on Rh(111) studied by low-energy electron diffraction and nuclear reaction analysis, Phys. Rev. B 75 (2007) 235434.

[283] L.C. Feldman, P.J. Silverman, I. Stensgaard, Si(001) surface studies using high energy ion scattering, Nucl. Instrum. Methods 168 (1980) 589-593.

[284] I. Stensgaard, L.C. Feldman, P.J. Silverman, Evidence of multilayer distortions in the reconstructed Si(001) surface, Surf. Sci. 102 (1981) 1-6.

[285] D.T. Jiang, G.W. Anderson, K. Griffiths, T.K. Sham, P.R. Norton, Adsorption of atomic hydrogen on Si(100)-2×1 at $400 \mathrm{~K}$, Phys. Rev. B 48 (1993) 4952-4955.

[286] M. Wilde, K. Fukutani, S. Koh, K. Sawano, Y. Shiraki, Quantitative coverage and stability of hydrogen-passivation layers on HF-etched $\mathrm{Si}_{(1-x)} \mathrm{Ge}_{x}$ surfaces, J. Appl. Phys. 98 (2005) 023503.

[287] R.J. Culbertson, L.C. Feldman, P.J. Silverman, R. Haight, Hydrogen adsorption on Si(111)-(7×7), J. Vac. Sci. Technol. 20 (1982) 868-871.

[288] M. Copel, R.J. Culbertson, R.M. Tromp, Relaxation and H coverage of ammonium fluoride treated Si(111), Appl. Phys. Lett. 65 (1994) 2344-2346.

[289] K. Takeyasu, K. Fukada, S. Ogura, M. Matsumoto, K. Fukutani, Two charged states of hydrogen on the $\mathrm{SrTiO}_{3}(001)$ surface, J. Chem. Phys. 140 (2014) 084703.

[290] I. Stensgaard, L.C. Feldman, P.J. Silverman, Reconstruction of the W(001) Surface and Its Reordering by Hydrogen Adsorption, Studied by MeV Ion Scattering, Phys. Rev. Lett. 42 (1979) 247-250.

[291] F. Traeger, M. Kauer, C. Wöll, D. Rogalla, H.W. Becker, Analysis of surface, subsurface, and bulk hydrogen in ZnO using nuclear reaction analysis, Phys. Rev. B 84 (2011) 075462.

[292] A. Winkler, A new accurate calibration method for thermal-desorption spectra, J. Vac. Sci. Technol. A 2 (1984) 1393-1394.

[293] A. Winkler, Absolute calibration of small gas amounts, J. Vac. Sci. Technol. A 5 (1987) 2430-2435.

[294] C. Wöll, The chemistry and physics of zinc oxide surfaces, Prog. Surf. Sci. 82 (2007) 55120.

[295] J. Strömquist, L. Bengtsson, M. Persson, B. Hammer, The dynamics of $\mathrm{H}$ absorption in and adsorption on $\mathrm{Cu}(111)$, Surf. Sci. 397 (1998) 382-394. 
[296] M. Blanco-Rey, J.I. Juaristi, R. Díez Muiño, H.F. Busnengo, G.J. Kroes, M. Alducin, Electronic Friction Dominates Hydrogen Hot-Atom Relaxation on Pd(100), Phys. Rev. Lett. 112 (2014) 103203.

[297] T. Kammler, J. Küppers, Interaction of H atoms with $\mathrm{Cu}(111)$ surfaces: Adsorption, absorption, and abstraction, J. Chem. Phys. 111 (1999) 8115-8123.

[298] C.D. Vurdu, Z.B. Guvenc, H(D) -> D(H)+Cu(111) collision system: Molecular dynamics study of surface temperature effects, J. Chem. Phys. 134 (2011) 164306.

[299] J.W. Davenport, G.J. Dienes, R.A. Johnson, Surface effects on the kinetics of hydrogen absorption by metals, Phys. Rev. B 25 (1982) 2165-2174.

[300] K. Nobuhara, H. Nakanishi, H. Kasai, A. Okiji, Interactions of atomic hydrogen with $\mathrm{Cu}(111), \mathrm{Pt}(111)$, and Pd(111), J. Appl. Phys. 88 (2000) 6897-6901.

[301] J. Greeley, M. Mavrikakis, Surface and Subsurface Hydrogen: Adsorption Properties on Transition Metals and Near-Surface Alloys, J. Phys. Chem. B 109 (2005) 3460-3471.

[302] J.L. Nie, H.Y. Xiao, X.T. Zu, First-principles study of $\mathrm{H}$ adsorption on and absorption in $\mathrm{Cu}(111)$ surface, Chem. Phys. 321 (2006) 48-54.

[303] M.F. Luo, G.R. Hu, Coverage-dependent absorption of atomic hydrogen into the subsurface of $\mathrm{Cu}(111)$ studied by density-functional-theory calculations, Surf. Sci. 603 (2009) 1081-1086.

[304] P. Ferrin, S. Kandoi, A.U. Nilekar, M. Mavrikakis, Hydrogen adsorption, absorption and diffusion on and in transition metal surfaces: A DFT study, Surf. Sci. 606 (2012) 679-689.

[305] H. Okuyama, T. Nakagawa, W. Siga, N. Takagi, M. Nishijima, T. Aruga, Subsurface Hydrogen at Pd(100) Induced by Gas-Phase Atomic Hydrogen, J. Phys. Chem. B 103 (1999) 7876-7881.

[306] P.J. Estrup, J. Anderson, Chemisorption of Hydrogen on Tungsten (100), J. Chem. Phys. 45 (1966) 2254-2260.

[307] T.E. Madey, Chemisorption of $\mathrm{H}_{2}$ on W(100): Absolute sticking probability, coverage, and electron stimulated desorption, Surf. Sci. 36 (1973) 281-294.

[308] D.A. King, G. Thomas, Displacive surface phases formed by hydrogen chemisorption on W\{001\}, Surf. Sci. 92 (1980) 201-236.

[309] P.W. Tamm, L.D. Schmidt, Condensation of Hydrogen on Tungsten, J. Chem. Phys. 55 (1971) 4253-4259.

[310] Y. Viswanath, G. Sanders, L.D. Schmidt, Adsorbate densities on (100)W, Surf. Sci. 59

(1976) 297-301.

[311] B.D. Barford, R.R. Rye, Adsorption of ethylene and hydrogen on tungsten single crystals: Flash desorption, adsorption kinetics, and work function changes, J. Chem. Phys. 60 (1974) 1046-1056.

[312] W. Ho, R.F. Willis, E.W. Plummer, Observation of Nondipole Electron Impact Vibrational Excitations: H on W(100), Phys. Rev. Lett. 40 (1978) 1463-1466.

[313] M.R. Barnes, R.F. Willis, Hydrogen-Adsorption-Induced Reconstruction of Tungsten (100): Observation of Surface Vibrational Modes, Phys. Rev. Lett. 41 (1978) 1729-1733.

[314] Y.J. Chabal, Electronic Damping of Hydrogen Vibration on the W(100) Surface, Phys. Rev. Lett. 55 (1985) 845-848.

[315] G. Witte, J.P. Toennies, C. Wöll, Comparison of surface phonon dispersion curves for clean and hydrogen covered Rh(111) surfaces, Surf. Sci. 323 (1995) 228-240.

[316] H. Yanagita, H. Fujioka, T. Aruga, N. Takagi, M. Nishijima, Vibrational spectra of hydrogen on the Rh(111) surface, Surf. Sci. 441 (1999) 507-514.

[317] T. Mitsui, M.K. Rose, E. Fomin, D.F. Ogletree, M. Salmeron, Hydrogen adsorption and diffusion on Pd(111), Surf. Sci. 540 (2003) 5-11.

[318] C.M. Mate, G.A. Somorjai, Delocalized quantum nature of hydrogen adsorbed on the Rh(111) crystal surface, Phys. Rev. B 34 (1986) 7417-7420. 
[319] W. Lai, D. Xie, A DFT investigation of potential energy surface and vibrational properties of hydrogen adsorbed on the Rh(111) surface, Surf. Sci. 550 (2004) 15-20. [320] M. Matsumoto, S. Ogura, K. Fukutani, T. Okano, M. Okada, Dynamical low-energy electron diffraction analyses of clean and H-adsorbed $\operatorname{Ir}(111)$ surfaces, J. Vac. Soc. Jpn. 49 (2006) 313-316.

[321] K. Nobuhara, H. Kasai, H. Nakanishi, W.A. Diño, Vibrational properties of hydrogen atom adsorbed on $\mathrm{Cu}(111)$ and on Ir(111) surfaces, J. Appl. Phys. 96 (2004) 5020-5025.

[322] K. Moritani, M. Okada, M. Nakamura, T. Kasai, Y. Murata, Hydrogen-exchange reactions via hot hydrogen atoms produced in the dissociation process of molecular hydrogen on Ir\{111\}, J. Chem. Phys. 115 (2001) 9947-9959.

[323] K. Christmann, O. Schober, G. Ertl, M. Neumann, Adsorption of hydrogen on nickel single crystal surfaces, J. Chem. Phys. 60 (1974) 4528-4540.

[324] T.N. Taylor, P.J. Estrup, The interaction of hydrogen with Ni(110), J. Vac. Sci. Technol. 11 (1974) 244-247.

[325] V. Penka, K. Christmann, G. Ertl, Ordered Low-Temperature Phases in the H/Ni(110) System, Surf. Sci. 136 (1984) 307-318.

[326] K.H. Rieder, T. Engel, Adatom Configurations of $\mathrm{H}(2 \times 6)$ and $\mathrm{H}(2 \times 1)$ on $\mathrm{Ni}(110)$ Analyzed Using He Diffraction, Phys. Rev. Lett. 45 (1980) 824-828.

[327] T. Engel, K.H. Rieder, A molecular-beam diffraction study of $\mathrm{H}_{2}$ adsorption on $\mathrm{Ni}(110)$, Surf. Sci. 109 (1981) 140-166.

[328] K.H. Rieder, Low-coverage ordered phases of hydrogen on Ni(110), Phys. Rev. B 27 (1983) 7799-7802.

[329] T.E. Jackman, K. Griffiths, W.N. Unertl, J.A. Davies, K.H. Gurtler, D.A. Harrington, P.R. Norton, Surface phases of $\mathrm{Ni}(110)$ induced by adsorption of deuterium, Surf. Sci. 179 (1987) 297-321.

[330] D.A. Harrington, P.R. Norton, Activated adsorption of deuterium on Ni(110): evidence for a high temperature desorption state, Surf. Sci. 195 (1988) L135-L144.

[331] G. Kresse, J. Hafner, First-principles study of the adsorption of atomic $\mathrm{H}$ on Ni (111), (100) and (110), Surf. Sci. 459 (2000) 287-302.

[332] B. Bhatia, D.S. Sholl, Chemisorption and diffusion of hydrogen on surface and subsurface sites of flat and stepped nickel surfaces, J. Chem. Phys. 122 (2005) 204707.

[333] A. Winkler, K.D. Rendulic, Adsorption-kinetics for hydrogen adsorption on nickel and coadsorption of hydrogen and oxygen, Surf. Sci. 118 (1982) 19-31.

[334] I. Stensgaard, R. Feidenhans'l, Absolute coverage measurements of deuterium on $\mathrm{Ni}$ (110), Surf. Sci. 131 (1983) L373-L378.

[335] T. Kammler, S. Wehner, J. Küppers, Interaction of thermal $\mathrm{H}$ atoms with $\mathrm{Ni}(100)-\mathrm{H}$ surfaces: through surface penetration and adsorbed hydrogen abstraction, Surf. Sci. 339 (1995) 125-134.

[336] S. Andersson, Vibrational excitations and structure of $\mathrm{H}_{2}, \mathrm{D}_{2}$ and $\mathrm{HD}$ absorbed on $\mathrm{Ni}(100)$, Chem. Phys. Lett. 55 (1978) 185-188.

[337] K.H. Rieder, H. Wilsch, Helium diffraction from Ni(100): A study of the clean surface and of hydrogen and carbon adsorption phases, Surf. Sci. 131 (1983) 245-257.

[338] I. Stensgaard, Adsorption position of light and heavy atoms on crystal surfaces determined with transmission channeling, Nucl. Instrum. Methods Phys. Res. B 15 (1986) 300-305.

[339] I. Stensgaard, F. Jakobsen, Adsorption-Site Location by Transmission Channeling: Deuterium on $\mathrm{Ni}(100)$, Phys. Rev. Lett. 54 (1985) 711-713.

[340] J. Greeley, M. Mavrikakis, A first-principles study of surface and subsurface $\mathrm{H}$ on and in $\mathrm{Ni}(111)$ : diffusional properties and coverage-dependent behavior, Surf. Sci. 540 (2003) 215229. 
[341] K. Christmann, R.J. Behm, G. Ertl, M.A. Vanhove, W.H. Weinberg, Chemisorption Geometry of Hydrogen on Ni(111) - Order and Disorder, J. Chem. Phys. 70 (1979) 4168-4184. [342] F. Besenbacher, I. Stensgaard, P. Vase, Absolute cross section for recoil detection of deuterium, Nucl. Instrum. Methods Phys. Res. B 15 (1986) 459-463.

[343] G. Gross, K.H. Rieder, He-diffraction investigations of the $(2 \times 2)$-phases of oxygen and hydrogen on Ni(111), Surf. Sci. 241 (1991) 33-38.

[344] L. Hammer, H. Landskron, W. Nichtl-Pecher, A. Fricke, K. Heinz, K. Müller, Hydrogeninduced restructuring of close-packed metal surfaces: $\mathrm{H} / \mathrm{Ni}(111)$ and $\mathrm{H} / \mathrm{Fe}(110)$, Phys. Rev. B 47 (1993) 15969-15972.

[345] T. Ito, K. Umezawa, S. Nakanishi, Hydrogen adsorption site on the Ni\{111\}-(2×2)-H: a TOF-LERS study, Appl. Surf. Sci. 147 (1999) 146-152.

[346] K. Umezawa, T. Ito, S. Nakanishi, Low-energy recoil scattering from hydrogen adsorbed on Ni (111), Nucl. Instrum. Methods Phys. Res. B 136-138 (1998) 1121-1124.

[347] F. Jensen, I. Stensgaard, F. Besenbacher, K. Mortensen, Applications of transmission channeling for adatom location on metal surfaces, Nucl. Instrum. Methods Phys. Res. B 48 (1990) 334-338.

[348] H. Premm, H. Polzl, A. Winkler, Dynamics and kinetics of subsurface absorption and desorption for the system hydrogen (deuterium)-Ni(111), Surf. Sci. 401 (1998) L444-L451. [349] M.J. Puska, R.M. Nieminen, Hydrogen chemisorbed on nickel surfaces: A wavemechanical treatment of proton motion, Surf. Sci. 157 (1985) 413-435.

[350] H. Okuyama, T. Ueda, T. Aruga, M. Nishijima, Overtones of H vibrations at $\mathrm{Ni}(111)$ : Formation of delocalized states, Phys. Rev. B 63 (2001) 233403.

[351] G.X. Cao, E. Nabighian, X.D. Zhu, Diffusion of Hydrogen on Ni(111) over a Wide Range of Temperature: Exploring Quantum Diffusion on Metals, Phys. Rev. Lett. 79 (1997) 36963699.

[352] A.D. Johnson, K.J. Maynard, S. Daley, S.T. Ceyer, Hydrogen embedded in Ni in an ultrahigh-vacuum environment, Abstr. Pap. Am. Chem. Soc. 201 (1991) 291-PHYS.

[353] A. Michaelides, P. Hu, A. Alavi, Physical origin of the high reactivity of subsurface hydrogen in catalytic hydrogenation, J. Chem. Phys. 111 (1999) 1343-1345.

[354] V. Ledentu, W. Dong, P. Sautet, Heterogeneous catalysis through subsurface sites, J. Am. Chem. Soc. 122 (2000) 1796-1801.

[355] X. Sha, B. Jackson, Ab initio and transition state theory studies of the energetics of $\mathrm{H}$ atom resurfacing on $\mathrm{Ni}(111)$, Chem. Phys. Lett. 357 (2002) 389-396.

[356] G. Henkelman, A. Arnaldsson, H. Jónsson, Theoretical calculations of $\mathrm{CH}_{4}$ and $\mathrm{H}_{2}$ associative desorption from $\mathrm{Ni}(111)$ : Could subsurface hydrogen play an important role?, J. Chem. Phys. 124 (2006) 044706.

[357] S. Wright, J.F. Skelly, A. Hodgson, Energy disposal during desorption of D-2 from the surface and subsurface region of $\mathrm{Ni}(111)$, Faraday Discuss. 117 (2000) 133-146.

[358] S.T. Ceyer, The Unique Chemistry of Hydrogen beneath the Surface: Catalytic Hydrogenation of Hydrocarbons, Acc. Chem. Res. 34 (2001) 737-744.

[359] S.P. Daley, A.L. Utz, T.R. Trautman, S.T. Ceyer, Ethylene hydrogenation on Ni(111) by bulk hydrogen, J. Am. Chem. Soc. 116 (1994) 6001-6002.

[360] A.D. Johnson, S.P. Daley, A.L. Lutz, S.T. Ceyer, The chemistry of bulk hydrogen: Reaction of hydrogen embedded in nickel with adsorbed $\mathrm{CH}_{3}$, Science 257 (1992) 223.

[361] R. Baer, Y. Zeiri, R. Kosloff, Hydrogen transport in nickel (111), Phys. Rev. B 55 (1997) 10952-10952.

[362] K. Christmann, G. Ertl, Interaction of Hydrogen with Pt(111) - Role of Atomic Steps, Surf. Sci. 60 (1976) 365-384.

[363] K. Christmann, G. Ertl, T. Pignet, Adsorption of Hydrogen on a Pt(111) Surface, Surf. Sci. 54 (1976) 365-392. 
[364] B. Poelsema, K. Lenz, G. Comsa, The dissociative adsorption of hydrogen on Pt(111): Actuation and acceleration by atomic defects, J. Chem. Phys. 134 (2011) 074703.

[365] B. Poelsema, K. Lenz, G. Comsa, The dissociative adsorption of hydrogen on defect'free' Pt(111), J. Phys.: Condensed Matter 22 (2010) 304006.

[366] H. Nakatsuji, Y. Matsuzaki, T. Yonezawa, Ab initio theoretical study on the reactions of a hydrogen molecule with small platinum clusters: A model for chemisorption on a Pt surface, J. Chem. Phys. 88 (1988) 5759-5769.

[367] A.C. Luntz, J.K. Brown, M.D. Williams, Molecular beam studies of $\mathrm{H}_{2}$ and $\mathrm{D}_{2}$ dissociative chemisorption on Pt(111), J. Chem. Phys. 93 (1990) 5240-5246.

[368] R.A. Olsen, G.J. Kroes, E.J. Baerends, Atomic and molecular hydrogen interacting with Pt(111), J. Chem. Phys. 111 (1999) 11155-11163.

[369] B. Poelsema, L.K. Verheij, G. Comsa, Temperature dependency of the initial sticking probability of $\mathrm{H}_{2}$ and $\mathrm{CO}$ on Pt(111), Surf. Sci. 152-153 (1985) 496-504.

[370] K. Umezawa, T. Ito, M. Asada, S. Nakanishi, P. Ding, W.A. Lanford, B. Hjörvarsson, Adsorption of hydrogen on the Pt(111) surface from low-energy recoil scattering, Surf. Sci. 387 (1997) 320-327.

[371] P.R. Norton, J.A. Davies, T.E. Jackman, Absolute coverage and isosteric heat of adsorption of deuterium on Pt(111) studied by nuclear microanalysis, Surf. Sci. 121 (1982) 103-110.

[372] B. Poelsema, G. Mechtersheimer, G. Comsa, The interaction of hydrogen with platinum(s)-9(111) × (111) studied with helium beam diffraction, Surf. Sci. 111 (1981) 519544.

[373] R.J. Madix, G. Ertl, K. Christmann, Pre-Exponential Factors for Hydrogen Desorption from Single-Crystal Metal-Surfaces, Chem. Phys. Lett. 62 (1979) 38-41.

[374] R.C. Baetzold, G.A. Somorjai, Preexponential factors in surface reactions, J. Catal. 45 (1976) 94-105.

[375] B. Poelsema, G. Mechtersheimer, G. Comsa, Anomalous preexponential factor for hydrogen desorption from platinum single crystals?, Surf. Sci. 111 (1981) L728-L732.

[376] W. Eberhardt, F. Greuter, E.W. Plummer, Bonding of H to Ni, Pd, and Pt Surfaces, Phys. Rev. Lett. 46 (1981) 1085-1088.

[377] P.J. Feibelman, D.R. Hamann, Theory of $\mathrm{H}$ bonding and vibration on Pt(111), Surf. Sci. 182 (1987) 411-422.

[378] L.J. Richter, W. Ho, Vibrational spectroscopy of $\mathrm{H}$ on Pt(111): Evidence for universally soft parallel modes, Phys. Rev. B 36 (1987) 9797-9800.

[379] J.E. Reutt, Y.J. Chabal, S.B. Christman, Hydrogen phonon-spectra on Pt(111) at T = 100K and 160-K, J. Vac. Sci. Technol. A 6 (1988) 816-819.

[380] A.P. Graham, A. Menzel, J.P. Toennies, Quasielastic helium atom scattering measurements of microscopic diffusional dynamics of $H$ and $D$ on the $\mathrm{Pt}(111)$ surface, J. Chem. Phys. 111 (1999) 1676-1685.

[381] Ş.C. Bădescu, K. Jacobi, Y. Wang, K. Bedürftig, G. Ertl, P. Salo, T. Ala-Nissila, S.C. Ying, Vibrational states of a H monolayer on the Pt(111) surface, Phys. Rev. B 68 (2003) 205401. [382] T. Roman, H. Nakanishi, W.A. Diño, H. Kasai, Hydrogen atom quantum migration on platinum, e-J. Surf. Sci. Nanotechnol. 4 (2006) 619-623.

[383] P. Légaré, A theoretical study of $\mathrm{H}$ surface and subsurface species on Pt(111), Surf. Sci. 559 (2004) 169-178.

[384] B. Hammer, J.K. Nørskov, Why gold is the noblest of all the metals, Nature 376 (1995) 238-240.

[385] G. Anger, A. Winkler, K.D. Rendulic, Adsorption and desorption-kinetics in the systems $\mathrm{H}_{2} / \mathrm{Cu}(111), \mathrm{H}_{2} / \mathrm{Cu}(110)$ and $\mathrm{H}_{2} / \mathrm{Cu}(100)$, Surf. Sci. 220 (1989) 1-17.

[386] B. Jackson, X. Sha, Z.B. Guvenc, Kinetic model for Eley-Rideal and hot atom reactions between $\mathrm{H}$ atoms on metal surfaces, J. Chem. Phys. 116 (2002) 2599-2608. 
[387] C.T. Rettner, D.J. Auerbach, Quantum - state distributions for the HD product of the direct reaction of $\mathrm{H}(\mathrm{D}) / \mathrm{Cu}(111)$ with $\mathrm{D}(\mathrm{H})$ incident from the gas phase, J. Chem. Phys. 104 (1996) 2732-2739.

[388] P.B. Lloyd, M. Swaminathan, J.W. Kress, B.J. Tatarchuk, Temperature programmed desorption study of the adsorption and absorption of hydrogen on and in $\mathrm{Cu}(111)$, Appl. Surf. Sci. 119 (1997) 267-274.

[389] F. Greuter, E. Ward Plummer, Chemisorption of atomic hydrogen on $\mathrm{Cu}(111)$, Solid State Commun. 48 (1983) 37-41.

[390] U. Bischler, P. Sandl, E. Bertel, T. Brunner, W. Brenig, Sticking, adsorption, and absorption of atomic $\mathrm{H}$ on $\mathrm{Cu}(110)$, Phys. Rev. Lett. 70 (1993) 3603-3606.

[391] U. Bischler, E. Bertel, Simple source of atomic hydrogen for ultrahigh vacuum applications, J. Vac. Sci. Technol. A 11 (1993) 458-460.

[392] G. Lee, E.W. Plummer, High-resolution electron energy loss spectroscopy study on chemisorption of hydrogen on $\mathrm{Cu}(111)$, Surf. Sci. 498 (2002) 229-236.

[393] M.F. Luo, D.A. MacLaren, W. Allison, Migration and abstraction of $\mathrm{H}$-atoms from the $\mathrm{Cu}(111)$ surface, Surf. Sci. 586 (2005) 109-114.

[394] M.F. Luo, D.A. MacLaren, I.G. Shuttleworth, W. Allison, Preferential sub-surface occupation of atomic hydrogen on $\mathrm{Cu}(111)$, Chem. Phys. Lett. 381 (2003) 654-659.

[395] I. Chorkendorff, P.B. Rasmussen, Reconstruction of $\mathrm{Cu}(100)$ by adsorption of atomic hydrogen, Surf. Sci. 248 (1991) 35-44.

[396] R. Spitzl, H. Niehus, B. Poelsema, G. Comsa, H-induced $(1 \times 2)$ reconstruction of the $\mathrm{Cu}(110)$ surface: structure and deconstruction kinetics, Surf. Sci. 239 (1990) 243-253.

[397] A.V. Mijiritskii, U. Wahl, M.H. Langelaar, D.O. Boerma, Direct determination of the lattice site of $\mathrm{H}$ atoms on the $(1 \times 2)$ reconstructed $\mathrm{Cu}(110)$ surface, Nucl. Instrum. Methods Phys. Res. B 136-138 (1998) 1097-1101.

[398] B.E. Hayden, D. Lackey, J. Schott, A vibrational study of the hydrogen induced reconstructions on $\mathrm{Cu}(110)$, Surf. Sci. 239 (1990) 119-126.

[399] Y. Xu, J. Greeley, M. Mavrikakis, Effect of Subsurface Oxygen on the Reactivity of the $\mathrm{Ag}(111)$ Surface, J. Am. Chem. Soc. 127 (2005) 12823-12827.

[400] G. Lee, E.W. Plummer, Interaction of hydrogen with the $A g(111)$ surface, Phys. Rev. B 51 (1995) 7250-7261.

[401] V. Zhukov, K.D. Rendulic, A. Winkler, Coadsorption of hydrogen and potassium on silver single crystal surfaces, Vacuum 47 (1996) 5-11.

[402] G.E. Gdowski, T.E. Felter, R.H. Stulen, Effect of surface temperature on the sorption of hydrogen by Pd(111), Surf. Sci. 181 (1987) L147-L155.

[403] E.M. McCash, S.F. Parker, J. Pritchard, M.A. Chesters, The adsorption of atomic hydrogen on $\mathrm{Cu}(111)$ investigated by reflection-absorption infrared spectroscopy, electron energy loss spectroscopy and low energy electron diffraction, Surf. Sci. 215 (1989) 363-377. [404] X.L. Zhou, J.M. White, B.E. Koel, Chemisorption of atomic hydrogen on clean and Clcovered Ag(111), Surf. Sci. 218 (1989) 201-210.

[405] F. Healey, R.N. Carter, A. Hodgson, The recombinative desorption of $D_{2}$ from $\mathrm{Ag}(111)$ Temperature-programmed desorption and low-energy-electron diffraction, Surf. Sci. 328 (1995) 67-79.

[406] M. Okada, M. Nakamura, K. Moritani, T. Kasai, Dissociative adsorption of hydrogen on thin Au films grown on Ir\{111\}, Surf. Sci. 523 (2003) 218-230.

[407] M. Okada, S. Ogura, W.A. Diño, M. Wilde, K. Fukutani, T. Kasai, Reactivity of gold thin films grown on iridium: Hydrogen dissociation, Applied Catalysis A: General 291 (2005) 55-61. [408] M. Okada, S. Ogura, W.A. Diño, M. Wilde, K. Fukutani, T. Kasai, Reactive gold thin films grown on iridium, Appl. Surf. Sci. 246 (2005) 68-71. 
[409] M. Tsunoda, K. Fukutani, Y. Murata, H. Yamashita, K. Komaki, K. Kobayashi, in: B. Lengeler, H. Lueth, W. Moench, J. Pollmann (Eds.) Formation of Semiconductor Interfaces, World Scientific, Singapore, 1994, pp. 162-165.

[410] A. Ruban, B. Hammer, P. Stoltze, H.L. Skriver, J.K. Nørskov, Surface electronic structure and reactivity of transition and noble metals, J. Mol. Catal. A 115 (1997) 421-429.

[411] S. Ogura, K. Fukutani, M. Wilde, M. Matsumoto, T. Okano, M. Okada, T. Kasai, W.A. Diño, Hydrogen adsorption on Ag and Au monolayers grown on Pt(111), Surf. Sci. 566 (2004) 755-760.

[412] P.T. Sprunger, E.W. Plummer, Interaction of hydrogen with the $\mathrm{Ag}(110)$ surface, Phys. Rev. B 48 (1993) 14436-14446.

[413] H. Iwai, K. Fukutani, Y. Murata, Behavior of atomic hydrogen on Au(001), Surf. Sci. 357 (1996) 663-666.

[414] A.G. Sault, R.J. Madix, C.T. Campbell, Adsorption of oxygen and hydrogen on Au(110)$(1 \times 2)$, Surf. Sci. 169 (1986) 347-356.

[415] H.H. Hwu, J. Eng, J.G. Chen, Ni/Pt(111) Bimetallic Surfaces: Unique Chemistry at Monolayer Ni Coverage, J. Am. Chem. Soc. 124 (2002) 702-709.

[416] B. Hammer, J.K. Nørskov, Electronic factors determining the reactivity of metal surfaces, Surf. Sci. 343 (1995) 211-220.

[417] K. Fukutani, S. Ogura, M. Wilde, M. Matsumoto, Doppler analysis of hydrogen motion on Pt(111)-Sn surface alloys using the ${ }^{1} \mathrm{H}\left({ }^{15} \mathrm{~N}, \alpha \gamma\right){ }^{12} \mathrm{C}$ nuclear reaction, Nucl. Instrum. Methods Phys. Res. B 261 (2007) 494-497.

[418] M.T. Paffett, R.G. Windham, Surface modification of Pt(111) by Sn adatoms: Evidence for the formation of ordered overlayers and surface alloys, Surf. Sci. 208 (1989) 34-54.

[419] L. Hammer, M. Kottcke, W. Weiß, A. Wimmer, D.M. Zehner, K. Heinz, Hydrogen on $M_{o_{x}} \operatorname{Re}_{1-x}(100)$ - the impact of alloying on the adsorption structure, Surf. Sci. 401 (1998) 455468.

[420] R. Döll, M. Kottcke, K. Heinz, L. Hammer, K. Müller, D.M. Zehner, Application of chemical tensor LEED to $\mathrm{Mo}_{x} \mathrm{Re}_{1-\mathrm{x}}(100)$ surfaces, Surf. Sci. 307-309 (1994) 434-439.

[421] M. Kottcke, B. Dötsch, L. Hammer, K. Heinz, K. Müller, D.M. Zehner, Deep layer oscillatory segregation and relaxation of substitutionally disordered $\mathrm{Mo}_{\mathbf{x}} \operatorname{Re}_{1-\mathrm{x}}(100)$ surfaces, Surf. Sci. 376 (1997) 319-329.

[422] R.S. Daley, T.E. Felter, M.L. Hildner, P.J. Estrup, Structural changes in the Mo(100) reconstruction, Phys. Rev. Lett. 70 (1993) 1295-1298.

[423] D.-M. Smilgies, P.J. Eng, I.K. Robinson, Triple chain model of the reconstructed Mo(001) surface, Phys. Rev. Lett. 70 (1993) 1291-1294.

[424] J.A. Prybyla, P.J. Estrup, Y.J. Chabal, Phase transitions, surface structures, and adsorbate bonding in the $\mathrm{H} / \mathrm{Mo}(100)$ chemisorption system, J. Chem. Phys. 94 (1991) 62746295.

[425] J.A. Prybyla, P.J. Estrup, S.C. Ying, Y.J. Chabal, S.B. Christman, Reconstructive phase transitions and effective adsorbate-adsorbate interactions: $\mathrm{H} / \mathrm{Mo}(100)$ and $\mathrm{H} / \mathrm{W}(100)$, Phys. Rev. Lett. 58 (1987) 1877-1880.

[426] M. Altman, J.W. Chung, P.J. Estrup, J.M. Kosterlitz, J. Prybyla, D. Sahu, S.C. Ying, Phase transformations of the H/W(110) and H/Mo(110) surfaces, J. Vac. Sci. Technol. A 5 (1987) 1045-1048.

[427] J.W. Chung, S.C. Ying, P.J. Estrup, Reconstruction of the W(110) surface induced by hydrogen adsorption, Phys. Rev. Lett. 56 (1986) 749-752.

[428] D.M. Riffe, G.K. Wertheim, P.H. Citrin, Chemical and reconstruction-induced surface core-level shifts: H on low-index W surfaces, Phys. Rev. Lett. 65 (1990) 219-222.

[429] J.F. van der Veen, F.J. Himpsel, D.E. Eastman, Chemisorption-induced 4f-core-electron binding-energy shifts for surface atoms of W(111), W(100), and Ta(111), Phys. Rev. B 25 (1982) 7388-7397. 
[430] J.C. Bertolini, J. Massardier, P. Ruiz, B. Tardy, $\mathrm{Pt}_{50} \mathrm{Ni}_{50}(110)$ alloy: Chemical surface reactivity with respect to the pure components, Surf. Sci. 211-212 (1989) 1053-1060.

[431] N.J. DiNardo, E.W. Plummer, The vibrational modes of hydrogen adsorbed on Ni(110), Surf. Sci. 150 (1985) 89-119.

[432] S. Jans, S. Kalbitzer, P. Oberschachtsiek, J.P.F. Sellschop, ${ }^{15} \mathrm{~N}$ Doppler spectroscopy of ${ }^{1} \mathrm{H}$ on diamond, Nucl. Instrum. Methods Phys. Res. B 85 (1994) 321-325.

[433] A.A. Shiryaev, D. Grambole, A. Rivera, F. Herrmann, On the interaction of molecular hydrogen with diamonds: An experimental study using nuclear probes and thermal desorption, Diamond Relat. Mater. 16 (2007) 1479-1485.

[434] K. Oura, M. Naitoh, J. Yamane, F. Shoji, Hydrogen-induced reordering of the Si(111)-3 $\times$ 3-Ag surface, Surf. Sci. 230 (1990) L151-L154.

[435] K. Sumitomo, T. Kobayashi, F. Shoji, K. Oura, I. Katayama, Hydrogen-mediated epitaxy of Ag on Si(111) as studied by low-energy ion scattering, Phys. Rev. Lett. 66 (1991) 11931196.

[436] T. Yamazaki, H. Asaoka, T. Taguchi, S. Yamamoto, D. Yamazaki, R. Maruyama, M. Takeda, S. Shamoto, Analysis of buried heterointerfacial hydrogen in highly latticemismatched epitaxy on silicon, Thin Solid Films 520 (2012) 3300-3303.

[437] M. Copel, R.M. Tromp, H coverage dependence of Si(001) homoepitaxy, Phys. Rev. Lett. 72 (1994) 1236-1239.

[438] Y. Kido, T. Nishimura, Y. Furukawa, Y. Nakayama, T. Yasue, T. Koshikawa, P.C. GoppeltLanger, S. Yamamoto, Z.Q. Ma, H. Naramoto, T. Ueda, Solid-phase epitaxial growth of Ge on H-terminated and oxidized Si(100) surfaces, Surf. Sci. 327 (1995) 225-232.

[439] T. Fujino, T. Fuse, E. Tazou, T. Nakano, K. Inudzuka, K. Goto, Y. Yamazaki, M. Katayama, K. Oura, In situ monitoring of hydrogen-surfactant effect during Ge growth on $\mathrm{Si}(001)$ using coaxial impact-collision ion scattering spectroscopy and time-of-flight elastic recoil detection analysis, Nucl. Instrum. Methods Phys. Res. B 161-163 (2000) 419-423.

[440] T. Fujino, T. Fuse, J.T. Ryu, K. Inudzuka, T. Nakano, K. Goto, Y. Yamazaki, M. Katayama, K. Oura, Ge thin film growth on Si(111) surface using hydrogen surfactant, Thin Solid Films 369 (2000) 25-28.

[441] E.T. Foley, A.F. Kam, J.W. Lyding, P. Avouris, Cryogenic UHV-STM Study of Hydrogen and Deuterium Desorption from Si(100), Phys. Rev. Lett. 80 (1998) 1336-1339.

[442] J.W. Lyding, K. Hess, G.C. Abeln, D.S. Thompson, J.S. Moore, M.C. Hersam, E.T. Foley, J. Lee, Z. Chen, S.T. Hwang, H. Choi, P. Avouris, I.C. Kizilyalli, Ultrahigh vacuum-scanning tunneling microscopy nanofabrication and hydrogen/deuterium desorption from silicon surfaces: implications for complementary metal oxide semiconductor technology, Appl. Surf. Sci. 130-132 (1998) 221-230.

[443] K. Takayanagi, Y. Tanishiro, M. Takahashi, S. Takahashi, Structural analysis of Si(111) $7 \times 7$ by UHV - transmission electron diffraction and microscopy, J. Vac. Sci. Technol. A 3 (1985) 1502-1506.

[444] G. Binning, H. Rohrer, C. Gerber, E. Weibel, Surface studies by scanning tunneling microscopy, Phys. Rev. Lett. 49 (1982) 57-61.

[445] Y.J. Chabal, Surface infrared spectroscopy, Surf. Sci. Rep. 8 (1988) 211-357.

[446] B. Hartmann, S. Kalbitzer, M. Behar, ${ }^{15} \mathrm{~N}$ nuclear-reaction analysis of ${ }^{1} \mathrm{H}$ on $<111>\mathrm{Si}$ surfaces, Nucl. Instrum. Methods Phys. Res. B 103 (1995) 494-501.

[447] Y.J. Chabal, G.S. Higashi, K. Raghavachari, V.A. Burrows, Infrared spectroscopy of $\mathrm{Si}(111)$ and $\mathrm{Si}(100)$ surfaces after HF treatment: Hydrogen termination and surface morphology, J. Vac. Sci. Technol. A 7 (1989) 2104-2109.

[448] Y.J. Chabal, K. Raghavachari, New Ordered Structure for the H-Saturated Si(100) Surface: The (3×1) Phase, Phys. Rev. Lett. 54 (1985) 1055-1058.

[449] Y.J. Chabal, K. Raghavachari, Surface Infrared Study of Si(100)-(2×1)H, Phys. Rev. Lett. 53 (1984) 282-285. 
[450] J.J. Boland, Structure of the H-saturated Si(100) surface, Phys. Rev. Lett. 65 (1990) 3325-3328.

[451] J.J. Boland, Role of bond-strain in the chemistry of hydrogen on the $\mathrm{Si}(100)$ surface, Surf. Sci. 261 (1992) 17-28.

[452] F. Owman, P. Mårtensson, STM study of structural defects on in situ prepared Si(111) 1×1-H surfaces, Surf. Sci. 324 (1995) 211-225.

[453] F. Owman, P. Mårtensson, STM study of Si(111) $1 \times 1-\mathrm{H}$ surfaces prepared by in situ hydrogen exposure, Surf. Sci. 303 (1994) L367-L372.

[454] G.S. Higashi, Y.J. Chabal, G.W. Trucks, K. Raghavachari, Ideal hydrogen termination of the Si (111) surface, Appl. Phys. Lett. 56 (1990) 656-658.

[455] X. Zhang, E. Garfunkel, Y.J. Chabal, S.B. Christman, E.E. Chaban, Stability of HF-etched Si(100) surfaces in oxygen ambient, Appl. Phys. Lett. 79 (2001) 4051-4053.

[456] T. Narusawa, W.M. Gibson, Summary Abstract: Hydrogen adsorption on Si(100) surface studied by MeV ion scattering, J. Vac. Sci. Technol. 17 (1980) 256-256.

[457] G.S. Higashi, R.S. Becker, Y.J. Chabal, A.J. Becker, Comparison of Si(111) surfaces prepared using aqueous solutions of $\mathrm{NH}_{4} \mathrm{~F}$ versus HF, Appl. Phys. Lett. 58 (1991) 1656-1658. [458] P. Dumas, Y.J. Chabal, Electron energy loss spectroscopy of H-terminated Si(111) and Si(100) prepared by chemical etching, J. Vac. Sci. Technol. A 10 (1992) 2160-2165.

[459] C. Stuhlmann, G. Bogdányi, H. Ibach, Surface phonons of the hydrogen-terminated Si(111)(1×1) surface, Phys. Rev. B 45 (1992) 6786-6792.

[460] C.C. Cheng, J.T. Yates, Jr., H-induced surface restructuring on Si(100): Formation of higher hydrides, Phys. Rev. B 43 (1991) 4041-4045.

[461] A. Ishizaka, Y. Shiraki, Low Temperature Surface Cleaning of Silicon and Its Application to Silicon MBE, J. Electrochem. Soc. 133 (1986) 666-671.

[462] T. Irisawa, S. Tokumitsu, T. Hattori, K. Nakagawa, S. Koh, Y. Shiraki, Ultrahigh roomtemperature hole Hall and effective mobility in $\mathrm{Si}_{0.3} \mathrm{Ge}_{0.7} / \mathrm{Ge}_{\mathrm{Si}} \mathrm{Si}_{0.3} \mathrm{Ge}_{0.7}$ heterostructures, Appl. Phys. Lett. 81 (2002) 847-849.

[463] V.A. Henrich, P.A. Cox, The Surface Science of Metal Oxides, University Press, Cambridge, 1994.

[464] H.-J. Freund, H. Kuhlenbeck, V. Staemmler, Oxide surfaces, Rep. Prog. Phys. 59 (1996) 283.

[465] M.A. Henderson, A surface science perspective on photocatalysis, Surf. Sci. Rep. 66 (2011) 185-297.

[466] U. Diebold, The surface science of titanium dioxide, Surf. Sci. Rep. 48 (2003) 53-229.

[467] Y. Wang, B. Meyer, X. Yin, M. Kunat, D. Langenberg, F. Traeger, A. Birkner, C. Wöll, Hydrogen Induced Metallicity on the ZnO(1010) Surface, Phys. Rev. Lett. 95 (2005) 266104. [468] B. Meyer, D. Marx, O. Dulub, U. Diebold, M. Kunat, D. Langenberg, C. Wöll, Partial Dissociation of Water Leads to Stable Superstructures on the Surface of Zinc Oxide, Angew. Chem. Int. Ed. 43 (2004) 6641-6645.

[469] D.M. Hofmann, A. Hofstaetter, F. Leiter, H. Zhou, F. Henecker, B.K. Meyer, S.B. Orlinskii, J. Schmidt, P.G. Baranov, Hydrogen: A Relevant Shallow Donor in Zinc Oxide, Phys. Rev. Lett. 88 (2002) 045504.

[470] C.H. Seager, S.M. Myers, Quantitative comparisons of dissolved hydrogen density and the electrical and optical properties of ZnO, J. Appl. Phys. 94 (2003) 2888-2894.

[471] A. Janotti, C.G. Van de Walle, Hydrogen multicentre bonds, Nature Mater. 6 (2007) 4447.

[472] M.D. McCluskey, S.J. Jokela, Defects in ZnO, J. Appl. Phys. 106 (2009) 071101.

[473] G. Brauer, W. Anwand, D. Grambole, W. Skorupa, Y. Hou, A. Andreev, C. Teichert, K.H. Tam, A.B. Djurišić, Non-destructive characterization of vertical ZnO nanowire arrays by slow positron implantation spectroscopy, atomic force microscopy, and nuclear reaction analysis, Nanotechnology 18 (2007) 195301. 
[474] J. Čížek, N. Žaludová, M. Vlach, S. Daniš, J. Kuriplach, I. Procházka, G. Brauer, W. Anwand, D. Grambole, W. Skorupa, R. Gemma, R. Kirchheim, A. Pundt, Defect studies of ZnO single crystals electrochemically doped with hydrogen, J. Appl. Phys. 103 (2008) 053508.

[475] K. Takeyasu, K. Fukada, M. Matsumoto, K. Fukutani, Control of the surface electronic structure of $\mathrm{SrTiO}_{3}(001)$ by modulation of the density of oxygen vacancies, J. Phys.:

Condensed Matter 25 (2013).

[476] S. Wilke, D. Hennig, R. Löber, M. Methfessel, M. Scheffler, Ab initio study of hydrogen adsorption on Pd(100), Surf. Sci. 307-309 (1994) 76-81.

[477] W. Dong, V. Ledentu, P. Sautet, A. Eichler, J. Hafner, Hydrogen adsorption on palladium: a comparative theoretical study of different surfaces, Surf. Sci. 411 (1998) 123136.

[478] J.F. Paul, P. Sautet, Density-functional periodic study of the adsorption of hydrogen on a palladium (111) surface, Phys. Rev. B 53 (1996) 8015-8027.

[479] A. Eichler, J. Hafner, G. Kresse, Hydrogen adsorption on the (100) surfaces of rhodium and palladium: the influence of non-local exchange - correlation interactions, J. Phys.:

Condensed Matter 8 (1996) 7659.

[480] V. Pallassana, M. Neurock, L.B. Hansen, B. Hammer, J.K. Nørskov, Theoretical analysis of hydrogen chemisorption on $\mathrm{Pd}(111), \operatorname{Re}(0001)$ and $\mathrm{Pd} \mathrm{d}_{\mathrm{ML}} / \operatorname{Re}(0001), \operatorname{Re}_{\mathrm{ML}} / \mathrm{Pd}(111)$

pseudomorphic overlayers, Phys. Rev. B 60 (1999) 6146-6154.

[481] G. Rupprechter, M. Morkel, H.-J. Freund, R. Hirschl, Sum frequency generation and density functional studies of $\mathrm{CO}-\mathrm{H}$ interaction and hydrogen bulk dissolution on $\mathrm{Pd}(111)$, Surf. Sci. 554 (2004) 43-59.

[482] S. Yamanaka, K. Higuchi, M. Miyake, Hydrogen solubility in zirconium alloys, J. Alloys Compds. 231 (1995) 503-507.

[483] C.S. Zhang, B.J. Flinn, K. Griffiths, P.R. Norton, Interaction of hydrogen with $\operatorname{Zr}(0001)$ at room temperature, J. Vac. Sci. Technol. A 10 (1992) 2560-2564.

[484] C.S. Zhang, B. Li, P.R. Norton, The initial stages of interaction of hydrogen with the $\operatorname{Zr}(10 \overline{1} 0)$ surface, Surf. Sci. 346 (1996) 206-221.

[485] S. Ogura, M. Okada, K. Fukutani, Near-Surface Accumulation of Hydrogen and CO Blocking Effects on a Pd-Au Alloy, J. Phys. Chem. C 117 (2013) 9366-9371.

[486] C.S. Zhang, B. Li, P.R. Norton, The study of hydrogen segregation on $\operatorname{Zr}(0001)$ and $\mathrm{Zr}(1010)$ surfaces by static secondary ion mass spectroscopy, work function, Auger electron spectroscopy and nuclear reaction analysis, J. Alloys Compds. 231 (1995) 354-363.

[487] G. Teeter, J.L. Erskine, Surface relaxation of Ti(0001): Influence of hydrogen contamination, Phys. Rev. B 61 (2000) 13929-13935.

[488] M.N. Huda, L. Kleinman, Density functional calculations of the influence of hydrogen adsorption on the surface relaxation of Ti(0001), Phys. Rev. B 71 (2005) 241406.

[489] Y. Hu, K. Griffiths, P.R. Norton, G. Bussière, J. Hepburn, Laser induced thermal desorption of hydrogen from $\operatorname{Zr}(0001)$ : Relationship to water dissociation and hydrogen dissolution, Surf. Sci. 601 (2007) 3645-3650.

[490] G. Bussiere, M. Musa, P.R. Norton, K. Griffiths, A.G. Brolo, J.W. Hepburn, Dynamics of $D_{2}$ released from the dissociation of $D_{2} \mathrm{O}$ on a zirconium surface, J. Chem. Phys. 124 (2006) 124704-124709.

[491] M. Kovar, K. Griffiths, R.V. Kasza, J.G. Shapter, P.R. Norton, V.P. Zhdanov, Kinetics of concurrent desorption and diffusion into the solid: D/Zr(0001), J. Chem. Phys. 106 (1997) 4797-4805.

[492] J.W. He, P.R. Norton, Adsorption and reaction of oxygen and deuterium on a $\mathrm{Pd}(110)$ surface, studied by $\Delta \phi$ and TDS, Surf. Sci. 230 (1990) 150-158.

[493] U. Memmert, J.W. He, K. Griffiths, W.N. Lennard, P.R. Norton, N.V. Richardson, T.E. Jackman, W.N. Unertl, $\mathrm{D}_{2}$ on $\mathrm{Pd}(110)$ : Surface and subsurface phases, absolute coverages, and interconversion, J. Vac. Sci. Technol. A 7 (1989) 2152-2154. 
[494] J.M. Lin, R.E. Gilbert, The interaction of hydrogen with polycrystalline zirconium. 1. Sticking coefficients and binding states, Appl. Surf. Sci. 18 (1984) 315-324.

[495] H. Okuyama, W. Siga, N. Takagi, M. Nishijima, T. Aruga, Path and mechanism of hydrogen absorption at Pd(100), Surf. Sci. 401 (1998) 344-354.

[496] G.E. Gdowski, R.H. Stulen, T.E. Felter, Isotopic differences in low-temperature sorption and desorption of hydrogen by Pd(111), J. Vac. Sci. Technol. A 5 (1987) 1103-1104.

[497] R.J. Behm, V. Penka, M.G. Cattania, K. Christmann, G. Ertl, Evidence for Subsurface Hydrogen on $\mathrm{Pd}(110)$ - an Intermediate between Chemisorbed and Dissolved Species, J. Chem. Phys. 78 (1983) 7486-7490.

[498] M.G. Cattania, V. Penka, R.J. Behm, K. Christmann, G. Ertl, Interaction of Hydrogen with a Palladium(110) Surface, Surf. Sci. 126 (1983) 382-391.

[499] R.J. Behm, K. Christmann, G. Ertl, Adsorption of Hydrogen on Pd(100), Surf. Sci. 99 (1980) 320-340.

[500] M.J. Gladys, I. Kambali, M.A. Karolewski, A. Soon, C. Stampfl, D.J. O'Connor, Comparison of hydrogen and deuterium adsorption on Pd(100), J. Chem. Phys. 132 (2010).

[501] S.H. Kim, H.L. Meyerheim, J. Barthel, J. Kirschner, J. Seo, J.S. Kim, Origin of anomalous surface lattice expansion in Pd(001), Phys. Rev. B 71 (2005) 205418.

[502] D. Tománek, Z. Sun, S.G. Louie, Ab initio calculation of chemisorption systems: $\mathrm{H}$ on $\mathrm{Pd}(001)$ and Pd(110), Phys. Rev. B 43 (1991) 4699-4713.

[503] E. Wicke, G.H. Nernst, Zustandsdiagramm und thermodynamisches Verhalten der Systeme $\mathrm{Pd} / \mathrm{H}_{2}$ und $\mathrm{Pd} / \mathrm{D}_{2}$ bei normalen Temperaturen; H/D-Trenneffekte, Ber. Bunsenges. Phys. Chem. 68 (1964) 224-235.

[504] H.W. Lewis, Straggling Effects on Resonant Yields, Phys. Rev. 125 (1962) 937-940. [505] H. Frieske, E. Wicke, Magnetic Susceptibility and Equilibrium Diagram of $\mathrm{PdH}_{\mathrm{n}}$, Ber. Bunsenges. Phys. Chem. 77 (1973) 48-52.

[506] F.A. Lewis, The Palladium-Hydrogen System, Platinum Metals Review 26 (1982) 70.

[507] Y. Fukai, H. Sugimoto, Diffusion of hydrogen in metals, Advances in Physics 34 (1985) 263-326.

[508] B.D. Kay, C.H.F. Peden, D.W. Goodman, Kinetics of hydrogen absorption by Pd(110), Phys. Rev. B 34 (1986) 817-822.

[509] A. Maeland, T.B. Flanagan, X-Ray and Thermodynamic Studies of the Absorption of Hydrogen by Gold-Palladium Alloys, J. Phys. Chem. 69 (1965) 3575-3581.

[510] S. Luo, D. Wang, T.B. Flanagan, Thermodynamics of Hydrogen in fcc Pd-Au Alloys, J. Phys. Chem. B 114 (2010) 6117-6125.

[511] K.H. Rieder, M. Baumberger, W. Stocker, Selective Transition of Chemisorbed Hydrogen to Subsurface Sites on Pd(110), Phys. Rev. Lett. 51 (1983) 1799-1802.

[512] J. Kuntze, S. Speller, W. Heiland, P. Deurinck, C. Creemers, A. Atrei, U. Bardi, Surface structure and segregation profile of the alloy $\mathrm{Au}_{3} \mathrm{Pd}(110)$ : Experiment and theory, Phys. Rev. B 60 (1999) 9010-9018.

[513] T.B. Flanagan, D. Wang, Hydrogen Permeation through fcc Pd-Au Alloy Membranes, J. Phys. Chem. C 115 (2011) 11618-11623.

[514] C.G. Sonwane, J. Wilcox, Y.H. Ma, Achieving optimum hydrogen permeability in PdAg and PdAu alloys, J. Chem. Phys. 125 (2006) 184714.

[515] W.R. Wampler, R.P. Doerner, Deuterium retention in tungsten from exposure to plasma, Physica Scripta, 2009 (2009) 014037.

[516] W.R. Wampler, P.M. Richards, Surface-controlled deuterium-palladium interactions, Phys. Rev. B 41 (1990) 7483-7490.

[517] S.M. Myers, P.M. Richards, W.R. Wampler, F. Besenbacher, lon-beam studies of hydrogen-metal interactions, J. Nucl. Mater. 165 (1989) 9-64.

[518] S.M. Myers, W.R. Wampler, F. Besenbacher, S.L. Robinson, N.R. Moody, lon beam studies of hydrogen in metals, Materials Science and Engineering, 69 (1985) 397-409. 
[519] S. Nagata, K. Takahiro, S. Yamaguchi, S. Yamamoto, B. Tsuchiya, H. Naramoto, Application of ERDA method to study hydrogen and helium in $\mathrm{Ti}, \mathrm{Zr}$ and $\mathrm{Nb}$ membranes, Nucl. Instrum. Methods Phys. Res. B 136-138 (1998) 680-684.

[520] M. Ogura, N. Yamaji, T. Higuchi, M. Imai, A. Itoh, N. Imanishi, K. Nakata, Thermal behavior of hydrogen in helium-implanted high-purity SUS316L, Nucl. Instrum. Methods Phys. Res. B 136-138 (1998) 483-487.

[521] M. Miyamoto, D. Nishijima, Y. Ueda, R.P. Doerner, H. Kurishita, M.J. Baldwin, S. Morito, K. Ono, J. Hanna, Observations of suppressed retention and blistering for tungsten exposed to deuterium-helium mixture plasmas, Nuclear Fusion 49 (2009) 065035.

[522] M.J. Baldwin, R.P. Doerner, W.R. Wampler, D. Nishijima, T. Lynch, M. Miyamoto, Effect of He on $D$ retention in $W$ exposed to low-energy, high-fluence ( $D, \mathrm{He}, \mathrm{Ar}$ ) mixture plasmas, Nuclear Fusion 51 (2011) 103021.

[523] W.D. Michalak, J.B. Miller, D.R. Alfonso, A.J. Gellman, Uptake, transport, and release of hydrogen from Pd(100), Surf. Sci. 606 (2012) 146-155.

[524] R.V. Bucur, V. Mecea, T.B. Flanagan, The kinetics of hydrogen (deuterium) sorption by thin palladium layers studied with a piezoelectric quartz crystal microbalance, Surf. Sci. 54 (1976) 477-488.

[525] M.A. Pick, M.G. Greene, The sticking coefficient of hydrogen on niobium, Surf. Sci. 93 (1980) L129-L134.

[526] M.A. Pick, Kinetics of hydrogen absorption-desorption by niobium, Phys. Rev. B 24 (1981) 4287-4294.

[527] I. Takagi, T. Sugihara, T. Sasaki, K. Moritani, H. Moriyama, Potential energy diagram for hydrogen near vanadium surface, J. Nucl. Mater. 329-333 (2004) 434-437.

[528] I. Takagi, H. Fujita, K. Higashi, Experiments on potential energy diagram for hydrogen isotopes on nickel surface, J. Nucl. Mater. 266-269 (1999) 697-702.

[529] I. Takagi, M. Matsuoka, H. Fujita, K. Shin, K. Higashi, In-situ depth-profiling of deuterium in nickel exposed to RF plasma, J. Nucl. Mater. 179-181 (1991) 319-321.

[530] A.T. Gee, B.E. Hayden, C. Mormiche, T.S. Nunney, The blocking of the step-mediated indirect channel to hydrogen dissociation by oxygen on Pt(533), Surf. Sci. 512 (2002) 165-172. [531] A.T. Gee, B.E. Hayden, C. Mormiche, T.S. Nunney, The role of steps in the dynamics of hydrogen dissociation on Pt(533), J. Chem. Phys. 112 (2000) 7660-7668.

[532] B. Hoffmann, H. Baumann, F. Rauch, Hydrogen uptake by palladium-implanted titanium studied by NRA and RBS, Nucl. Instrum. Methods Phys. Res. B 15 (1986) 361-366.

[533] J. Rydén, B. Hjörvarsson, T. Ericsson, E. Karlsson, A. Krozer, B. Kasemo, Unusual kinetics of hydride formation in Mg-Pd sandwiches, studied by hydrogen profiling and quartz crystal microbalance measurements, J. Less Common Metals 152 (1989) 295-309.

[534] M. Strongin, M. El-Batanouny, M.A. Pick, Structure of Pd overlayers on Nb and Ta and the relationship to hydrogen uptake, Phys. Rev. B 22 (1980) 3126-3129.

[535] M.A. Pick, J.W. Davenport, M. Strongin, G.J. Dienes, Enhancement of Hydrogen Uptake Rates for Nb and Ta by Thin Surface Overlayers, Phys. Rev. Lett. 43 (1979) 286-289.

[536] T. Wang, D. Grambole, R. Grötzschel, F. Herrmann, U. Kreißig, F. Eichhorn, G. Brauer, W. Möller, Mobility and retention of implanted hydrogen in Ti225 titanium alloy, Surf. Coatings Technol. 158-159 (2002) 139-145.

[537] T.S. Wang, D. Grambole, F. Herrmann, H.B. Peng, S.W. Wang, Hydrogen 3D-distribution and the kinetics in a Ti/H system studied by micro-ERDA, NRA and XRD, Surf. Interf. Analysis 39 (2007) 52-57.

[538] B. Tsuchiya, S. Nagata, K. Toh, T. Shikama, Measurement of the transport of hydrogen isotopes near the surface of metal-hydride and deuteride using elastic recoil detection technique, Nucl. Instrum. Methods Phys. Res. B 249 (2006) 370-373. 
[539] J.N. Huiberts, R. Griessen, R.J. Wijngaarden, M. Kremers, C. Van Haesendonck, Logarithmic Divergence of the Electrical Resistivity in the Metal Hydride $\mathrm{YH}_{3-\delta}$, Phys. Rev. Lett. 79 (1997) 3724-3727.

[540] A. Remhof, G. Song, K. Theis-Bröhl, H. Zabel, Reversible loading of epitaxial Y(00.1) films with hydrogen, Phys. Rev. B 56 (1997) R2897-R2899.

[541] M. Kremers, N.J. Koeman, R. Griessen, P.H.L. Notten, R. Tolboom, P.J. Kelly, P.A. Duine, Optical transmission spectroscopy of switchable yttrium hydride films, Phys. Rev. B 57 (1998) 4943-4949.

[542] T.J. Richardson, J.L. Slack, B. Farangis, M.D. Rubin, Mixed metal films with switchable optical properties, Appl. Phys. Lett. 80 (2002) 1349-1351.

[543] T.J. Richardson, J.L. Slack, R.D. Armitage, R. Kostecki, B. Farangis, M.D. Rubin, Switchable mirrors based on nickel--magnesium films, Appl. Phys. Lett. 78 (2001) 3047-3049. [544] I. Bogdanović Radović, Z. Medunić, M. Jakšić, Z. Siketić, N. Skukan, Ion-induced electron emission ERDA with a nuclear microprobe, Nucl. Instrum. Methods Phys. Res. B 231 (2005) 123-129.

[545] D. Dujmic, M. Jakšić, N. Soić, T. Tadić, I. Bogdanović, 3D hydrogen profiling using a proton microbeam, Nucl. Instrum. Methods Phys. Res. B 111 (1996) 126-132.

[546] K. Sasa, K. Furuno, Y. Yamato, H. Ohshima, S. Ishii, T. Komatsubara, M. Kurosawa, The Tsukuba high-energy nuclear microprobe for hydrogen analysis of mineral samples, Nucl. Instrum. Methods Phys. Res. B 210 (2003) 48-53.

[547] T. Komatsubara, K. Sasa, K. Okumoto, S. Ishii, Y. Yamato, K. Satou, K. Furuno, M. Kurosawa, Development of ERCS hydrogen analysis for melt inclusions in erupted magmatic samples, Nucl. Instrum. Methods Phys. Res. B 251 (2006) 237-245.

[548] G. Dollinger, A. Bergmaier, A. Hauptner, S. Dietzel, G.A. Drexler, C. Greubel, V. Hable, P. Reichart, R. Krücken, T. Cremer, A.A. Friedl, Hydrogen microscopy and analysis of DNA repair using focused high energy ion beams, Nucl. Instrum. Methods Phys. Res. B 249 (2006) 270277. [549] P. Reichart, G. Dollinger, A. Bergmaier, G. Datzmann, A. Hauptner, H.J. Körner, R. Krücken, 3D hydrogen microscopy with sub-ppm detection limit, Nucl. Instrum. Methods Phys. Res. B 219-220 (2004) 980-987.

[550] P. Reichart, G. Datzmann, A. Hauptner, R. Hertenberger, C. Wild, G. Dollinger, Science 306 (2004) 1537.

[551] D. Sekiba, H. Yonemura, S. Ogura, M. Matsumoto, Y. Kitaoka, Y. Yokoyama, H. Matsuzaki, T. Narusawa, K. Fukutani, Development of micro-beam NRA for hydrogen mapping: Observation of fatigue-fractured surface of glassy alloys, Nucl. Instrum. Methods Phys. Res. B 269 (2011) 627-631.

[552] D. Sekiba, H. Yonemura, T. Nebiki, M. Wilde, S. Ogura, H. Yamashita, M. Matsumoto, J. Kasagi, Y. Iwamura, T. Itoh, H. Matsuzaki, T. Narusawa, K. Fukutani, Development of microbeam NRA for 3D-mapping of hydrogen distribution in solids: Application of tapered glass capillary to $6 \mathrm{MeV}{ }^{15} \mathrm{~N}$ ion, Nucl. Instrum. Methods Phys. Res. B 266 (2008) 4027-4036. [553] D. Sekiba, H. Yonemura, T. Nebiki, S. Ogura, M. Wilde, M. Matsumoto, T. Okano, J. Kasagi, T. Narusawa, S. Kuribayashi, Y. Iwamura, T. Itoh, H. Matsuzaki, K. Fukutani, Development and application of micro-beam nuclear reaction analysis for observation of hydrogen distribution, J. Vac. Soc. Jpn. 50 (2007) 574.

[554] Y. Mizuno, A. Tanaka, K. Takahiro, T. Takano, Y. Yamauchi, T. Okada, S. Yamaguchi, T. Homma, Hydrogen outgasing from titanium-modified layers with various surface treatments, J. Vac. Sci. Technol. A 19 (2001) 2571-2577.

[555] D. Grambole, T. Wang, F. Herrmann, F. Eichhorn, Hydrogen redistribution in titanium due to bending stress studied by micro ERDA, Nucl. Instrum. Methods Phys. Res. B 210 (2003) 526-530. 
[556] Y. Yokoyama, M. Wilde, K. Fukutani, A. Inoue, Alternative strain aging effect for fatigue of ductile bulk glassy alloys, Materials Transactions 48 (2007) 1261-1265.

[557] S. Blässer, J. Steiger, A. Weidinger, In-situ hydrogen charging of thin $\mathrm{Nb}$ films and depth profiling with the ${ }^{1} \mathrm{H}\left({ }^{15} \mathrm{~N}, \alpha \gamma\right){ }^{12} \mathrm{C}$ nuclear reaction, Nucl. Instrum. Methods Phys. Res. B 85 (1994) 24-27.

[558] J. Steiger, S. Blässer, A. Weidinger, Solubility of hydrogen in thin niobium films, Phys. Rev. B 49 (1994) 5570-5574.

[559] G. Reisfeld, N.M. Jisrawi, M.W. Ruckman, M. Strongin, Hydrogen absorption by thin $\mathrm{Pd} / \mathrm{Nb}$ films deposited on glass, Phys. Rev. B 53 (1996) 4974-4979.

[560] E.J. Grier, O. Kolosov, A.K. Petford-Long, R.C.C. Ward, M.R. Wells, B. Hjörvarsson, Structural changes to epitaxial (0001) holmium layers during hydrogen loading, J. Phys. D: Appl. Phys. 33 (2000) 894.

[561] T. Burkert, A. Miniotas, B. Hjörvarsson, Hydrogen-induced changes of the local structure in Fe/V (001) superlattices, Phys. Rev. B 63 (2001) 125424.

[562] A. Remhof, D. Labergerie, G. Song, C. Sutter, K. Theis-Bröhl, H. Zabel, B. Hjörvarsson, Hydrogen in thin epitaxial metal films and superlattices: structure, magnetism, and transport, J. Magn. Magn. Mater. 198-199 (1999) 264-266.

[563] B. Hjörvarsson, G. Andersson, E. Karlsson, Metallic superlattices: quasi twodimensional playground for hydrogen, J. Alloys Compds. 253-254 (1997) 51-57.

[564] G. Andersson, B. Hjörvarsson, P. Isberg, Influence of compressive biaxial strain on the hydrogen uptakeof ultrathin single-crystal vanadium layers, Phys. Rev. B 55 (1997) 17741781.

[565] G. Andersson, B. Hjörvarsson, H. Zabel, Hydrogen-induced lattice expansion of vanadium in a Fe/V (001) single-crystal superlattice, Phys. Rev. B 55 (1997) 15905-15911.

[566] F. Stillesjö, B. Hjörvarsson, B. Rodmacq, Studies of interdiffusion and alloy formation in Fe/V multilayers using hydrogen as a local probe, J. Magn. Magn. Mater. 126 (1993) 102-104. [567] A. Weidinger, D. Nagengast, C. Rehm, F. Klose, B. Pietzak, Metallic multilayers and hydrogen, Thin Solid Films 275 (1996) 48-53.

[568] D. Nagengast, J. Erxmeyer, F. Klose, C. Rehm, P. Kuschnerus, G. Dortmann, A. Weidinger, Hydrogen-charging of Nb/Fe multilayers, J. Alloys Compds. 231 (1995) 307-309. [569] G. Song, A. Remhof, D. Labergerie, H. Zabel, Kinetics of hydrogen absorption and desorption in epitaxial thin niobium films, Phys. Rev. B 66 (2002) 045407.

[570] G. Song, A. Remhof, D. Labergerie, C. Sutter, H. Zabel, Hydrogen in thin epitaxial Nb films, J. Alloys Compds. 293-295 (1999) 476-479.

[571] S. Yamamoto, H. Naramoto, Hydrogen distribution characterization in $\mathrm{Cu}(111) / \mathrm{Nb}(110)$ multilayer using ion beam analysis, Nucl. Instrum. Methods Phys. Res. B 161-163 (2000) 605608.

[572] J. Rydén, B. Hjörvarsson, T. Ericsson, E. Karlsson, A. Chamberod, B. Rodmacq, Hydrogenated Fe-Ti superlattices studied by hydrogen depth profiling, Mössbauer spectroscopy and X-ray diffraction, J. Less Common Metals 172-174 (1991) 784-791. [573] A. Pundt, R. Kirchheim, Hydrogen in Metals: Microstructural Aspects, Annu. Rev. Mater. Res. 36 (2006) 555-608.

[574] J.L. Slack, J.C.W. Locke, S.-W. Song, J. Ona, T.J. Richardson, Metal hydride switchable mirrors: Factors influencing dynamic range and stability, Sol. Energy Mater. Sol. Cells 90 (2006) 485-490.

[575] A.T.M. van Gogh, S.J. van der Molen, J.W.J. Kerssemakers, N.J. Koeman, R. Griessen, Performance enhancement of metal-hydride switchable mirrors using $\mathrm{Pd} / \mathrm{AlO}_{\mathrm{x}}$ composite cap layers, Appl. Phys. Lett. 77 (2000) 815-817.

[576] C. Chacon, E. Johansson, B. Hjörvarsson, C. Zlotea, Y. Andersson, Growth and hydrogen uptake of Mg-Y thin films, J. Appl. Phys. 97 (2005) 104903. 
[577] J. Emil, C. Cyril, Z. Claudia, A. Yvonne, H. Björgvin, Hydrogen uptake and optical properties of sputtered Mg-Ni thin films, J. Phys: Condensed Matter 16 (2004) 7649.

[578] A. Baldi, M. Gonzalez-Silveira, V. Palmisano, B. Dam, R. Griessen, Destabilization of the Mg-H System through Elastic Constraints, Phys. Rev. Lett. 102 (2009) 226102.

[579] J. Steiger, O. Boebel, M. Briere, A. Weidinger, P. Ziegler, Hydrogen profiling of Nb-Hf$\mathrm{Nb}$ layers by the ${ }^{15} \mathrm{~N}$ method, Nucl. Instrum. Methods Phys. Res. B 50 (1990) 31-34.

[580] G.G. Libowitz, Binary Metal Hydrides, W.A. Benjamin, New York, Amsterdam, 1965.

[581] S. Yamamoto, H. Naramoto, Y. Aoki, Analysis of hydrogen in $\mathrm{Nb}$ thin films and $\mathrm{Nb} / \mathrm{Cu}$ multilayers, using ion beams, J. Alloys Compds. 253-254 (1997) 66-69.

[582] G. Andersson, K. Aits, B. Hjörvarsson, Hydrogen uptake of thin epitaxial vanadium (001) films, J. Alloys Compds. 334 (2002) 14-19.

[583] S. Olsson, B. Hjörvarsson, E.B. Svedberg, K. Umezawa, H-H interactions in Nb/W(110) superlattices, Phys. Rev. B 66 (2002) 155433.

[584] S. Ólafsson, B. Hjörvarsson, F. Stillesjö, E. Karlsson, J. Birch, J.E. Sundgren, Charge transfer at interfaces in $\mathrm{Mo}_{x} \mathrm{~V}_{1-\mathrm{x}} / \mathrm{V}$ superlattices, Phys. Rev. B 52 (1995) 10792-10795. [585] P. Kesten, A. Pundt, G. Schmitz, M. Weisheit, H.U. Krebs, R. Kirchheim, H- and D distribution in metallic multilayers studied by 3-dimensional atom probe analysis and secondary ion mass spectrometry, J. Alloys Compds. 330-332 (2002) 225-228.

[586] J.N. Huiberts, J.H. Rector, R.J. Wijngaarden, S. Jetten, D. de Groot, B. Dam, N.J. Koeman, R. Griessen, B. Hjörvarsson, S. Olafsson, Y.S. Cho, Synthesis of yttriumtrihydride films for exsitu measurements, J. Alloys Compds. 239 (1996) 158-171.

[587] M.C. Huisman, M.R. Jongerden, S.J. van der Molen, R.D. Vis, lon beam analysis of oxidation and hydrogenation of switchable mirrors, Nucl. Instrum. Methods Phys. Res. B 181 (2001) 389-393.

[588] M.C. Huisman, S.J. van der Molen, R.D. Vis, Elastic recoil detection analysis for the determination of hydrogen concentration profiles in switchable mirrors, Nucl. Instrum. Methods Phys. Res. B 158 (1999) 451-457.

[589] B. Bogdanović, K. Bohmhammel, B. Christ, A. Reiser, K. Schlichte, R. Vehlen, U. Wolf, Thermodynamic investigation of the magnesium-hydrogen system, J. Alloys Compds. 282 (1999) 84-92.

[590] F. Stillesjø, S. Olafsson, B. Hjörvarsson, E. Karlsson, Hydride formation in $\mathrm{Mg} / \mathrm{Ni}-$ sandwiches studied by hydrogen profiling and volumetric measurements, Z. Phys. Chem. 181 (1993) 353-358.

[591] M. Johansson, O. Lytken, I. Chorkendorff, The sticking probability for $\mathrm{H}_{2}$ on some transition metals at a hydrogen pressure of 1 bar, J. Chem. Phys. 128 (2008) 034706-034711. [592] S. Bao, Y. Yamada, K. Tajima, M. Okada, K. Yoshimura, Optical property and cycling durability of polytetrafluoroethylene top-covered and metal buffer layer inserted $\mathrm{Mg}-\mathrm{Ni}$ switchable mirror, Sol. Energy Mater. Sol. Cells 93 (2009) 1642-1646.

[593] S. Bao, K. Tajima, Y. Yamada, M. Okada, K. Yoshimura, Metal buffer layer inserted switchable mirrors, Sol. Energy Mater. Sol. Cells 92 (2008) 216-223.

[594] K. Tajima, H. Hotta, Y. Yamada, M. Okada, K. Yoshimura, Electrochromic switchable mirror glass with controllable reflectance, Appl. Phys. Lett. 100 (2012) 091906.

[595] K. Tajima, H. Hotta, Y. Yamada, M. Okada, K. Yoshimura, Degradation studies of electrochromic all-solid-state switchable mirror glass under various constant temperature and relative humidity conditions, Sol. Energy Mater. Sol. Cells 94 (2010) 2411-2415.

[596] K. Tajima, Y. Yamada, M. Okada, K. Yoshimura, Surface Coating of Electrochromic Switchable Mirror Glass Based on Mg-Ni Thin Film for High Durability in the Environment, Appl. Phys. Express 3 (2010) 042201.

[597] D. Sekiba, M. Horikoshi, S. Abe, S. Ishii, Mg segregation in Mg-rich Mg--Ni switchable mirror studied by Rutherford backscattering, elastic recoil detection analysis, and nuclear reaction analysis, J. Appl. Phys. 106 (2009) 114912. 
[598] W.P. Kalisvaart, E.J. Luber, E. Poirier, C.T. Harrower, A. Teichert, D. Wallacher, N. Grimm, R. Steitz, H. Fritzsche, D. Mitlin, Probing the Room Temperature Deuterium Absorption Kinetics in Nanoscale Magnesium Based Hydrogen Storage Multilayers Using Neutron Reflectometry, X-ray Diffraction, and Atomic Force Microscopy, J. Phys. Chem. C 116 (2012) 5868-5880.

[599] C.J. Chung, S.C. Lee, J.R. Groves, E.N. Brower, R. Sinclair, B.M. Clemens, Interfacial Alloy Hydride Destabilization in Mg/Pd Thin Films, Phys. Rev. Lett. 108 (2012).

[600] A. Pundt, C. Sachs, M. Winter, M.T. Reetz, D. Fritsch, R. Kirchheim, Hydrogen sorption in elastically soft stabilized Pd-clusters, J. Alloys Compds. 293-295 (1999) 480-483.

[601] A. Pundt, M. Suleiman, C. Bähtz, M.T. Reetz, R. Kirchheim, N.M. Jisrawi, Hydrogen and Pd-clusters, Mater. Sci. Eng. B 108 (2004) 19-23.

[602] C. Sachs, A. Pundt, R. Kirchheim, M. Winter, M.T. Reetz, D. Fritsch, Solubility of hydrogen in single-sized palladium clusters, Phys. Rev. B 64 (2001) 075408.

[603] M. Yamauchi, R. Ikeda, H. Kitagawa, M. Takata, Nanosize Effects on Hydrogen Storage in Palladium, J. Phys. Chem. C 112 (2008) 3294-3299.

[604] G.C. Bond, Metal-Catalysed Reactions of Hydrocarbons, Springer, New York, 2005. [605] H.J. Freund, Model systems in heterogeneous catalysis: Selectivity studies at the atomic level, Top. Catal. 48 (2008) 137-144.

[606] R.M. Jaeger, H. Kuhlenbeck, H.J. Freund, M. Wuttig, W. Hoffmann, R. Franchy, H. Ibach, Formation of a well-ordered aluminium oxide overlayer by oxidation of $\mathrm{NiAl}(110)$, Surf. Sci. 259 (1991) 235-252.

[607] M. Baumer, H.J. Freund, Metal deposits on well-ordered oxide films, Prog. Surf. Sci. 61 (1999) 127-198.

[608] A.M. Doyle, S.K. Shaikhutdinov, H.J. Freund, Alkene chemistry on the palladium surface: nanoparticles vs single crystals, J. Catal. 223 (2004) 444-453.

[609] A.M. Doyle, S.K. Shaikhutdinov, S.D. Jackson, H.J. Freund, Hydrogenation on metal surfaces: Why are nanoparticles more active than single crystals?, Angew. Chem. Int. Ed. 42 (2003) 5240-5243.

[610] M. Morkel, G. Rupprechter, H.J. Freund, Finite size effects on supported Pd nanoparticles: Interaction of hydrogen with $\mathrm{CO}$ and $\mathrm{C}_{2} \mathrm{H}_{4}$, Surf. Sci. 588 (2005) L209-L219. [611] S. Shaikhutdinov, M. Heemeier, M. Baumer, T. Lear, D. Lennon, R.J. Oldman, S.D. Jackson, H.J. Freund, Structure-reactivity relationships on supported metal model catalysts: Adsorption and reaction of ethene and hydrogen on $\mathrm{Pd} / \mathrm{Al}_{2} \mathrm{O}_{3} / \mathrm{NiAl}(110)$, J. Catal. 200 (2001) 330-339.

[612] M. Wilde, K. Fukutani, Low-Temperature Growth of Au on H-Terminated Si(111): Instability of Hydrogen at the Au/Si Interface Revealed by Non-Destructive Ultra-Shallow HDepth Profiling, Jpn. J. Appl. Phys., 42 (2003) 4650-4654.

[613] K.H. Hansen, T. Worren, S. Stempel, E. Laegsgaard, M. Baumer, H.J. Freund, F. Besenbacher, I. Stensgaard, Palladium nanocrystals on $\mathrm{Al}_{2} \mathrm{O}_{3}$ : Structure and adhesion energy, Phys. Rev. Lett. 83 (1999) 4120-4123.

[614] D. Matsumura, Y. Okajima, Y. Nishihata, J.i. Mizuki, Fast and real-time observation of hydrogen absorption kinetics for palladium nanoparticles, J. Alloys Compds. 509 Supplement 2 (2011) S849-S852.

[615] M. Khanuja, B.R. Mehta, P. Agar, P.K. Kulriya, D.K. Avasthi, Hydrogen induced lattice expansion and crystallinity degradation in palladium nanoparticles: Effect of hydrogen concentration, pressure, and temperature, J. Appl. Phys. 106 (2009) 093515.

[616] W. Vogel, W. He, Q.-H. Huang, Z. Zou, X.-G. Zhang, H. Yang, Palladium nanoparticles "breathe" hydrogen; a surgical view with X-ray diffraction, Int. J. Hydrog. Energy 35 (2010) 8609-8620. 
[617] M. Wilde, K. Fukutani, W. Ludwig, B. Brandt, J.H. Fischer, S. Schauermann, H.J. Freund, Influence of Carbon Deposition on the Hydrogen Distribution in Pd Nanoparticles and Their Reactivity in Olefin Hydrogenation, Angew. Chem. Int. Ed. 47 (2008) 9289-9293.

[618] S. Schauermann, N. Nilius, S. Shaikhutdinov, H.J. Freund, Nanoparticles for Heterogeneous Catalysis: New Mechanistic Insights, Acc. Chem. Res. 46 (2013) 1673-1681. [619] W. Ludwig, A. Savara, R.J. Madix, S. Schauermann, H.-J. Freund, Subsurface Hydrogen Diffusion into Pd Nanoparticles: Role of Low-Coordinated Surface Sites and Facilitation by Carbon, J. Phys. Chem. C 116 (2012) 3539-3544.

[620] K.M. Neyman, S. Schauermann, Hydrogen Diffusion into Palladium Nanoparticles: Pivotal Promotion by Carbon, Angew. Chem. Int. Ed. 49 (2010) 4743-4746.

[621] B. Kleinsorge, S.E. Rodil, G. Adamopoulos, J. Robertson, D. Grambole, W. Fukarek, Hydrogen and disorder in diamond-like carbon, Diamond Relat. Mater. 10 (2001) 965-969. [622] P. Danesh, B. Pantchev, B. Schmidt, Infrared absorption strengths of ion-implanted hydrogenated amorphous silicon, Thin Solid Films 516 (2008) 3383-3386.

[623] P. Danesh, B. Pantchev, B. Schmidt, D. Grambole, Molecular hydrogen in amorphous silicon with high internal stress, Jpn. J. Appl. Phys. 46 (2007) 5050-5052.

[624] P. Danesh, B. Pantchev, B. Schmidt, D. Grambole, Hydrogen solubility limit in hydrogenated amorphous silicon, Semiconductor Sci. Technol. 19 (2004) 1422-1425.

[625] P. Danesh, B. Pantchev, K. Antonova, E. Liarokapis, B. Schmidt, D. Grambole, J. Baran, Hydrogen bonding and structural order in hydrogenated amorphous silicon prepared with hydrogen-diluted silane, J. Physics D: Appl. Phys. 37 (2004) 249-254.

[626] B. Pantchev, P. Danesh, K. Antonova, B. Schmidt, D. Grambole, J. Baran, Effect of film thickness on hydrogen content in $a-\mathrm{Si}: \mathrm{H}$, J. Mater. Sci.: Mater. Electron. 14 (2003) 751-752.

[627] P. Danesh, B. Pantchev, D. Grambole, B. Schmidt, Effect of film thickness on hydrogenated amorphous silicon grown with hydrogen diluted silane, Appl. Phys. Lett. 80 (2002) 2463-2465.

[628] P. Danesh, B. Pantchev, D. Grambole, B. Schmidt, Depth distributions of hydrogen and intrinsic stress in $a$-Si : $\mathrm{H}$ films prepared from hydrogen-diluted silane, J. Appl. Phys. 90 (2001) 3065-3068.

[629] B. Pantchev, P. Danesh, I. Savatinova, E. Liarokapis, B. Schmidt, D. Grambole, The etfect of structural disorder on mechanical stress in $a$-Si : H films, J. Physics D-Appl. Phys. 34 (2001) 2589-2592.

[630] V.K. Kudoyarova, G.M. Gusinsky, L.A. Rassadin, I.V. Kudryavtsev, Hydrogen depth profile measurement in $a-\mathrm{Si}_{1-\mathrm{X}} \mathrm{C}_{\mathrm{x}}: \mathrm{H}$ films by elastic recoil detection, Appl. Surf. Sci. 50 (1991) 173-176.

[631] N. Moncoffre, G. Barbier, E. Leblond, P. Martin, H. Jaffrezic, Diffusion studies using ion beam analysis, Nucl. Instrum. Methods Phys. Res. B 140 (1998) 402-408.

[632] J. Portmann, C. Haug, R. Brenn, J. Schneider, K. Rottner, R. Helbig, Determination of hydrogen in $6 \mathrm{H}-\mathrm{SiC}$ epitaxial layers by the $15 \mathrm{~N}$ nuclear reaction analysis technique, Nucl. Instrum. Methods Phys. Res. B 155 (1999) 132-136.

[633] A. Markwitz, M. Bachmann, H. Baumann, K. Bethge, E. Krimmel, P. Misaelides, Hydrogen profiles of thin PVD silicon nitride films using elastic recoil detection analysis, Nucl. Instrum. Methods Phys. Res. B 68 (1992) 218-222.

[634] K.H. Ecker, U. Reinholz, M. Volbracht, H.P. Weise, Analysis of hydrogen in technical layers, Nucl. Instrum. Methods Phys. Res. B 136-138 (1998) 1277-1280.

[635] S. Kumar, V.S. Raju, Compositional analysis of silicon nitride films on Si and GaAs by backscattering spectrometry and nuclear resonance reaction analysis, Nucl. Instrum. Methods Phys. Res. B 226 (2004) 631-636.

[636] Z.Y. Liu, S. Ito, M. Wilde, K. Fukutani, I. Hirozawa, T. Koganezawa, A hydrogen storage layer on the surface of silicon nitride films, Appl. Phys. Lett. 92 (2008). 
[637] H. Yasui, K. Awazu, H. Naramoto, Hydrogen analysis of BCN films with resonant nuclear reactions, Surf. Coatings Technol. 196 (2005) 221-225.

[638] A. Kimura, Y. Nakatani, K. Yamada, T. Suzuki, Hydrogen detection in CVD diamond films by elastic recoil detection analysis, Diamond Relat. Mater. 8 (1999) 37-41.

[639] A. Bergmaier, G. Dollinger, A. Aleksov, P. Gluche, E. Kohn, Deuterium depth profiles at CVD diamond surfaces, Surf. Sci. 481 (2001) L433-L436.

[640] S.M. Myers, C.H. Seager, Interaction of defects and H in proton-irradiated GaN(Mg, H), J. Appl. Phys. 97 (2005) 093517.

[641] V.M.F. Hammer, A. Beran, D. Endisch, F. Rauch, OH concentrations in natural titanites determined by FTIR spectroscopy and nuclear reaction analysis, Eur. J. Mineralogy 8 (1996) 281.

[642] E. Libowitzky, G.R. Rossman, An IR absorption calibration for water in minerals, Am. Mineralogist 82 (1997) 1111.

[643] W. Kulisch, T. Sasaki, F. Rossi, C. Popov, C. Sippel, D. Grambole, Hydrogen incorporation in ultrananocrystalline diamond/amorphous carbon films, phys. stat. sol. (Rapid Research Letters) 2 (2008) 77-79.

[644] T.E. Derry, C.C.P. Madiba, J.P.F. Sellschop, Oxygen and hydrogen on the surface of diamond, Nucl. Instrum. Methods Phys. Res. 218 (1983) 559-562.

[645] J.P.F. Sellschop, S.H. Connell, C.C.P. Madiba, E. Sideras-haddad, S. Kalbitzer, S. Jans, P. Oberschachtsiek, K. Bharuth-Ram, J. Schneider, R. Kiefl, The nature of the state of hydrogen on the surface and in the bulk of natural and synthetic diamond (using ion beam techniques), Vacuum 45 (1994) 397-402.

[646] P. Trocellier, B. Nens, C. Engelmann, Measurements of the hydrogen, sodium and aluminum concentration versus depth in the near surface region of glasses by resonant nuclear reactions, Nucl. Instrum. Methods Phys. Res. 197 (1982) 15-25.

[647] W.A. Lanford, C. Burman, R.H. Doremus, Y. Mehrotra, T. Wassick, Nuclear reaction analysis of glass surfaces: the study of the reaction between water and glass, Mater. Sci. Res. 15 (1983) 549-570.

[648] W.A. Lanford, K. Davis, P. Lamarche, T. Laursen, R. Groleau, R.H. Doremus, Hydration of soda-lime glass, J. Non-Crystalline Solids 33 (1979) 249-266.

[649] K. Cummings, W.A. Lanford, M. Feldmann, Weathering of glass in moist and polluted air, Nucl. Instrum. Methods Phys. Res. B 136 (1998) 858-862.

[650] A. Agarwal, M. Tomozawa, W.A. Lanford, Effect of stress on water diffusion in silica glass at various temperatures, J. Non-Crystalline Solids 167 (1994) 139-148.

[651] J.E. Ericson, Anthropology Papers 23 (1982) 299.

[652] O. Dersch, F. Rauch, Water uptake of quartz investigated by means of ion-beam analysis, Fresenius' J. Analytical Chem. 365 (1999) 114-116.

[653] M. Mäder, C. Neelmeijer, Proton beam examination of glass - an analytical contribution for preventive conservation, Nucl. Instrum. Methods Phys. Res. B 226 (2004) 110-118.

[654] M. Mäder, D. Grambole, F. Herrmann, C. Neelmeijer, M. Schreiner, G. Woisetschläger, Non-destructive evaluation of glass corrosion states, Nucl. Instrum. Methods Phys. Res. B 136-138 (1998) 863-868.

[655] R.A. Livingston, J.S. Schweitzer, C. Rolfs, H.-W. Becker, S. Kubsky, Characterization of the induction period in tricalcium silicate hydration by nuclear resonance reaction analysis, J. Mater. Res. 16 (2001) 687-693.

[656] J.S. Schweitzer, R.A. Livingston, C. Rolfs, H.-W. Becker, S. Kubsky, T. Spillane, M. Castellote, P.G. de Viedma, Nanoscale studies of cement chemistry with ${ }^{15} \mathrm{~N}$ resonance reaction analysis, Nucl. Instrum. Methods Phys. Res. B 241 (2005) 441-445.

[657] J.S. Schweitzer, R.A. Livingston, C. Rolfs, H.W. Becker, S. Kubsky, lon beam analysis of the hydration of tricalcium silicate, Nucl. Instrum. Methods Phys. Res. B 207 (2003) 80-84. 
[658] D.R. Bell, P.D. Ihringer, G.R. Rossman, Quantitative analysis of trance $\mathrm{OH}$ in garnet and pyroxene, Am. Mineralogist 80 (1995) 465.

[659] D.R. Bell, G.R. Rossman, Water in Earth's mantle: the role of nominally anhydrous minerals, Science 255 (1992) 1391.

[660] J. Maldener, A. Hosch, K. Langer, F. Rauch, Hydrogen in some natural garnets studied by nuclear reaction analysis and vibrational spectroscopy, Phys. Chem. Minerals 30 (2003) 337-344.

[661] D.R. Bell, G.R. Rossman, J. Maldener, D. Endisch, F. Rauch, Hydroxide in kyanite: A quantitative determination of the absolute amount and calibration of the IR spectrum, Am. Mineralogist 89 (2004) 998-1003.

[662] D.R. Bell, G.R. Rossman, J. Maldener, D. Endisch, F. Rauch, Hydroxide in olivine: A quantitative determination of the absolute amount and calibration of the IR spectrum, J. Geophys. Res. Solid Earth 108 (2003) 2105.

[663] G.R. Rossman, Analytical Methods for Measuring Water in Nominally Anhydrous Minerals, Rev. Mineralogy Geochem. 62 (2006) 1-28.

[664] R.H. Doremus, Diffusion of water in silica glass, J. Mater. Res. 10 (1995) 2379-2389.

[665] R.H. Doremus, Glass Science, Wiley-Interscience, New York, 1994.

[666] R.H. Doremus, Interdiffusion of hydrogen and alkali ions in a glass surface, J. NonCrystalline Solids 19 (1975) 137-144.

[667] A. Temmink, O. Anderson, K. Bange, H. Hantsche, X. Yu, $4 \mathrm{f}$ level shifts of tungsten and colouration state of $a-\mathrm{WO}_{3}$, Vacuum 41 (1990) 1144-1146.

[668] S.K. Deb, Optical and photoelectric properties and colour centres in thin films of tungsten oxide, Philos. Mag. 27 (1973) 801-822.

[669] C.M. Lambert, T.R. Omstread, P.C. Yu, Chemical and Optical Properties of Electrochromic Nickel Oxide Films, Proceedings SPIE 15 (1995) 562.

[670] W. Wagner, F. Rauch, C. Ottermann, K. Bange, In-depth profiling of hydrogen in oxidic multilayer systems, Surf. Interf. Analysis 16 (1990) 331-334.

[671] J. Krauser, F. Wulf, M.A. Briere, J. Steiger, D. Bräunig, Study of hydrogen incorporation in MOS-structures after various process steps using nuclear reaction analysis (NRA),

Microelectron. Eng. 22 (1993) 65-68.

[672] S.C. Li, S.P. Murarka, Electrical characteristics and hydrogen concentration of chemical vapor deposited silicon dioxide films: Effect of water treatment, J. Appl. Phys. 72 (1992)

4214-4219.

[673] K. Maser, J. Krauser, H. Berger, D. Bräunig, Hydrogen peak concentration in the SiO2Si transition zone as a function of oxide film thickness, Microelectron. Eng. 28 (1995) 129-132. [674] S. Scharf, J. Krauser, M. Störring, F. Wulf, D. Bräunig, Hydrogen concentration and interface state generation due to ionizing radiation in aluminum and polysilicon gate MOS devices, Microelectron. Eng. 28 (1995) 353-356.

[675] J. Krauser, A. Weidinger, D. Bräunig, in: E.H. Poindexter, C.R. Helms (Eds.) The Physics and Chemistry of $\mathrm{SiO}_{2}$ and of the $\mathrm{Si} / \mathrm{SiO}_{2}$ Interface-3 The Electrochemical Society, Pennington (NJ), 1996, p. 59.

[676] J. Krauser, A. Weidinger, D. Bräunig, in: H.Z. Massoud, E.H. Poindexter, C.R. Helms (Eds.) The Physics and Chemistry of $\mathrm{SiO}_{2}$ and of the $\mathrm{Si} / \mathrm{SiO}_{2}$ Interface-3 The Electrochemical Society, Pennington (NJ), 1996, p. 184.

[677] I.J.R. Baumvol, E.P. Gusev, F.C. Stedile, J. F. L. Freire, M.L. Green, D. Brasen, On the behavior of deuterium in ultrathin $\mathrm{SiO}_{2}$ films upon thermal annealing, Appl. Phys. Lett. 72 (1998) 450-452.

[678] I.J.R. Baumvol, C. Rolfs, lon beam analysis of ultrathin dielectric films, Nucl. Instrum. Methods Phys. Res. B 99 (1995) 431-435.

[679] M. Wilde, K. Fukutani, Z.Y. Liu, S. Fujieda, Behavior of hydrogen near $\mathrm{SiO}_{2} / \mathrm{Si}(001)$ interfaces probed by resonant nuclear reaction analysis, J. Vac. Soc. Jpn. 46 (2003) 607. 
[680] M. Wilde, M. Matsumoto, K. Fukutani, Z.Y. Liu, K. Ando, Y. Kawashima, S. Fujieda, Influence of $\mathrm{H}_{2}$-annealing on the hydrogen distribution near $\mathrm{SiO}_{2} / \mathrm{Si}(100)$ interfaces revealed by in situ nuclear reaction analysis, J. Appl. Phys. 92 (2002) 4320-4329.

[681] Z. Liu, S. Fujieda, K. Terashima, M. Wilde, K. Fukutani, Hydrogen redistribution induced by negative-bias-temperature stress in metal-oxide-silicon diodes, Appl. Phys. Lett. 81 (2002) 2397-2399.

[682] Y. Kawashima, Z.Y. Liu, K. Terashima, K. Hamada, K. Fukutani, M. Wilde, S. Aoyagi, M. Kudo, SIMS characterization of hydrogen transport through $\mathrm{SiO}_{2}$ by low-temperature hydrogen annealing, Appl. Surf. Sci. 212 (2003) 804-808.

[683] S.M. Myers, G.A. Brown, A.G. Revesz, H.L. Hughes, Deuterium interactions with ion implanted $\mathrm{SiO}_{2}$ layers in silicon, J. Appl. Phys. 73 (1993) 2196-2206.

[684] J. Krauser, A.G. Revesz, H.L. Hughes, Nuclear reaction analysis of hydrogen in the buried $\mathrm{SiO}_{2}$ layer of $\mathrm{Si} / \mathrm{SiO}_{2} / \mathrm{Si}$ structures, J. Non-Crystalline Solids 296 (2001) 143-145.

[685] Z. Liu, T. Saito, T. Matsuda, K. Ando, I. Shu, M. Wilde, K. Fukutani, Hydrogen distribution in oxide-nitride-oxide stacks and correlation with data retention of MONOS memories, Reliability Physics Symposium (IRPS), IEEE International (2008) 705-706.

[686] Z. Liu, S. Fujieda, F. Hayashi, M. Shimizu, M. Nakata, H. Ishigaki, M. Wilde, K. Fukutani, Influence of Hydrogen Permeability of Liner Nitride Film on Program/Erase Endurance of Split-Gate Type Flash EEPROMS, Reliability Physics Symposium (IRPS), IEEE International (2007) 190-196.

[687] R. Beyer, H. Burghardt, R. Reich, E. Thomas, D. Grambole, F. Herrmann, T. Scholz, J. Albrecht, D.R.T. Zahn, T. Gessner, Trapping Behavior of Thin Siliconoxynitride Layers Prepared by Rapid Thermal Processing, MRS Online Proceedings Library 428 (1996) 421. [688] R. Beyer, H. Burghardt, G. Prösch, E. Thomas, R. Reich, D. Grambole, F. Herrmann, G. Weidner, H. Syhre, K. Dittmar, A Study of the Compositional Structure and Electrical Behaviour of Thin Silicon Oxynitride Layers Prepared by Rapid Thermal Processing, physica status solidi (a) 145 (1994) 447-452.

[689] Z. Liu, S. Ito, S. Hiroshima, S. Koyama, M. Makabe, M. Wilde, K. Fukutani, Mobile and stable hydrogen species in the interface layer between poly silicon and gate oxynitride, Reliability Physics Symposium (IRPS), IEEE International (2010) 417-423.

[690] Z. Liu, S. Ito, T. Ide, M. Nakata, H. Ishigaki, M. Makabe, M. Wilde, K. Fukutani, H. Mitoh, Y. Kamigaki, Indications for an ideal interface structure of oxynitride tunnel dielectrics, Reliability Physics Symposium (IRPS), IEEE International (2009) 902-906.

[691] Z. Liu, H. Ishigaki, S. Ito, T. Ide, M. Makabe, M. Wilde, K. Fukutani, M. Kimura, V.A. Miha, $\mathrm{H}$. Yoshikawa, Correlation of electrical properties with interface structures of CVD oxidebased oxynitride tunnel dielectrics, in: Integrated Reliability Workshop (IRW '09) Final Report, IEEE International (2009) 145-147.

[692] S.M. Myers, Interaction of deuterium gas with dry $\mathrm{SiO}_{2}$ on $\mathrm{Si}$ : An ion-beam study, J. Appl. Phys. 61 (1987) 5428-5437.

[693] F.J. Himpsel, F.R. McFeely, A. Taleb-Ibrahimi, J.A. Yarmoff, G. Hollinger, Microscopic structure of the $\mathrm{SiO}_{2} / \mathrm{Si}$ interface, Phys. Rev. B 38 (1988) 6084-6096.

[694] C.R. Helms, E.H. Poindexter, The silicon-silicon dioxide system: Its microstructure and imperfections, Reports on Progress in Physics, 57 (1994) 791.

[695] K.H. Ecker, J. Krauser, A. Weidinger, H.P. Weise, K. Maser, Nuclear reaction analysis of hydrogen migration in silicon dioxide films on silicon under ${ }^{15} \mathrm{~N}$ ion irradiation, Nucl. Instrum. Methods Phys. Res. B 161 (2000) 682-685.

[696] K. Maser, U. Mohr, R. Leihkauf, K. Ecker, U. Beck, D. Grambole, R. Grötzschel, F. Herrmann, J. Krauser, A. Weidinger, Hydrogen migration in wet-thermally grown silicon dioxide layers due to high dose ${ }^{15} \mathrm{~N}$ ion beam irradiation, Microelectron. Eng. 48 (1999) 139142. 
[697] B. Pantchev, P. Danesh, B. Schmidt, D. Grambole, W. Moller, lon beam-induced hydrogen migration in a $\mathrm{SiO}_{2} / a-\mathrm{Si}: \mathrm{H} / \mathrm{SiO}_{2}$ layer stack, Semiconductor Sci. Technol. 24 (2009) 035012.

[698] D.A. Buchanan, A.D. Marwick, D.J. DiMaria, L. Dori, Hot-electron-induced hydrogen redistribution and defect generation in metal-oxide-semiconductor capacitors, J. Appl. Phys. 76 (1994) 3595-3608.

[699] K. Nakajima, R. Imaizumi, M. Suzuki, K. Kimura, Accumulation of hydrogen near the interface between ultrathin $\mathrm{SiO}_{2}$ and $\mathrm{Si}(001)$ under ion irradiation in high-resolution elastic recoil detection, Nucl. Instrum. Methods Phys. Res. B 249 (2006) 425-428.

[700] F.J. Feigl, R. Gale, H. Chew, C.W. Magee, D.R. Young, Current-induced hydrogen migration and interface trap generation in aluminum-silicon dioxide-silicon capacitors, Nucl. Instrum. Methods Phys. Res. B 1 (1984) 348-354.

[701] R. Gale, F.J. Feigl, C.W. Magee, D.R. Young, Hydrogen migration under avalanche injection of electrons in Si metal-oxide-semiconductor capacitors, J. Appl. Phys. 54 (1983) 6938-6942.

[702] After large ${ }^{15} \mathrm{~N}$ fluences $\left(>2 \times 10^{16} \mathrm{~cm}^{-2}\right)$ on thin uncapped $\mathrm{SiO}_{2} / \mathrm{Si}$ a decrease of the nearinterfacial $\mathrm{H}$ concentration may occur after the initial pileup has completed $[107 ; 695 ; 696]$, presumably due to $\mathrm{H}$ desorption from the sample. Since such large ${ }^{15} \mathrm{~N}$ fluences are not necessary in routine analysis of typical $\mathrm{H}$ impurity concentrations in dielectric films [680; 697], we shall not consider this behavior further.

[703] E.H. Poindexter, P.J. Caplan, Characterization of $\mathrm{Si} / \mathrm{SiO}_{2}$ interface defects by electron spin resonance, Prog. Surf. Sci. 14 (1983) 201-294.

[704] P.J. Caplan, E.H. Poindexter, B.E. Deal, R.R. Razouk, ESR centers, interface states, and oxide fixed charge in thermally oxidized silicon wafers, J. Appl. Phys. 50 (1979) 5847-5854.

[705] K.L. Brower, Kinetics of $\mathrm{H}_{2}$ passivation of $\mathrm{Pb}_{b}$ centers at the (111) $\mathrm{Si}^{-} \mathrm{SiO}_{2}$ interface, Phys. Rev. B 38 (1988) 9657-9666.

[706] G.D. Wilk, R.M. Wallace, J.M. Anthony, High-k gate dielectrics: Current status and materials properties considerations, J. Appl. Phys. 89 (2001) 5243-5275.

[707] C. Driemeier, L. Miotti, I.J.R. Baumvol, C. Radtke, E.P. Gusev, M.J. Kim, R.M. Wallace, Interaction of $\mathrm{HfO}_{2} / \mathrm{SiO}_{2} / \mathrm{Si}$ structures with deuterium gas, Appl. Phys. Lett. 88 (2006) 041918. [708] C. Driemeier, I.J.R. Baumvol, Some mechanisms for the incorporation of hydrogen in $\mathrm{Hf}$-based gate dielectric films on Si, physica status solidi (a) 204 (2007) 945-950.

[709] L.R.C. Fonseca, J. A. L. Xavier, J. M. Ribeiro, C. Driemeier, I.J.R. Baumvol, Hydrogen trapping in oxygen-deficient hafnium silicates, J. Appl. Phys. 102 (2007) 044108.

[710] R.P. Pezzi, L. Miotti, K.P. Bastos, G.V. Soares, C. Driemeier, I.J.R. Baumvol, P. Punchaipetch, G. Pant, B.E. Gnade, R.M. Wallace, A. Rotondaro, J.M. Visokay, J.J. Chambers, L. Colombo, Hydrogen and deuterium incorporation and transport in hafnium-based dielectric films on silicon, Appl. Phys. Lett. 85 (2004) 3540-3542.

[711] S. Skordas, F. Papadatos, G. Nuesca, J.J. Sullivan, E.T. Eisenbraun, A.E. Kaloyeros, Lowtemperature metalorganic chemical vapor deposition of $\mathrm{Al}_{2} \mathrm{O}_{3}$ for advanced complementary metal-oxide semiconductor gate dielectric applications, J. Mater. Res. 18 (2003) 1868-1876. [712] C. Driemeier, R.M. Wallace, I.J.R. Baumvol, Oxygen species in $\mathrm{HfO}_{2}$ films: An in situ xray photoelectron spectroscopy study, J. Appl. Phys. 102 (2007) 024112.

[713] F. Ojeda, I. Montero, F. Abel, J.M. Albella, Influence of Hydrogen Incorporation on the Structure and Stoichiometry of Chemically Vapor Deposited Silica Films, Chem. Mater. 13 (2001) 3986-3992.

[714] B. Schmidt, D. Grambole, F. Herrmann, Impact of ambient atmosphere on asimplanted amorphous insulating layers, Nucl. Instrum. Methods Phys. Res. B 191 (2002) 482486. 
[715] Z. Liu, S. Ito, T. Saito, S.W. Chang, A. Ogawa, S. Horii, T. Horikawa, M. Wilde, K. Fukutani, T. Chikyow, Impact of air-induced poly-Si/oxynitride interface layer degradation on gateedge leakage, Reliability Physics Symposium (IRPS), IEEE International (2011) 3F.2.1-3F.2.7. [716] Z. Liu, F. Hayashi, S. Fujieda, M. Wilde, K. Fukutani, Reliability driven guideline for BEOL Optimization: Protecting MOS stacks from hydrogen-related impurity penetration, IC Design \& Technology (ICICDT), IEEE International (2012) 1-4.

[717] K.L. Brower, S.M. Myers, Chemical kinetics of hydrogen and (111) $\mathrm{Si}_{-S i O}$ interface defects, Appl. Phys. Lett. 57 (1990) 162-164.

[718] E. Cartier, J.H. Stathis, D.A. Buchanan, Passivation and depassivation of silicon dangling bonds at the $\mathrm{Si} / \mathrm{SiO}_{2}$ interface by atomic hydrogen, Appl. Phys. Lett. 63 (1993) 1510-1512.

[719] A. Stesmans, V.V. Afanas'ev, Hydrogen-induced thermal interface degradation in (111) $\mathrm{Si} / \mathrm{SiO}_{2}$ revealed by electron-spin resonance, Appl. Phys. Lett. 72 (1998) 2271-2273. [720] D.K. Schroder, J.A. Babcock, Negative bias temperature instability: Road to cross in deep submicron silicon semiconductor manufacturing, J. Appl. Phys. 94 (2003) 1-18. [721] D.K. Schroder, Negative bias temperature instability: What do we understand?, Microelectronics Reliability 47 (2007) 841-852.

[722] D.L. Griscom, Diffusion of radiolytic molecular hydrogen as a mechanism for the postirradiation buildup of interface states in $\mathrm{SiO}_{2}$-on-Si structures, J. Appl. Phys. 58 (1985) 25242533.

[723] R.E. Stahlbush, A.H. Edwards, D.L. Griscom, B.J. Mrstik, Post-irradiation cracking of $\mathrm{H}_{2}$ and formation of interface states in irradiated metal-oxide-semiconductor field-effect transistors, J. Appl. Phys. 73 (1993) 658-667.

[724] S. Ogawa, N. Shiono, Generalized diffusion-reaction model for the low-field chargebuildup instability at the $\mathrm{Si}_{-} \mathrm{SiO}_{2}$ interface, Phys. Rev. B 51 (1995) 4218-4230.

[725] C.E. Blat, E.H. Nicollian, E.H. Poindexter, Mechanism of negative-bias-temperature instability, J. Appl. Phys. 69 (1991) 1712-1720.

[726] T.U. Kampen, W. Mönch, Lead contacts on Si(111):H-1 $\times 1$ surfaces, Surf. Sci. 331-333 (1995) 490-495.

[727] W. Mönch, Electronic properties of ideal and interface-modified metal-semiconductor interfaces, J. Vac. Sci. Technol. B 14 (1996) 2985-2993.

[728] W. Mönch, Electronic Properties of Semiconductor Interfaces, Springer, Berlin, 2004.

[729] H. Nienhaus, D. Krix, S. Glass, Varying the Schottky barrier of thin film Mg/H:p-Si(111) contacts: Properties and applications, J. Vac. Sci. Technol. A 25 (2007) 950-954.

[730] T.U. Kampen, R.F. Schmitsdorf, W. Mönch, Silver schottky contacts on Si(111): H-(1×1) surfaces prepared by wet-chemical etching, Appl. Phys. A 60 (1995) 391-394.

[731] N. Masamichi, S. Fumiya, O. Kenjiro, Hydrogen-termination effects on the growth of $\mathrm{Ag}$ thin films on Si(111) surfaces, Surf. Sci. 242 (1991) 152-156.

[732] M. Naitoh, A. Watanabe, S. Nishigaki, An STM observation of silver growth on hydrogen-terminated Si(111) surfaces, Surf. Sci. 357-358 (1996) 140-144.

[733] A. Nishiyama, G. ter Horst, P.M. Zagwijn, G.N. van den Hoven, J.W.M. Frenken, F. Garten, A.R. Schlatmann, J. Vrijmoeth, Growth mode and interface structure of Ag on the HF-treated Si(111):H surface, Surf. Sci. 350 (1996) 229-238.

[734] H. Jeong, S. Jeong, Diffusion of Ag adatom on the H-terminated and clean Si(111) surfaces: A first-principles study, Phys. Rev. B 71 (2005) 035310.

[735] $\mathrm{H}$. Jeong, S. Jeong, Segregation of $\mathrm{H}$ as a surfactant during the formation of an $\mathrm{Ag}$ cluster on H-terminated Si(111): First-principles total-energy calculations, Phys. Rev. B 73 (2006) 125343.

[736] J.H. Han, H.N. Hwang, H.G. Jee, B. Kim, S. Chung, Y.D. Kim, C.C. Hwang, Interaction of Ag with the Si(111)1×1-H surface, Surf. Sci. 604 (2010) 853-856.

[737] Y. Aoki, L. Shi, T. Sugimoto, H. Hirayama, H adsorption at Ag/Si interfaces in epitaxially grown $\mathrm{Ag}(111)$ films on Si(111)7×7 substrates, Surf. Sci. 604 (2010) 420-423. 
[738] Z.H. Lu, K. Griffiths, P.R. Norton, T.K. Sham, Stabilities of different hydrides at the Au/hydrogenated-Si interface, Phys. Rev. B 44 (1991) 5648-5650.

[739] C. Grupp, A. Taleb-Ibrahimi, Au/H:Si(111)-(1×1) interface versus Au/Si(111)-(7×7), Phys. Rev. B 57 (1998) 6258-6261.

[740] K. Budde, E. Abram, V. Yeh, M.C. Tringides, Uniform, self-organized, seven-step height $\mathrm{Pb} / \mathrm{Si}(111)-(7 \times 7)$ islands at low temperatures, Phys. Rev. B 61 (2000) R10602-R10605. [741] M. Hupalo, M.C. Tringides, Correlation between height selection and electronic structure of the uniform height Pb/Si(111) islands, Phys. Rev. B 65 (2002) 115406.

[742] Y.-F. Zhang, J.-F. Jia, Z. Tang, T.-Z. Han, X.-C. Ma, Q.-K. Xue, Growth, stability and morphology evolution of $\mathrm{Pb}$ films on $\mathrm{Si}(111)$ prepared at low temperature, Surf. Sci. 596 (2005) L331-L338.

[743] T.L. Chan, C.Z. Wang, M. Hupalo, M.C. Tringides, K.M. Ho, Quantum Size Effect on the Diffusion Barriers and Growth Morphology of Pb/Si(111), Phys. Rev. Lett. 96 (2006) 226102. [744] J.J. Yeh, J. Hwang, K. Bertness, D.J. Friedman, R. Cao, I. Lindau, Growth of the room temperature $\mathrm{Au} / \mathrm{Si}(111)-7 \times 7$ interface, Phys. Rev. Lett. 70 (1993) 3768-3771.

[745] J. Terry, R. Cao, C. Wigren, P. Pianetta, Photoemission study of Au, Ge, and $\mathrm{O}_{2}$ deposition on $\mathrm{NH}_{4} \mathrm{~F}$ etched Si(111), J. Vac. Sci. Technol. A 12 (1994) 1869-1875.

[746] J.H. Kim, G. Yang, S. Yang, A.H. Weiss, Study of the growth and stability of ultra-thin films of Au deposited on Si(100) and Si(111), Surf. Sci. 475 (2001) 37-46.

[747] L.A. Gheber, M. Hershfinkel, G. Gorodetsky, V. Volterra, Investigation of the composition and electrical properties of gold-H-terminated silicon (111) interface, Thin Solid Films 320 (1998) 228-235.

[748] T.-W. Pi, A.B. Yang, C.G. Olson, D.W. Lynch, Photoemission study of Au on $a-\mathrm{Si}: \mathrm{H}$, Phys. Rev. B 42 (1990) 9566-9574.

[749] S.-H. Lee, G.S. Hwang, Diffusion and clustering of Au adatoms on H-terminated Si(111)(1×1): A first principles study, J. Chem. Phys. 131 (2009) 144702.

[750] J.G. Yu, C.S. Zhang, J.L. Luo, P.R. Norton, Investigation of the Effect of Hydrogen on the Passive Film on Iron by Surface Analysis Techniques, J. Electrochem. Soc. 150 (2003) B68-B75. [751] C.S. Zhang, J.L. Luo, D. Munoz-Paniagua, P.R. Norton, The hydroxylation of passive oxide films on $\mathrm{X}-70$ steel by dissolved hydrogen studied by nuclear reaction analysis, Auger electron spectroscopy, $\mathrm{X}$-ray photoelectron spectroscopy and secondary ion mass spectroscopy, Thin Solid Films 503 (2006) 149-157.

[752] B. Hjörvarsson, A. Stierle, G. Song, H. Zabel, Hydrogen affinity at $\mathrm{Cr} / \alpha-\mathrm{Cr}_{2} \mathrm{O}_{3}$ metal/oxide interfaces studied by the ${ }^{1} \mathrm{H}\left({ }^{15} \mathrm{~N}, \alpha \gamma\right){ }^{12} \mathrm{C}$ nuclear resonance reaction, Vacuum 52 (1999) 291-294.

[753] W.A. Lanford, R.S. Alwitt, C.K. Dyer, Hydrogen profiles of anodic aluminum-oxide films, J. Electrochem. Soc. 127 (1980) 405-411.

[754] L. Iglesias-Rubianes, P. Skeldon, G.E. Thompson, U. Kreissig, D. Grambole, H. Habazaki, K. Shimizu, Behaviour of hydrogen impurity in aluminium alloys during anodizing, Thin Solid Films 424 (2003) 201-207.

[755] D. Khatamian, Hydrogen traps in the oxide-alloy interface region of $\mathrm{Zr}-\mathrm{Nb}$ alloys, J. Alloys Compds. 231 (1995) 722-729.

[756] A. Stern, D. Khatamian, T. Laursen, G.C. Weatherly, J.M. Perz, Hydrogen and deuterium profiling at the surface of zirconium alloys: II. The effects of oxidation, J. Nucl. Mater. 148 (1987) 257-265.

[757] J.P. Abriata, J. Garcés, R. Versaci, The O-Zr (Oxygen-Zirconium) system, Bulletin of Alloy Phase Diagrams 7 (1986) 116-124.

[758] R. Pretorius, M. Peisach, J.W. Mayer, Hydrogen and deuterium depth profiling by elastic recoil detection analysis, Nucl. Instrum. Methods Phys. Res. B 35 (1988) 478-483. 
[759] S. Nagata, S. Yamaguchi, Y. Fujino, Y. Hori, N. Sugiyama, K. Kamada, Depth resolution and recoil cross section for analyzing hydrogen in solids using elastic recoil detection with ${ }^{4} \mathrm{He}$ beam, Nucl. Instrum. Methods Phys. Res. B 6 (1985) 533-537.

[760] Y. Wang, J. Chen, F. Huang, The calculation of the differential cross sections for recoil protons in ${ }^{4} \mathrm{He}-\mathrm{p}$ Scattering, Nucl. Instrum. Methods Phys. Res. B 17 (1986) 11-14.

[761] E. Szilágyi, F. Pászti, A. Manuaba, C. Hajdu, E. Kótai, Cross section measurements of the ${ }^{1} \mathrm{H}\left({ }^{4} \mathrm{He},{ }^{4} \mathrm{He}\right){ }^{1} \mathrm{H}$ elastic recoil reaction for ERD analysis, Nucl. Instrum. Methods Phys. Res. B 43 (1989) 502-506.

[762] J.E.E. Baglin, A.J. Kellock, M.A. Crockett, A.H. Shih, Absolute cross section for hydrogen forward scattering, Nucl. Instrum. Methods Phys. Res. B 64 (1992) 469-474.

[763] J.F. Browning, J.C. Banks, W.R. Wampler, B.L. Doyle, Cross-sections for the elastic recoil of hydrogen isotopes for high energy helium ions, Nucl. Instrum. Methods Phys. Res. B 219220 (2004) 317-322.

[764] A.J. Kellock, J.E.E. Baglin, Absolute cross section for $D\left({ }^{4} \mathrm{He}, \mathrm{D}\right)^{4} \mathrm{He}$ forward scattering, Nucl. Instrum. Methods Phys. Res. B 79 (1993) 493-497.

[765] W. Assmann, J.A. Davies, G. Dollinger, J.S. Forster, H. Huber, T. Reichelt, R. Siegele, ERDA with very heavy ion beams, Nucl. Instrum. Methods Phys. Res. B 118 (1996) 242-250.

[766] G. Dollinger, M. Boulouednine, A. Bergmaier, T. Faestermann, C.M. Frey, Limits in elastic recoil detection analysis with heavy ions, Nucl. Instrum. Methods Phys. Res. B 118 (1996) 291-300.

[767] J.S. Williams, W. Möller, On the determination of optimum depth-resolution conditions for rutherford backscattering analysis, Nucl. Instrum. Methods 157 (1978) 213-221.

[768] J.P. Stoquert, G. Guillaume, M. Hage-Ali, J.J. Grob, C. Ganter, P. Siffert, Determination of concentration profiles by elastic recoil detection with a $\triangle \mathrm{E}-\mathrm{E}$ gas telescope and high energy incident heavy ions, Nucl. Instrum. Methods Phys. Res. B 44 (1989) 184-194.

[769] W. Assmann, P. Hartung, H. Huber, P. Staat, H. Steffens, C. Steinhausen, Setup for materials analysis with heavy ion beams at the Munich MP tandem, Nucl. Instrum. Methods Phys. Res. B 85 (1994) 726-731.

[770] E. Szilágyi, Energy spread in ion beam analysis, Nucl. Instrum. Methods Phys. Res. B 161-163 (2000) 37-47.

[771] E. Szilágyi, F. Pászti, V. Quillet, F. Abel, Optimization of the depth resolution in ERDA of $\mathrm{H}$ using ${ }^{12} \mathrm{C}$ ions, Nucl. Instrum. Methods Phys. Res. B 85 (1994) 63-67.

[772] F. Pászti, E. Szilágyi, E. Kótai, Optimization of the depth resolution in elastic recoil detection, Nucl. Instrum. Methods Phys. Res. B 54 (1991) 507-512.

[773] J.P. Thomas, M. Fallavier, A. Ziani, Light elements depth-profiling using time-of-flight and energy detection of recoils, Nucl. Instrum. Methods Phys. Res. B 15 (1986) 443-452.

[774] S.C. Gujrathi, S. Bultena, Depth profiling of hydrogen using the high efficiency ERD-TOF technique, Nucl. Instrum. Methods Phys. Res. B 64 (1992) 789-795.

[775] W.M.A. Bik, C.T.A.M. de Laat, F.H.P.M. Habraken, On the use of a $\Delta E-E$ telescope in elastic recoil detection, Nucl. Instrum. Methods Phys. Res. B 64 (1992) 832-835.

[776] R.G. Elliman, H. Timmers, T.R. Ophel, T.D.M. Weijers, L.S. Wielunski, G.L. Harding, Simultaneous hydrogen detection with an ERD gas ionization detector, Nucl. Instrum. Methods Phys. Res. B 161 (2000) 231-234.

[777] V.M. Prozesky, C.L. Churms, J.V. Pilcher, K.A. Springhorn, R. Behrisch, ERDA measurement of hydrogen isotopes with a Delta-E-E telescope, Nucl. Instrum. Methods Phys. Res. B 84 (1994) 373-379.

[778] J.F. Browning, R.A. Langley, B.L. Doyle, J.C. Banks, W.R. Wampler, High accuracy, high energy He-ERD analysis of H, D and T, Nucl. Instrum. Methods Phys. Res. B 161-163 (2000) 211-215. 
[779] G.G. Ross, B. Terreault, G. Gobeil, G. Abel, C. Boucher, G. Veilleux, Inexpensive, quantitative hydrogen depth-profiling for surface probes, J. Nucl. Mater. 128-129 (1984) 730-733.

[780] G.G. Ross, L. Leblanc, Depth-profiling of hydrogen and helium isotopes by means of the ERD E × B technique, Nucl. Instrum. Methods Phys. Res. B 62 (1992) 484-492.

[781] G.G. Ross, L. Leblanc, B. Terreault, J.F. Pageau, P.A. Gollier, Nuclear microanalysis by means of $350 \mathrm{keV}$ Van de Graaf accelerator, Nucl. Instrum. Methods Phys. Res. B 66 (1992) $17-22$.

[782] T. Enders, M. Rilli, H.D. Carstanjen, A high-resolution electrostatic spectrometer for the investigation of near-surface layers in solids by high-resolution Rutherford backscattering with MeV ions, Nucl. Instrum. Methods Phys. Res. B 64 (1992) 817-824.

[783] W.A. Lanford, B. Anderberg, H. Enge, B. Hjörvarsson, Compact broad range magnetic spectrometer for use in ion beam analysis, Nucl. Instrum. Methods Phys. Res. B 136-138 (1998) 1177-1182.

[784] C.R. Gossett, Use of a magnetic spectrometer to profile light elements by elastic recoil detection, Nucl. Instrum. Methods Phys. Res. B 15 (1986) 481-485.

[785] W.A. Lanford, S. Bedell, S. Amadon, A. Haberl, W. Skala, B. Hjörvarsson, Characteristics of Albany's compact high resolution magnetic spectrometer, Nucl. Instrum. Methods Phys.

Res. B 161 (2000) 202-206. 